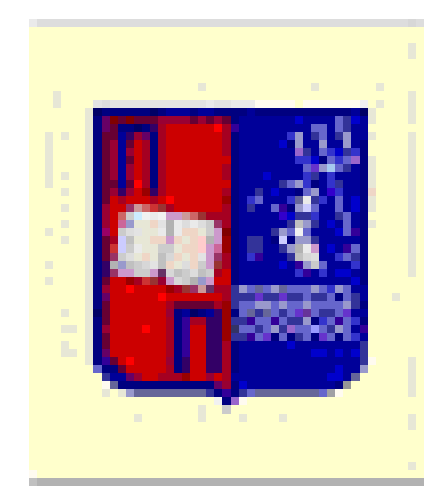

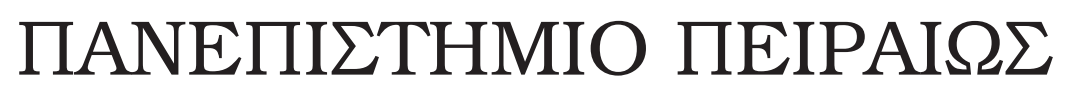

\author{
ТМНМА ПАНРОФОРІКН $\Sigma$
}

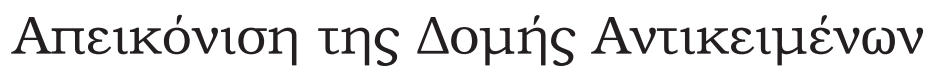

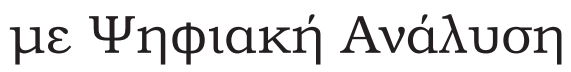

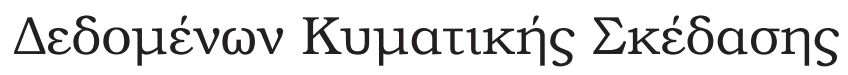

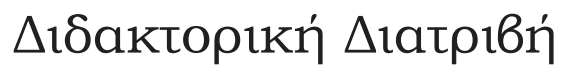 \\ Kwvotavtivou Г. Гkuptń
}

ПEIPAIA 2002 



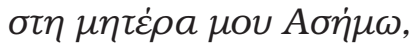

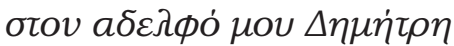

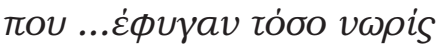

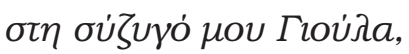

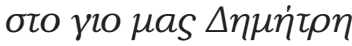

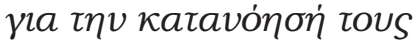





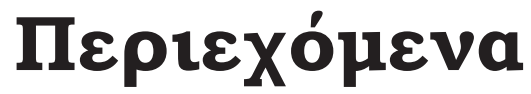

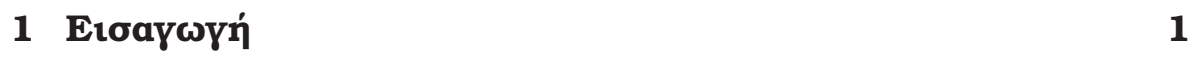

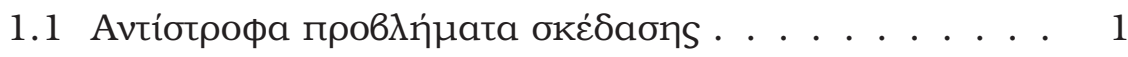

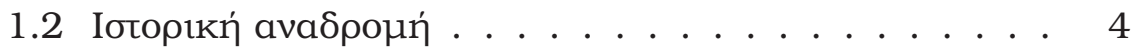

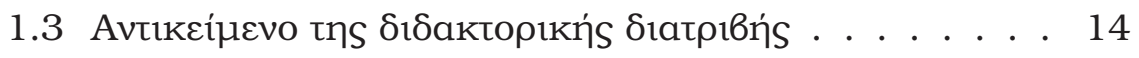

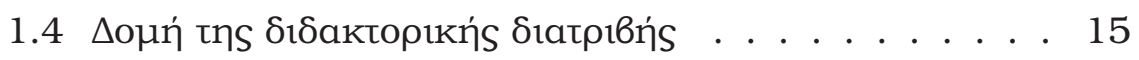

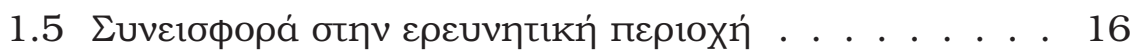

1.6 Evxaprotiss . . . . . . . . . . . . . . . . 16

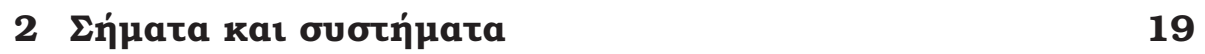

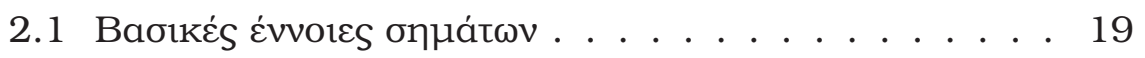

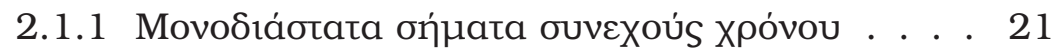

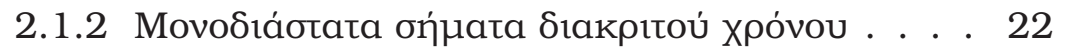

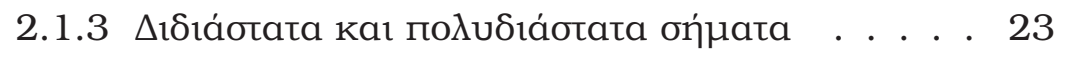

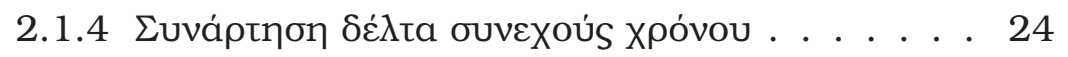

$2.1 .5 \Delta \dot{\lambda} \lambda$ ta tou Kronecker . . . . . . . . . . . . . 28

2.2 Metaбхпналıонoí Fourier . . . . . . . . . . 30

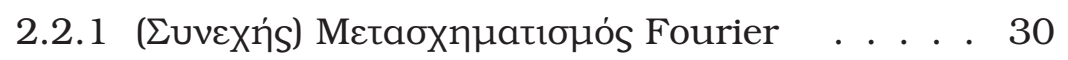

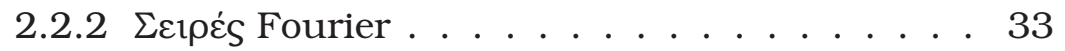




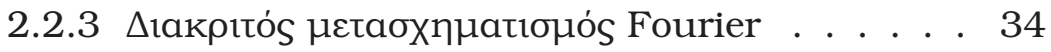

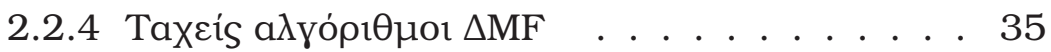

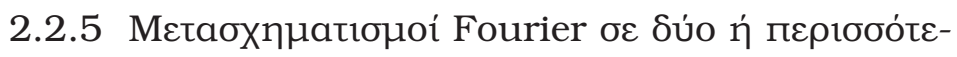

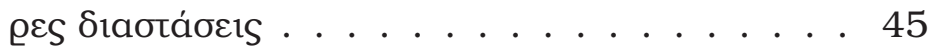

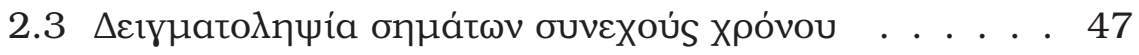

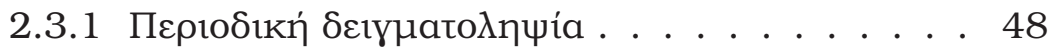

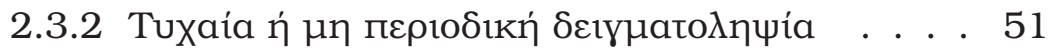

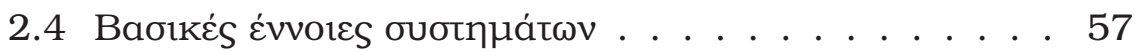

2.4.1 H évvora tou ouotńfuatos . . . . . . . . . . 57

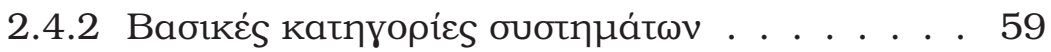

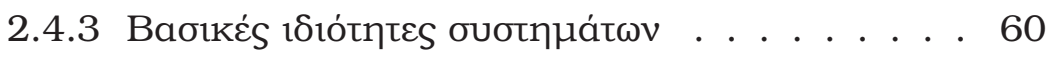

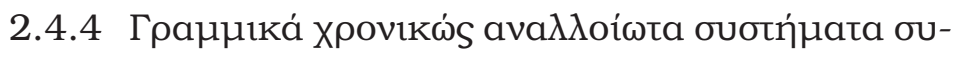

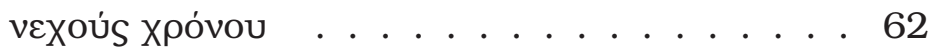

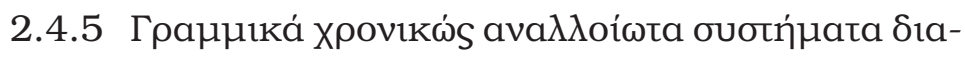
xpltoú xpóvou . . . . . . . . . . 66

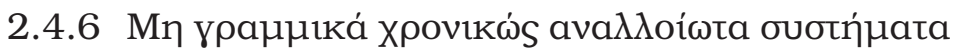
Volterra ............... 66

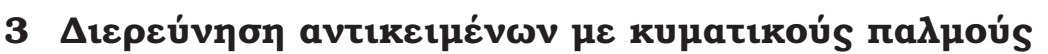

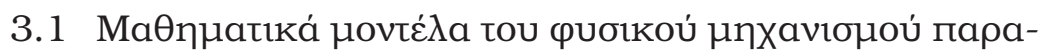

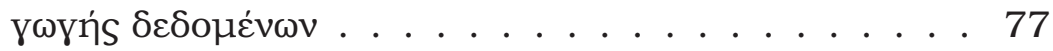

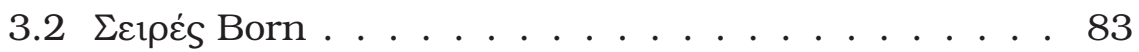

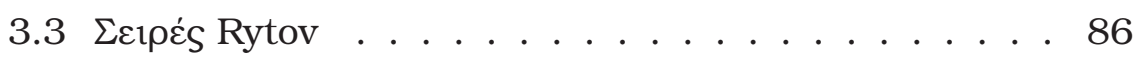

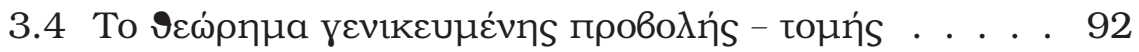

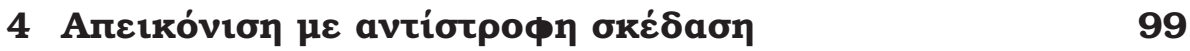


4.1 Eıбаушүท́ . . . . . . . . . . . . . . . . . . 99

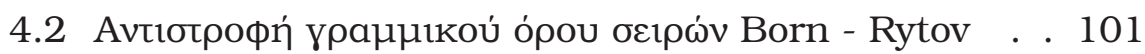

4.3 Avtıброфи́ бєıрás Born . . . . . . . . . . . . . . 103

4.4 Avtıброфи́ бєıрás Rytov . . . . . . . . . . . . . 110

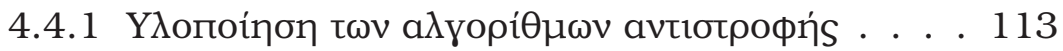

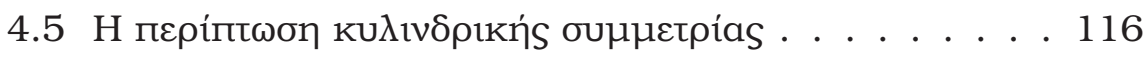

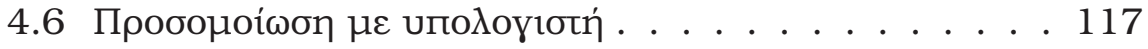

4.6.1 A'пробоноíоп . . . . . . . . . . . . 118

4.6 .2 B' пробоноí

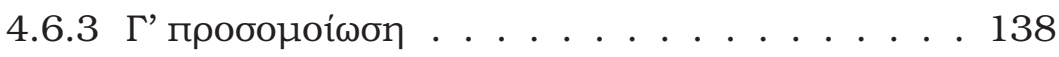

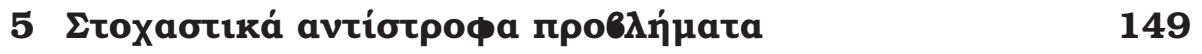

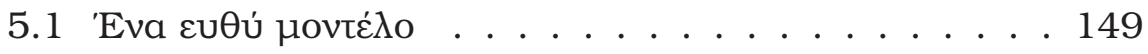

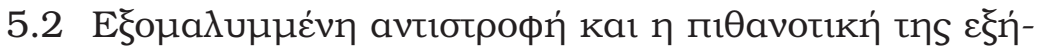

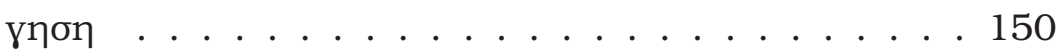

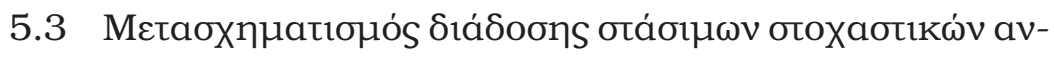

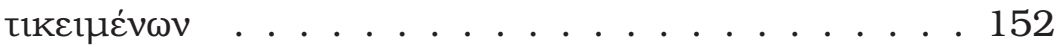

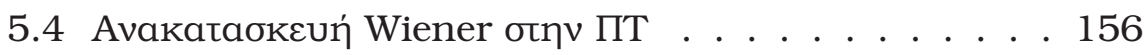

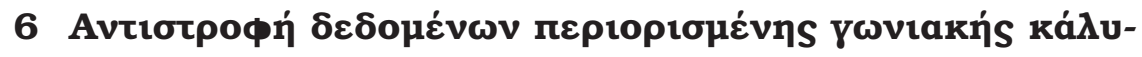
$\Psi \eta S$

6.1 Eıбаушүи́ . . . . . . . . . . . . . . . . 165

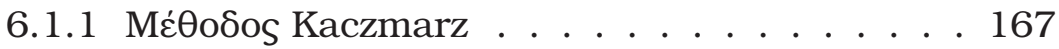

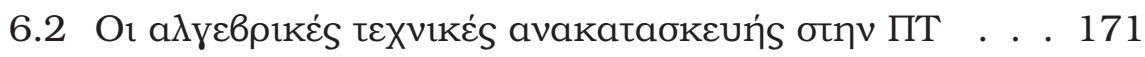

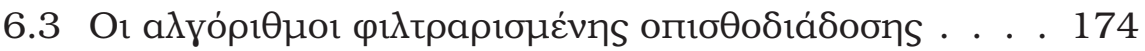




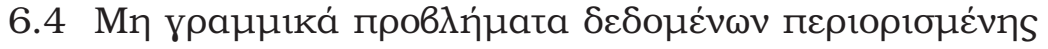

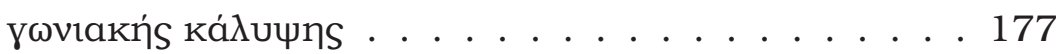

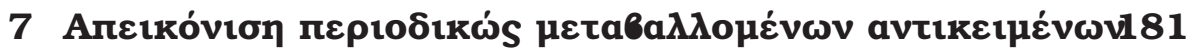

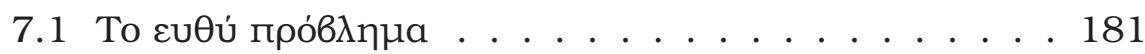

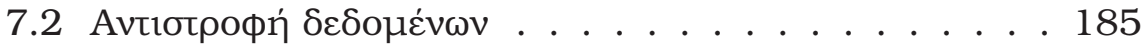

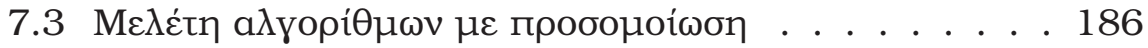

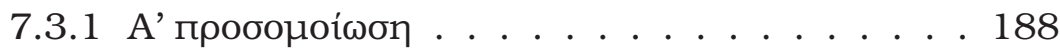

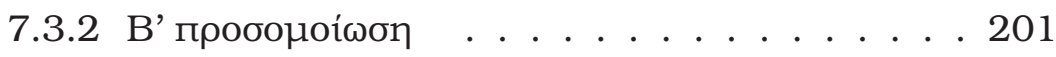

$7.3 .3 \Gamma^{\prime}$ проооноí⿴囗п . . . . . . . . . . . 207

8 Erí̉oyos 213 


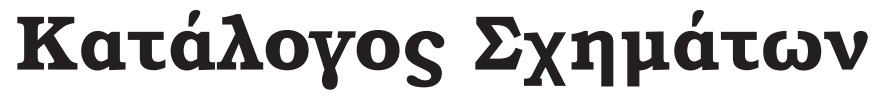

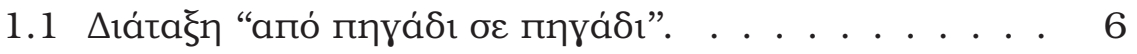

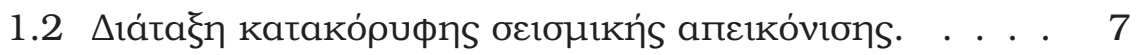

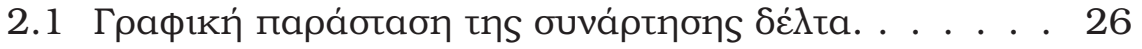

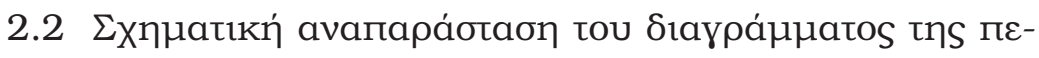

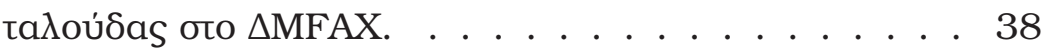

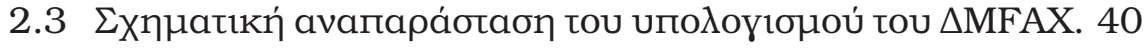

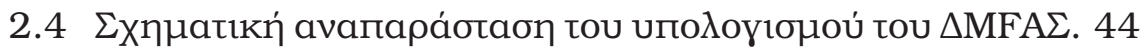

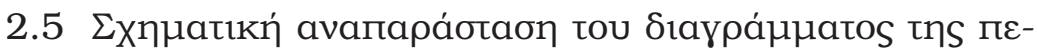

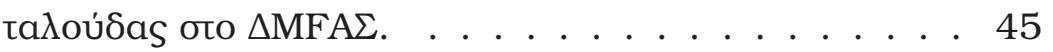

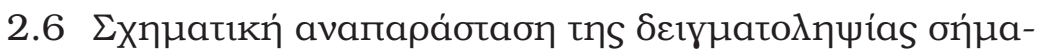
tos ouvexoús Xoóvou. . . . . . . . . . . . . . . . 49

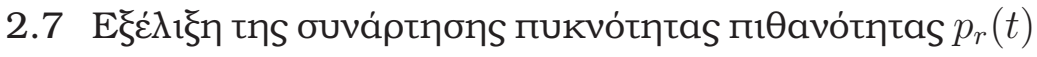

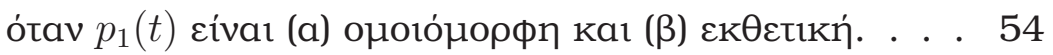

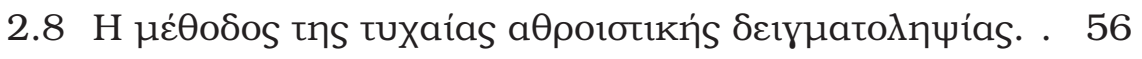

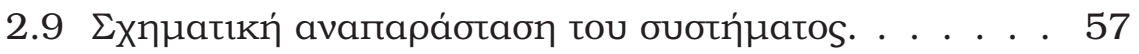

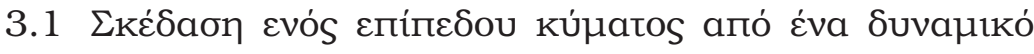

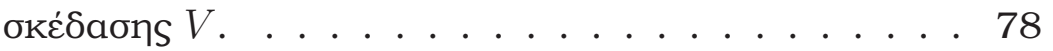




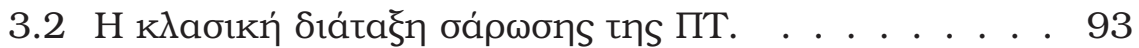

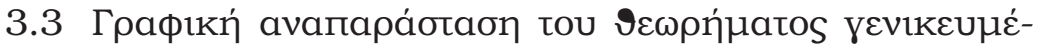

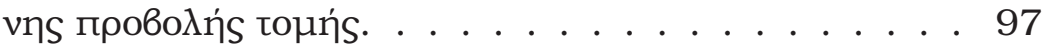

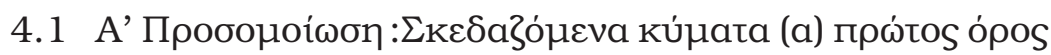

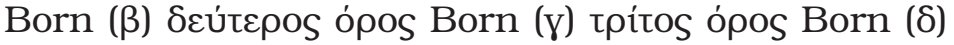

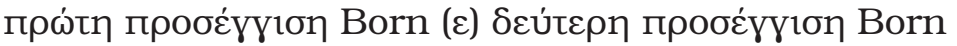

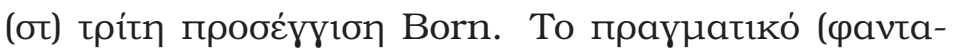

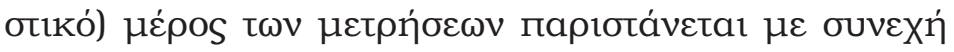

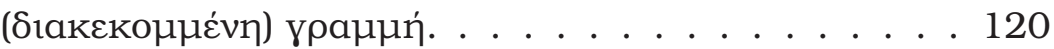

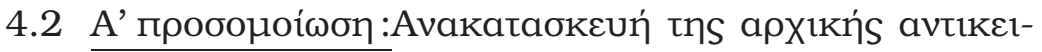

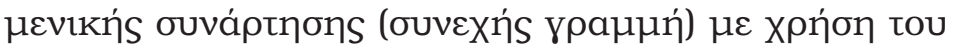

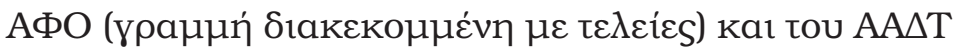

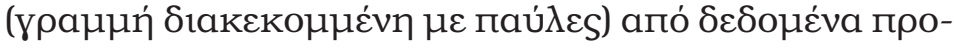

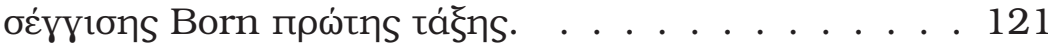

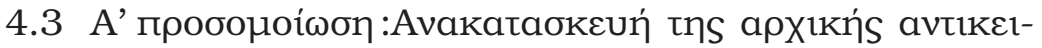

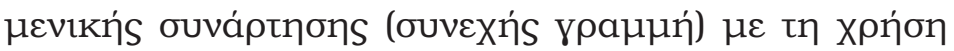

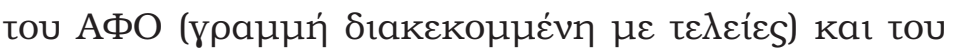

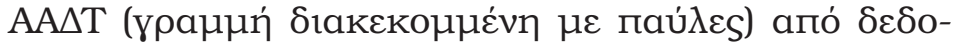

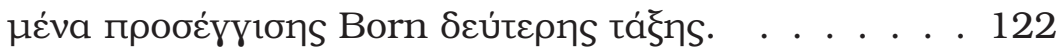

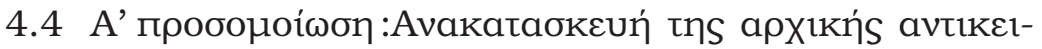

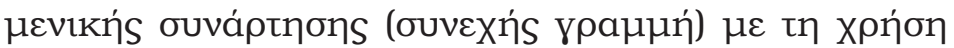

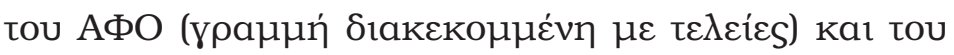

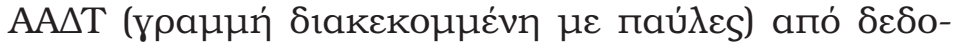

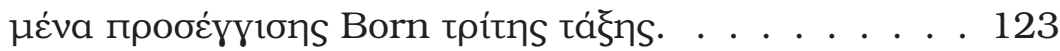




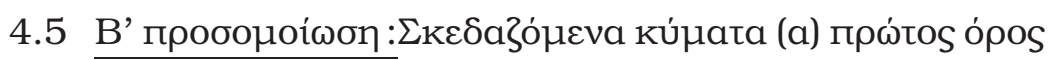

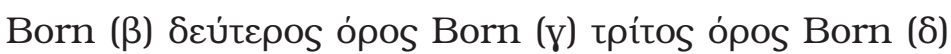

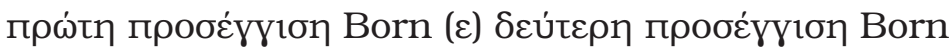

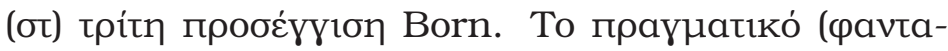

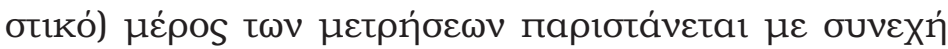

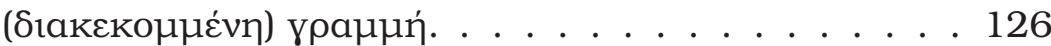

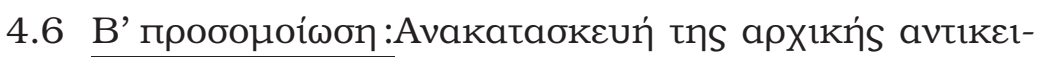

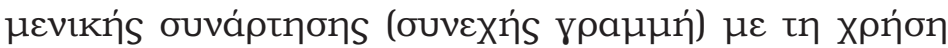

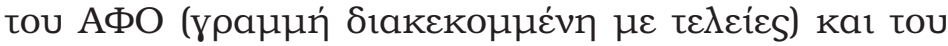

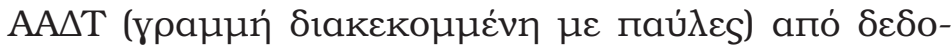

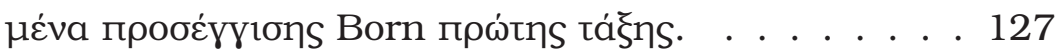

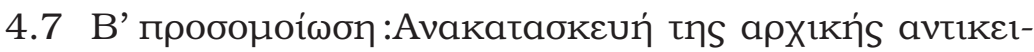

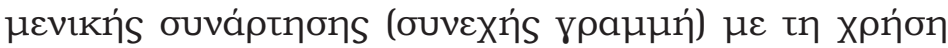

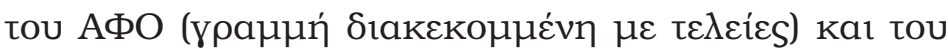

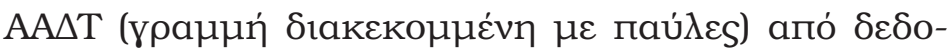

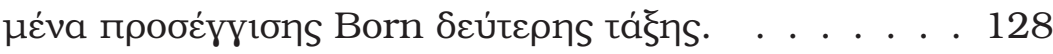

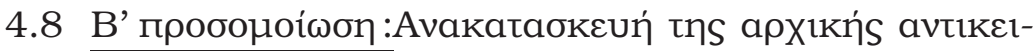

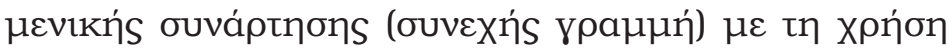

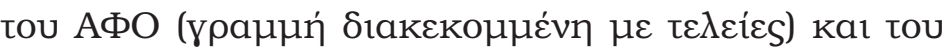

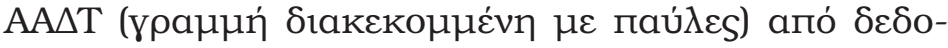

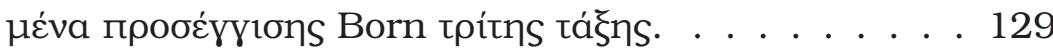




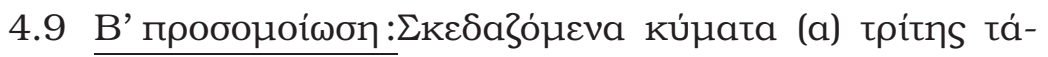

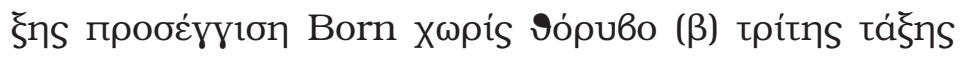

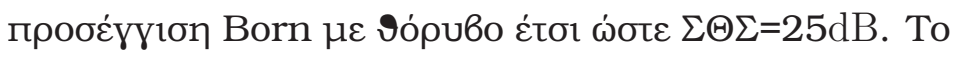

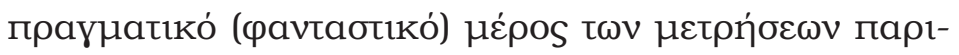

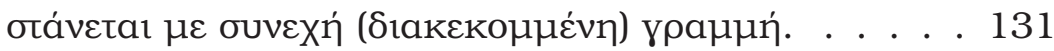

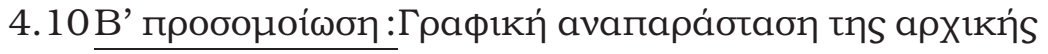

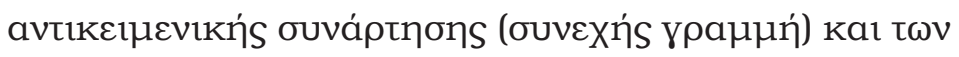

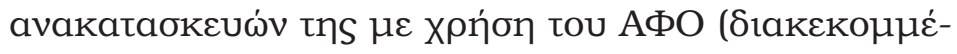

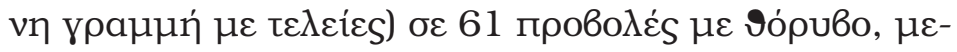

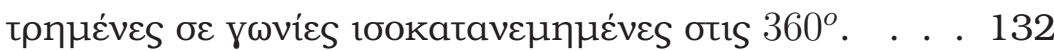

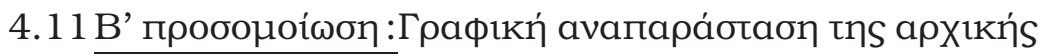

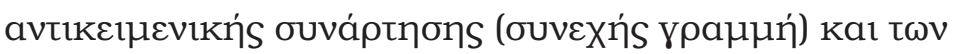

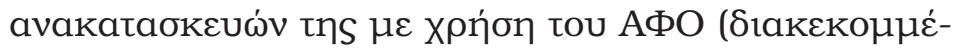

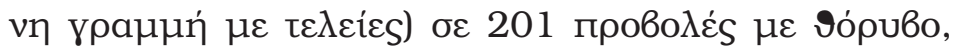

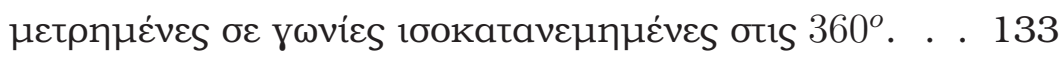

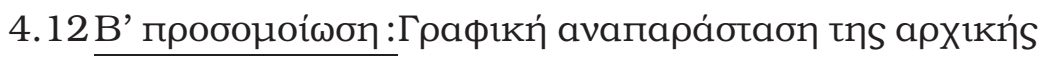

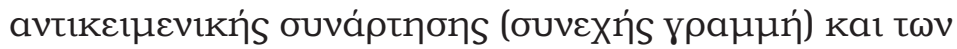

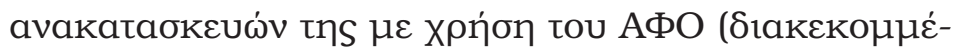

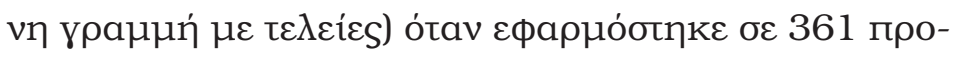

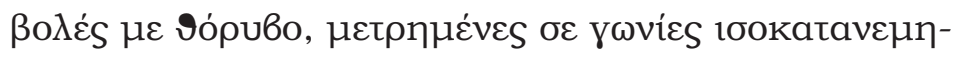

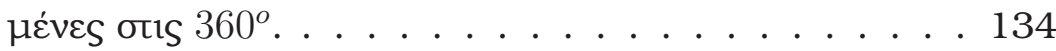




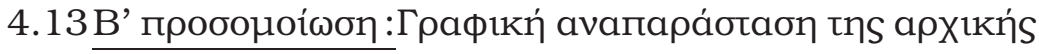

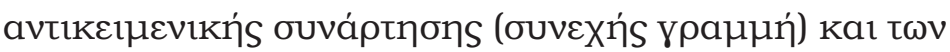

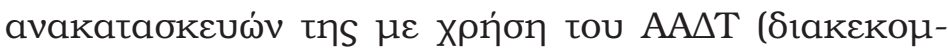

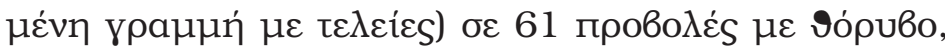

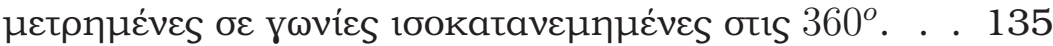

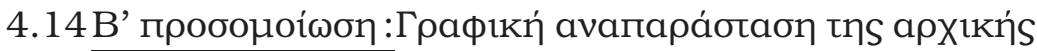

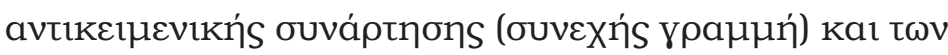

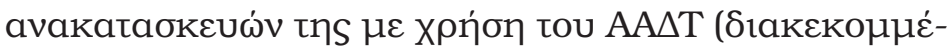

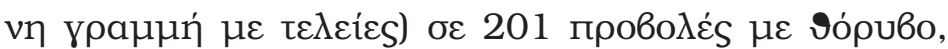

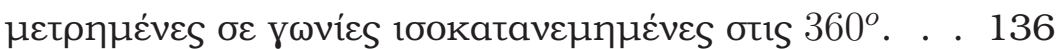

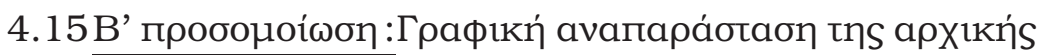

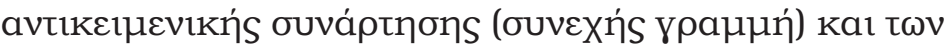

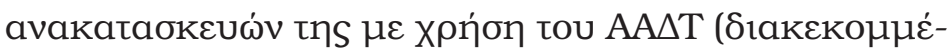

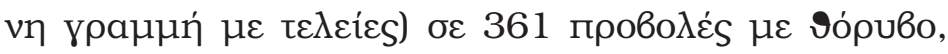

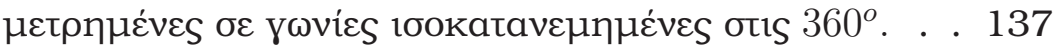

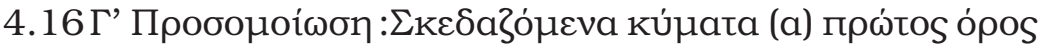

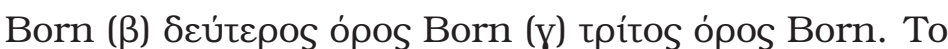

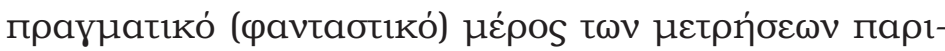

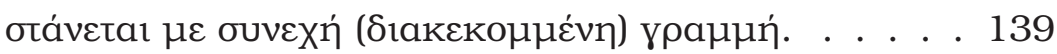

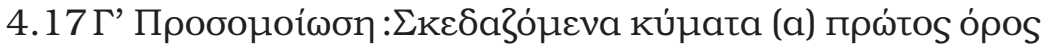

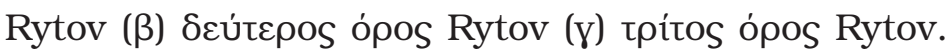

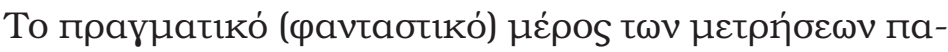

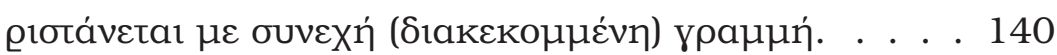




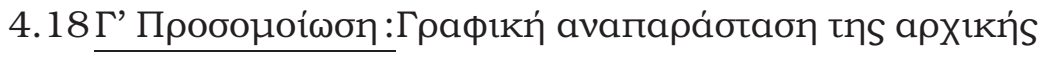

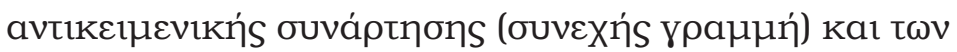

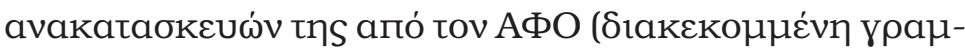

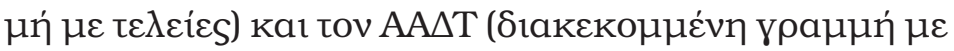

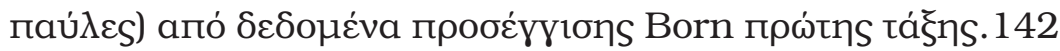

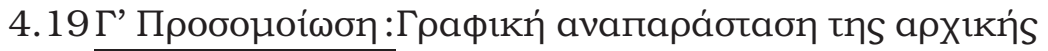

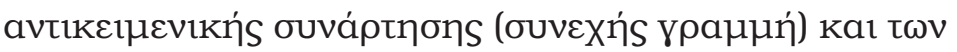

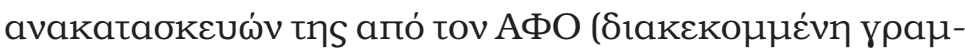

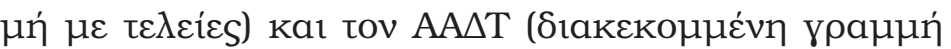

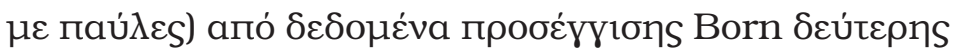
táłns. . . . . . . . . . . . . . . . . . . 143

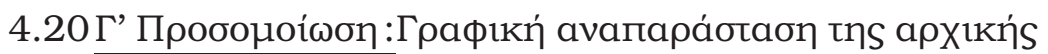

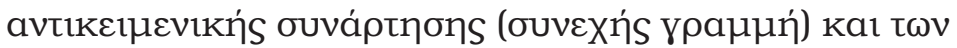

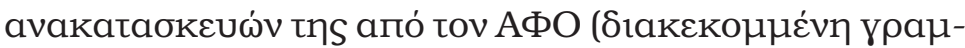

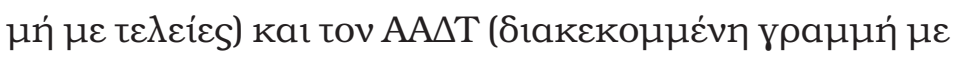

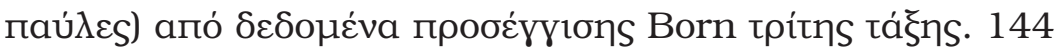

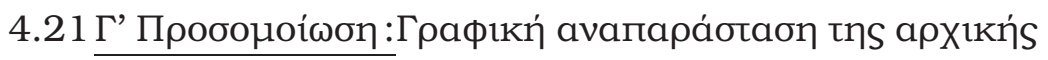

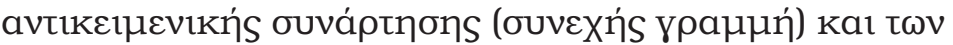

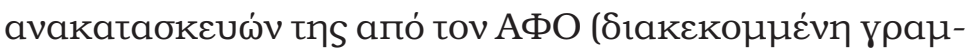

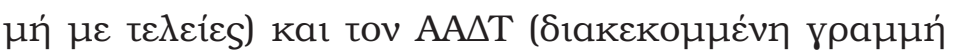

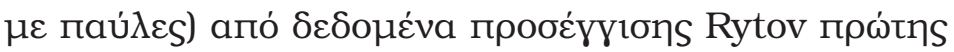
táłns. . . . . . . . . . . . . . . . . . . . . 145 


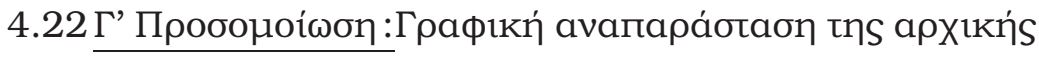

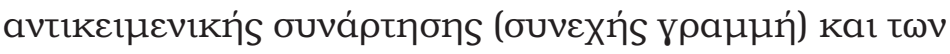

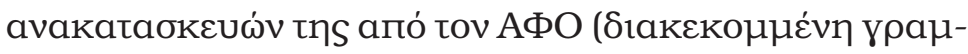

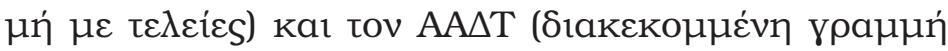

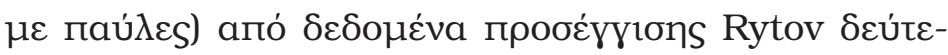

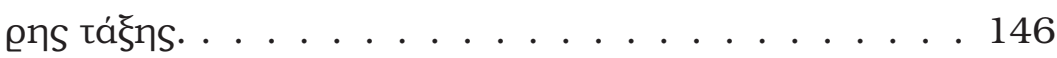

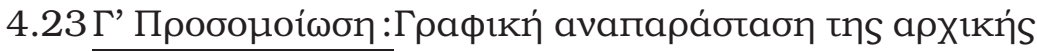

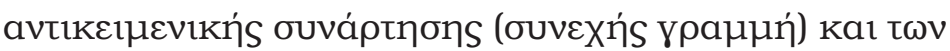

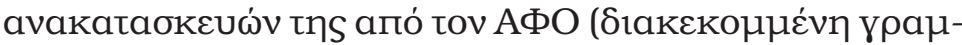

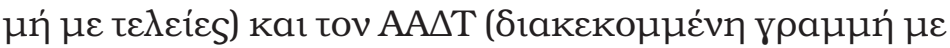

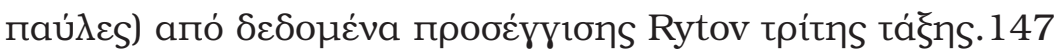

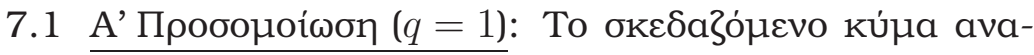

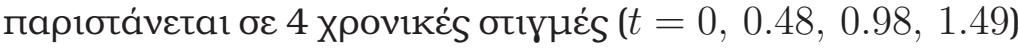

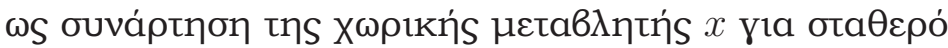
$y=0 \ldots \ldots \ldots \ldots \ldots$

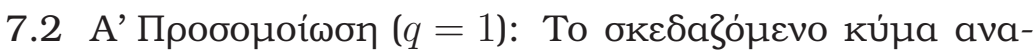

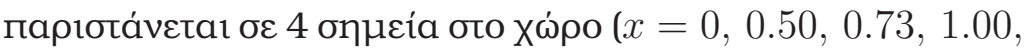

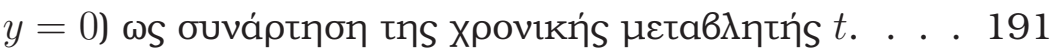

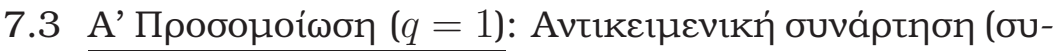

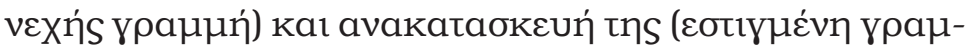

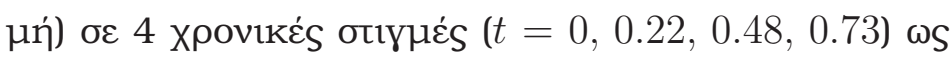

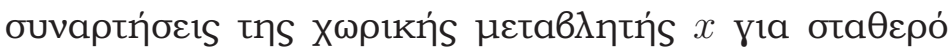
$y=0 \ldots \ldots \ldots \ldots \ldots 2 \ldots \ldots \ldots$ 


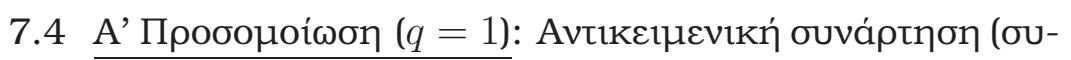

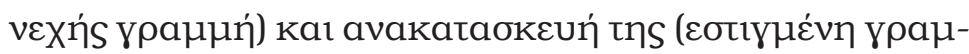

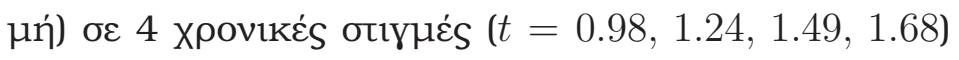

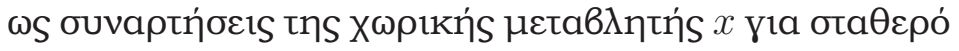
$y=0 . \ldots \ldots \ldots \ldots \ldots \ldots$

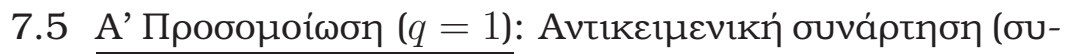

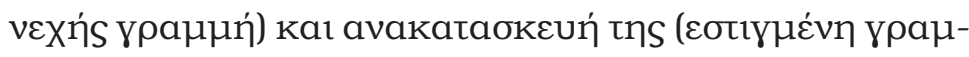

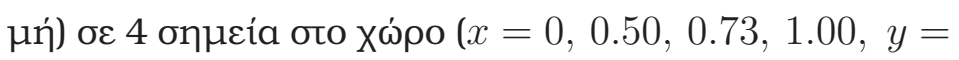

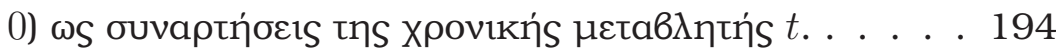

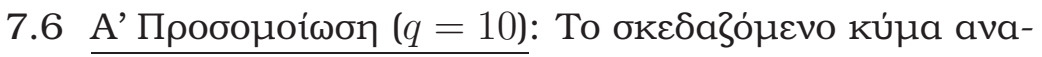

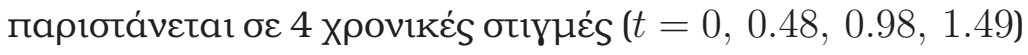

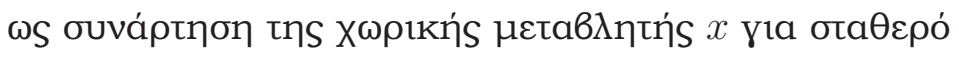
$y=0 \ldots \ldots \ldots \ldots \ldots \ldots$

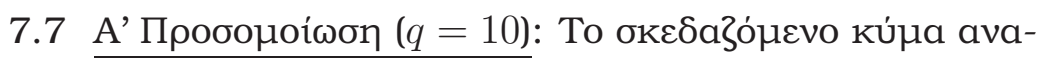

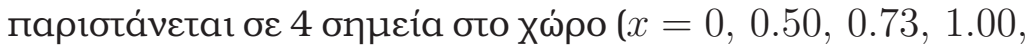

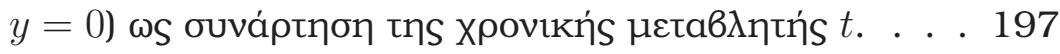

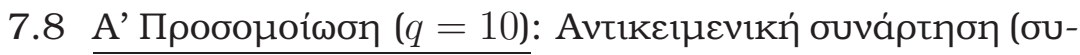

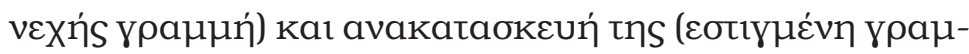

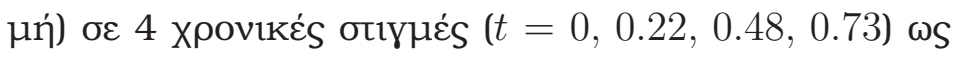

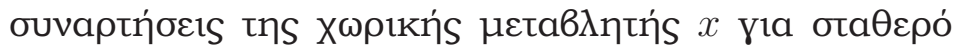
$y=0 \ldots \ldots \ldots \ldots \ldots \ldots \ldots$ 


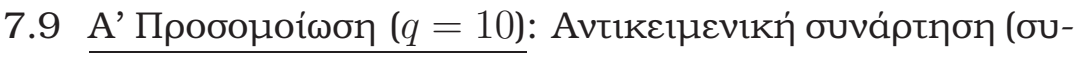

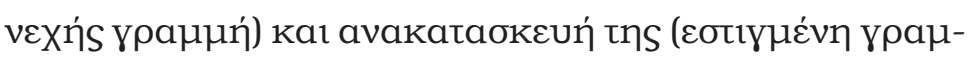

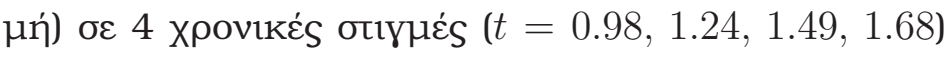

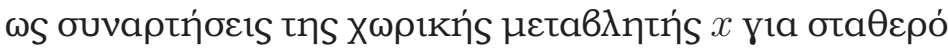
$y=0 \ldots \ldots \ldots \ldots \ldots \ldots$

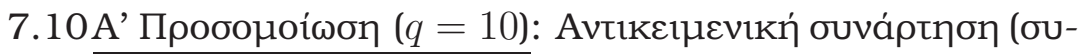

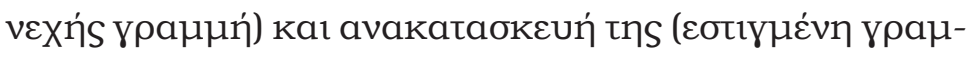

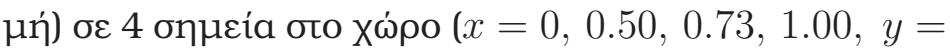

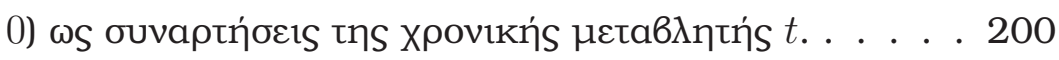

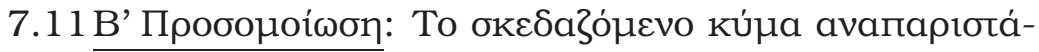

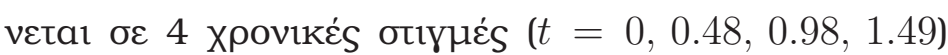

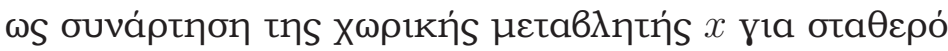

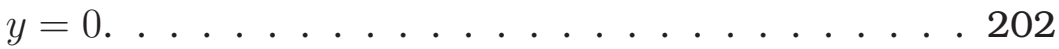

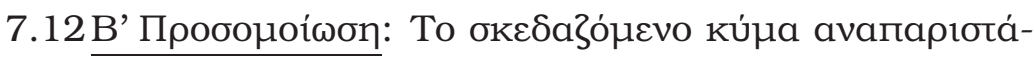

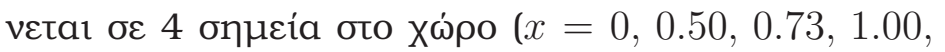

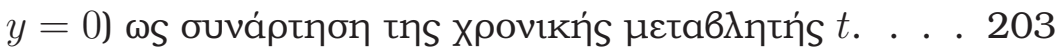

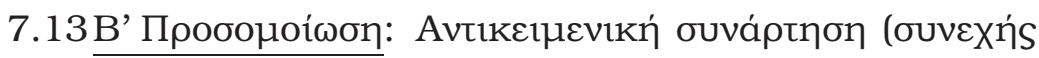

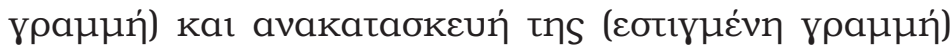

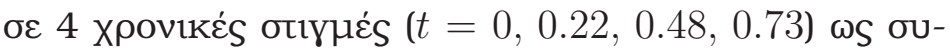

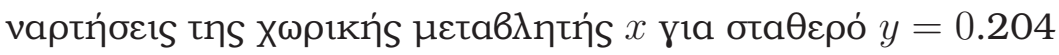

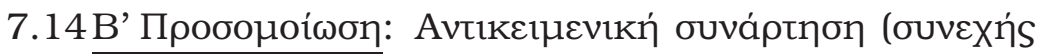

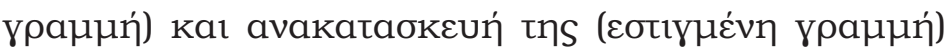

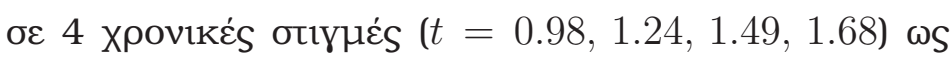

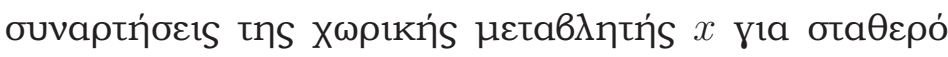
$y=0 \ldots \ldots \ldots \ldots \ldots \ldots \ldots$ 


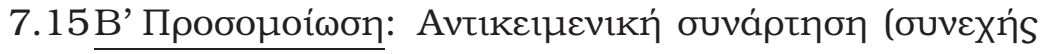

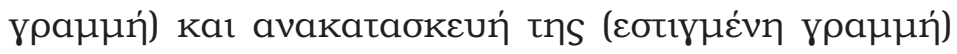

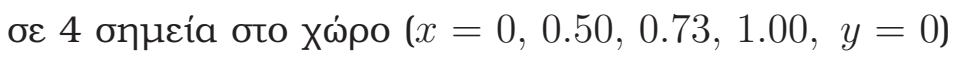

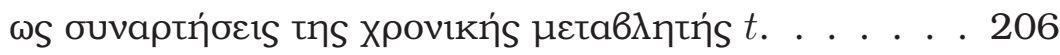

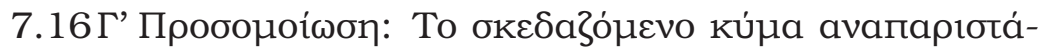

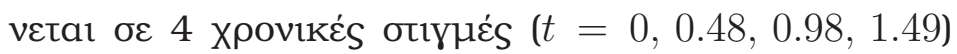

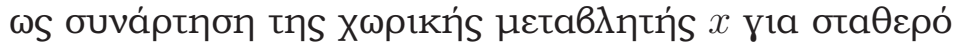
$y=0 \ldots \ldots \ldots \ldots \ldots \ldots \ldots$

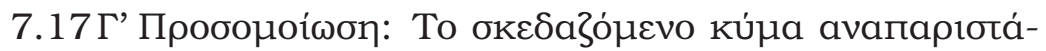

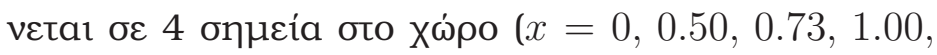

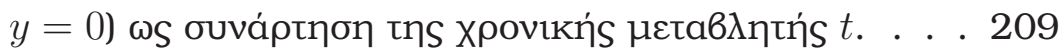

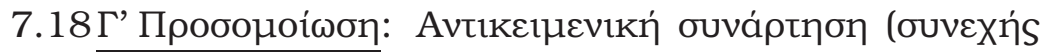

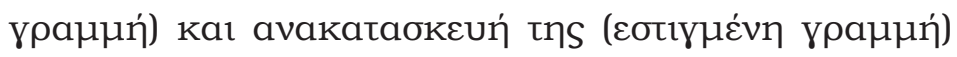

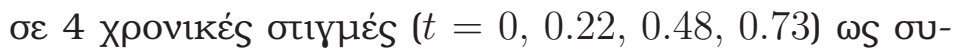

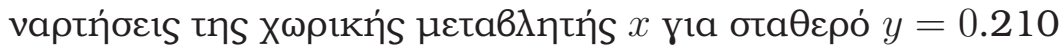

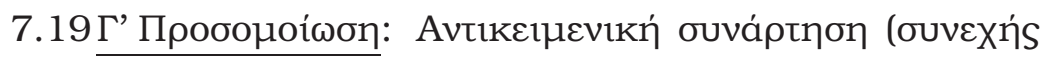

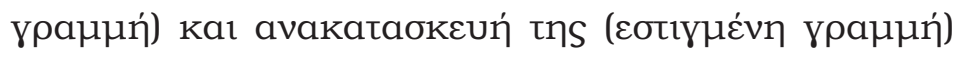

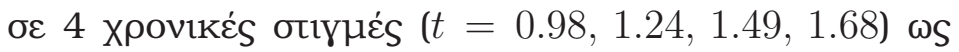

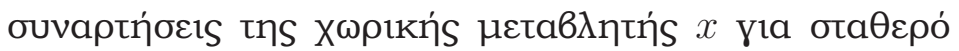
$y=0 \ldots \ldots \ldots \ldots \ldots \ldots \ldots \ldots$

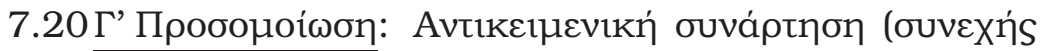

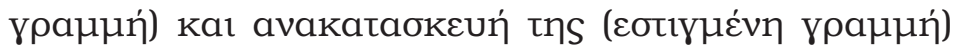

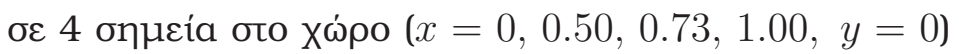

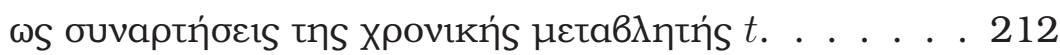


ПINAKA $\Sigma$ AKPONYMISN

\begin{tabular}{|c|c|}
\hline $\mathrm{E} \Pi \Sigma$ & 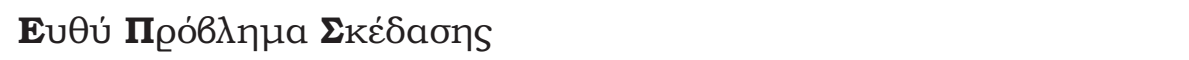 \\
\hline $\mathrm{A} \Pi \Sigma$ & 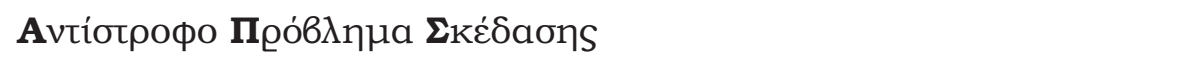 \\
\hline$\Pi \mathrm{T}$ & 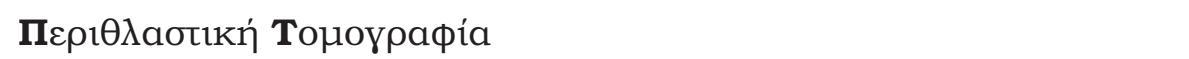 \\
\hline$\Theta Г П Т ~$ & 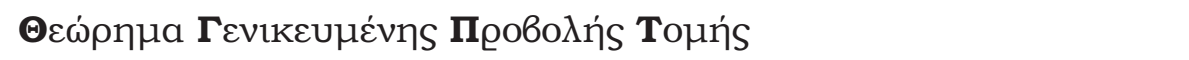 \\
\hline TMF & Taxús Metaoxnuatıouós Fourier \\
\hline АФО & 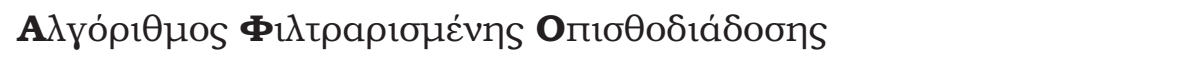 \\
\hline MF & 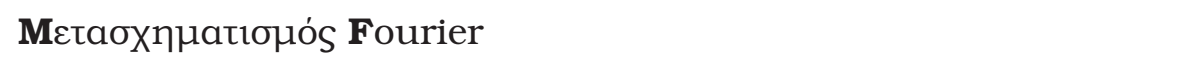 \\
\hline $\mathrm{AMF}$ & Avtiópoфos Mctaoxnuatıouós Fourier \\
\hline$\Delta \mathrm{MF}$ & 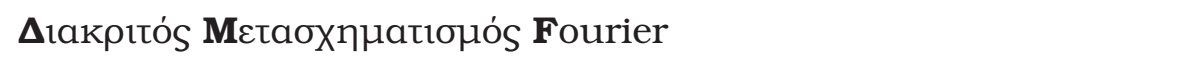 \\
\hline $\mathrm{A} \triangle \mathrm{MF}$ & 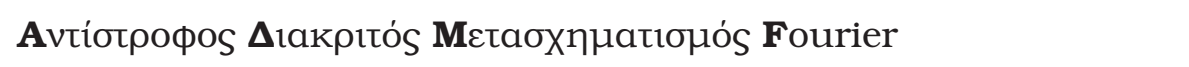 \\
\hline$\triangle \mathrm{MFAX}$ & 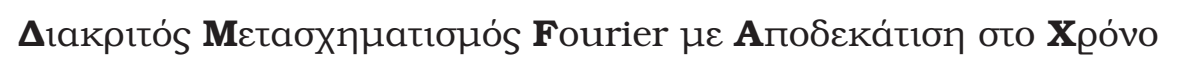 \\
\hline$\triangle \mathrm{MFA} \Sigma$ & 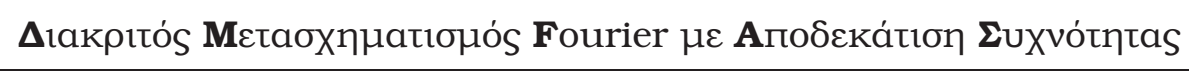 \\
\hline$\Gamma X A \Sigma$ & 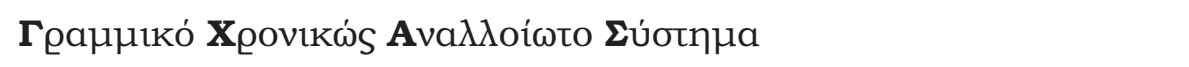 \\
\hline $\mathrm{AA} \triangle \mathrm{T}$ & 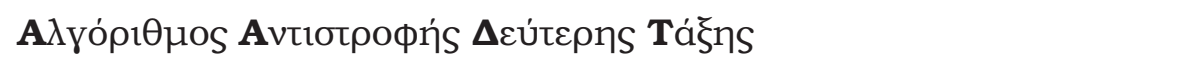 \\
\hline$\Sigma \Theta \Sigma$ & 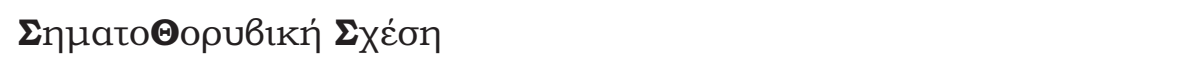 \\
\hline MTP & 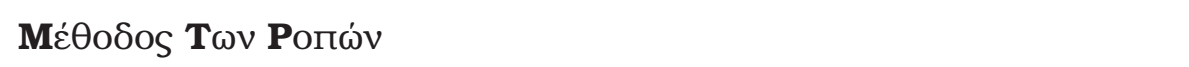 \\
\hline ATA & $\mathbf{A} \lambda y \varepsilon b \rho 1 k \varepsilon \dot{S} \mathbf{T} \varepsilon x v 1 k \varepsilon \dot{S}$ Avakataokeuńs \\
\hline TETA & 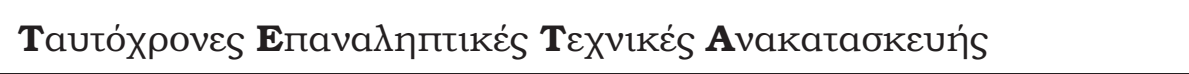 \\
\hline ППГК & 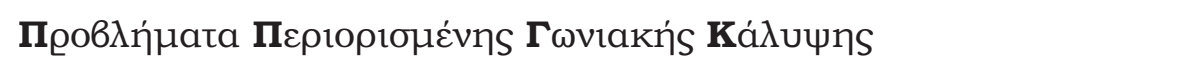 \\
\hline
\end{tabular}


ПINAKA $\Sigma \Sigma \mathrm{YMBO} \Lambda \mathrm{I} \Sigma \mathrm{M} \Omega \mathrm{N}$

\begin{tabular}{|c|c|}
\hline$k$ & 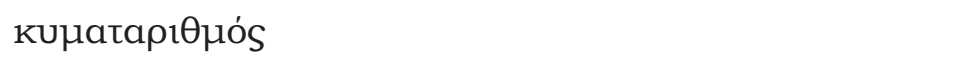 \\
\hline$\lambda$ & 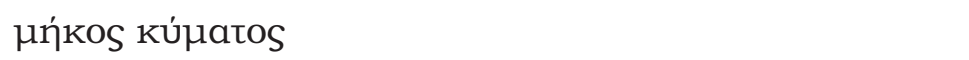 \\
\hline$c_{0}$ & 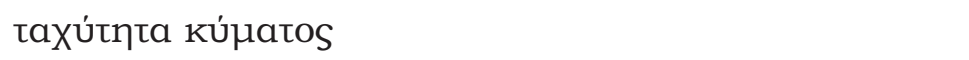 \\
\hline$\hat{u}$ & 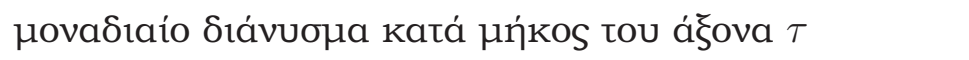 \\
\hline$\hat{v}$ & 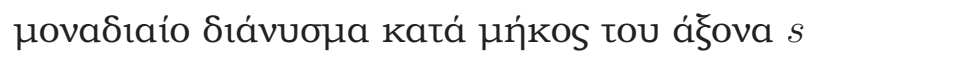 \\
\hline$\theta$ & 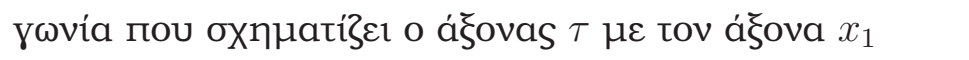 \\
\hline$f$ & 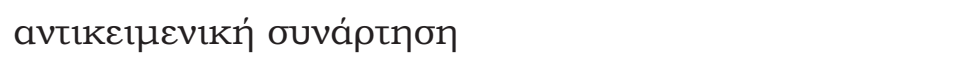 \\
\hline$\tilde{f}$ & 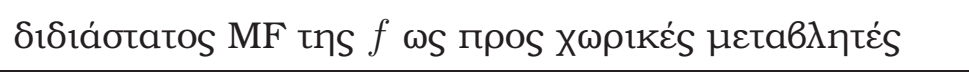 \\
\hline$\hat{f}$ & 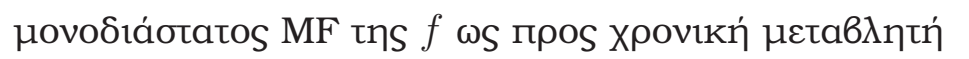 \\
\hline$P_{\theta} f$ & 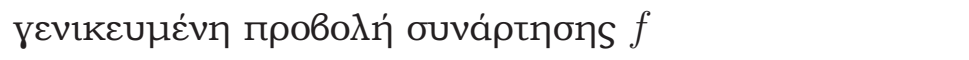 \\
\hline$f_{\text {rec }}$ & 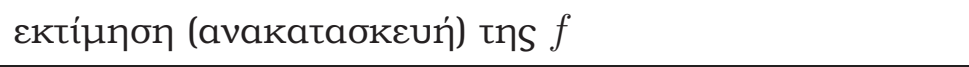 \\
\hline$f_{s}$ & 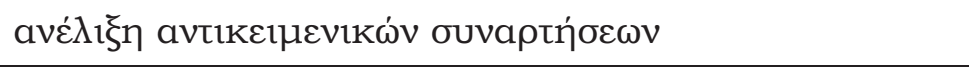 \\
\hline$f_{s, \text { rec }}$ & avakataokeuń tns $f_{s}$ \\
\hline$\psi$ & 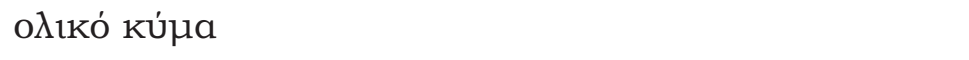 \\
\hline$\psi_{0}$ & проопіптоv кúja \\
\hline$\psi^{s}$ & 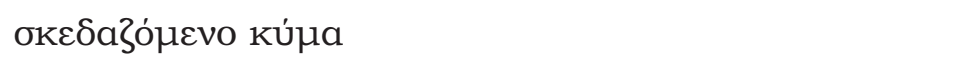 \\
\hline$\psi_{n}$ & $n$-oбtós ópos бeıрás Born \\
\hline$W_{n}$ & n-ootós ópos oєıрás Rytov \\
\hline$B_{\theta}$ & 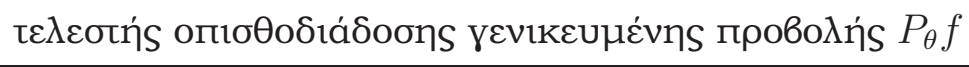 \\
\hline $\mathcal{B}$ & 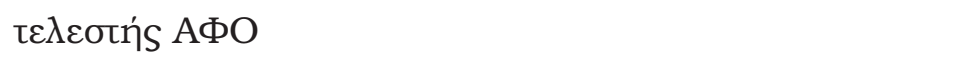 \\
\hline $\mathcal{G}_{n}$ & 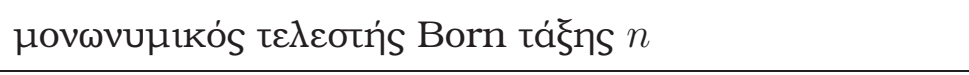 \\
\hline $\mathcal{W}_{n}$ & 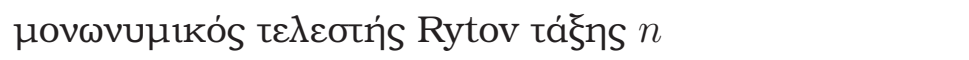 \\
\hline
\end{tabular}

xvii 
xviii 


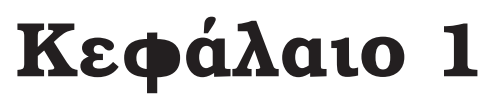

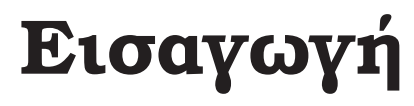

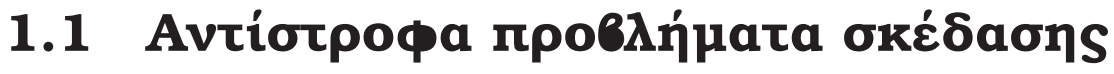

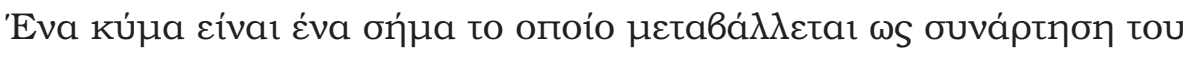
X

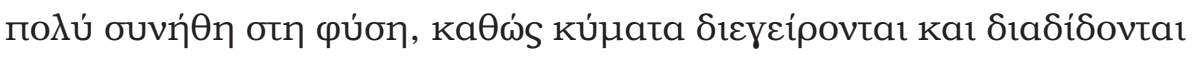

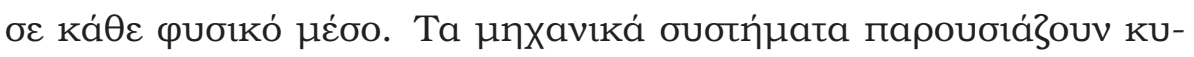

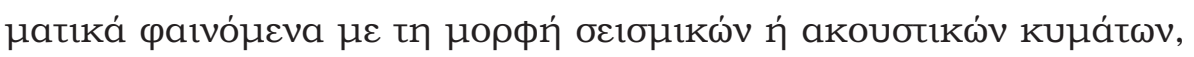

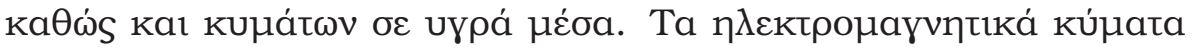

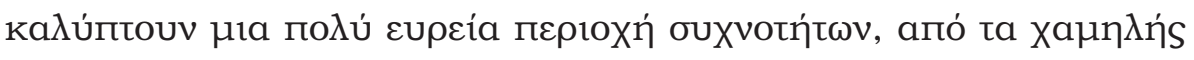

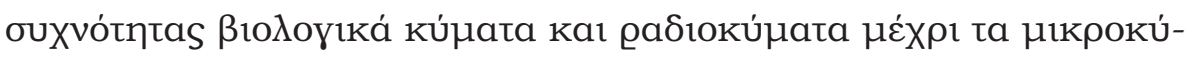

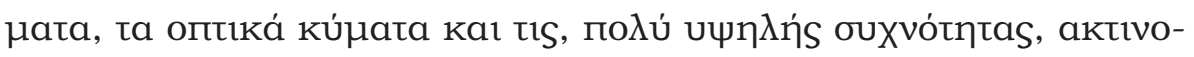

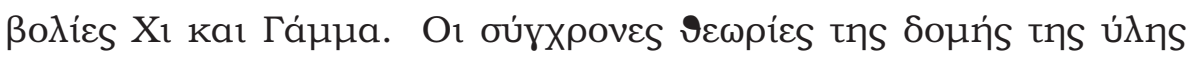

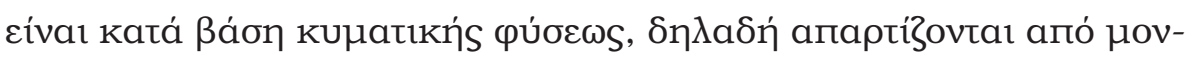

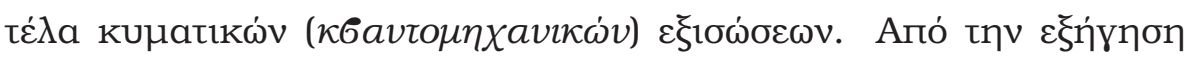

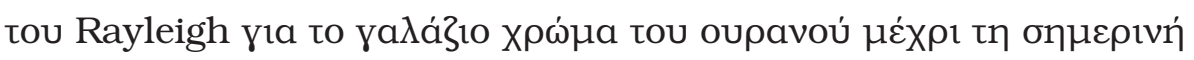




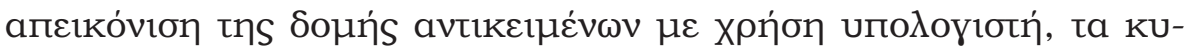

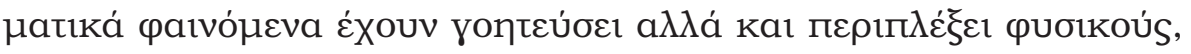

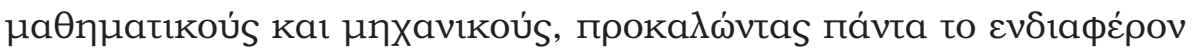
tous.

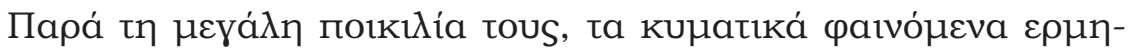

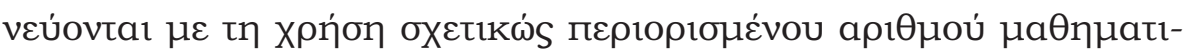

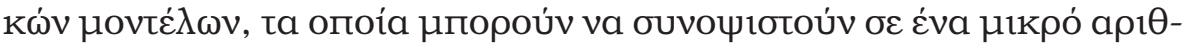

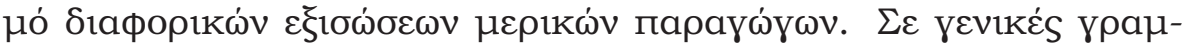

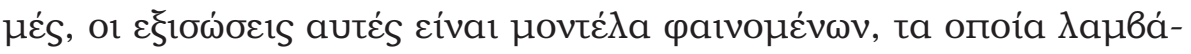

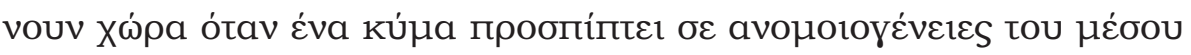

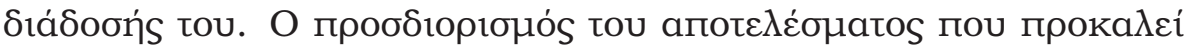

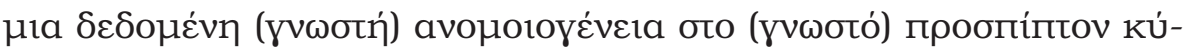

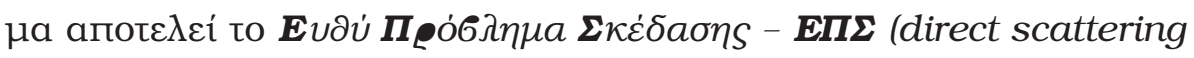

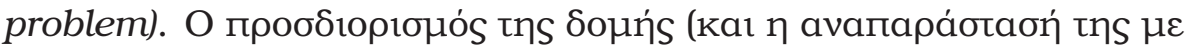

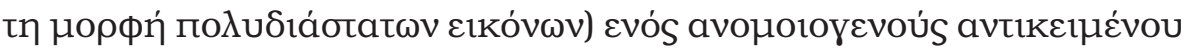

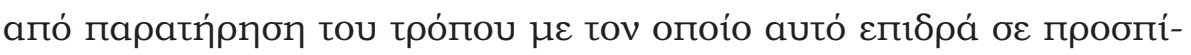

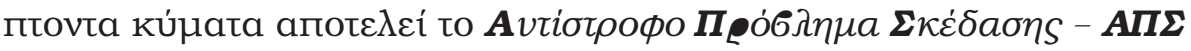

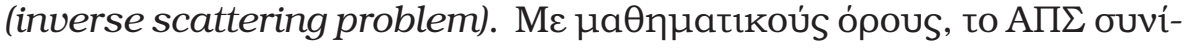

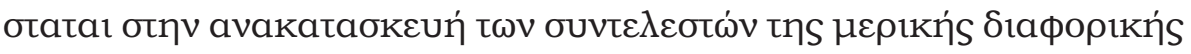

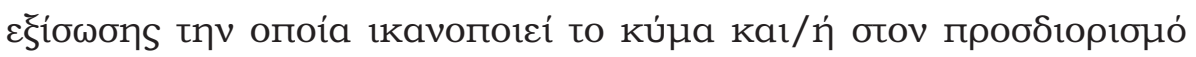

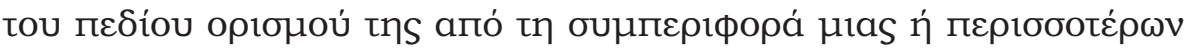

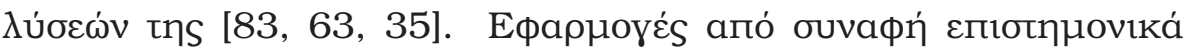

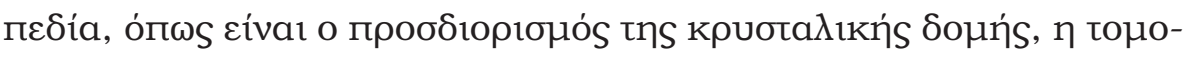

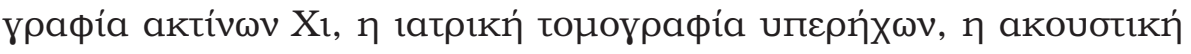

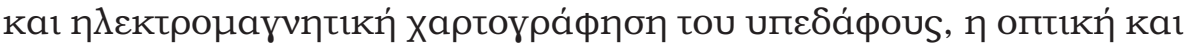




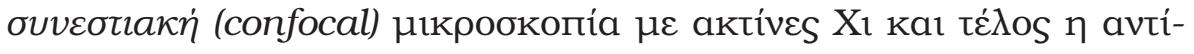

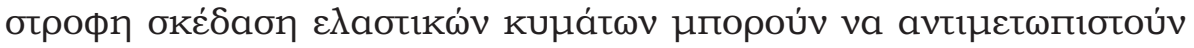

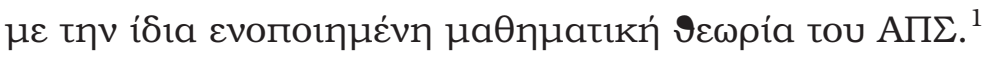

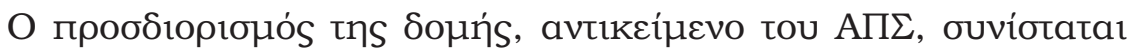

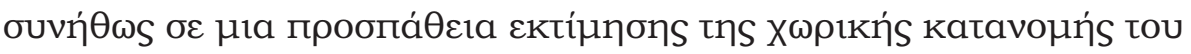

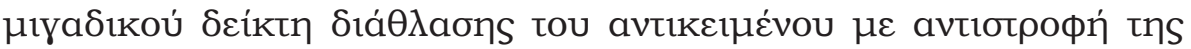

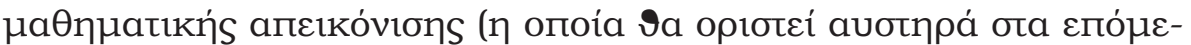

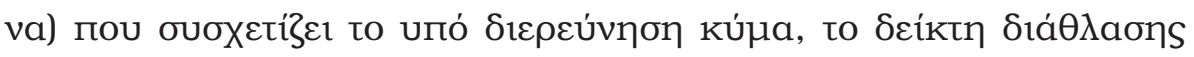

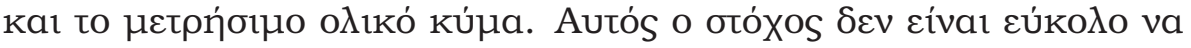

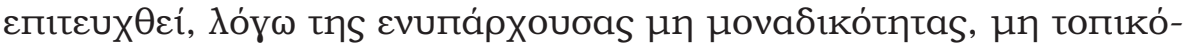

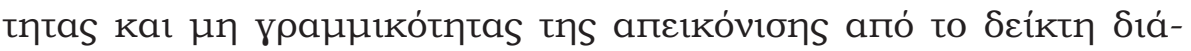

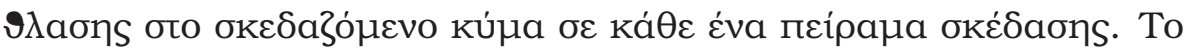

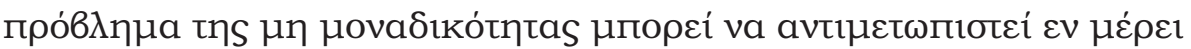

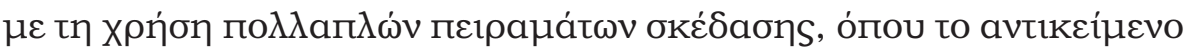

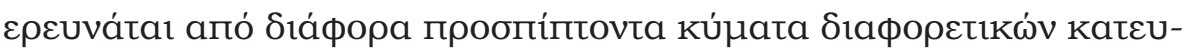

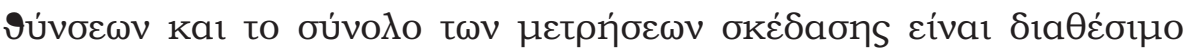

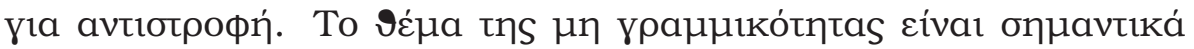

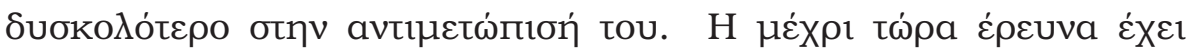

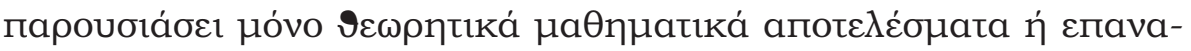

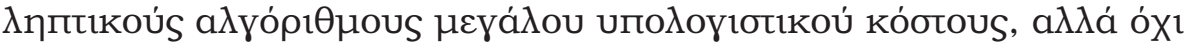

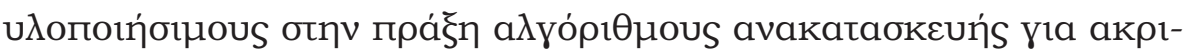

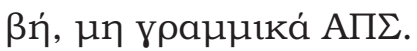

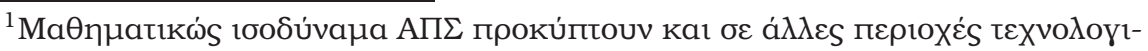

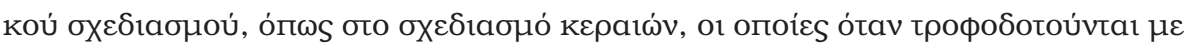

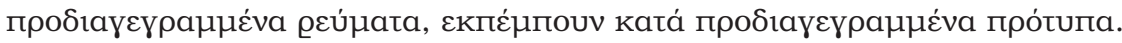




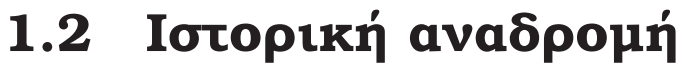

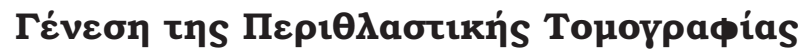

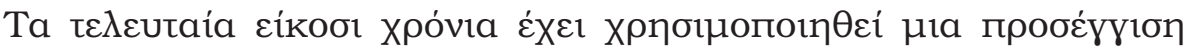

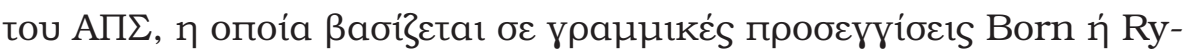

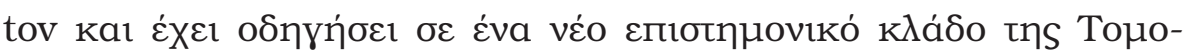

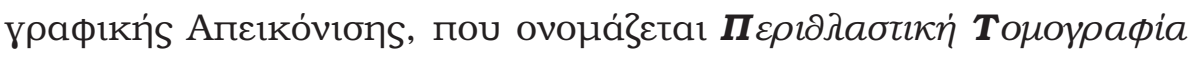

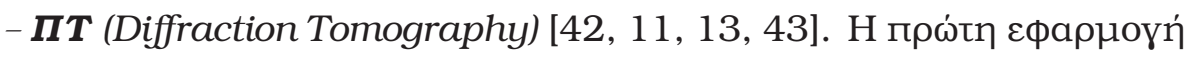

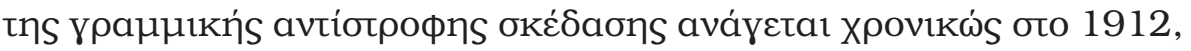

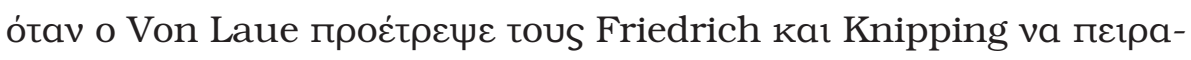

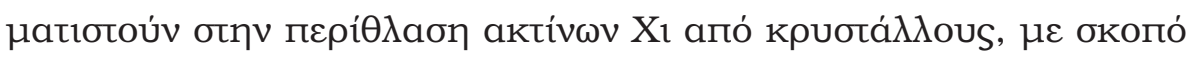

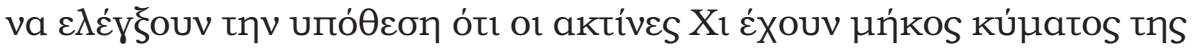

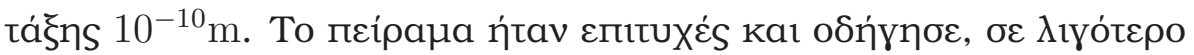

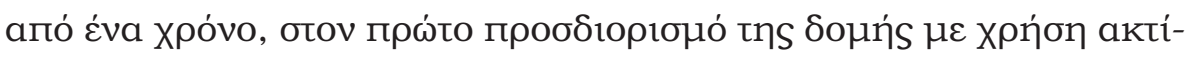

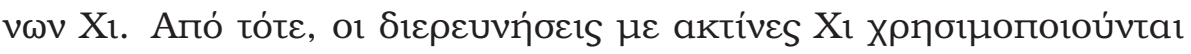

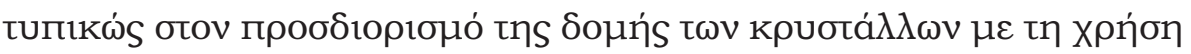

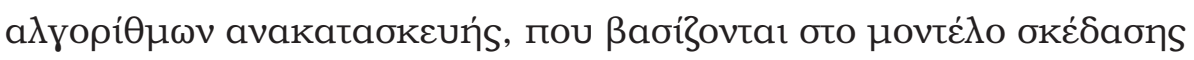

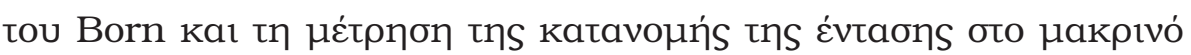

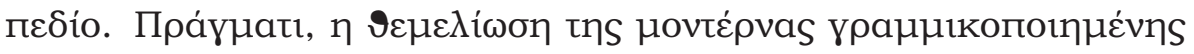

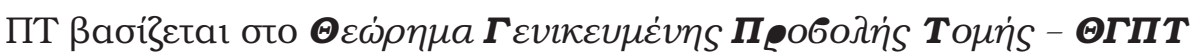

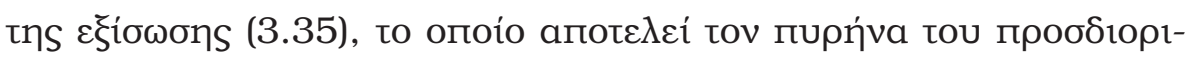

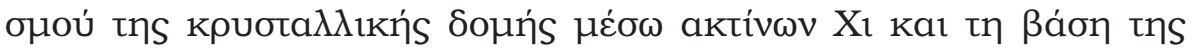

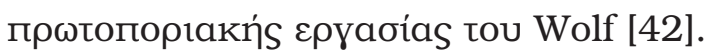

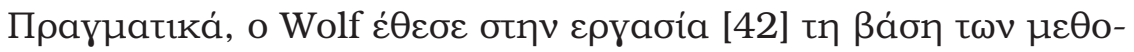

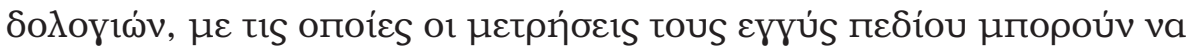




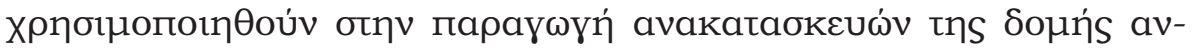

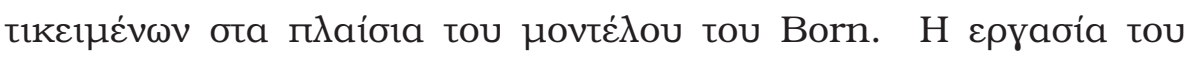

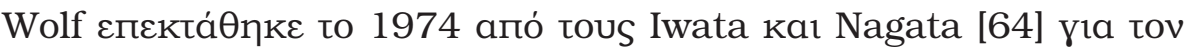

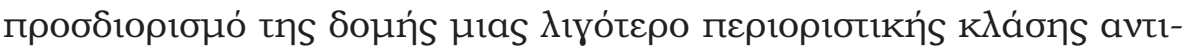

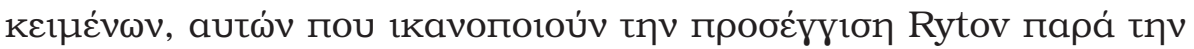

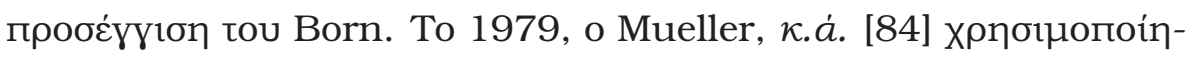

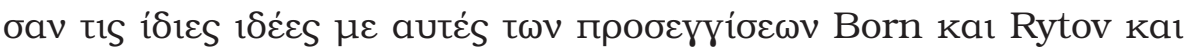

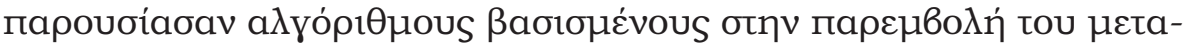

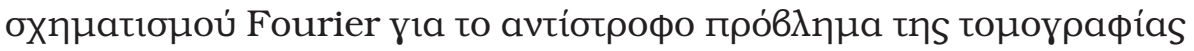

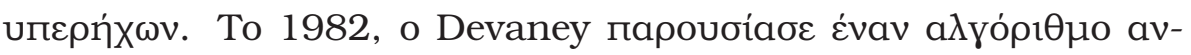

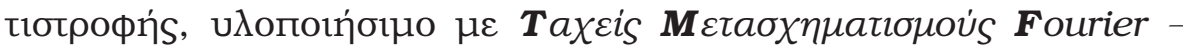

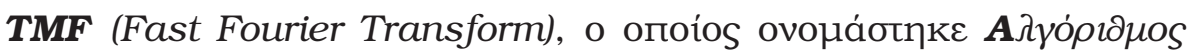

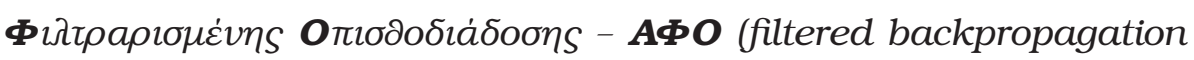

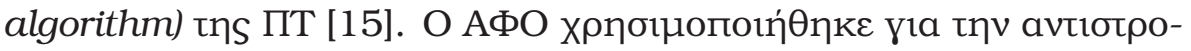

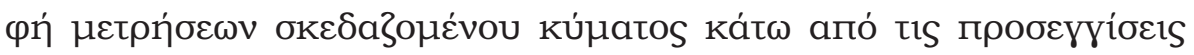

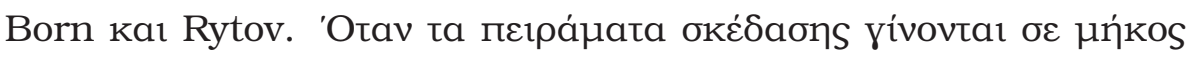

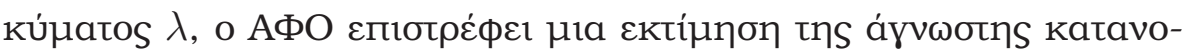

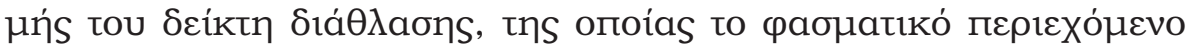

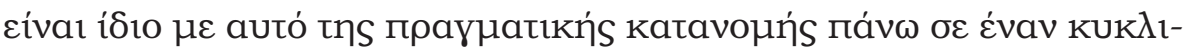

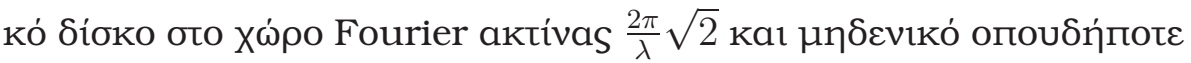

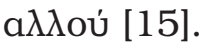

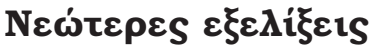

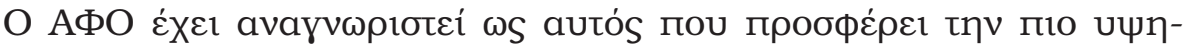

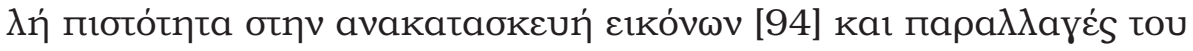


غ́xouv пароuбıабєí aпó tov Devaney [12], tous Witten א.á. [62,

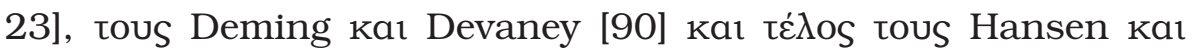

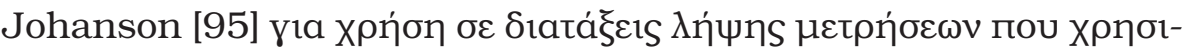

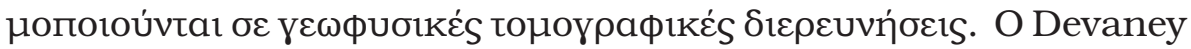

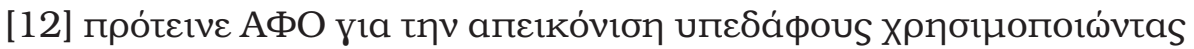

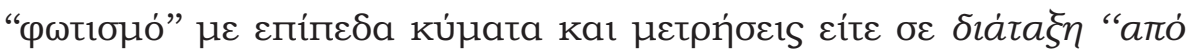

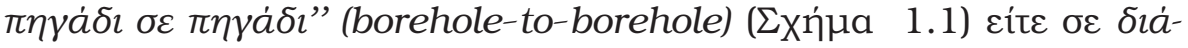

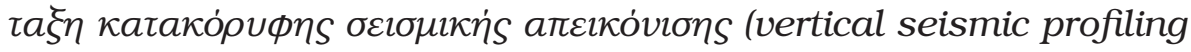

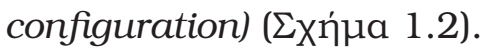

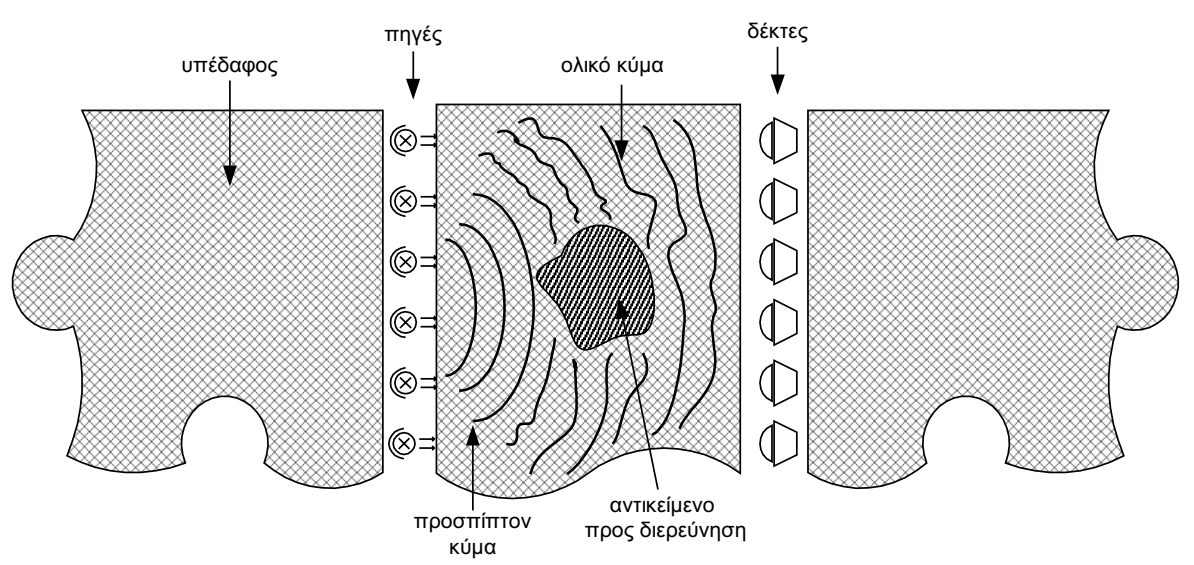

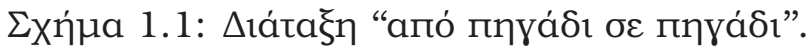

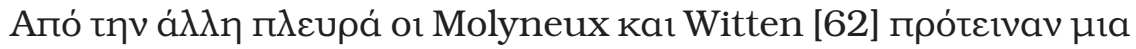

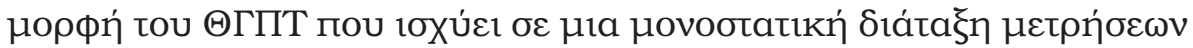

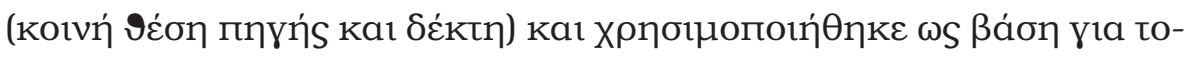

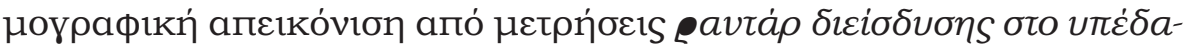
५os (ground penetrating radar). Akoגoú $\theta \omega s$, oı Witten, Molyneux 


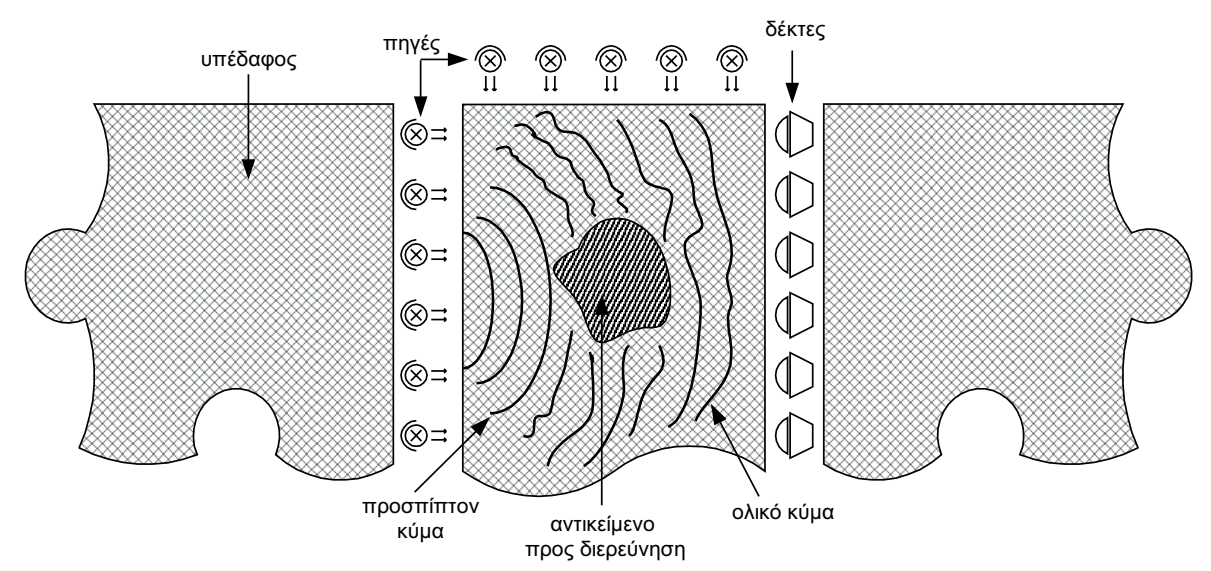

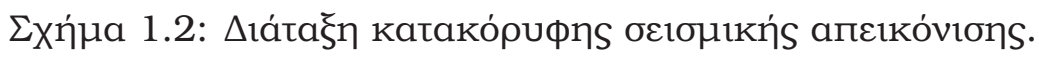

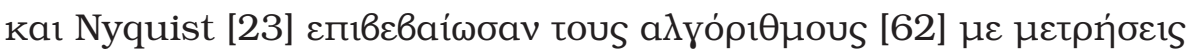

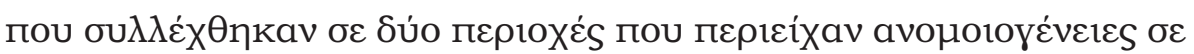

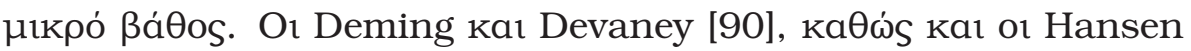

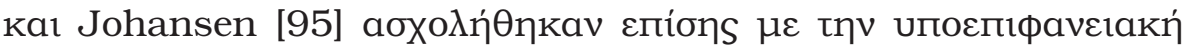

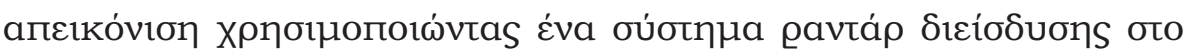

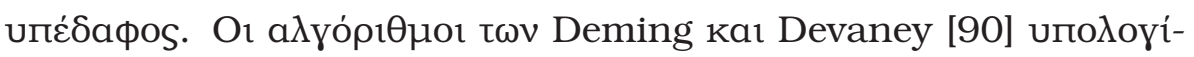

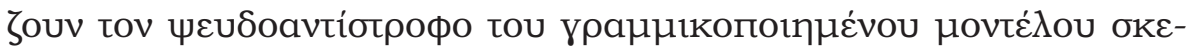

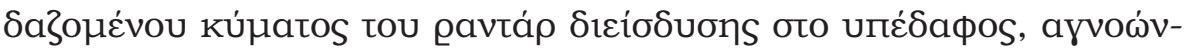

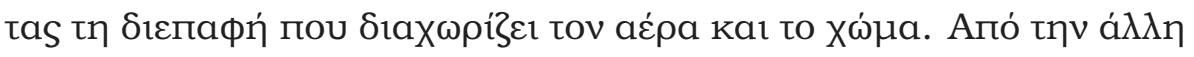

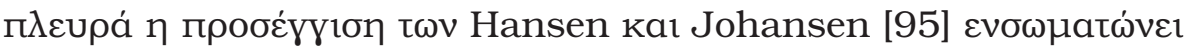

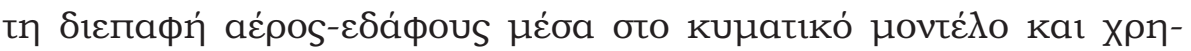

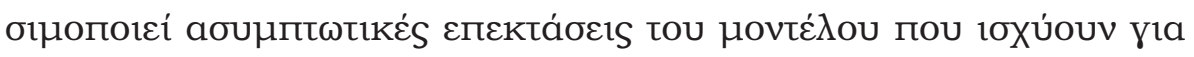

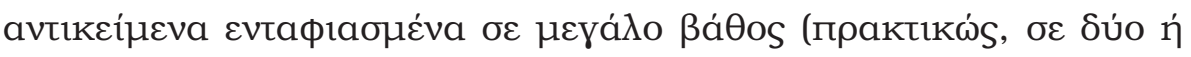

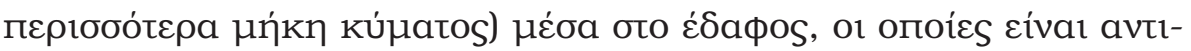




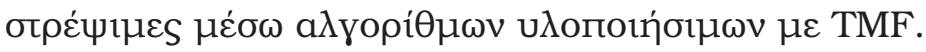

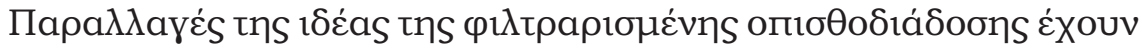

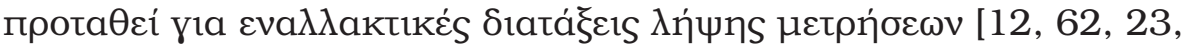

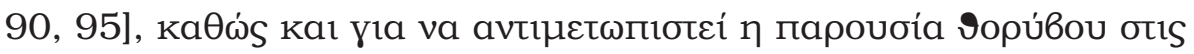

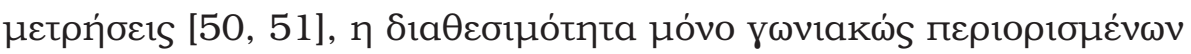

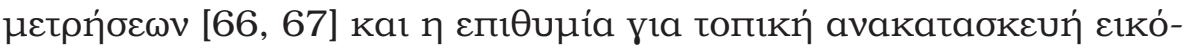

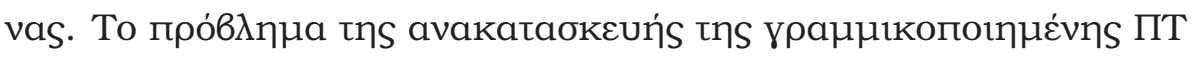

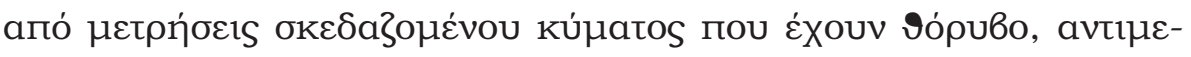

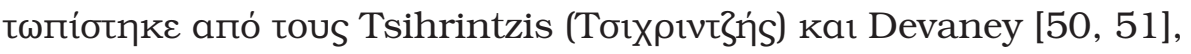

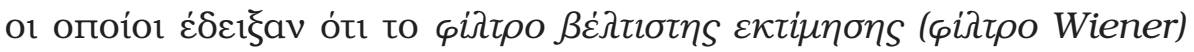

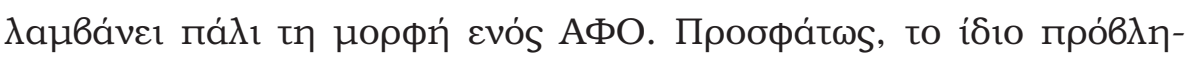

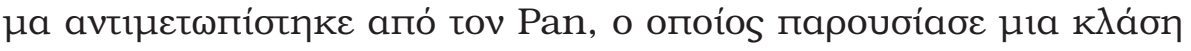

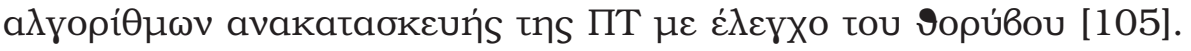

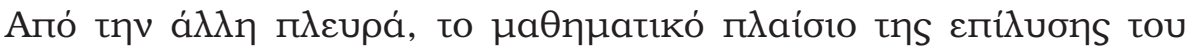

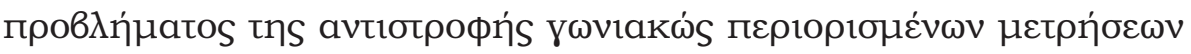

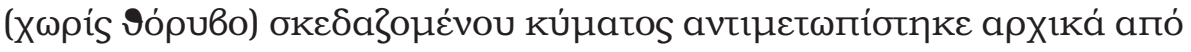
tov Devaney [15] kaı a

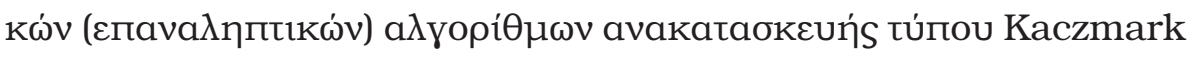

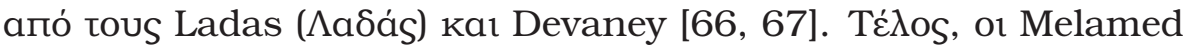
kaı Heyman [100], kaӨஸ́s kaı or Melamed, Heyman kaı Felsen

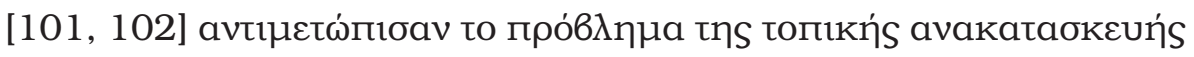

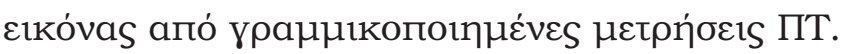

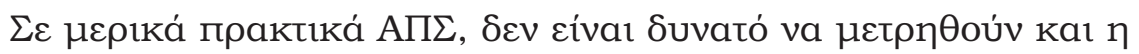

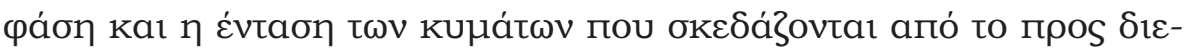

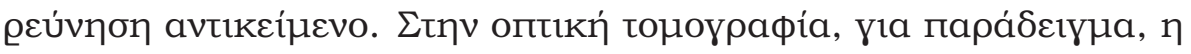




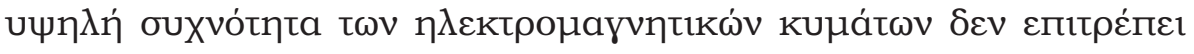

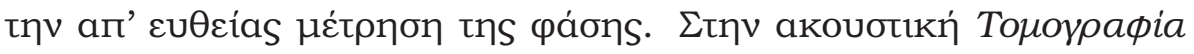

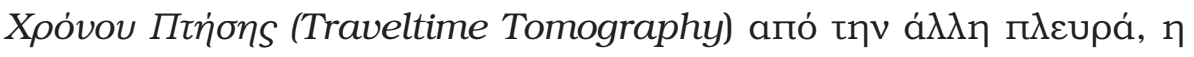

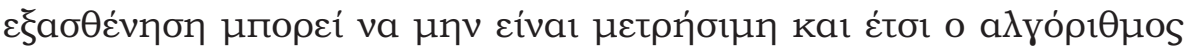

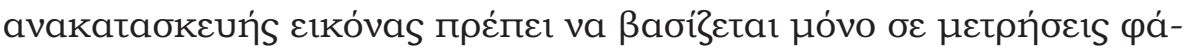

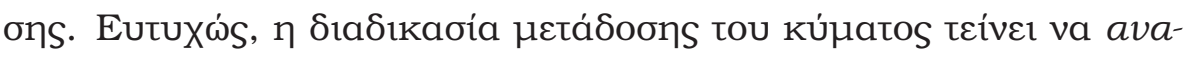

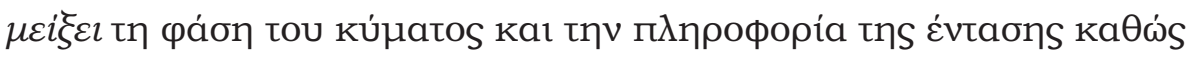

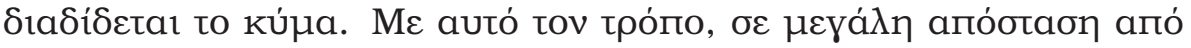

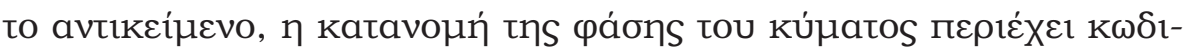

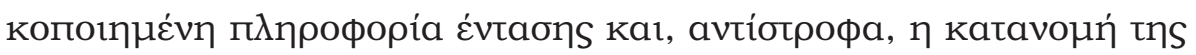

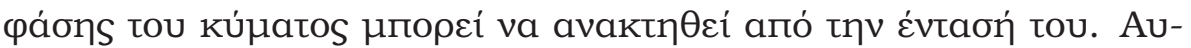

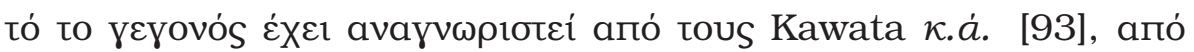

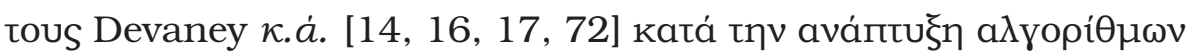
avakataokeuńs عıkóvas, kaӨஸ́S kaı anó tous Tsihrintzis (Toıxpıv-

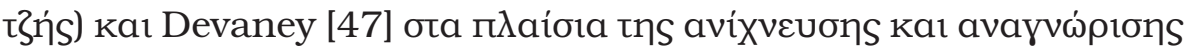

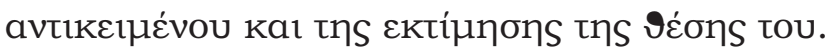

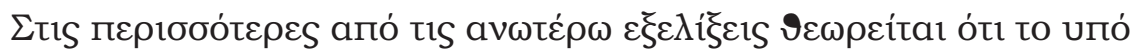

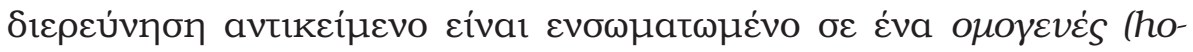

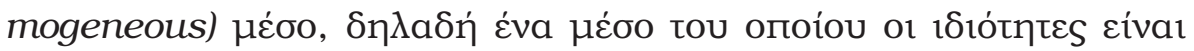

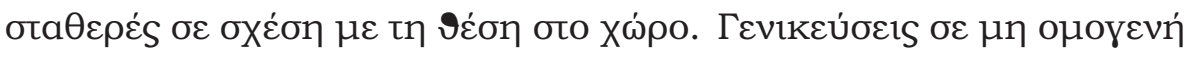

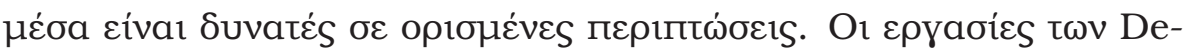

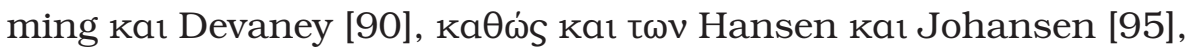

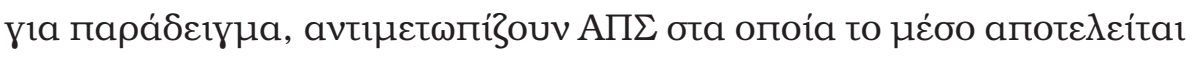

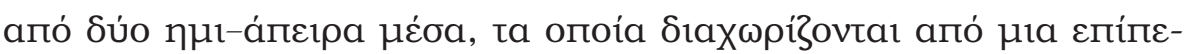

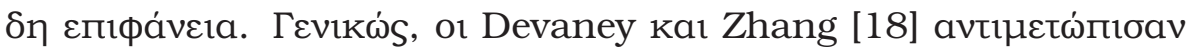




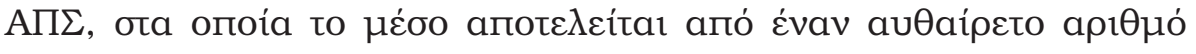

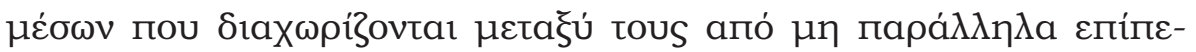

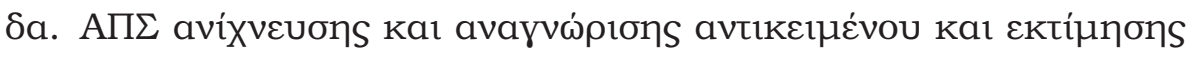

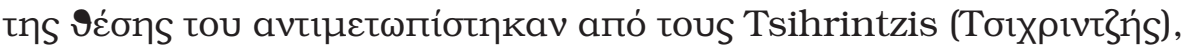

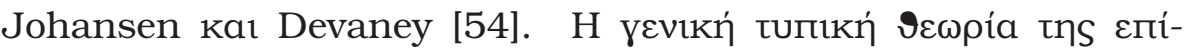

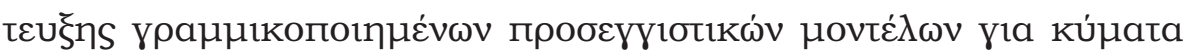

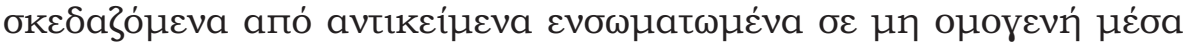

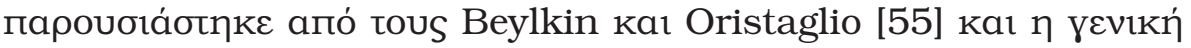

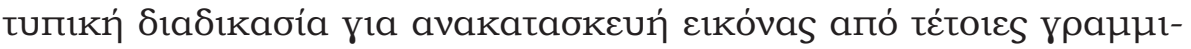

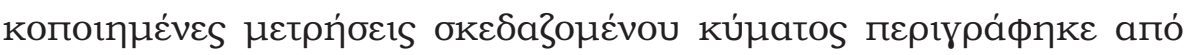
tous Devaney kaı Oristaglio oто [22].

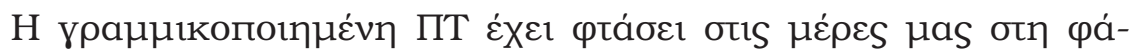

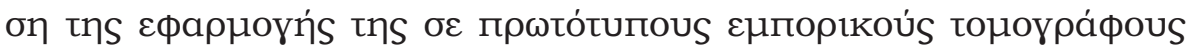

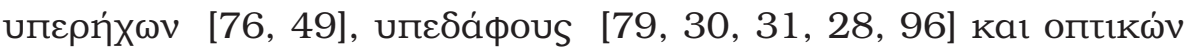

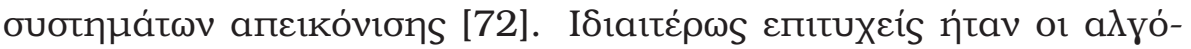

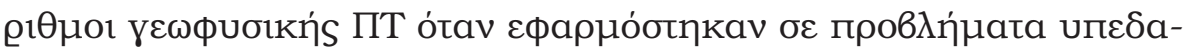

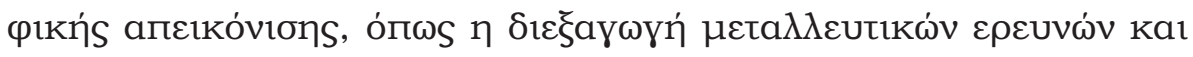

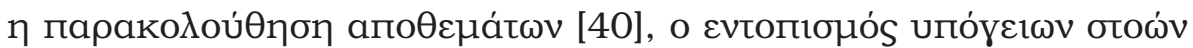

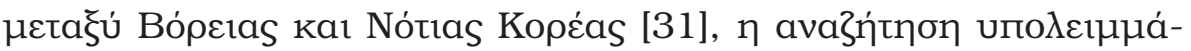

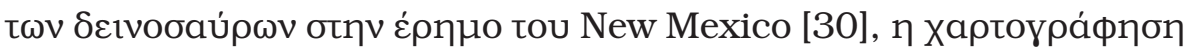

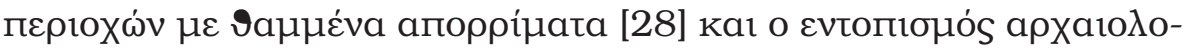

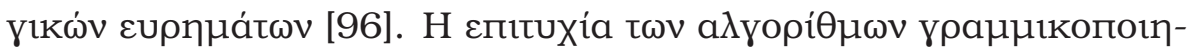

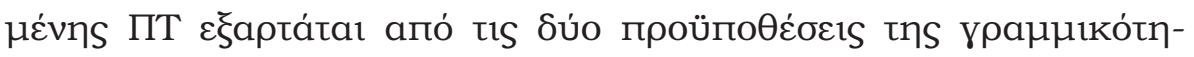

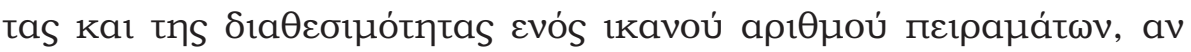

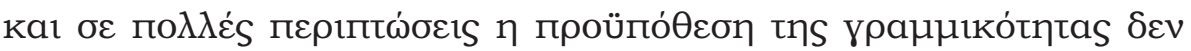




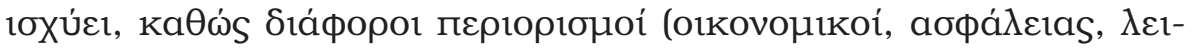

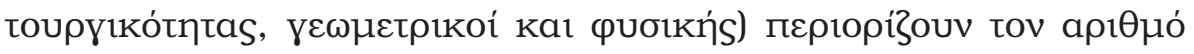

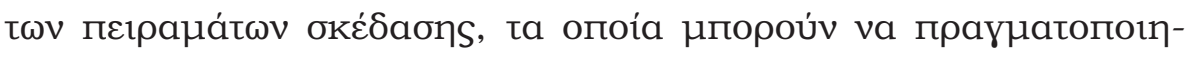

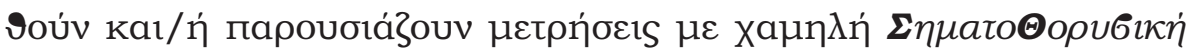

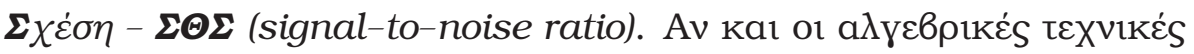

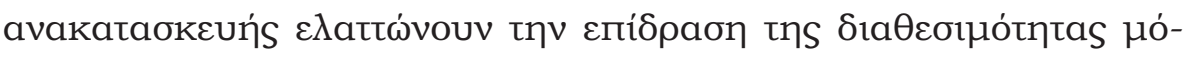

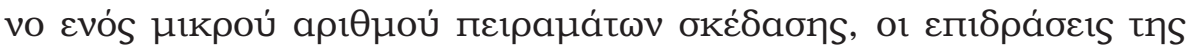

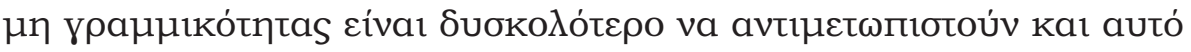

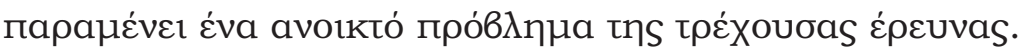

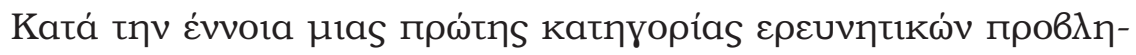

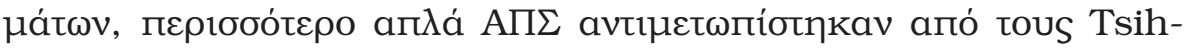

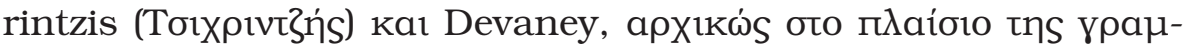

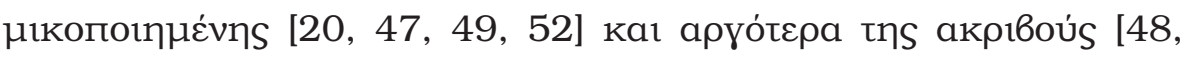

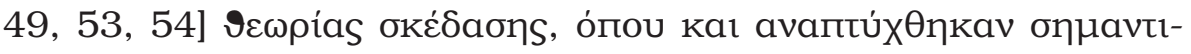

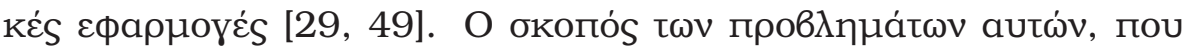

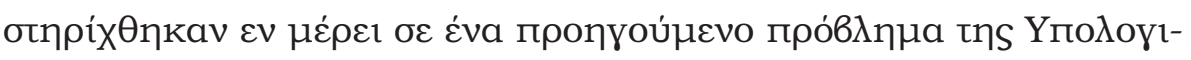

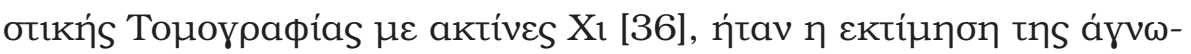

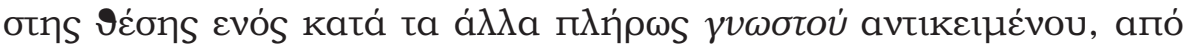

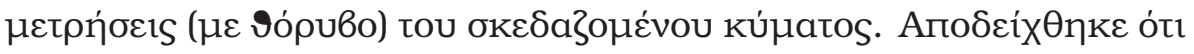

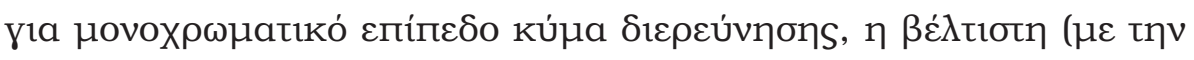

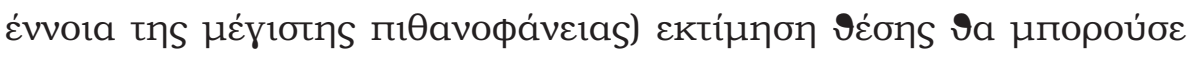

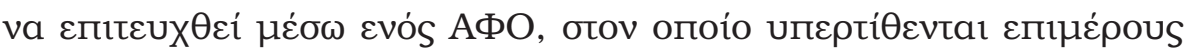

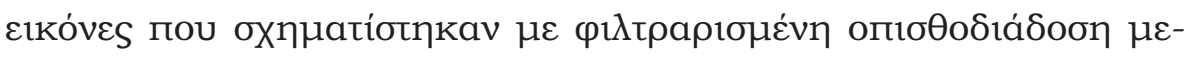

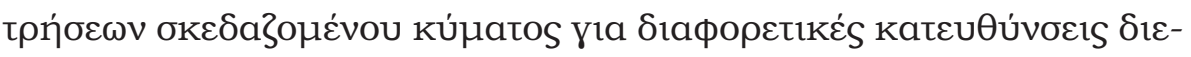

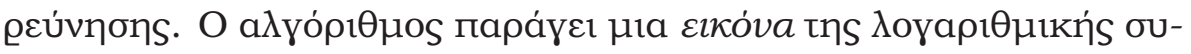




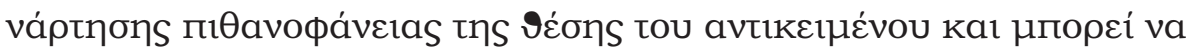

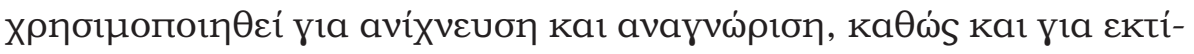

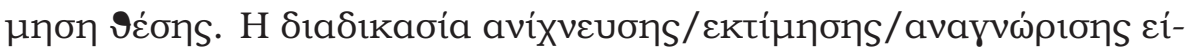

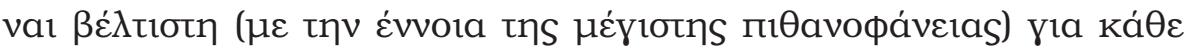

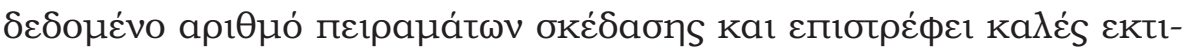

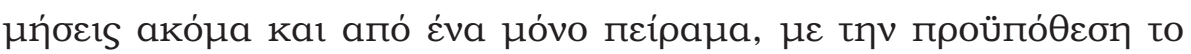

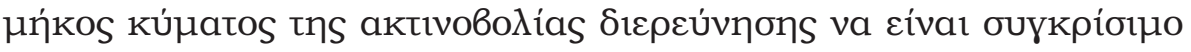

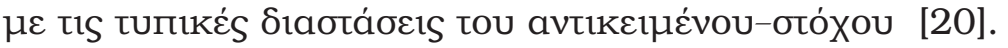

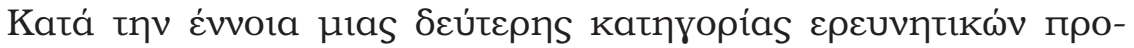

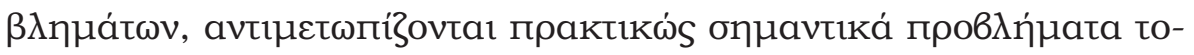

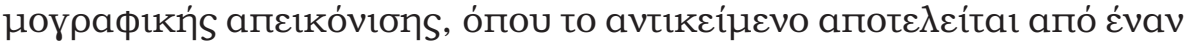

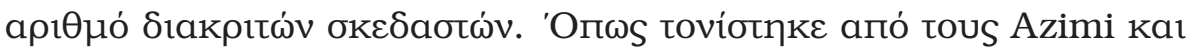

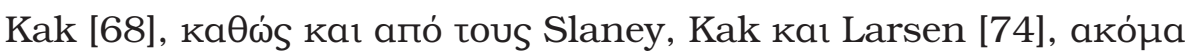

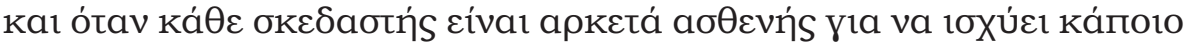

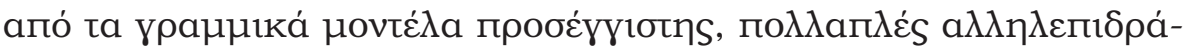

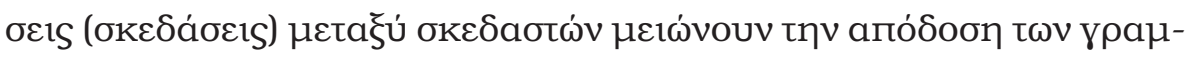

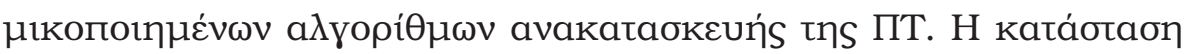

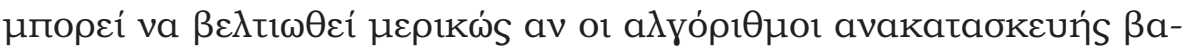

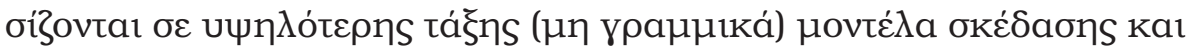

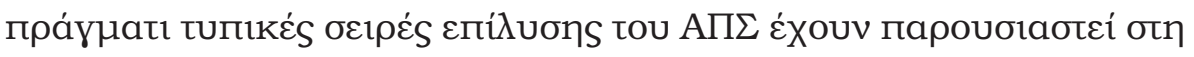

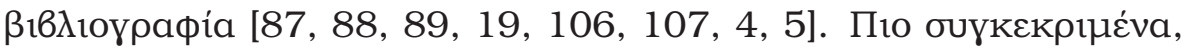

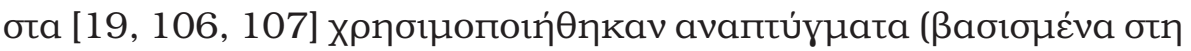

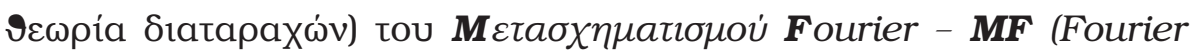

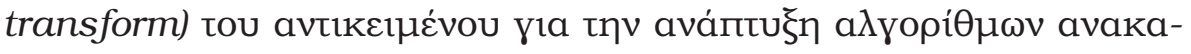

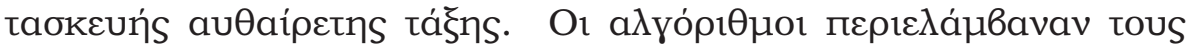




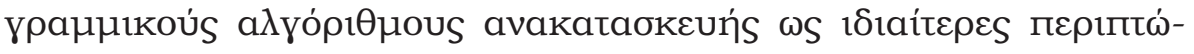

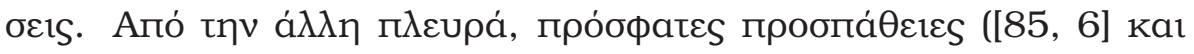

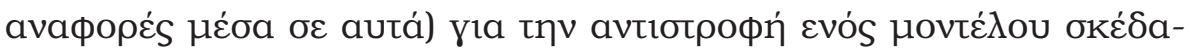

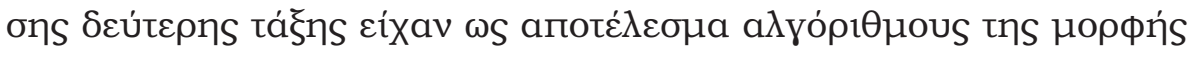

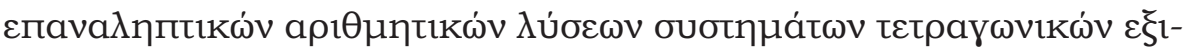

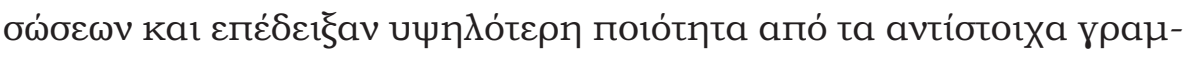

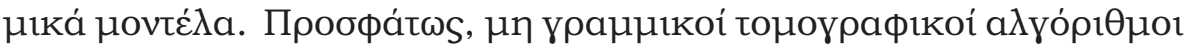

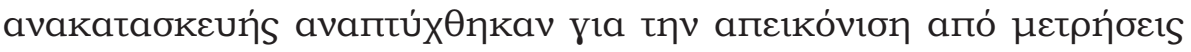

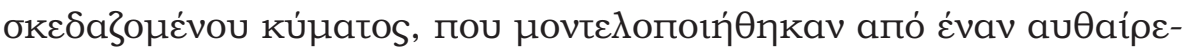

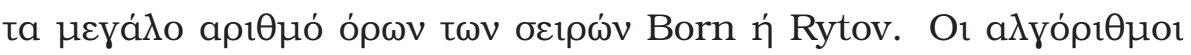

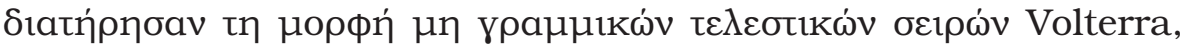

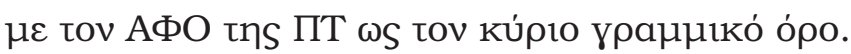

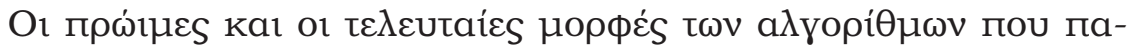

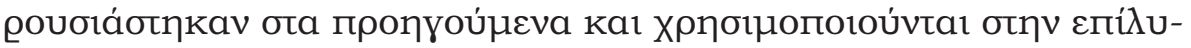

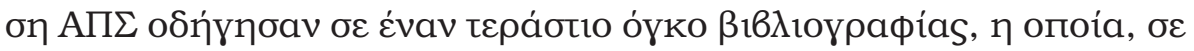

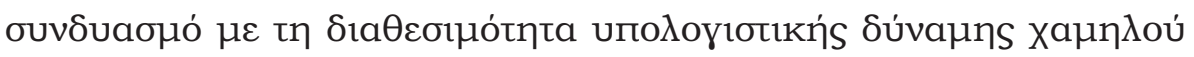

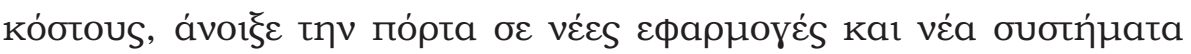

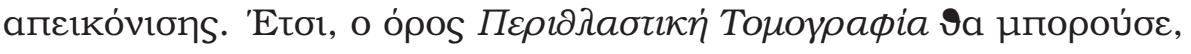

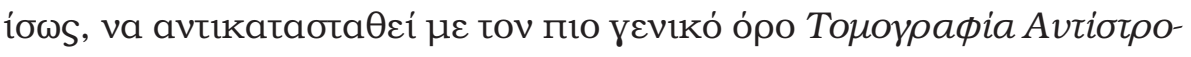

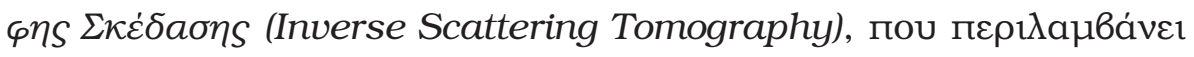

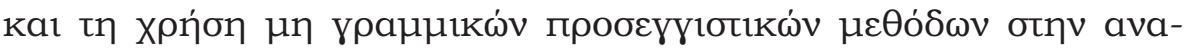

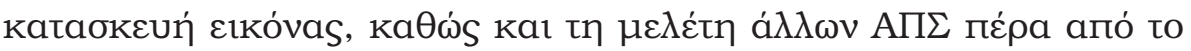

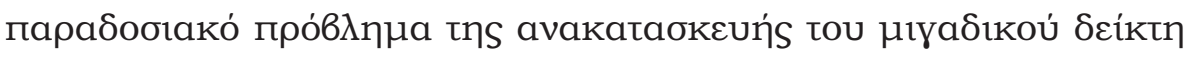

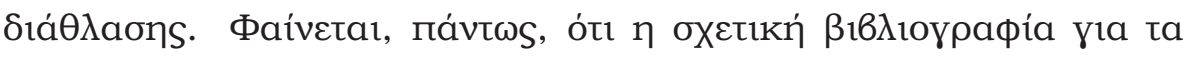

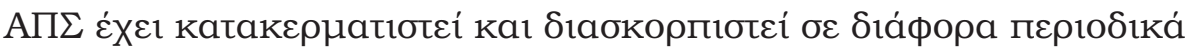




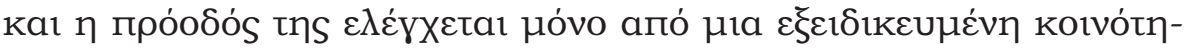

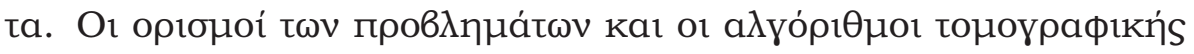

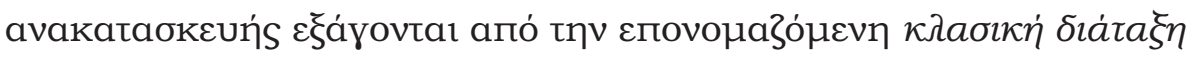

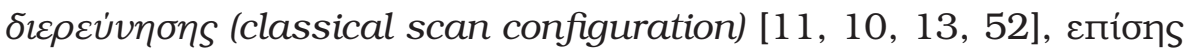

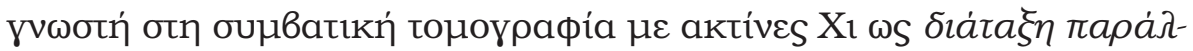

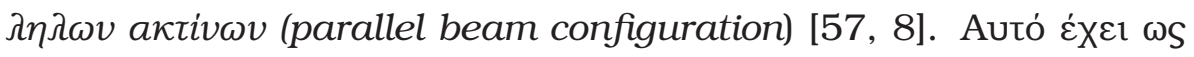

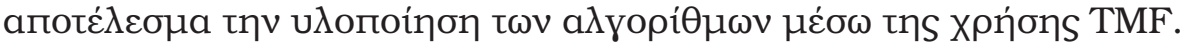

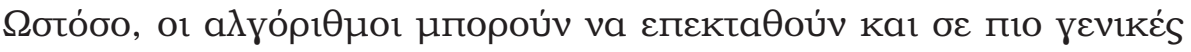

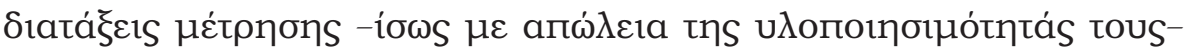

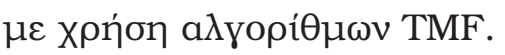

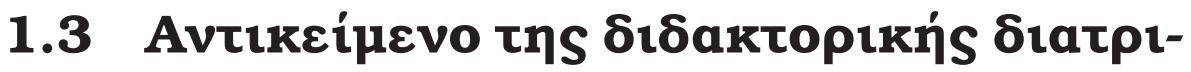 Bńs}

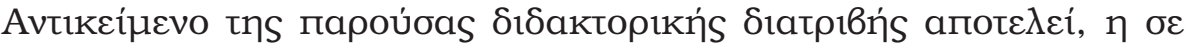

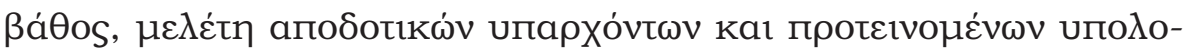

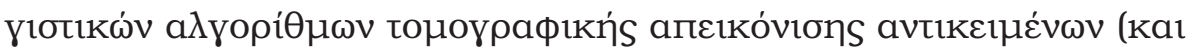

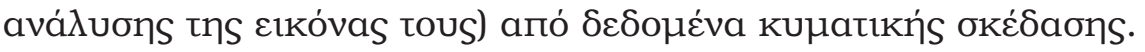

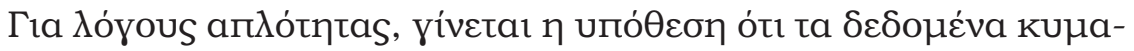

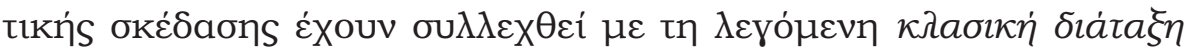

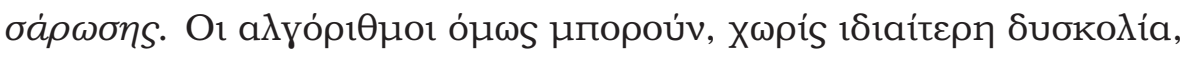

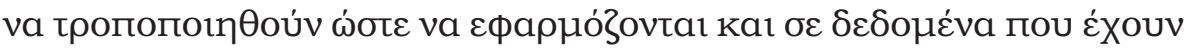

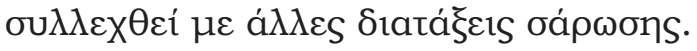




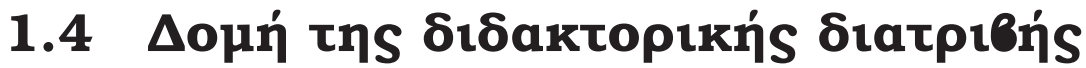

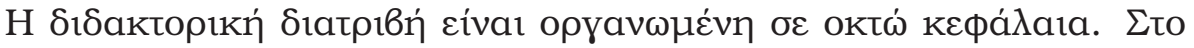

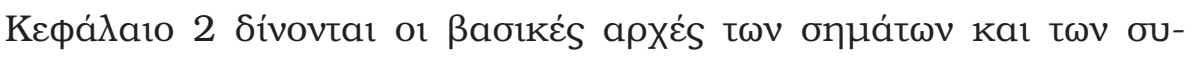

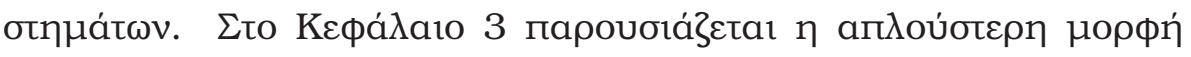

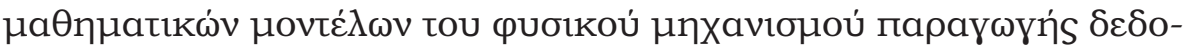

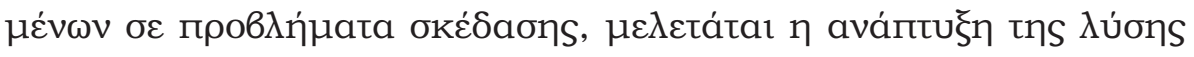

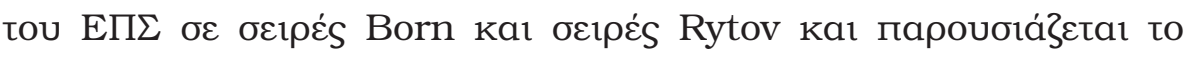

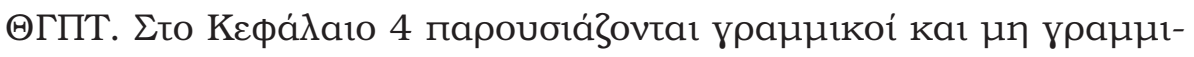

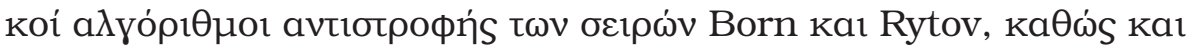

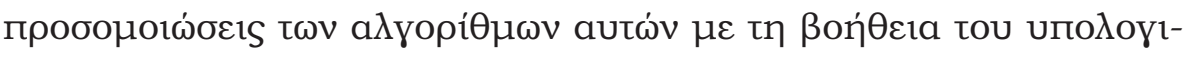

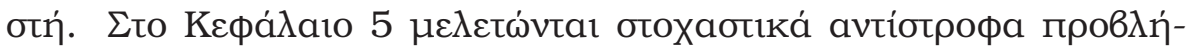

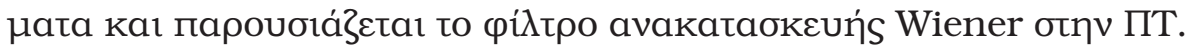

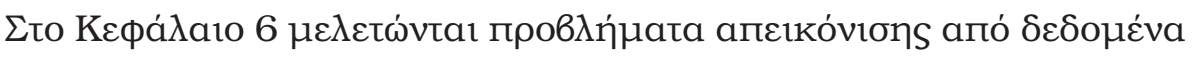

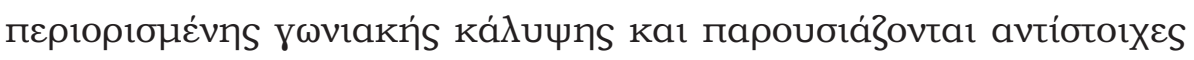

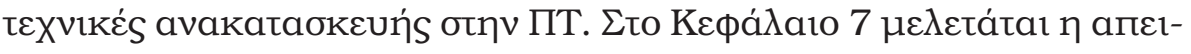

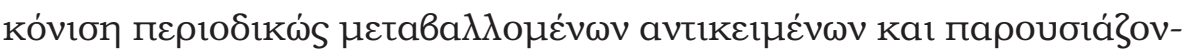

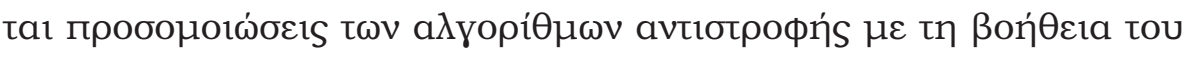

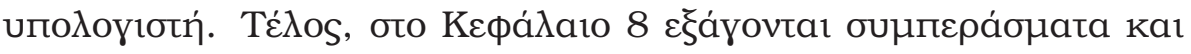

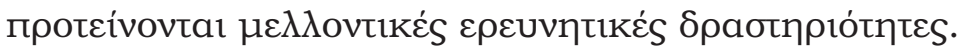




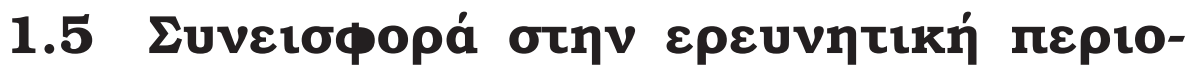 X́n}

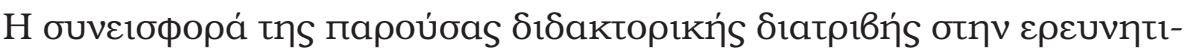

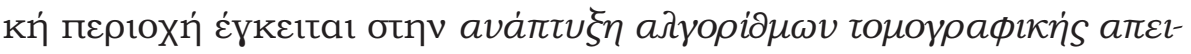

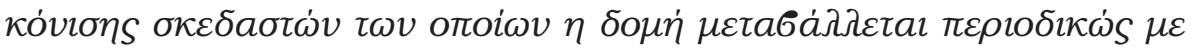

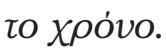

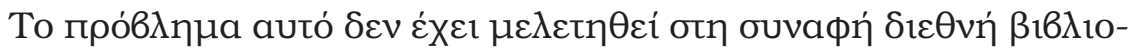

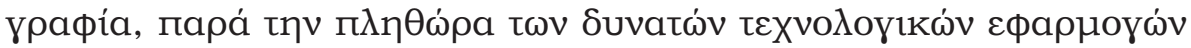

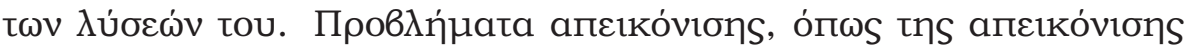

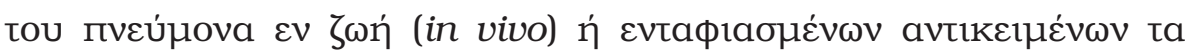

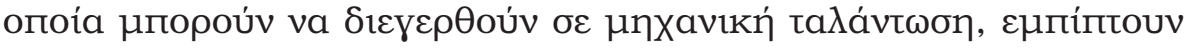

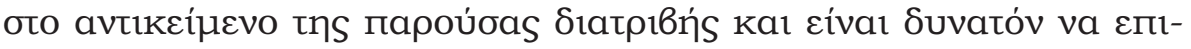

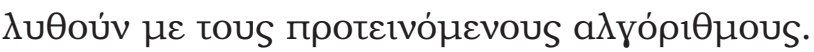

\subsection{Euxaprotís}

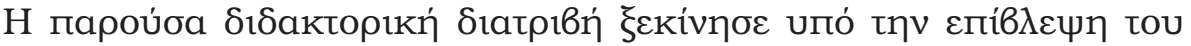

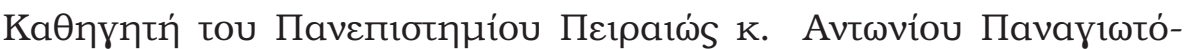

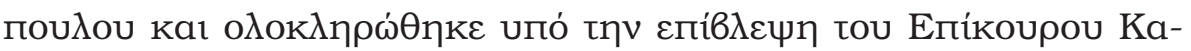

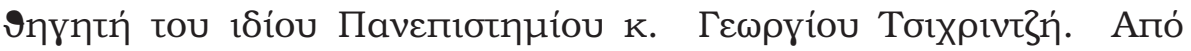

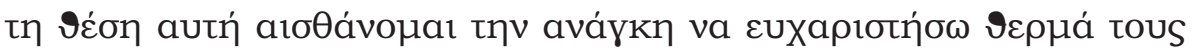

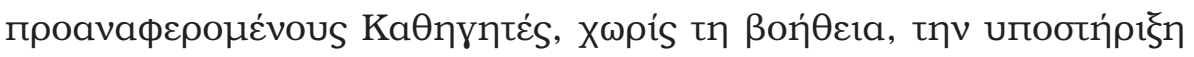

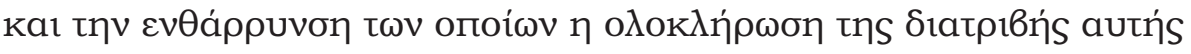

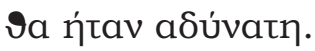




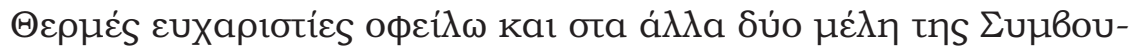

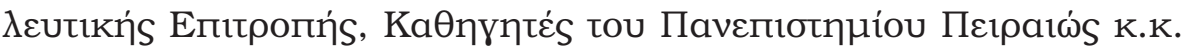

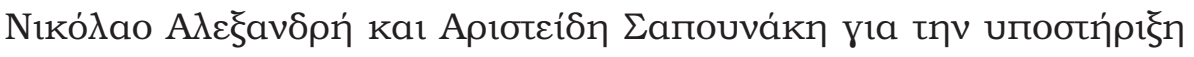

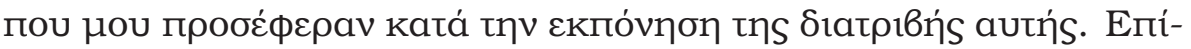

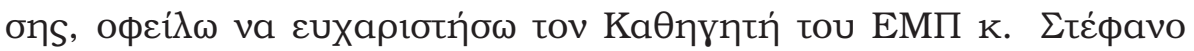

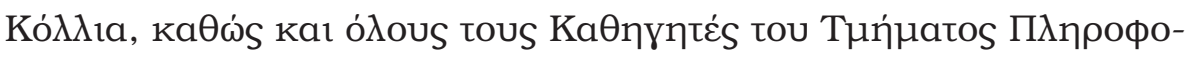

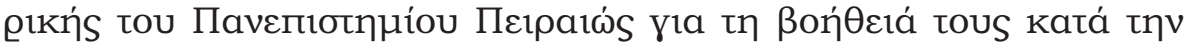

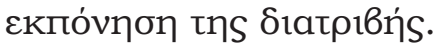

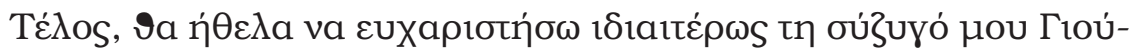

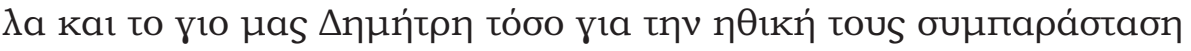

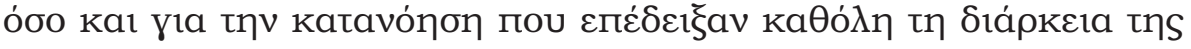

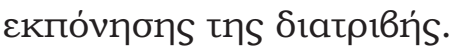




\section{Keфádato 2}

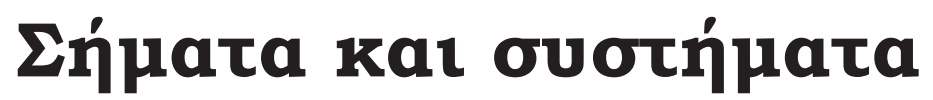

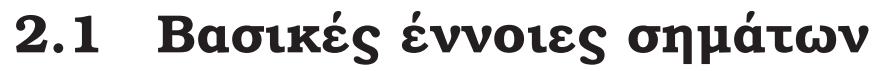

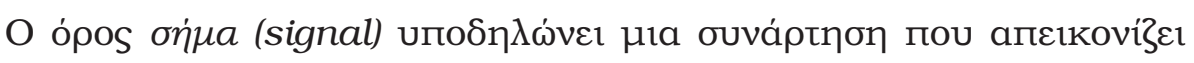

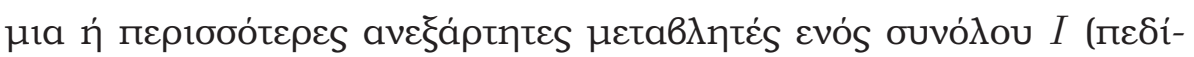

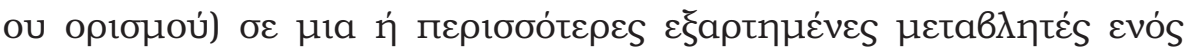

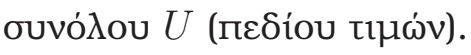

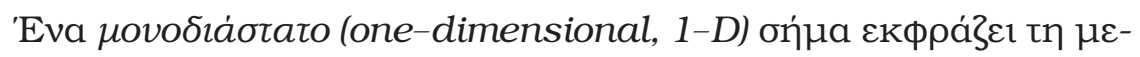

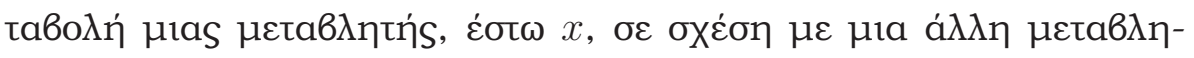

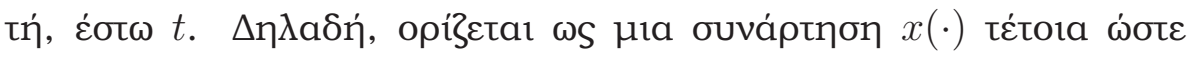

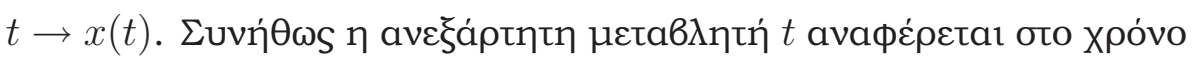

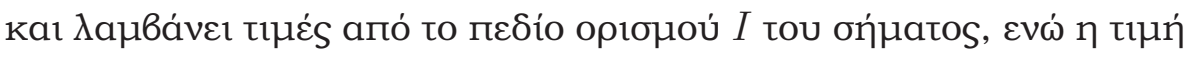

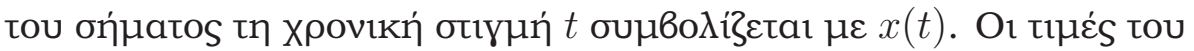

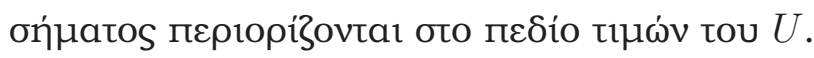

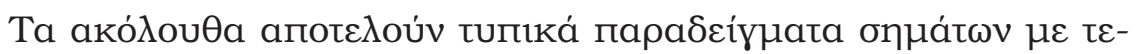

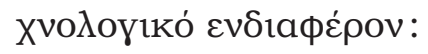




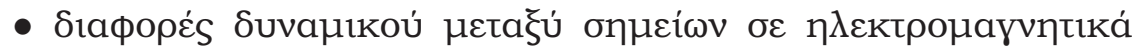

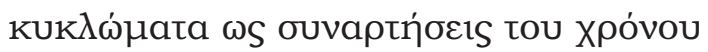

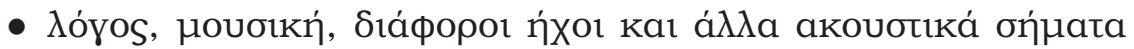

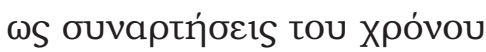

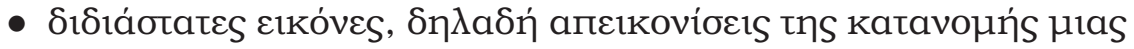

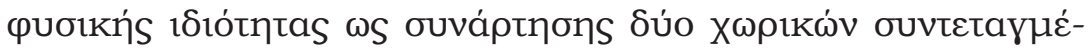
$v \omega v$

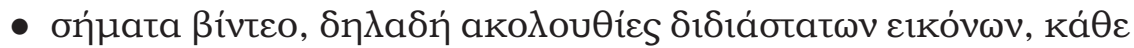

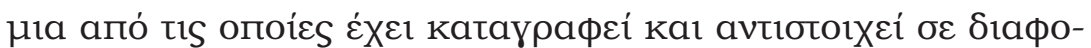

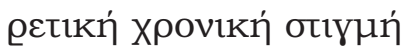

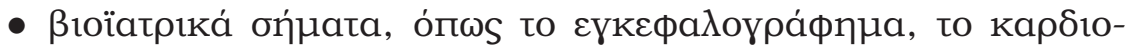

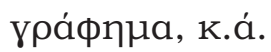

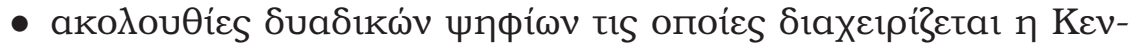

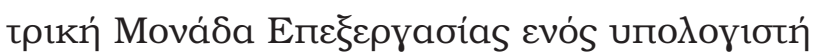

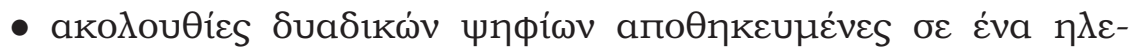

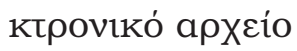

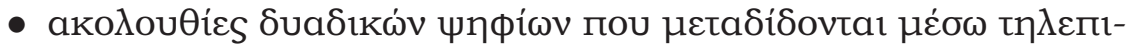

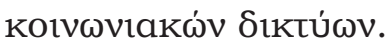

Ta бท́

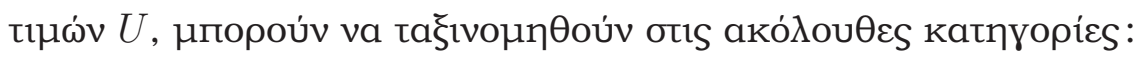

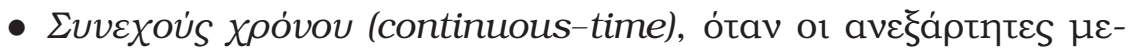

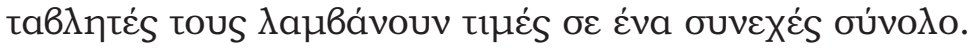




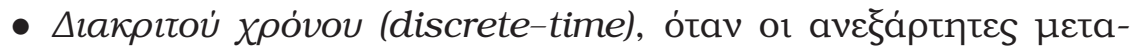

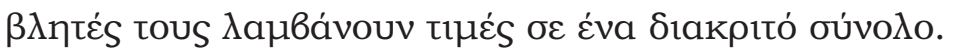

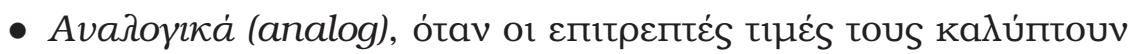

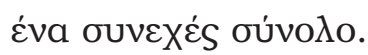

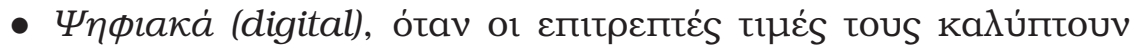

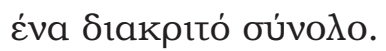

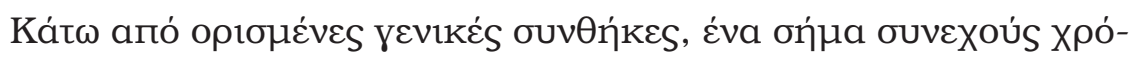

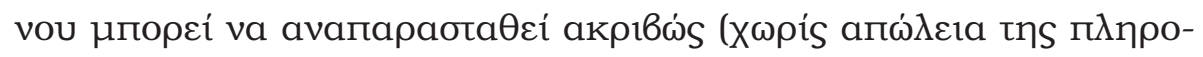

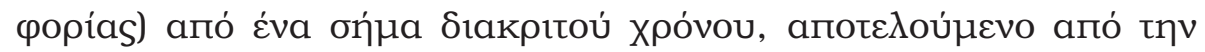

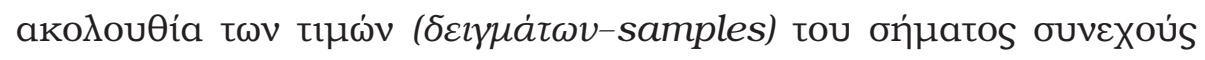

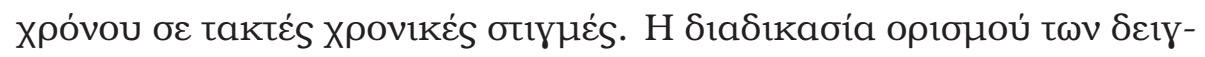

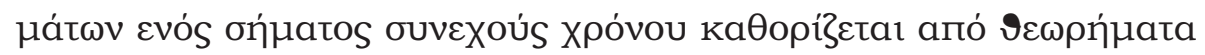

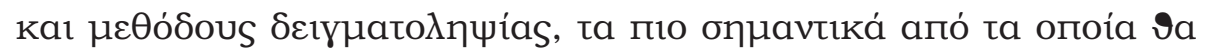

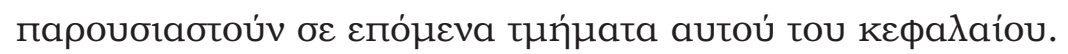

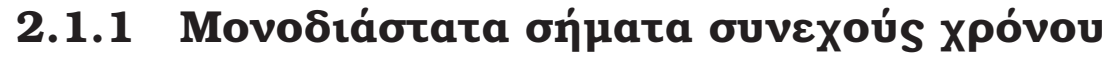

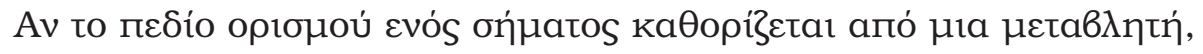

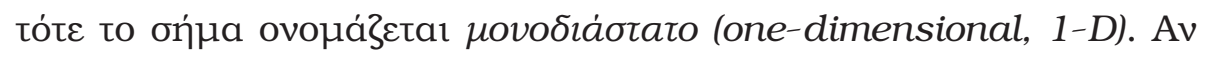

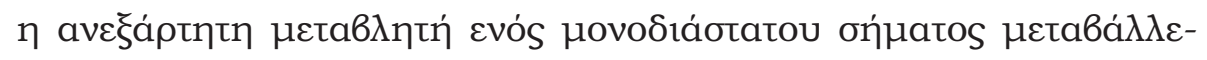

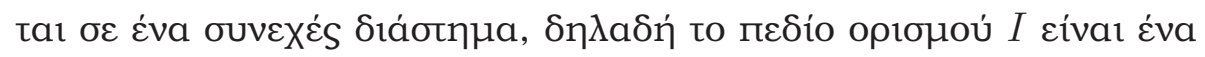

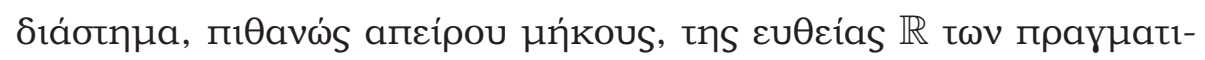

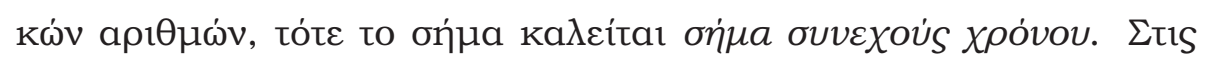

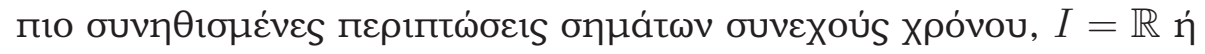




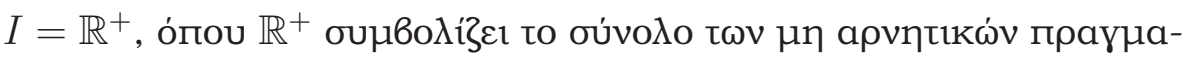

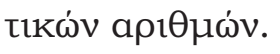

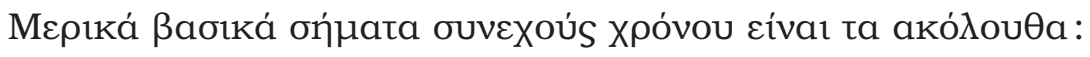

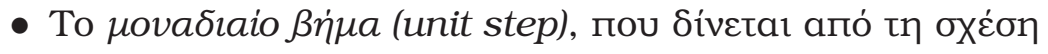

$$
u_{s}(t)= \begin{cases}1, & t \geq 0 \\ 0, & t<0 .\end{cases}
$$

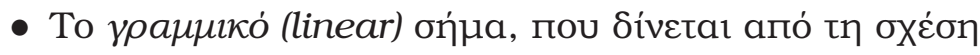

$$
u_{r}(t)= \begin{cases}t, & t \geq 0 \\ 0, & t<0 .\end{cases}
$$

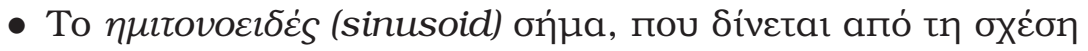

$$
x(t)=\sin \omega t, \quad t \in \mathbb{R} .
$$

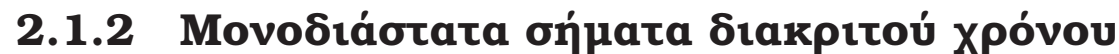

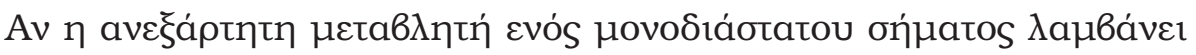

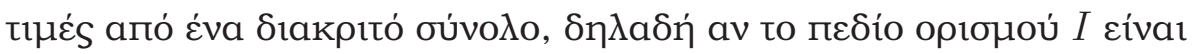

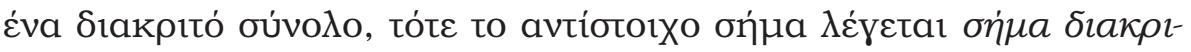

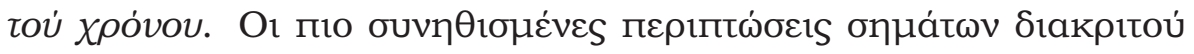

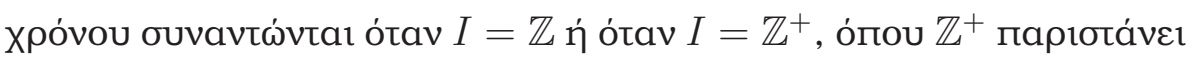

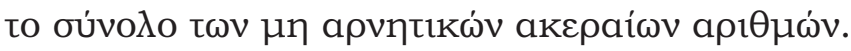

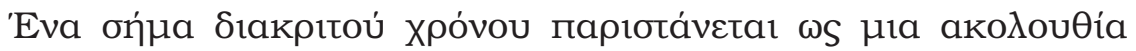

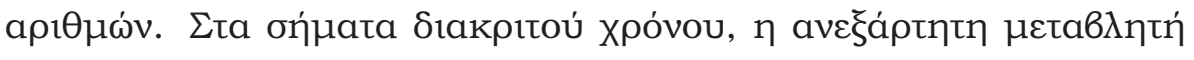

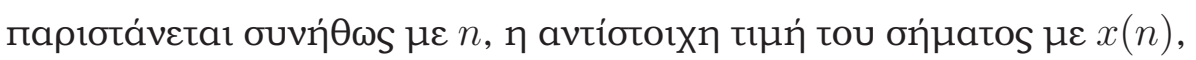

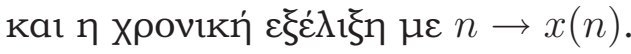




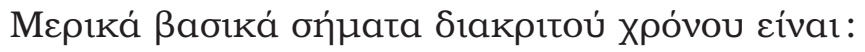

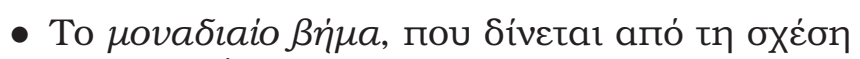
$u_{s}(n)=\left\{\begin{array}{ll}1, & n \geq 0 \\ 0, & n<0\end{array}\right.$ о́поч $n \in \mathbb{Z}$

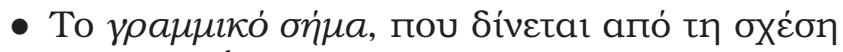
$u_{r}(n)=\left\{\begin{array}{ll}n, & n \geq 0 \\ 0, & n<0,\end{array} \quad\right.$ о́поч $n \in \mathbb{Z}$

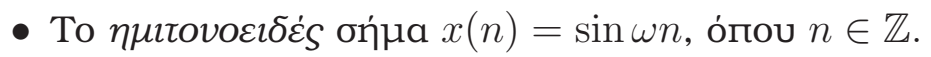

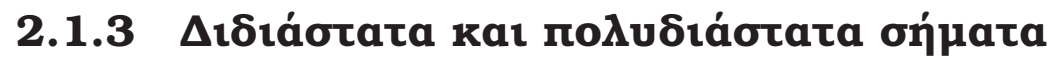

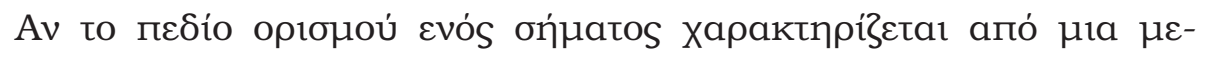

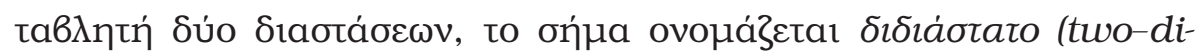

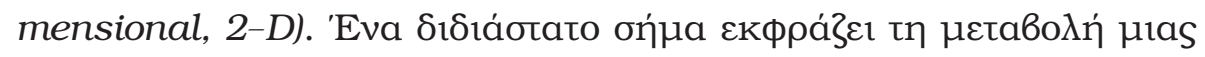

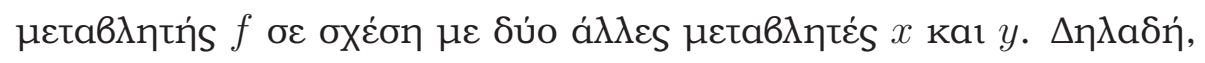

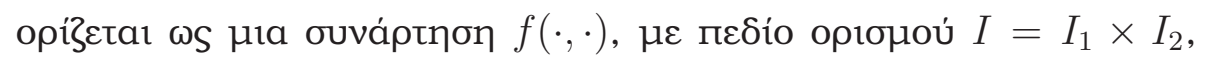

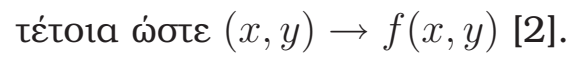

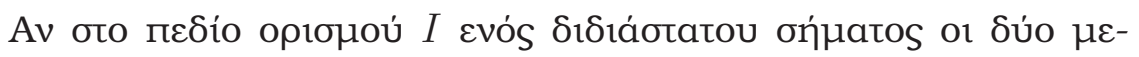

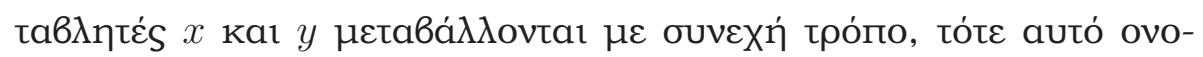

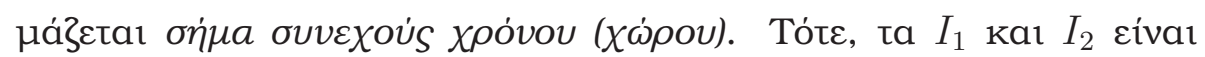

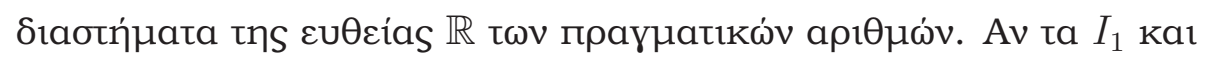

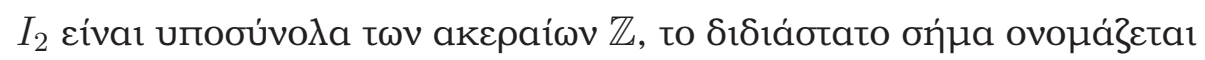

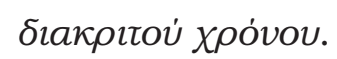




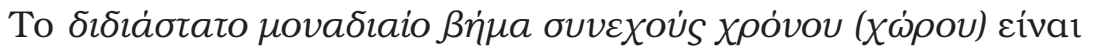

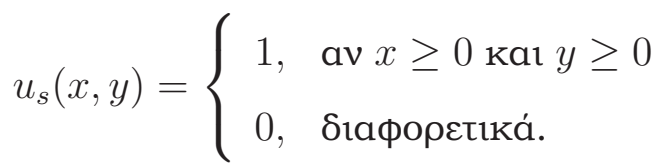

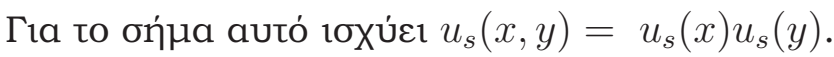

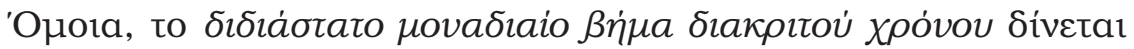
aпó tov túпо

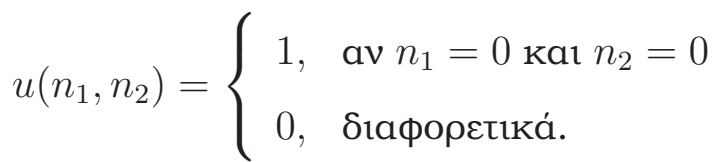

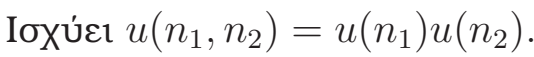

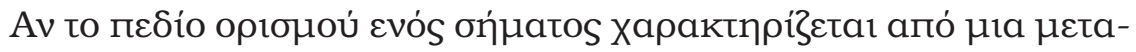

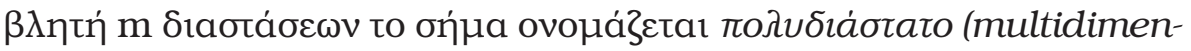

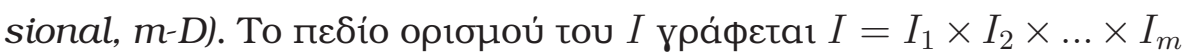

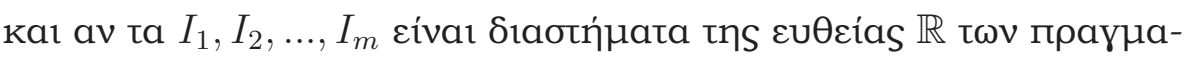

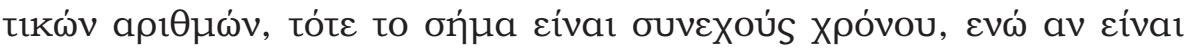

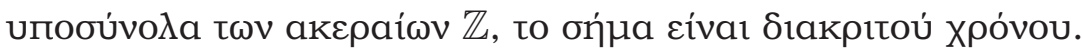

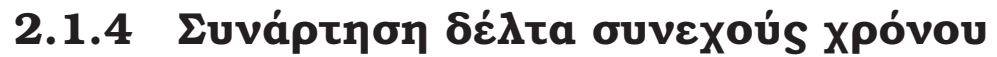

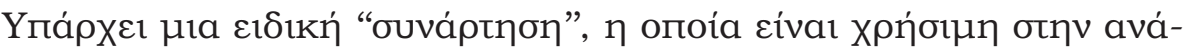

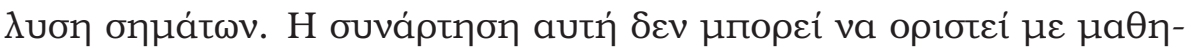

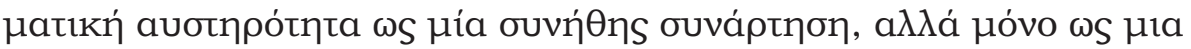

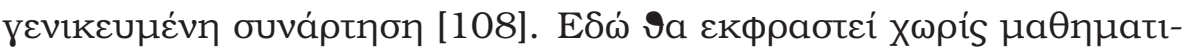

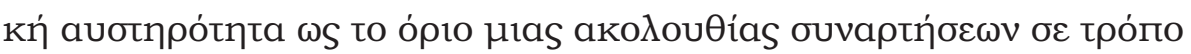

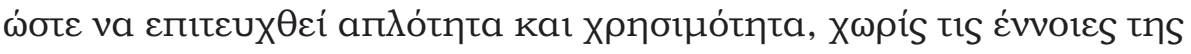

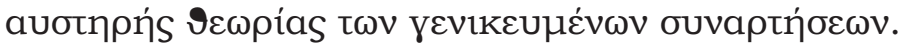




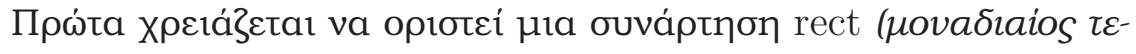

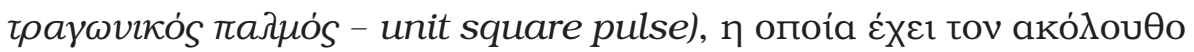
тúпо

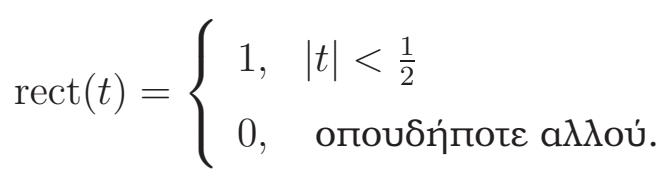

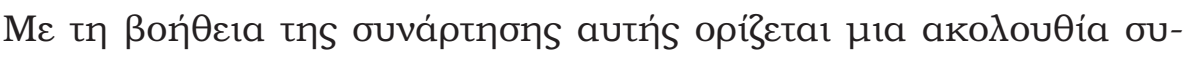

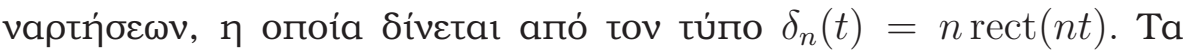

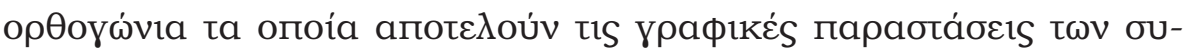

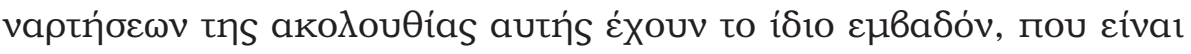

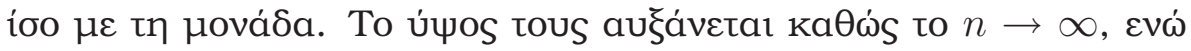

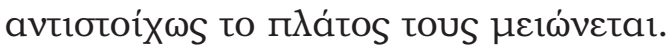

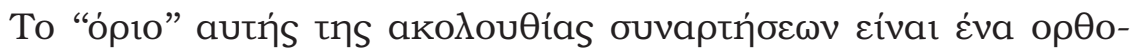

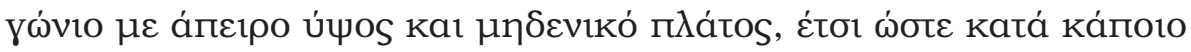

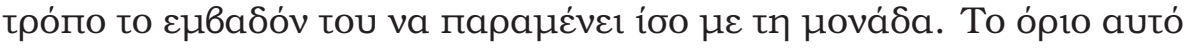

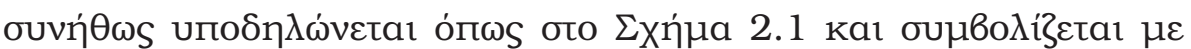
$\delta(t)$. 

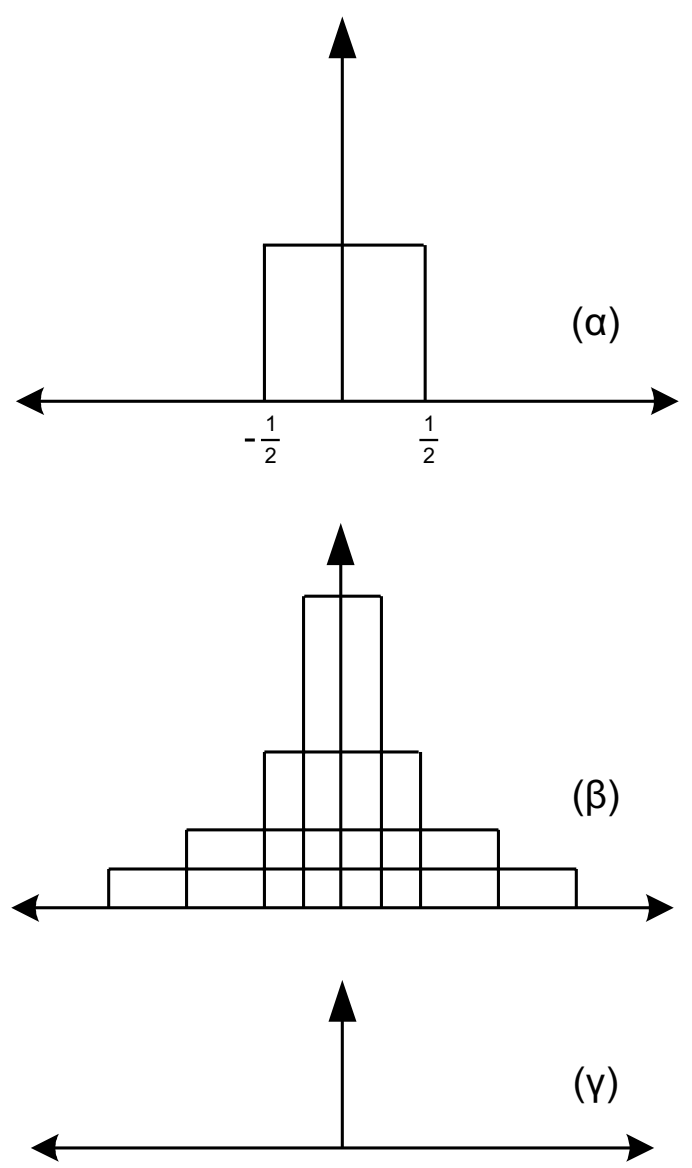

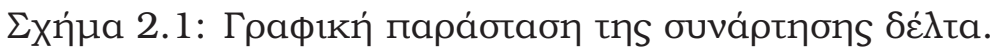




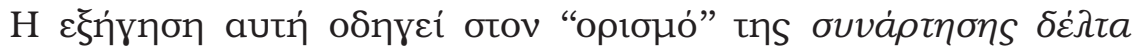

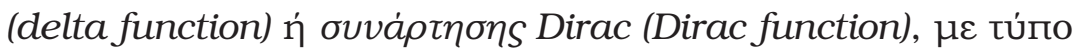

$$
\delta(t)=\lim _{n \rightarrow \infty} \delta_{n}(t)
$$

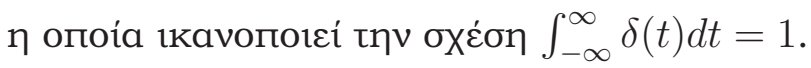

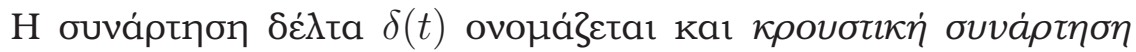

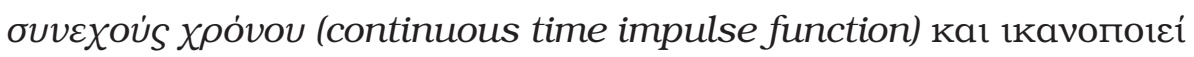

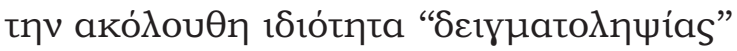

$$
\int_{-\infty}^{\infty} x(t) \delta\left(t-t^{\prime}\right) d t=x\left(t^{\prime}\right)
$$

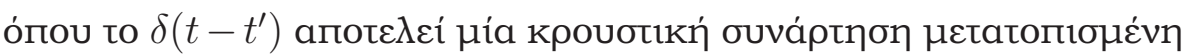

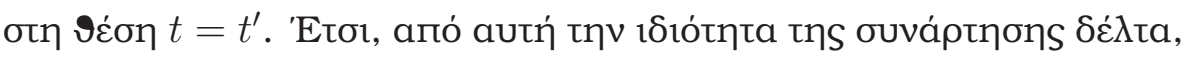

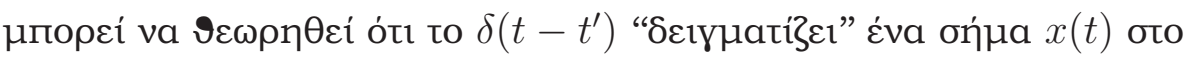
oпuкio $t=t^{\prime}$.

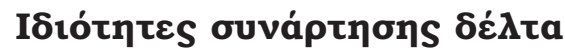

1. $\int_{-\infty}^{\infty} \delta(t) d t=\lim _{n \rightarrow \infty} \int_{-\infty}^{\infty} \delta_{n}(t) d t=1$

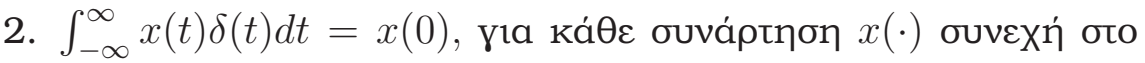
$t=0$.

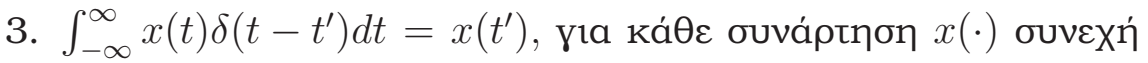
бто $t=t^{\prime}$.

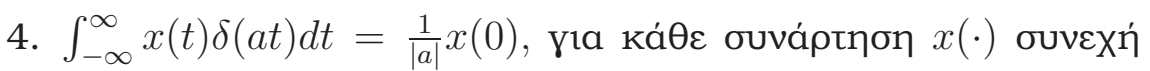
бто $t=0$. 'Ара $\delta(a t)=\frac{1}{|a|} \delta(t)$. 
5. $\delta(t)=\frac{1}{2 \pi} \int_{-\infty}^{\infty} e^{-i \omega t} d \omega=\int_{-\infty}^{\infty} e^{-i 2 \pi s t} d s$.

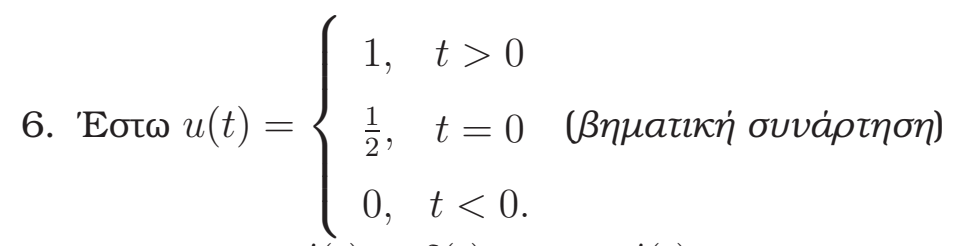

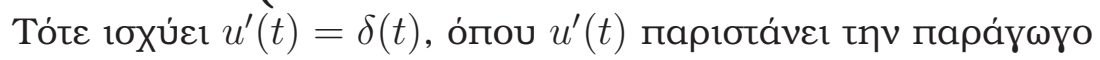
tns $u(t)$.

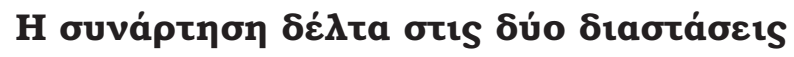

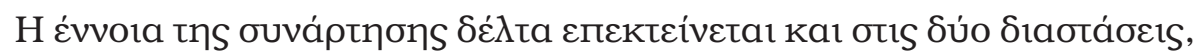

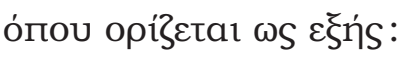

$$
\delta(x, y)=\delta(x) \delta(y)
$$

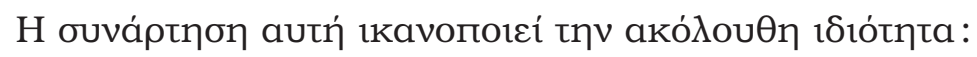

$$
\int_{-\infty}^{\infty} \int_{-\infty}^{\infty} f(x, y) \delta(x, y) d x d y=f(0,0)
$$

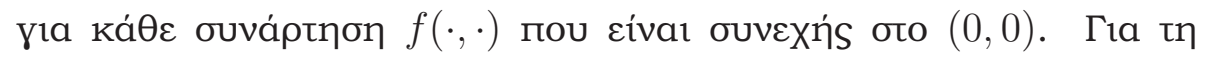

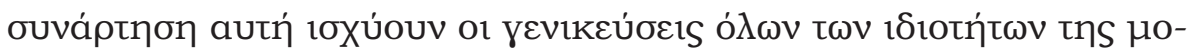

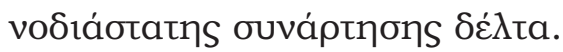

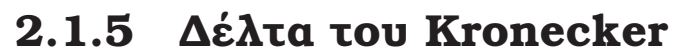

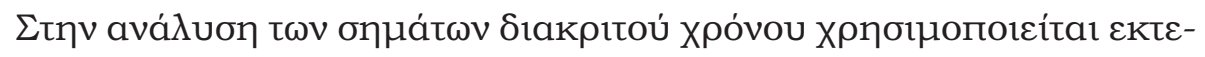

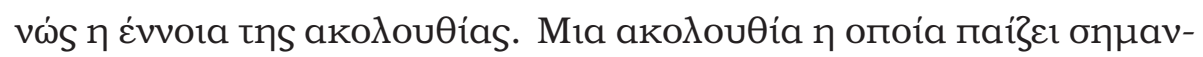

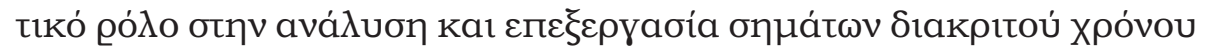




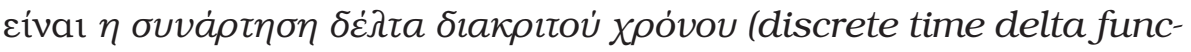

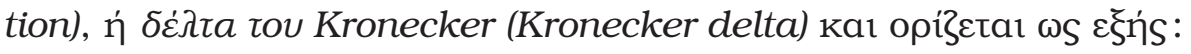

$$
\delta(n)= \begin{cases}1, & n=0 \\ 0, & n \neq 0 .\end{cases}
$$

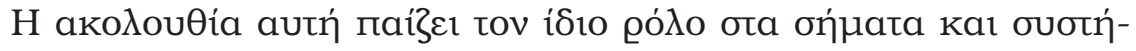

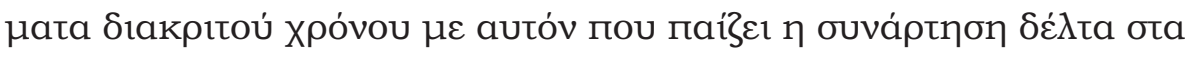

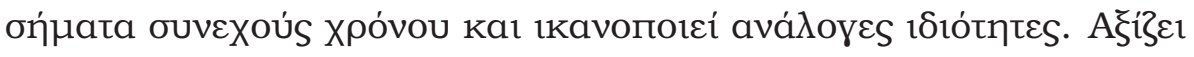

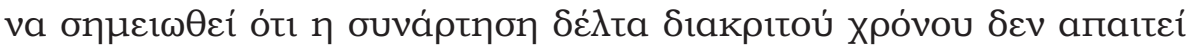

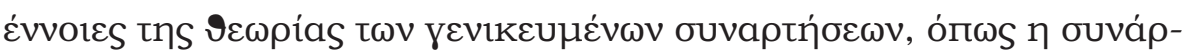

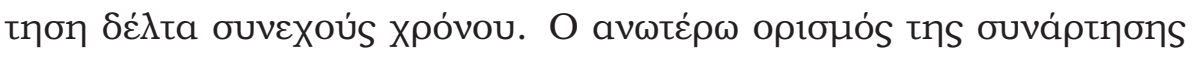

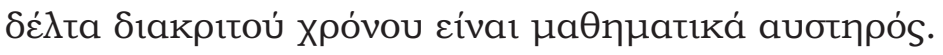

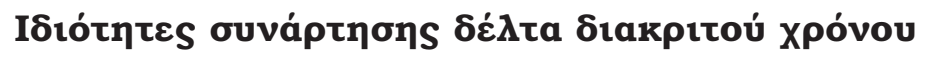

1. $\sum_{n=-\infty}^{\infty} \delta(n)=1$.

2. $\sum_{n=-\infty}^{\infty} x(n) \delta(n)=x(0)$.

3. $\sum_{n=-\infty}^{\infty} x(n) \delta\left(n-n^{\prime}\right)=x\left(n^{\prime}\right)$.

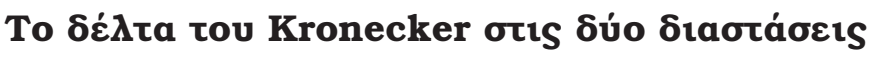

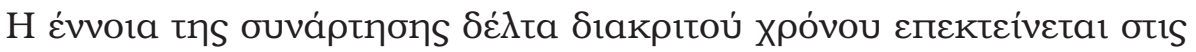

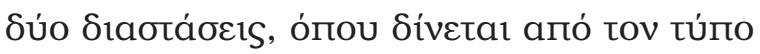

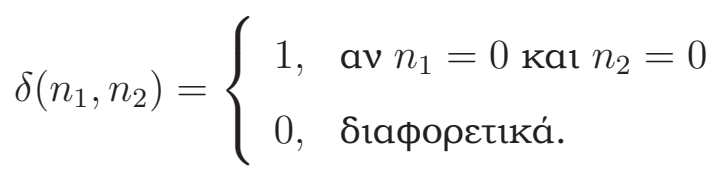




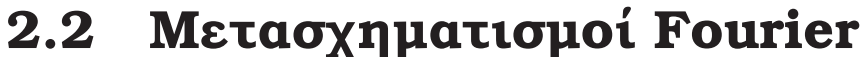

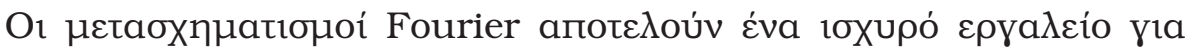

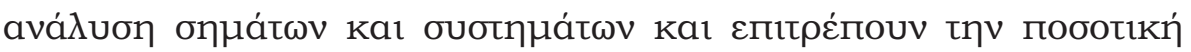

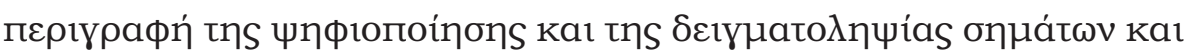

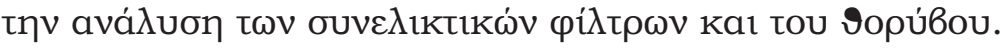

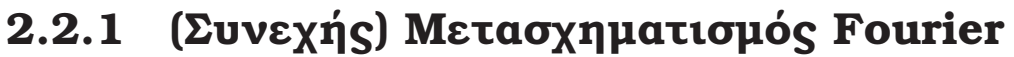

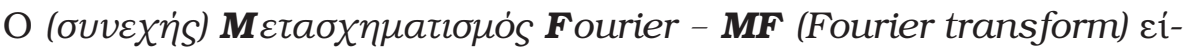

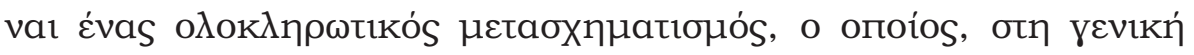

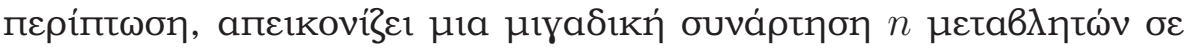

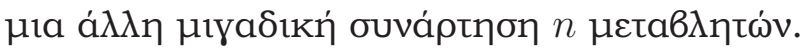

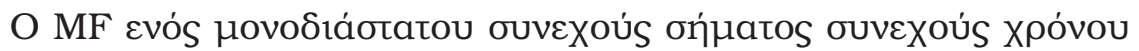

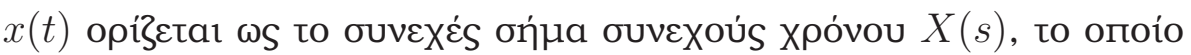

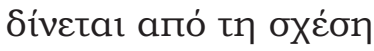

$$
\mathcal{F}\{x(t)\}=X(s)=\int_{-\infty}^{\infty} x(t) e^{i 2 \pi s t} d t
$$

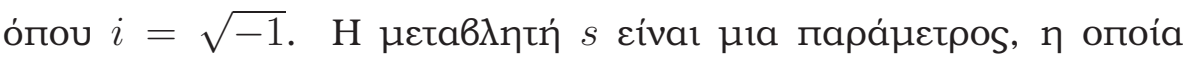

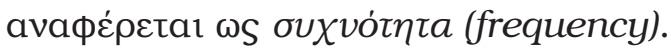

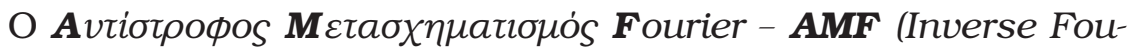

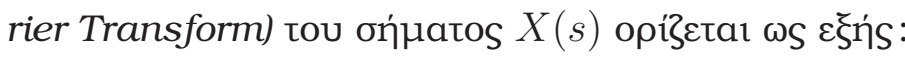

$$
\mathcal{F}^{-1}\{X(s)\}=\int_{-\infty}^{\infty} X(s) e^{-i 2 \pi s t} d s
$$

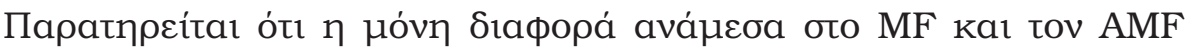

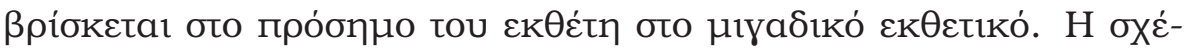




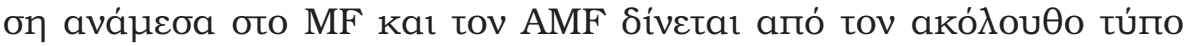
avtıброфńs:

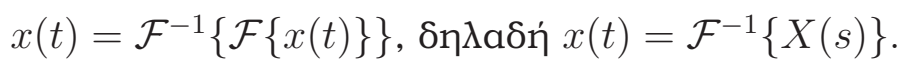

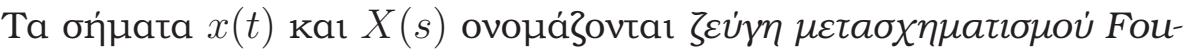

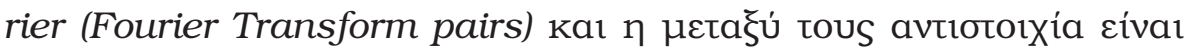

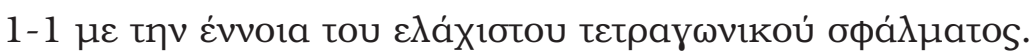

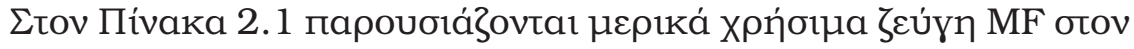
опоі́ $\operatorname{sinc}(\xi) \equiv \frac{\sin (\pi \xi)}{\pi \xi} \mu \varepsilon \operatorname{sinc}(0)=1$.

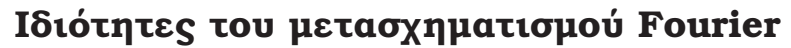

1. Граниккótпта

$$
\mathcal{F}\left\{\alpha_{1} x_{1}(t)+\alpha_{2} x_{2}(t)\right\}=\alpha_{1} \mathcal{F}\left\{x_{1}(t)\right\}+\alpha_{2} \mathcal{F}\left\{x_{2}(t)\right\} .
$$

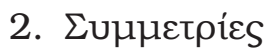

(a') $x(t)=x(-t) \Rightarrow X(s)=X(-s)$.

( $\left.\beta^{\prime}\right) x(t)=-x(-t) \Rightarrow X(s)=-X(-s)$.

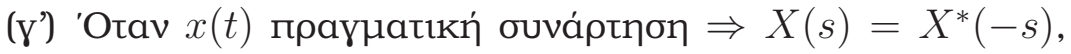

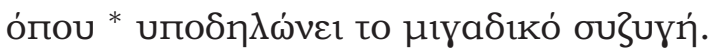

3. Мвtatónıon

$$
g(t)=x(t-a) \Rightarrow G(s)=e^{i 2 \pi a} X(s) .
$$




\begin{tabular}{|c|c|c|}
\hline ONOMA & $x(t)$ & $X(s)$ \\
\hline 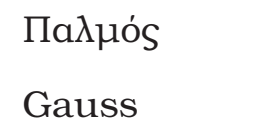 & $e^{-\pi t^{2}}$ & $e^{-\pi s^{2}}$ \\
\hline 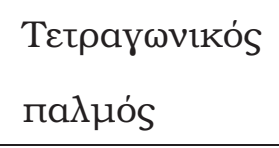 & $\Pi(t)= \begin{cases}1, & |t| \leq 1 \\
0, & \mathrm{a} \lambda \lambda \mathrm{t \omega}_{\mathrm{S}}\end{cases}$ & $2 \operatorname{sinc}(2 \mathrm{~s})=\frac{\sin (2 \pi \mathrm{s})}{\pi \mathrm{s}}$ \\
\hline 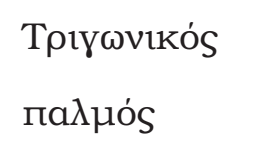 & $\Lambda(t)= \begin{cases}1-|t|, & |t| \leq 1 \\
0, & |t|>1\end{cases}$ & $(\operatorname{sinc})^{2}(\mathrm{~s})=\frac{\sin ^{2}(\pi \mathrm{s})}{(\pi \mathrm{s})^{2}}$ \\
\hline $\begin{array}{l}\text { Ф日ivov } \\
\varepsilon k \theta \varepsilon t 1 k o ́\end{array}$ & $e^{\alpha|t|}, \quad \alpha<0$ & $\frac{-2 \alpha}{\alpha^{2}+4 \pi^{2} s^{2}}$ \\
\hline 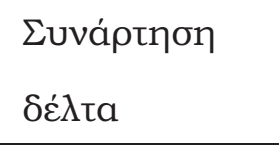 & $\delta(t)$ & 1 \\
\hline 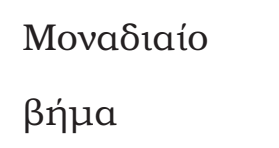 & $u_{s}(t)$ & $\frac{1}{2}\left[\delta(s)+\frac{i}{\pi s}\right]$ \\
\hline 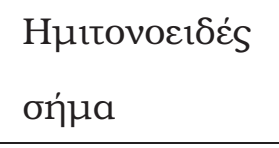 & $\sin \left(2 \pi s_{0}\right)$ & $-\frac{i}{2}\left[\delta\left(s+s_{0}\right)-\delta\left(s-s_{0}\right)\right]$ \\
\hline 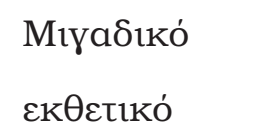 & $e^{i 2 \pi s_{0}}$ & $\delta\left(s-s_{0}\right)$ \\
\hline
\end{tabular}

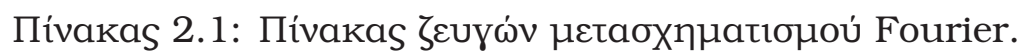


4. Opoiótnta

$$
g(t)=x(a t) \Rightarrow G(s)=\frac{1}{|a|} X\left(\frac{s}{a}\right) .
$$

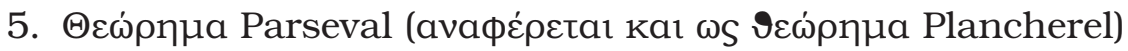

$$
\int_{-\infty}^{\infty}|x(t)|^{2} d t=\int_{-\infty}^{\infty}|X(s)|^{2} d s
$$

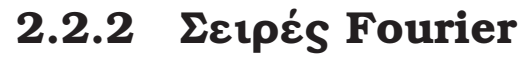

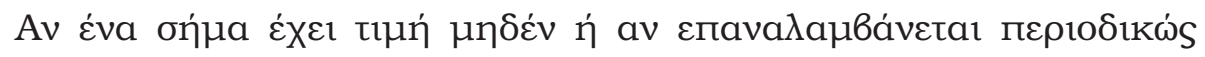

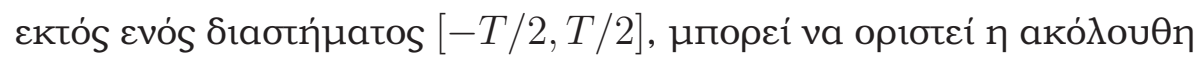
oєџó Fourier (Fourier series):

$$
X_{n}=\int_{-\frac{T}{2}}^{\frac{T}{2}} x(t) e^{i 2 \pi n \frac{t}{T}} d t=\left.\mathcal{F}\{x(t)\}\right|_{s=\frac{n}{T}}, n=0, \pm 1, \pm 2, \ldots
$$

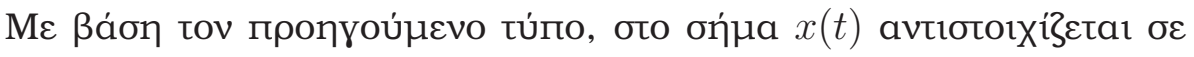

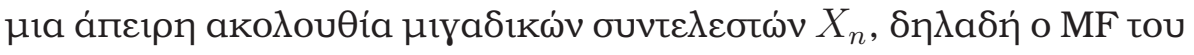
бท́

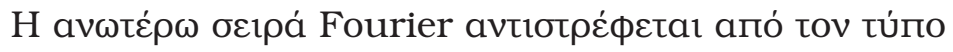

$$
x(t)=\frac{1}{T} \sum_{n=-\infty}^{\infty} X_{n} e^{-i 2 \pi n \frac{t}{T}} .
$$

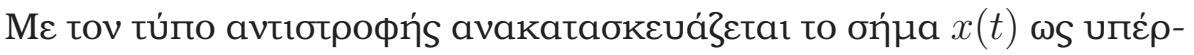

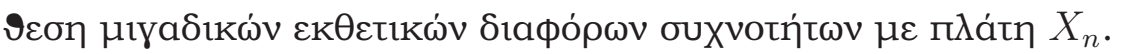

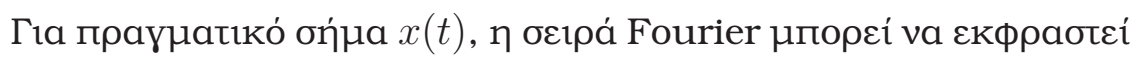

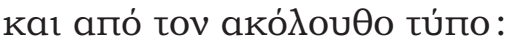

$$
x(t)=\frac{a_{0}}{2}+\sum_{n=1}^{\infty} a_{n} \cos \left(\frac{2 \pi n t}{T}\right)+\sum_{n=1}^{\infty} b_{n} \sin \left(\frac{2 \pi n t}{T}\right),
$$


о́поu

$$
\begin{aligned}
& a_{n}=\frac{2}{T} \int_{-\frac{T}{2}}^{\frac{T}{2}} x(t) \cos \left(\frac{2 \pi n t}{T}\right) d t, n=0,1,2, \ldots \\
& b_{n}=\frac{2}{T} \int_{-\frac{T}{2}}^{\frac{T}{2}} x(t) \sin \left(\frac{2 \pi n t}{T}\right) d t, n=1,2,3, \ldots .
\end{aligned}
$$

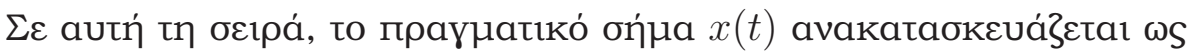

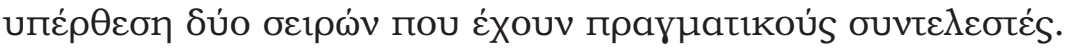

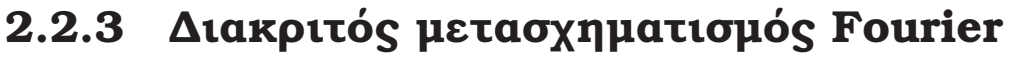

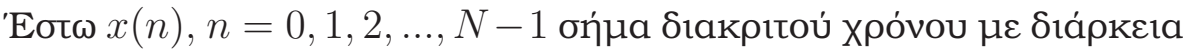

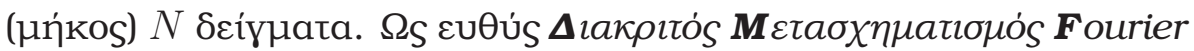
- $\boldsymbol{\Delta M}$ M (Discrete Fourier Transform) tou ońjuatos autoú opí̧દtaı to

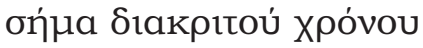

$$
X(m)=\sum_{n=0}^{N-1} x(n) e^{i 2 \pi \frac{m n}{N}}, m=0,1,2, \ldots, N-1 .
$$

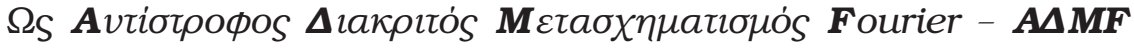
(Inverse Discrete Fourier Transform) tou oń $0,1,2, \ldots, N-1$ opí̧єtaı to ớ

$$
g(n)=\frac{1}{N} \sum_{m=0}^{N-1} X(m) e^{-i 2 \pi \frac{m n}{N}}, n=0,1,2, \ldots, N-1 .
$$

Ioxúє1 $g(n)=x(n), n=0,1,2, \ldots, N-1$.

O $\Delta \mathrm{MF} \mu п о \rho \varepsilon i$ va

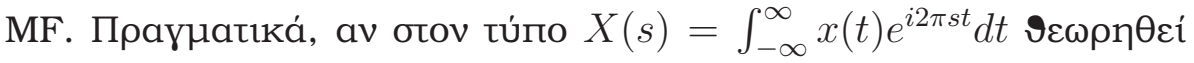
$s=\frac{m}{\Delta t}$, ouváyetaı ótı

$$
X\left(\frac{m}{\Delta t}\right)=\int_{-\infty}^{\infty} x(t) e^{i 2 \pi \frac{m t}{\Delta t}} d t .
$$




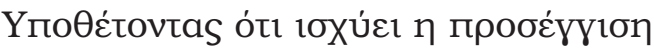

$$
X\left(\frac{m}{\Delta t}\right)=\int_{-\infty}^{\infty} x(t) e^{i 2 \pi \frac{m t}{\Delta t}} d t \approx \Delta t \sum_{n=0}^{N-1} x(n \Delta t) e^{i 2 \pi \frac{m n}{N}}
$$

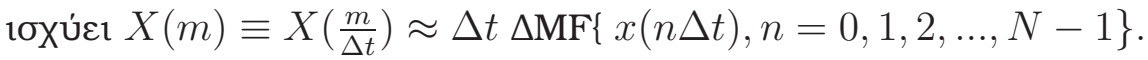

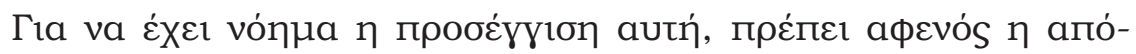

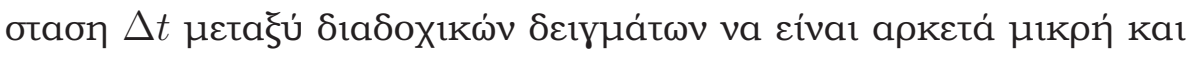

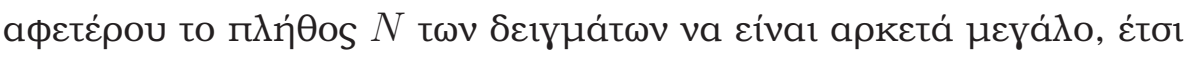

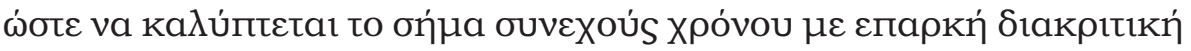

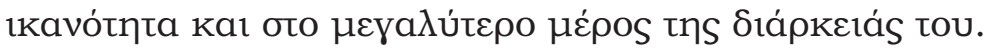

\subsubsection{Taxeis aגyópı $\theta$ por $\Delta \mathrm{MF}$}

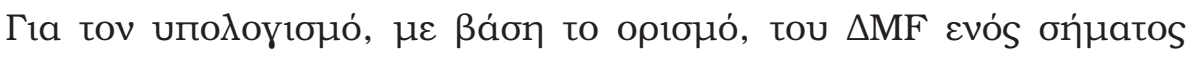

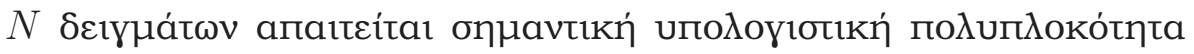

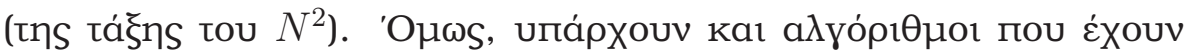

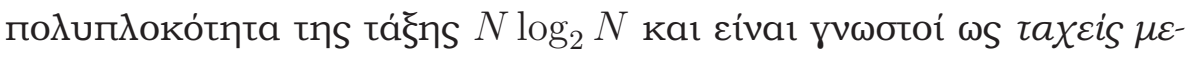

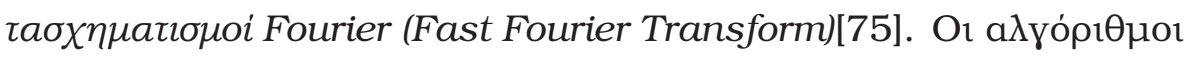

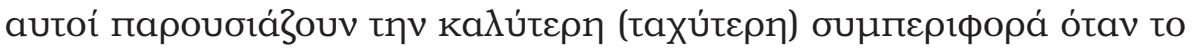

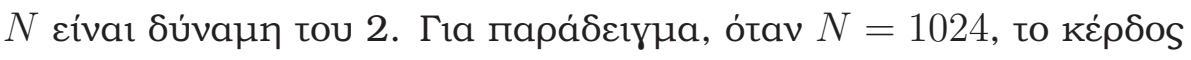

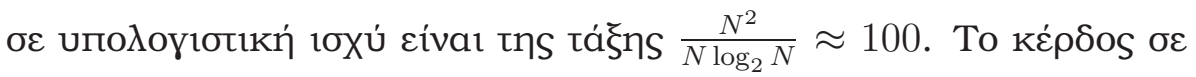

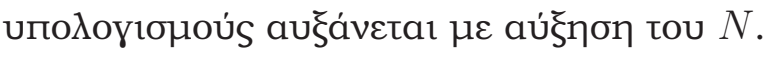

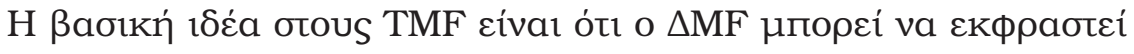

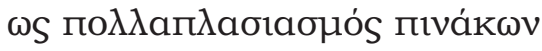




$$
\left[\begin{array}{c}
X_{0} \\
\vdots \\
X_{N-1}
\end{array}\right]=\underline{\underline{W}}\left[\begin{array}{c}
x_{0} \\
\vdots \\
x_{N-1}
\end{array}\right] \text {, о́поч } \underline{\underline{W}}=\left[W_{n m}\right]_{N \times N}=\left[e^{i \frac{2 \pi n m}{N}}\right]_{N \times N} \text {. }
$$

'Opoia

$$
\left[\begin{array}{c}
x_{0} \\
\vdots \\
x_{N-1}
\end{array}\right]=\frac{1}{N} \underline{\underline{W}}^{\dagger}\left[\begin{array}{c}
X_{0} \\
\vdots \\
X_{N-1}
\end{array}\right]
$$

$\mu \varepsilon{\underline{\underline{W^{\dagger}}}}^{\dagger}{\underline{\underline{W^{*}}}}^{\top \top}={\underline{\underline{W^{*}}}}^{*}$.

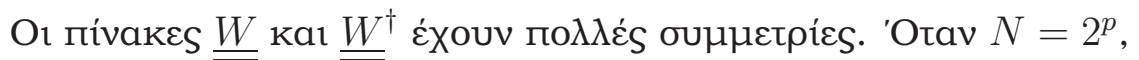

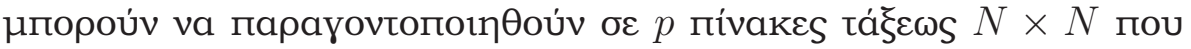

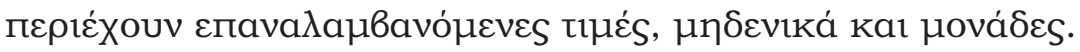

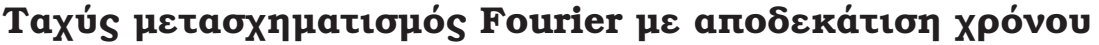

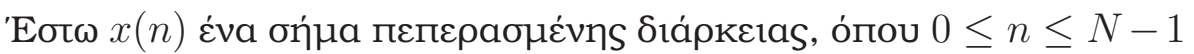

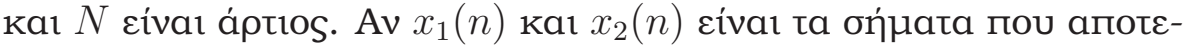

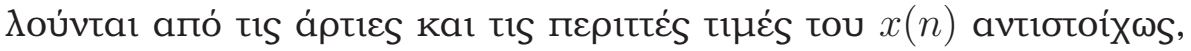

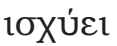

$$
\begin{gathered}
x_{1}(n)=x(2 n), n=0,1,2, \ldots, \frac{N}{2}-1, \\
x_{2}(n)=x(2 n+1), n=0,1,2, \ldots, \frac{N}{2}-1 .
\end{gathered}
$$

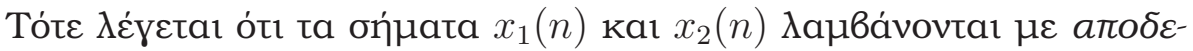

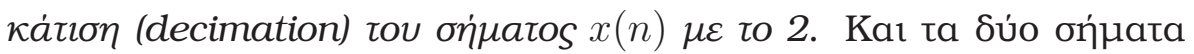

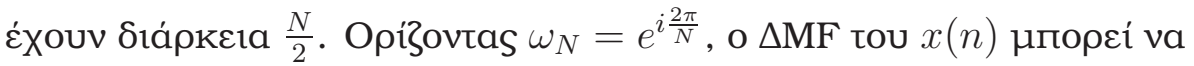

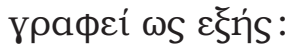




$$
X(k)=\sum_{n=0,2,4, \ldots} x(n) \omega_{N}^{n k}+\sum_{n=1,3,5, \ldots} x(n) \omega_{N}^{n k}
$$

ń

$$
X(k)=\sum_{n=0}^{\frac{N}{2}-1} x(2 n) \omega_{N}^{2 n k}+\sum_{n=0}^{\frac{N}{2}-1} x(2 n+1) \omega_{N}^{2(n+1) k} .
$$

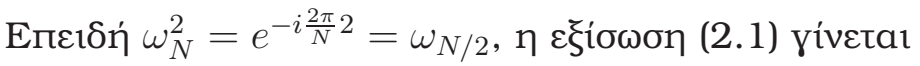

$$
X(k)=\sum_{n=0}^{\frac{N}{2}-1} x_{1}(n) \omega_{N / 2}^{n k}+\omega_{N}^{k} \sum_{n=0}^{\frac{N}{2}-1} x_{2}(n) \omega_{N / 2}^{n k},
$$

опо́єє

$$
X(k)=X_{1}(k)+\omega_{N}^{k} X_{2}(k), \quad k=0,1,2, \ldots, \frac{N}{2}-1 .
$$

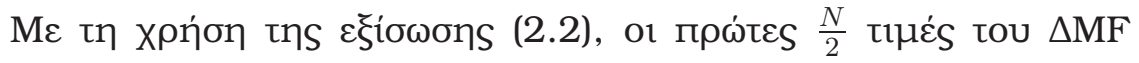

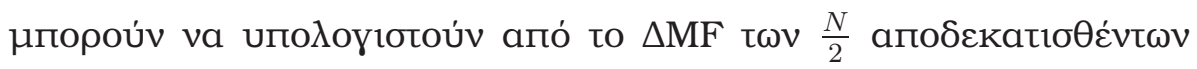

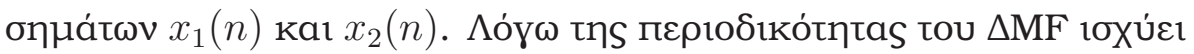

$$
X_{1}\left(k+\frac{N}{2}\right)=X_{1}(k), \quad X_{2}\left(k+\frac{N}{2}\right)=X_{2}(k) .
$$

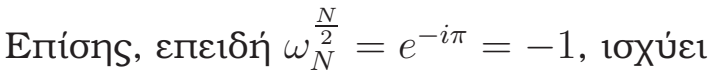

$$
\omega_{N}^{k+\frac{N}{2}}=\omega_{N}^{k} \omega_{N}^{\frac{N}{2}}=-\omega_{N}^{k}
$$

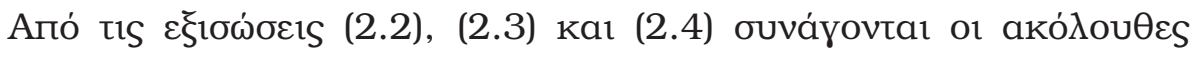

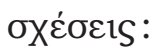

$$
\begin{aligned}
X(k) & =X_{1}(k)+\omega_{N}^{k} X_{2}(k), & 0 \leq k \leq \frac{N}{2}-1 \\
X\left(k+\frac{N}{2}\right) & =X_{1}(k)-\omega_{N}^{k} X_{2}(k), & 0 \leq k \leq \frac{N}{2}-1 .
\end{aligned}
$$




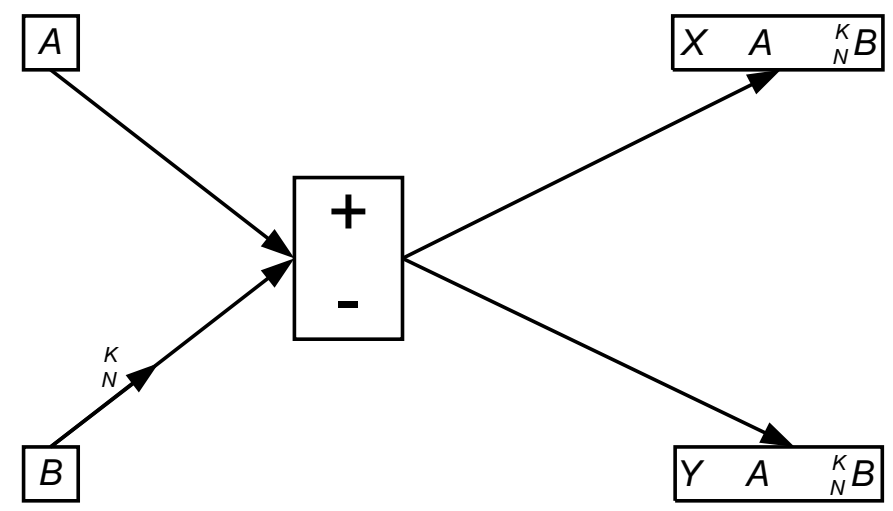

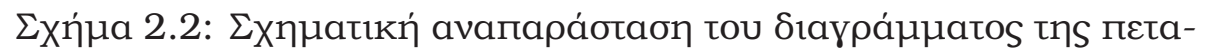

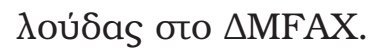

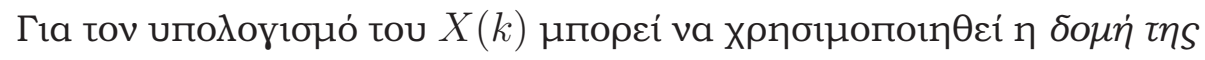

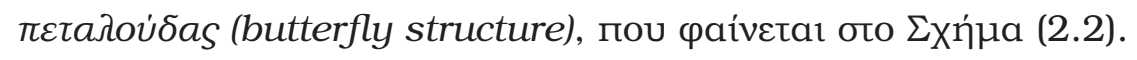

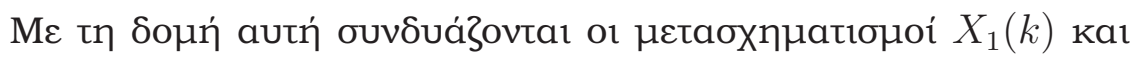

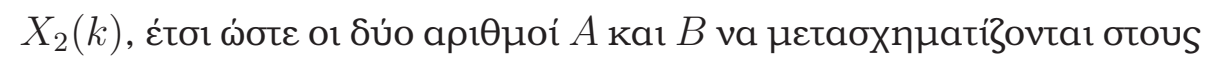
$X$ kaı $Y$ oú $\phi \omega v a ~ \mu \varepsilon$ tov kavóva

$$
\begin{aligned}
& X=A+\omega_{N}^{k} B \\
& Y=A-\omega_{N}^{k} B .
\end{aligned}
$$

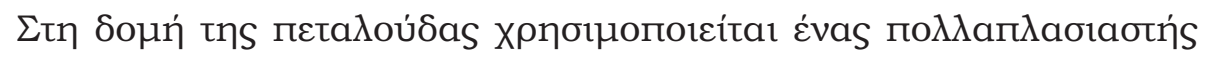
ka1 évas aӨporotńs.

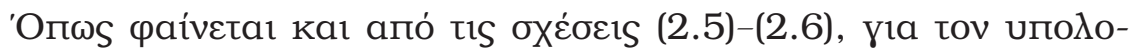

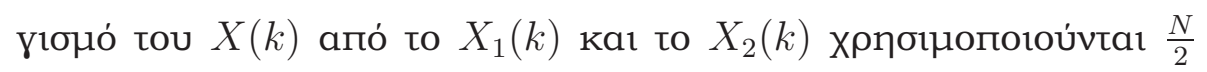

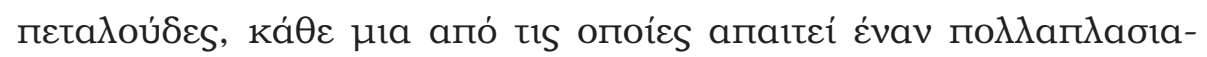

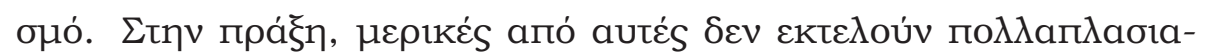




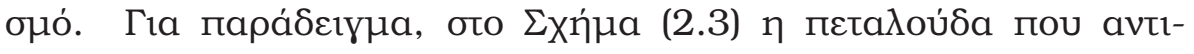

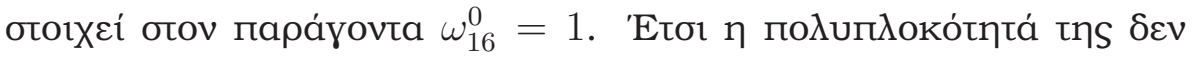

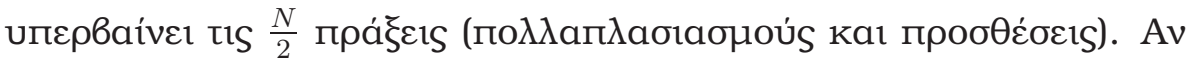

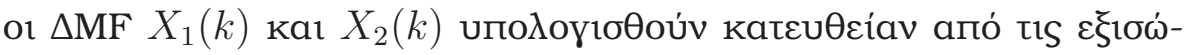

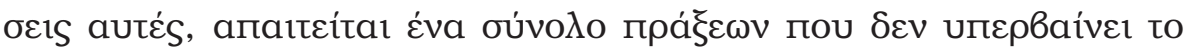

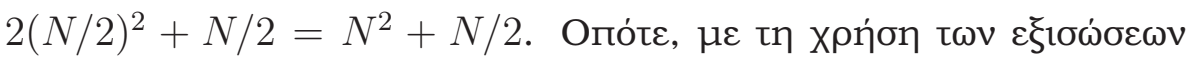

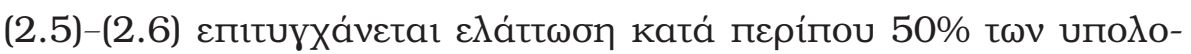

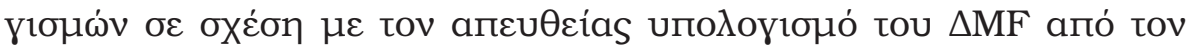
opıouó tou.

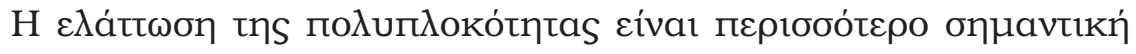

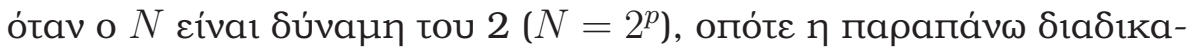

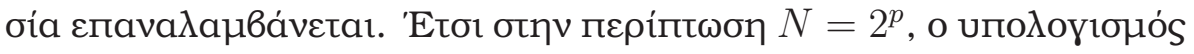

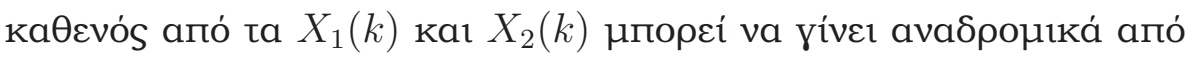

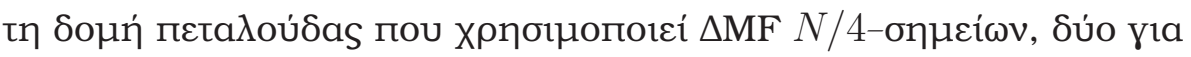

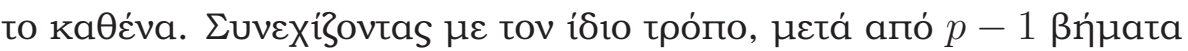

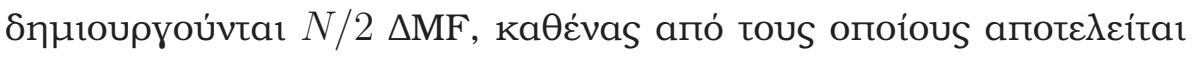
aпó 2 onjeía.

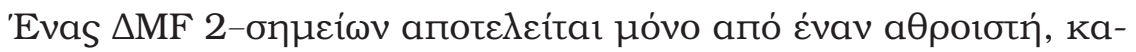
จ่́s $\omega_{2}^{0}=1, \omega_{2}^{1}=-1 \mathrm{ka}$

$$
\begin{aligned}
& X(0)=x(0)+x(1) \\
& X(1)=x(0)-x(1) .
\end{aligned}
$$

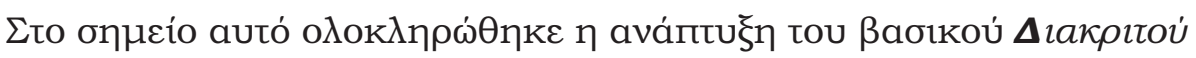

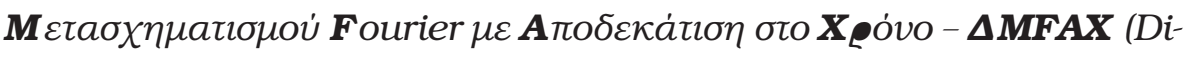

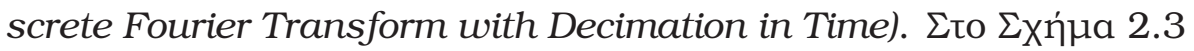




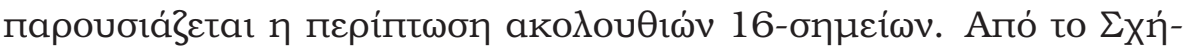

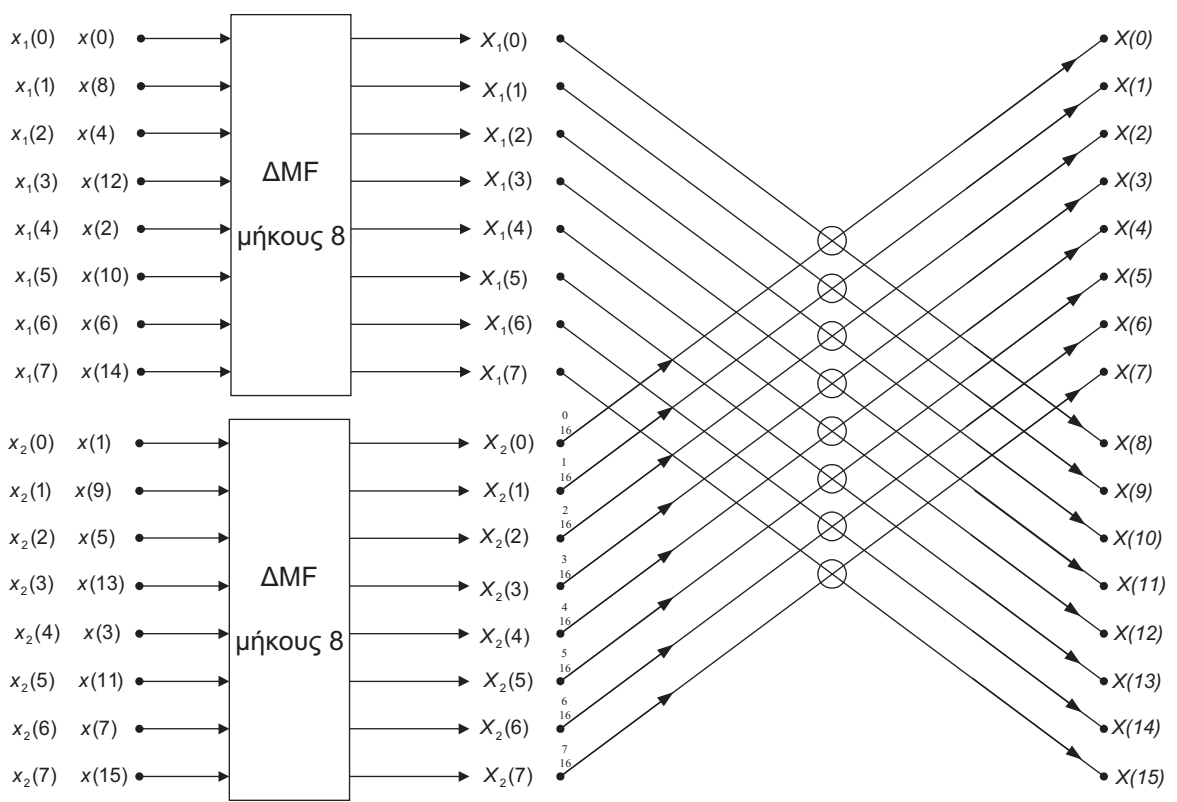

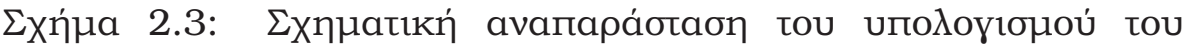
$\triangle$ MFAX.

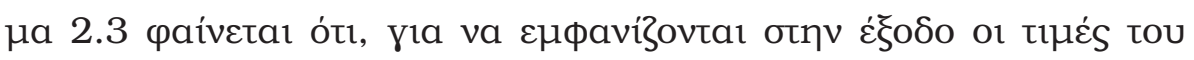

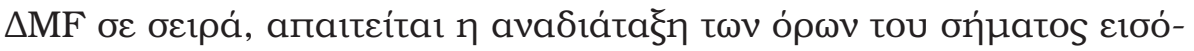

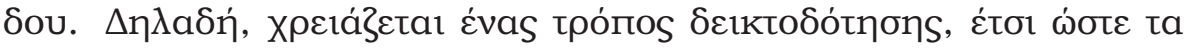

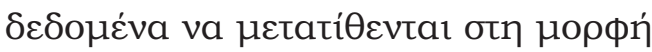

$$
\begin{aligned}
& (x(0), x(8), x(4), x(12), x(2), x(10), x(6), x(14), \\
& x(1), x(9), x(5), x(13), x(32), x(11), x(7), x(15)) .
\end{aligned}
$$

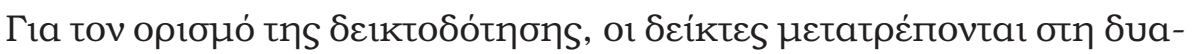

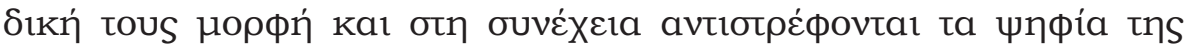

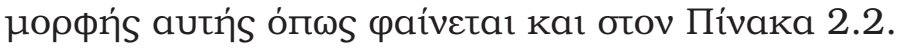




\begin{tabular}{|c|c|c|c|}
\hline 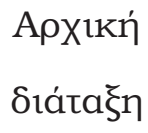 & 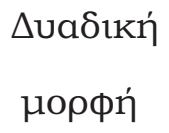 & 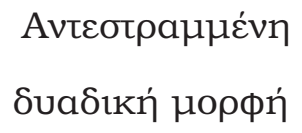 & 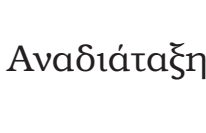 \\
\hline 0 & 0000 & 0000 & 0 \\
\hline 1 & 0001 & 1000 & 8 \\
\hline 2 & 0010 & 0100 & 4 \\
\hline 3 & 0011 & 1100 & 12 \\
\hline 4 & 0100 & 0010 & 2 \\
\hline 5 & 0101 & 1010 & 10 \\
\hline 6 & 0110 & 0110 & 6 \\
\hline 7 & 0111 & 1110 & 14 \\
\hline 8 & 1000 & 0001 & 1 \\
\hline 9 & 1001 & 1001 & 9 \\
\hline 10 & 1010 & 0101 & 5 \\
\hline 11 & 1011 & 1101 & 13 \\
\hline 12 & 1100 & 0011 & 3 \\
\hline 13 & 1101 & 1011 & 11 \\
\hline 14 & 1110 & 0111 & 7 \\
\hline 15 & 1111 & 1111 & 15 \\
\hline
\end{tabular}

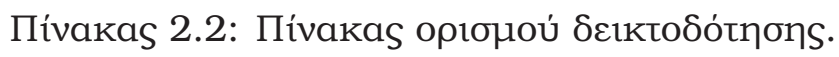




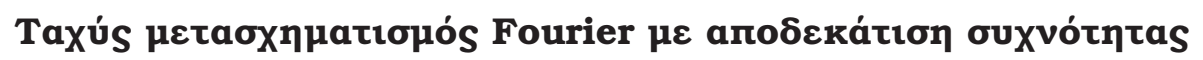

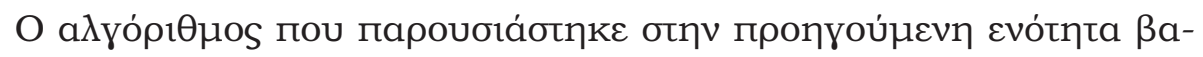

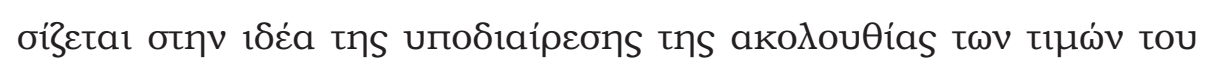

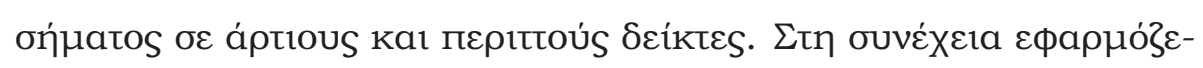

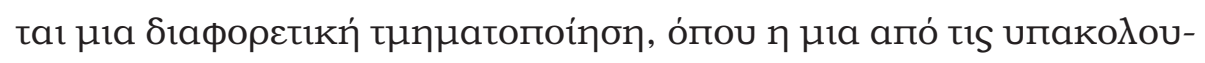

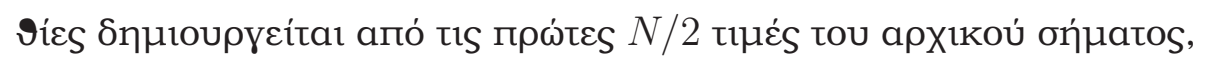

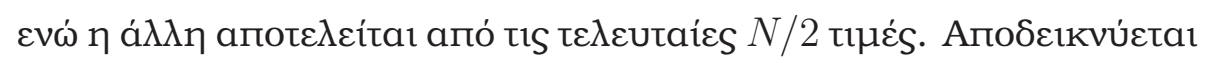

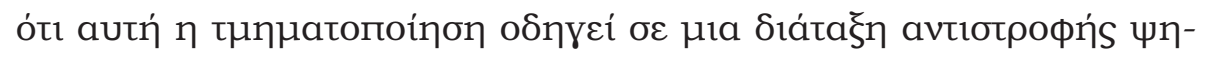

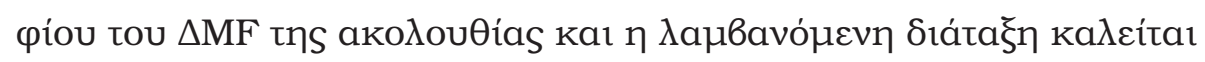

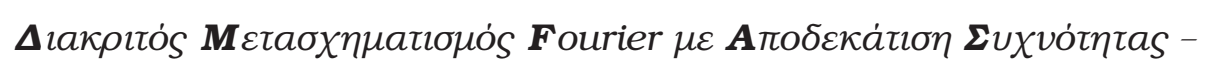
$\triangle$ MFA (Discrete Fourier Transform with Decimation in Frequency).

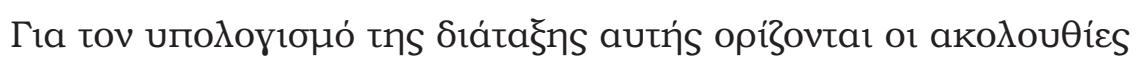

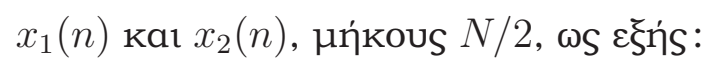

$$
\begin{gathered}
x_{1}(n)=x(n), \quad n=0,1,2, \ldots ., \frac{N}{2}-1 \\
x_{2}(n)=x\left(n+\frac{N}{2}\right), \quad n=0,1,2, \ldots ., \frac{N}{2}-1 .
\end{gathered}
$$

Tóte o $\Delta \mathrm{MF}$ yívetal

$$
X(k)=\sum_{n=0}^{\frac{N}{2}-1} x(n) \omega_{N}^{n k}+\sum_{n=\frac{N}{2}}^{N-1} x(n) \omega_{N}^{n k},
$$

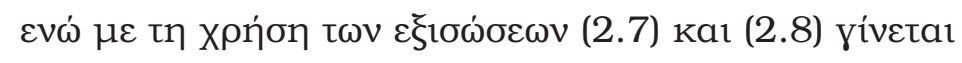

$$
X(k)=\sum_{n=0}^{\frac{N}{2}-1}\left[x_{1}(n)+e^{-i \pi k} x_{2}(n)\right] \omega_{N}^{n k} .
$$




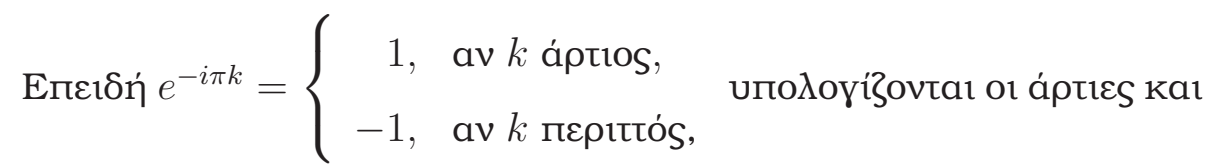

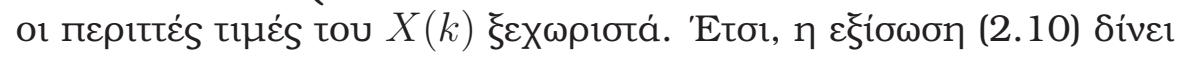

$$
\begin{aligned}
X(2 k) & =\sum_{n=0}^{\frac{N}{2}-1}\left[x_{1}(n)+x_{2}(n)\right] \omega_{\frac{N}{2}}^{n k} \\
X(2 k+1) & =\sum_{n=0}^{\frac{N}{2}-1}\left[x_{1}(n)-x_{2}(n)\right] \omega_{N}^{n k} \omega_{\frac{N}{2}}^{n k} .
\end{aligned}
$$

Av $\tau \varepsilon \theta \varepsilon i$

$$
f(n)=x_{1}(n)+x_{2}(n), \quad n=0,1,2, \ldots, \frac{N}{2}-1
$$

kal

$$
g(n)=\left[x_{1}(n)-x_{2}(n)\right] \omega_{N}^{n k}, \quad n=0,1,2, \ldots, \frac{N}{2}-1,
$$

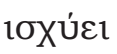

$$
X(2 k)=F(k), \quad X(2 k+1)=G(k), \quad k=0,1,2, \ldots, \frac{N}{2}-1 .
$$

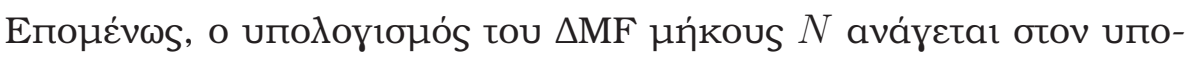

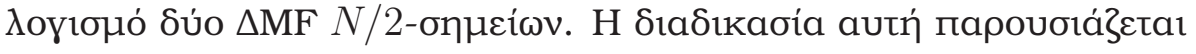

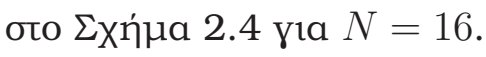

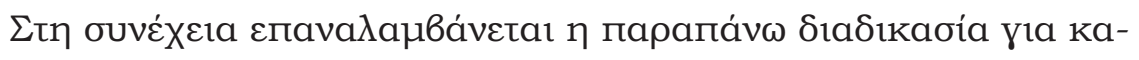

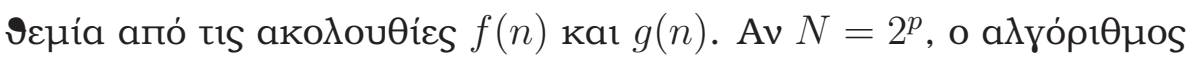

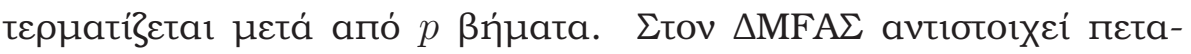

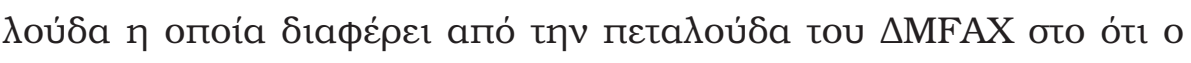

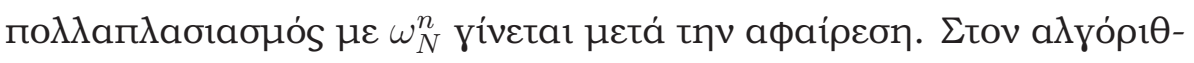

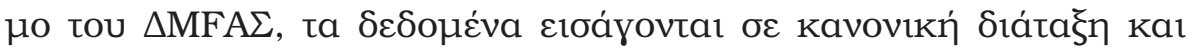




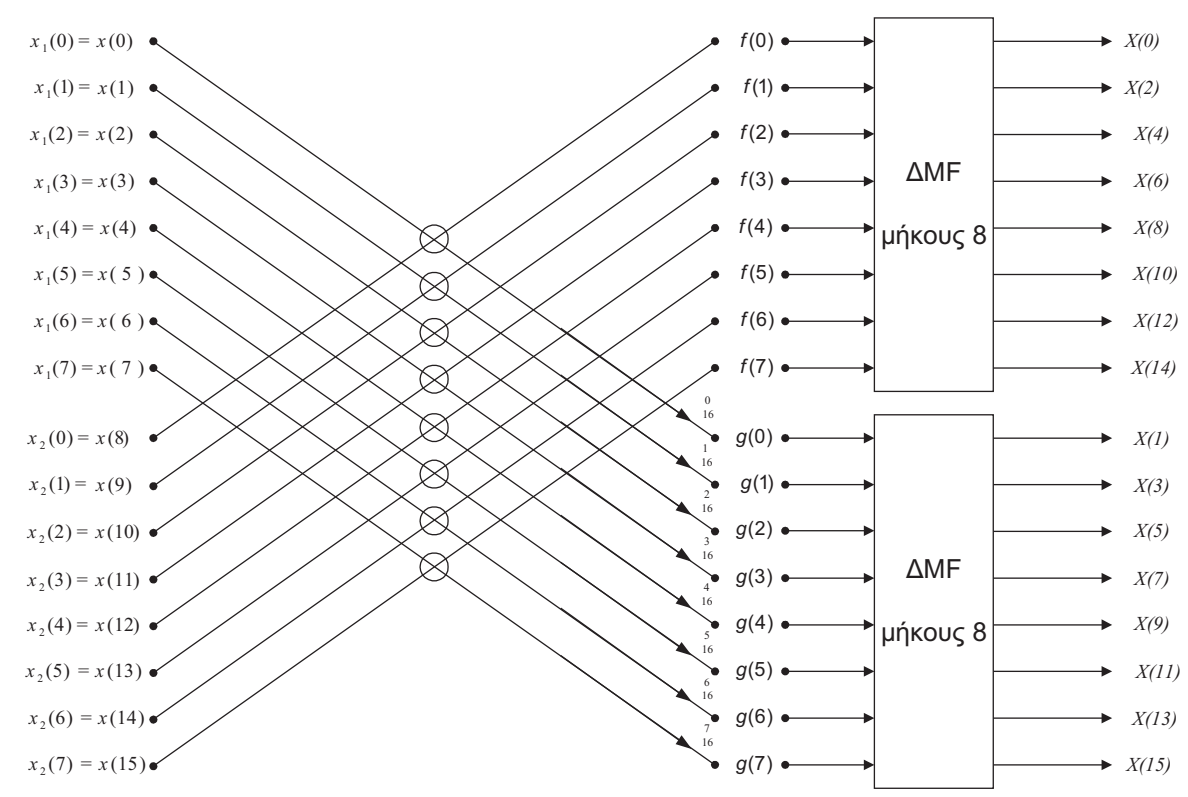

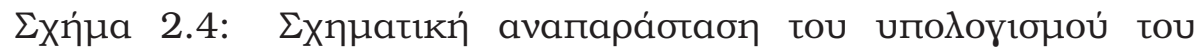
$\triangle \mathrm{MFA} \Sigma$.

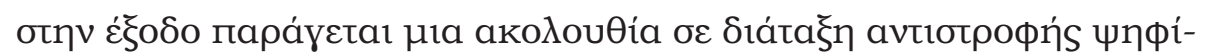

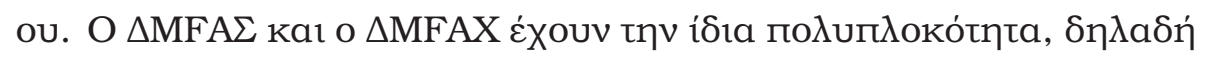

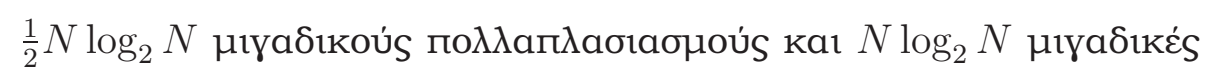

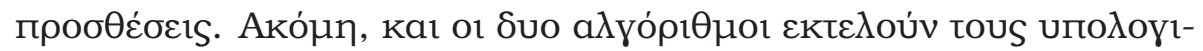

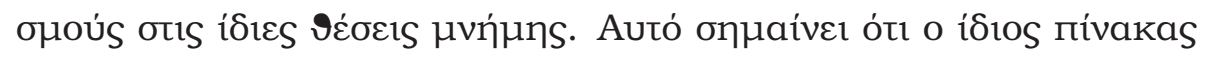

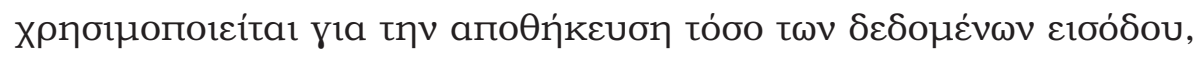

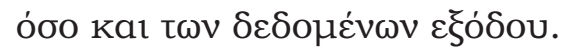




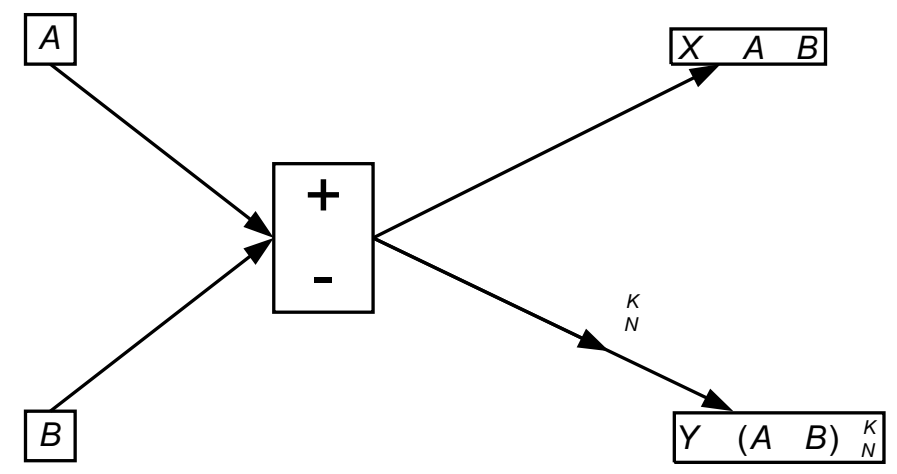

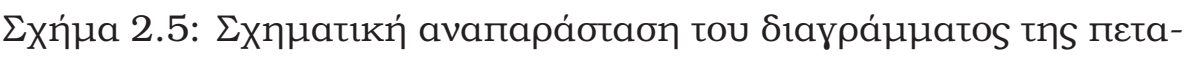

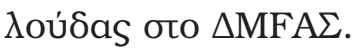

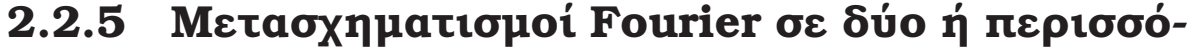

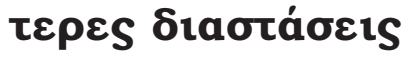

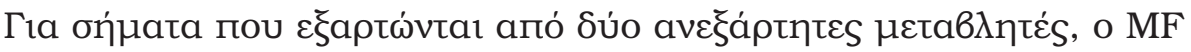
ka1 o avtiotpoфós tou opißovtal wS

$$
F(u, v)=\int_{-\infty}^{\infty} \int_{-\infty}^{\infty} f(x, y) e^{i 2 \pi(u x+v y)} d x d y
$$

Kal

$$
f(x, y)=\int_{-\infty}^{\infty} \int_{-\infty}^{\infty} F(u, v) e^{-i 2 \pi(u x+v y)} d u d v
$$

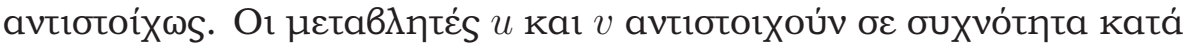

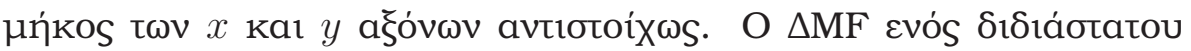

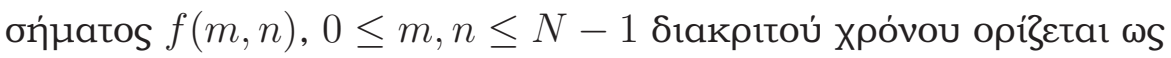

$$
F(k, l)=\sum_{m=0}^{N-1} \sum_{n=0}^{N-1} f(m, n) e^{i 2 \pi \frac{k m+l n}{N}}, 0 \leq k, l \leq N-1
$$




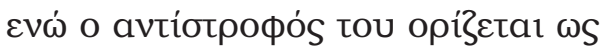

$$
f(m, n)=\frac{1}{N^{2}} \sum_{k=0}^{N-1} \sum_{l=0}^{N-1} F(k, l) e^{-i 2 \pi \frac{k m+l n}{N}}, 0 \leq m, n \leq N-1 .
$$

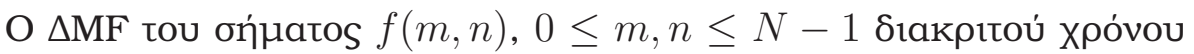

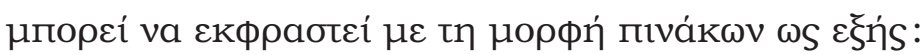

$$
\underline{\underline{f}}=[f(m, n)], \underline{\underline{F}}=[F(k, l)], \underline{\underline{W}}=\left[w_{k n}\right]=\left[e^{i 2 \pi \frac{k n}{N}}\right] .
$$

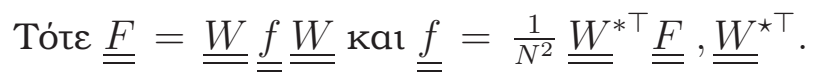

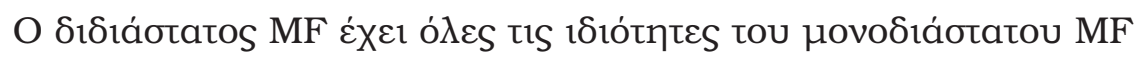

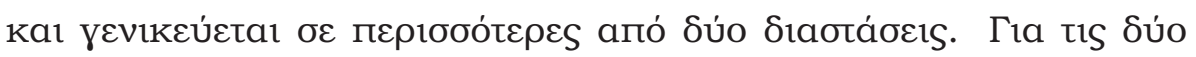

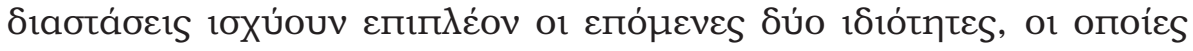

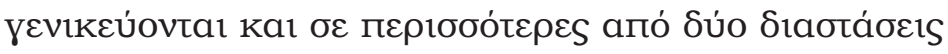

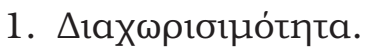

Av $f(x, y)=f_{1}(x) f_{2}(y)$, tóte $F(u, v)=F_{1}(u) F_{2}(v) . \Delta \eta \lambda a \delta \eta ́ n$,

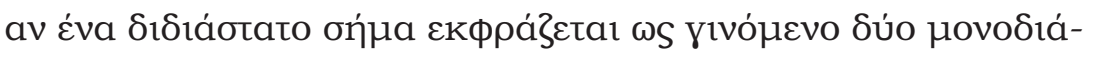

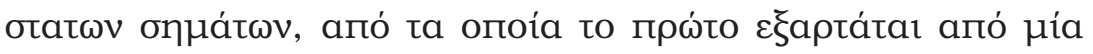

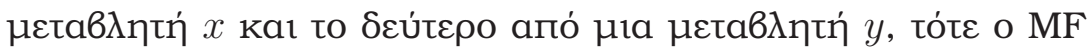

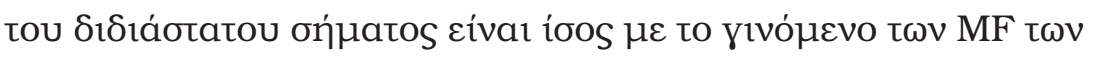

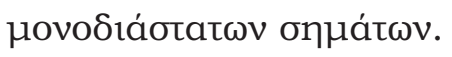

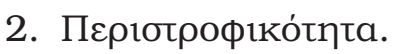

Av $g(x, y)=f(x \cos \theta+y \sin \theta,-x \sin \theta+y \cos \theta)$, tótع $G(u, v)=F(u \cos \theta+v \sin \theta,-u \sin \theta+v \cos \theta) . \Delta \eta \lambda a \delta \dot{n}, a v$

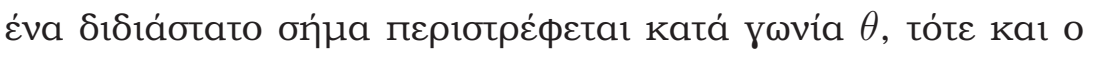

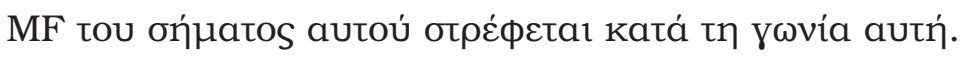




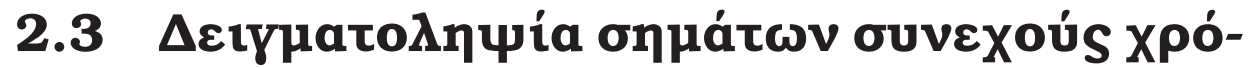 VOU}

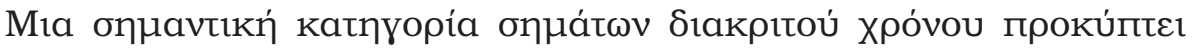

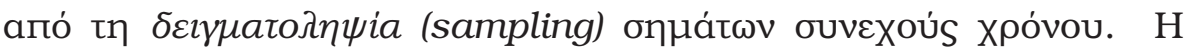

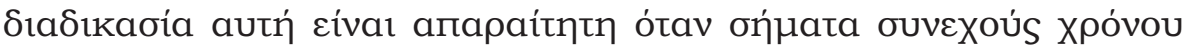

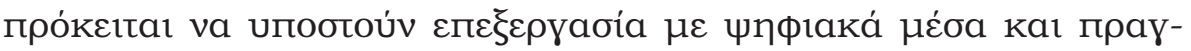

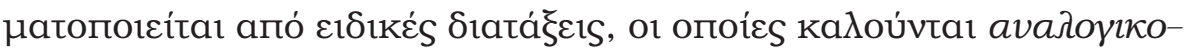

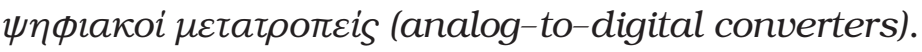

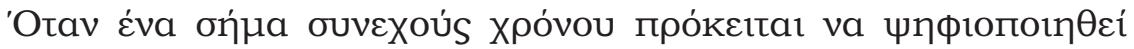

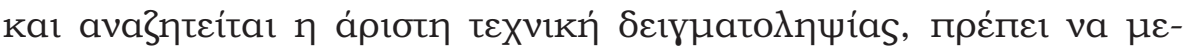

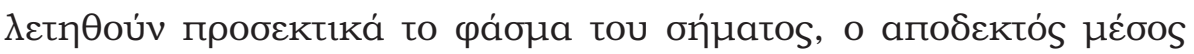

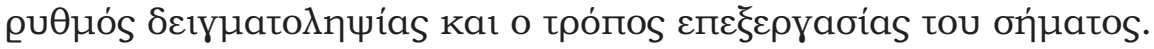

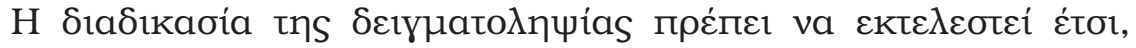

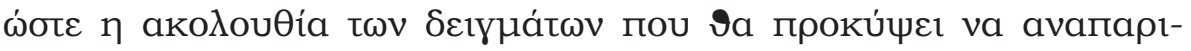

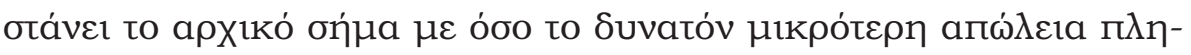

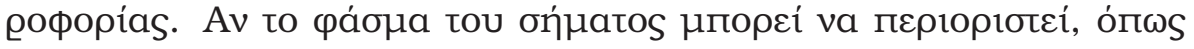

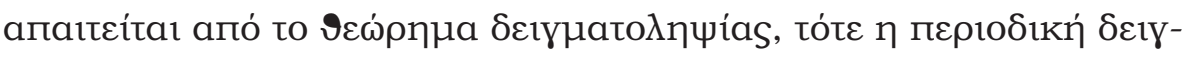

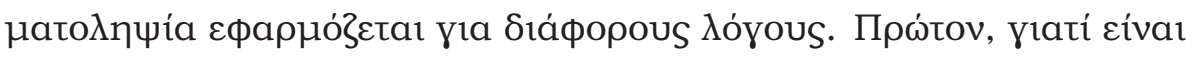

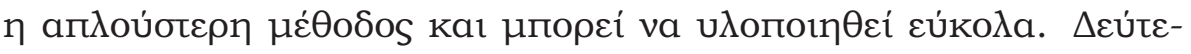

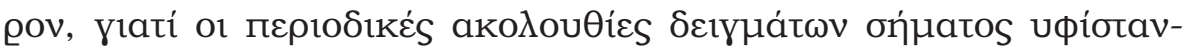

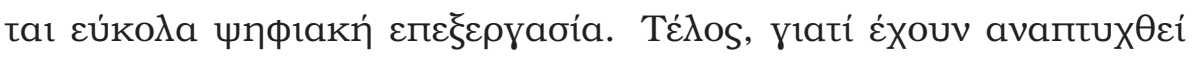

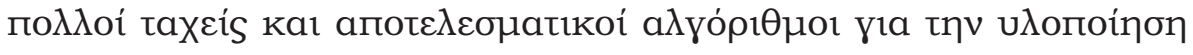

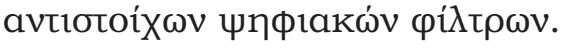




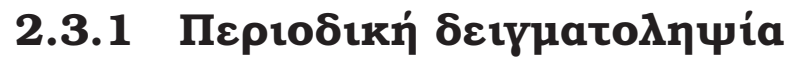

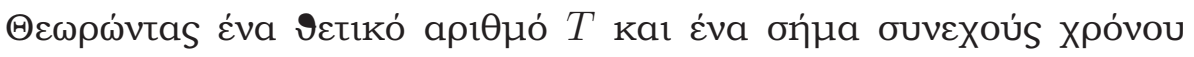
$x(t)$, גa

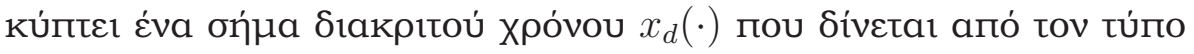

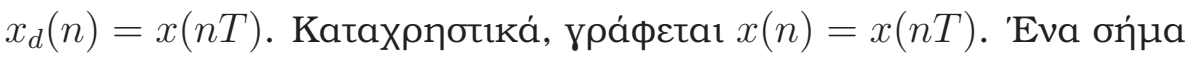

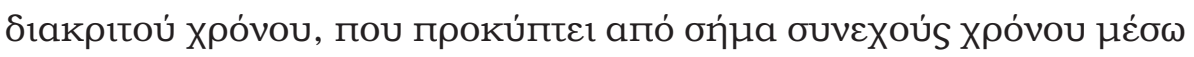

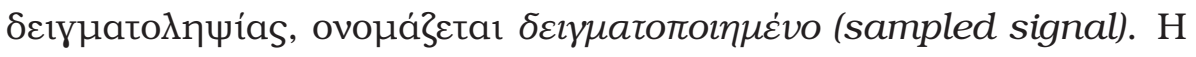

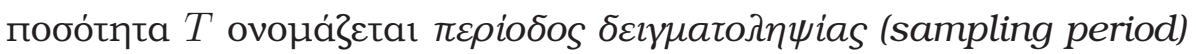

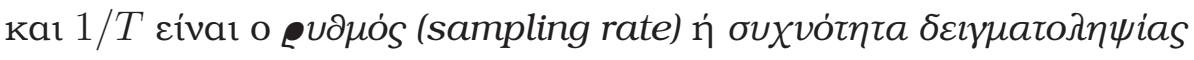
(sampling frequency).

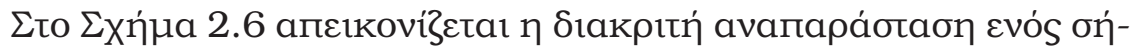

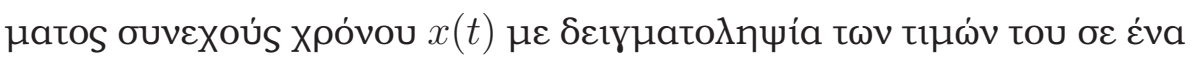

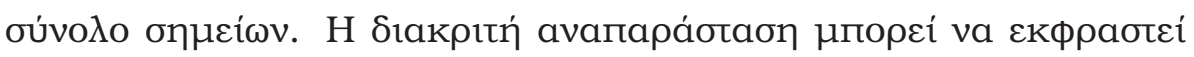

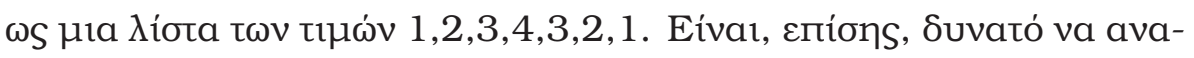

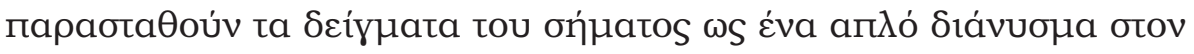

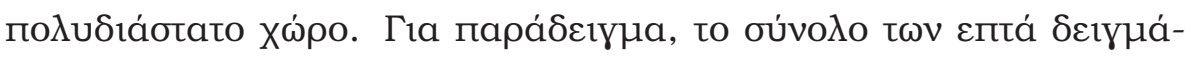

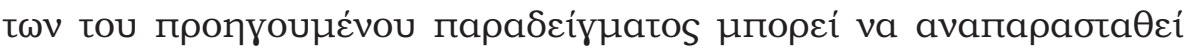

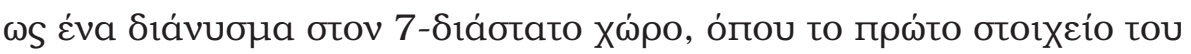

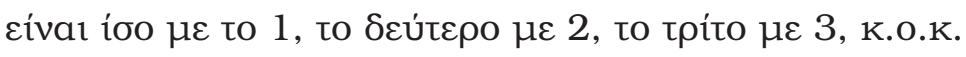

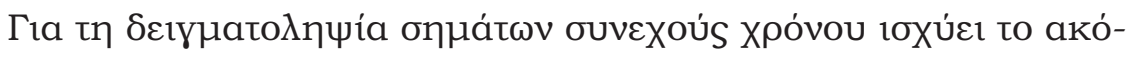

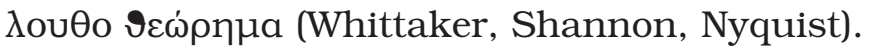



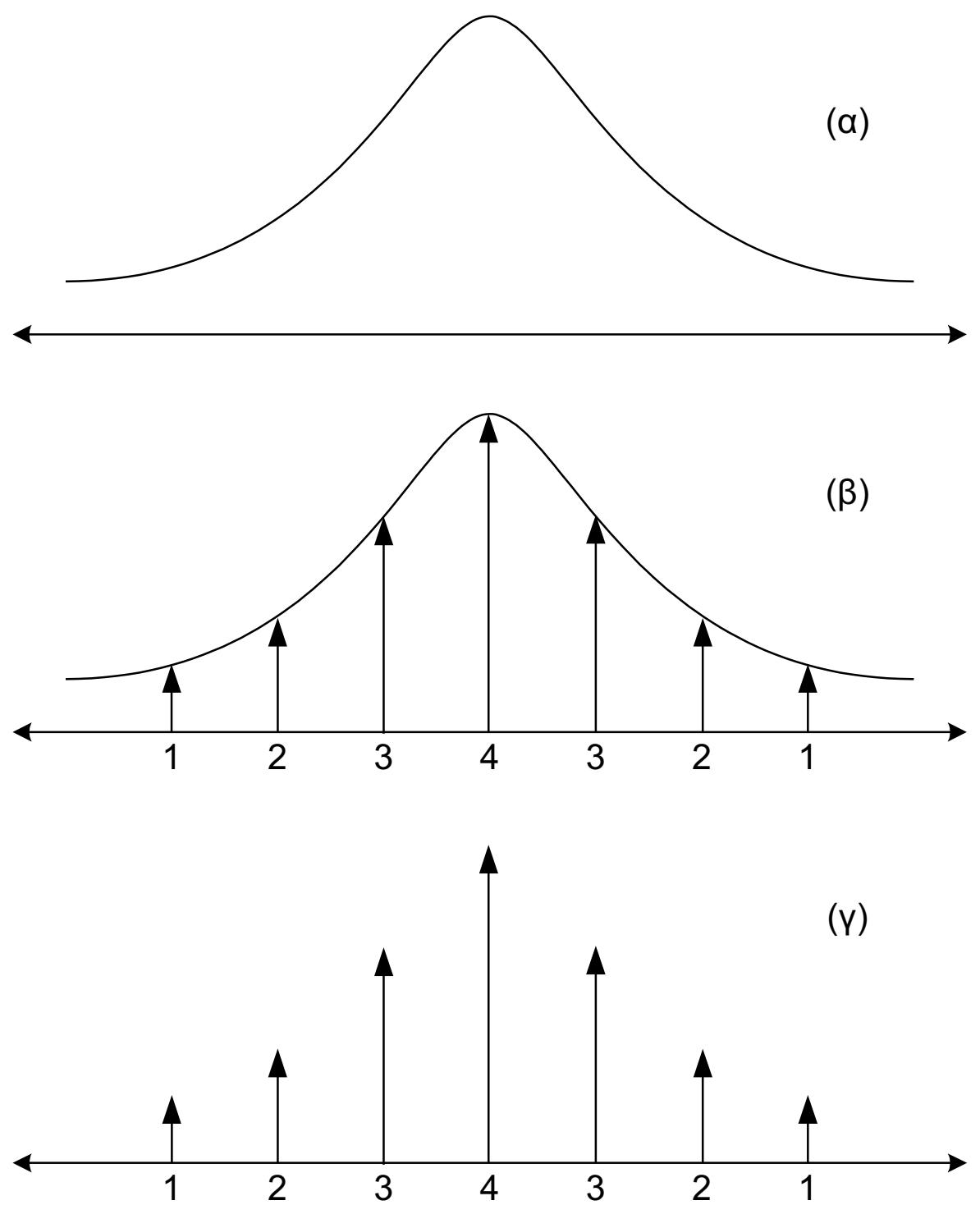

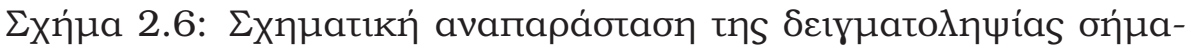

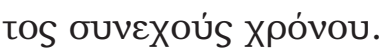




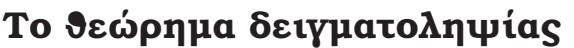

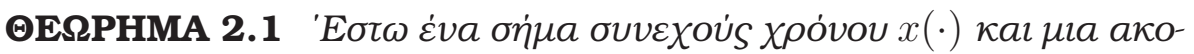

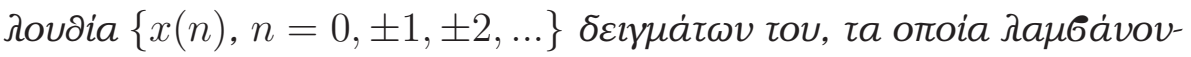

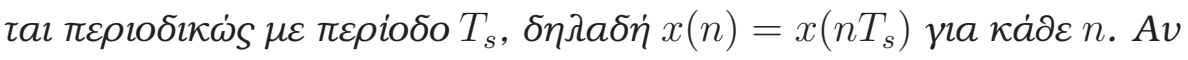

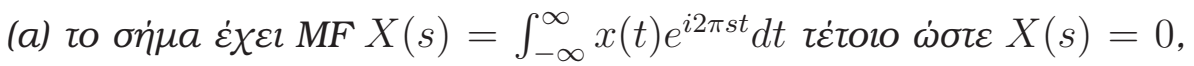

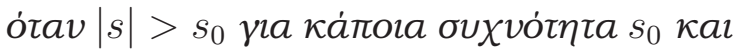

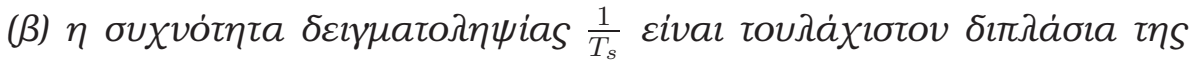

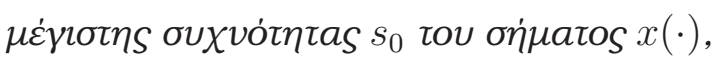

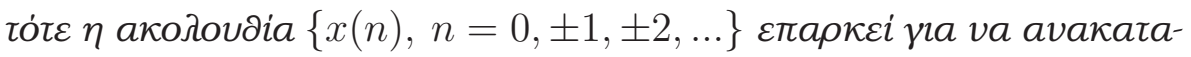

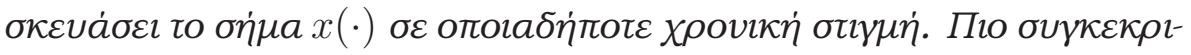
$\mu \dot{\varepsilon} v a:$

$$
x(t)=\sum_{n=-\infty}^{\infty} x(n) \frac{\sin \frac{\pi\left(t-n T_{s}\right)}{T_{s}}}{\frac{\pi\left(t-n T_{s}\right)}{T_{s}}}=\sum_{n=-\infty}^{\infty} x\left(n T_{s}\right) \operatorname{sinc}\left(\frac{t-n T_{s}}{T_{s}}\right)
$$

ó $\pi$ ov $\operatorname{sinc}(u)=\frac{\sin (\pi u)}{\pi u}$.

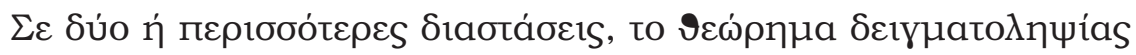

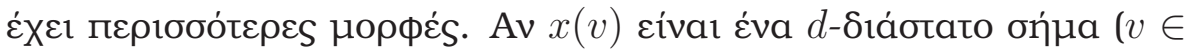

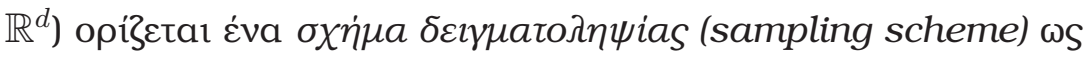

$$
x_{n_{1} \ldots n_{d}}=x\left(n_{1} v_{1}+\ldots+n_{d} v_{d}\right),
$$

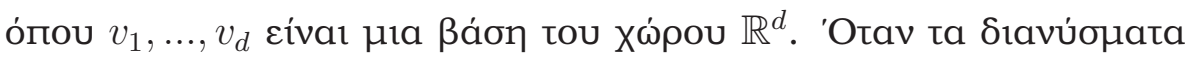

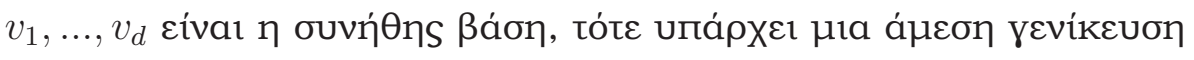

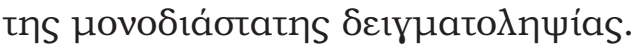




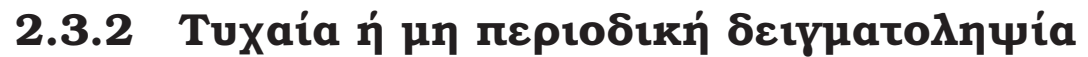

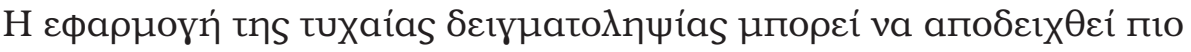

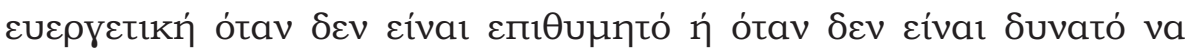

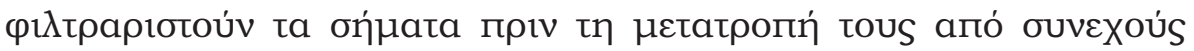

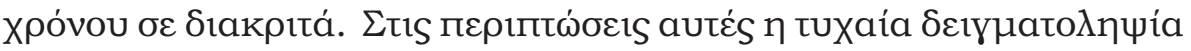

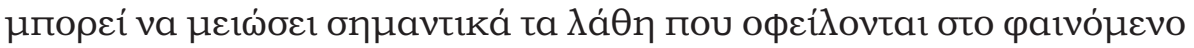

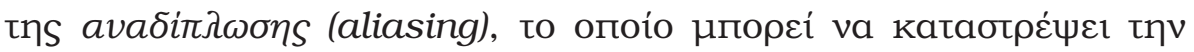

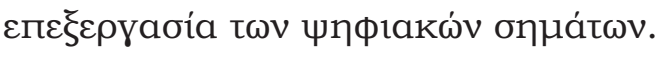

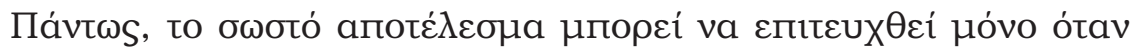

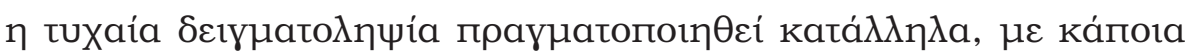

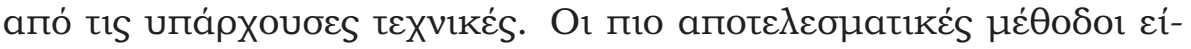

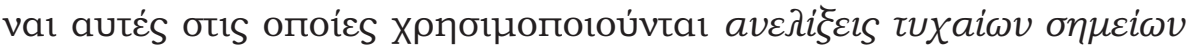
(random point processes).

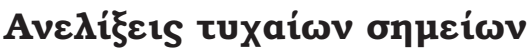

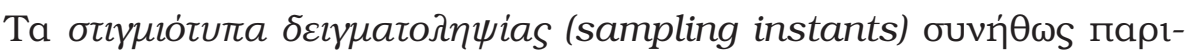

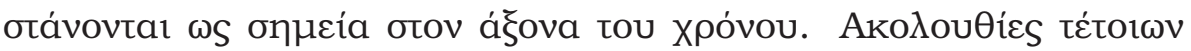

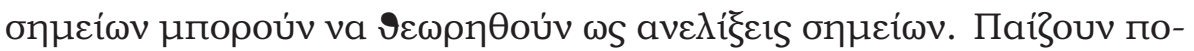

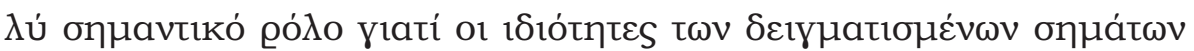

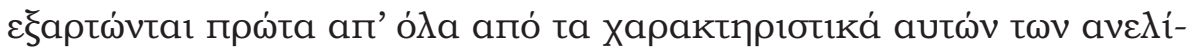
$\xi \varepsilon \omega v$.

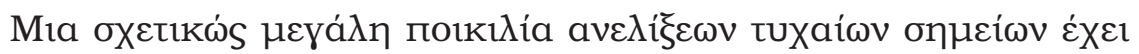

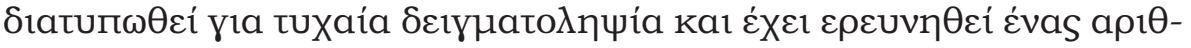

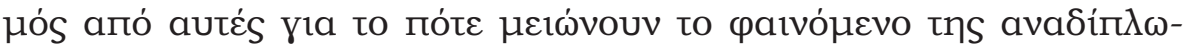




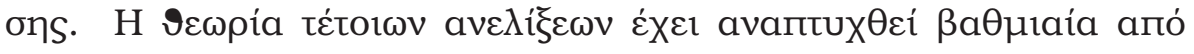
tous Beutler kaı Leneman [45, 46], kaӨஸ́s kaı anó to Masry [41].

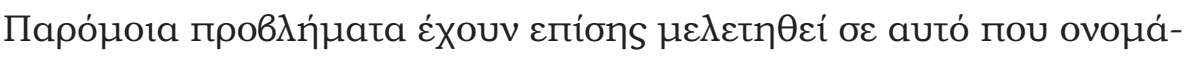

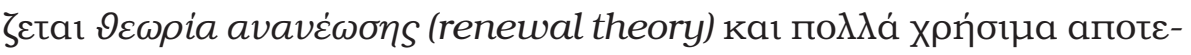

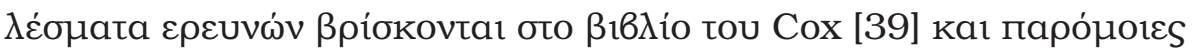

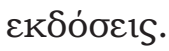

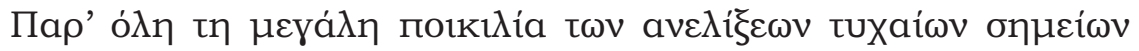

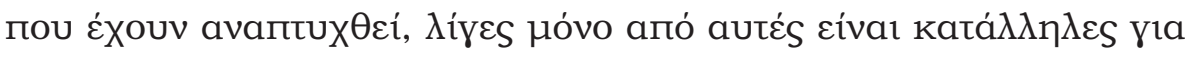

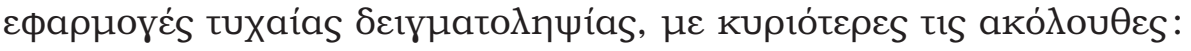

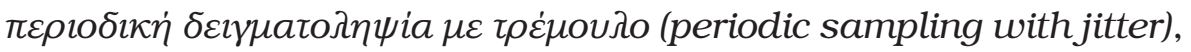

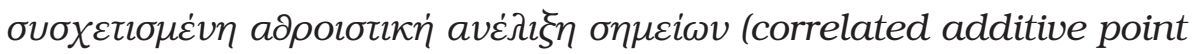

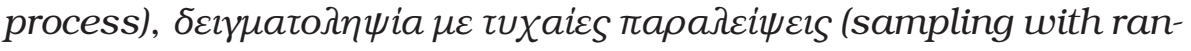

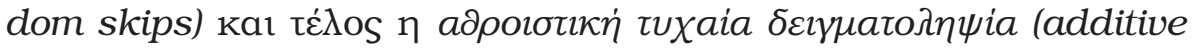

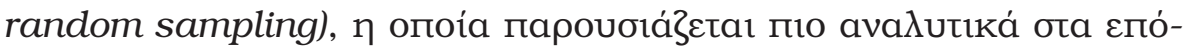
$\mu \varepsilon v a$.

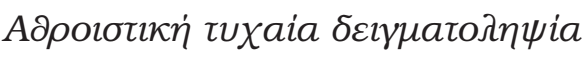

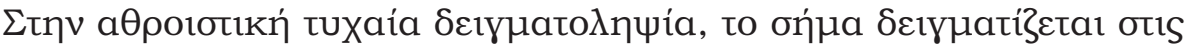

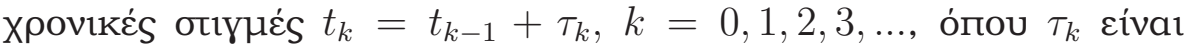

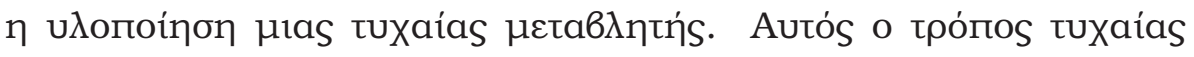

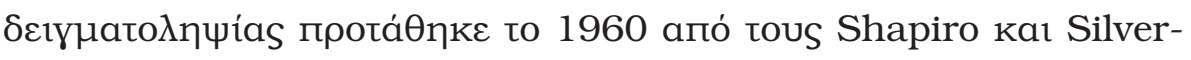

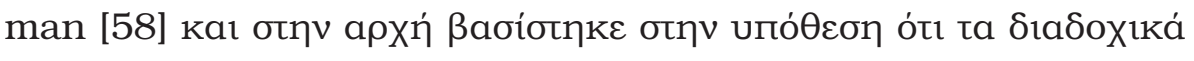

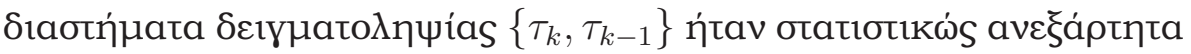

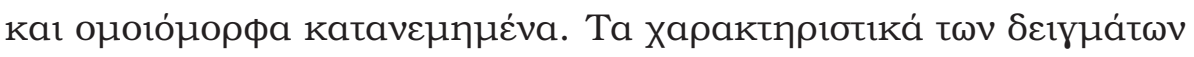

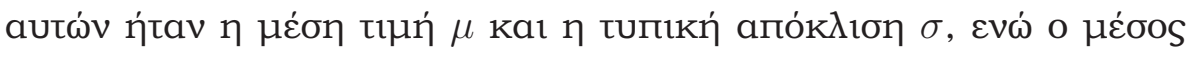

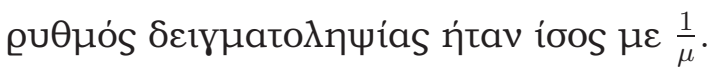




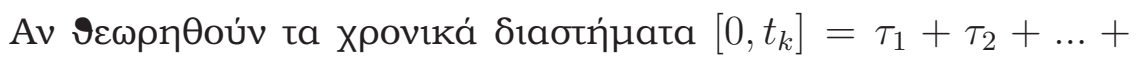

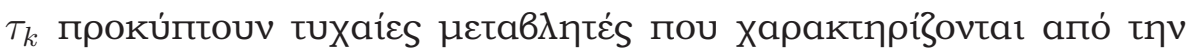

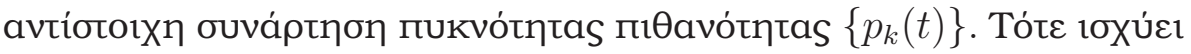

$$
\begin{aligned}
& p_{1}(t)=p_{\tau}(t) \\
& p_{2}(t)=p_{1}(t) * p_{\tau}(t) \\
& \ldots \ldots \ldots \ldots \ldots \ldots \ldots \ldots \ldots \ldots \\
& p_{k}(t)=p_{k-1}(t) * p_{\tau}(t),
\end{aligned}
$$

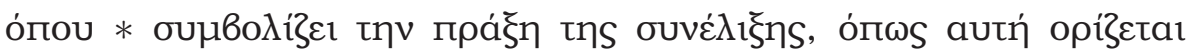

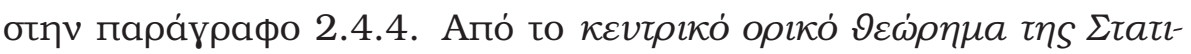

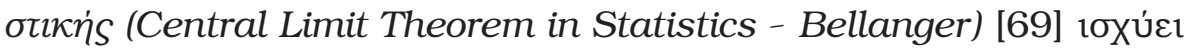

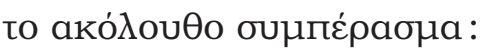

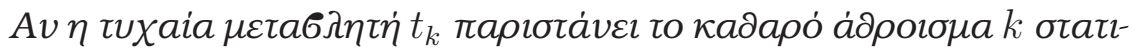

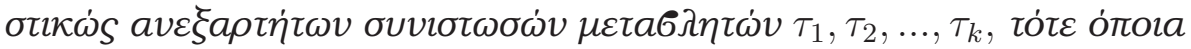

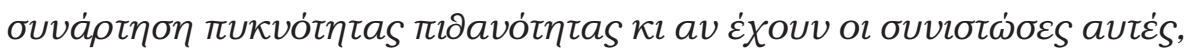
$\eta$ бuvá

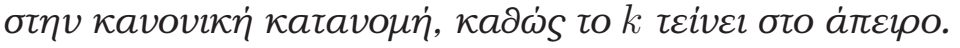

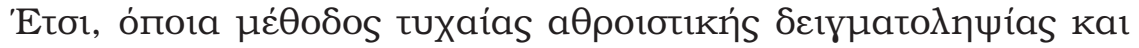

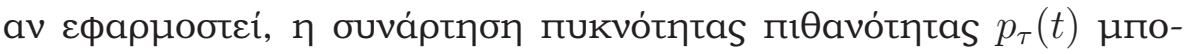

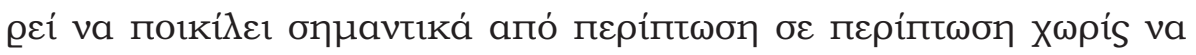

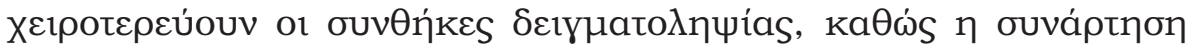

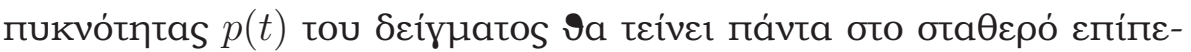

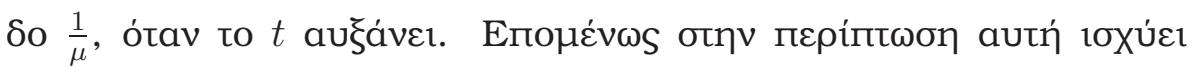
$p(t)=\frac{1}{\mu}$. 

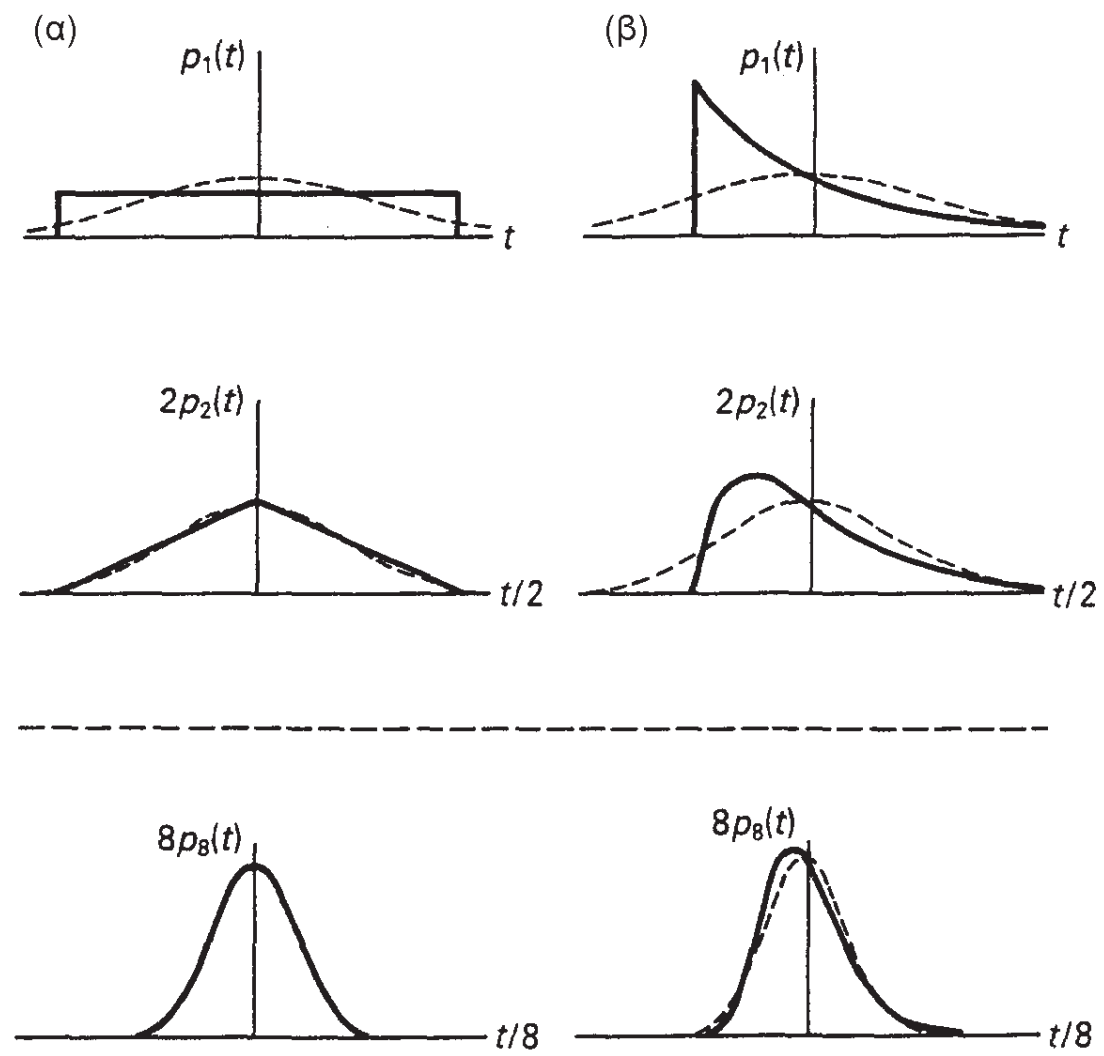

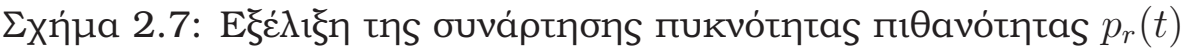

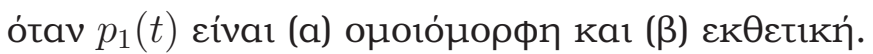




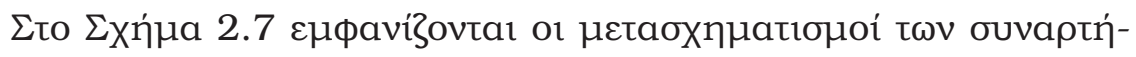

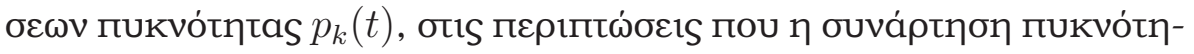

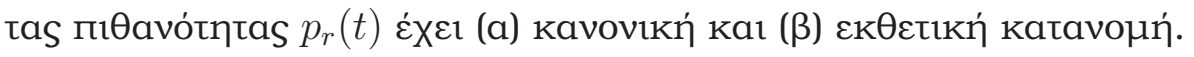

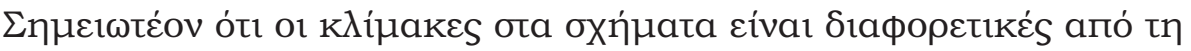

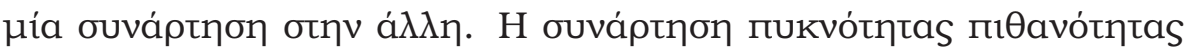

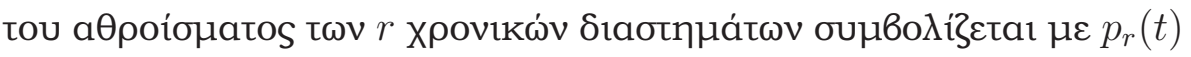
Ka1 ol ava

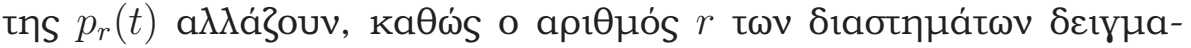

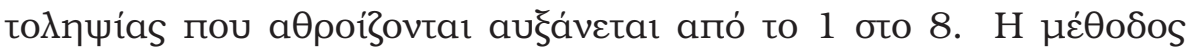

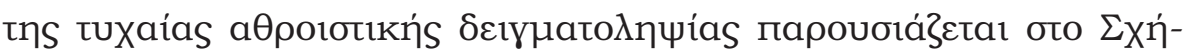

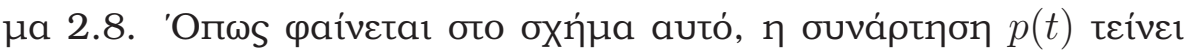

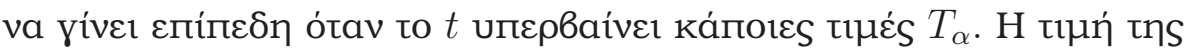

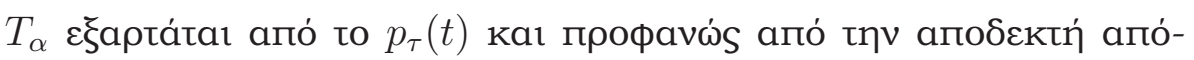

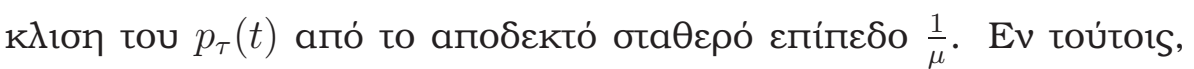

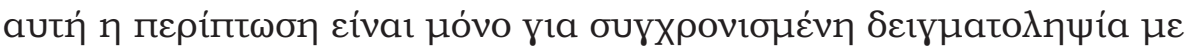

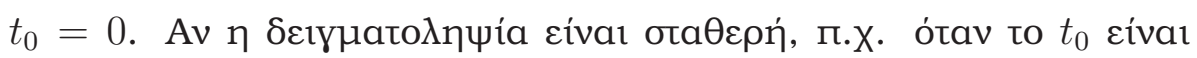

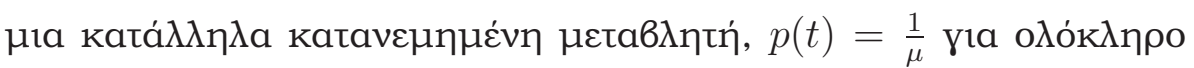

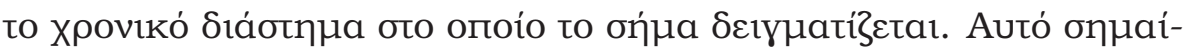

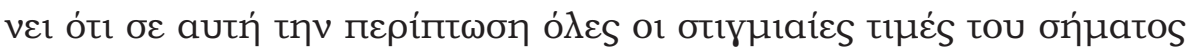

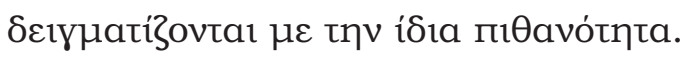



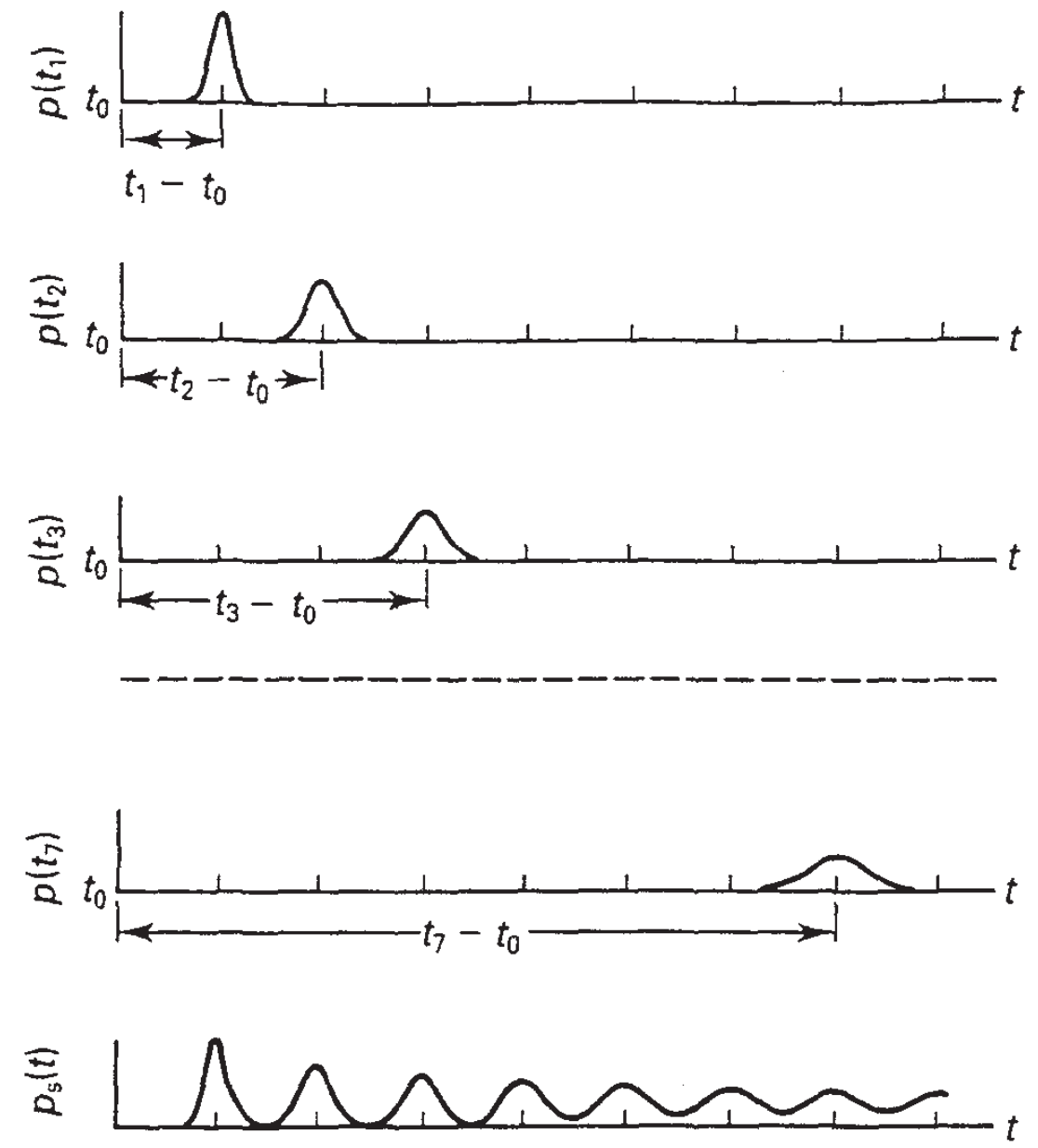

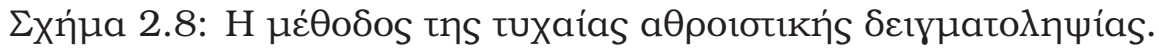




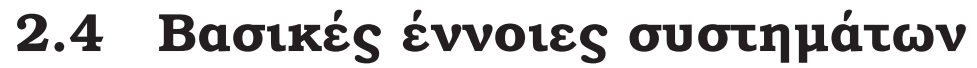

\subsubsection{H Évvola tou ouotńpatos}

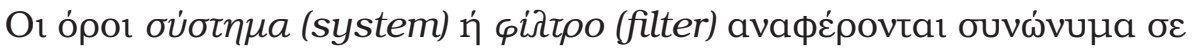

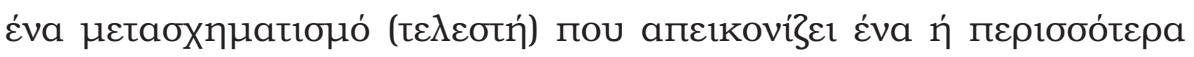

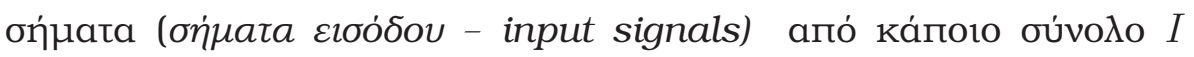

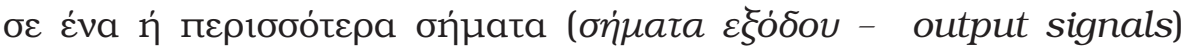

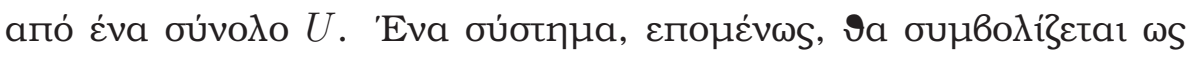

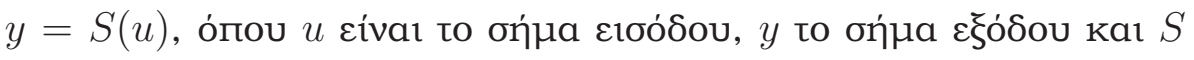

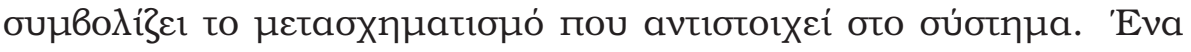

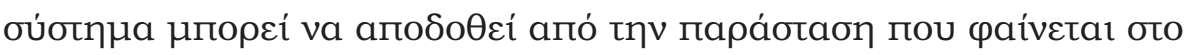

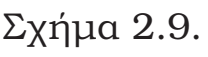

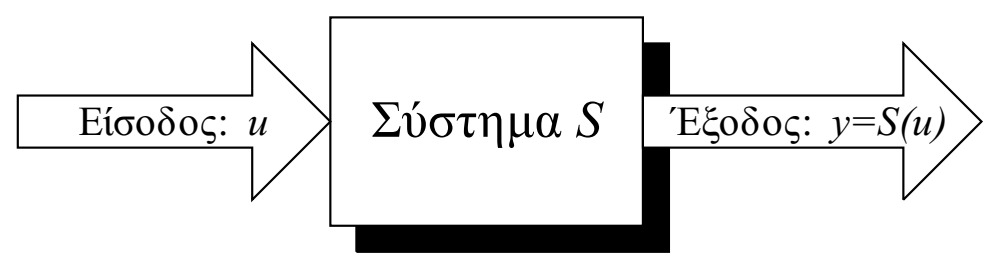

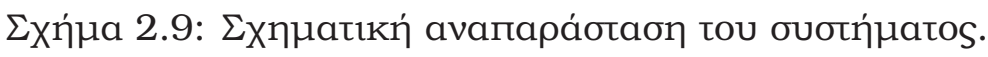

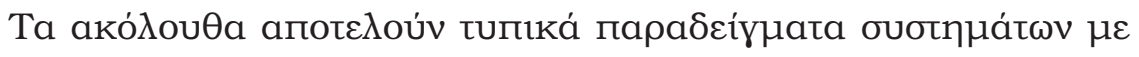

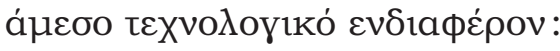

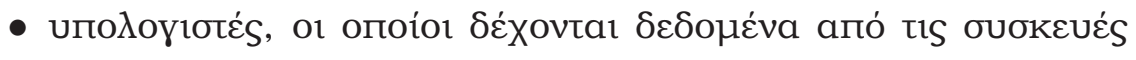

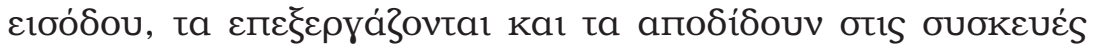
$\varepsilon \xi ̧$ óóou 


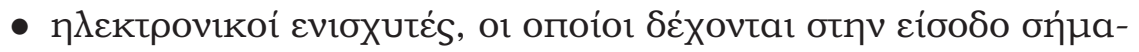

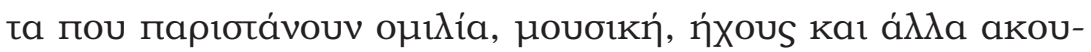

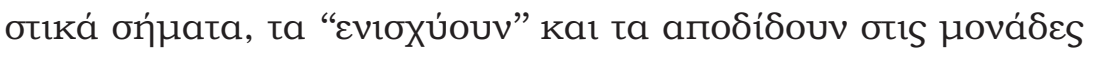

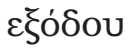

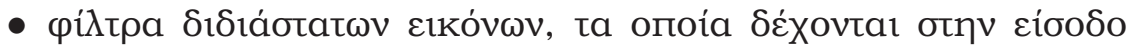

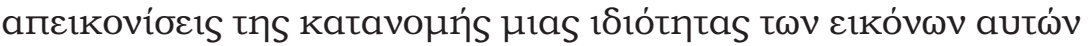

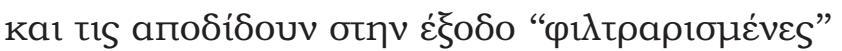

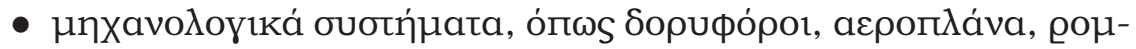

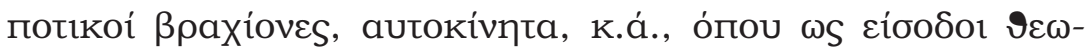

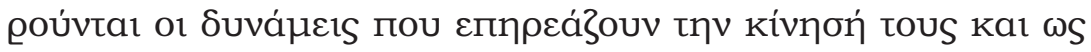

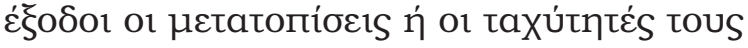

- бuбtń

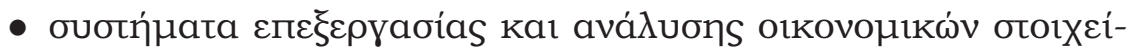
$\omega v$

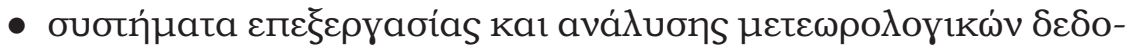
$\mu \varepsilon \dot{v \omega \nu}$

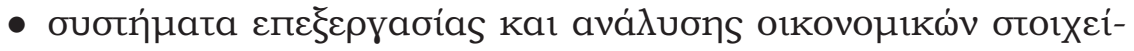
$\omega v$

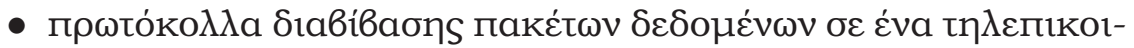

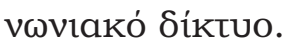




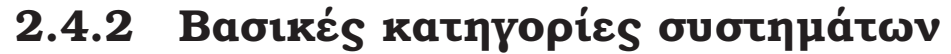

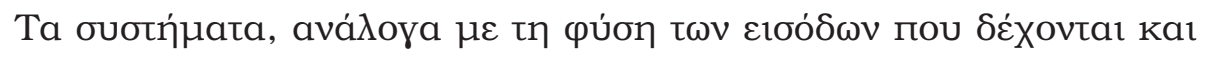

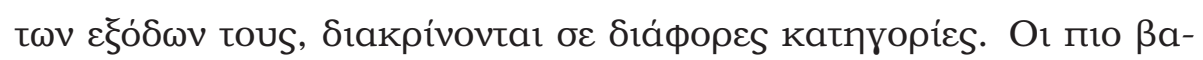

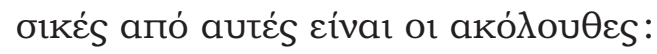

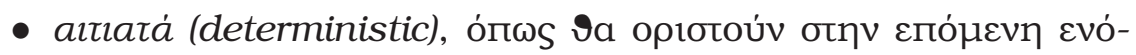
tᄁта 2.4.3

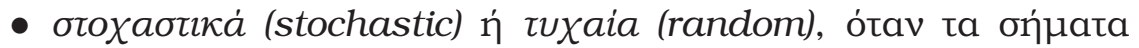

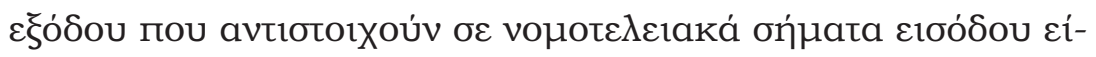
vaı бтохабıкá

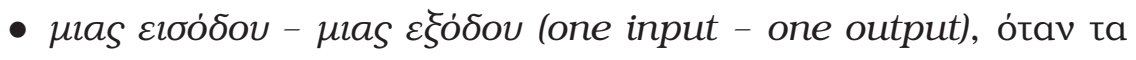

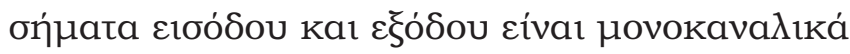

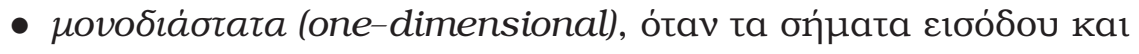

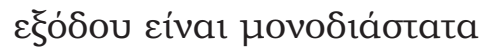

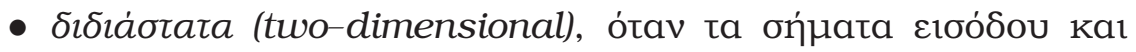

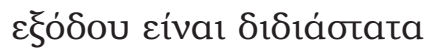

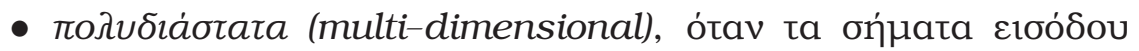

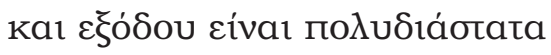

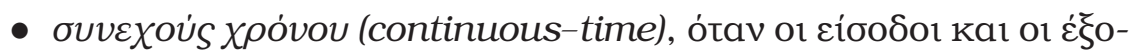

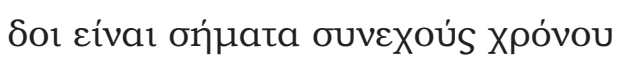

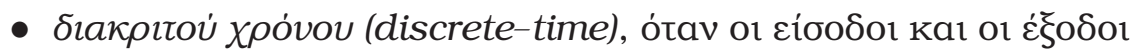

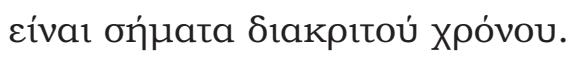




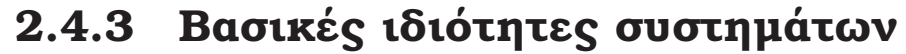

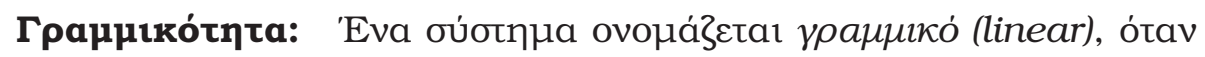

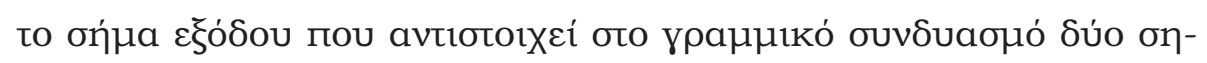

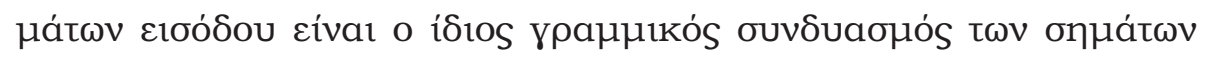

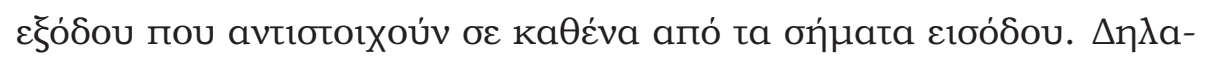

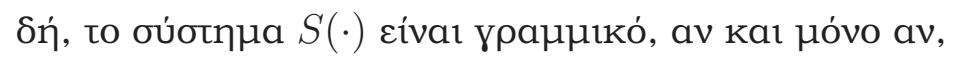

$$
S\left(\alpha_{1} u_{1}+\alpha_{2} u_{2}\right)=\alpha_{1} S\left(u_{1}\right)+\alpha_{2} S\left(u_{2}\right)
$$

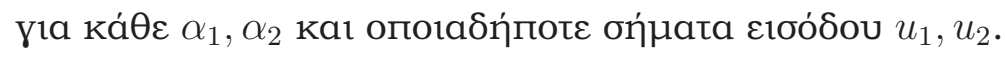

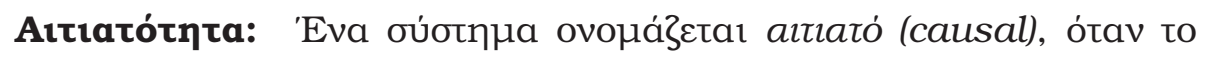

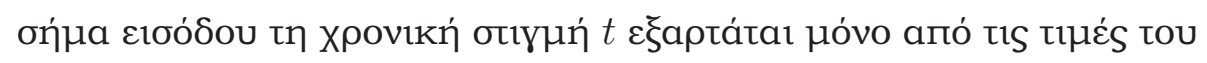

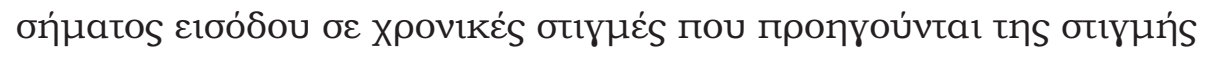

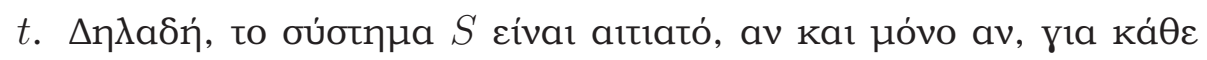
X

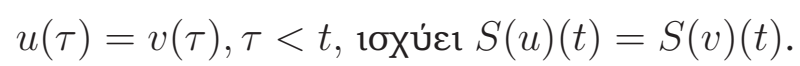

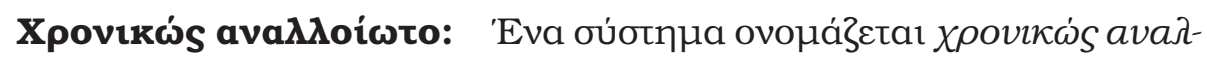

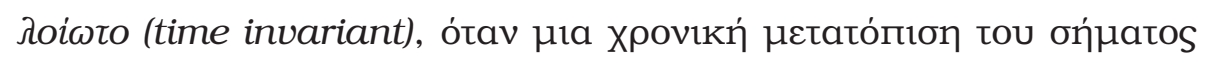

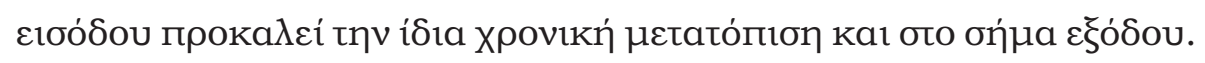

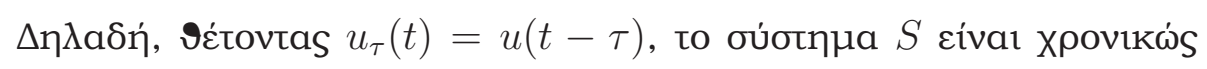

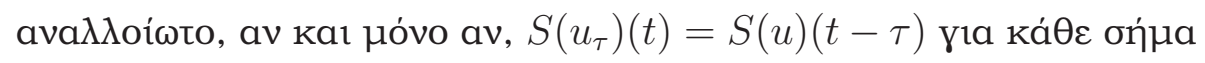

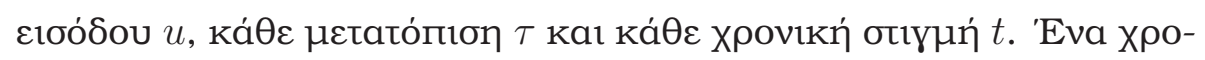

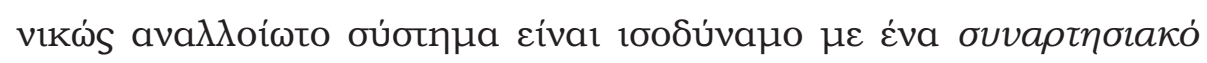

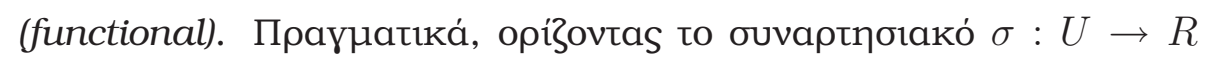




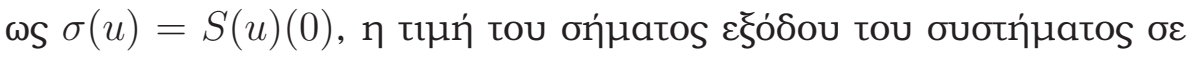

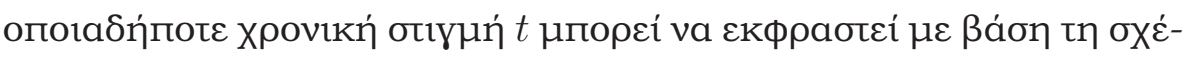

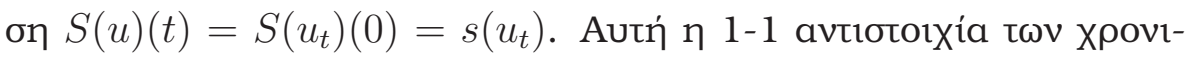

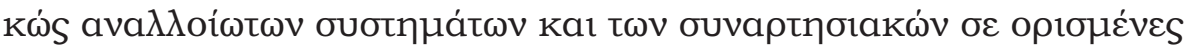

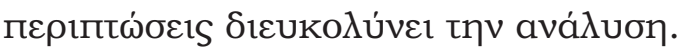

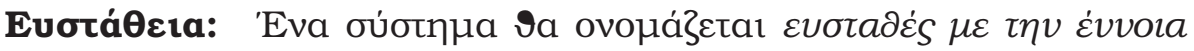

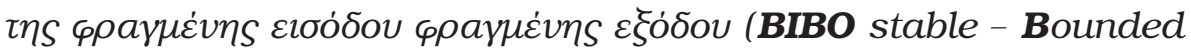

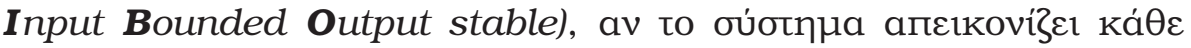

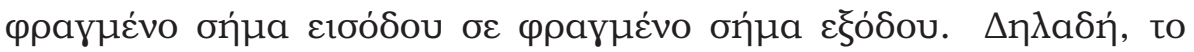

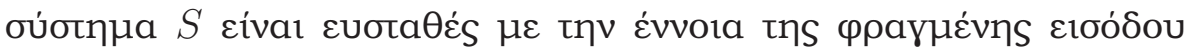

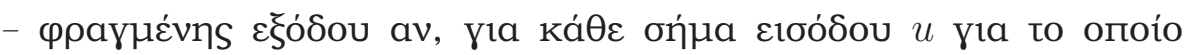

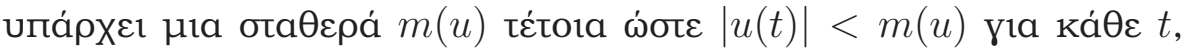

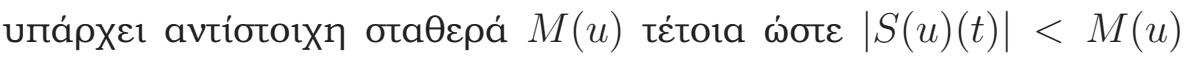
Vıa ká $\theta \varepsilon t$.

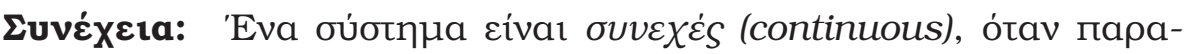

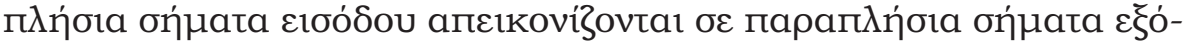

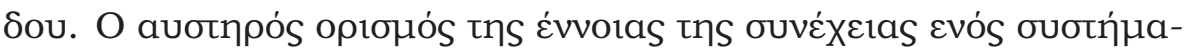

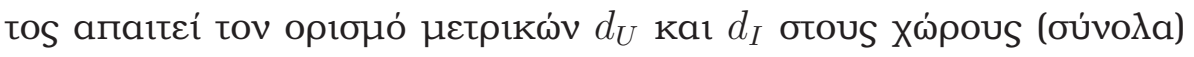

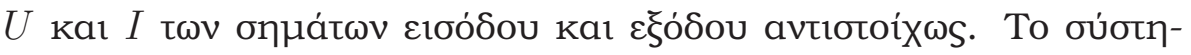

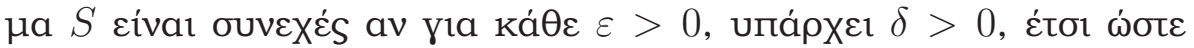

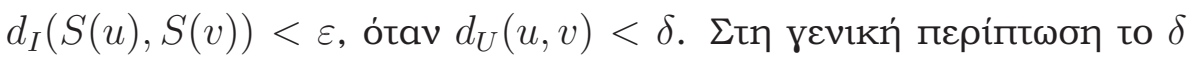

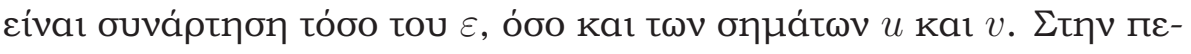

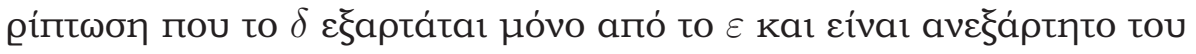




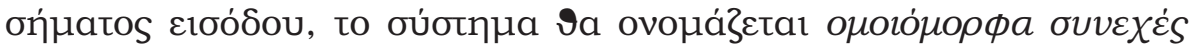
(uniformly continuous).

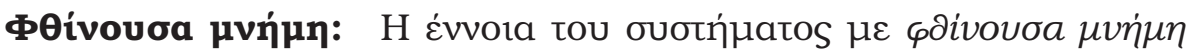

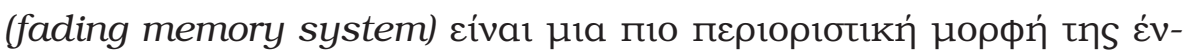

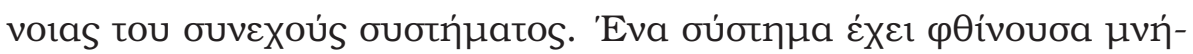

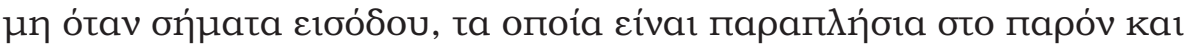

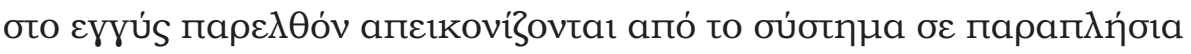

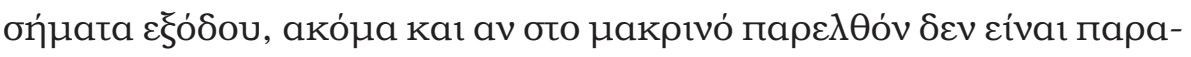

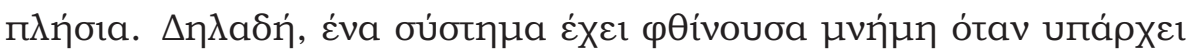

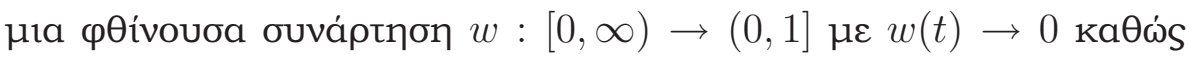

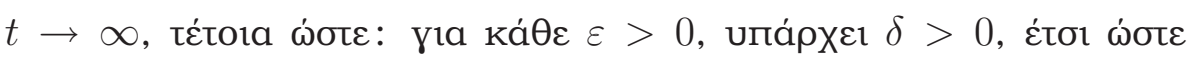
$d_{I}(S(u), S(v))<\varepsilon$, ótav $d_{U}\left(u_{w}, v_{w}\right)<\delta$, о́поu $u_{w}(t)=u(t) w(-t)$ kaı $v_{w}(t)=v(t) w(-t)$.

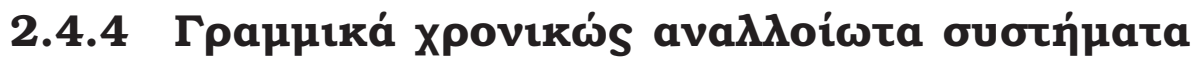 ouvexoús Xpóvou}

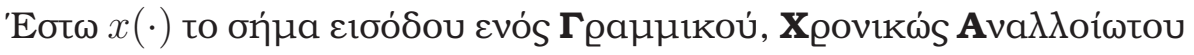

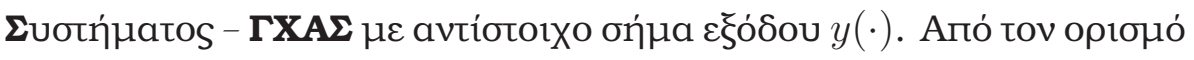

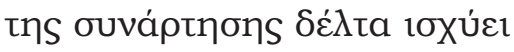

$$
x(t)=\int_{-\infty}^{\infty} x(\tau) \delta(t-\tau) d \tau, \text { Vıa ká } \theta \varepsilon t .
$$

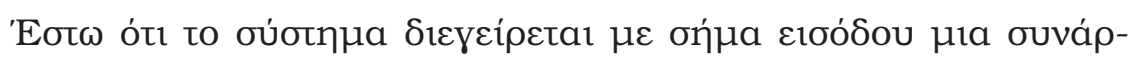

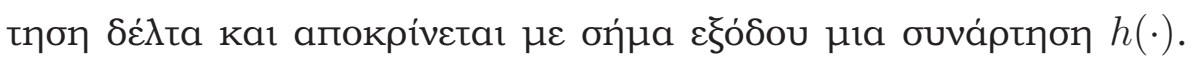

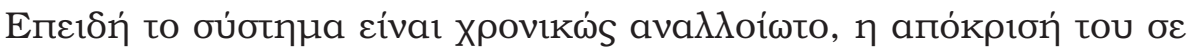




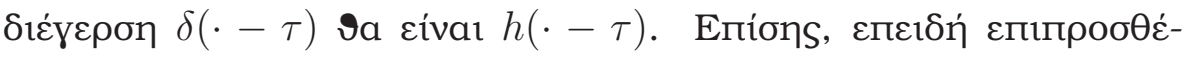

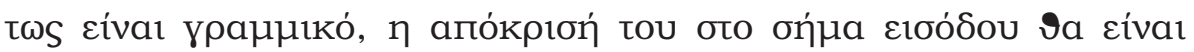

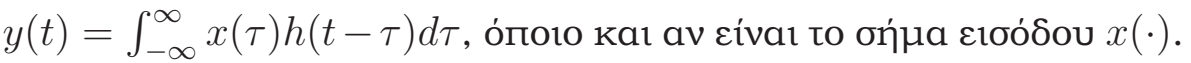

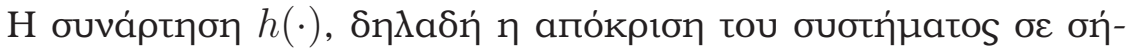

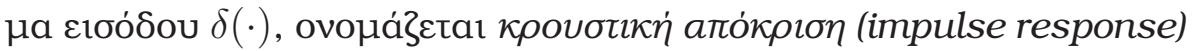
tou ouotń

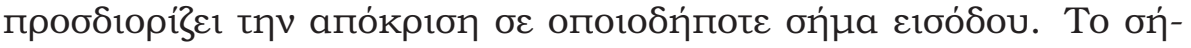

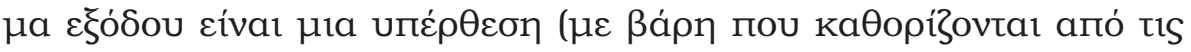

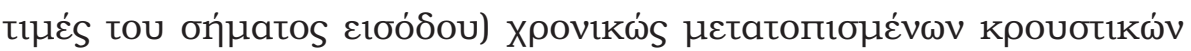

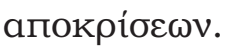

Ioxúc1

$$
y(t)=\int_{-\infty}^{\infty} x(\tau) h(t-\tau) d \tau=\int_{-\infty}^{\infty} x(t-\tau) h(\tau) d \tau .
$$

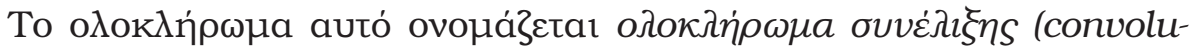

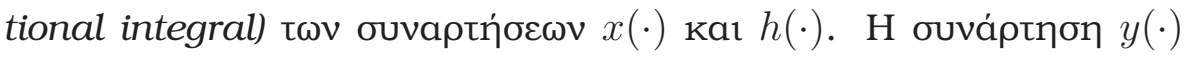

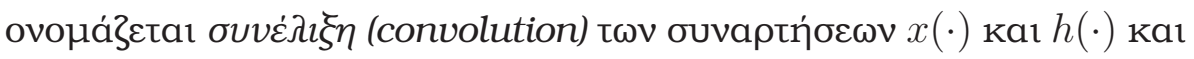

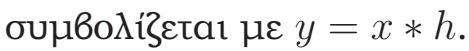

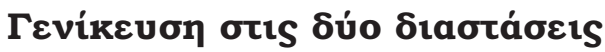

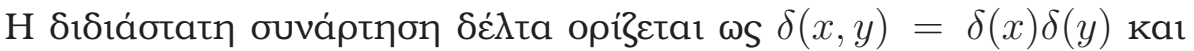

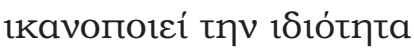

$$
\int_{-\infty}^{\infty} u(x, y) \delta(x, y)=u(0,0)
$$

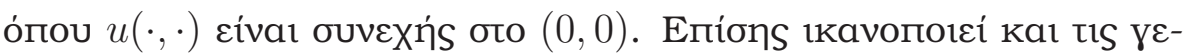

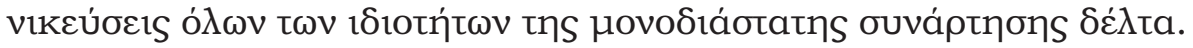




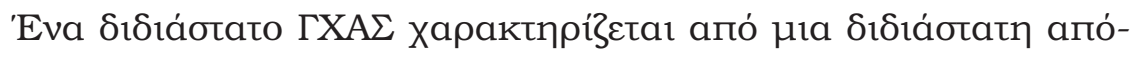

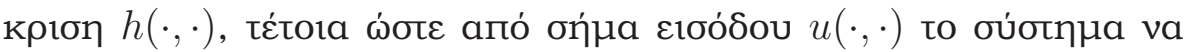

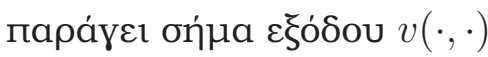

$$
\begin{aligned}
v(x, y) & =\int_{-\infty}^{\infty} u\left(x^{\prime}, y^{\prime}\right) h\left(x-x^{\prime}, y-y^{\prime}\right) d x^{\prime} d y^{\prime} \\
& =\int_{-\infty}^{\infty} u\left(x-x^{\prime}, y-y^{\prime}\right) h\left(x^{\prime}, y^{\prime}\right) d x^{\prime} d y^{\prime} .
\end{aligned}
$$

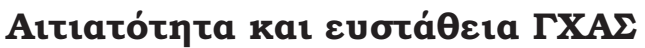

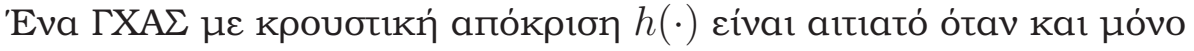

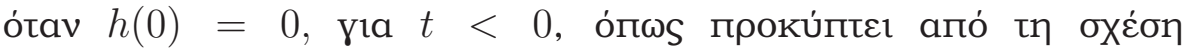
$y(t)=\int_{-\infty}^{\infty} x(t-\tau) h(\tau) d \tau$.

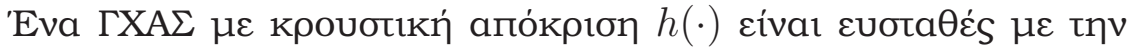

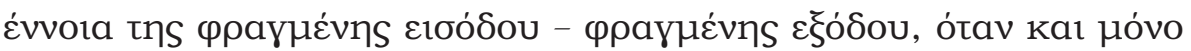
ótav

$$
\int_{-\infty}^{\infty}|h(\tau)| d \tau<\infty
$$

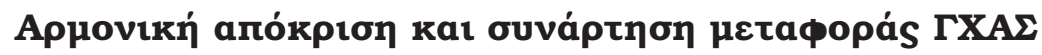

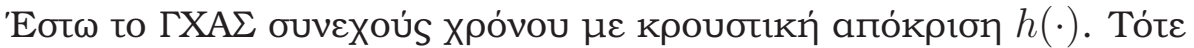

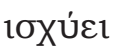

$$
y(t)=\int_{-\infty}^{\infty} x(t-\tau) h(\tau) d \tau=\int_{-\infty}^{\infty} x(\tau) h(t-\tau) d \tau .
$$

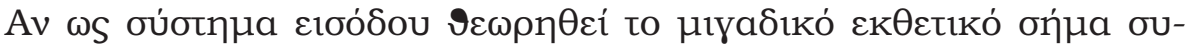

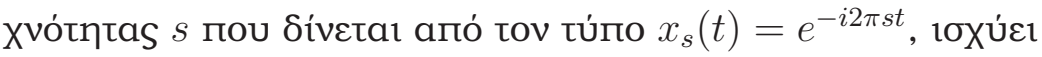

$$
y_{s}(t)=\int_{-\infty}^{\infty} e^{-i 2 \pi s(t-\tau)} h(\tau) d \tau=e^{-i 2 \pi s t} \int_{-\infty}^{\infty} e^{i 2 \pi s \tau} h(\tau) d \tau .
$$


Епє1ठи́

$$
\int_{-\infty}^{\infty} e^{i 2 \pi s \tau} h(\tau) d \tau=H(s)
$$

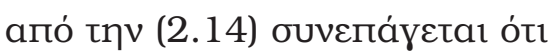

$$
y_{s}(t)=H(s) x_{s}(t)
$$

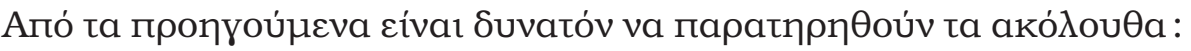

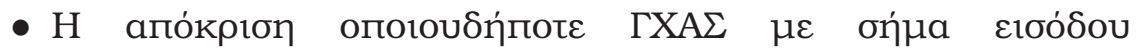

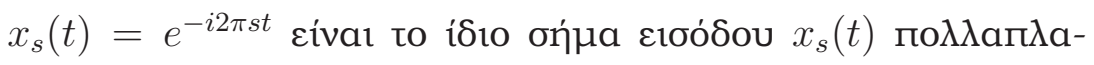
бıаo

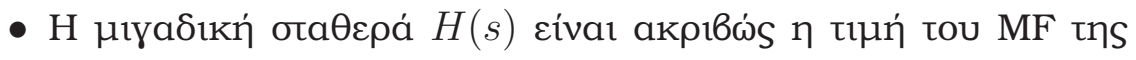

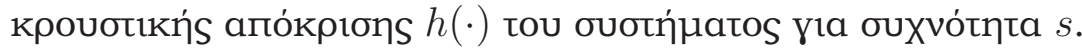

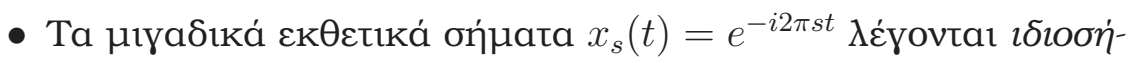

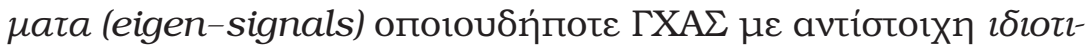

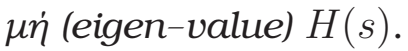

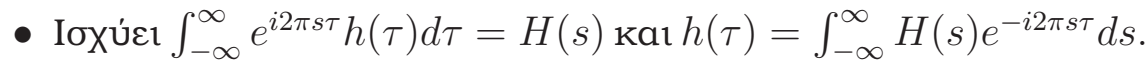

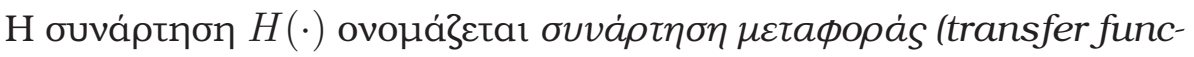
tion) tou ouotńpatos.

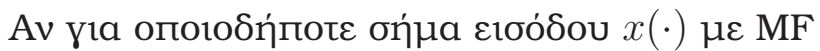

$$
X(s)=\int_{-\infty}^{\infty} x(t) e^{i 2 \pi s t} d t
$$

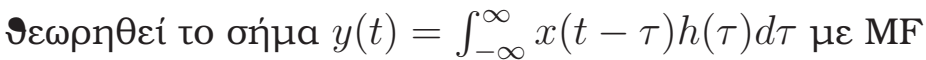

$$
Y(s)=\int_{-\infty}^{\infty} y(t) e^{i 2 \pi s t} d t,
$$




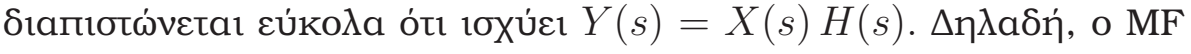

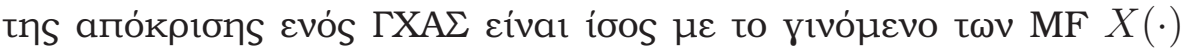

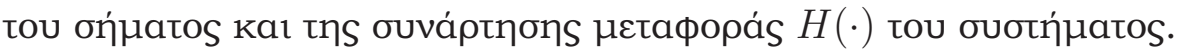

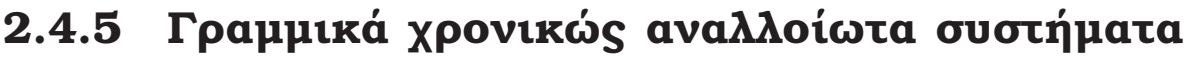

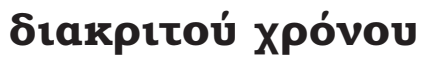

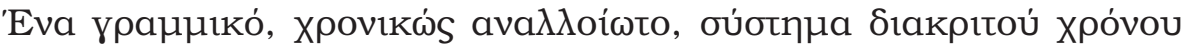

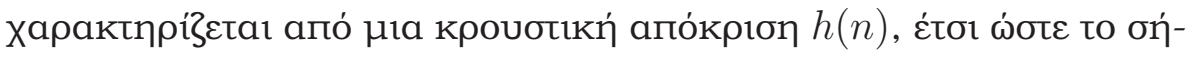

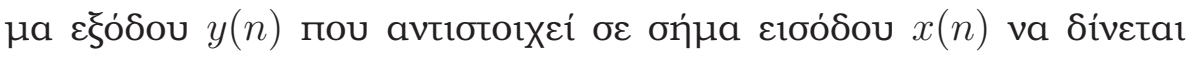

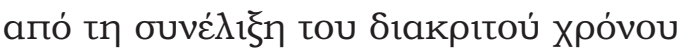

$$
y(n)=\sum_{n^{\prime}=-\infty}^{\infty} x\left(n^{\prime}\right) h\left(n-n^{\prime}\right)=\sum_{n^{\prime}=-\infty}^{\infty} x\left(n-n^{\prime}\right) h\left(n^{\prime}\right) .
$$

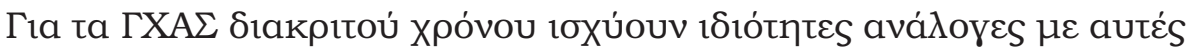

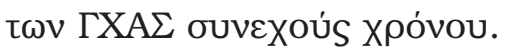

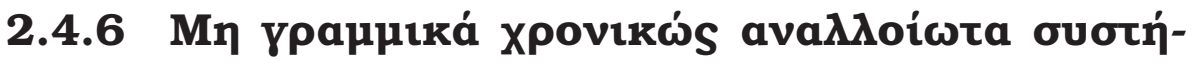 paca Volterra}

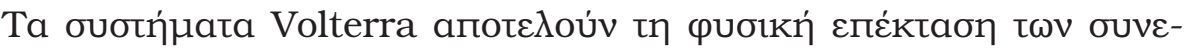

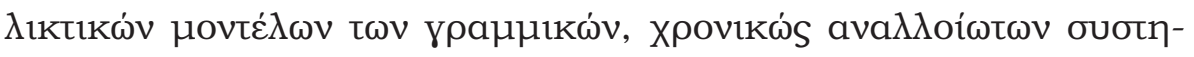

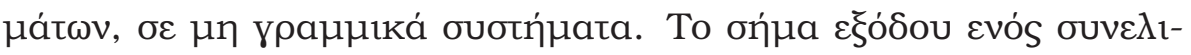

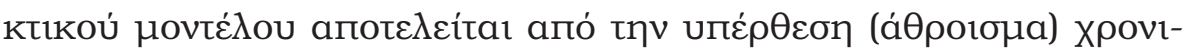

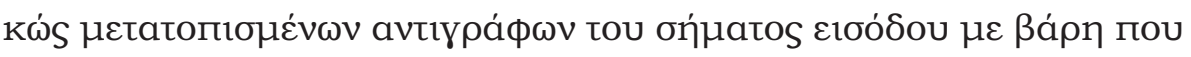

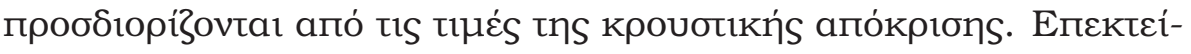

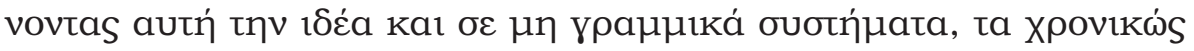




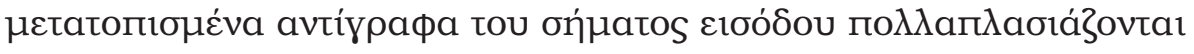

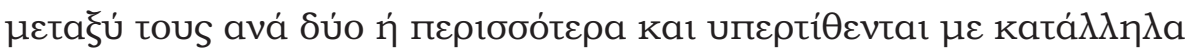
ßápๆ.

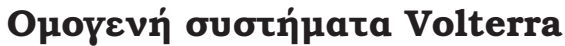

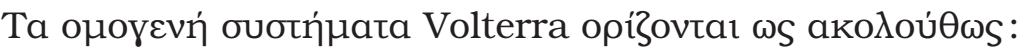

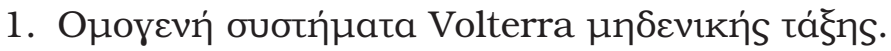

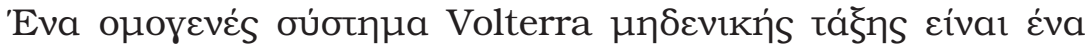

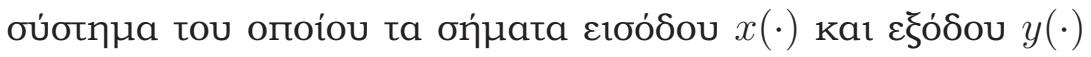

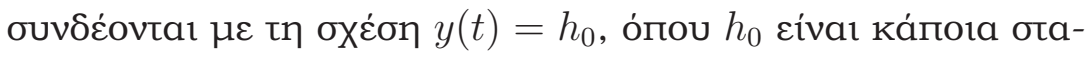

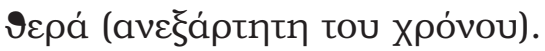

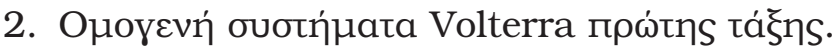

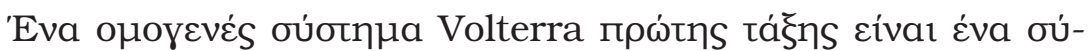

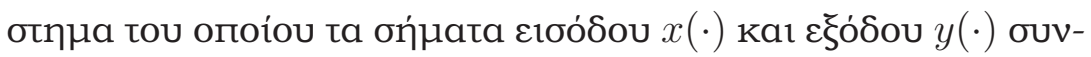

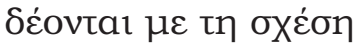

$$
y(t)=\int_{-\infty}^{\infty} x(\tau) h_{1}(t-\tau) d \tau=\int_{-\infty}^{\infty} x(t-\tau) h_{1}(\tau) d \tau
$$

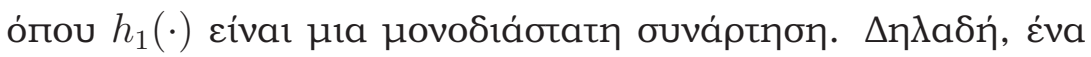

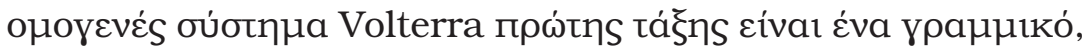

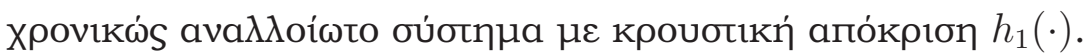
Гıa ta ouotń

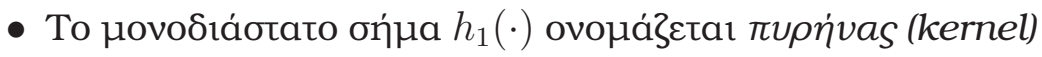
tou ouorńfatos. 


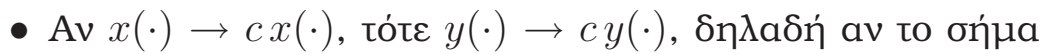

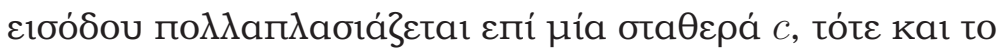

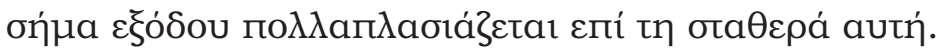

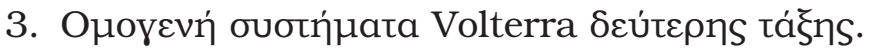

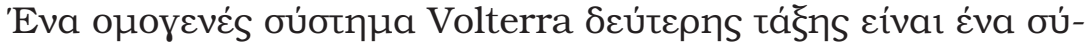

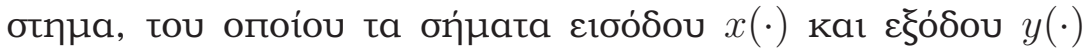

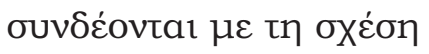

$$
\begin{aligned}
y(t) & =\int_{-\infty}^{\infty} \int_{-\infty}^{\infty} x\left(\tau_{1}\right) x\left(\tau_{2}\right) h_{2}\left(t-\tau_{1}, t-\tau_{2}\right) d \tau_{1} d \tau_{2} \\
& =\int_{-\infty}^{\infty} \int_{-\infty}^{\infty} x\left(t-\tau_{1}\right) x\left(t-\tau_{2}\right) h_{2}\left(\tau_{1}, \tau_{2}\right) d \tau_{1} d \tau_{2},
\end{aligned}
$$

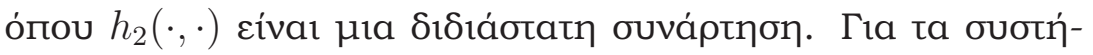

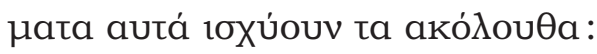

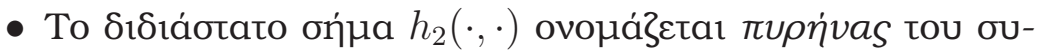
oтńpatos.

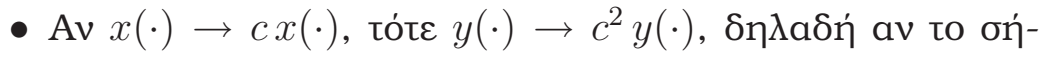

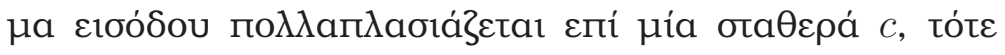

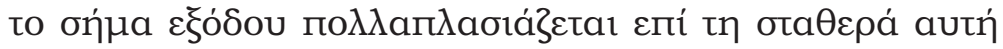

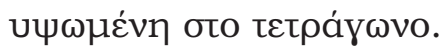

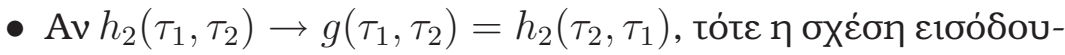

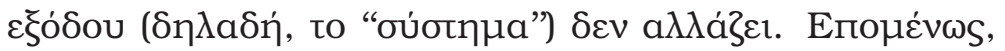

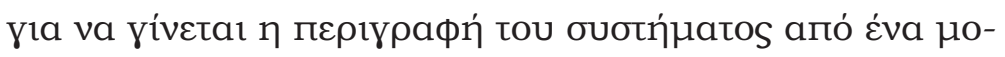

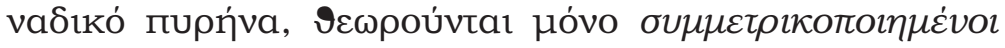
пuрńves $h_{2}^{\sigma}\left(\tau_{1}, \tau_{2}\right)=\frac{1}{2}\left[h_{2}\left(\tau_{1}, \tau_{2}\right)+h_{2}\left(\tau_{2}, \tau_{1}\right)\right]$. 


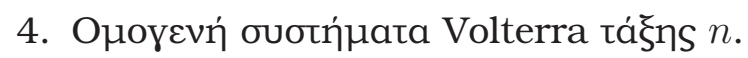

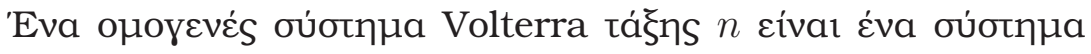

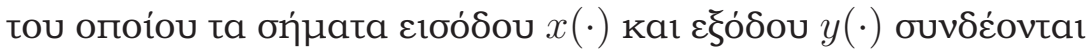

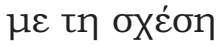

$$
\begin{aligned}
y(t) & =\int_{-\infty}^{\infty} \ldots \int_{-\infty}^{\infty} x\left(\tau_{1}\right) \ldots x\left(\tau_{n}\right) h_{n}\left(t-\tau_{1}, \ldots, t-\tau_{n}\right) d \tau_{1} \ldots d \tau_{n} \\
& =\int_{-\infty}^{\infty} \ldots \int_{-\infty}^{\infty} x\left(t-\tau_{1}\right) \ldots x\left(t-\tau_{n}\right) h_{n}\left(\tau_{1}, \ldots, \tau_{n}\right) d \tau_{1} \ldots d \tau_{n},
\end{aligned}
$$

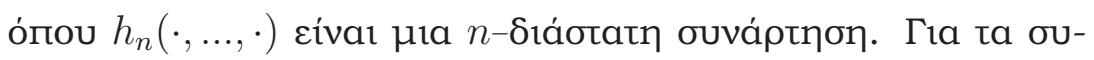

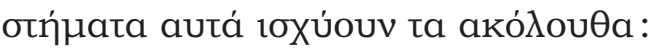

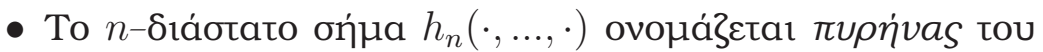
ovotńpatos.

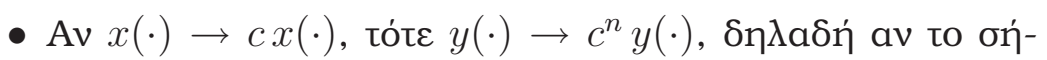

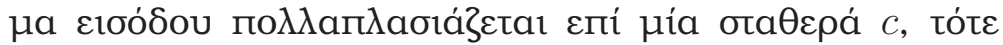

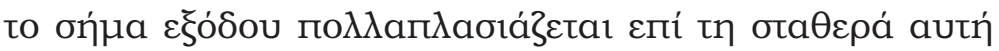

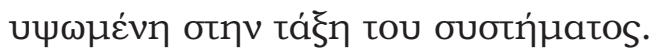

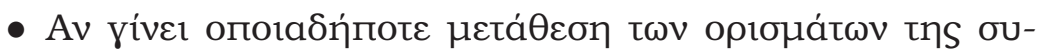

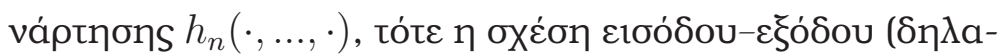

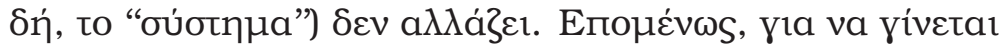

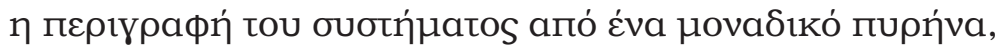

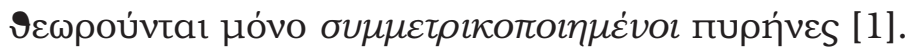




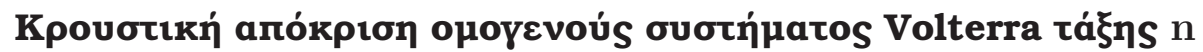

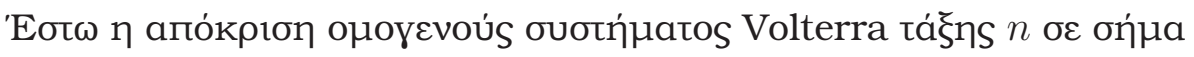

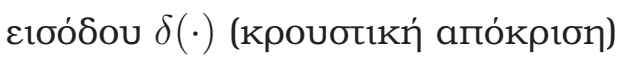

$$
\begin{aligned}
y(t) & =\int_{-\infty}^{\infty} \ldots \int_{-\infty}^{\infty} \delta\left(\tau_{1}\right) \ldots \delta\left(\tau_{n}\right) h_{n}\left(t-\tau_{1}, \ldots, t-\tau_{n}\right) d \tau_{1} \ldots d \tau_{n} \\
& =h_{n}\left(t_{1}, \ldots, t_{n}\right) .
\end{aligned}
$$

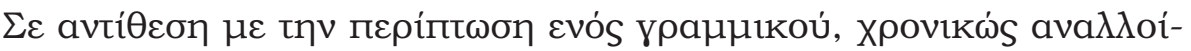

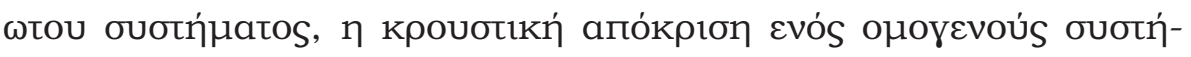

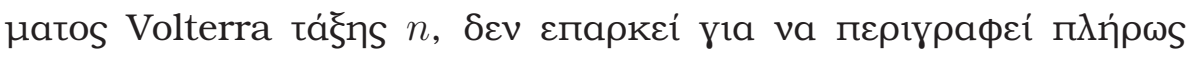

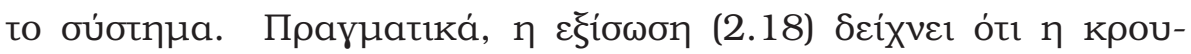

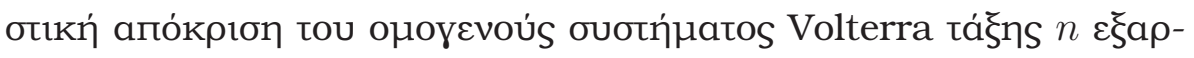

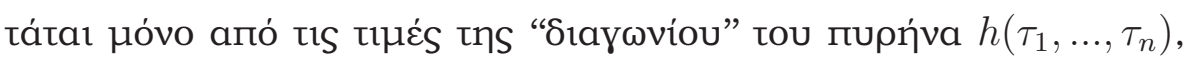
$\tau_{1}=\ldots=\tau_{n}=t$.

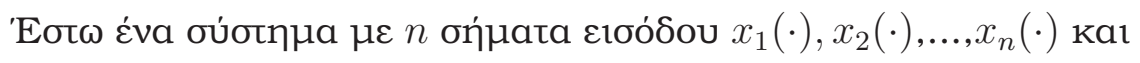

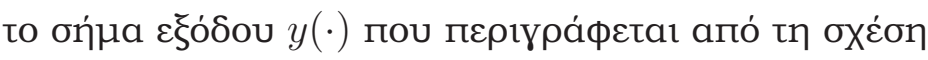

$$
y_{x_{1} x_{2} \ldots x_{n}}(t)=\int_{-\infty}^{\infty} \ldots \int_{-\infty}^{\infty} x_{1}\left(\tau_{1}\right) \ldots x_{n}\left(\tau_{n}\right) h_{n}\left(t-\tau_{1}, \ldots, t-\tau_{n}\right) d \tau_{1} \ldots d \tau_{n} .
$$

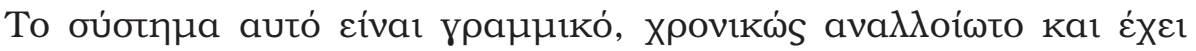

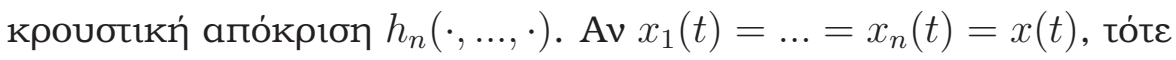

$$
\begin{aligned}
& y_{x_{1} x_{2} \ldots x_{n}}(t)= \\
& =y(t)=\int_{-\infty}^{\infty} \ldots \int_{-\infty}^{\infty} x\left(\tau_{1}\right) \ldots x\left(\tau_{n}\right) h_{n}\left(t-\tau_{1}, \ldots, t-\tau_{n}\right) d \tau_{1} \ldots d \tau_{n} .
\end{aligned}
$$

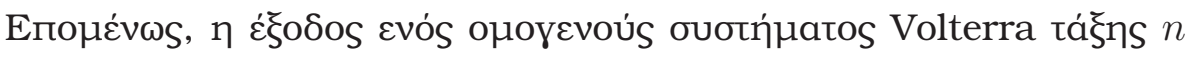

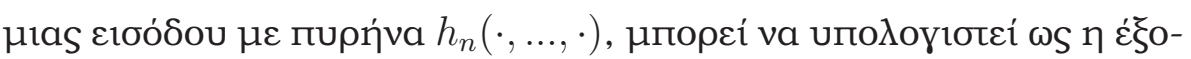




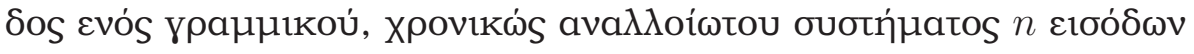
$\mu \varepsilon$ крочотикń апо́крıоп $h_{n}(\cdot, \ldots, \cdot)$.

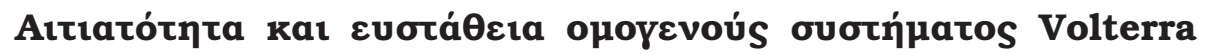

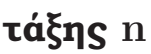

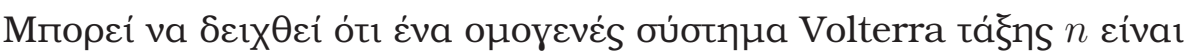

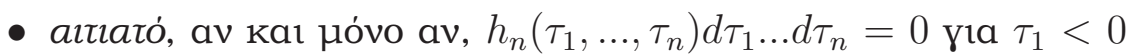
ท́ $\tau_{2}<0$ ท́ ... ท́ $\tau_{n}<0$

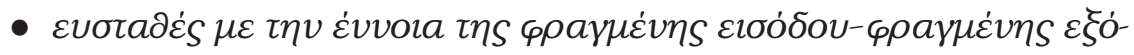

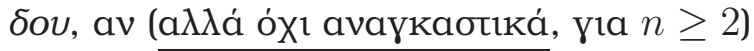

$$
\int_{-\infty}^{\infty} \ldots \int_{-\infty}^{\infty}\left|h_{n}\left(t-\tau_{1}, \ldots, t-\tau_{n}\right)\right| d \tau_{1} \ldots d \tau_{n}<\infty
$$

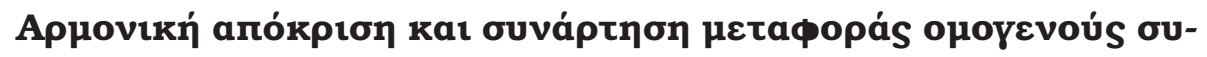

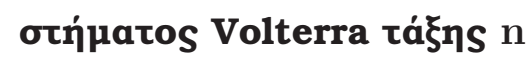

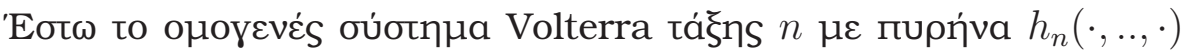
kaı oń

$$
y_{s}(t)=e^{-i 2 \pi n s t} H_{n}(s, \ldots, s),
$$

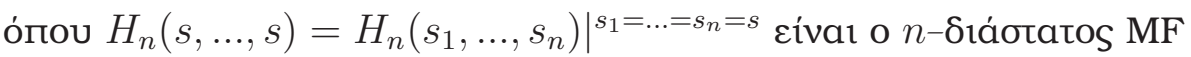

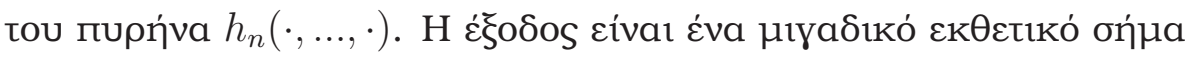

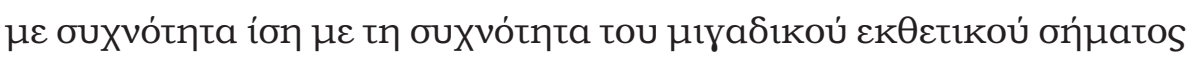

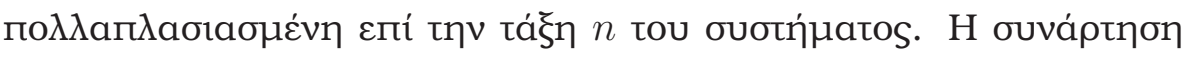

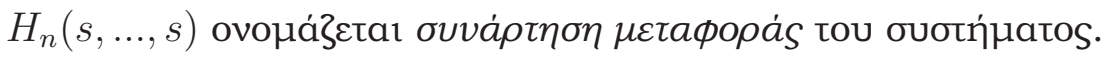




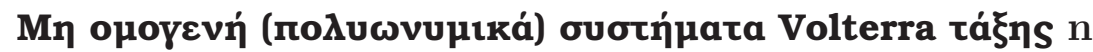

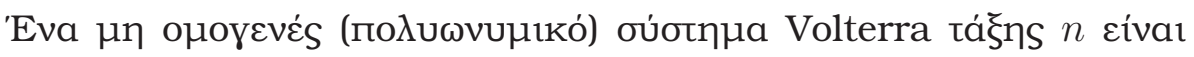

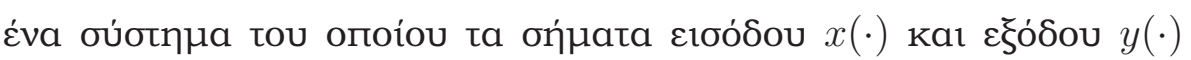

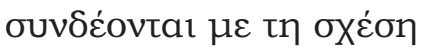

$y(t)=h_{0}+\sum_{k=1}^{n} \int_{-\infty}^{\infty} \ldots \int_{-\infty}^{\infty} x\left(\tau_{1}\right) \ldots x\left(\tau_{k}\right) h_{k}\left(t-\tau_{1}, \ldots, t-\tau_{k}\right) d \tau_{1} \ldots d \tau_{k}$,

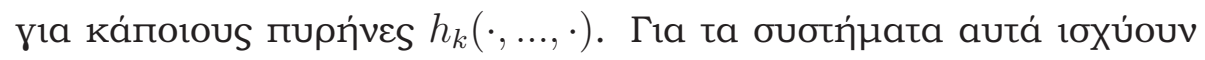

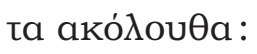

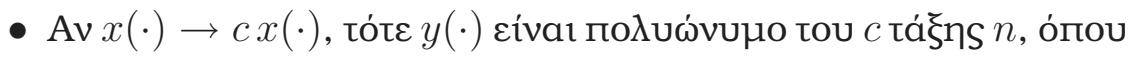

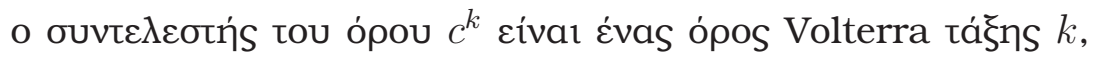
$k=0,1,2, \ldots, n$.

- H kpouotıkń anókpıon tou ouotńjatos cívaı

$$
h_{0}+\sum_{k=1}^{n} h_{k}(t, \ldots, t)
$$

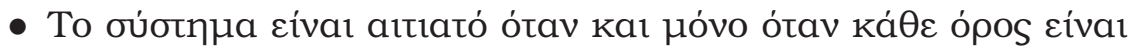
aıtıatós.

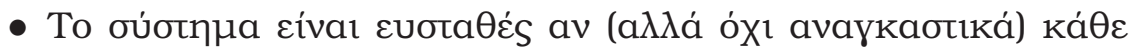

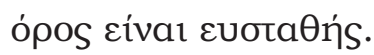

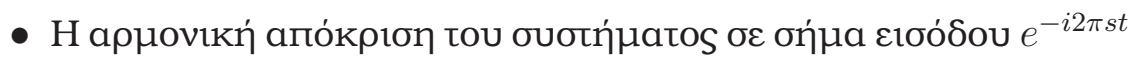
síval

$$
h_{0}+\sum_{k=1}^{n} H_{k}(s, \ldots, s) e^{-i 2 \pi s t}
$$




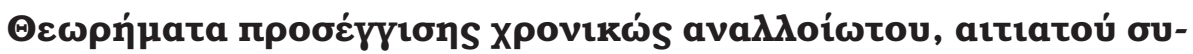

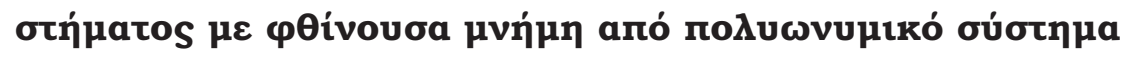

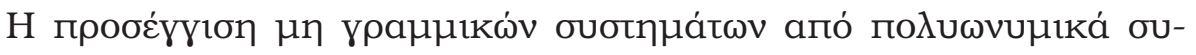

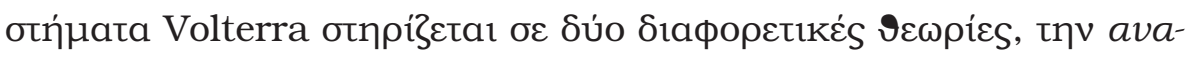

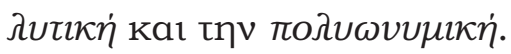

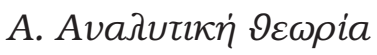

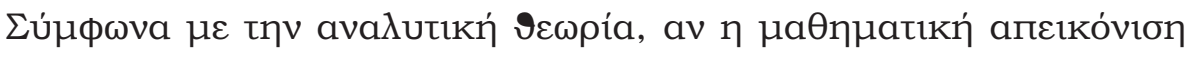
aпó to oń

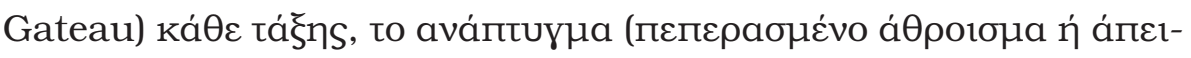

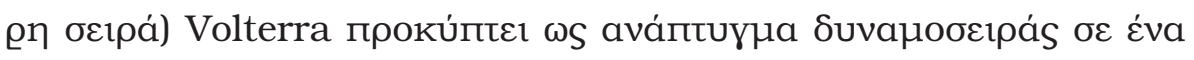

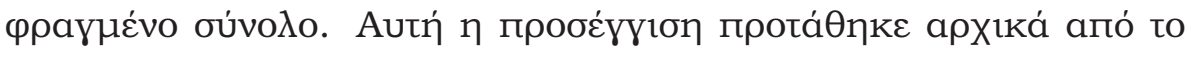

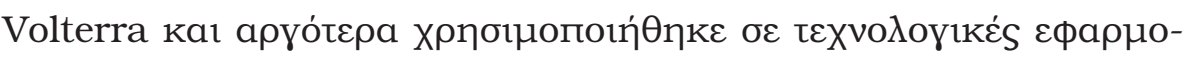

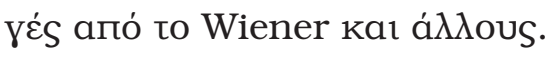

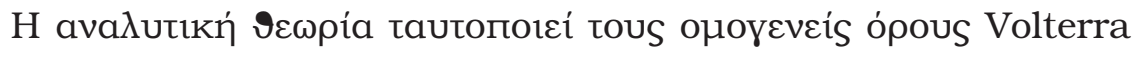

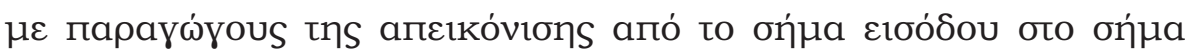

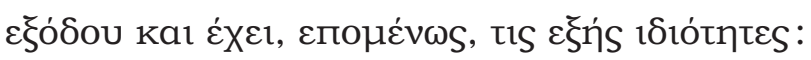

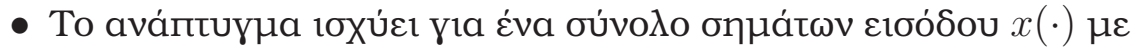

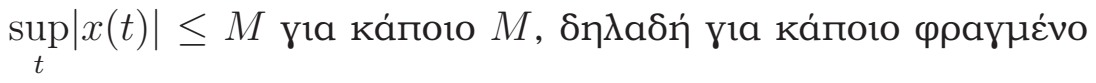

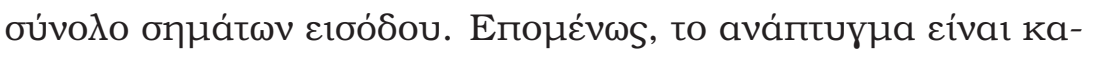

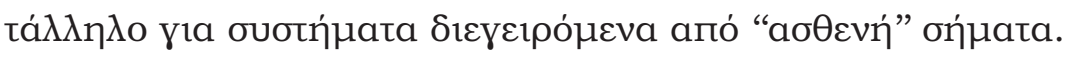

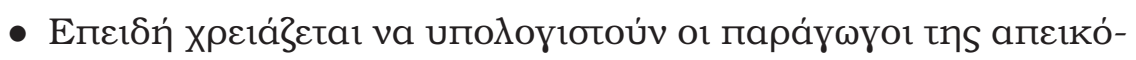
vıons aпó to oń

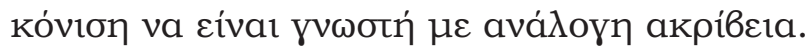




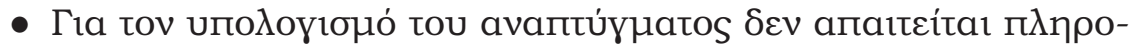

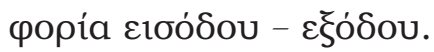

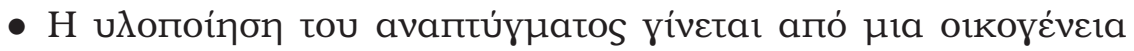

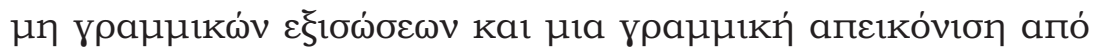

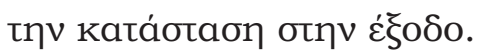

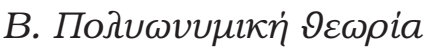

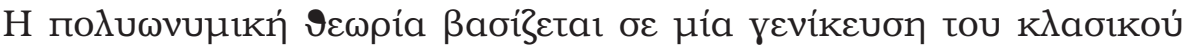

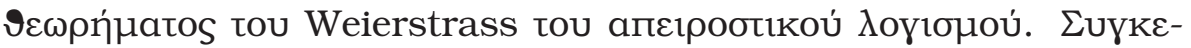

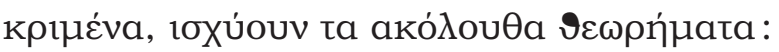

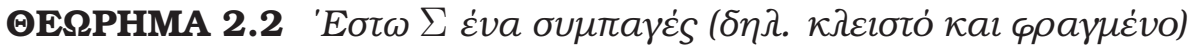

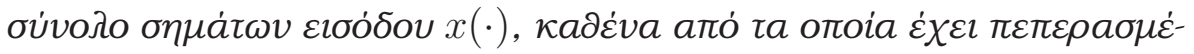

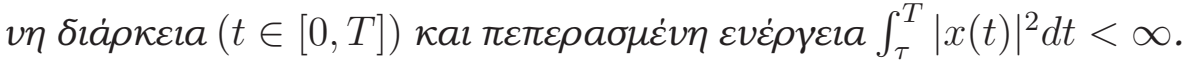

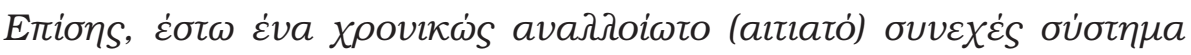

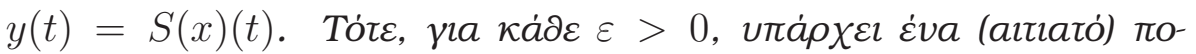

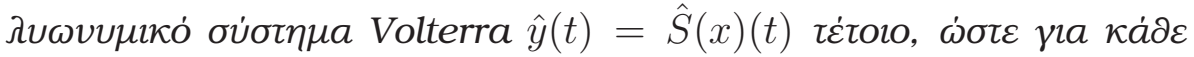

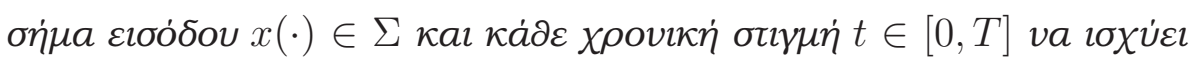
$|y(t)-\hat{y}(t)|<\varepsilon$.

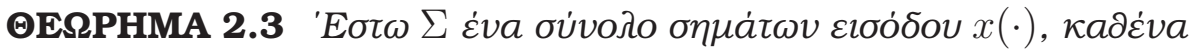

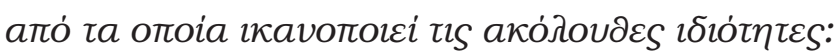

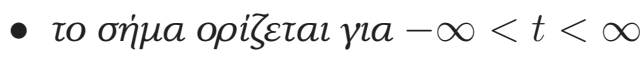

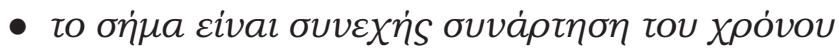

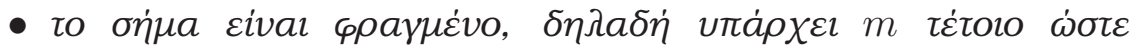

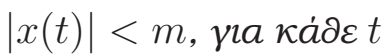




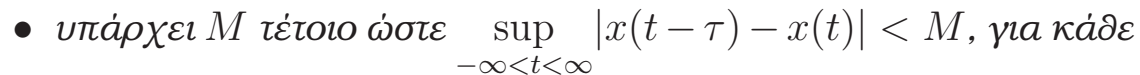
$\tau>0$.

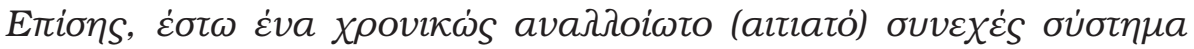

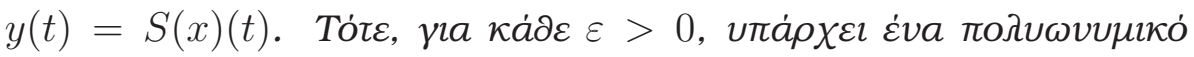

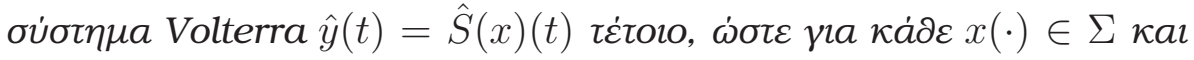

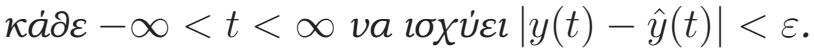

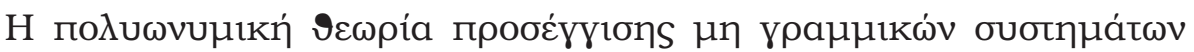

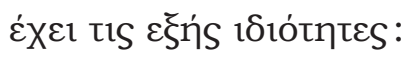

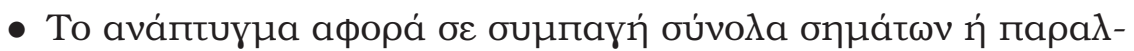
גayés tous.

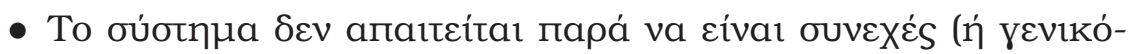

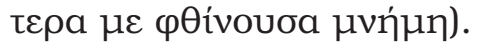

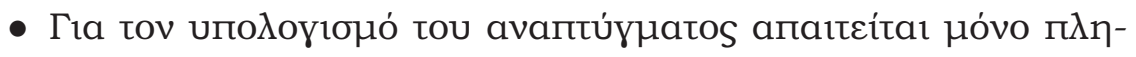

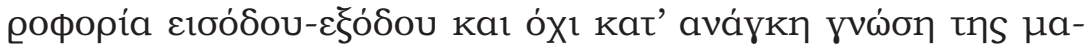

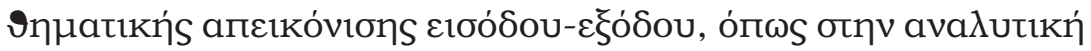

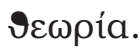

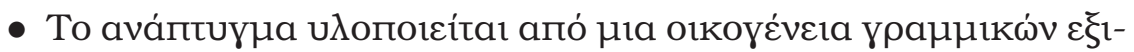

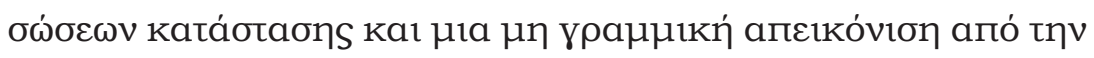

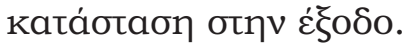




\section{Keфádaı 3}

\section{$\Delta 1 \varepsilon \rho \varepsilon u ́ v \eta \sigma \eta ~ a v \tau 1 k \varepsilon ı \varepsilon ́ v \omega v ~ \mu \varepsilon$ kupattkoús raגpoús}

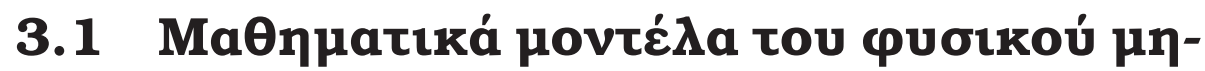

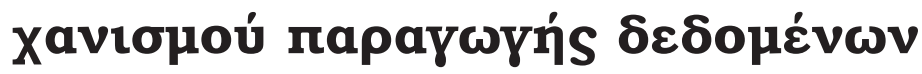

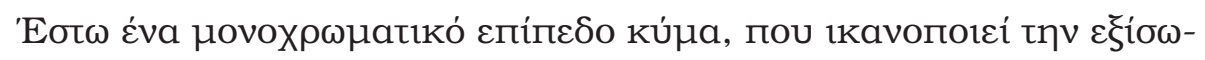

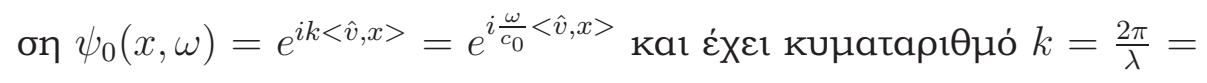

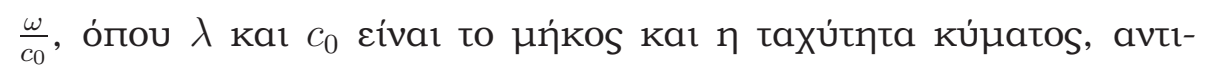

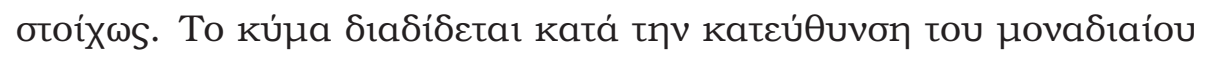

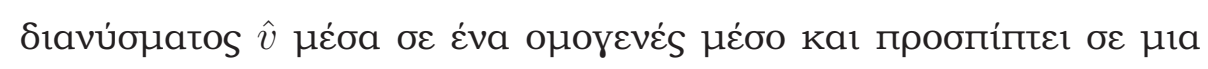

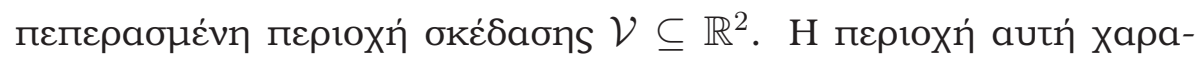

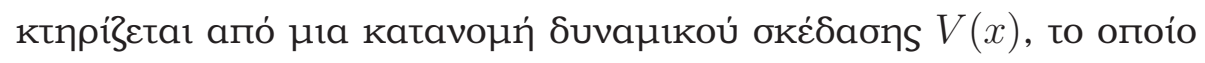

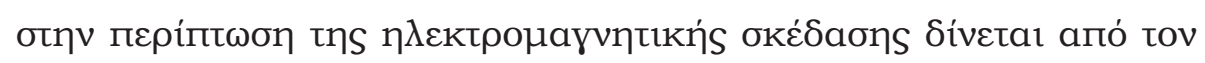

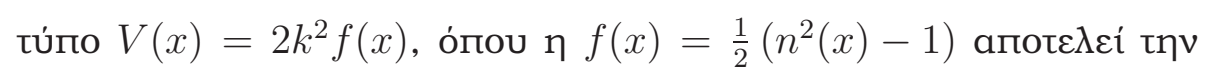




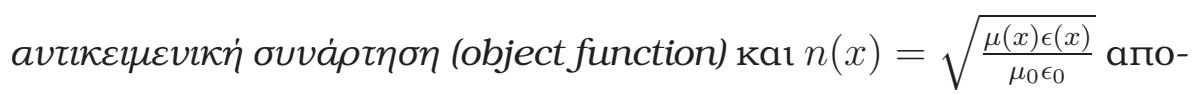

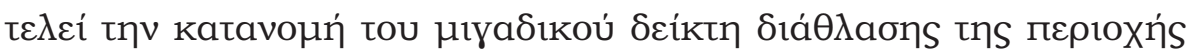

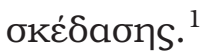

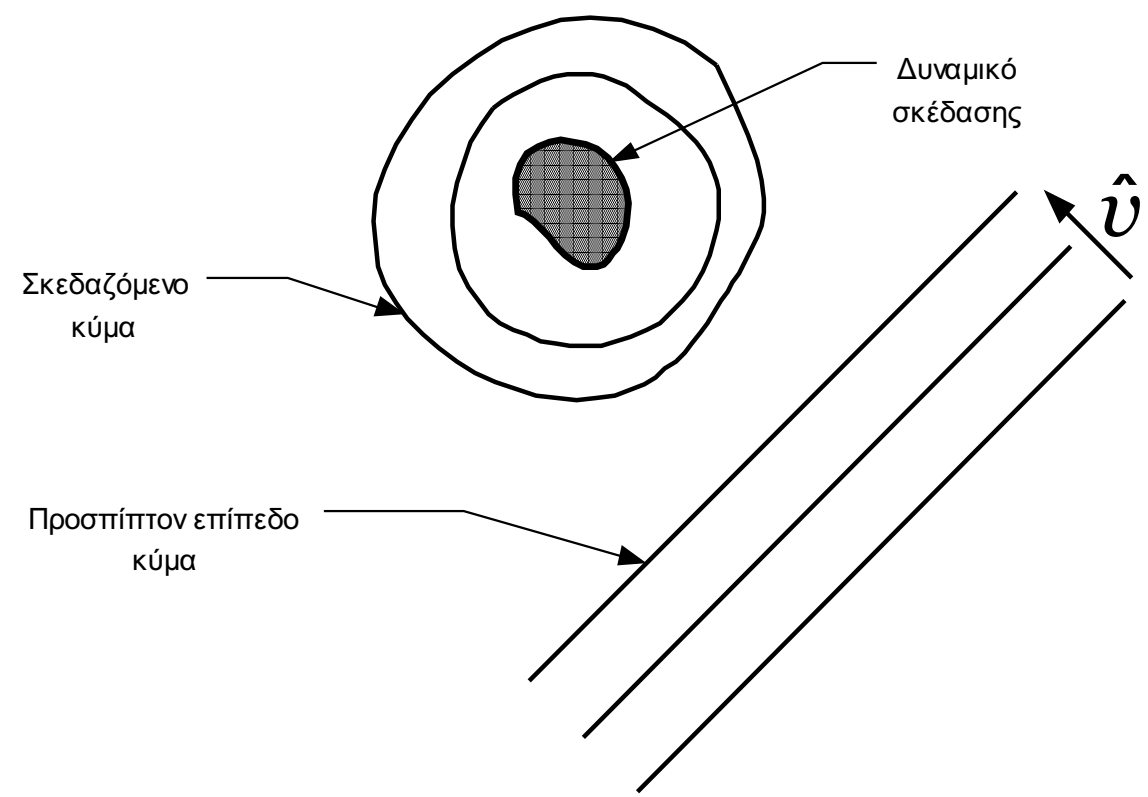

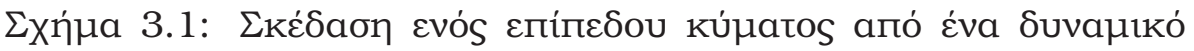

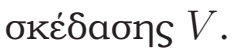

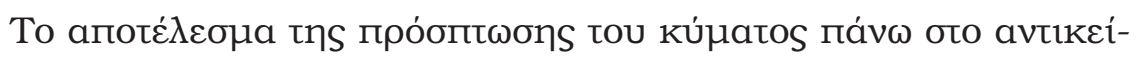

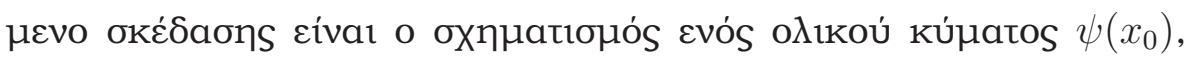

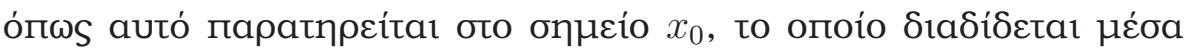

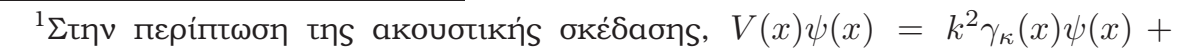
$\nabla\left[\gamma_{\rho}(x) \nabla \psi(x)\right]$, о́поч $\gamma_{k}(x)=\frac{\kappa(x)-\kappa_{0}}{\kappa_{0}}, \gamma_{\rho}(x)=\frac{\rho(x)-\rho_{0}}{\rho_{0}} \mu \varepsilon \kappa(x)$ каи $\rho(x)$ va

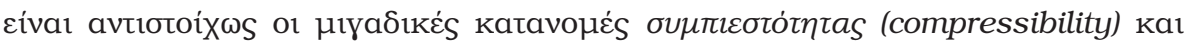

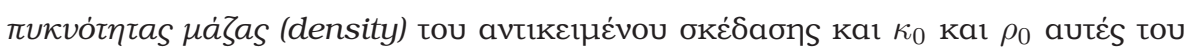

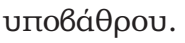




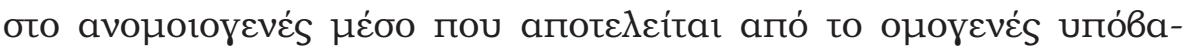

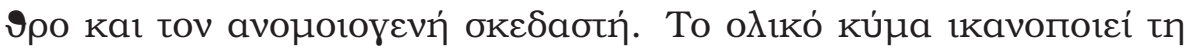

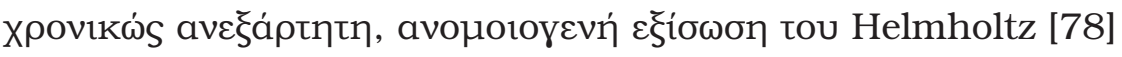

$$
\left(\nabla_{x_{0}}^{2}+k^{2}\right) \psi\left(x_{0}\right)=-V\left(x_{0}\right) \psi\left(x_{0}\right)
$$

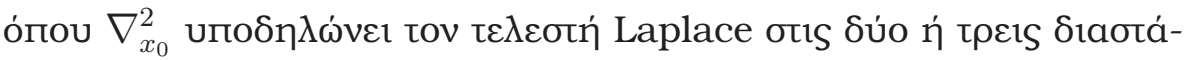
ocis.

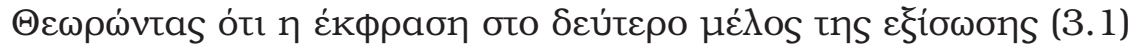

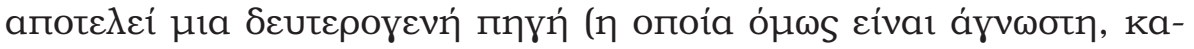

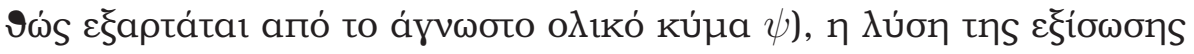

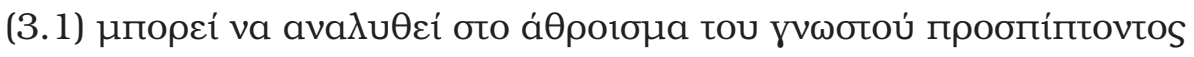

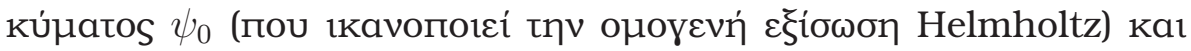

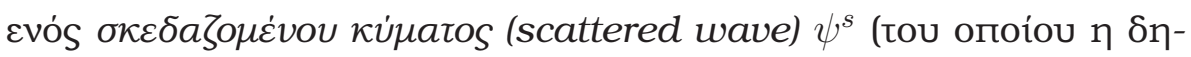

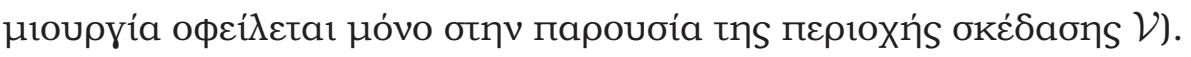

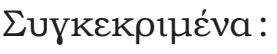

$$
\psi\left(x_{0}\right)=\psi_{0}\left(x_{0}\right)+\psi^{s}\left(x_{0}\right),
$$

о́поч

$$
\psi^{s}\left(x_{0}\right)=-\int_{\mathcal{V}} V\left(x^{\prime}\right) \psi\left(x^{\prime}\right) G\left(x_{0}-x^{\prime}\right) d x^{\prime} .
$$

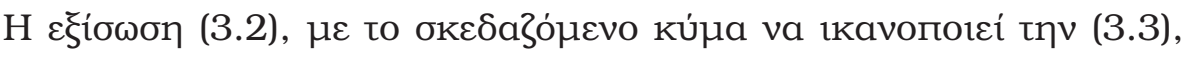

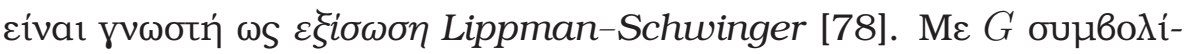

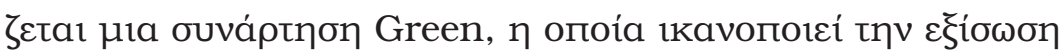

$$
\left(\nabla^{2}+k^{2}\right) G\left(x, x^{\prime}\right)=\delta\left(x-x^{\prime}\right) .
$$

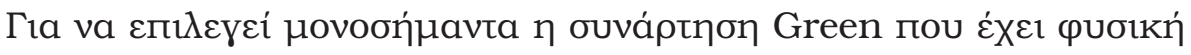

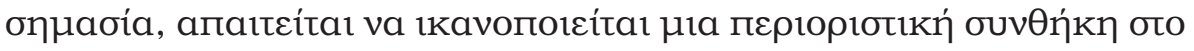




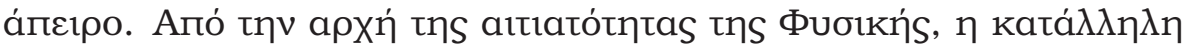

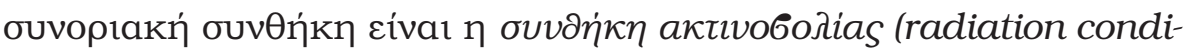

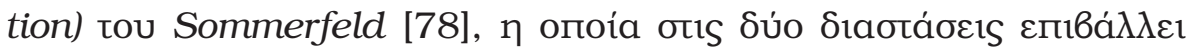

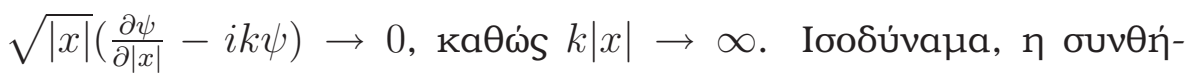

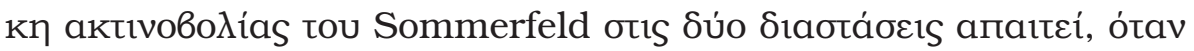

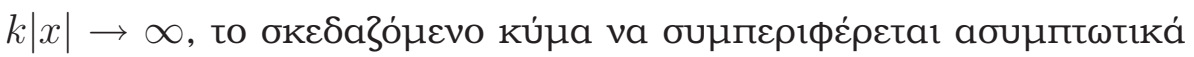

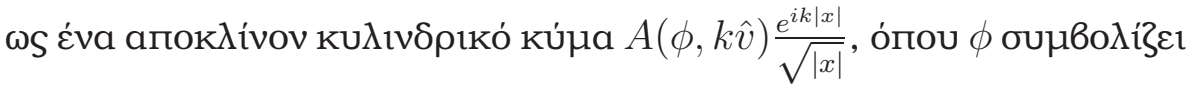

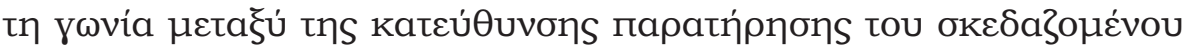

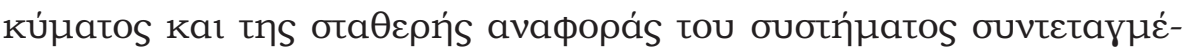

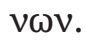

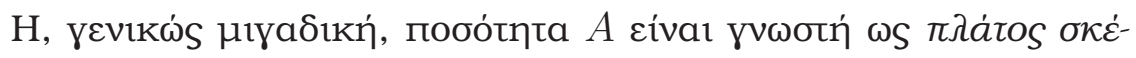

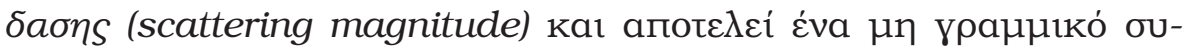

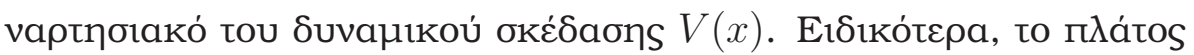

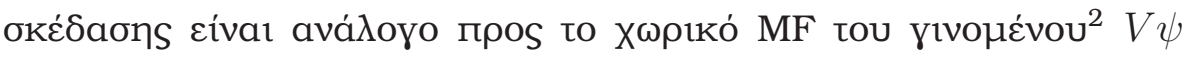

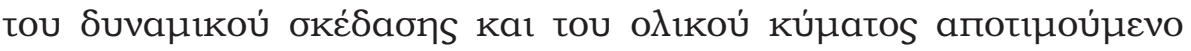

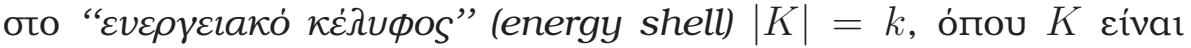

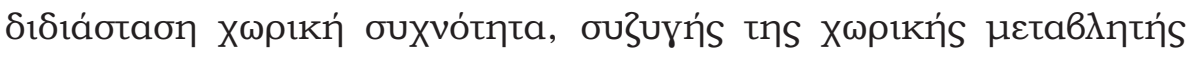

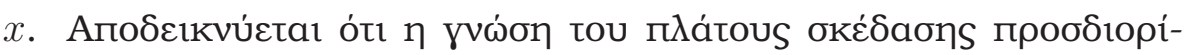

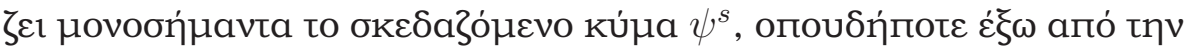

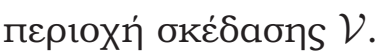

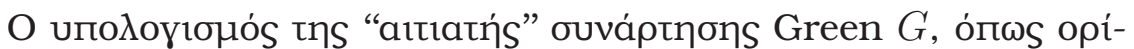

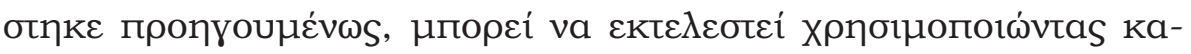

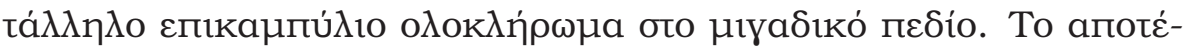

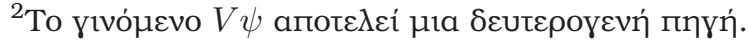




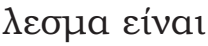

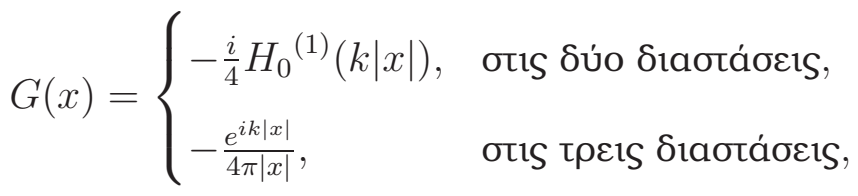

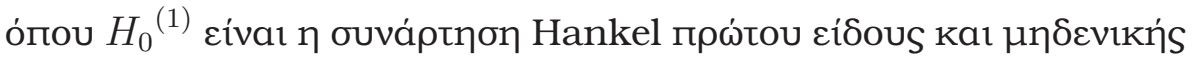
táłnns.

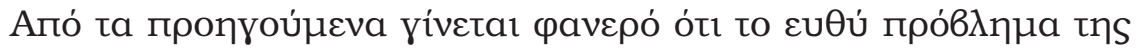

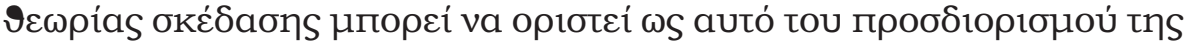

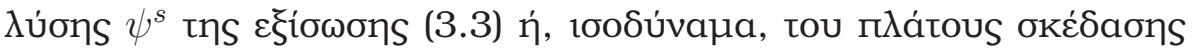

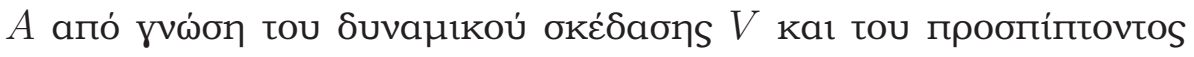

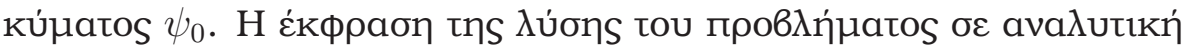

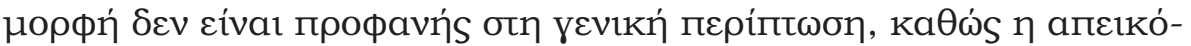

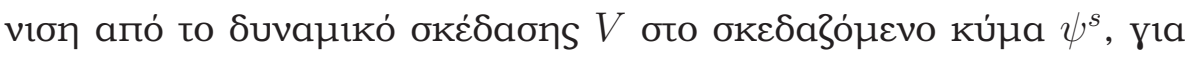

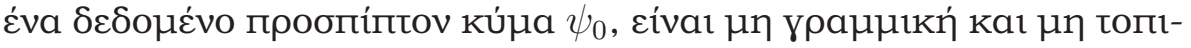

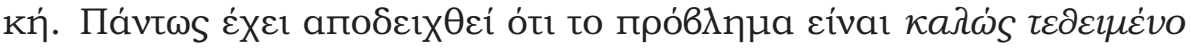

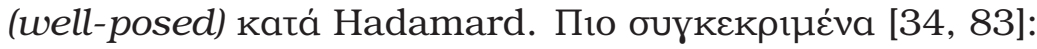

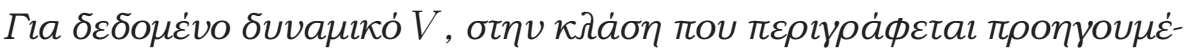

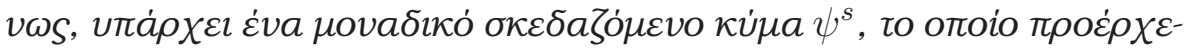

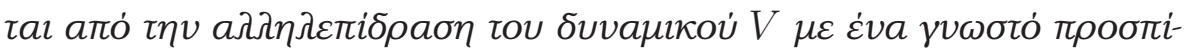

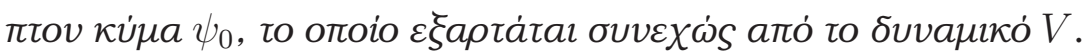

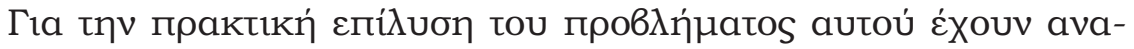

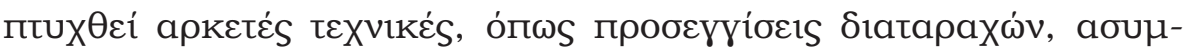

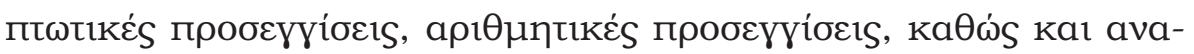

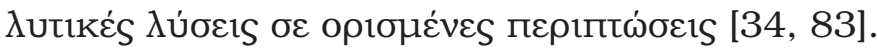

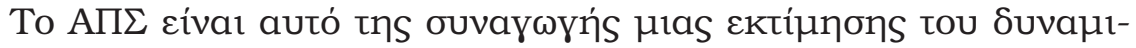




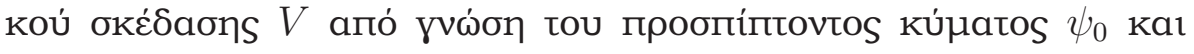

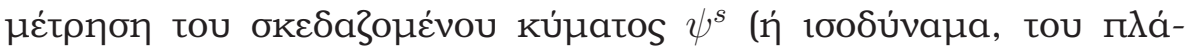

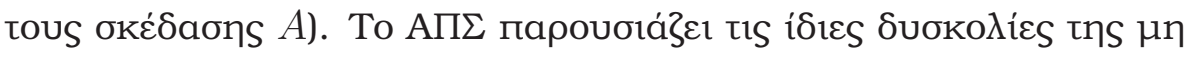

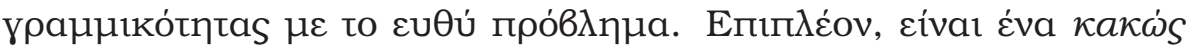

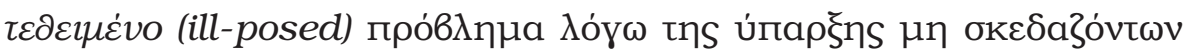

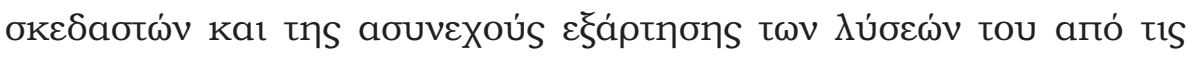

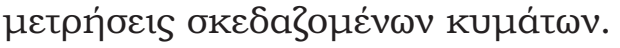

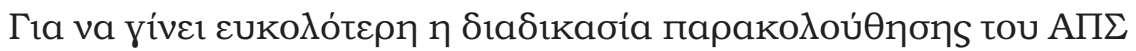

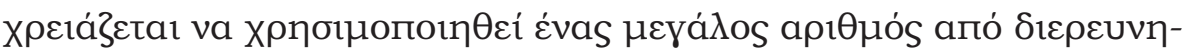

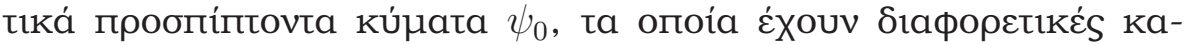

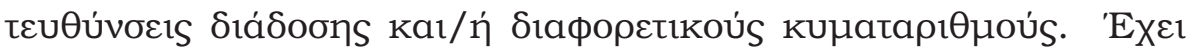

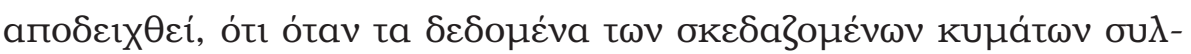

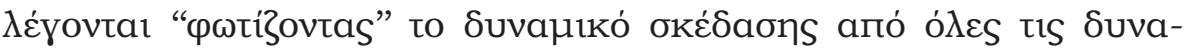

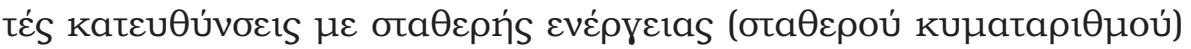

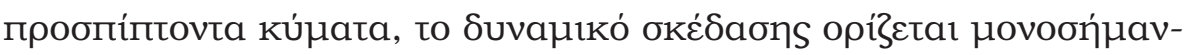

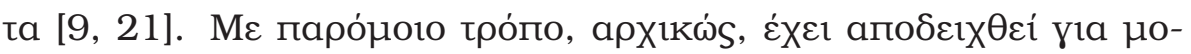

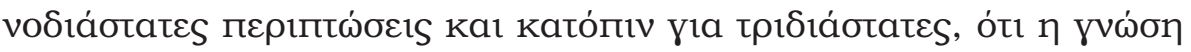

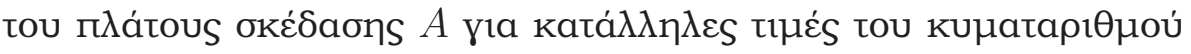

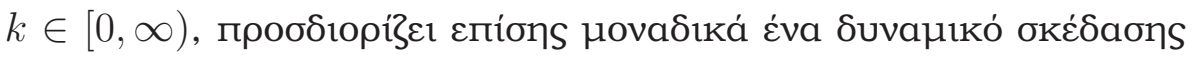

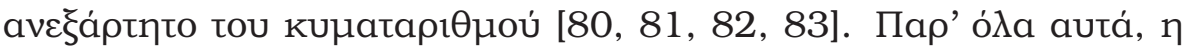

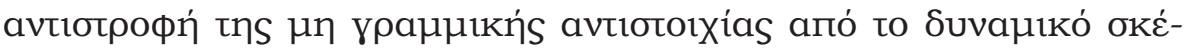

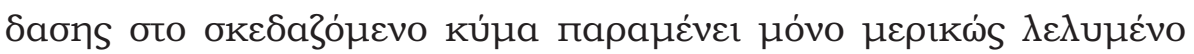

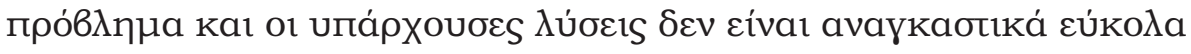

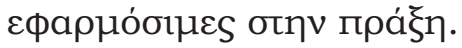




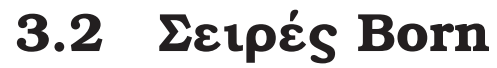

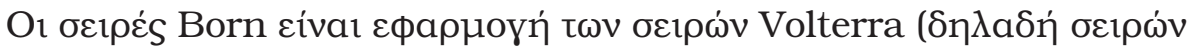

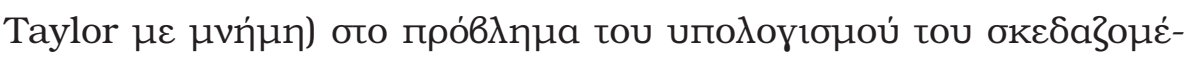

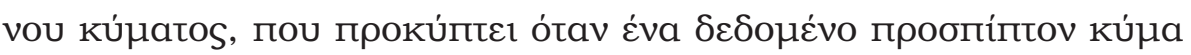

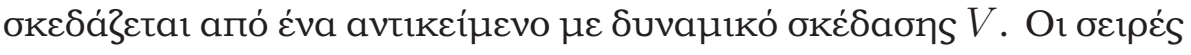

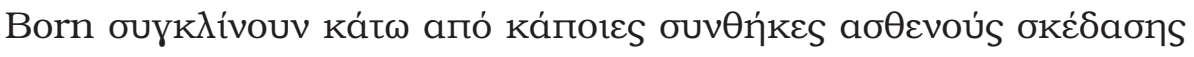

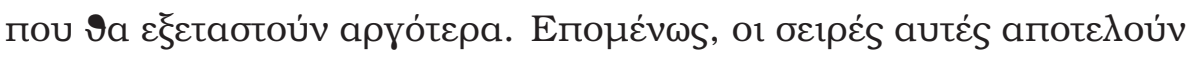

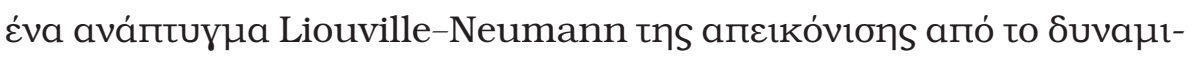

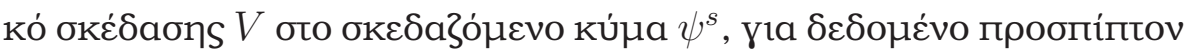

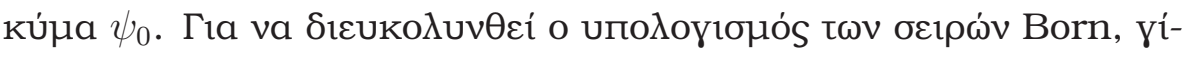

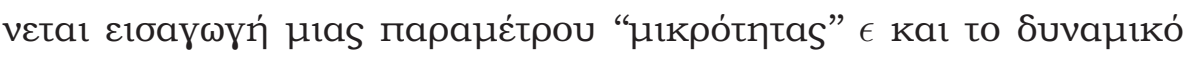

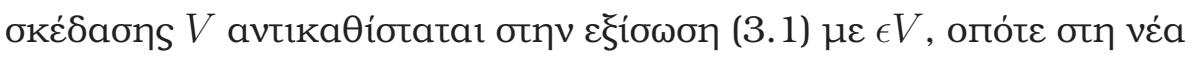

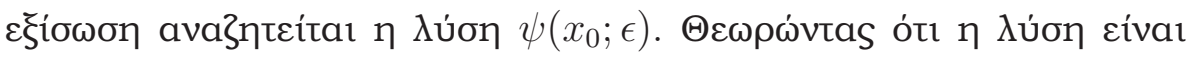

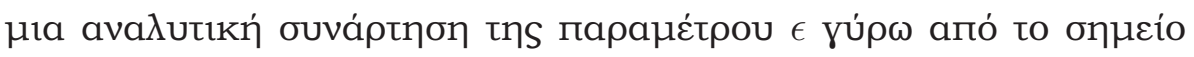

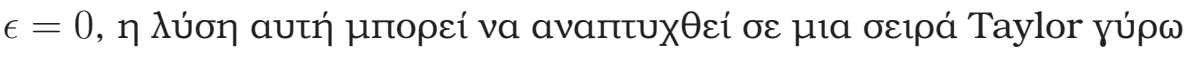
aпó to $\epsilon=0$,

$$
\psi\left(x_{0} ; \epsilon\right)=\psi_{0}\left(x_{0}\right)+\epsilon \psi_{1}\left(x_{0}\right)+\epsilon^{2} \psi_{2}\left(x_{0}\right)+\ldots,
$$

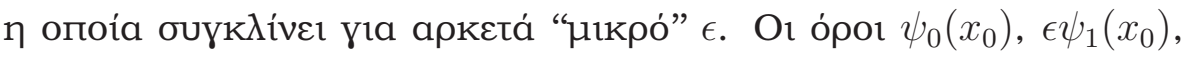

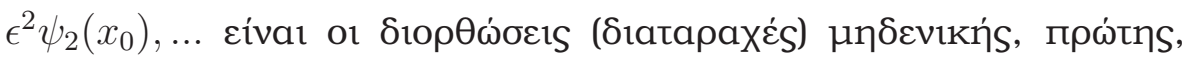

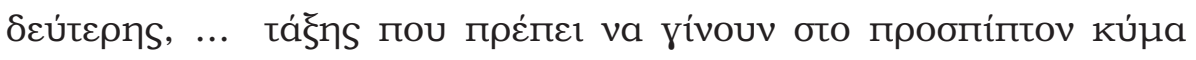

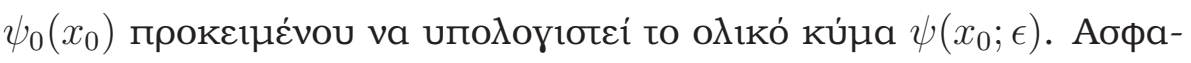

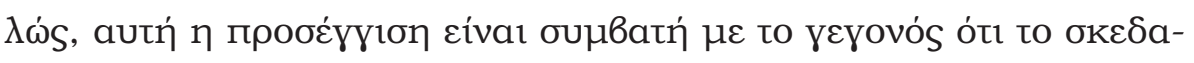

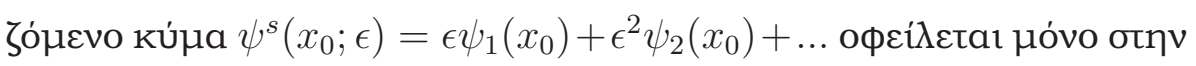

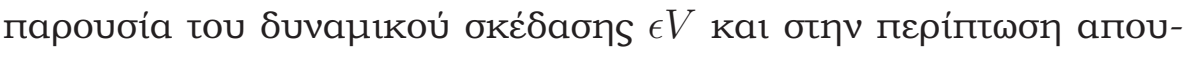




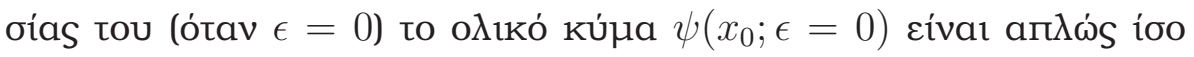

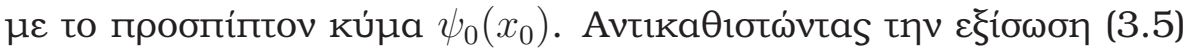

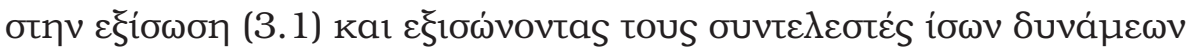

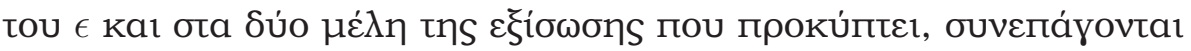

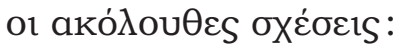

$$
\begin{aligned}
\left(\nabla_{x_{0}}^{2}+k^{2}\right) \psi_{0}\left(x_{0}\right) & =0 \\
\left(\nabla_{x_{0}}^{2}+k^{2}\right) \psi_{1}\left(x_{0}\right) & =-V\left(x_{0}\right) \psi_{0}\left(x_{0}\right) \\
\left(\nabla_{x_{0}}^{2}+k^{2}\right) \psi_{2}\left(x_{0}\right) & =-V\left(x_{0}\right) \psi_{1}\left(x_{0}\right) \\
& \vdots \\
\left(\nabla_{x_{0}}^{2}+k^{2}\right) \psi_{n}\left(x_{0}\right) & =-V\left(x_{0}\right) \psi_{n-1}\left(x_{0}\right) \\
& \vdots
\end{aligned}
$$

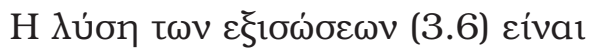

$$
\begin{aligned}
\psi_{1}\left(x_{0}\right) & =-\int_{\mathcal{V}} V\left(x^{\prime}\right) \psi_{0}\left(x^{\prime}\right) G\left(x_{0}-x^{\prime}\right) d x^{\prime} \\
\psi_{2}\left(x_{0}\right) & =-\int_{\mathcal{V}} V\left(x^{\prime}\right) \psi_{1}\left(x^{\prime}\right) G\left(x_{0}-x^{\prime}\right) d x^{\prime} \\
& \vdots \\
\psi_{n}\left(x_{0}\right) & =-\int_{\mathcal{V}} V\left(x^{\prime}\right) \psi_{n-1}\left(x^{\prime}\right) G\left(x_{0}-x^{\prime}\right) d x^{\prime} \\
& \vdots
\end{aligned}
$$

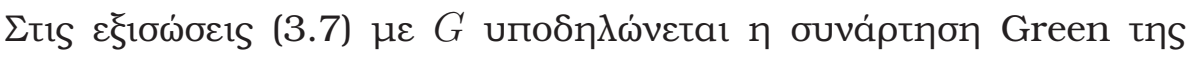

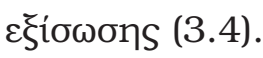

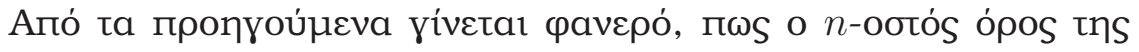

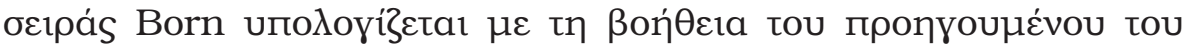




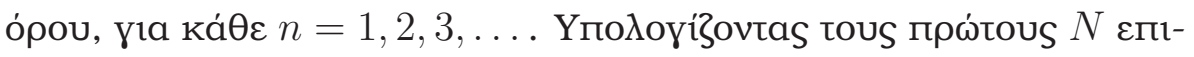

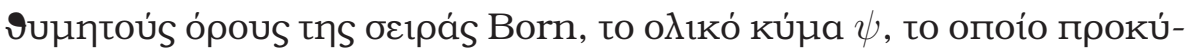

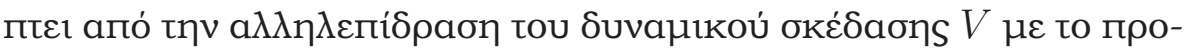

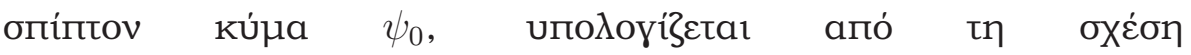

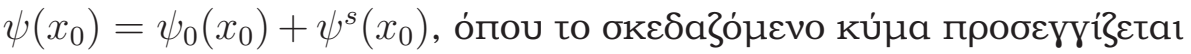

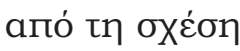

$$
\psi^{s}\left(x_{0}\right) \approx \psi_{1}\left(x_{0}\right)+\psi_{2}\left(x_{0}\right)+\ldots+\psi_{N}\left(x_{0}\right) .
$$

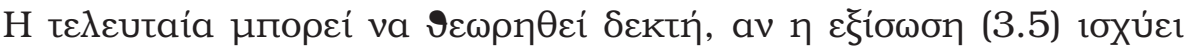
ka1 үıа $\epsilon=1$.

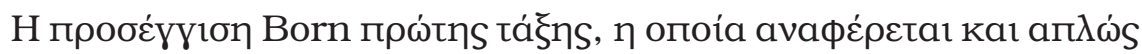

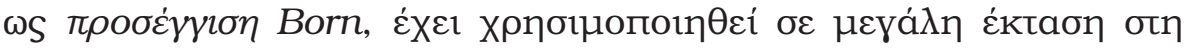

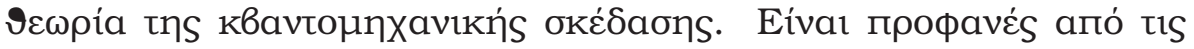

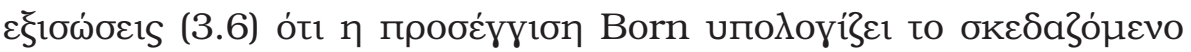

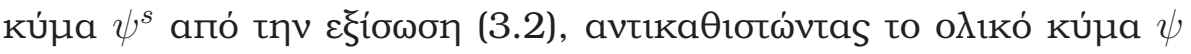

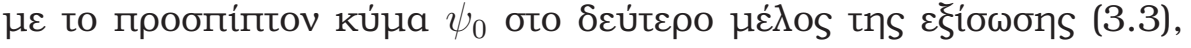

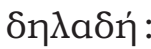

$$
\psi^{s}\left(x_{0}\right) \approx \psi_{1}\left(x_{0}\right)=-\int_{\mathcal{V}} V\left(x^{\prime}\right) \psi_{0}\left(x^{\prime}\right) G\left(x_{0}-x^{\prime}\right) d x^{\prime}
$$

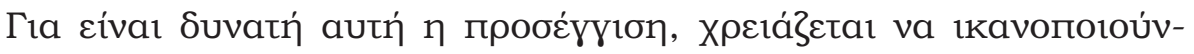

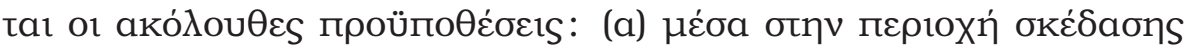

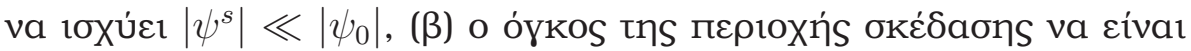

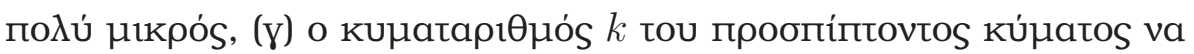

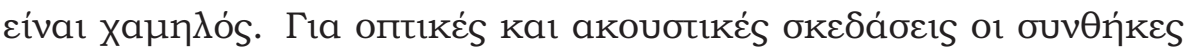

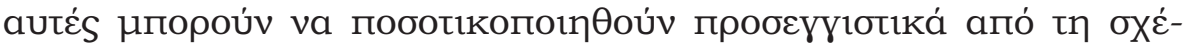


on $V_{m} k D \ll 1$, óпоu $V_{m}=\max _{x \in \mathcal{V}}|V(x)|$ kaı $D$ cívaı $\eta$ aktiva tou

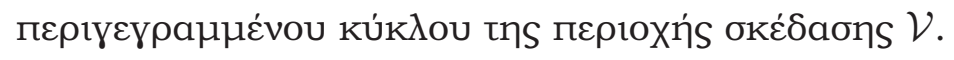

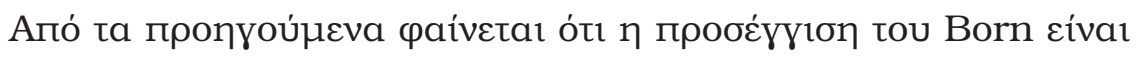

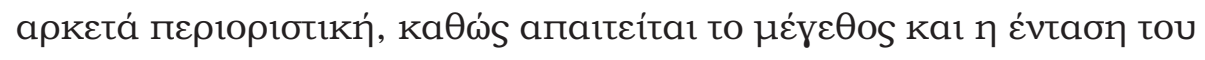

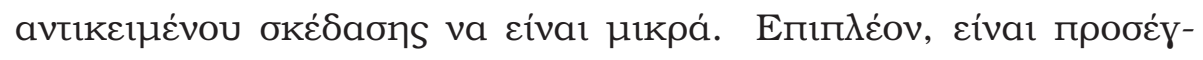

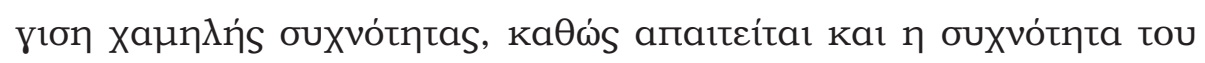

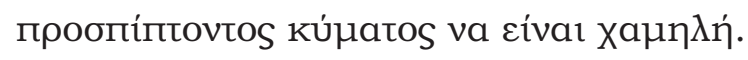

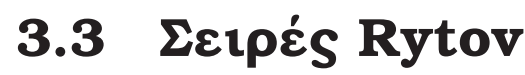

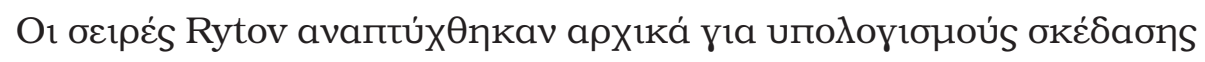

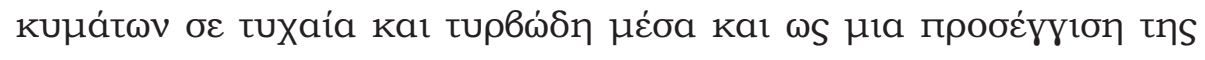

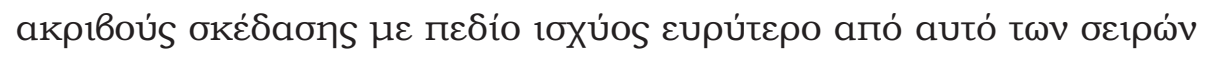

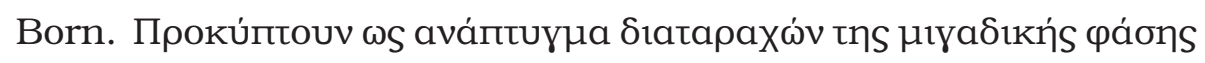
(

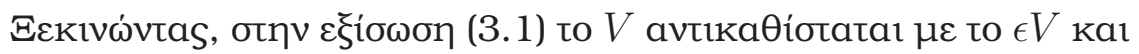

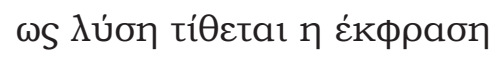

$$
\psi\left(x_{0} ; \epsilon\right)=e^{i k W\left(x_{0} ; \epsilon\right)}
$$

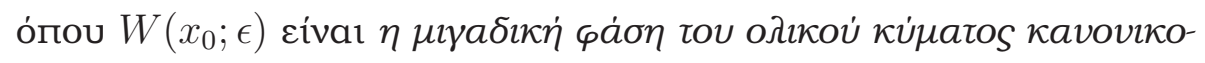

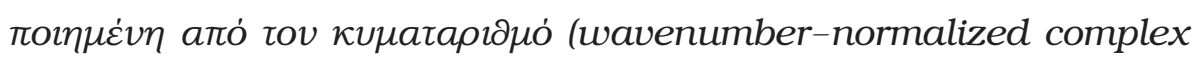

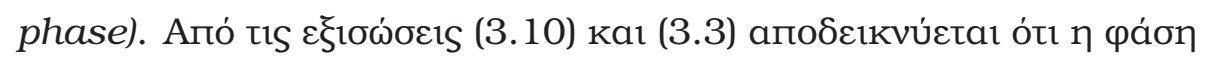

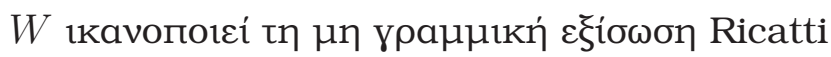

$$
\nabla W \cdot \nabla W+\frac{1}{i k} \nabla^{2} W-1=\frac{\epsilon}{k^{2}} V
$$




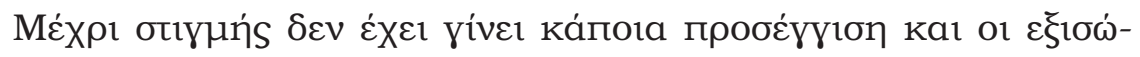

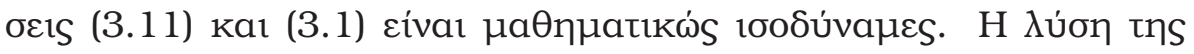

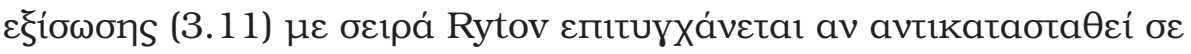

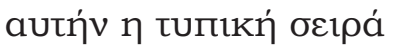

$$
W\left(x_{0} ; \epsilon\right)=W_{0}\left(x_{0}\right)+\epsilon W_{1}\left(x_{0}\right)+\epsilon^{2} W_{2}\left(x_{0}\right)+\ldots
$$

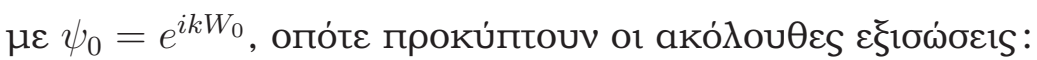

$$
\begin{aligned}
\nabla_{x_{0}} W_{0} \cdot \nabla_{x_{0}} W_{0}+\frac{1}{i k} \nabla_{x_{0}}^{2} W_{0} & =1 \\
2 \nabla_{x_{0}} W_{0} \cdot \nabla_{x_{0}} W_{1}+\frac{1}{i k} \nabla_{x_{0}}^{2} W_{1} & =\frac{1}{i k} V \\
2\left(\nabla_{x_{0}} W_{0} \cdot \nabla_{x_{0}} W_{2}+\nabla_{x_{0}} W_{1} \cdot \nabla_{x_{0}} W_{1}\right)+\frac{1}{i k} \nabla_{x_{0}}^{2} W_{2} & =0 \\
2\left(\nabla_{x_{0}} W_{0} \cdot \nabla_{x_{0}} W_{n}+\nabla_{x_{0}} W_{1} \cdot \nabla_{x_{0}} W_{n-1}+\ldots\right)+\frac{1}{i k} \nabla_{x_{0}}^{2} W_{n} & =0
\end{aligned}
$$

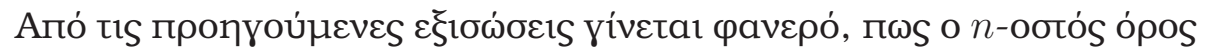

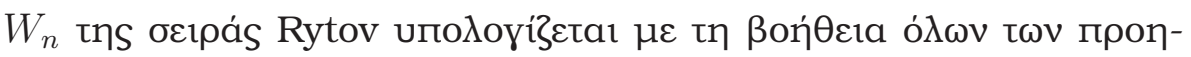
yoú $\mu \varepsilon v \omega v$ ó $\rho \omega v W_{n-1}, W_{n-2}, \ldots, W_{0}$, yıa $n=1,2,3, \ldots$.

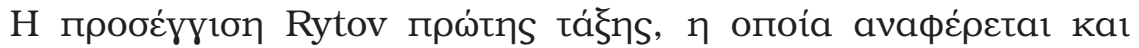

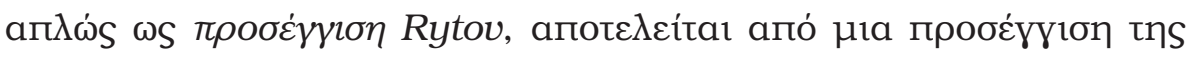

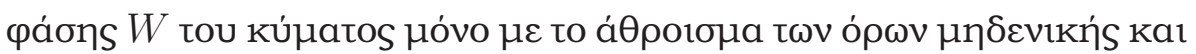

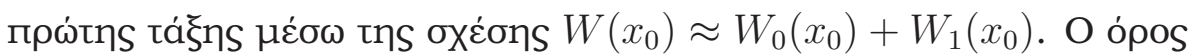




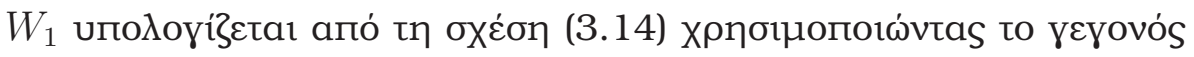

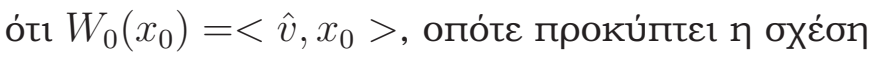

$$
W_{1}=e^{-i k W_{0}} F
$$

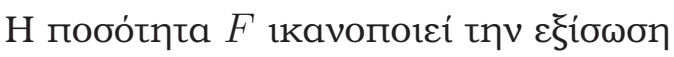

$$
\left(\nabla_{x_{0}}^{2}+k^{2}\right) F=-\frac{V}{i k} e^{-i k W_{0}}
$$

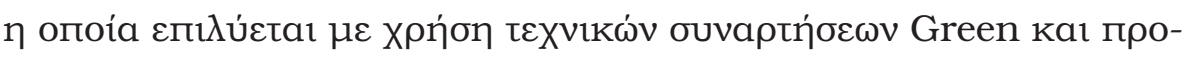

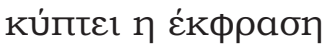

$$
F\left(x_{0}\right)=-\frac{1}{i k} \int_{\mathcal{V}} V\left(x^{\prime}\right) G\left(x_{0}-x^{\prime}\right) e^{i k W_{0}\left(x^{\prime}\right)} d x^{\prime} .
$$

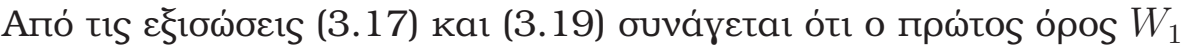

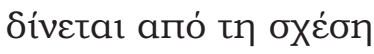

$$
W_{1}\left(x_{0}\right)=-\frac{e^{-i k W_{0}\left(x_{0}\right)}}{i k} \int_{\mathcal{V}} V\left(x^{\prime}\right) G\left(x_{0}-x^{\prime}\right) e^{i k W_{0}\left(x^{\prime}\right)} d x^{\prime} .
$$

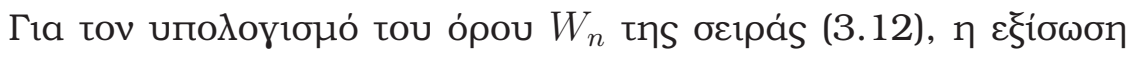

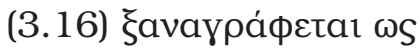

$$
2 \nabla W_{0} \cdot \nabla W_{n}+\frac{1}{i k} \nabla^{2} W_{n}=-\sum_{j=1}^{n-1} \nabla W_{j} \cdot \nabla W_{n-j}
$$

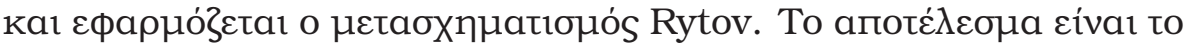

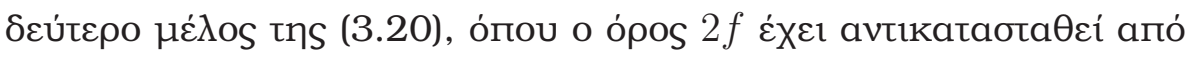

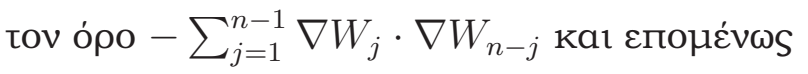

$$
\begin{aligned}
W_{n}\left(x_{0}\right)= & \frac{k e^{-i k W_{0}\left(x_{0}\right)}}{i} \int_{\mathcal{V}} d x^{\prime} \\
& \cdot\left[-\sum_{j=1}^{n-1} \nabla W_{j} \cdot \nabla W_{n-j}\right]\left(x^{\prime}\right) G\left(x_{0}-x^{\prime}\right) e^{i k W_{0}\left(x^{\prime}\right)} .
\end{aligned}
$$




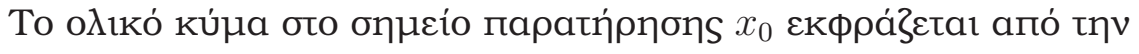

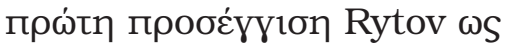

$$
\begin{aligned}
& \psi\left(x_{0}\right) \approx e^{-i k\left[W_{0}\left(x_{0}\right)+W_{1}\left(x_{0}\right)\right]}= \\
& =\exp \left\{i k<\hat{v}, x_{0}>-e^{-i k<\hat{v}, x_{0}>} \int_{\mathcal{V}} V\left(x^{\prime}\right) G\left(x_{0}-x^{\prime}\right) e^{i k<\hat{v}, x^{\prime}>} d x^{\prime}\right\} .
\end{aligned}
$$

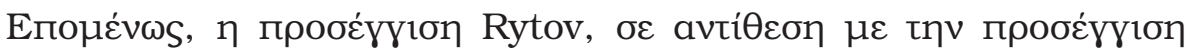

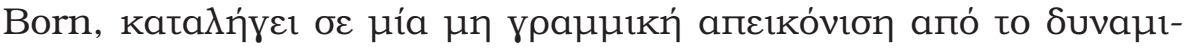

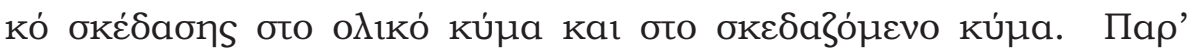

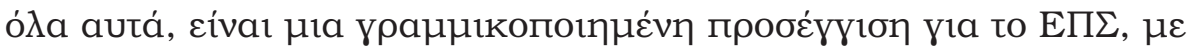

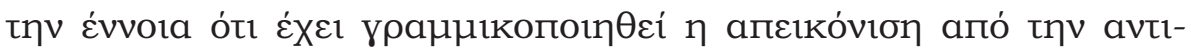

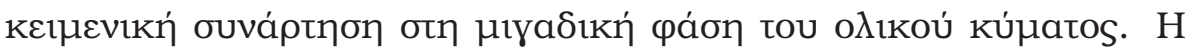

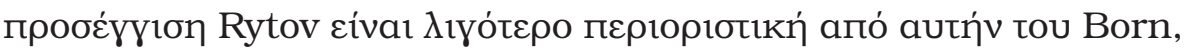

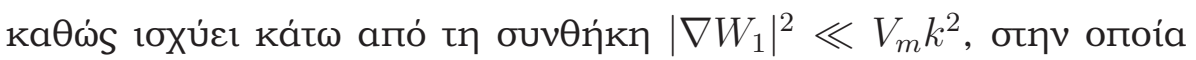

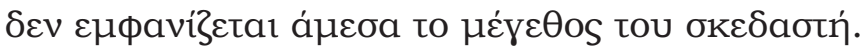

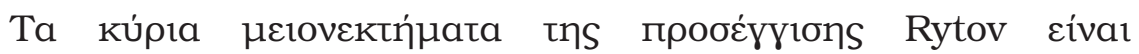

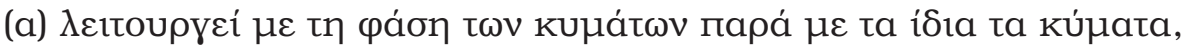

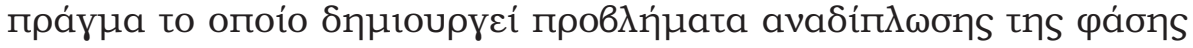

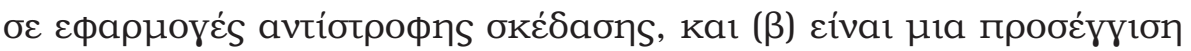

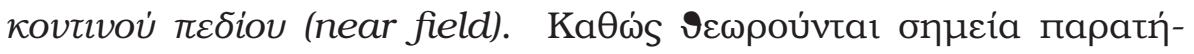

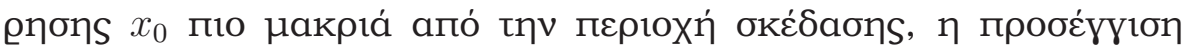

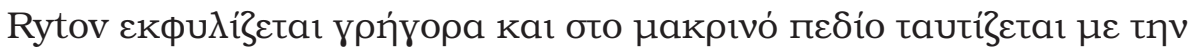

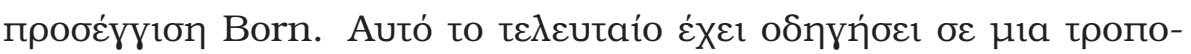

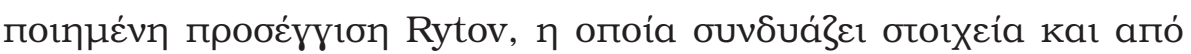

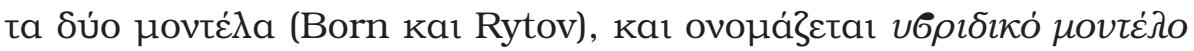


(hybrid model).

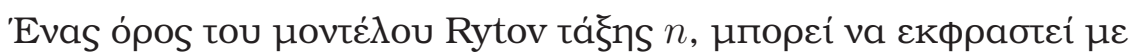

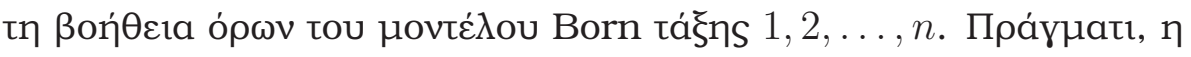

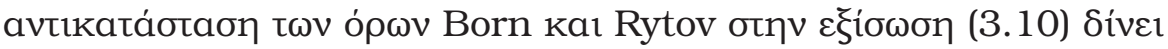

$$
\sum_{n=0}^{\infty} \epsilon^{n} \psi_{n}=\exp \left(i k \sum_{n=0}^{\infty} \epsilon^{n} W_{n}\right),
$$

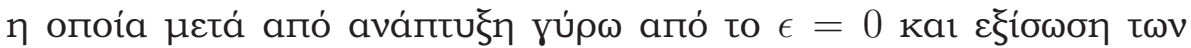

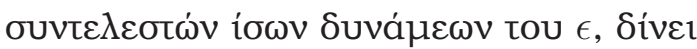

$$
\begin{aligned}
W_{0} & =\frac{1}{i k} \log \psi_{0} \\
W_{1} & =\frac{1}{i k} \frac{\psi_{1}}{\psi_{0}} \\
W_{2} & =\frac{1}{i k}\left[\frac{\psi_{2}}{\psi_{0}}-\frac{1}{2}\left(\frac{\psi_{1}}{\psi_{0}}\right)^{2}\right] \\
W_{3} & =\frac{1}{i k}\left[\frac{\psi_{3}}{\psi_{0}}-\frac{\psi_{2}}{\psi_{0}} \frac{\psi_{1}}{\psi_{0}}+\frac{\psi_{1}^{3}}{3 !}\right] \\
& \vdots
\end{aligned}
$$

Парó

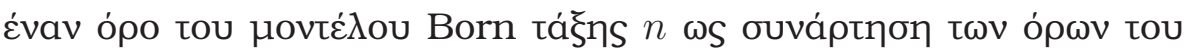

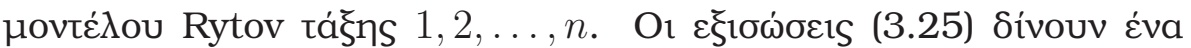

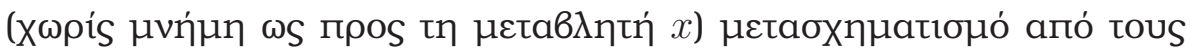
ópous Born otous ópous Rytov.

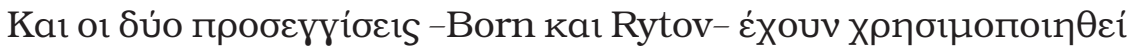

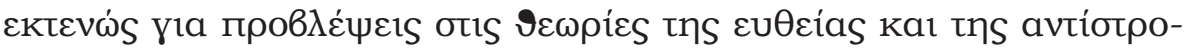

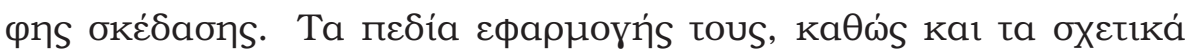

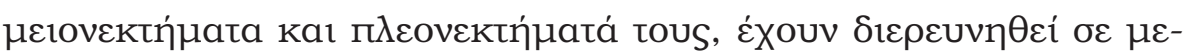

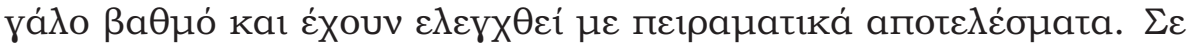




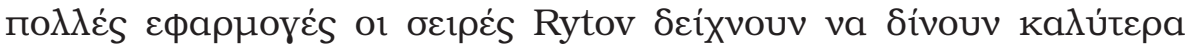

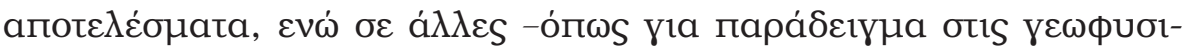

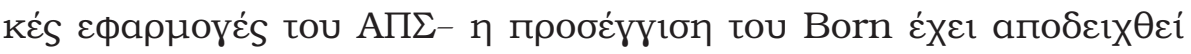

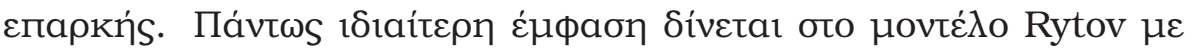

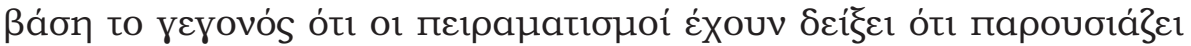

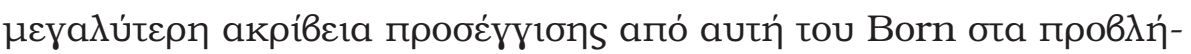

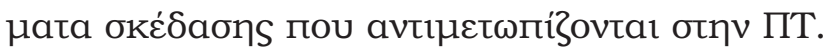

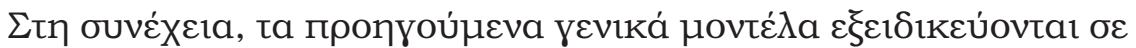

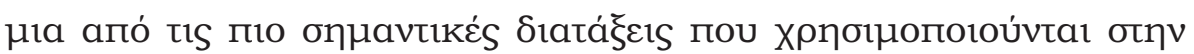

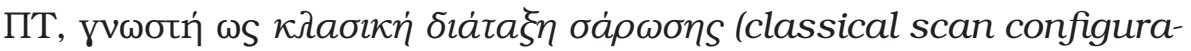

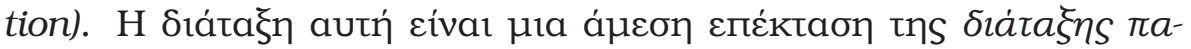

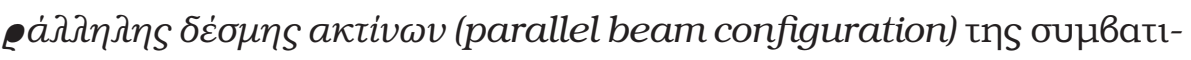

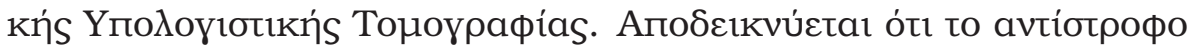

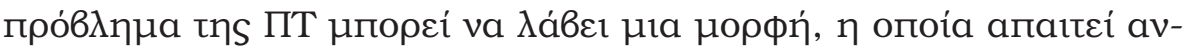

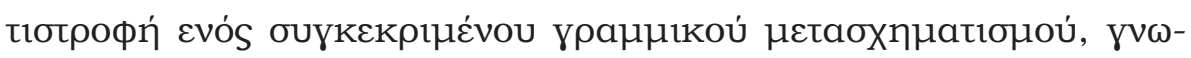

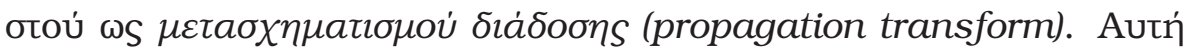

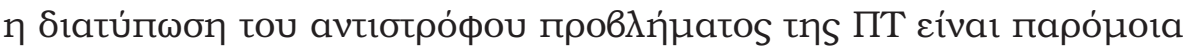

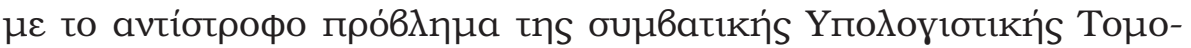

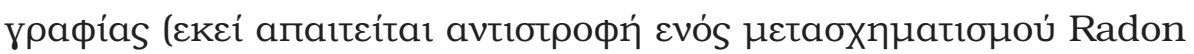

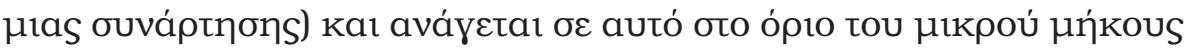

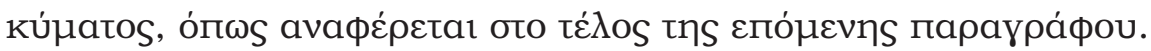




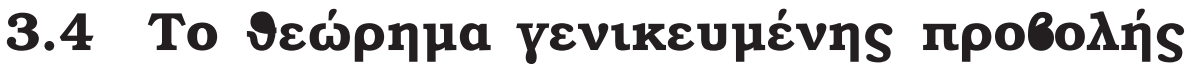 - тори́s}

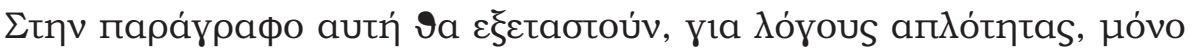

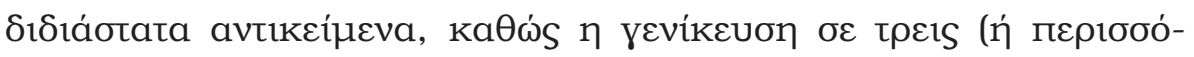

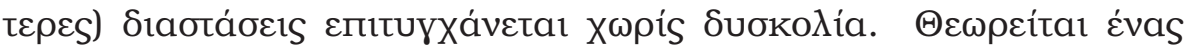

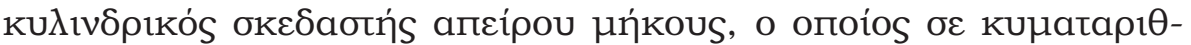

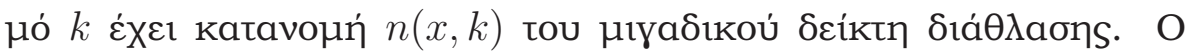

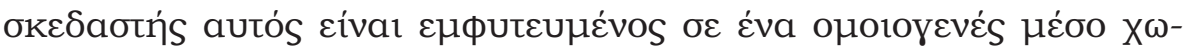

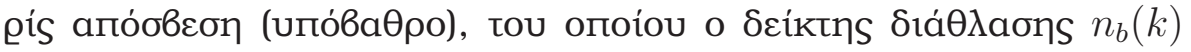

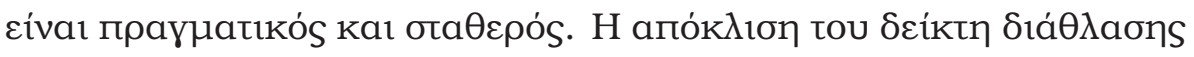

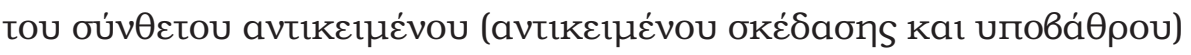

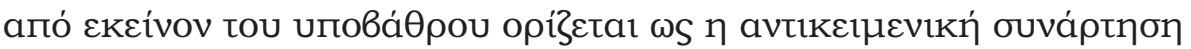
$f(x), x \in \mathbb{R}^{2}$. Пıо очүкєкрџне́va ${ }^{3}$

$$
f(x)=\frac{1}{2}\left(\frac{n^{2}(x, k)}{n^{2}{ }_{b}(k)}-1\right) \approx \frac{n(x, k)}{n_{b}(k)}-1 .
$$

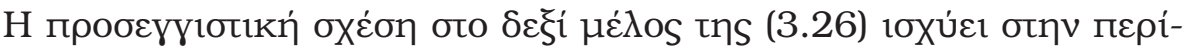

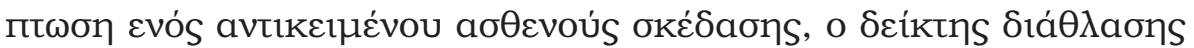

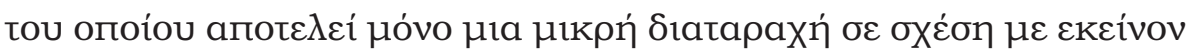

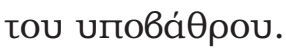

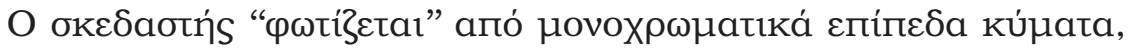

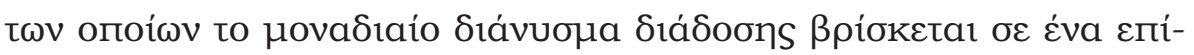

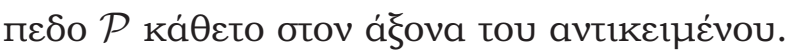

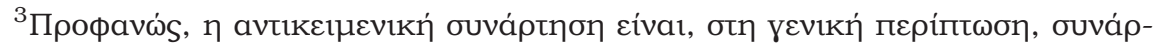

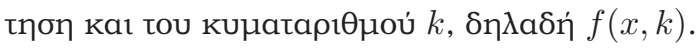




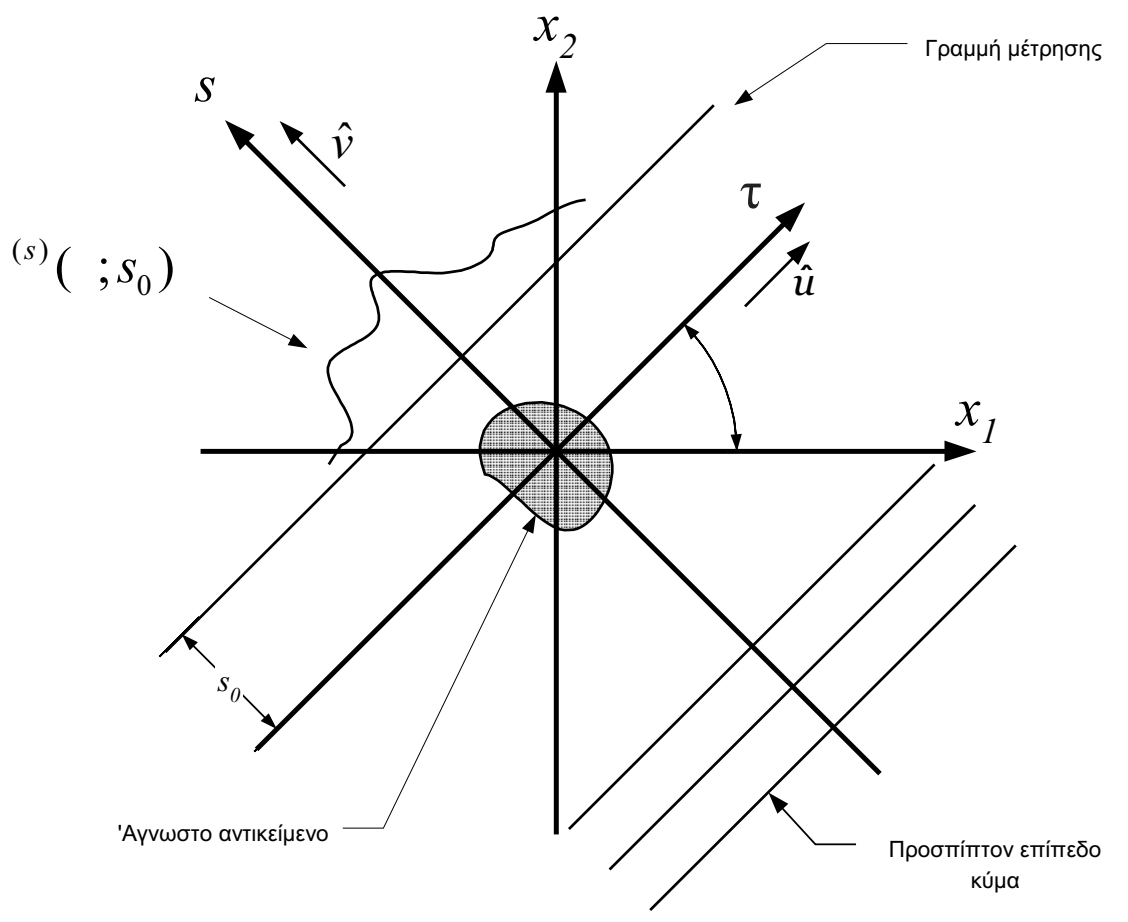

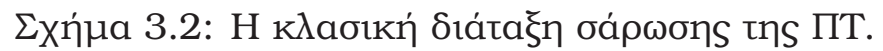

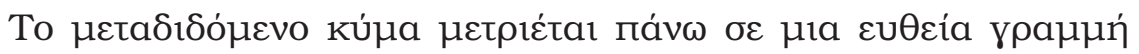

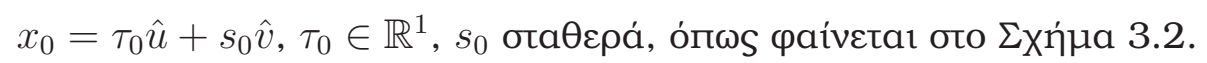

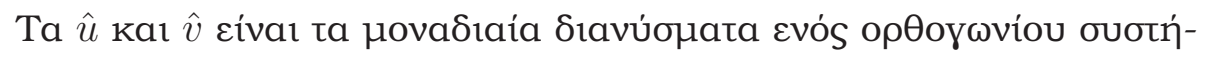

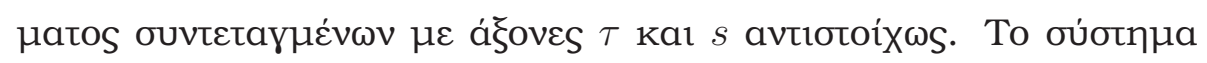

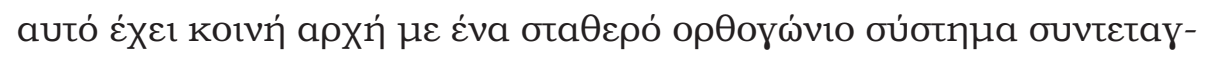

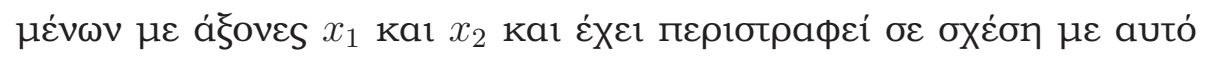

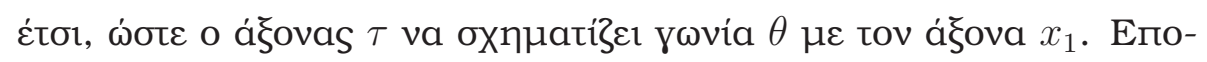
$\mu \varepsilon \dot{v \omega S ~ 10 X u ́ \varepsilon 1 ~}$ 


$$
\tau=x_{1} \cos \theta+x_{2} \sin \theta \text { kaı } s=-x_{1} \sin \theta+x_{2} \cos \theta .
$$

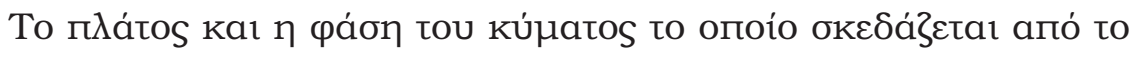

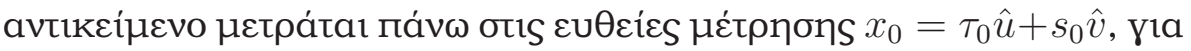

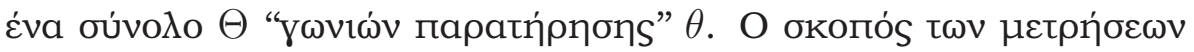

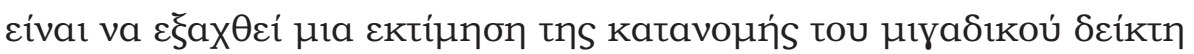

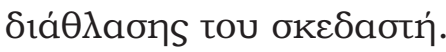

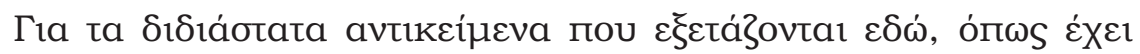

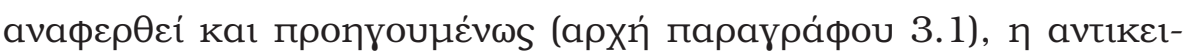

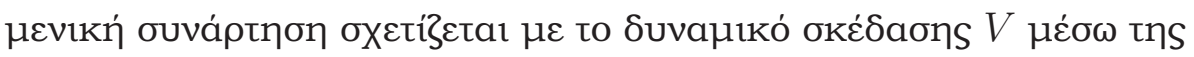

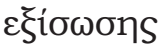

$$
V=2 k^{2} f
$$

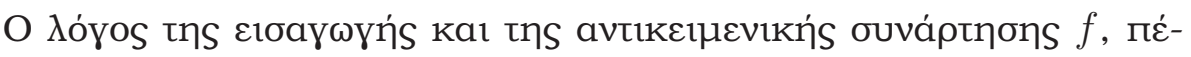

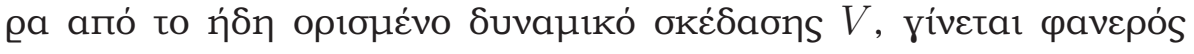

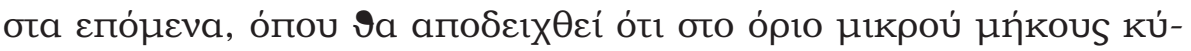

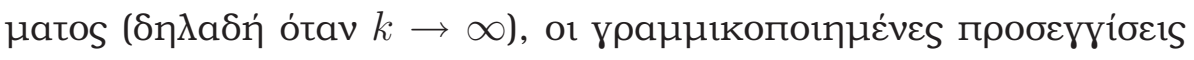

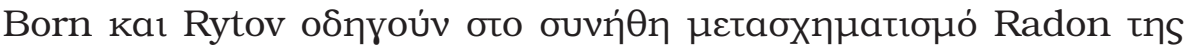

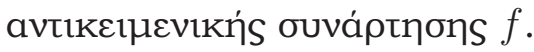

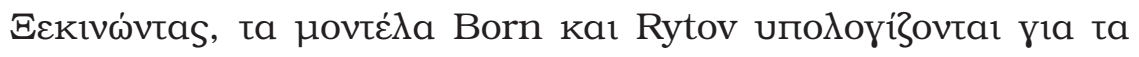

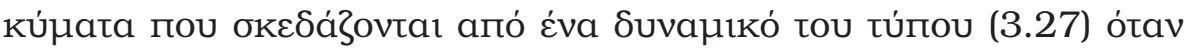

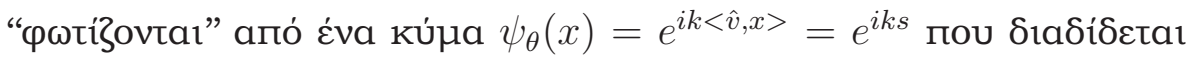

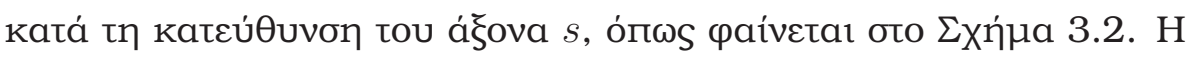

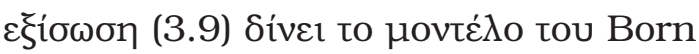

$$
\psi_{\theta}^{B}\left(x_{0}\right)=-2 k^{2} \int_{V} G\left(x_{0}-x^{\prime}\right) f\left(x^{\prime}\right) e^{i k<\hat{v}, x^{\prime}>} d x^{\prime},
$$




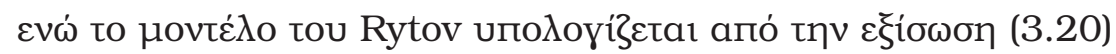

$$
W_{\theta}^{R}\left(x_{0}\right)=-2 k^{2} e^{-i k s_{0}} \int_{V} G\left(x_{0}-x^{\prime}\right) f\left(x^{\prime}\right) e^{i k<\hat{v}, x^{\prime}>} d x^{\prime} .
$$

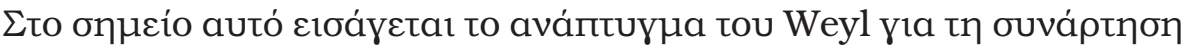

Green $G$

$$
G\left(\tau^{\prime} \hat{u}+s^{\prime} \hat{v}\right)=-\frac{i}{4 \pi} \int_{-\infty}^{\infty} d p e^{i p \tau^{\prime}} \frac{e^{i m\left|s^{\prime}\right|}}{m} \approx-\frac{i}{4 \pi} \int_{-k}^{k} d p e^{i p \tau^{\prime}} \frac{e^{i m\left|s^{\prime}\right|}}{m},
$$

о́поч $m=\left\{\begin{array}{cc}\sqrt{k^{2}-p^{2}}, & \text { vıa }|p| \leq k \\ i \sqrt{p^{2}-k^{2}}, & \text { vıa }|p|>k\end{array}\right.$

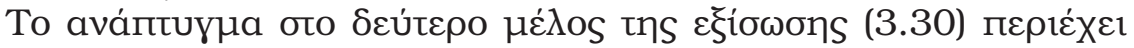

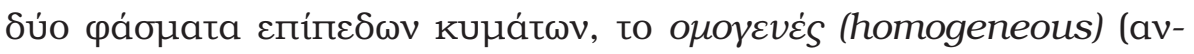

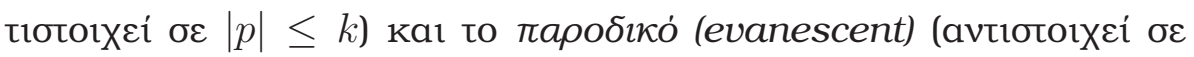

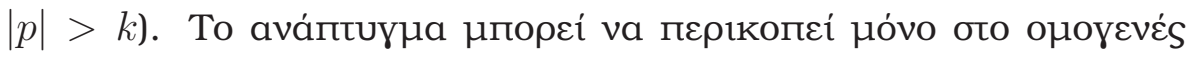

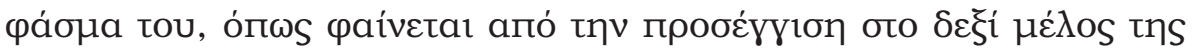

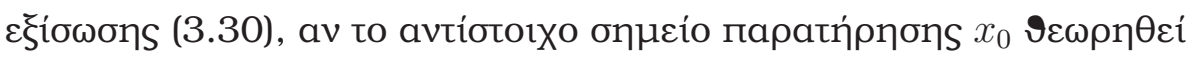

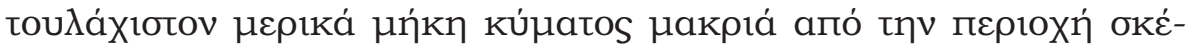

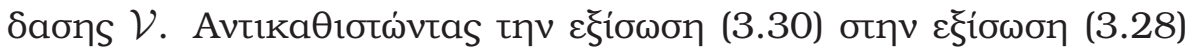
ouváyetal ótı

$$
\begin{aligned}
\psi_{\theta}^{B}\left(\tau_{0} ; s_{0}\right) \approx & \\
& \approx \frac{2 i k^{2}}{4 \pi} \iint_{\mathcal{V}} d \tau^{\prime} d s^{\prime} \int_{-k}^{k} d p e^{i p\left(\tau_{0}-\tau^{\prime}\right)} \frac{e^{i m\left(s_{0}-s^{\prime}\right)}}{m} f\left(\tau^{\prime} \hat{u}+s^{\prime} \hat{v}\right) e^{i k s^{\prime}}= \\
& =\frac{i k^{2}}{2 \pi} \int_{-k}^{k} d p e^{i p \tau_{0}} \frac{e^{i m s_{0}}}{m} \iint_{\mathcal{V}} d \tau^{\prime} d s^{\prime} e^{-i p \tau^{\prime}} e^{-i(m-k) s^{\prime}} f\left(\tau^{\prime} \hat{u}+s^{\prime} \hat{v}\right)= \\
& =\frac{i k^{2}}{2 \pi} \int_{-k}^{k} d p e^{i p \tau_{0}} \frac{e^{i m s_{0}}}{m} \tilde{f}[p \hat{u}+(m-k) \hat{v}]
\end{aligned}
$$




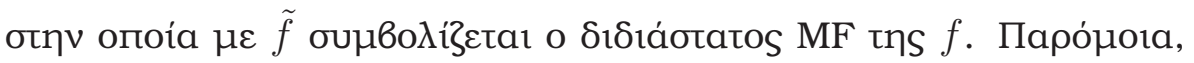

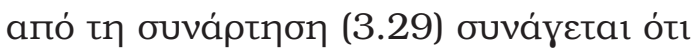

$$
W_{\theta}^{R}\left(\tau_{0} ; s_{0}\right) \approx \frac{k}{2 \pi} \int_{-k}^{k} d p e^{i p \tau_{0}} \frac{e^{i(m-k) s_{0}}}{m} \tilde{f}[p \hat{u}+(m-k) \hat{v}] .
$$

Opißovtas ws

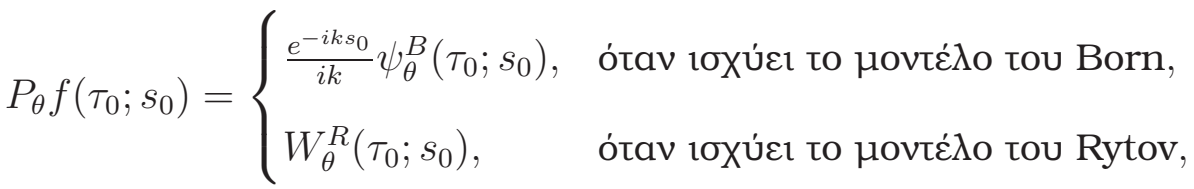

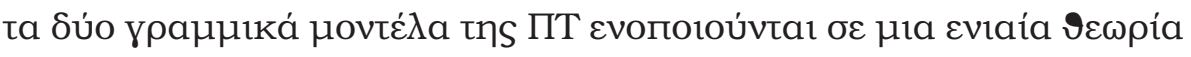

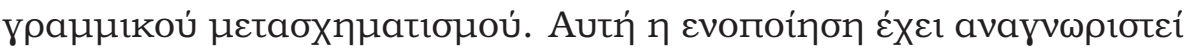

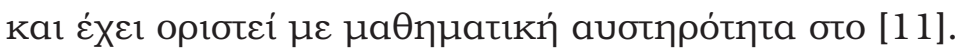

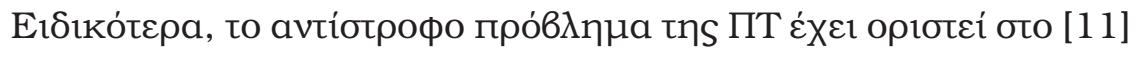

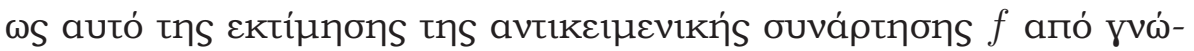

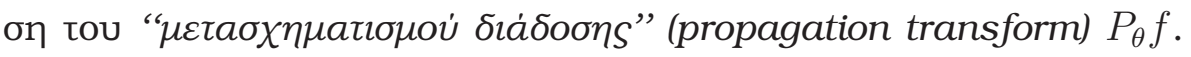

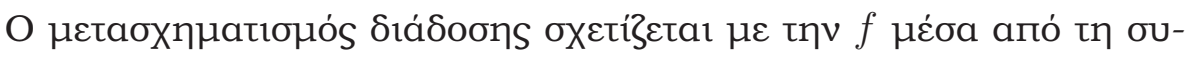
vé $\lambda_{1} \xi \eta$

$$
P_{\theta} f\left(\tau_{0} ; s_{0}\right)=\iint_{\mathcal{V}} d \tau^{\prime} d s^{\prime} \Gamma_{\theta}\left(\tau_{0}-\tau^{\prime}, s_{0}-s^{\prime}\right) f\left(\tau^{\prime} \hat{u}+s^{\prime} \hat{v}\right)
$$

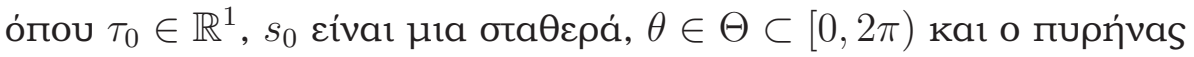

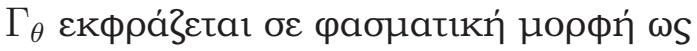

$$
\Gamma_{\theta}\left(\tau^{\prime}, s^{\prime}\right)=\frac{1}{2 \pi} \int_{-k}^{k} d p \frac{k}{m} e^{\left[i p \tau^{\prime}+(m-k) s^{\prime}\right]} .
$$

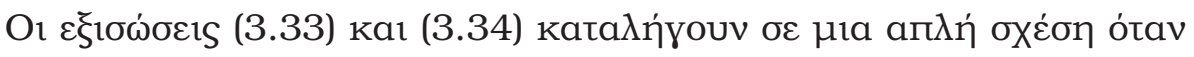

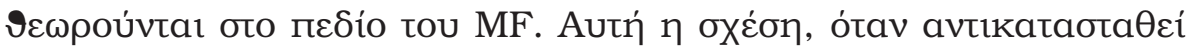




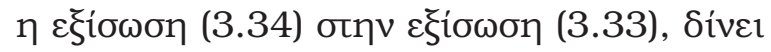

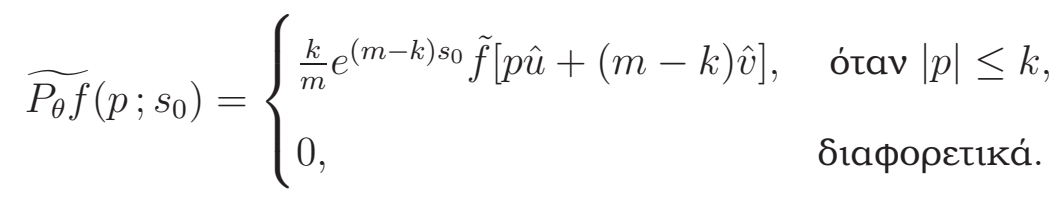

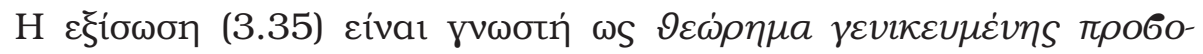

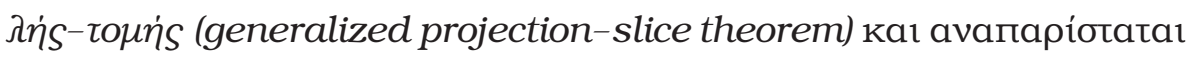

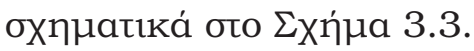

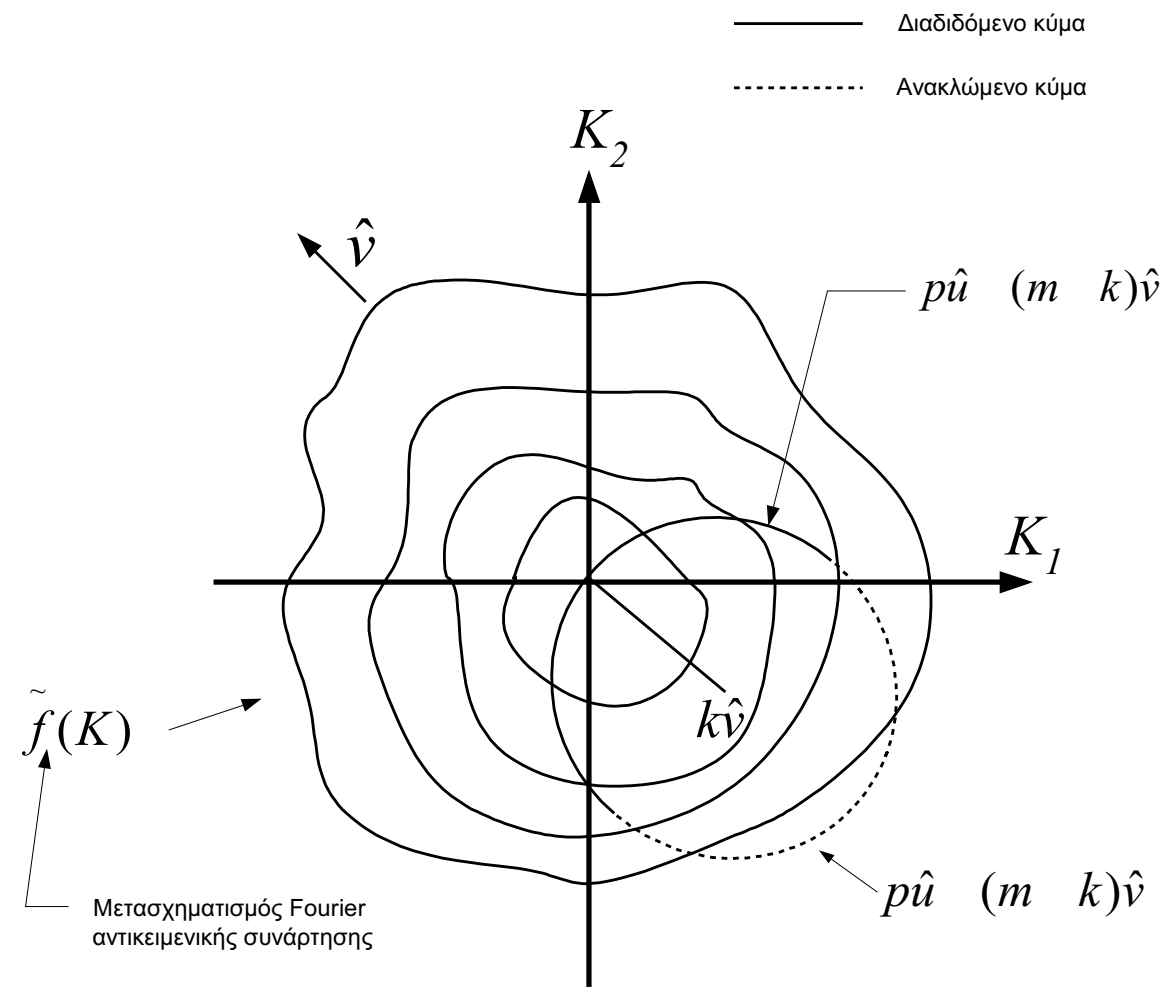

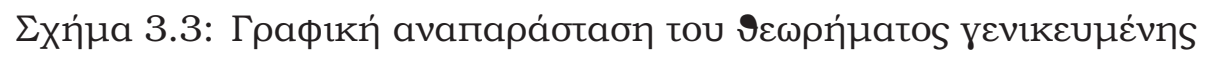

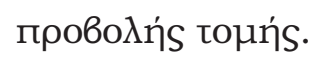




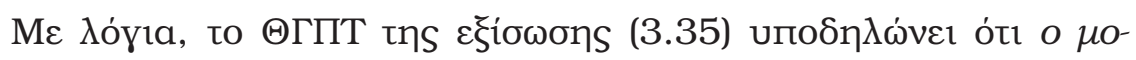

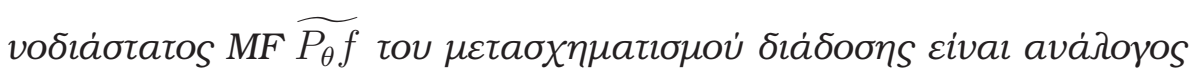

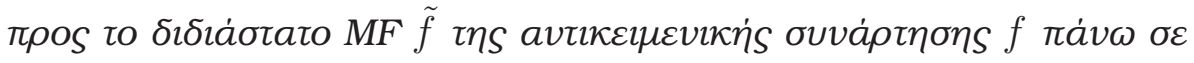

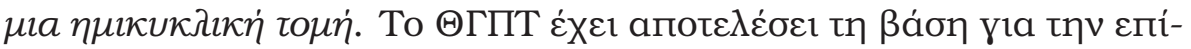

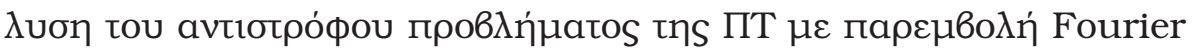

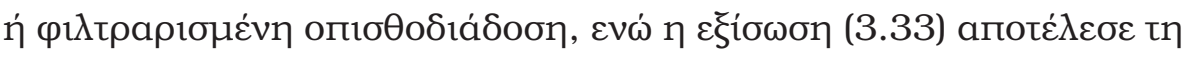

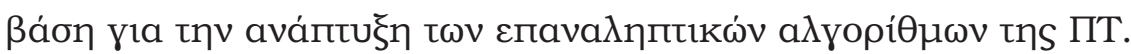

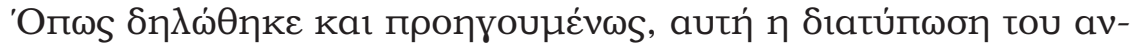

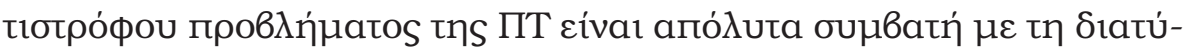

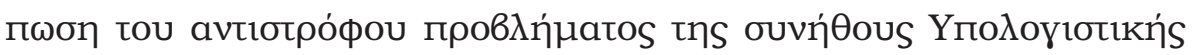

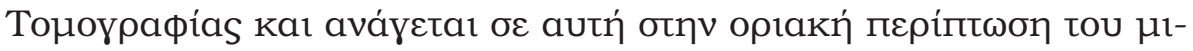

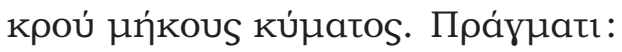

$$
k \rightarrow \infty,\left(\frac{k}{m}\right) \rightarrow 1,(m-k) \rightarrow 0,
$$

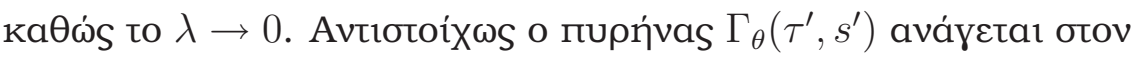

$$
\Gamma_{\theta}\left(\tau^{\prime}, s^{\prime}\right) \approx \frac{1}{2 \pi} \int_{-\infty}^{\infty} d p e^{i p \tau^{\prime}}=\delta\left(\tau^{\prime}\right), \operatorname{ka} \theta \dot{\boldsymbol{S}} \lambda \rightarrow 0
$$

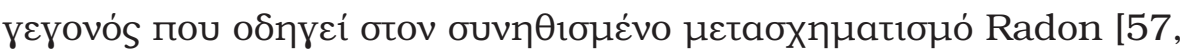
$44]$

$$
\begin{aligned}
P_{\theta} f\left(\tau_{0}\right) & =\iint_{\mathcal{V}} d \tau^{\prime} d s^{\prime} \delta\left(\tau_{0}-\tau^{\prime}\right) f\left(\tau^{\prime} \hat{u}+s^{\prime} \hat{v}\right) \\
& =\int_{-\infty}^{\infty} d s^{\prime} f\left(\tau_{0} \hat{u}+s^{\prime} \hat{v}\right) .
\end{aligned}
$$




\section{KeфáAaro 4}

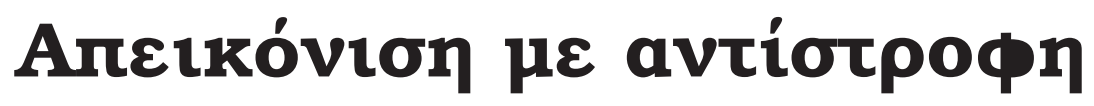

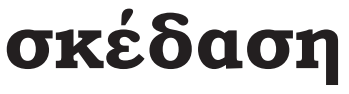

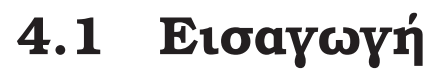

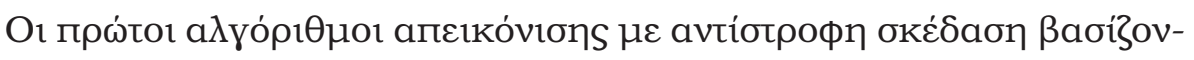

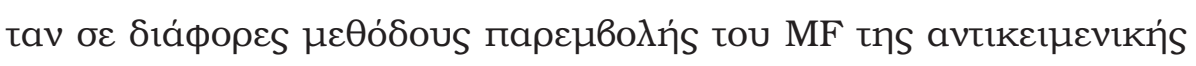

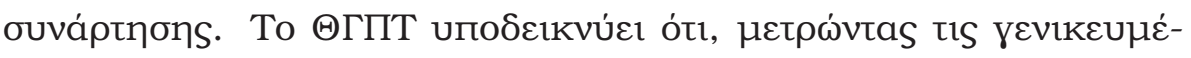

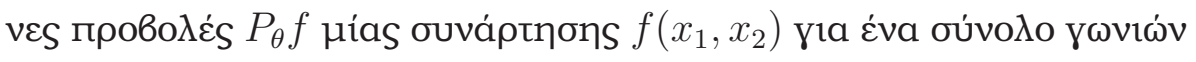

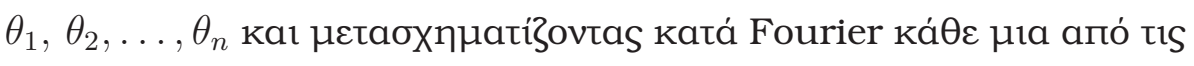

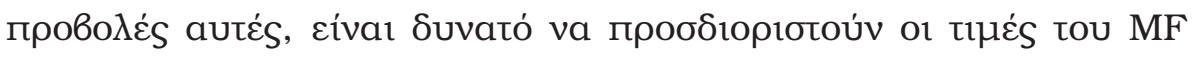

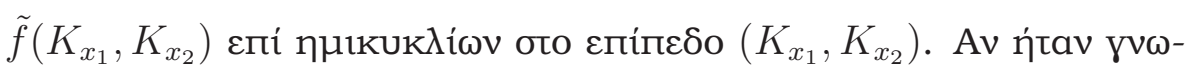

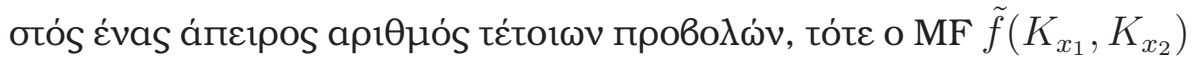

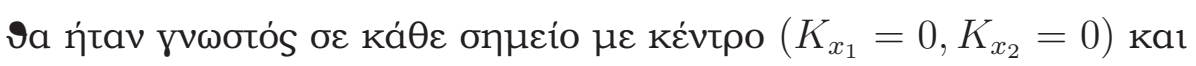

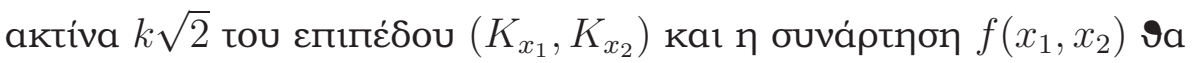




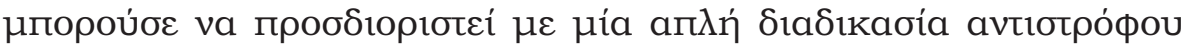
MF.

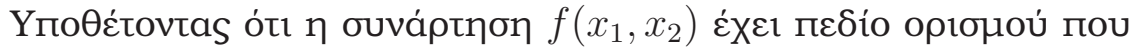

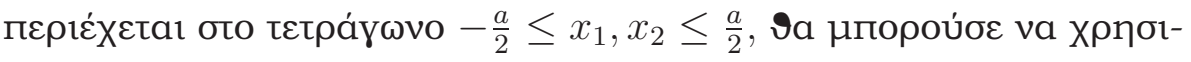

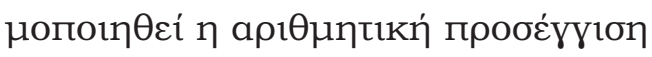

$$
f\left(x_{1}, x_{2}\right) \approx \frac{1}{a^{2}} \sum_{m=-\frac{N}{2}}^{\frac{N}{2}} \sum_{n=-\frac{N}{2}}^{\frac{N}{2}} \tilde{f}\left(\frac{m}{a}, \frac{n}{a}\right) e^{-i 2 \pi\left(\frac{m x_{1}}{a}+\frac{n x_{2}}{a}\right)}
$$

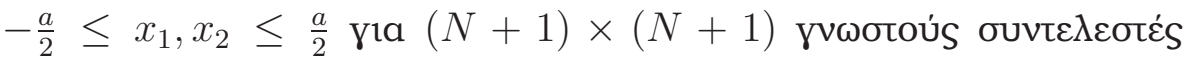

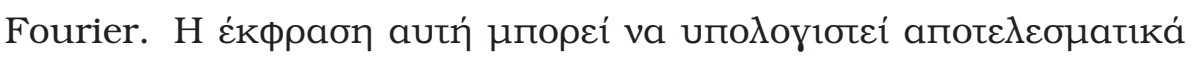

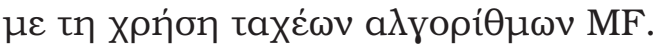

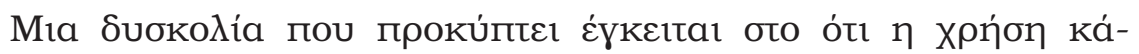

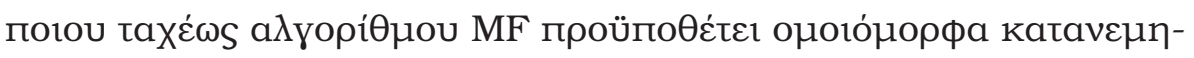

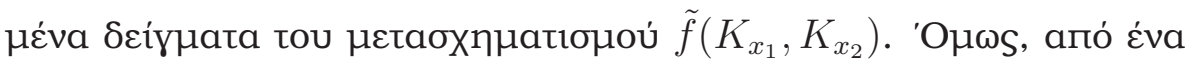

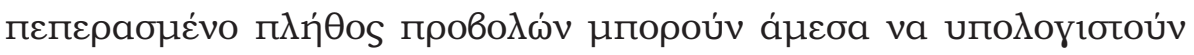

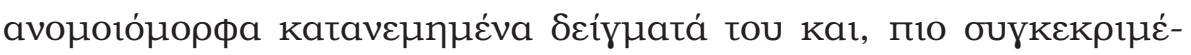

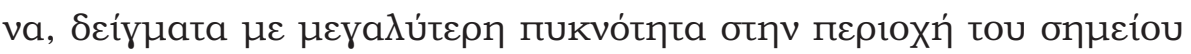

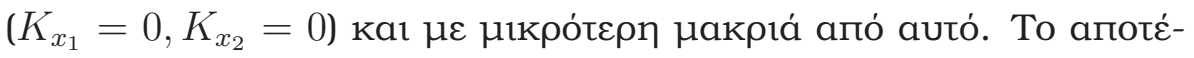

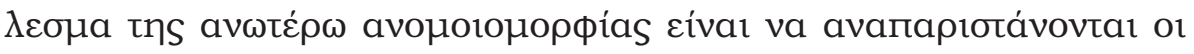

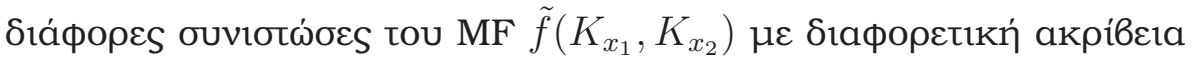

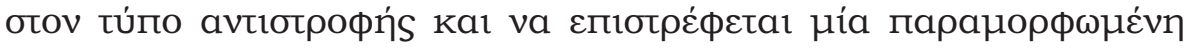

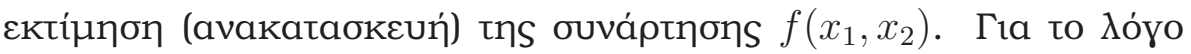

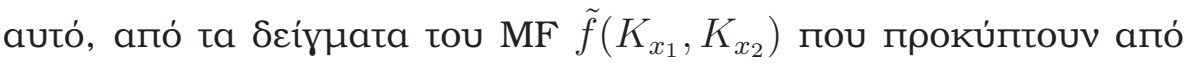

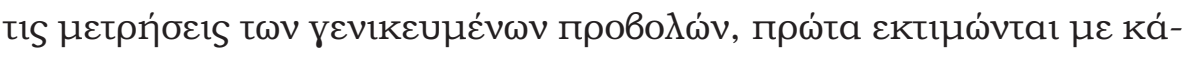

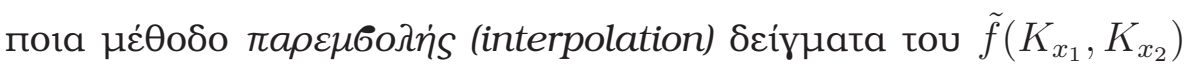




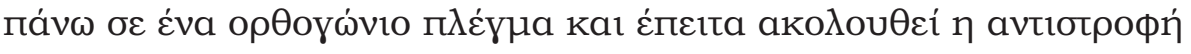

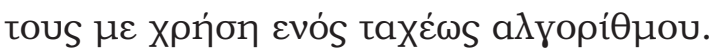

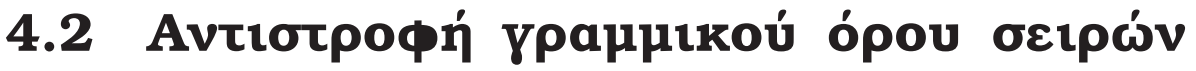 Born - Rytov}

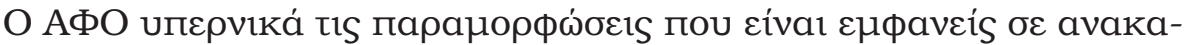

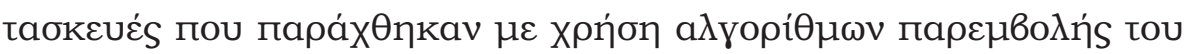

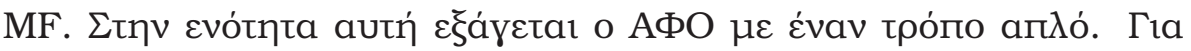

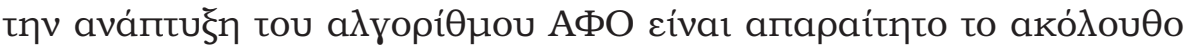
$\lambda$ ńнนa:

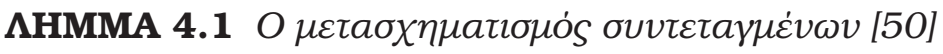

$$
\begin{aligned}
& K(p, \theta) \equiv p \hat{u}+(m-k) \hat{v}= \\
= & {[p \cos \theta-(m-k) \sin \theta] \hat{x}_{1}+[p \sin \theta+(m-k) \cos \theta] \hat{x}_{2}, }
\end{aligned}
$$

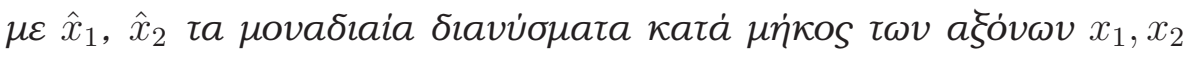

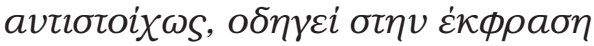

$$
\delta\left[p \hat{u}+(m-k) \hat{v}-p^{\prime} \hat{u}^{\prime}-\left(m^{\prime}-k\right) \hat{v}^{\prime}\right] d p^{\prime}=\frac{2 \delta\left(p-p^{\prime}\right) \delta\left(\theta-\theta^{\prime}\right)}{\left|\frac{k p}{m}\right|} d p^{\prime} .
$$

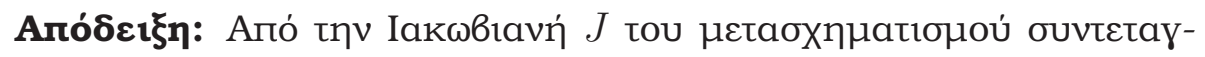

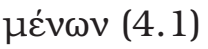

$$
J=\operatorname{det}\left(\begin{array}{cc}
\frac{\partial K_{1}}{\partial p} & \frac{\partial K_{1}}{\partial \theta} \\
\frac{\partial K_{2}}{\partial p} & \frac{\partial K_{2}}{\partial \theta}
\end{array}\right)=\frac{k p}{m}
$$




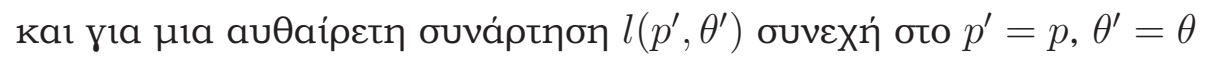
ouváyetal

$$
\begin{array}{r}
\int_{0}^{2 \pi} d \theta^{\prime} \int_{-k}^{k} d p^{\prime} l\left(p^{\prime}, \theta^{\prime}\right) \delta\left[p \hat{u}+(m-k) \hat{v}-p^{\prime} \hat{u}^{\prime}-\left(m^{\prime}-k\right) \hat{v}^{\prime}\right]= \\
=2 \int_{\mathcal{D}} d K^{\prime} \frac{l\left[K^{\prime}\left(p^{\prime}, \theta^{\prime}\right)\right]}{\frac{\left|k p^{\prime}\right|}{\left|m^{\prime}\right|}} \delta\left(K-K^{\prime}\right)=2 \frac{l[K(p, \theta)]}{\frac{|k p|}{|m|}}
\end{array}
$$

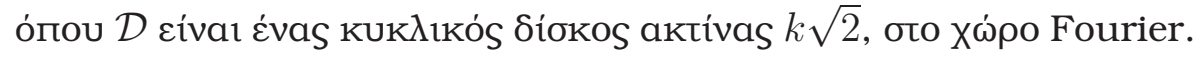

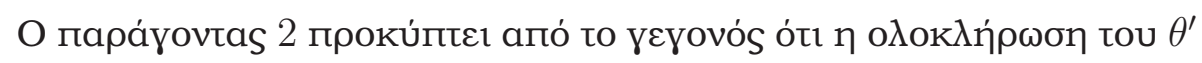

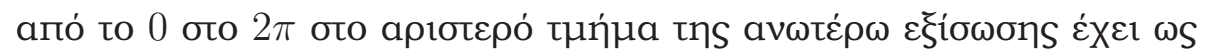

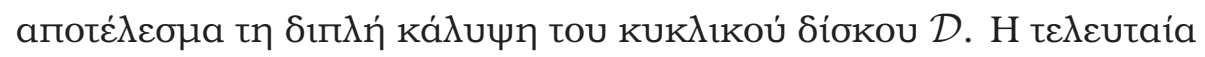

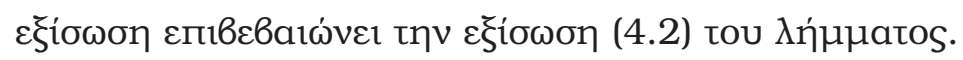

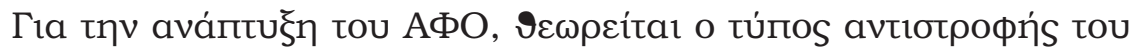
MF

$$
\begin{aligned}
f_{\mathrm{rec}}(x) & =\frac{1}{(2 \pi)^{2}} \int_{|K|<k \sqrt{2}} d K e^{i p t} e^{i<K, x>} \tilde{f}(K)= \\
& =\frac{1}{2} \frac{1}{(2 \pi)^{2}} \int_{0}^{2 \pi} d \theta \int_{-k}^{k} d p|p| e^{i p t} e^{i(m-k) s} \frac{k|p|}{m} \tilde{f}[p \hat{u}+(m-k) \hat{v}]
\end{aligned}
$$

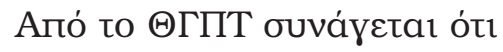

$$
\frac{k}{m} e^{i(m-k) s_{0}} \tilde{f_{\text {rec }}}[p \hat{u}+(m-k) \hat{v}]=\widetilde{P_{\theta} f}\left(p, s_{0}\right)
$$

ń

$$
\frac{k}{m} \tilde{f_{\text {rec }}}[p \hat{u}+(m-k) \hat{v}]=e^{-i(m-k) s_{0}} \widetilde{P_{\theta} f}\left(p, s_{0}\right) .
$$




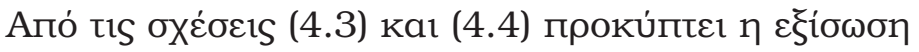

$$
f_{\mathrm{rec}}(x)=\frac{1}{2} \frac{1}{(2 \pi)^{2}} \int_{0}^{2 \pi} d \theta \int_{-k}^{k} d p|p| e^{i p \tau} e^{i(m-k)\left(s-s_{0}\right)} \widetilde{P_{\theta} f}\left(p, s_{0}\right),
$$

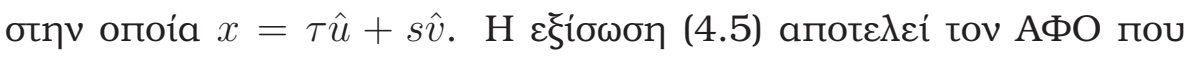

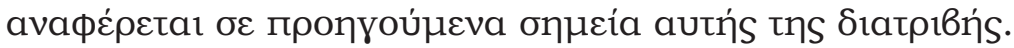

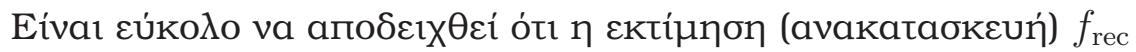

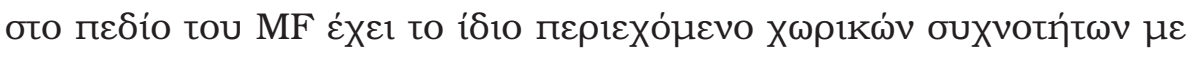

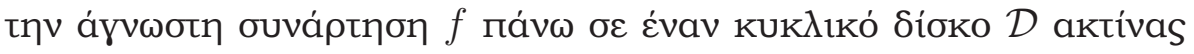

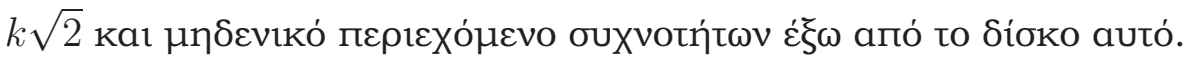

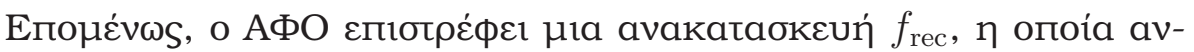

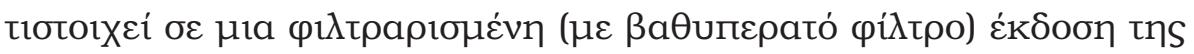

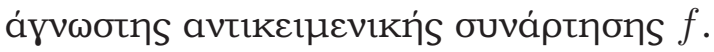

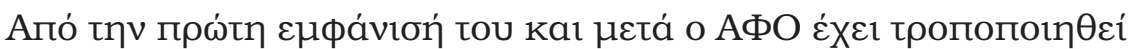

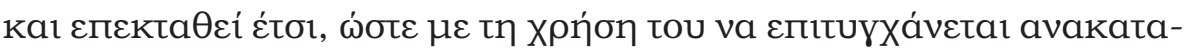

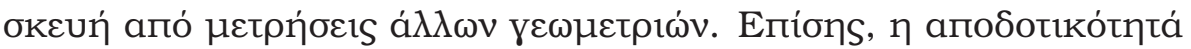

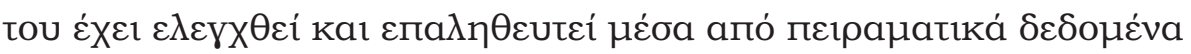

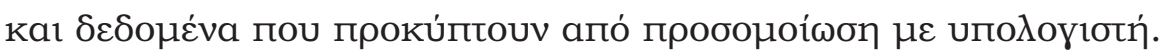

\subsection{Avtıotpoфń oetpás Born}

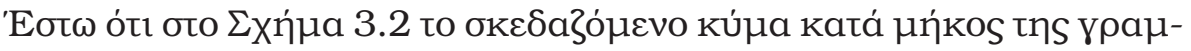

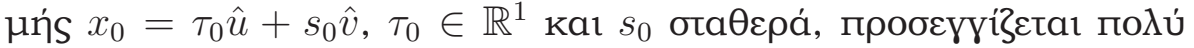

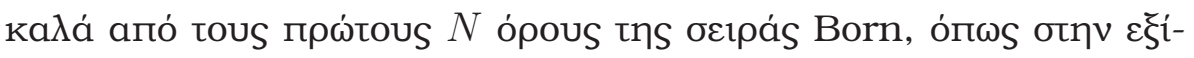

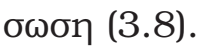

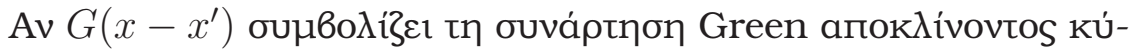




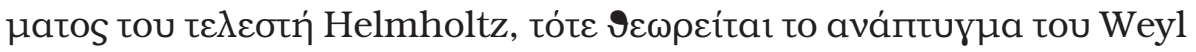

$$
\begin{aligned}
G\left[\left(\tau-\tau^{\prime}\right) \hat{u}+\left(s-s^{\prime}\right) \hat{v}\right] & =-\frac{i}{4 \pi} \int_{-\infty}^{\infty} d p e^{i p\left(\tau-\tau^{\prime}\right)} \frac{e^{i m\left|s-s^{\prime}\right|}}{m} \\
& \approx-\frac{i}{4 \pi} \int_{-k}^{k} d p e^{i p\left(\tau-\tau^{\prime}\right)} \frac{e^{i m\left|s-s^{\prime}\right|}}{m} \\
& \equiv G_{L}\left[\left(\tau-\tau^{\prime}\right) \hat{u}+\left(s-s^{\prime}\right) \hat{v}\right]
\end{aligned}
$$

о́поч

$$
m= \begin{cases}\sqrt{k^{2}-p^{2}}, & \text { ótav }|p| \leq k \\ i \sqrt{p^{2}-k^{2}}, & \text { ótav }|p|>k .\end{cases}
$$

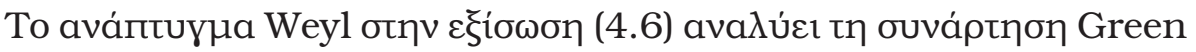

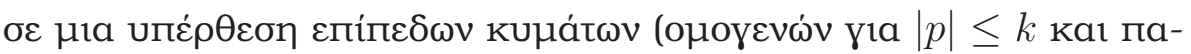

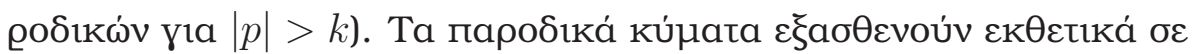

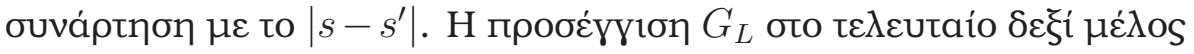

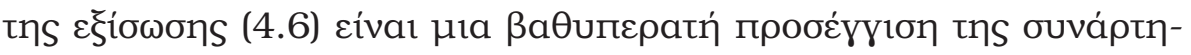

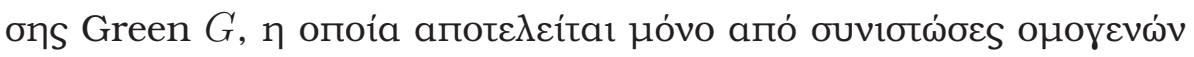

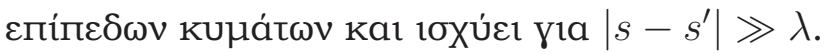

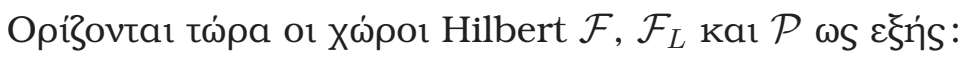

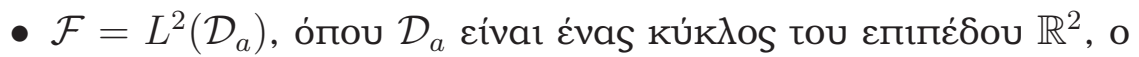

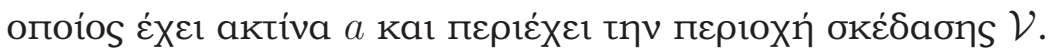

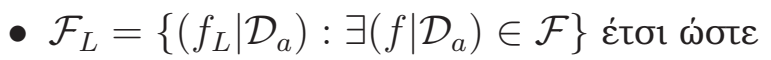

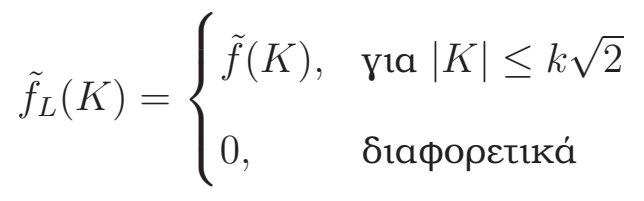




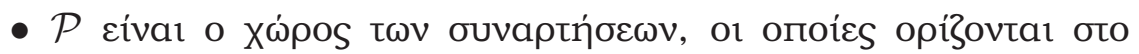

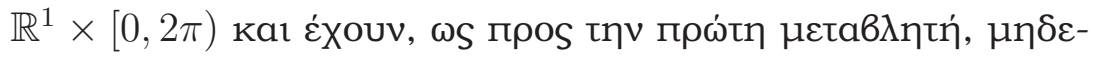

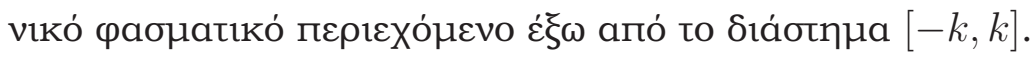

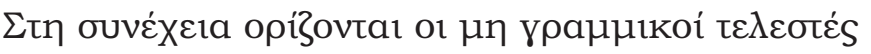

$$
\mathcal{G}_{n}: \mathcal{F}_{L} \rightarrow \mathcal{P}
$$

о́поu

$$
\left(\mathcal{G}_{n} f_{L}\right)\left(\tau_{0}, \theta\right)=\frac{e^{-i k s_{0}}}{i k} \psi_{n}^{L}\left(\tau_{0} \hat{u}+s_{0} \hat{v}, \theta\right)
$$

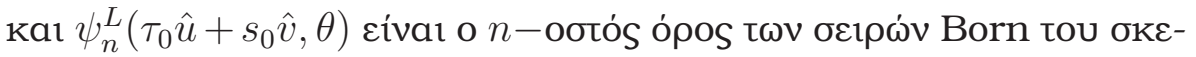

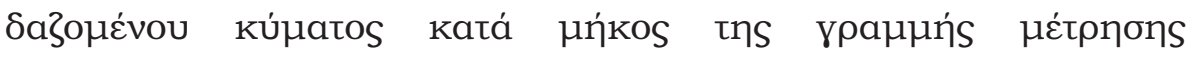

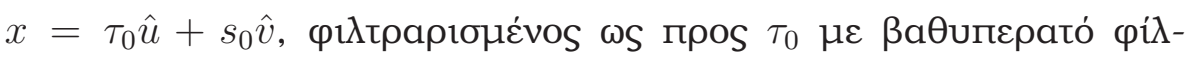

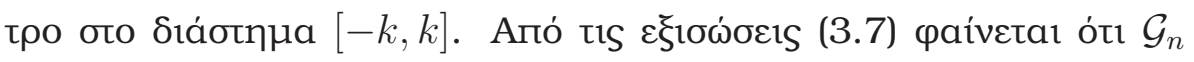

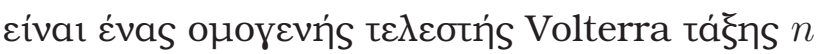

$$
\begin{aligned}
& \left(\mathcal{G}_{n} f_{L}\right)\left(\tau_{0}, \theta\right)= \\
& \quad=\frac{e^{-i k s_{0}}\left(-2 k^{2}\right)^{n}}{i k} \cdot \int_{\mathcal{V}} d x^{(1)} G_{L}\left(x_{0}-x^{(1)}\right) \cdot \int_{\mathcal{V}} d x^{(2)} G\left(x^{(1)}-x^{(2)}\right) \cdot \ldots \\
& \ldots \cdot \int_{\mathcal{V}} d x^{(n)} G\left(x^{(n-1)}-x^{(n)}\right) e^{i k<\hat{v}, x^{(n)}>} f\left(x^{(1)}\right) \cdot f\left(x^{(2)}\right) \cdot \ldots \cdot f\left(x^{(n)}\right) .
\end{aligned}
$$

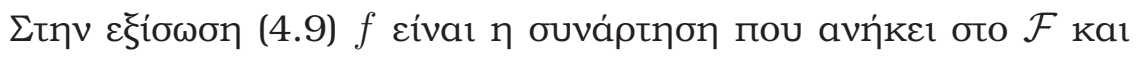

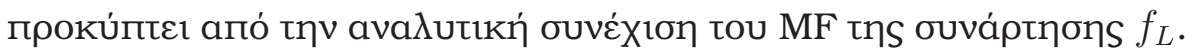

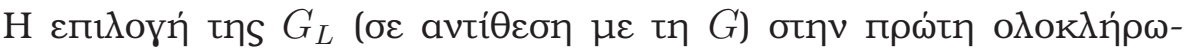

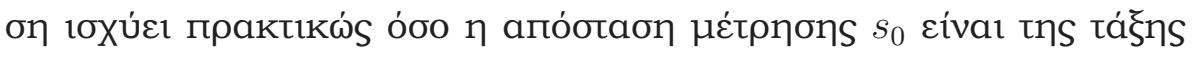

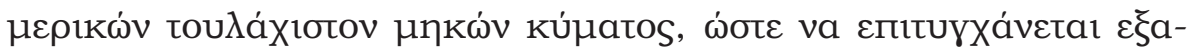

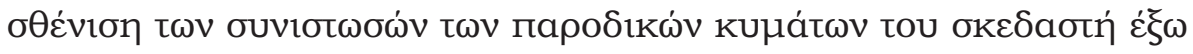




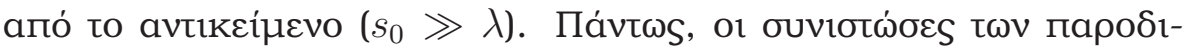

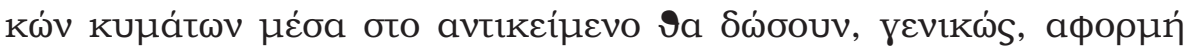

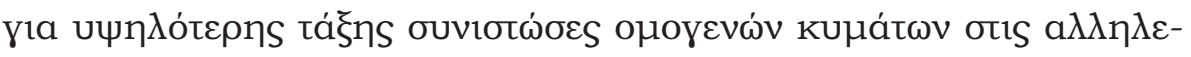

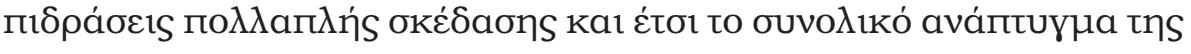

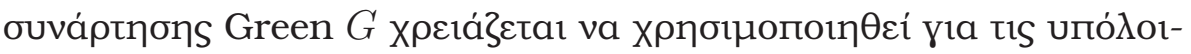

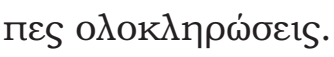

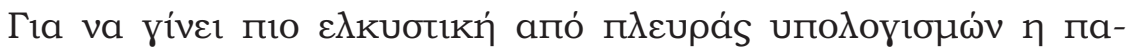

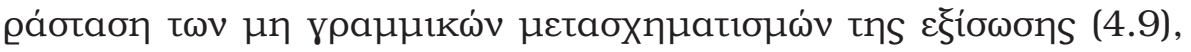

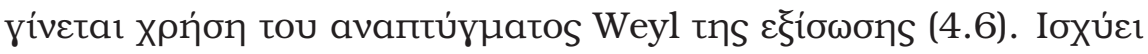

$$
\begin{aligned}
\left.\left(\mathcal{G}_{n} f_{L}\right)\left(\tau_{0}, \theta\right)\right)= & \frac{e^{-i k s_{0}}}{i k} \frac{2 i k^{2}}{4 \pi} \iint_{\mathcal{V}} d \tau^{(1)} d s^{(1)} \int_{-k}^{k} d p \cdot \\
& \cdot e^{i p\left(\tau_{0}-\tau^{(1)}\right)} \frac{e^{i m\left(s_{0}-s^{(1)}\right)}}{m} f\left(\tau^{(1)} \hat{u}+s^{(1)} \hat{v}\right) e^{i k s^{(1)}}= \\
= & \frac{k e^{-i p s_{0}}}{2 \pi} \int_{-k}^{k} d p e^{i p \tau_{0}} \frac{e^{i m s_{0}}}{m} \iint_{\mathcal{V}} d \tau^{(1)} d s^{(1)} . \\
& \cdot e^{-i p \tau^{(1)}} e^{-i(m-k) s^{(1)}} f\left(\tau^{(1)} \hat{u}+s^{(1)} \hat{v}\right)= \\
= & \frac{1}{2 \pi} \int_{-k}^{k} d p e^{i p \tau_{0}} \frac{k e^{i(m-k) s_{0}}}{m} \tilde{f}[p \hat{u}+(m-k) \hat{v}],
\end{aligned}
$$

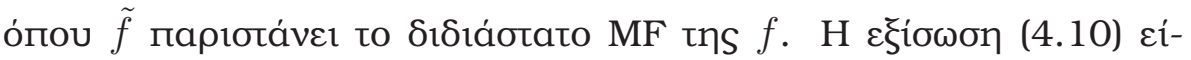

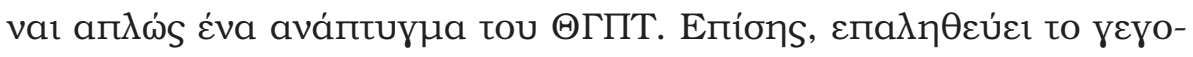

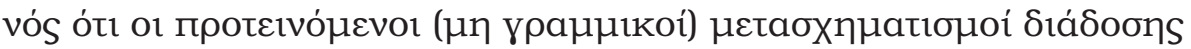

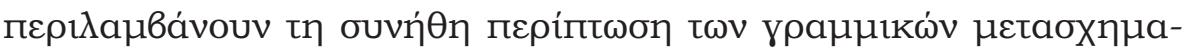

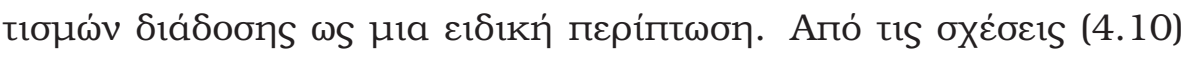

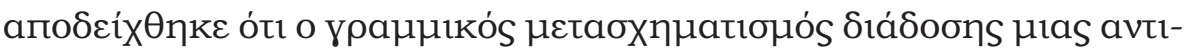

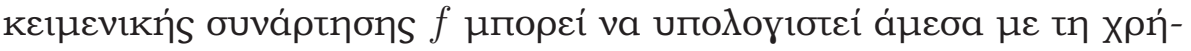




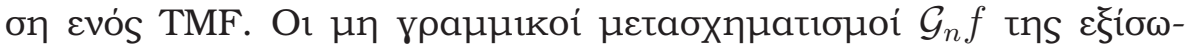

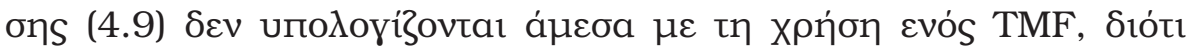

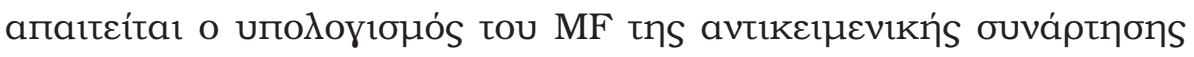

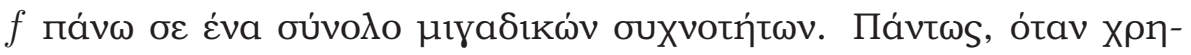

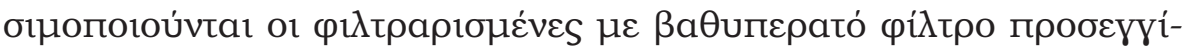

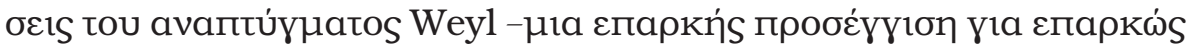

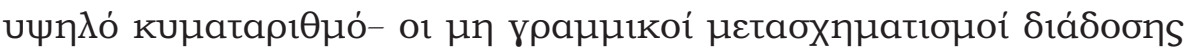

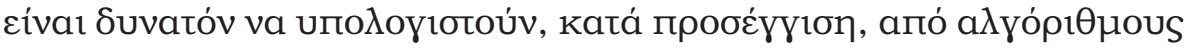
поч ßaбißovtaı бє TMF.

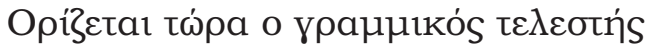

$$
\mathcal{B}: \mathcal{P} \rightarrow \mathcal{F}_{L}
$$

$\mu \varepsilon$

$$
(\mathcal{B} d)(x=\tau \hat{u}+s \hat{v})=\frac{1}{2} \frac{1}{(2 \pi)^{2}} \int_{0}^{2 \pi} d \theta \int_{-k}^{k} d p|p| \cdot e^{i p \tau} e^{i(m-k)\left(s-s_{0}\right)} \tilde{d}(p, \theta) .
$$

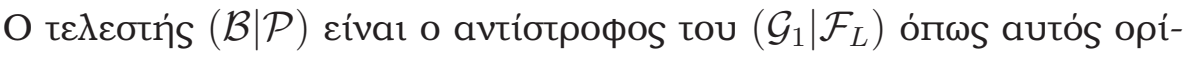

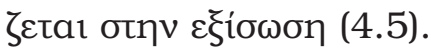

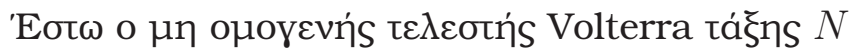

$$
\mathcal{G}^{N}=\mathcal{G}_{1}+\mathcal{G}_{2}+\ldots+\mathcal{G}_{N}: \mathcal{F}_{L} \rightarrow \mathcal{P}
$$

о́поч

$$
\left(\mathcal{G}^{N} f_{L}\right)\left(\tau_{0}, \theta\right)=\frac{e^{-i k s_{0}}}{i k}\left(\psi_{1}^{L}+\psi_{2}^{L}+\ldots+\psi_{N}^{L}\right)\left(\tau_{0} \hat{u}+s_{0} \hat{v}, \theta\right)
$$

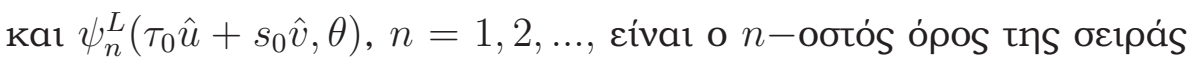

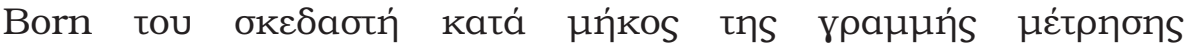




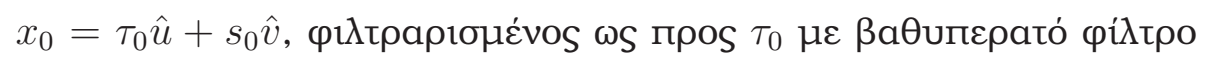

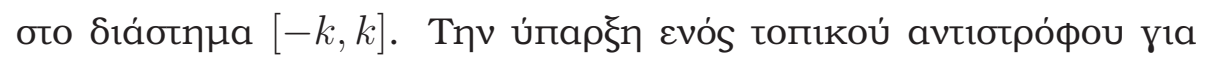

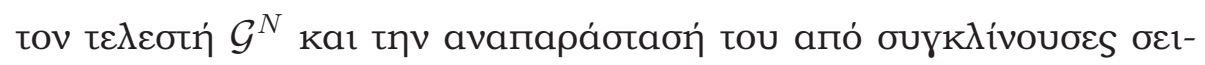

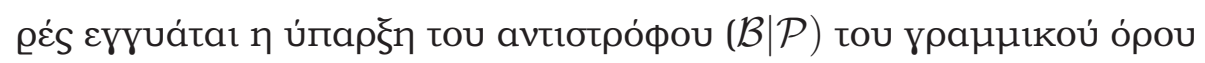

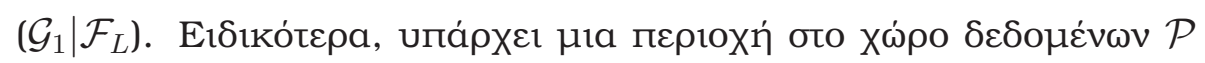

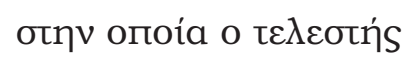

$$
\mathcal{B}_{1}+\mathcal{B}_{2}+\ldots+\mathcal{B}_{n}+\ldots
$$

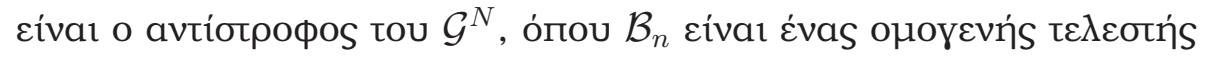

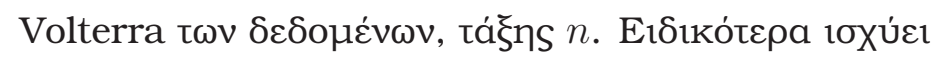

$$
\begin{aligned}
\mathcal{B}_{1} & =\mathcal{B} \\
\mathcal{B}_{2} & =-\mathcal{B G}_{2} \mathcal{B} \\
\mathcal{B}_{3} & =\mathcal{B G}_{2} \mathcal{B G}_{2} \mathcal{B}-\mathcal{B G}_{3} \mathcal{B} \\
& \vdots
\end{aligned}
$$

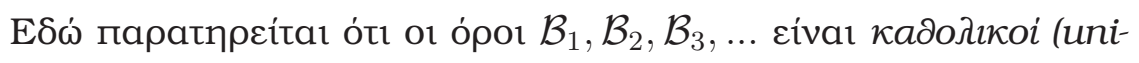

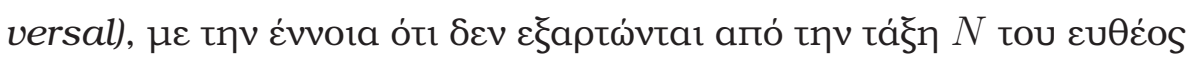

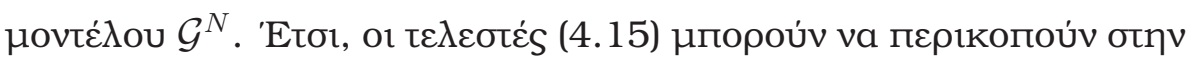

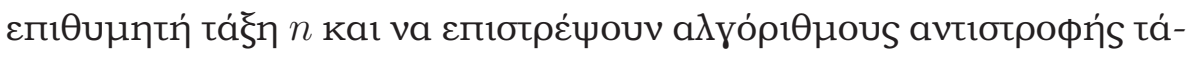

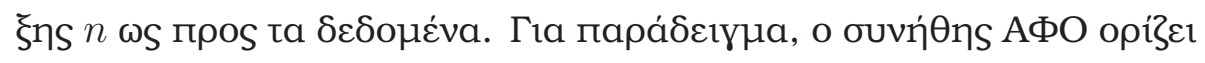

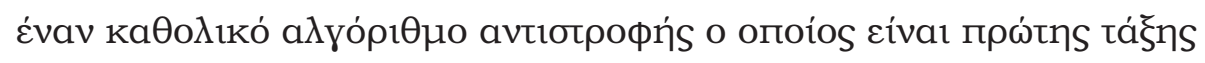

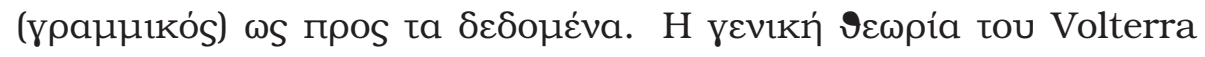

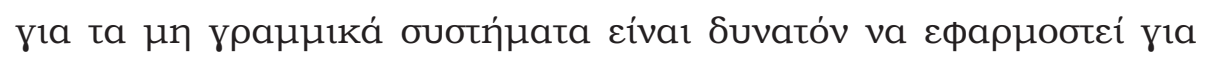

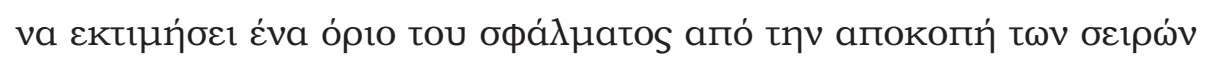

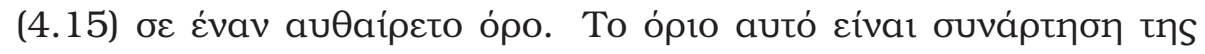




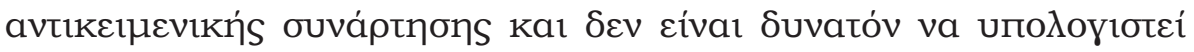

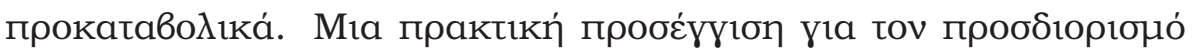

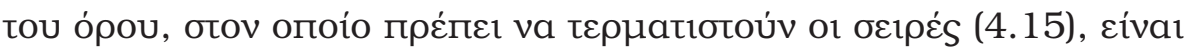

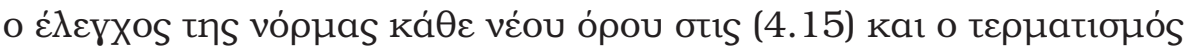

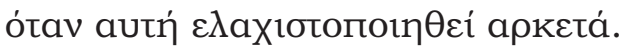

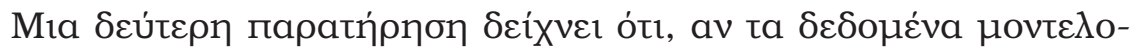

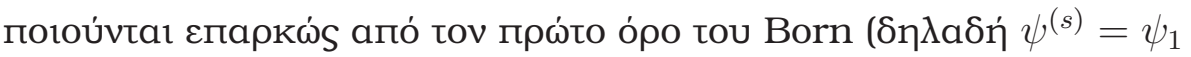

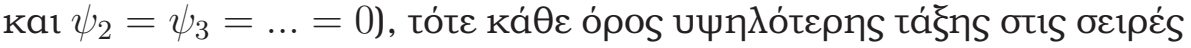

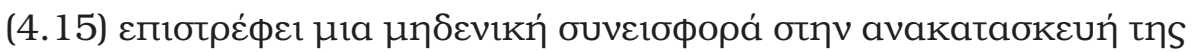

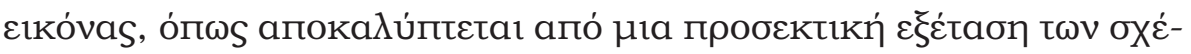

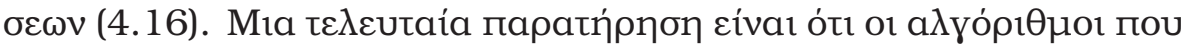

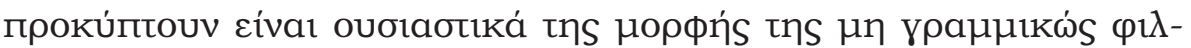

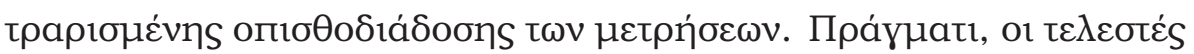

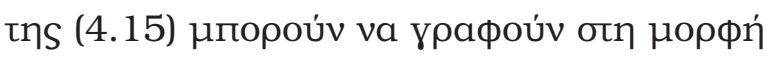

$$
\mathcal{B}\left(\mathcal{I}-\mathcal{G}_{2} \mathcal{B}+\mathcal{G}_{2} \mathcal{B} \mathcal{G}_{2} \mathcal{B}-\mathcal{G}_{3} \mathcal{B}-\ldots\right)
$$

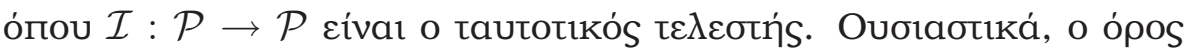

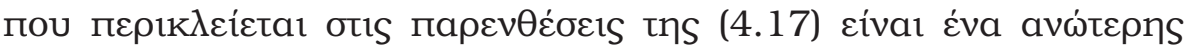

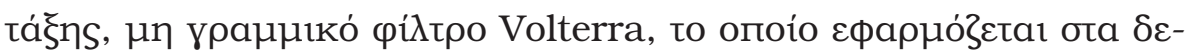

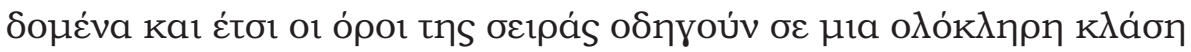

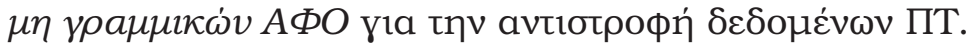




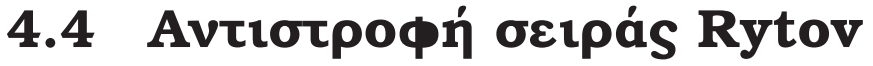

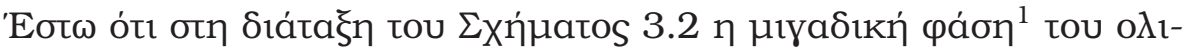

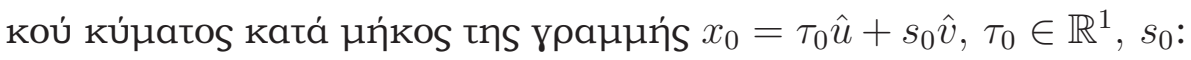

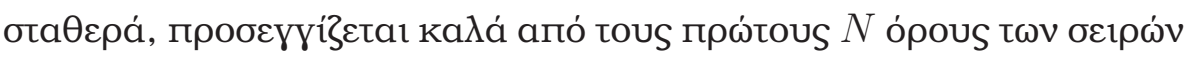

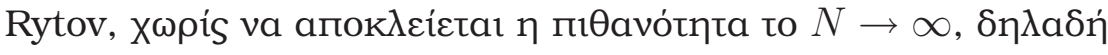

$$
W\left(x_{0}\right) \approx W_{0}\left(x_{0}\right)+W_{1}\left(x_{0}\right)+\ldots+W_{N}\left(x_{0}\right) .
$$

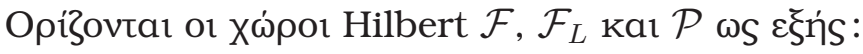

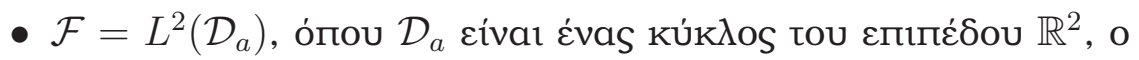

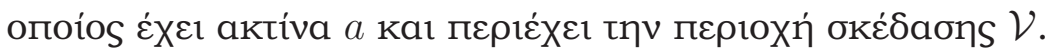

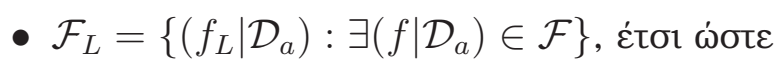

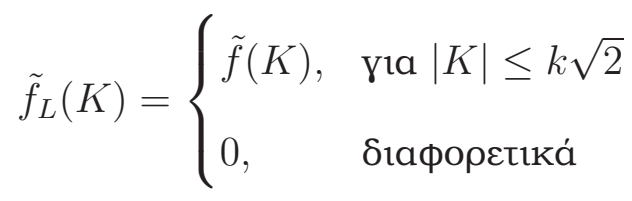

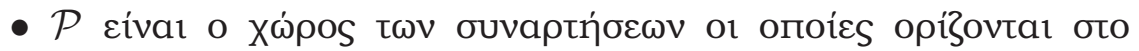

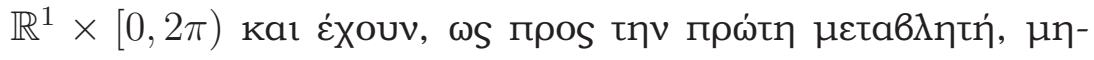

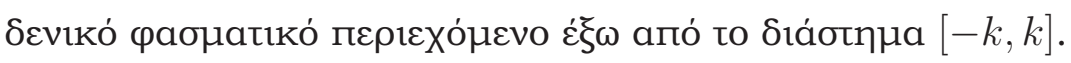

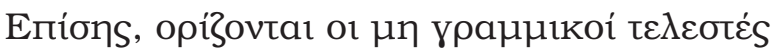

$$
\mathcal{W}_{n}: \mathcal{F}_{L} \rightarrow \mathcal{P}
$$

óпоu

$$
\left(\mathcal{W}_{n} f_{L}\right)\left(\tau_{0}, \theta\right)=W_{n}^{L}\left(\tau_{0} \hat{u}+s_{0} \hat{v}, \theta\right)
$$

\footnotetext{
${ }^{1} \Delta \eta \lambda a \delta$ o
} 


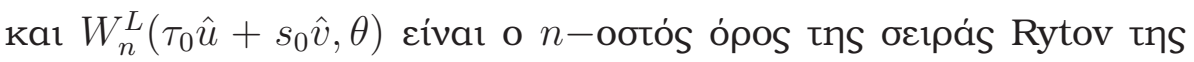

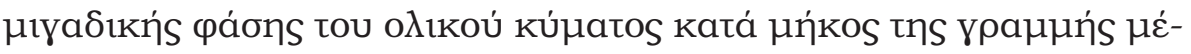

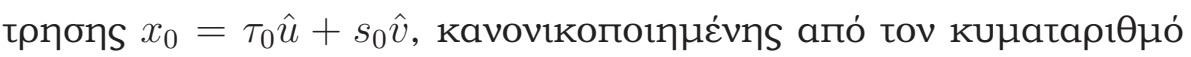

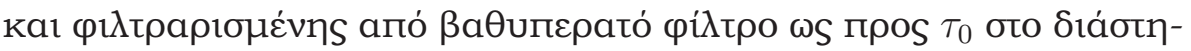

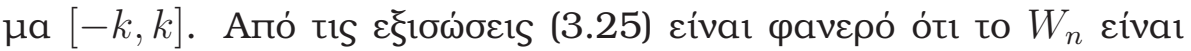

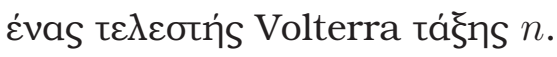

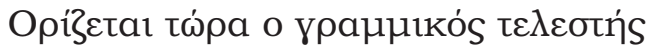

$$
\mathcal{B}: \mathcal{P} \rightarrow \mathcal{F}_{L}
$$

$\mu \varepsilon$

$(\mathcal{B} d)(x=\tau \hat{u}+s \hat{v})=\frac{1}{2} \frac{1}{(2 \pi)^{2}} \int_{0}^{2 \pi} d \theta \int_{-k}^{k} d p|p| e^{i p \tau} e^{i(m-k)\left(s-s_{0}\right)} \tilde{d}(p, \theta)$.

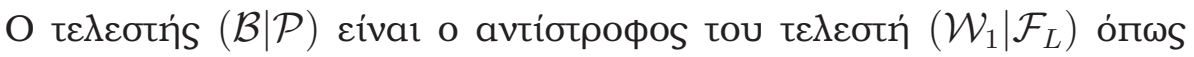

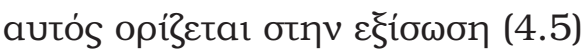

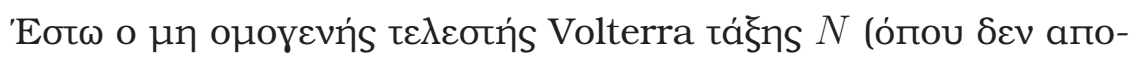

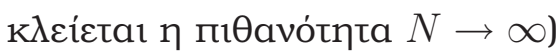

$$
\mathcal{W}^{N}=\mathcal{W}_{1}+\mathcal{W}_{2}+\ldots+\mathcal{W}_{N}: \mathcal{F}_{L} \rightarrow \mathcal{P}
$$

о́поч

$$
\left(\mathcal{W}^{N} f_{L}\right)\left(\tau_{0}, \theta\right)=\frac{e^{i k s_{0}}}{i k}\left(W_{1}^{L}+W_{2}^{L}+\ldots+W_{N}^{L}\right)\left(\tau_{0} \hat{u}+s_{0} \hat{v}, \theta\right)
$$

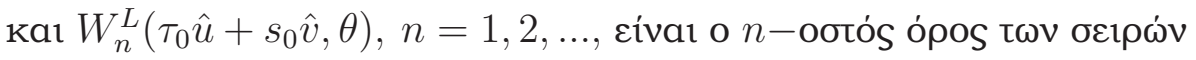

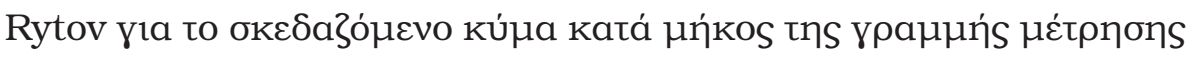

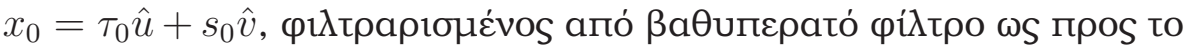

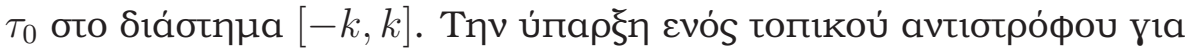




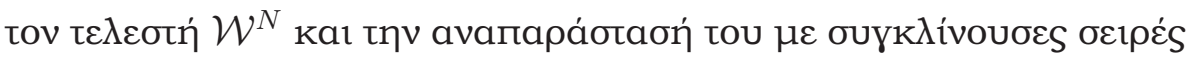

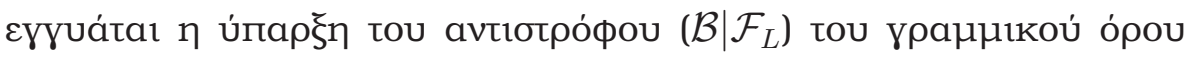

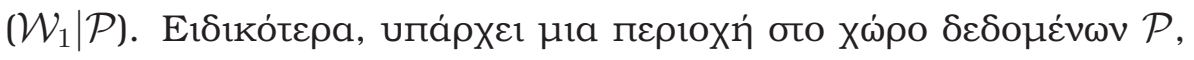

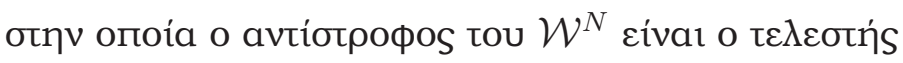

$$
\mathcal{B}_{1}+\mathcal{B}_{2}+\ldots+\mathcal{B}_{n}+\ldots
$$

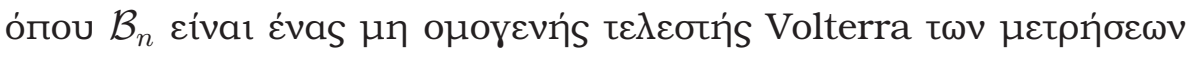
táł̆ns $n$.

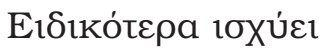

$$
\begin{aligned}
\mathcal{B}_{1} & =\mathcal{B} \\
\mathcal{B}_{2} & =-\mathcal{B W}_{2} \mathcal{B} \\
\mathcal{B}_{3} & =\mathcal{B W}_{2} \mathcal{B W}_{2} \mathcal{B}-\mathcal{B W}_{3} \mathcal{B} \\
& \vdots
\end{aligned}
$$

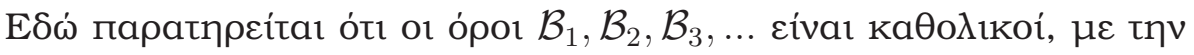

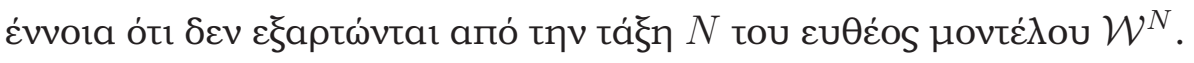

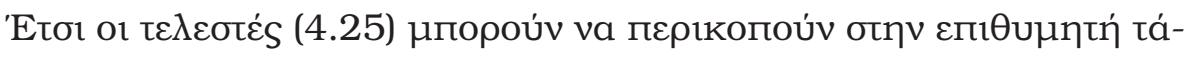

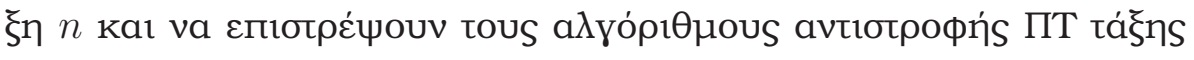

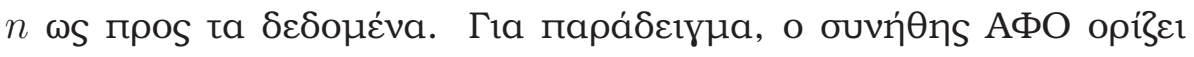

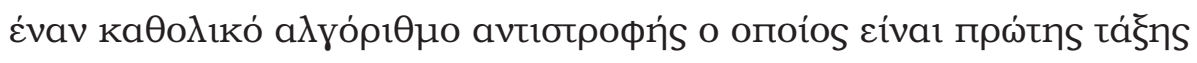

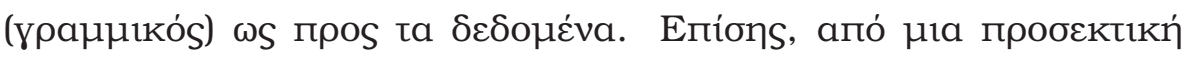

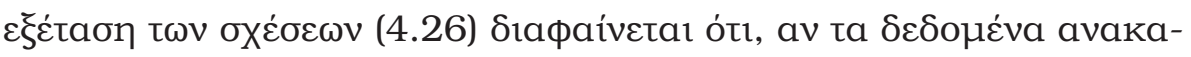

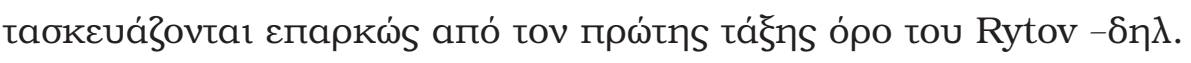

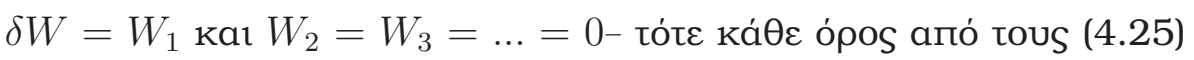

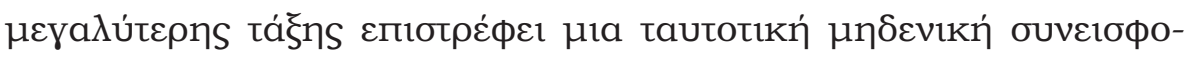




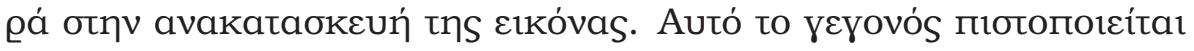

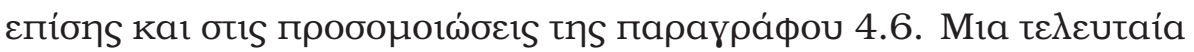

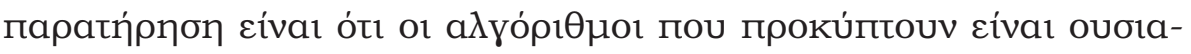

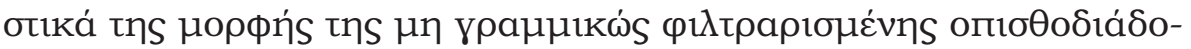

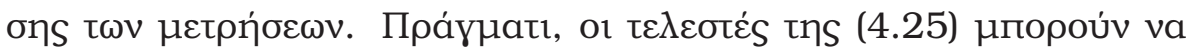

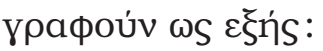

$$
\mathcal{B}\left(\mathcal{I}-\mathcal{W}_{2} \mathcal{B}+\mathcal{W}_{2} \mathcal{B} \mathcal{W}_{2} \mathcal{B}-\mathcal{W}_{3} \mathcal{B}-\ldots\right)
$$

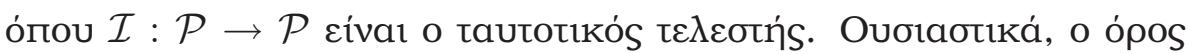

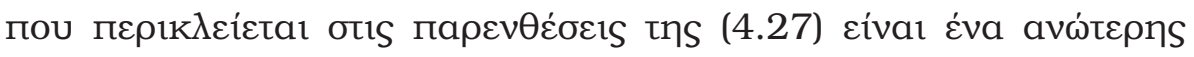

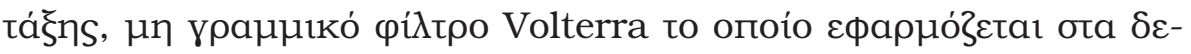

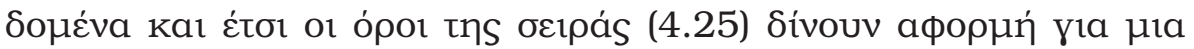

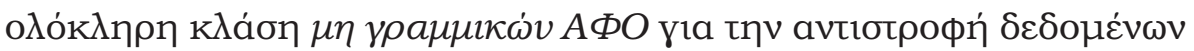
tทs ПТ.

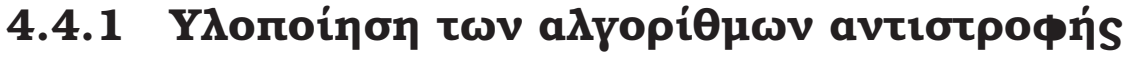

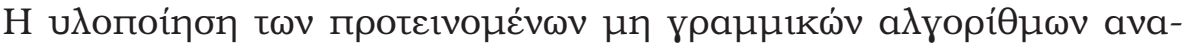

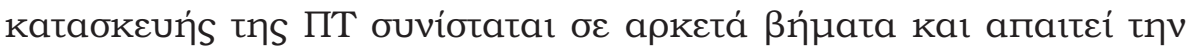

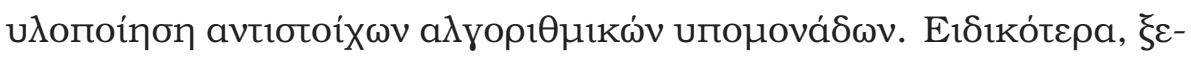

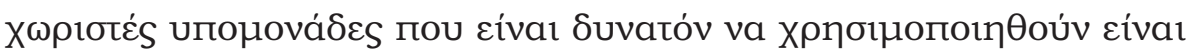

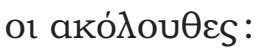

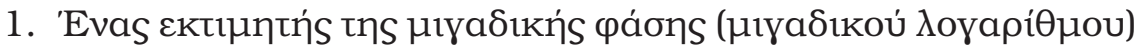

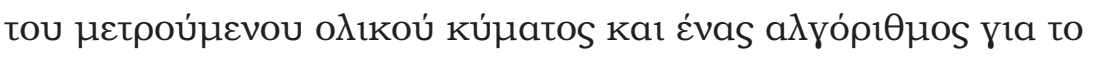

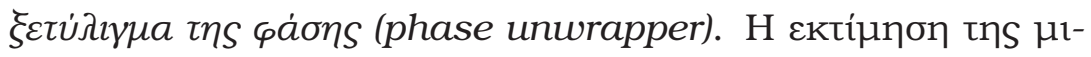

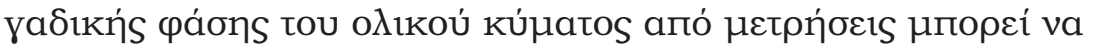




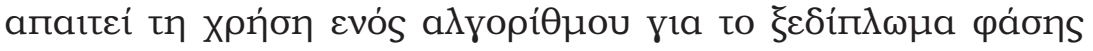

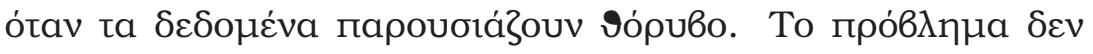

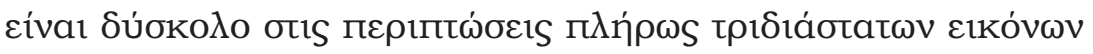

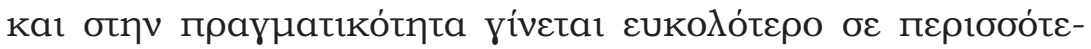

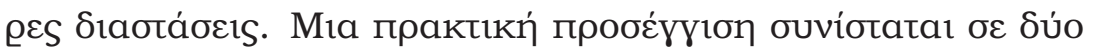

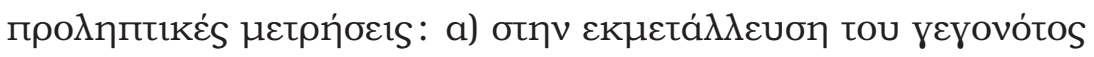

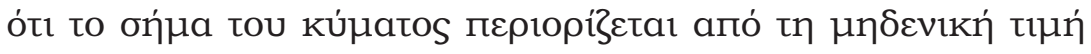

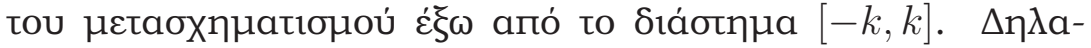

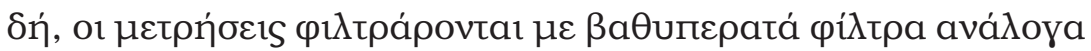

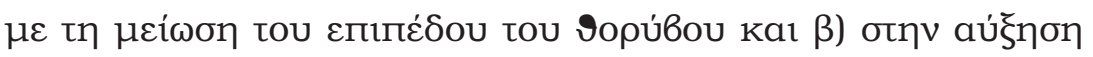

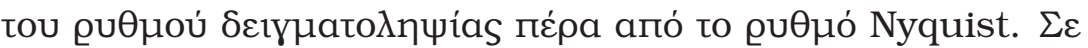

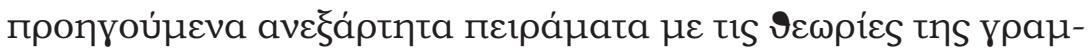

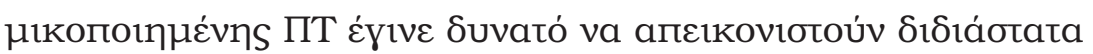

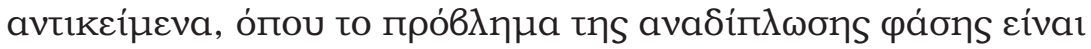

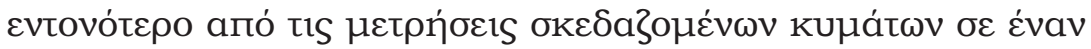

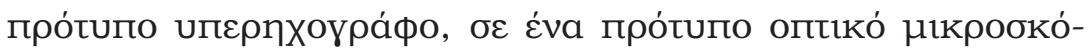

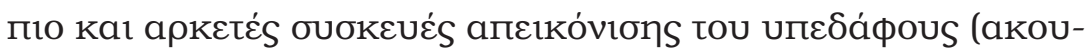

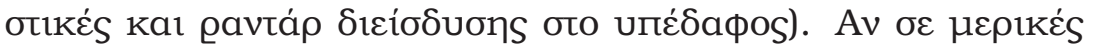

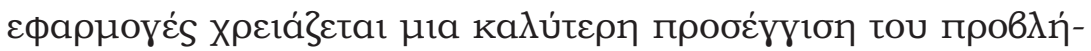

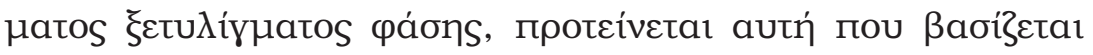

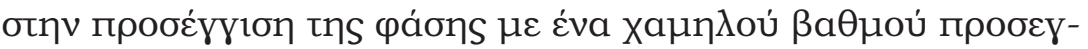

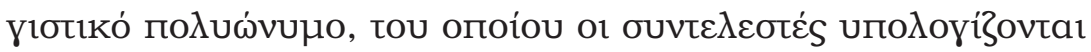

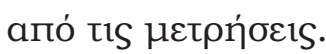

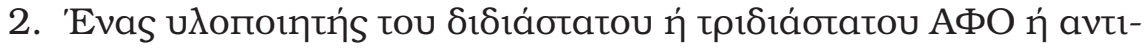




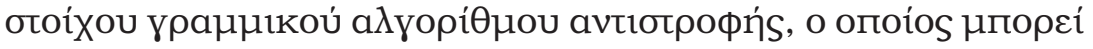

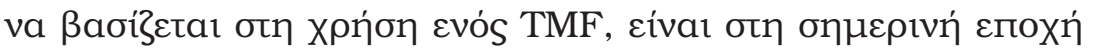

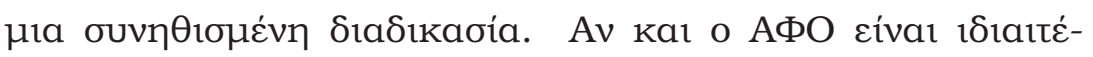

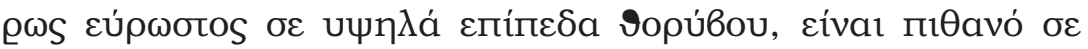

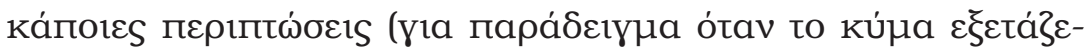

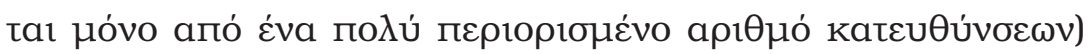

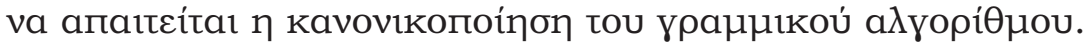

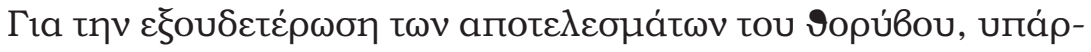

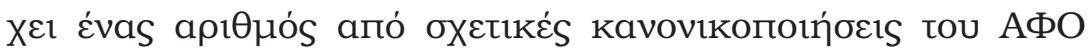

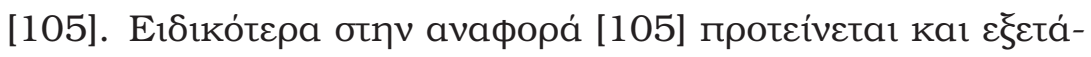

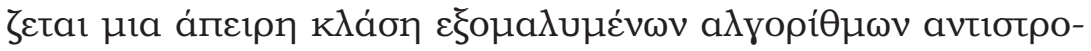

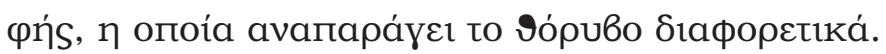

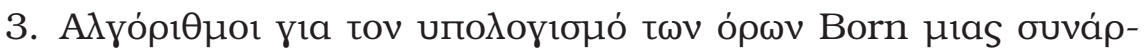

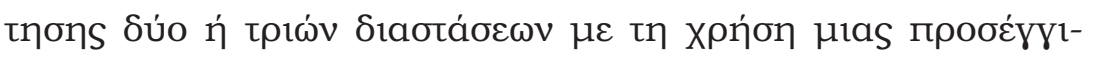

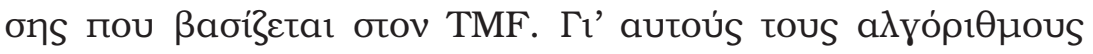

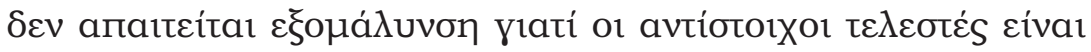

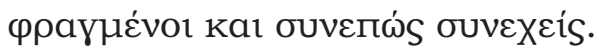

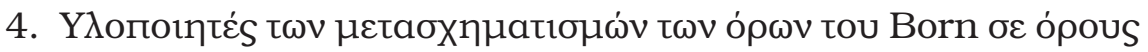
tou Rytov.

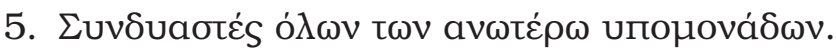




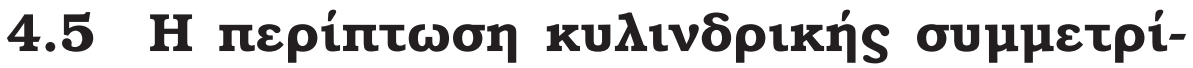 as}

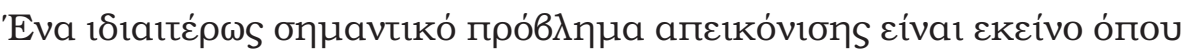

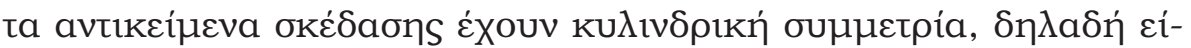

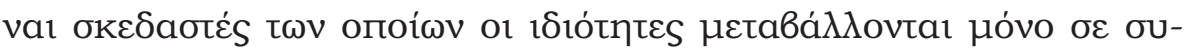

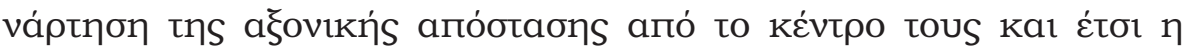

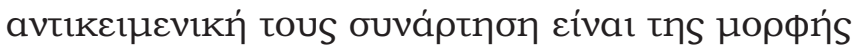

$$
f(x)=f_{r}(r), r=|x| .
$$

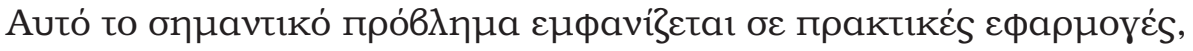

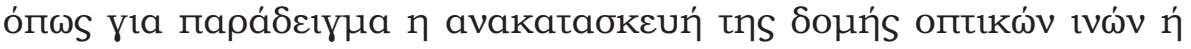

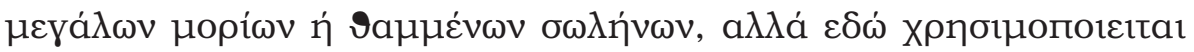

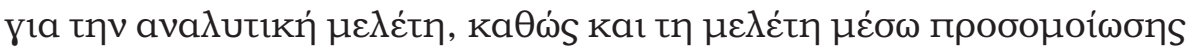

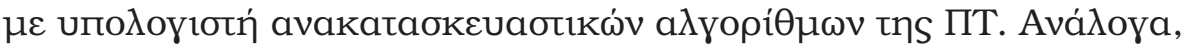

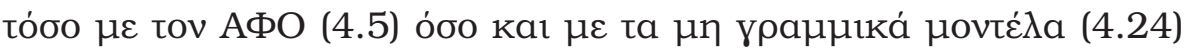

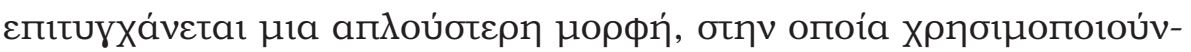

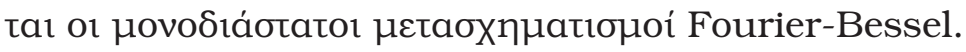

Пра́yนatı, av

$$
\tilde{f}_{r}(|K|)=2 \pi \int_{0}^{\infty} d r r J_{0}(|K| r) f_{r}(r)
$$

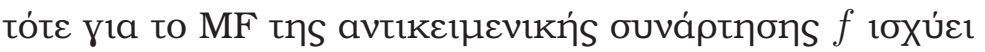

$$
\tilde{f}(|K|)=\tilde{f}_{r}(|K|)
$$




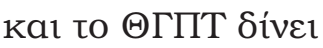

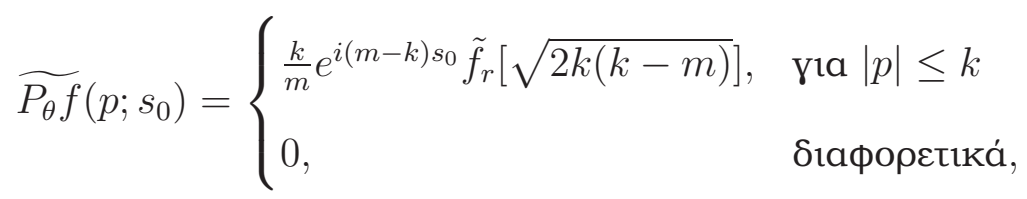

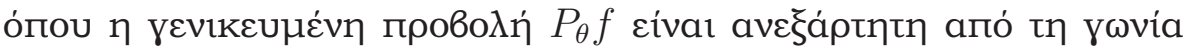

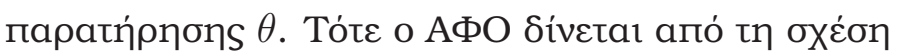

$$
f_{\text {rec }}(x)=\frac{1}{2 \pi} \int_{0}^{k} d p p e^{i(k-m) s_{0}} J_{0}[r \sqrt{2 k(k-m)}] \widetilde{P_{\theta} f}\left(p ; s_{0}\right), r=|x|,
$$

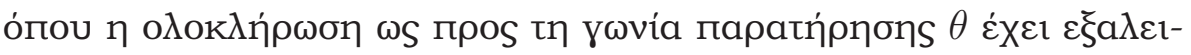

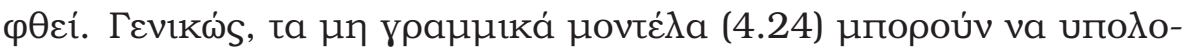

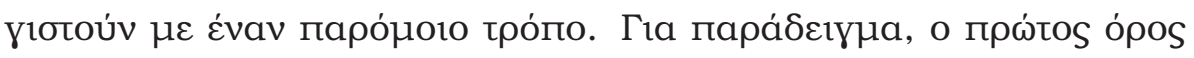
tou Born Sívetaı aпó tov túпo

$$
\tilde{\psi}_{1}\left(p \hat{u}+s_{0} \hat{v}\right)= \begin{cases}\frac{i k^{2}}{m} e^{i m s_{0}} \tilde{f}_{r}[r \sqrt{2 k(k-m)}], & \text { Vıa }|p| \leq k \\ \frac{i k^{2}}{m} e^{i m s_{0}} \tilde{f}_{r}(|p| \sqrt{2}) & \text { Vıa }|p|>k,\end{cases}
$$

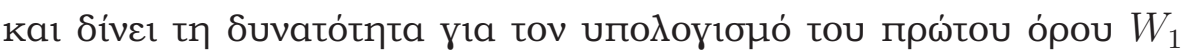

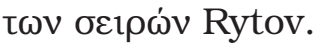

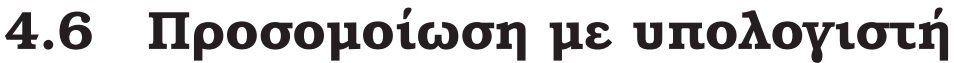

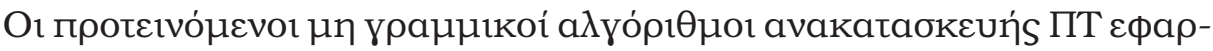

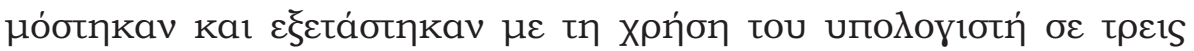

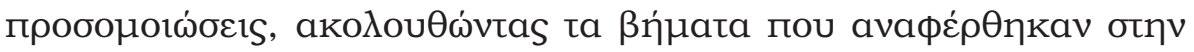

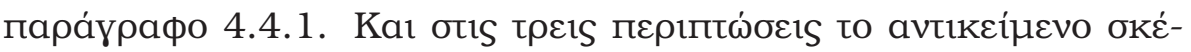

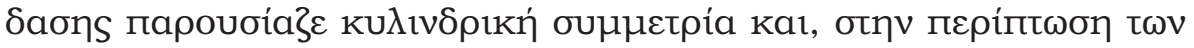




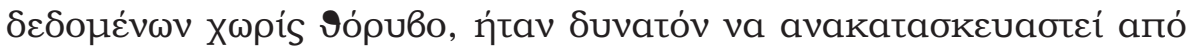

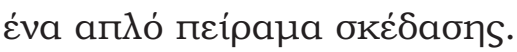

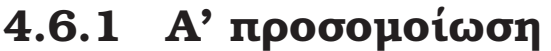

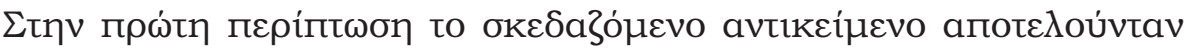

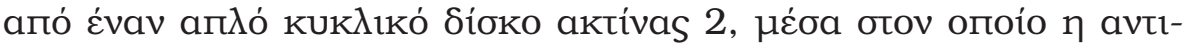

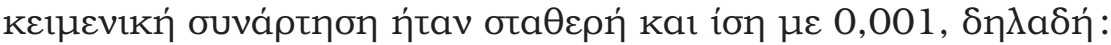

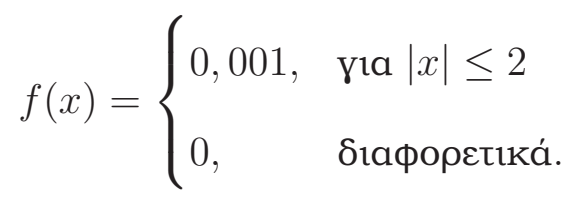

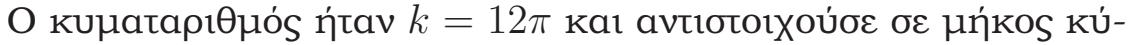

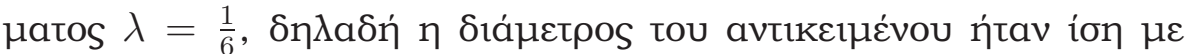

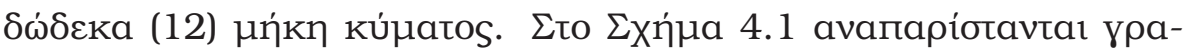

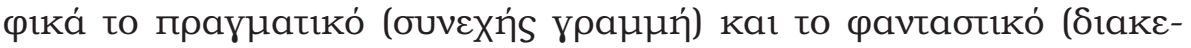

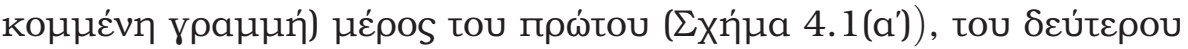

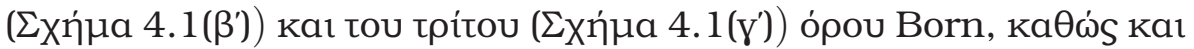

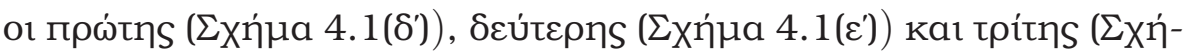

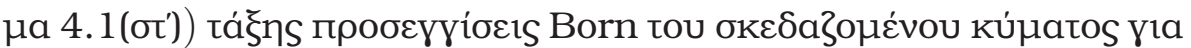

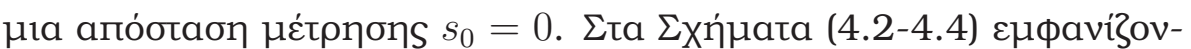

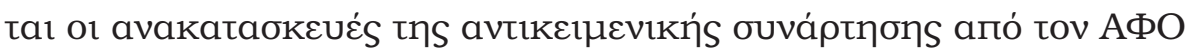

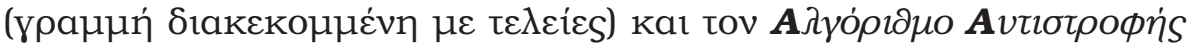

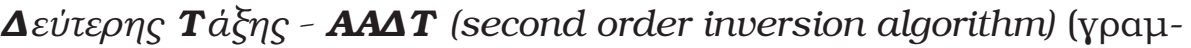

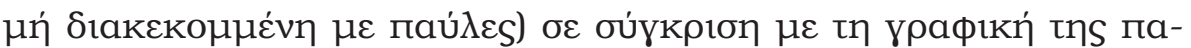

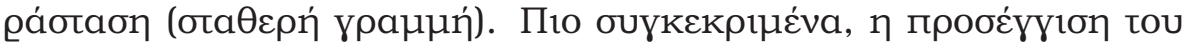

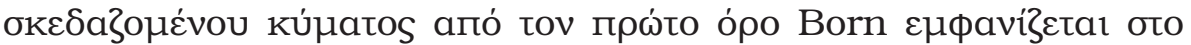




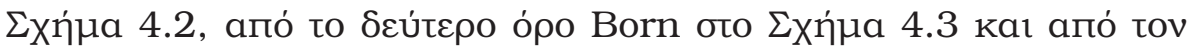

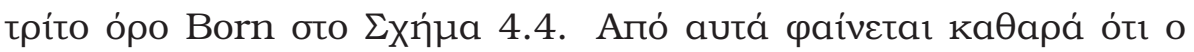

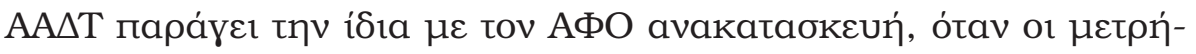

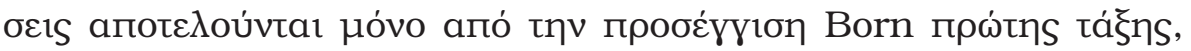

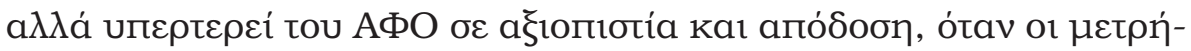

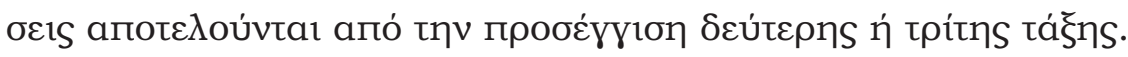



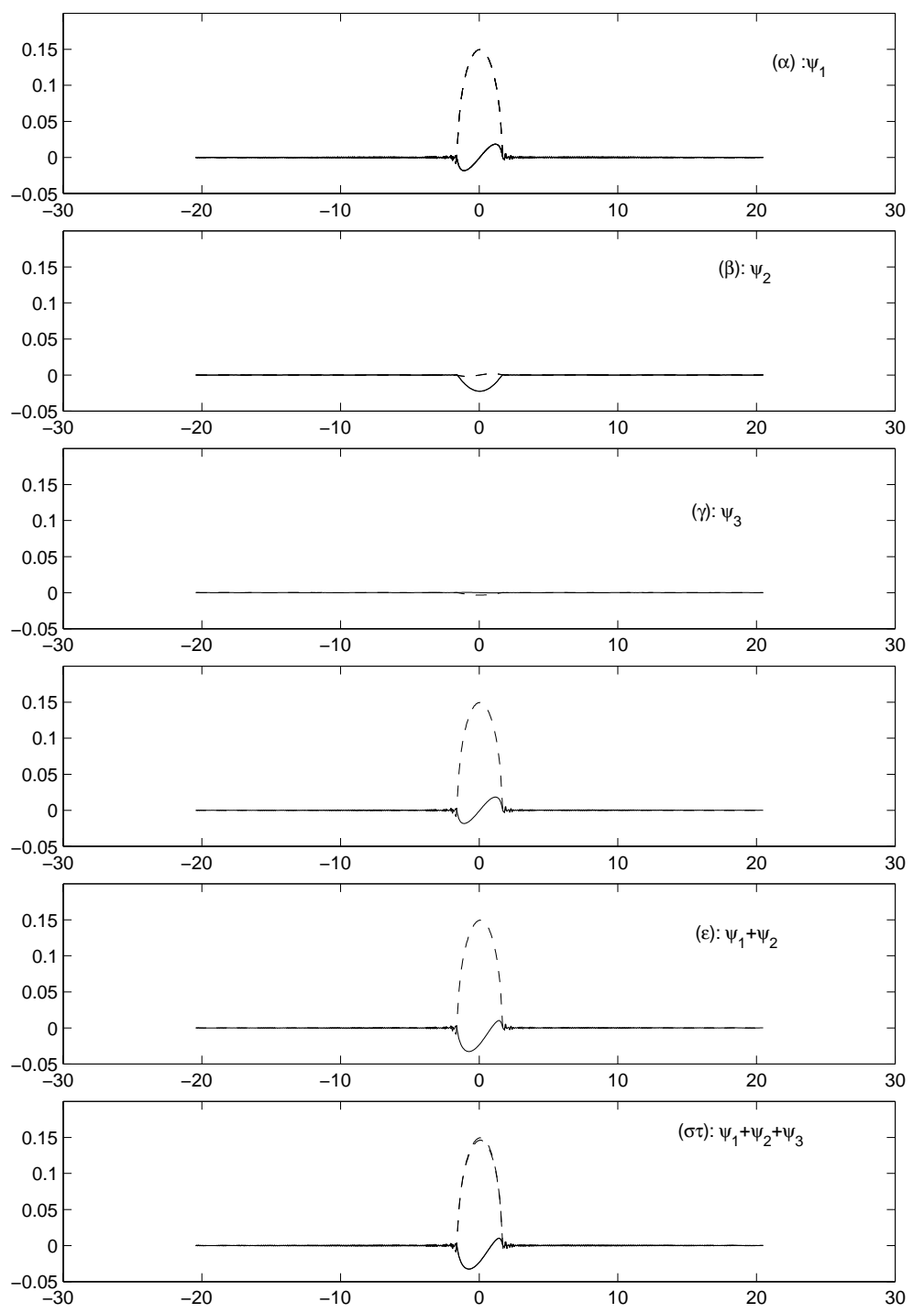

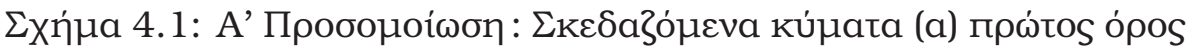

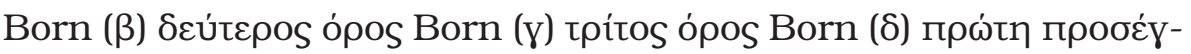

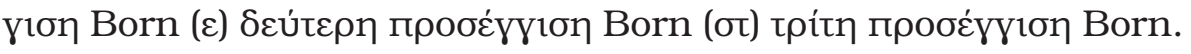

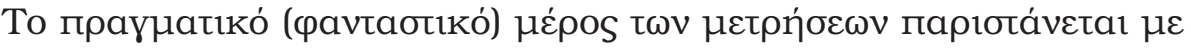

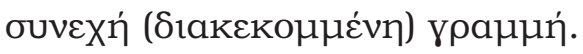




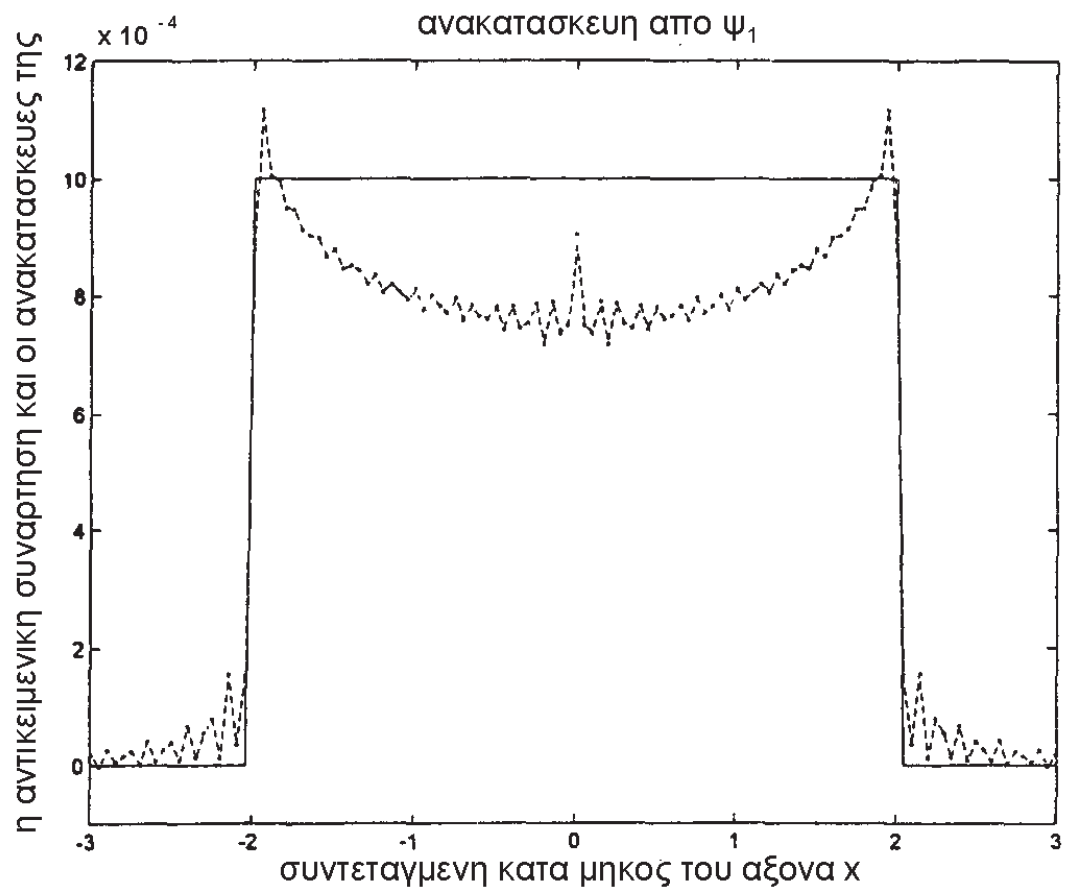

(a)

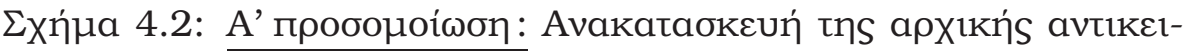

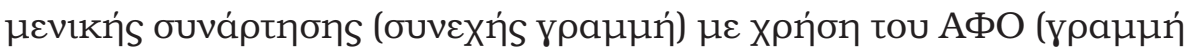

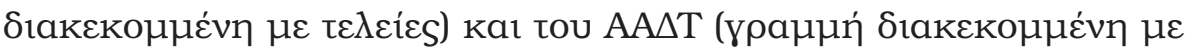

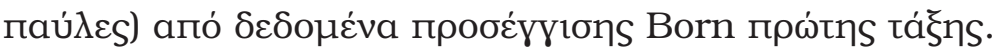




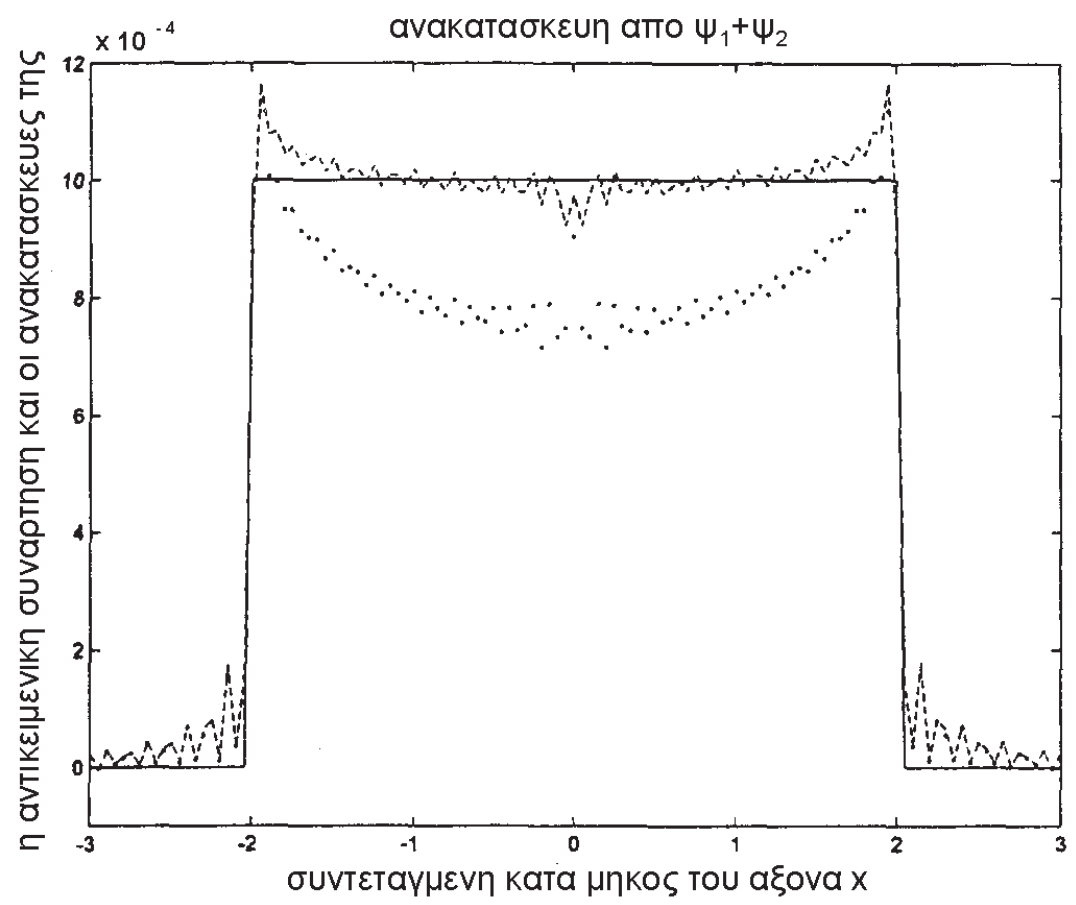

$(\beta)$

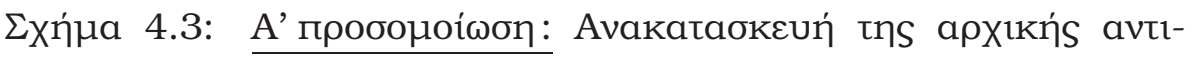

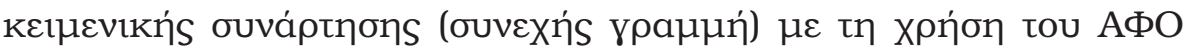

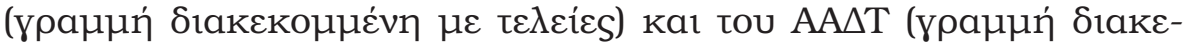

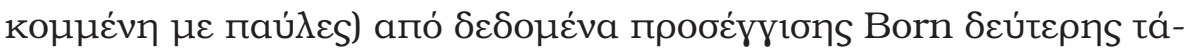
छns. 


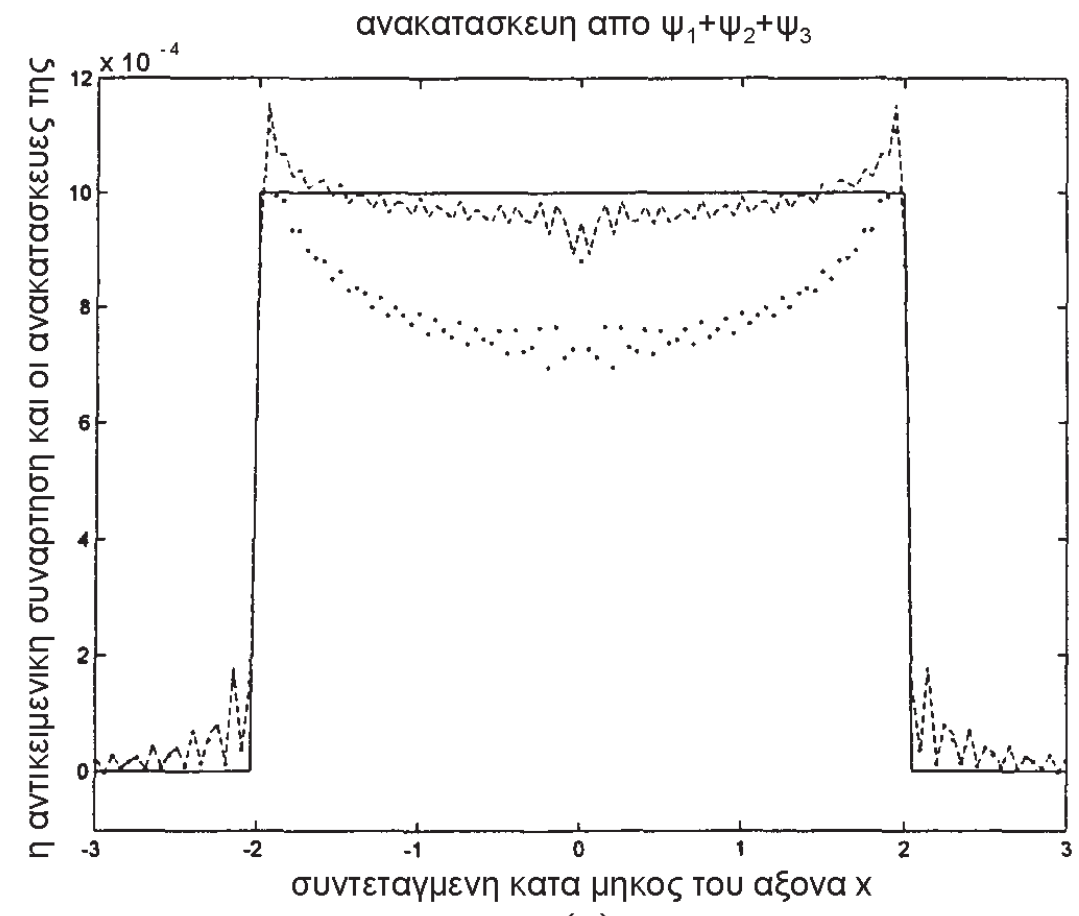

( $\mathrm{Y})$

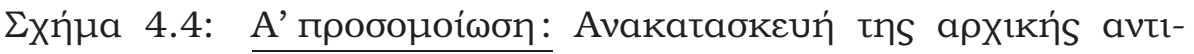

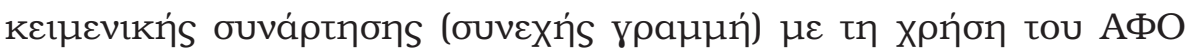

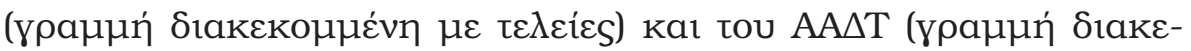

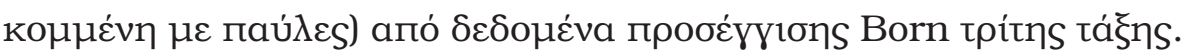




\subsection{2 В' пробороí⿴囗п}

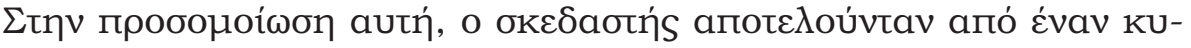

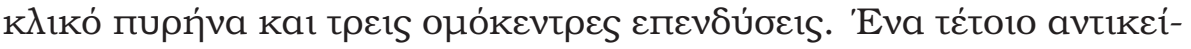

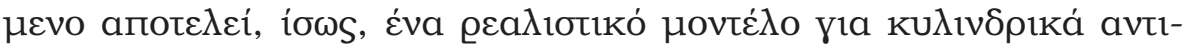

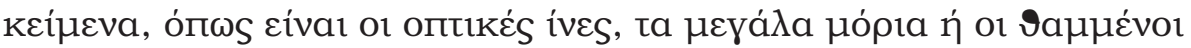

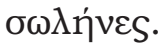

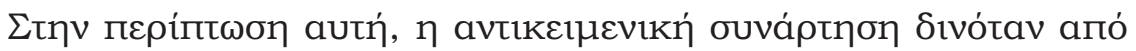

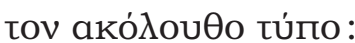

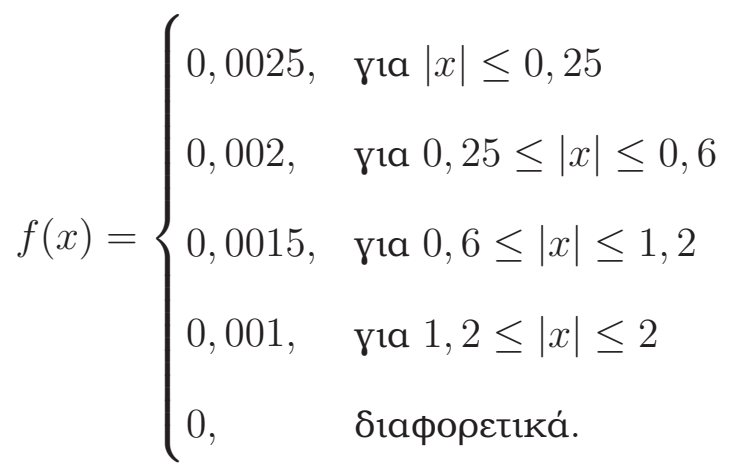

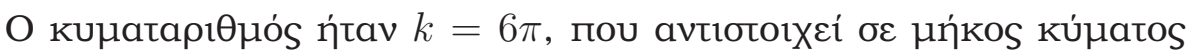

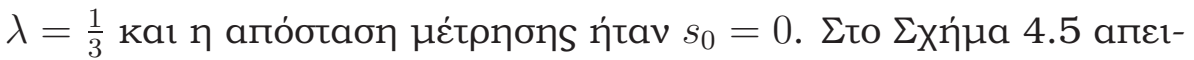

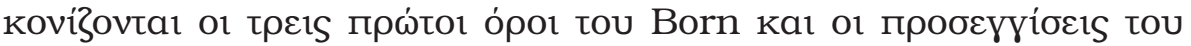

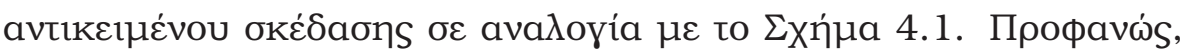

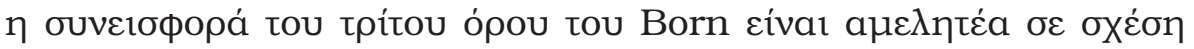

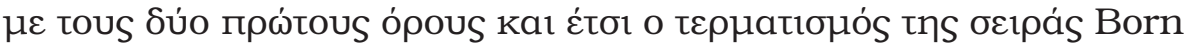

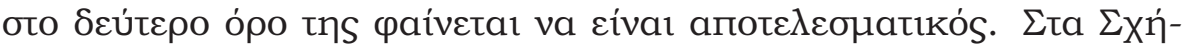

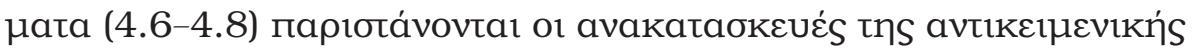

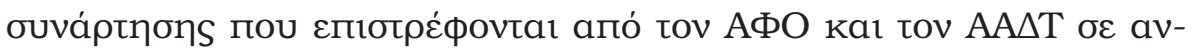

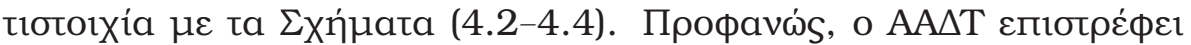




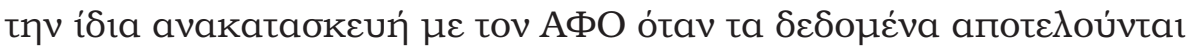

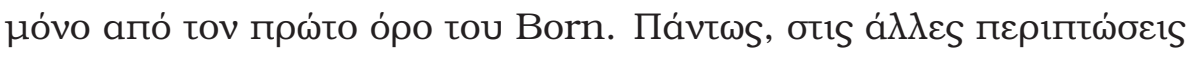

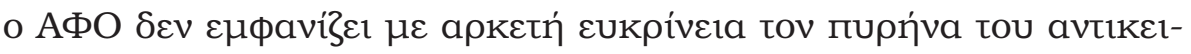

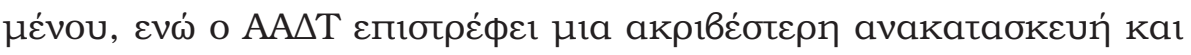

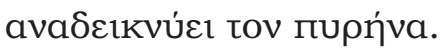

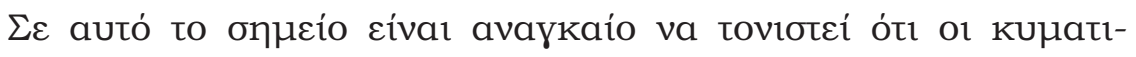

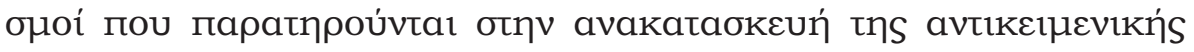

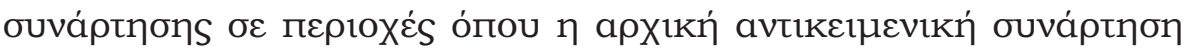

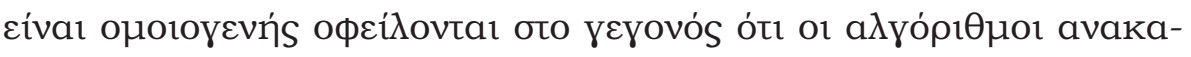

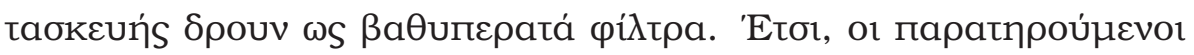

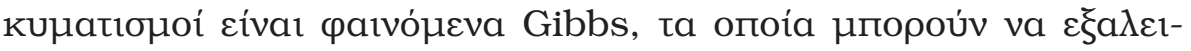

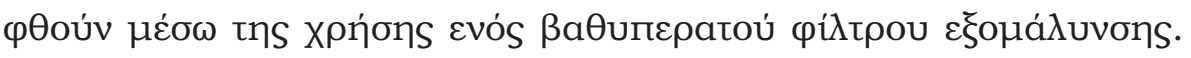

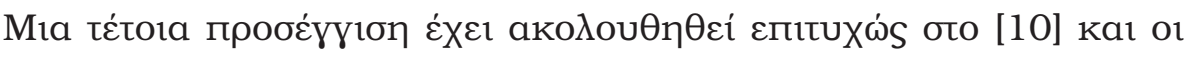

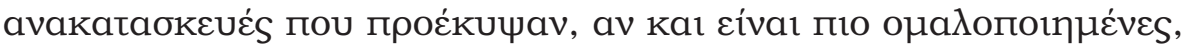

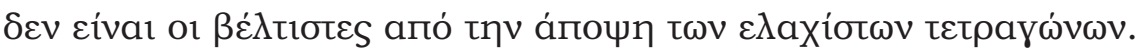



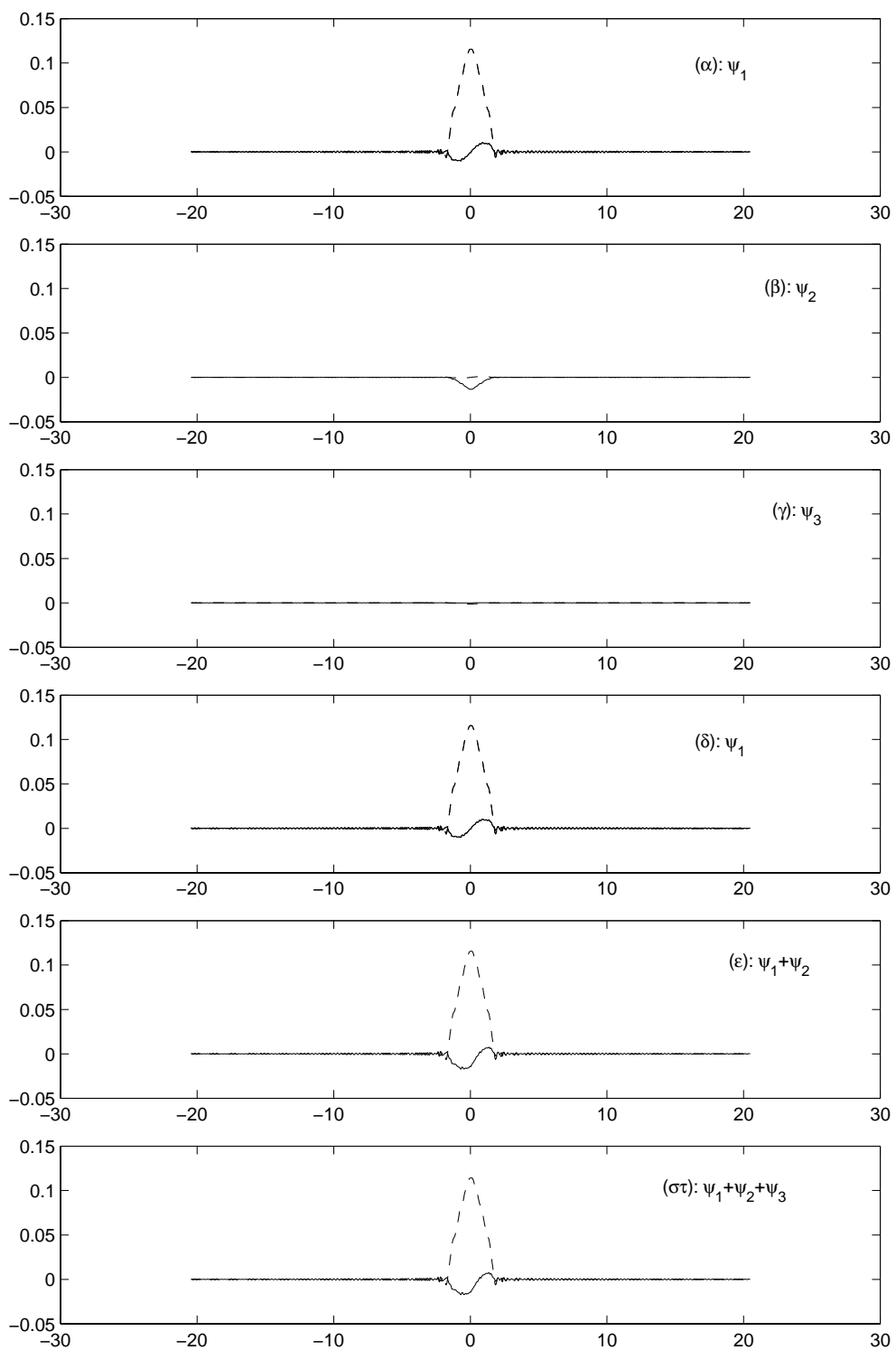

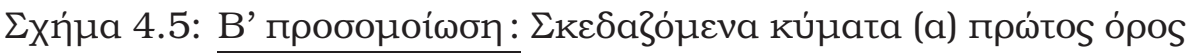

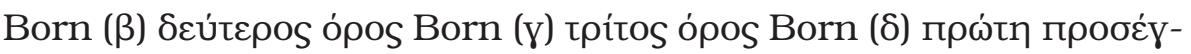

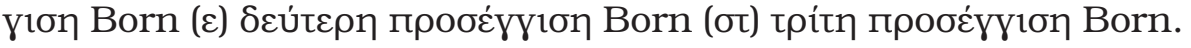

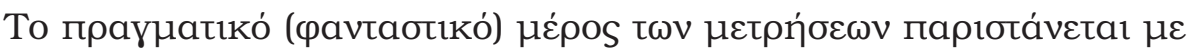

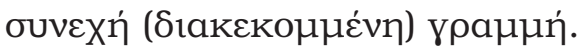




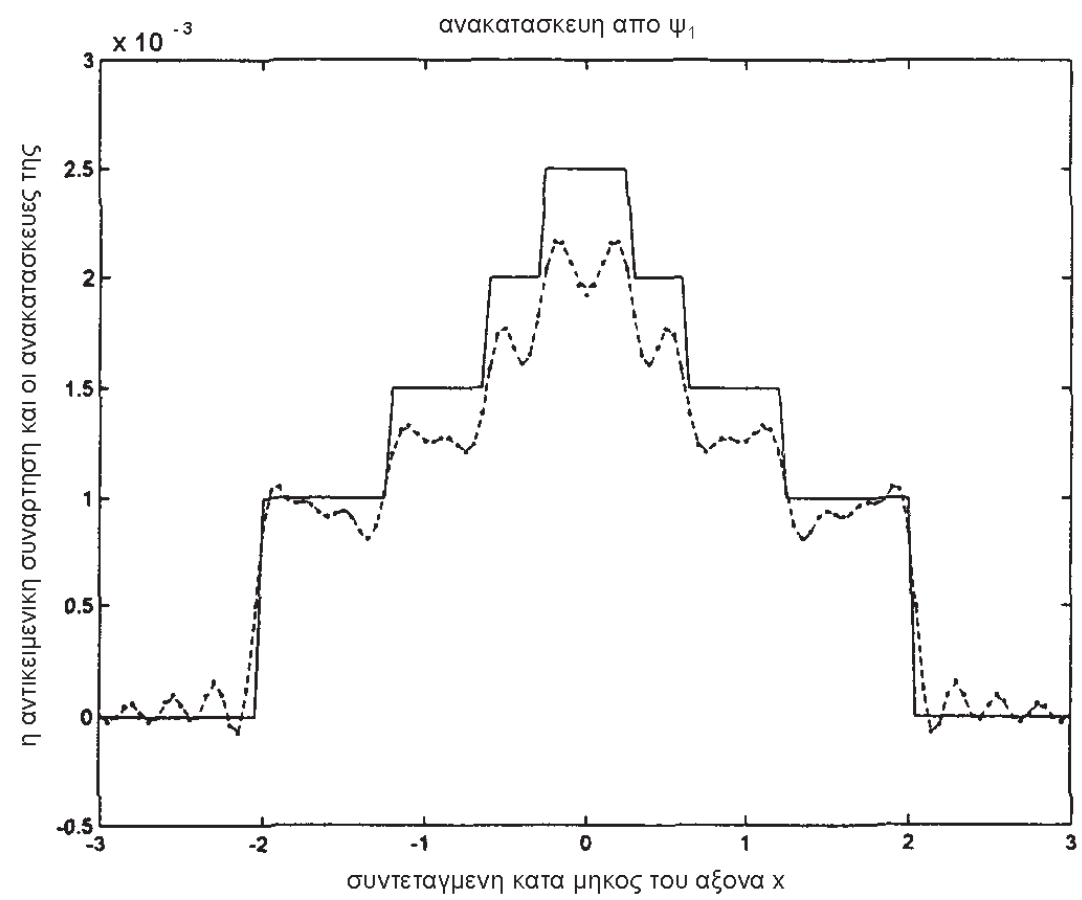

(a)

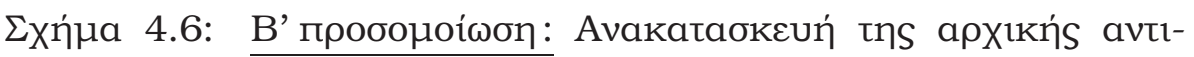

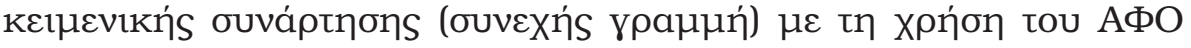

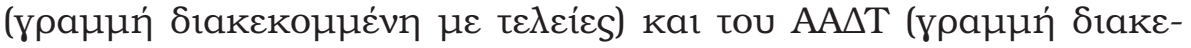

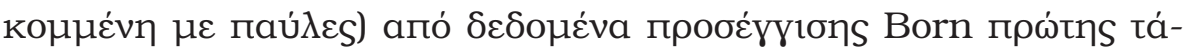
乡ns. 


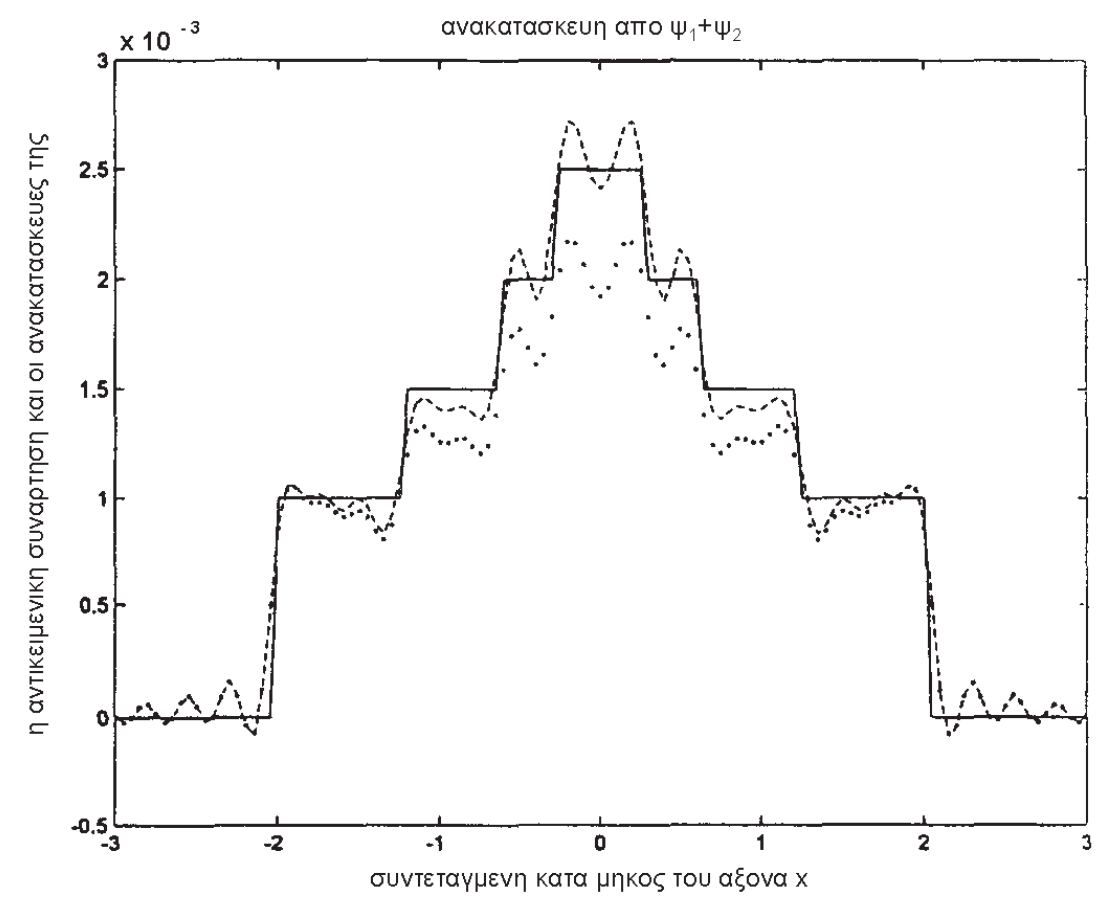

( $\beta)$

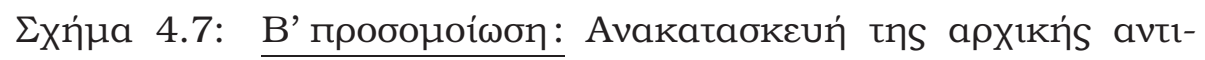

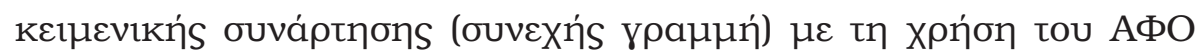

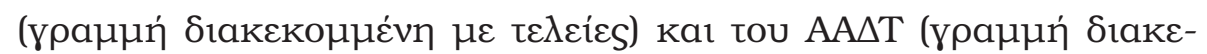

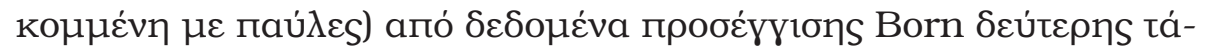
乡ns. 


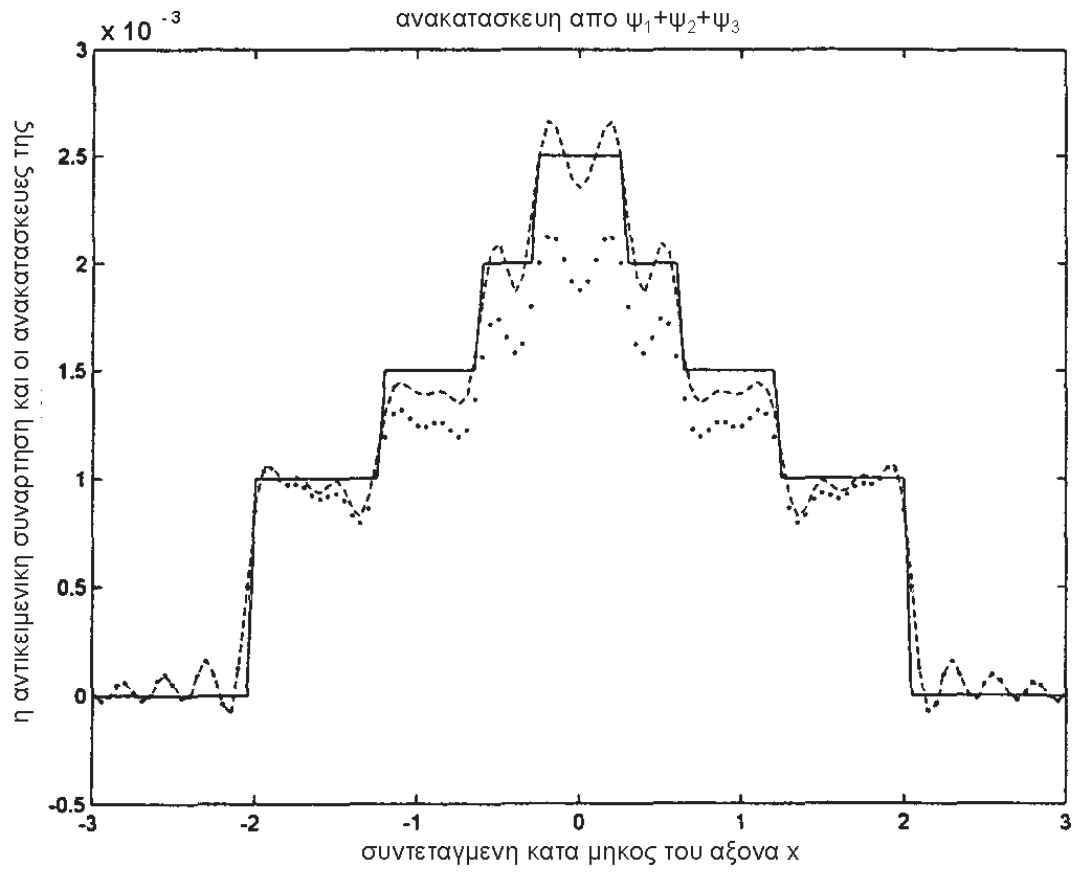

(

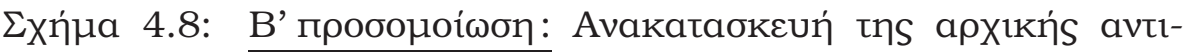

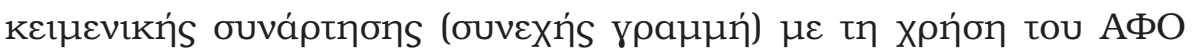

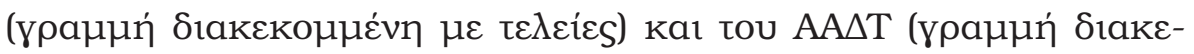

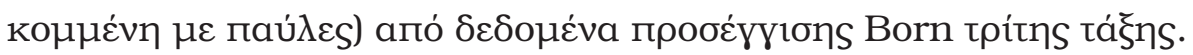




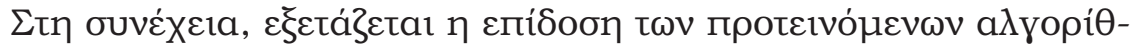

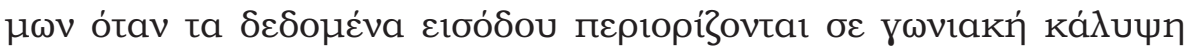

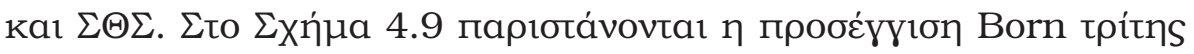

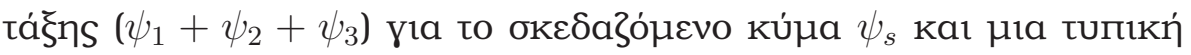

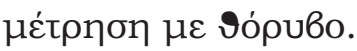

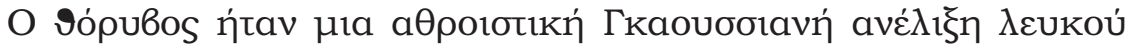

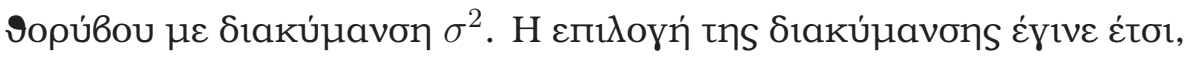

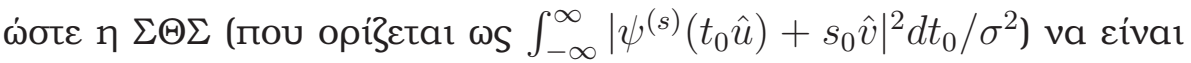

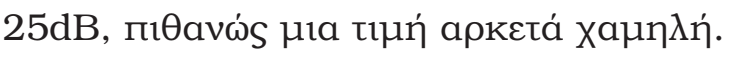

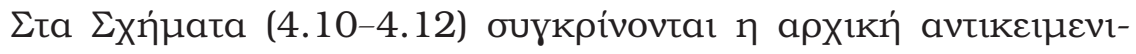

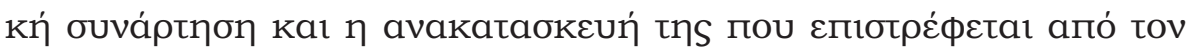

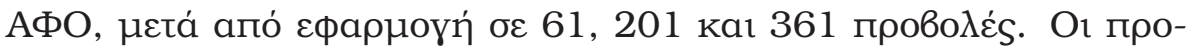

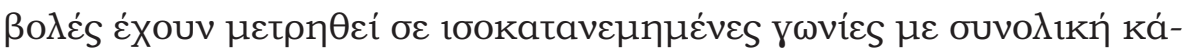

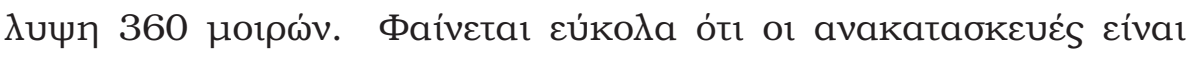

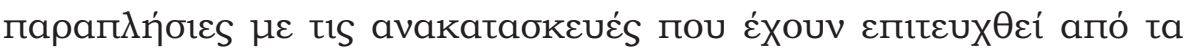

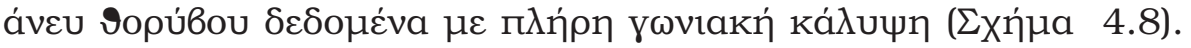

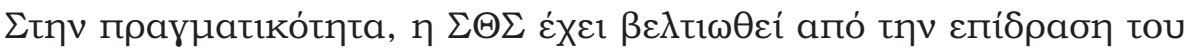

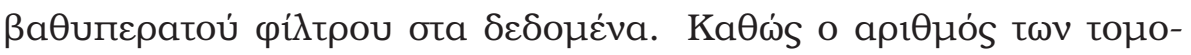

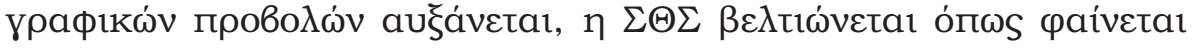

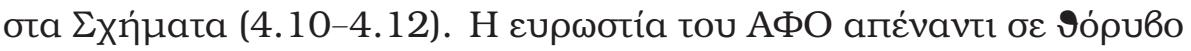

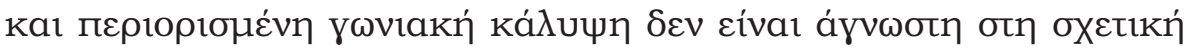
$\beta 16 \lambda$ ıорафі́a [10].

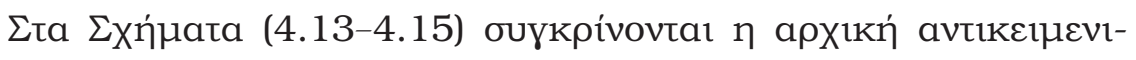

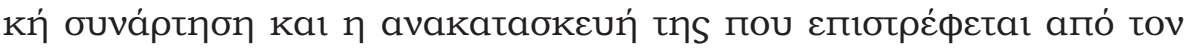

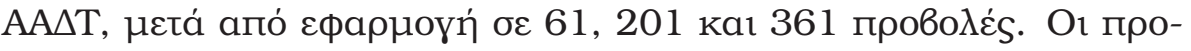



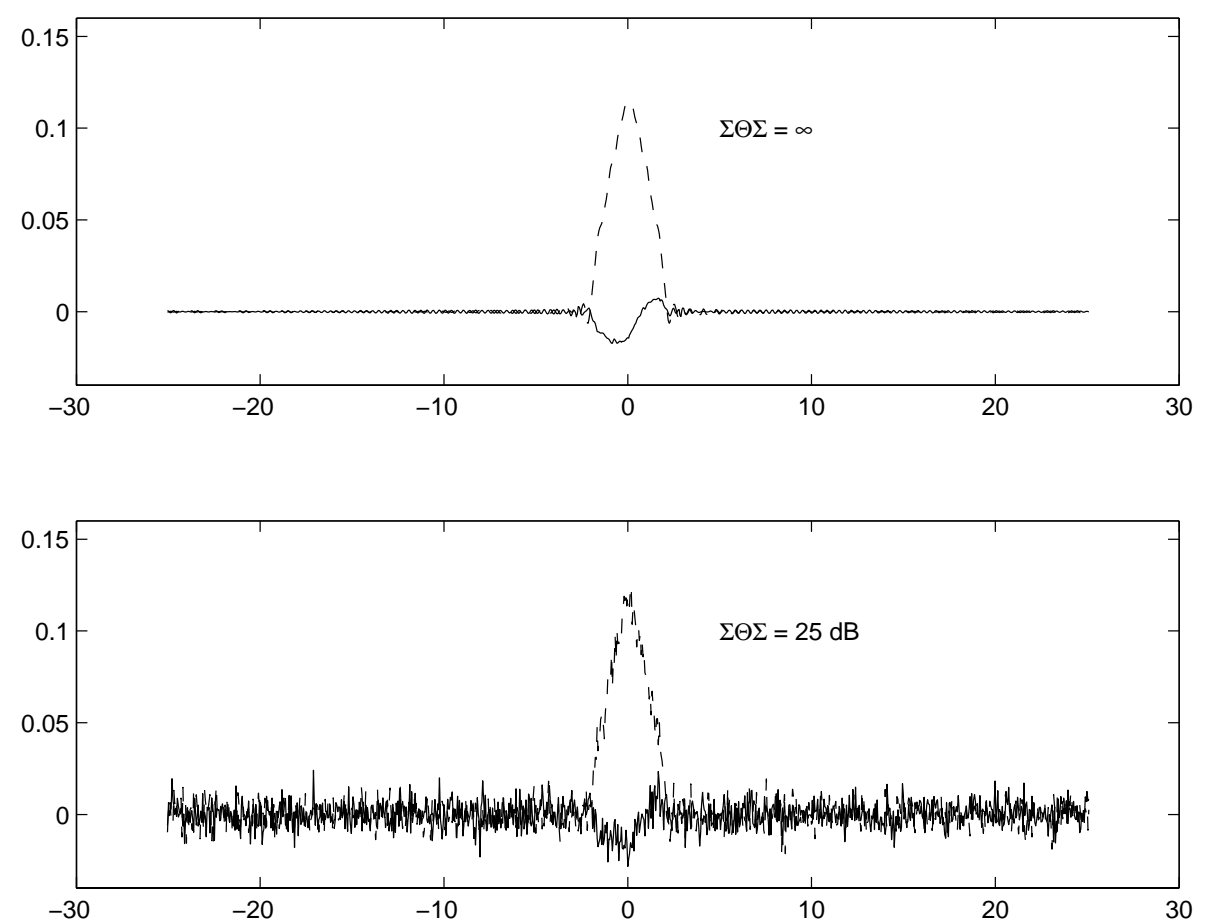

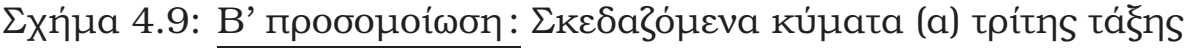

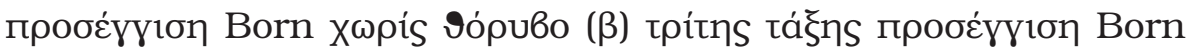

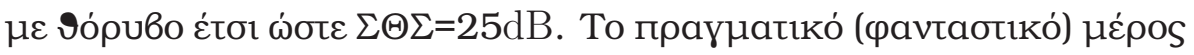

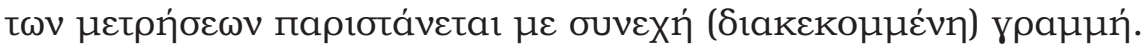

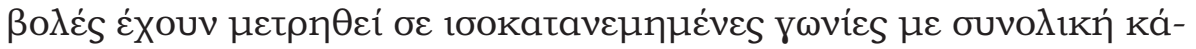

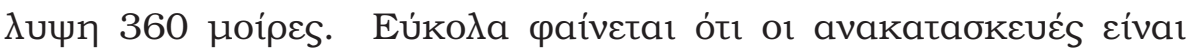

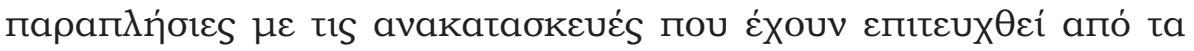

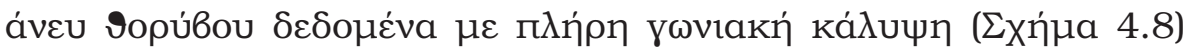

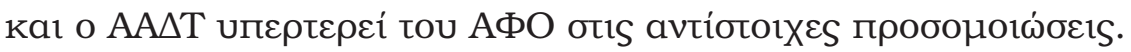




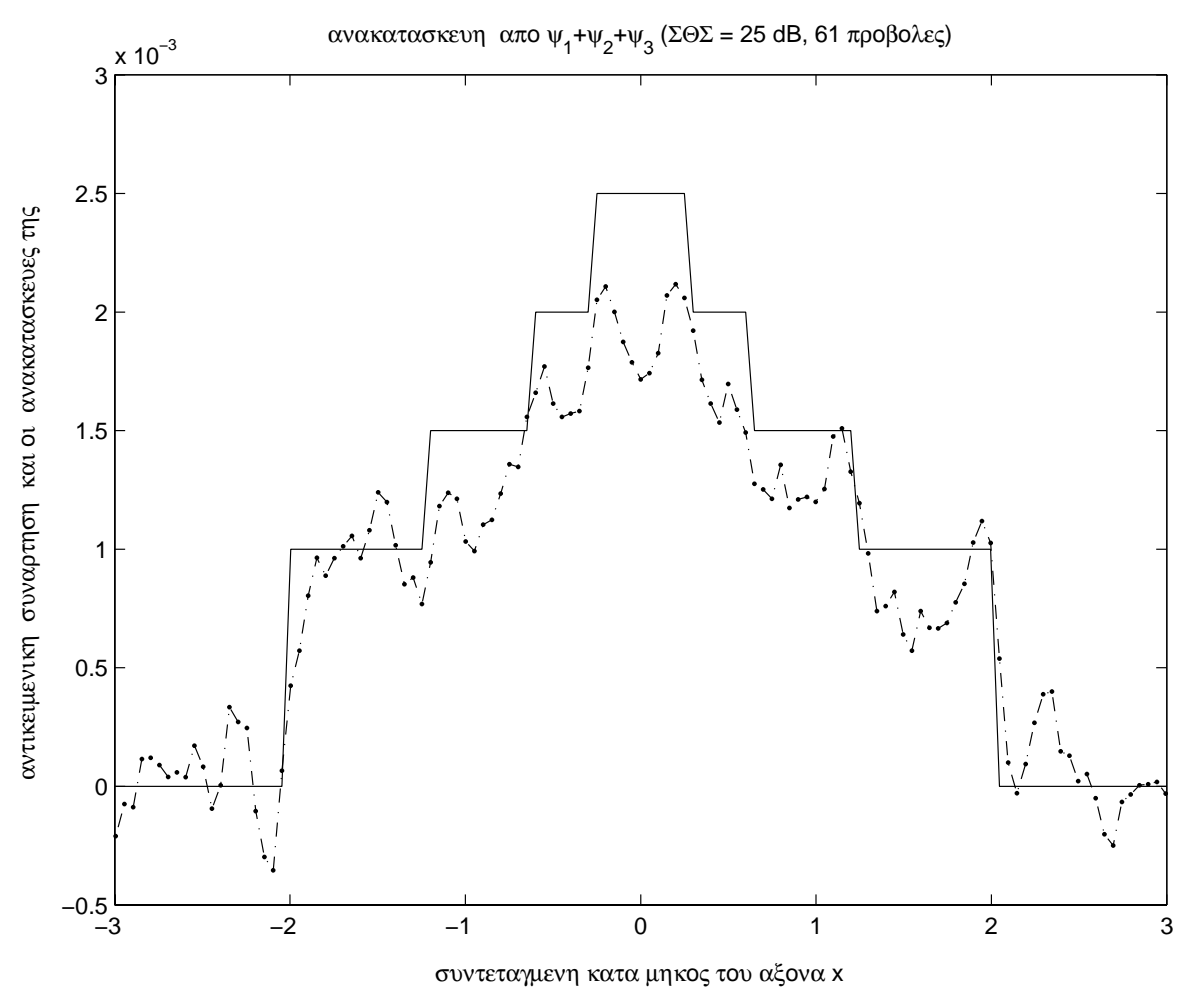

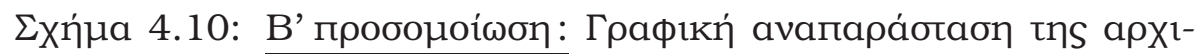

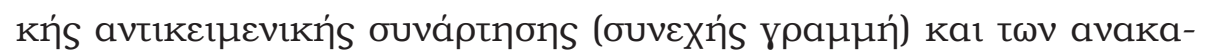

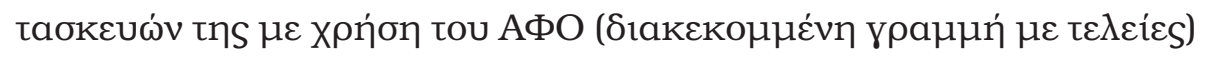

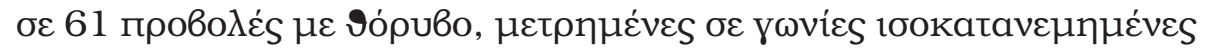
ois $360^{\circ}$. 


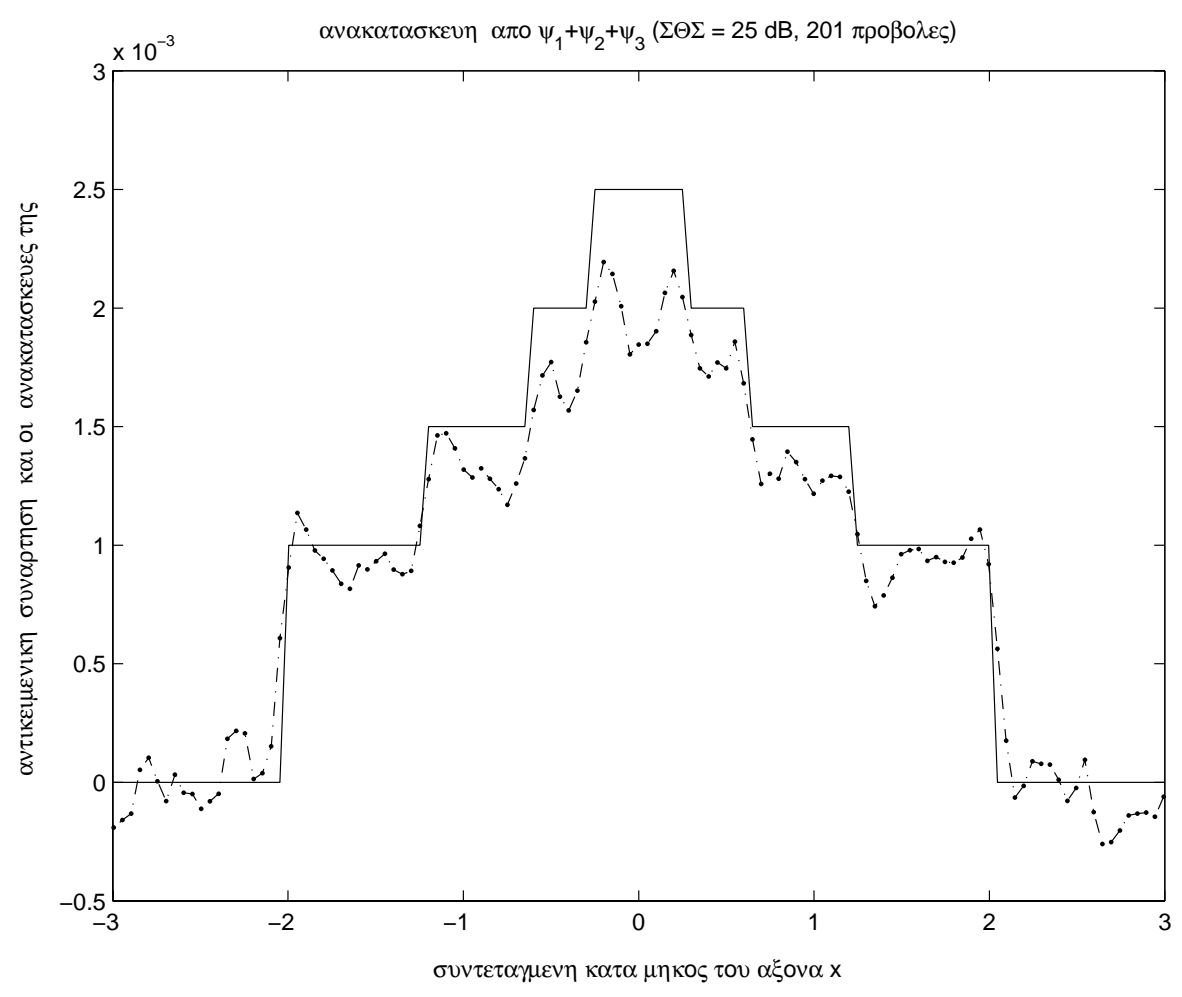

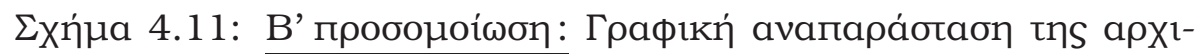

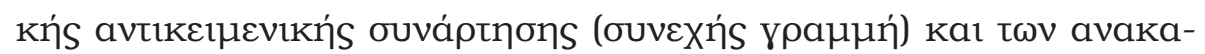

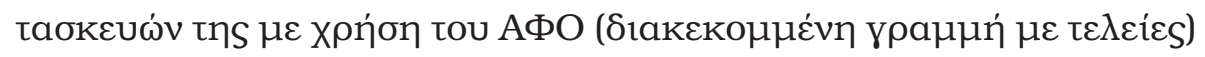

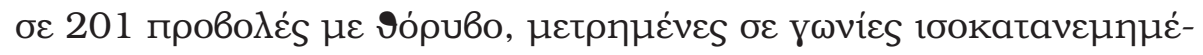
ves $\sigma \mathrm{is} 360^{\circ}$. 


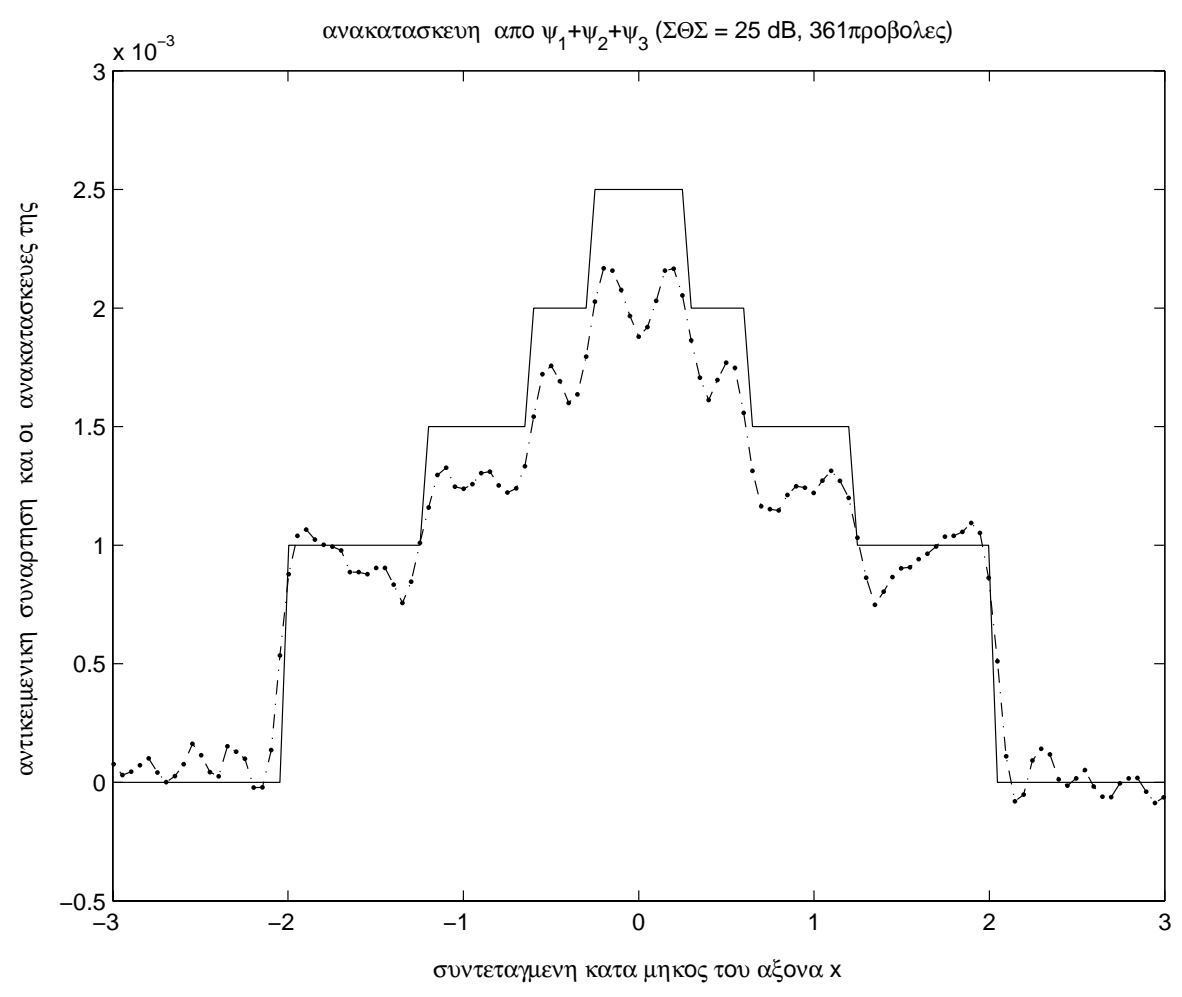

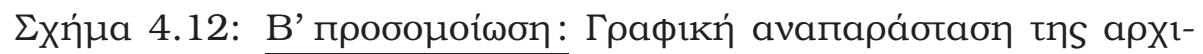

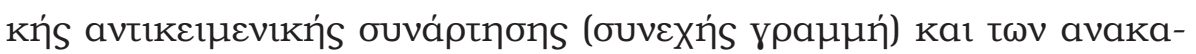

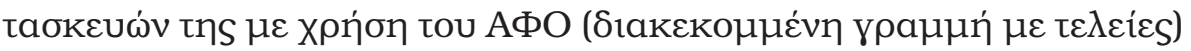

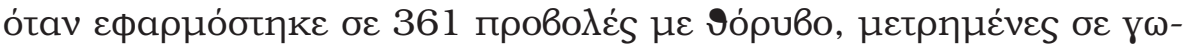

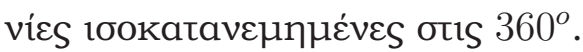




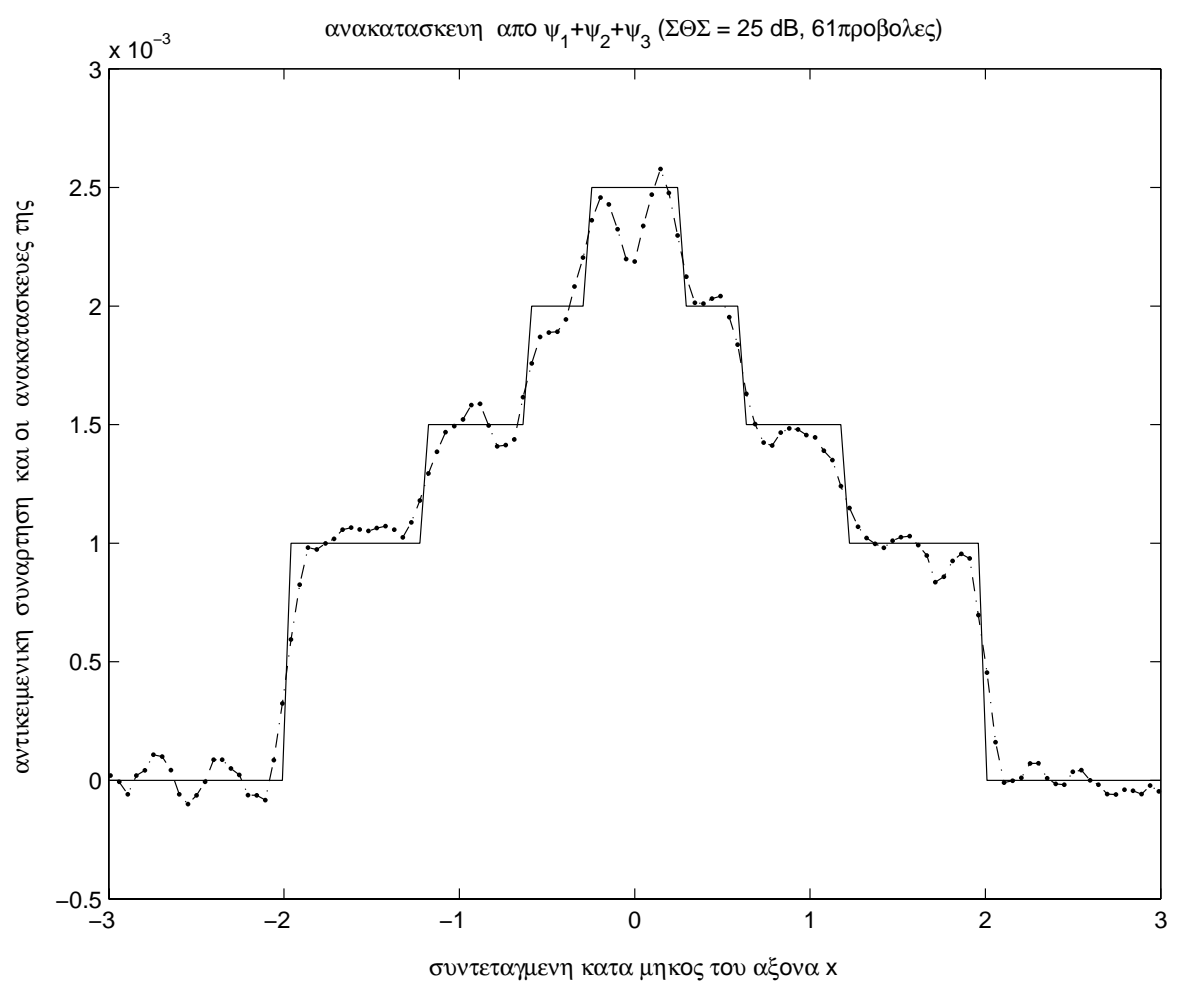

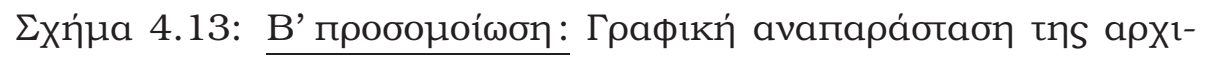

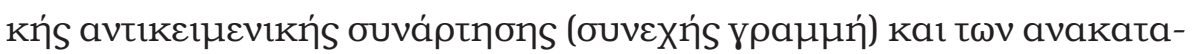

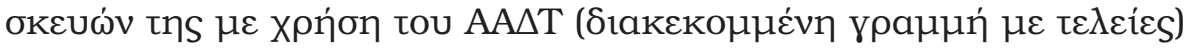

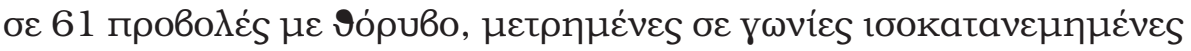
otis $360^{\circ}$. 


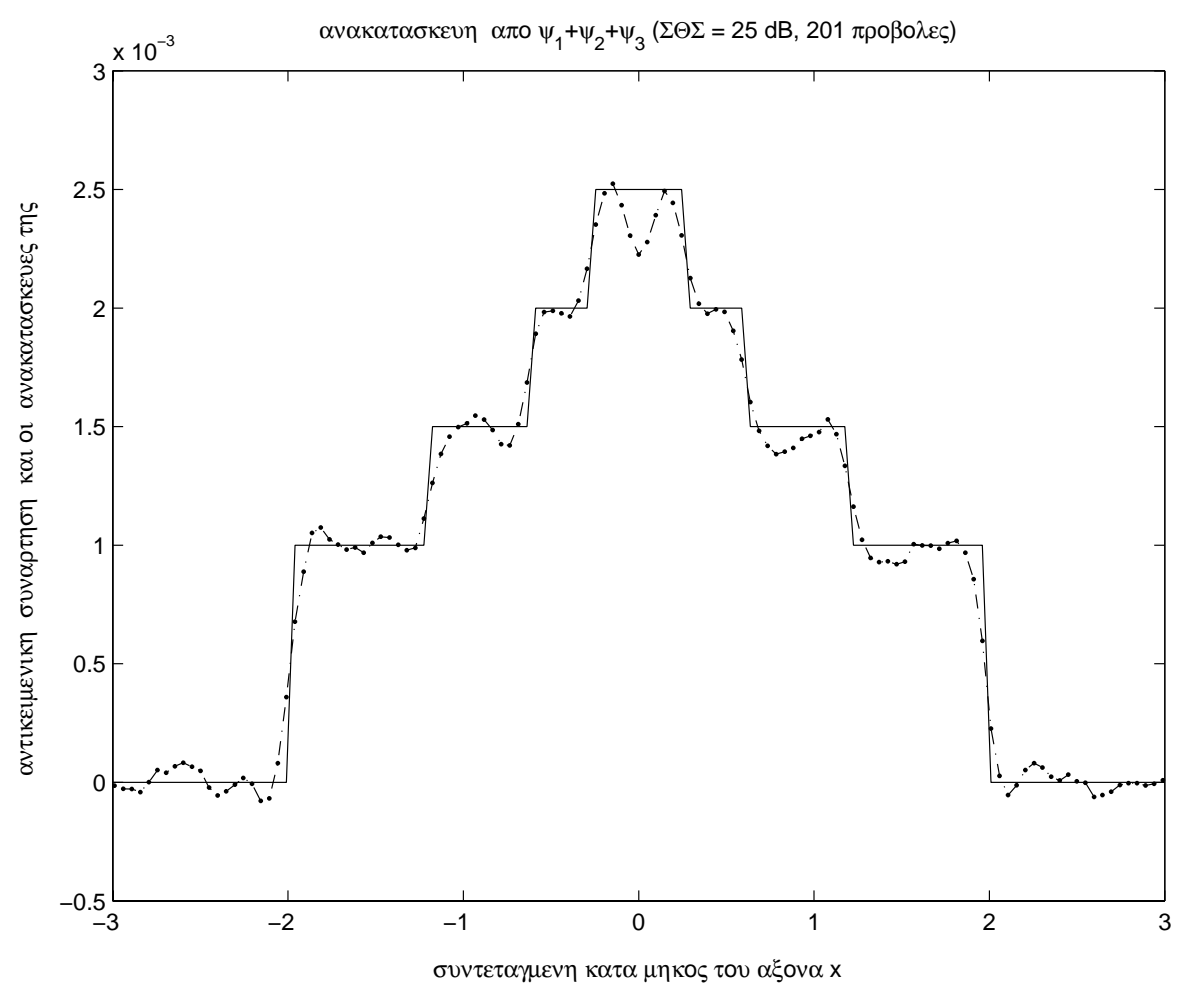

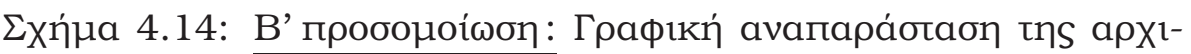

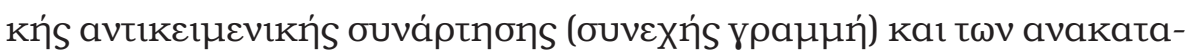

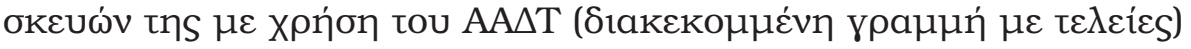

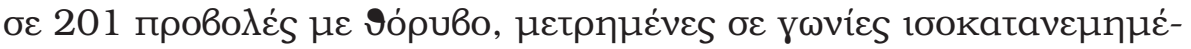
ves otıs $360^{\circ}$. 


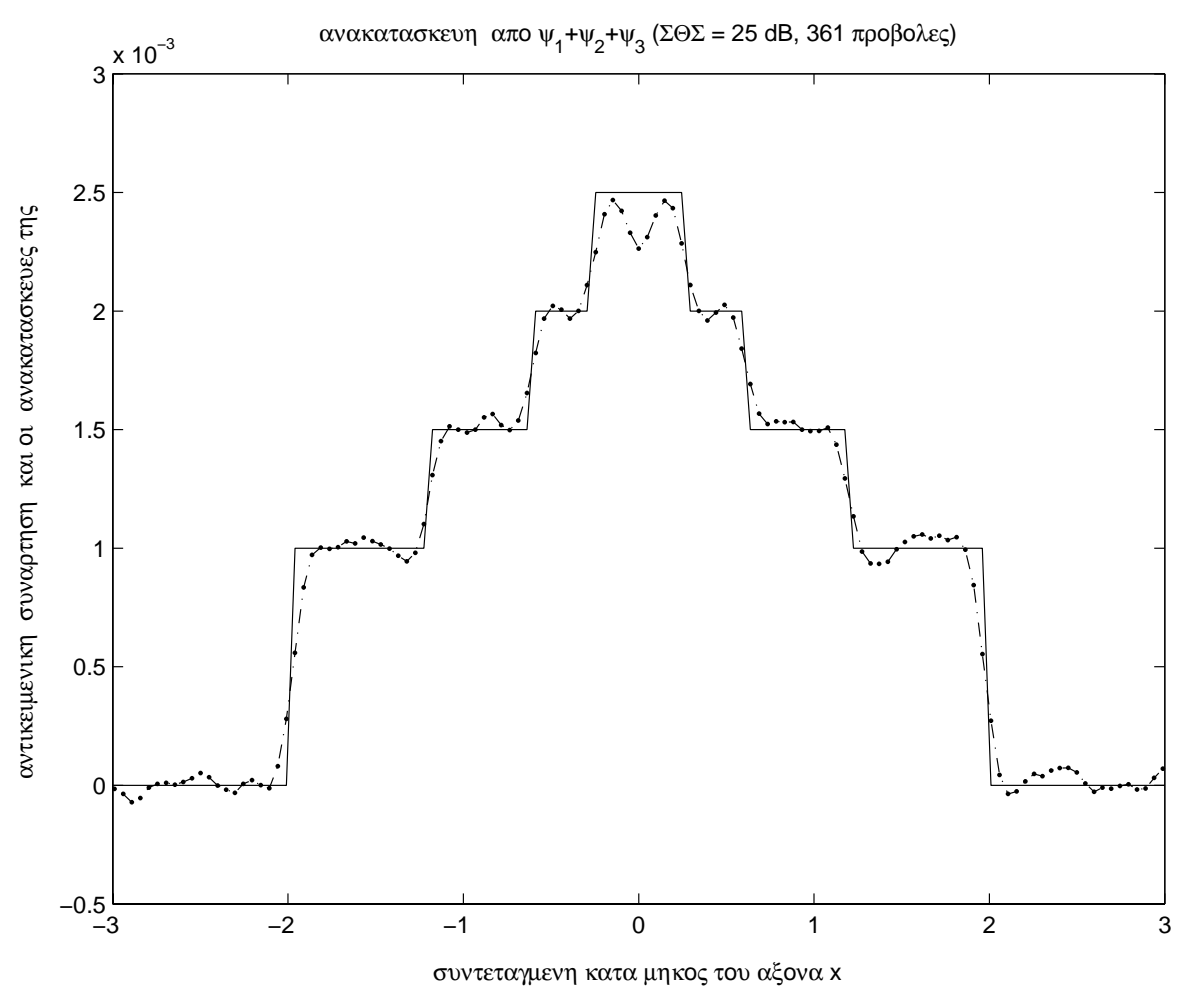

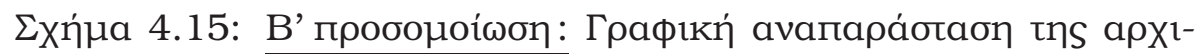

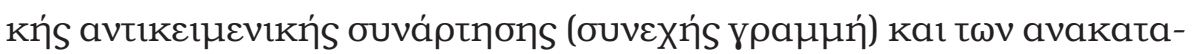

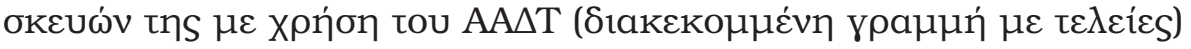

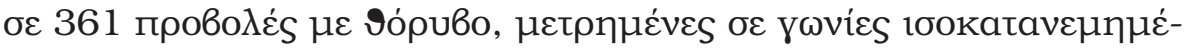
ves otıs $360^{\circ}$. 


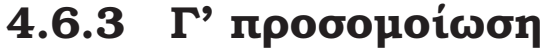

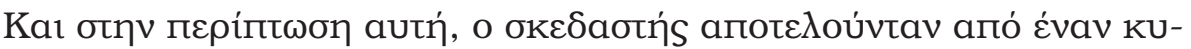

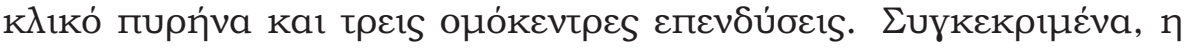

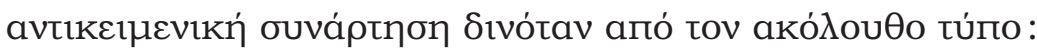

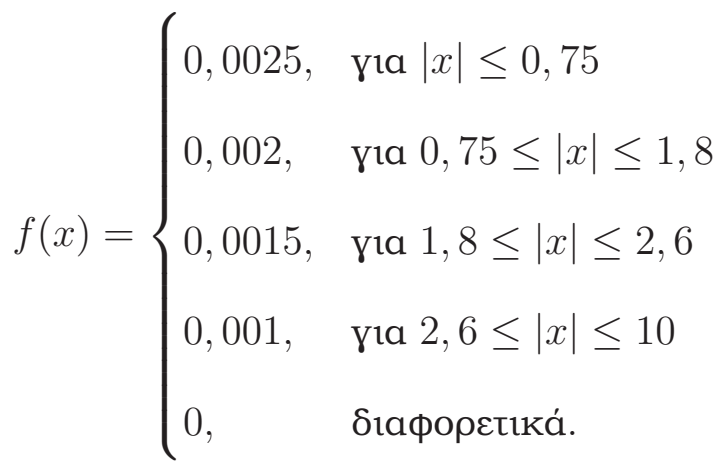

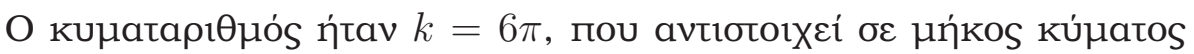

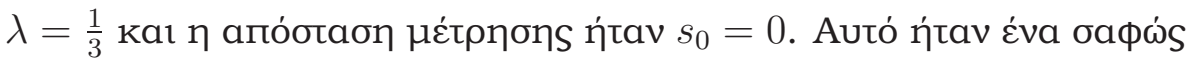

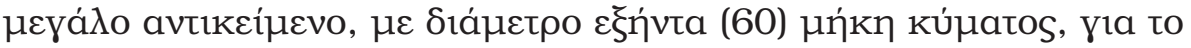

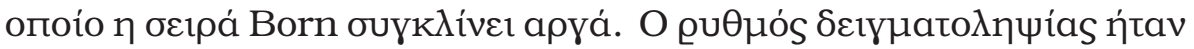

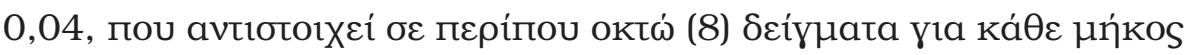

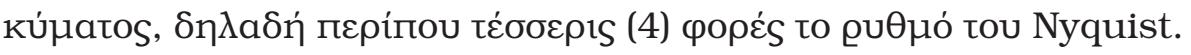

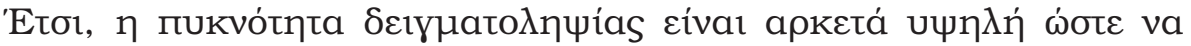

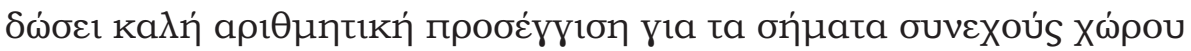

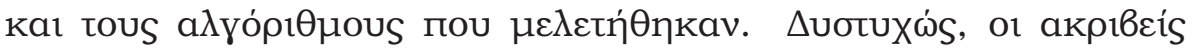

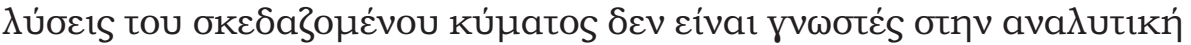

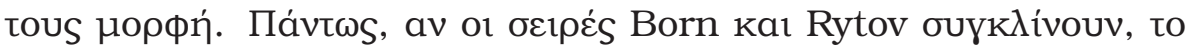

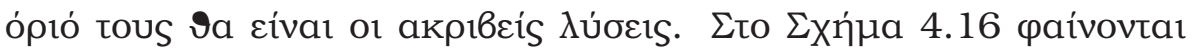

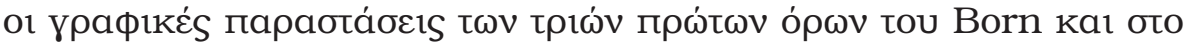

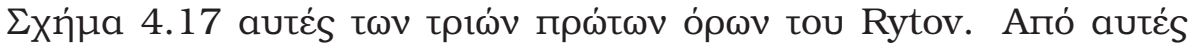




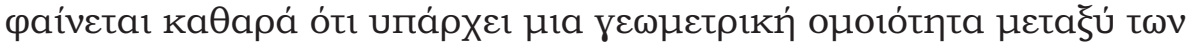
ópwv tou Born kaı tou Rytov.
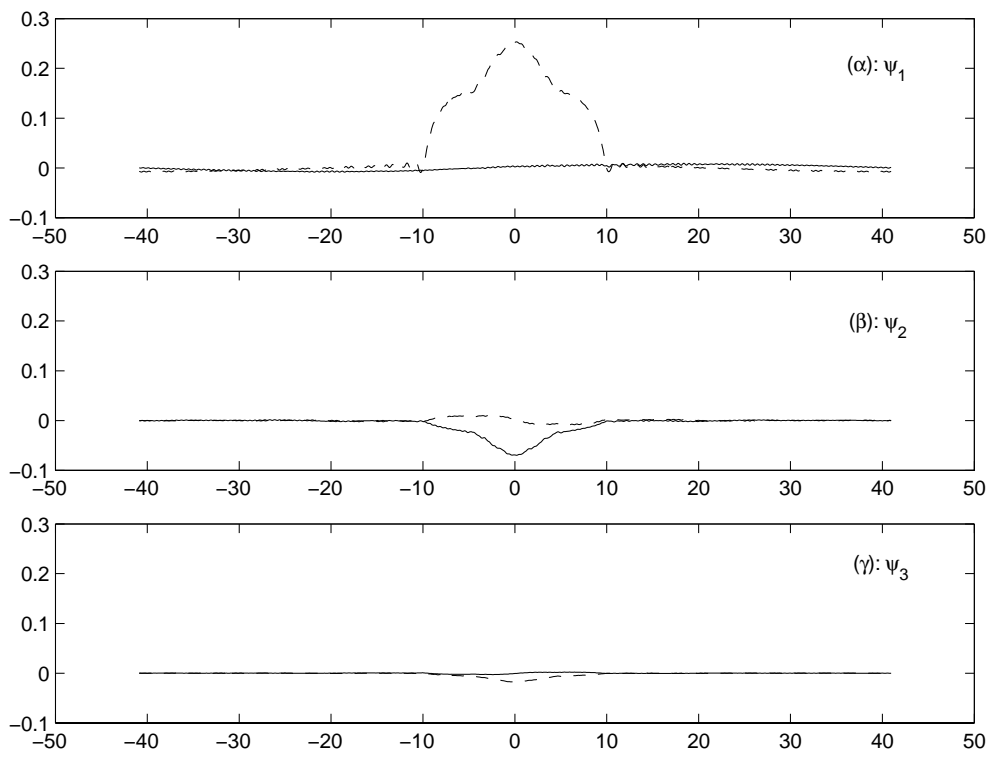

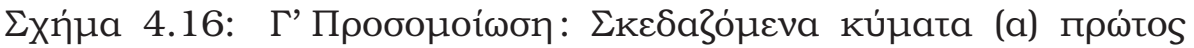

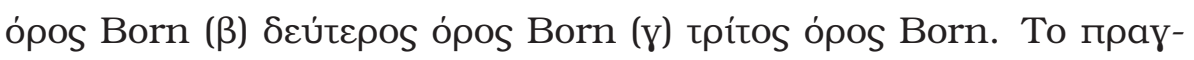

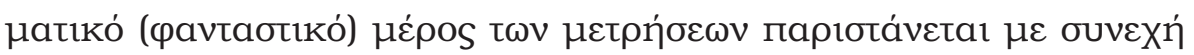

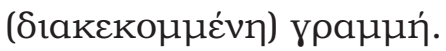

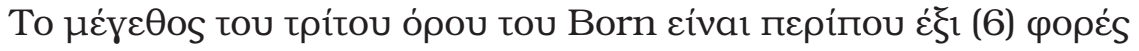

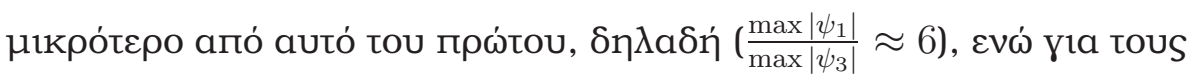

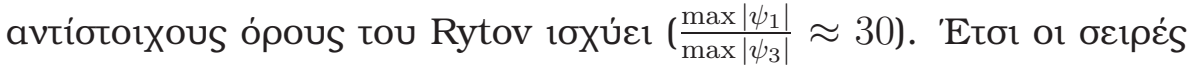

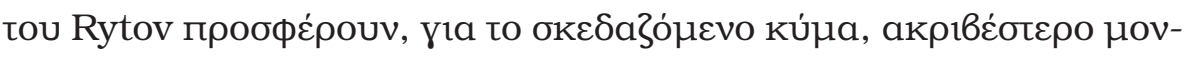

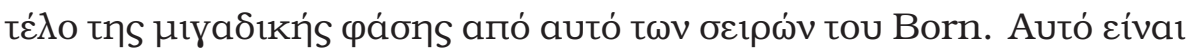

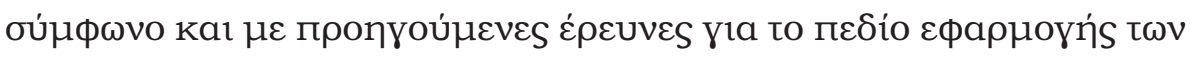

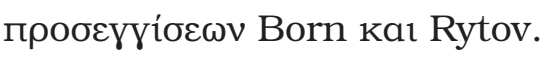



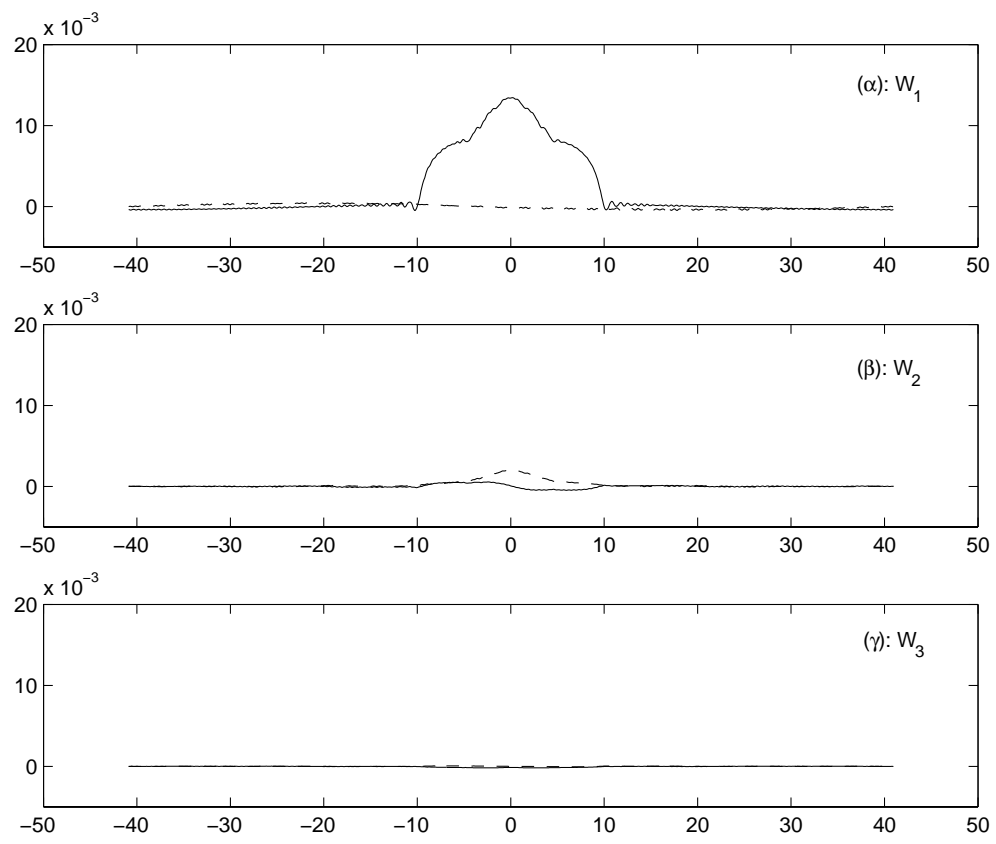

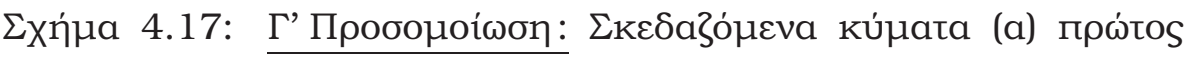

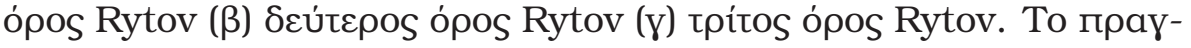

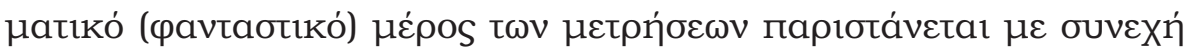

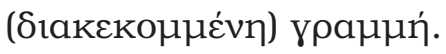




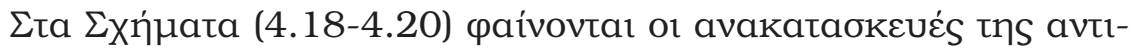

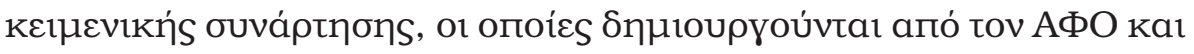

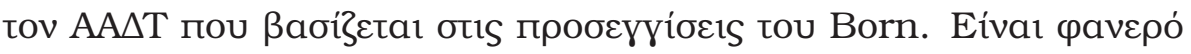

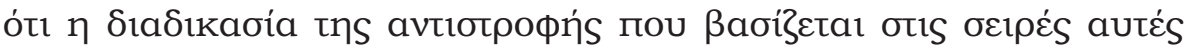

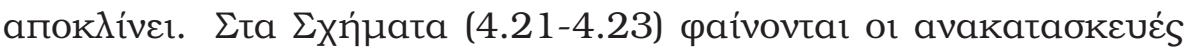

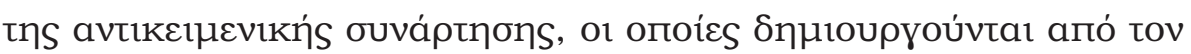

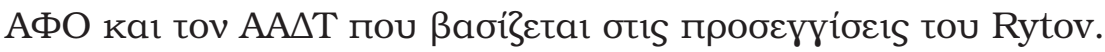

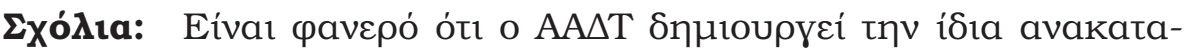

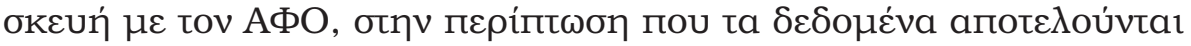

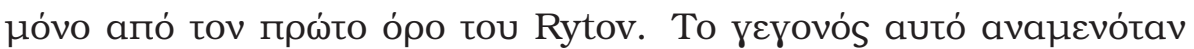

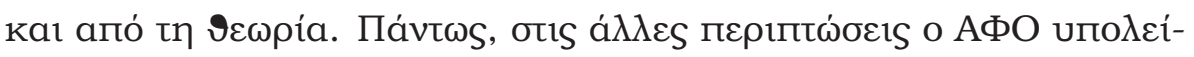

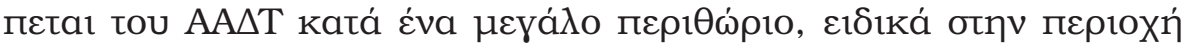

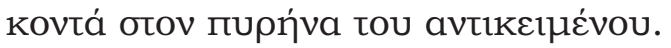




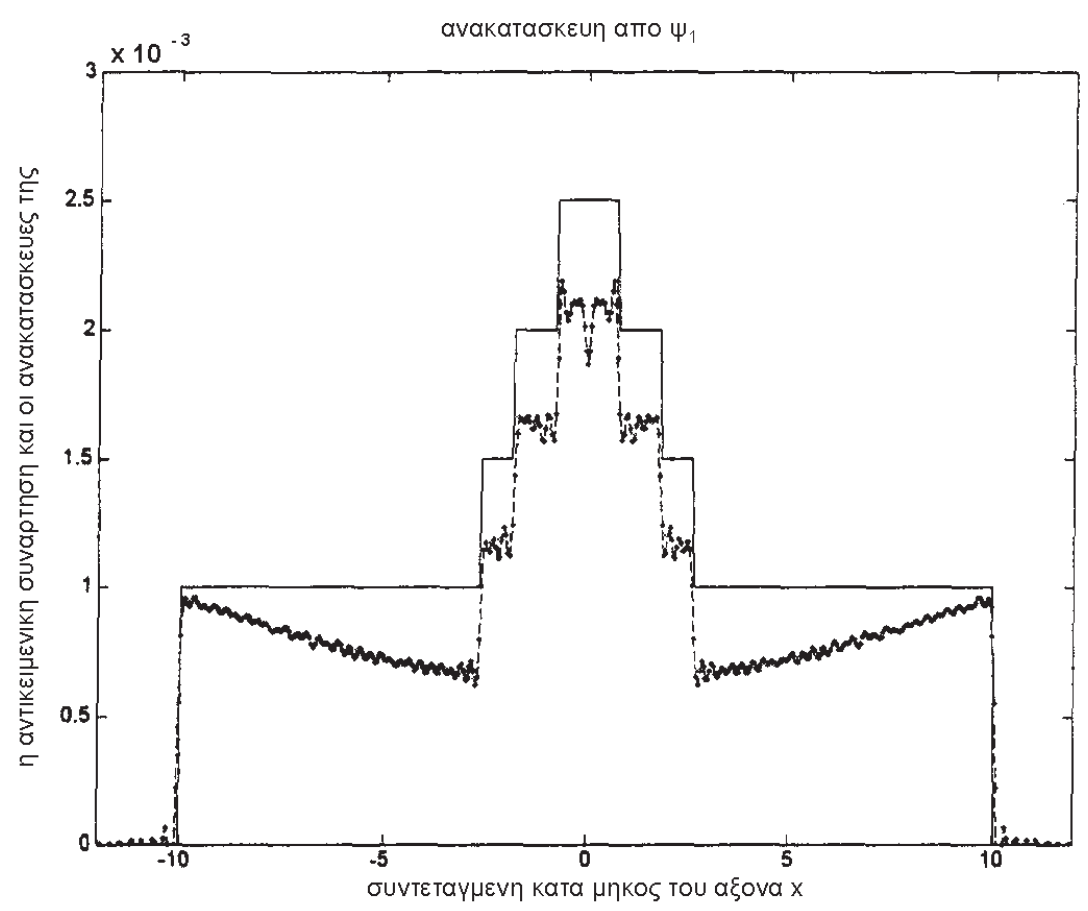

(a)

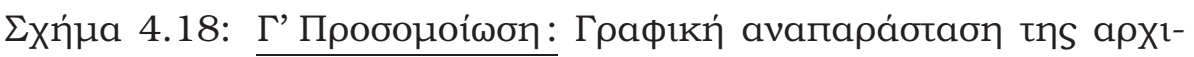

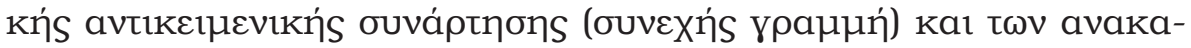

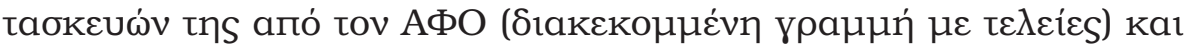

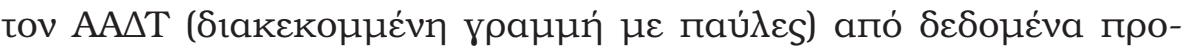

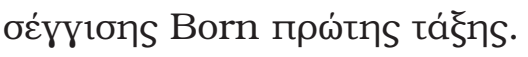




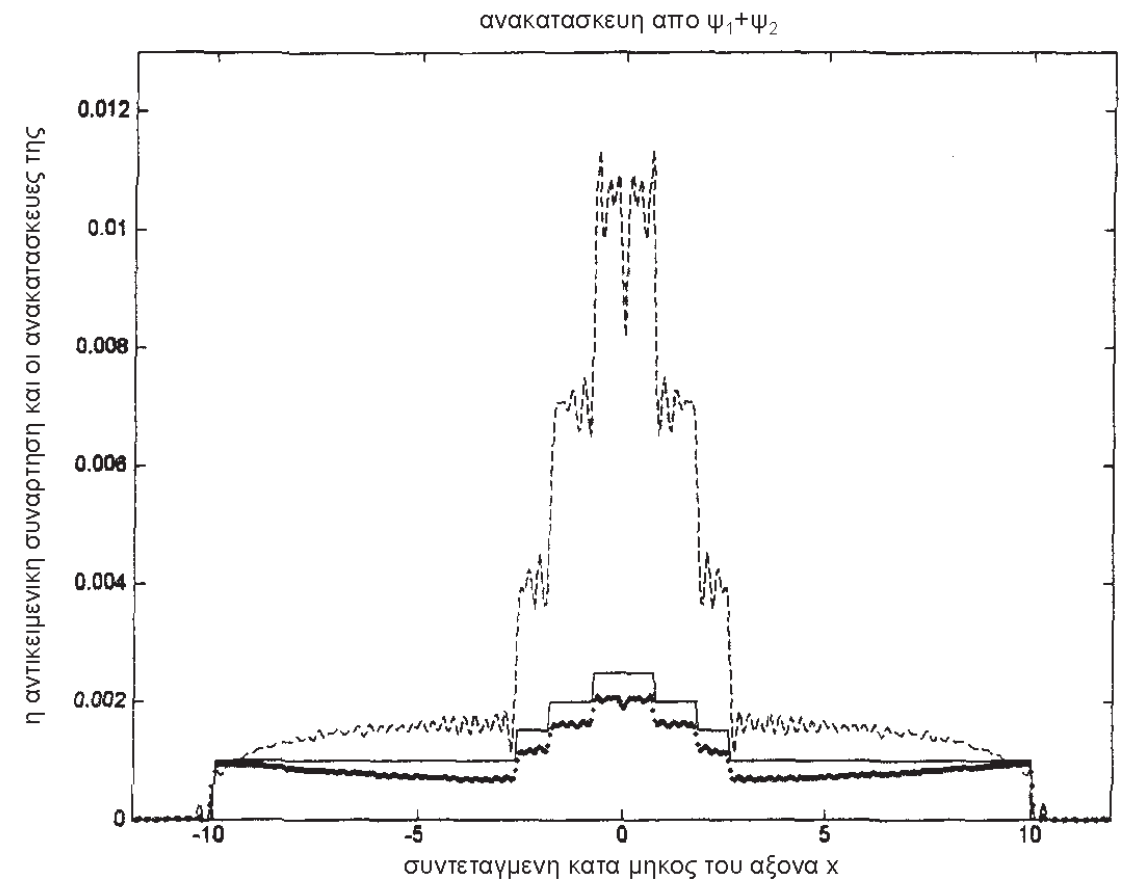

$(\beta)$

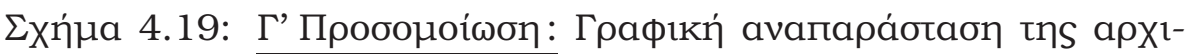

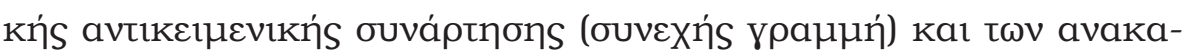

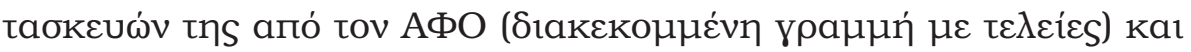

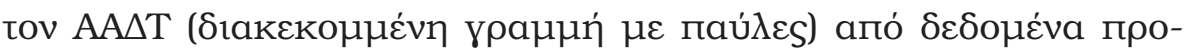

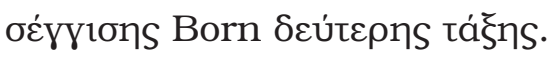




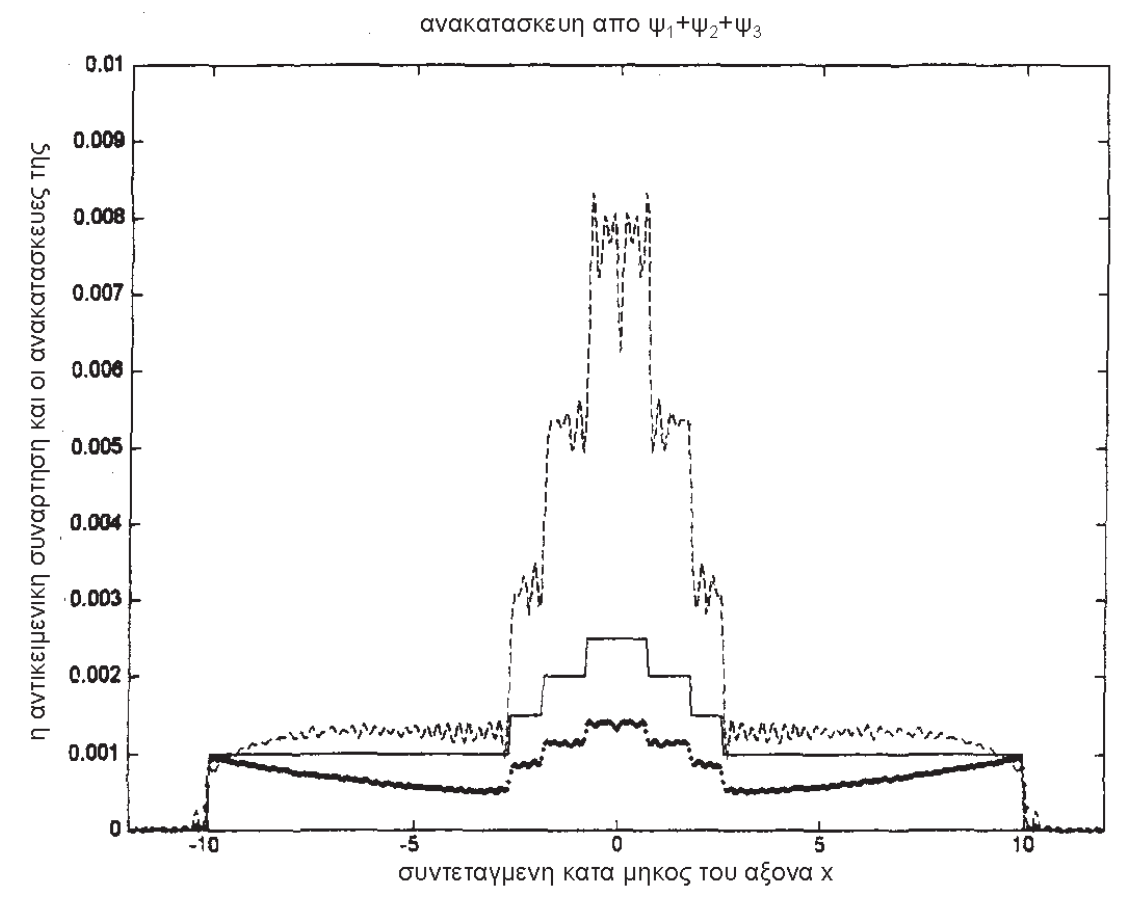

(v)

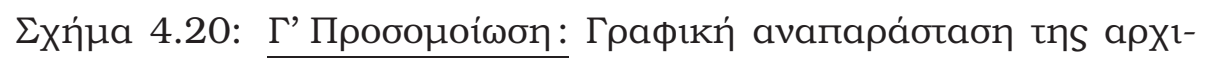

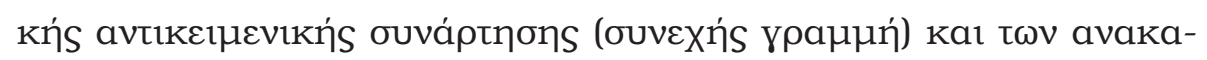

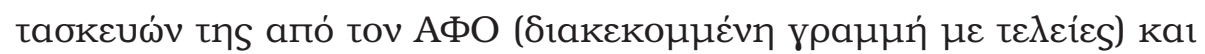

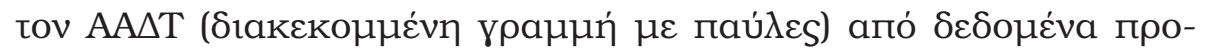

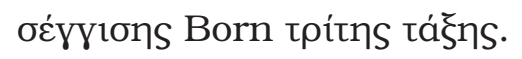




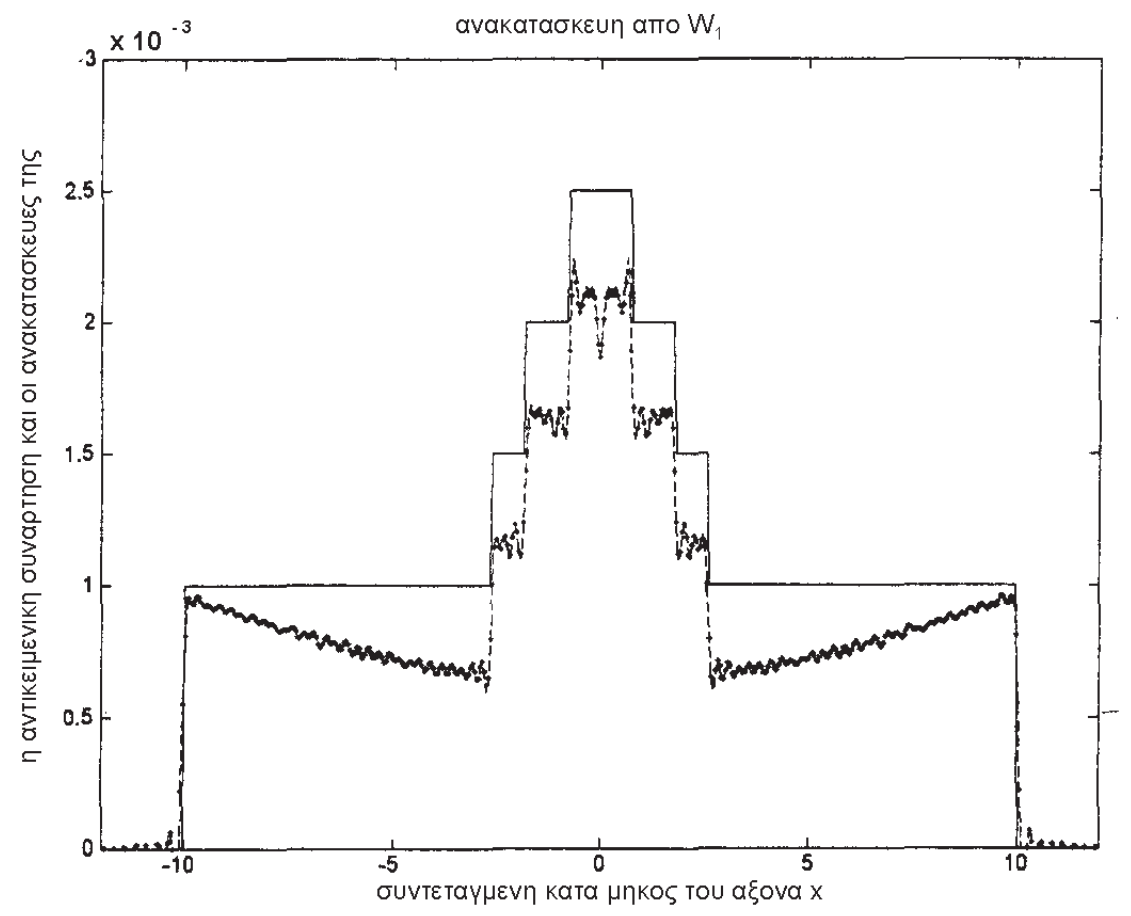

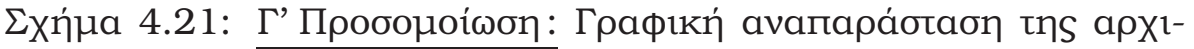

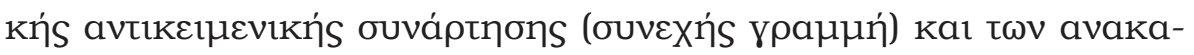

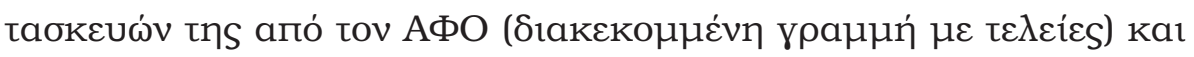

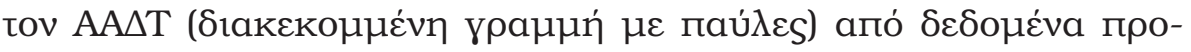

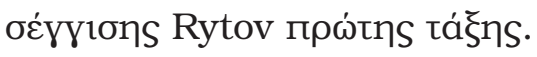




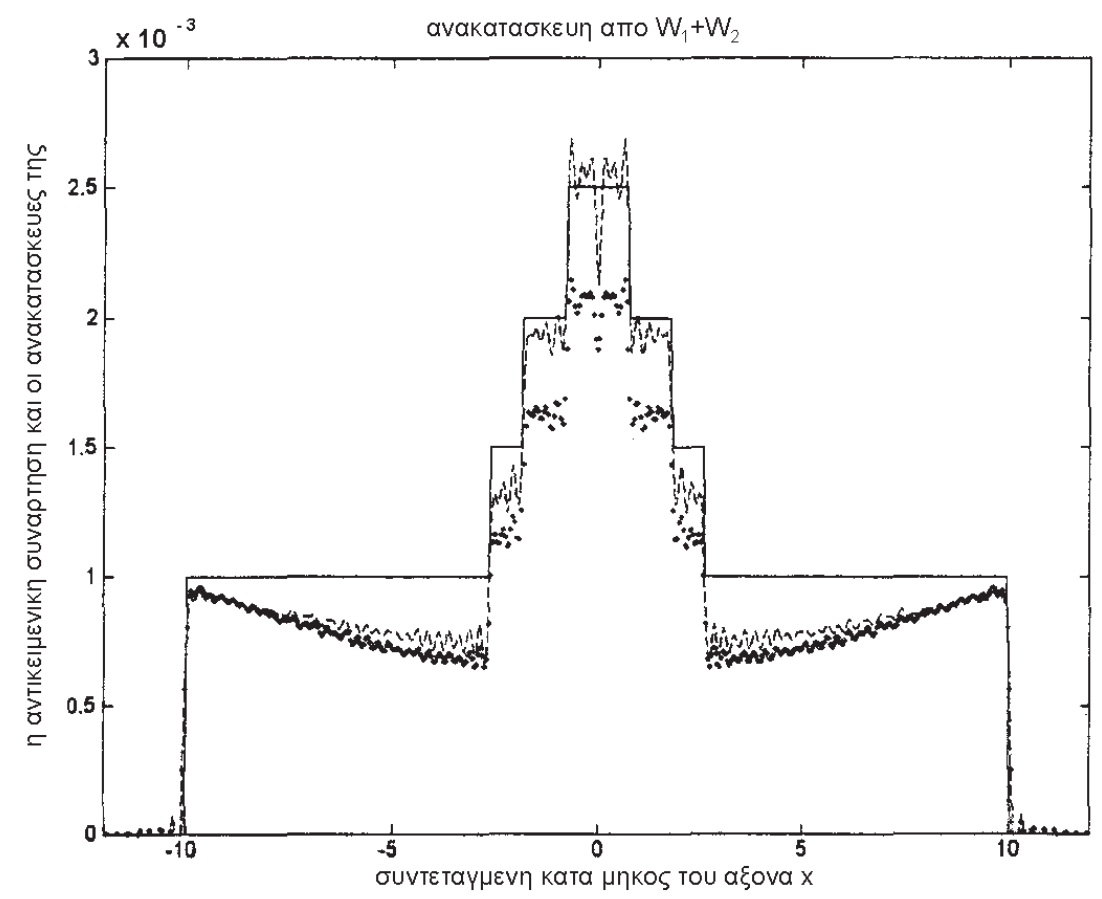

( $\beta)$

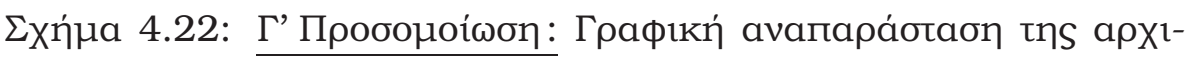

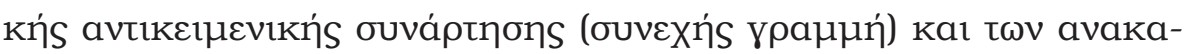

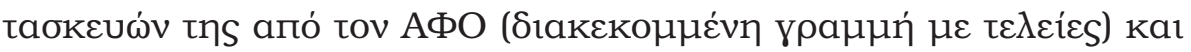

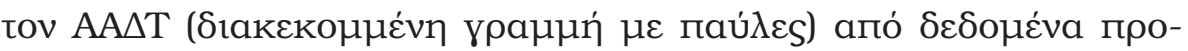

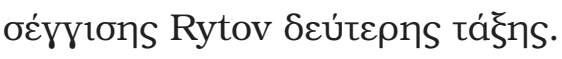




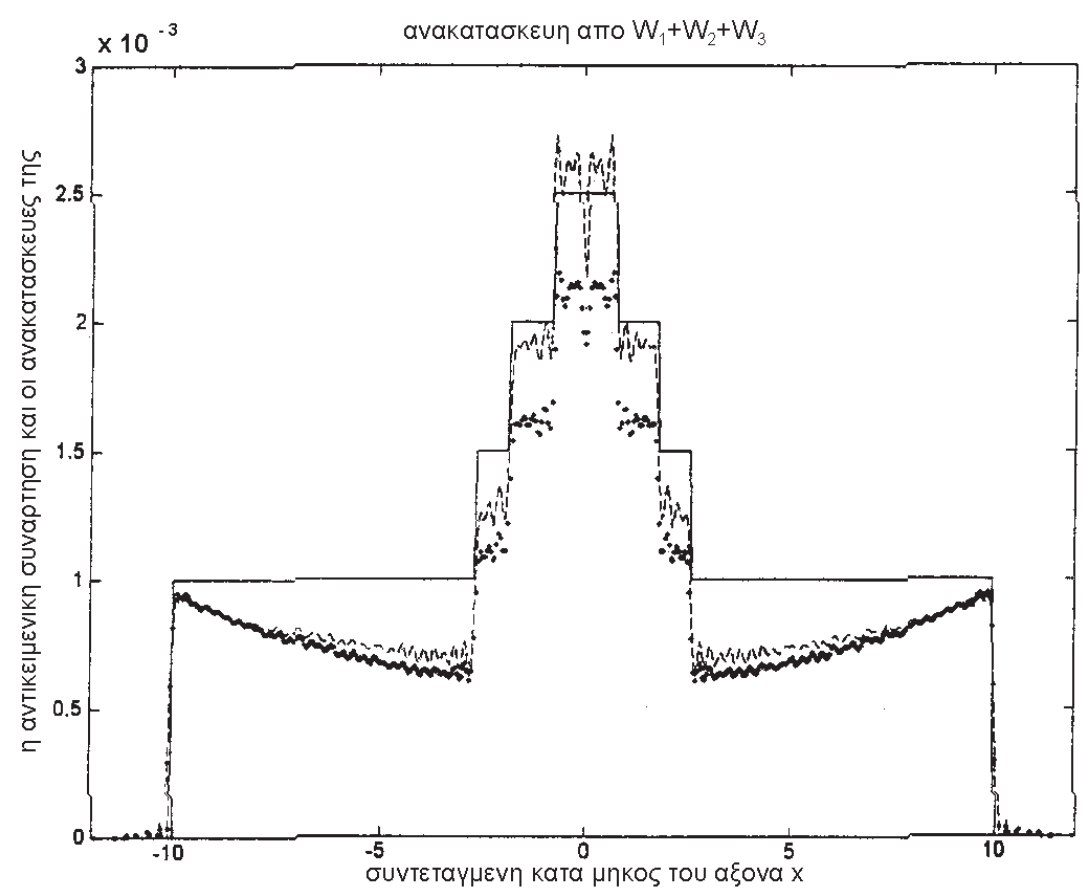

(

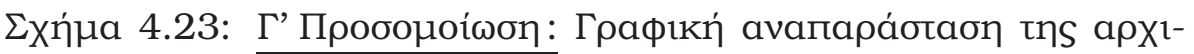

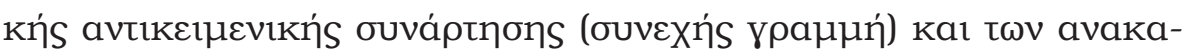

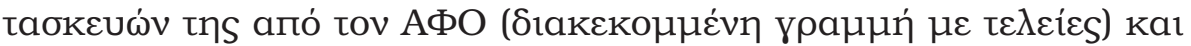

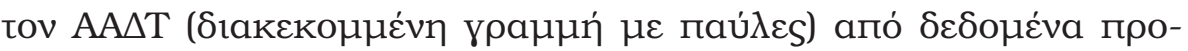

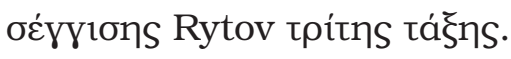




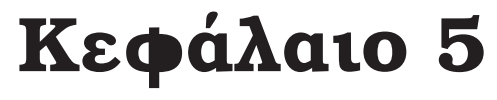

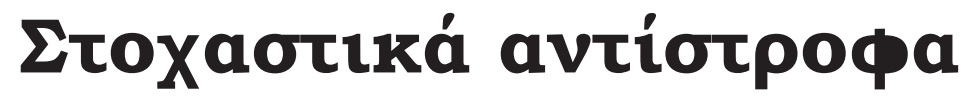

\section{rpobגńpata}

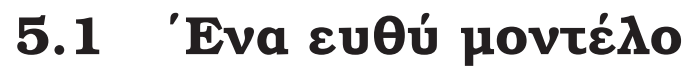

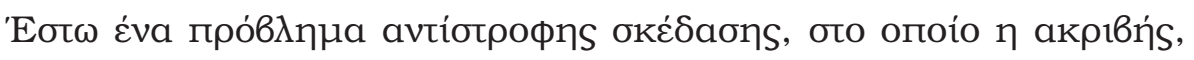

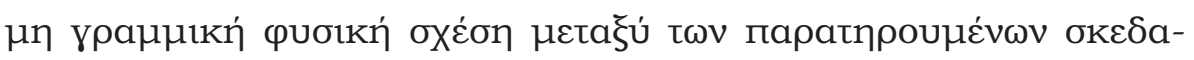

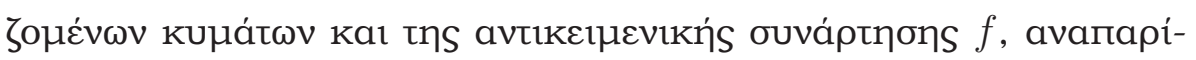

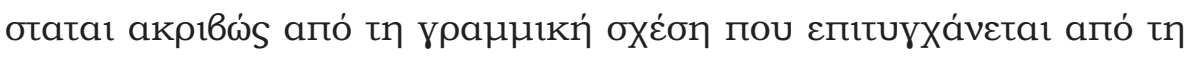

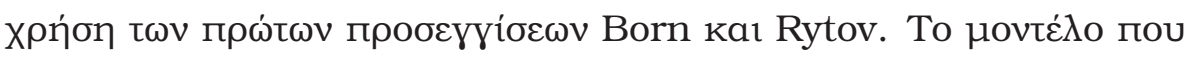

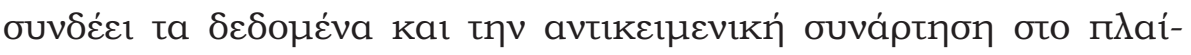

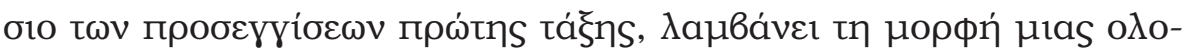

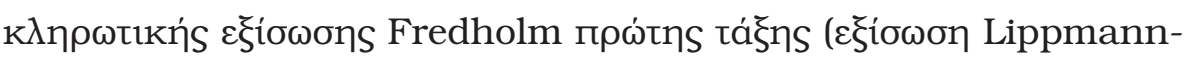

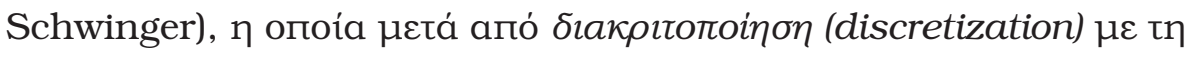

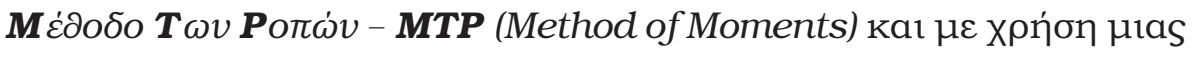




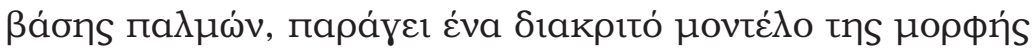

$$
\underline{y}_{i}=\underline{\underline{T}}_{i} \underline{f}+\underline{n}_{i}
$$

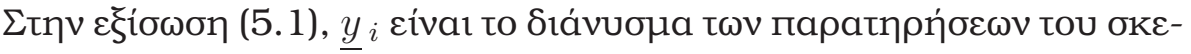

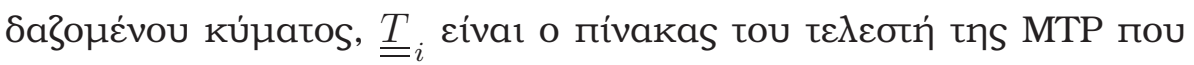

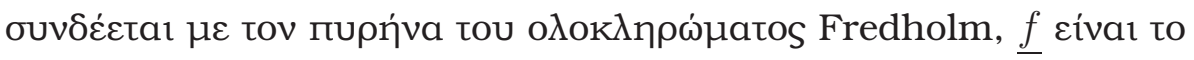

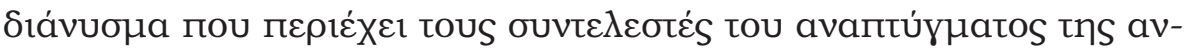

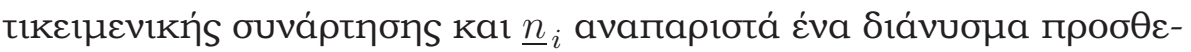

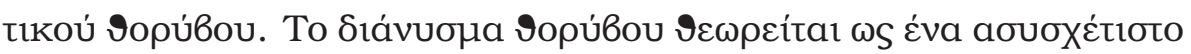

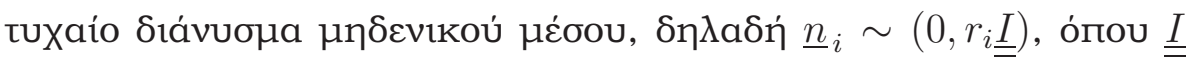

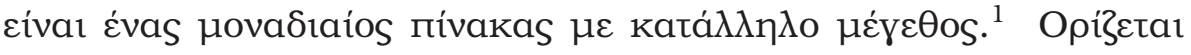

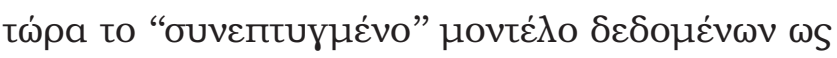

$$
\underline{y}=\underline{\underline{T}} \underline{f}+\underline{n}
$$

о́поч $\underline{y}=\left[\begin{array}{llll}\underline{y}_{1}^{T} & \underline{y}_{2}^{T} & \cdots & \underline{y}_{K}^{T}\end{array}\right]^{T}$, кaı ta $\underline{\underline{T}}$ kaı $\underline{n}$ opí̧ovtaı кatá $\lambda \lambda \eta \lambda a$.

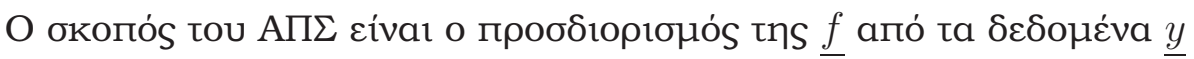

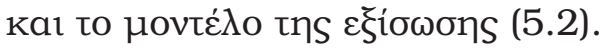

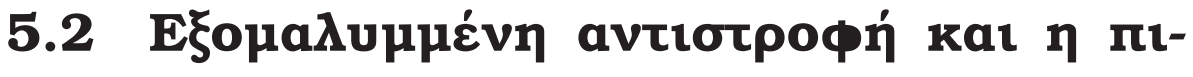

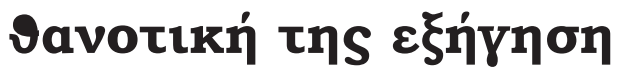

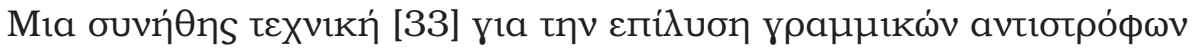

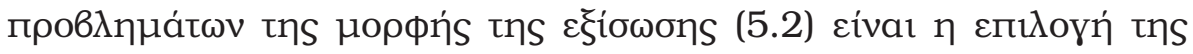

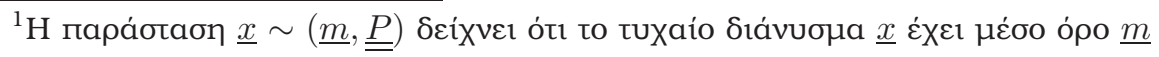
kaı пívaka ouvઠıakúpavons $\underline{\underline{P}}$.
} 


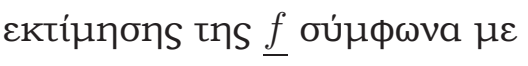

$$
\underline{f}_{\mathrm{rec}}=\arg \min _{\underline{f}}\|\underline{y}-\underline{\underline{T}} \underline{f}\|_{\mathcal{R}^{-1}}^{2}+\|\underline{f}\|_{L^{T} L}^{2}
$$

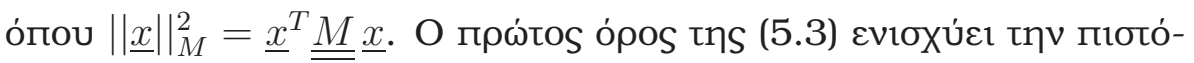

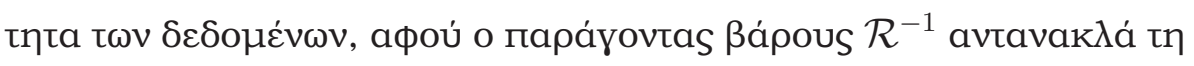

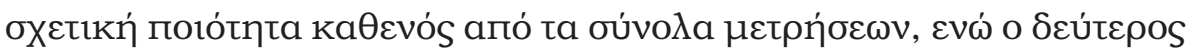

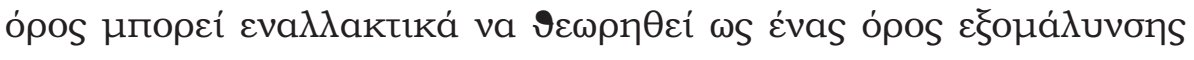

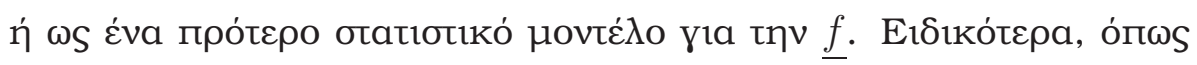

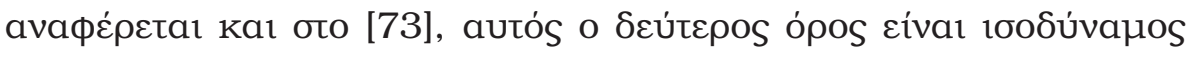

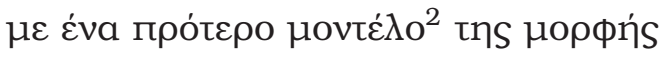

$$
\underline{\underline{L}} \underline{f}=\underline{w}, \quad \underline{w} \sim(0, \underline{\underline{I}}) .
$$

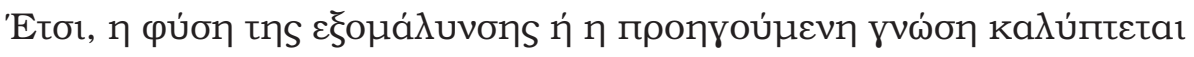

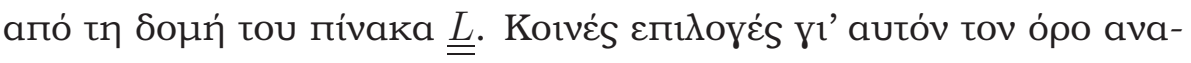

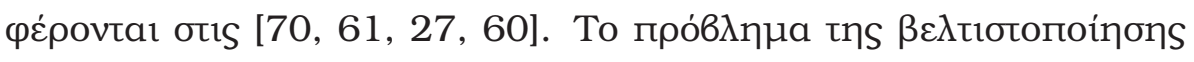

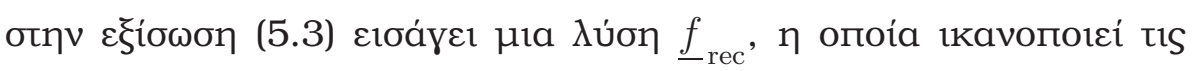

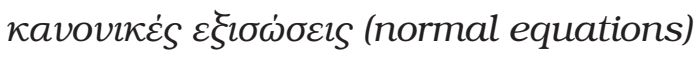

$$
\left(\underline{\underline{T}}^{T} \underline{\underline{\mathcal{R}}}^{-1} \underline{\underline{T}}+\underline{\underline{L}}^{T} \underline{\underline{L}}\right) \underline{f}_{\mathrm{rec}}=\underline{\underline{T}}^{T} \underline{\underline{\mathcal{R}}}^{-1} \underline{y}
$$

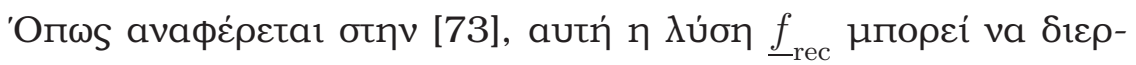

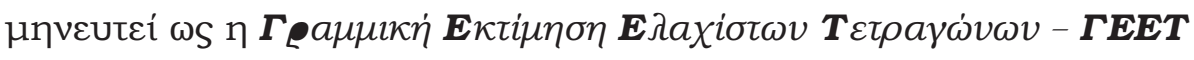

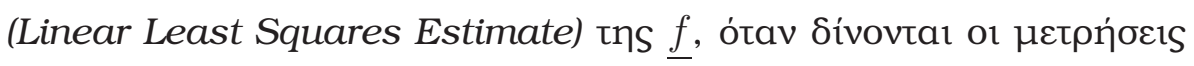

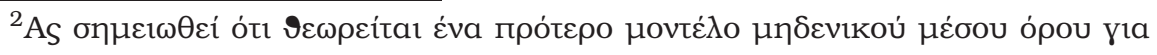

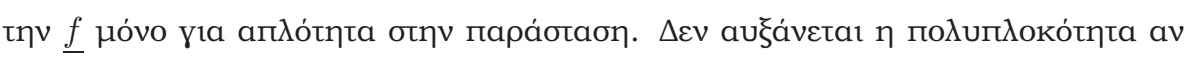

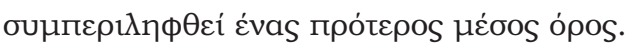




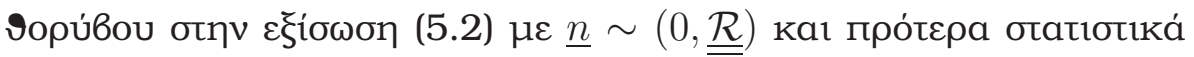

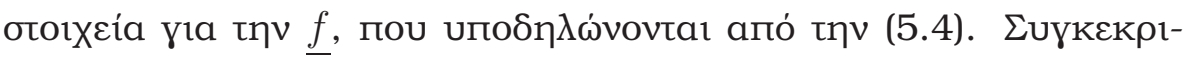

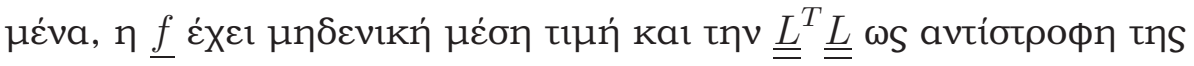

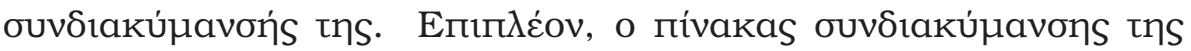

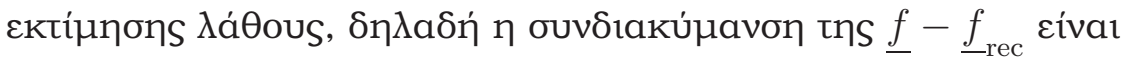

$$
\mathcal{E}\left\{\left(\underline{f}-\underline{f}_{\mathrm{rec}}\right)\left(\underline{f}-\underline{f}_{\mathrm{rec}}\right)^{T}\right\}=\left(\underline{\underline{T}}^{T} \underline{\underline{\mathcal{R}}}^{-1} \underline{\underline{T}}+\underline{\underline{L}}^{T}\right)^{-1}
$$

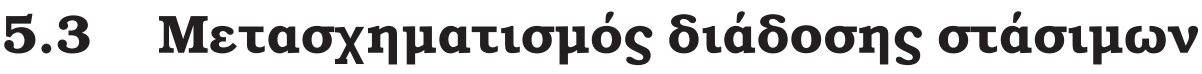

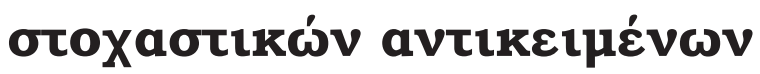

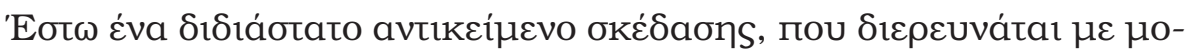

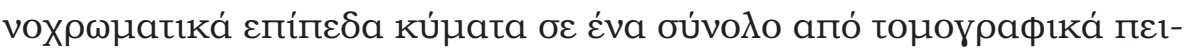

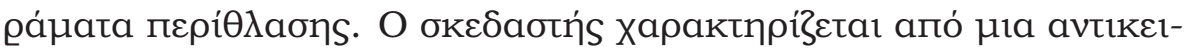

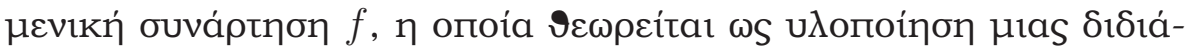

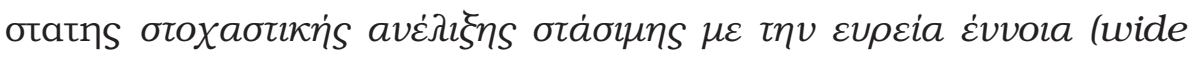

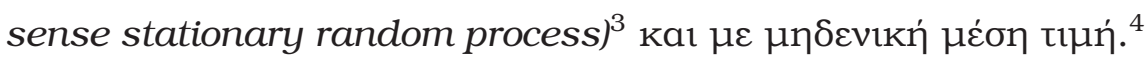

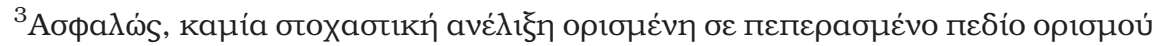

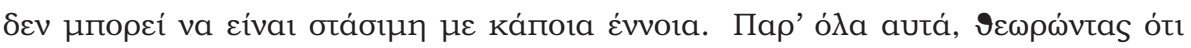

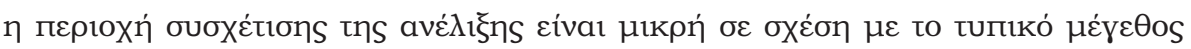

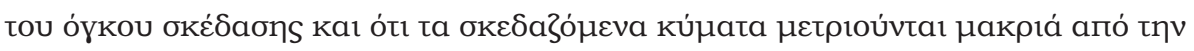

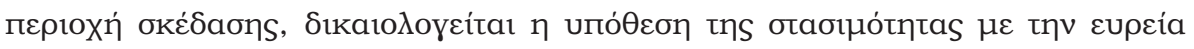

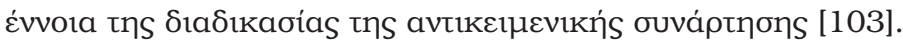

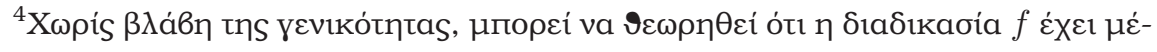

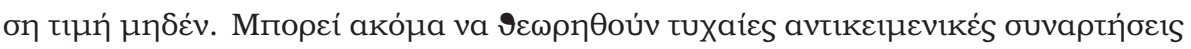

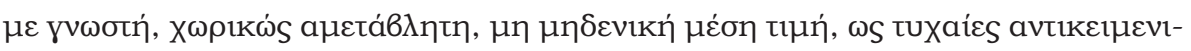

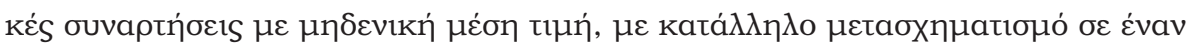




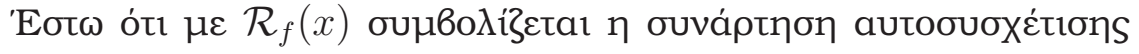

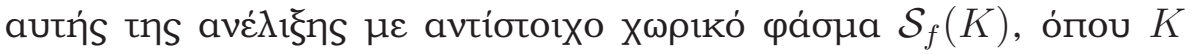

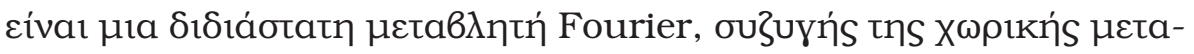

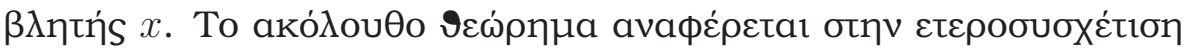

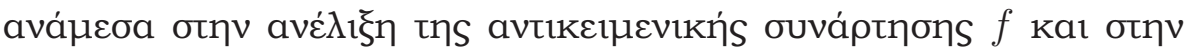

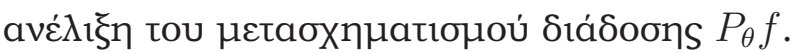

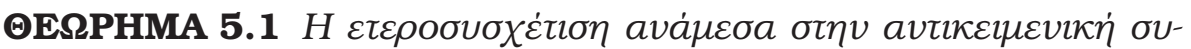

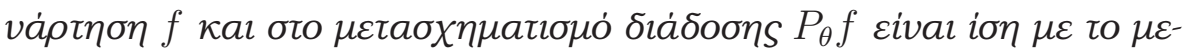

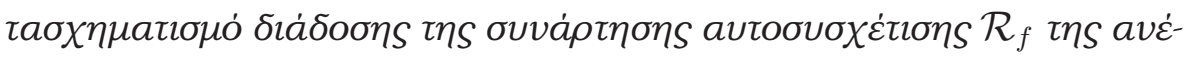

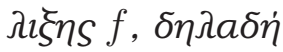

$$
\mathcal{E}\left\{f(\tau \hat{u}+s \hat{v}) \overline{P_{\theta} f}\left(\tau_{0} ; s_{0}\right)\right\}=P_{\theta} \mathcal{R}_{f}\left(\tau-\tau_{0} ; s-s_{0}\right) .
$$

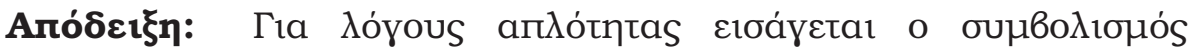

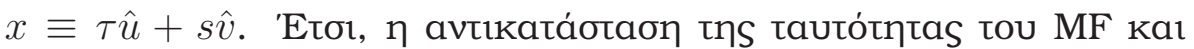

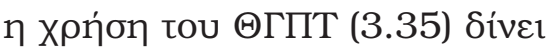

$$
\begin{aligned}
& \mathcal{E}\left\{f(x) \overline{P_{\theta} f}\left(\tau_{0} ; s_{0}\right)\right\}= \\
& =\frac{1}{(2 \pi)^{3}} \int d K \int_{-k}^{k} d p e^{i<K, x>} e^{-i p \tau_{0}} \mathcal{E}\left\{\tilde{f}(K) \overline{\widetilde{P_{\theta} f}}\left(p ; s_{0}\right)\right\}= \\
& =\frac{1}{(2 \pi)^{3}} \int d K \int_{-k}^{k} d p e^{i<K, x>} e^{-i p \tau_{0}} \frac{k}{m} e^{-i(m-k) s_{0}} . \\
& \cdot \mathcal{E}\{\tilde{f}(K) \overline{\tilde{f}}[p \hat{u}+(m-k) \hat{v}]\} .
\end{aligned}
$$

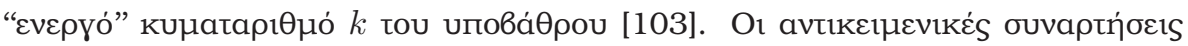

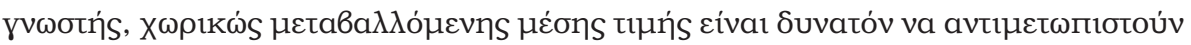

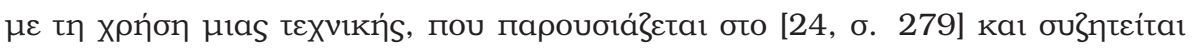

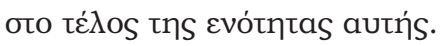




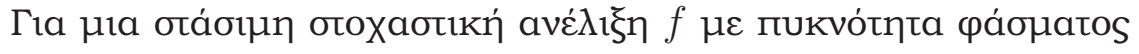

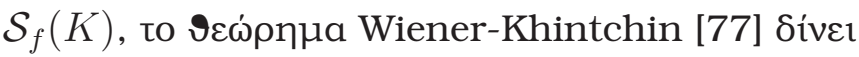

$$
\mathcal{E}\{\tilde{f}(K) \overline{\tilde{f}}[p \hat{u}+(m-k) \hat{v}]\}=(2 \pi)^{2} \mathcal{S}_{f}(K) \delta[K-p \hat{u}-(m-k) \hat{v}] .
$$

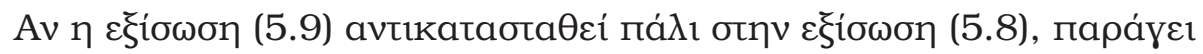

$$
\begin{aligned}
& \mathcal{E}\left\{f(x) \overline{P_{\theta} f}\left(\tau_{0} ; s_{0}\right)\right\}= \\
= & \frac{1}{2 \pi} \int_{-k}^{k} d p e^{i<p \hat{u}+(m-k) \hat{v}, x>} e^{-i p \tau_{0}} \frac{k}{m} e^{-i(m-k) s_{0}} \mathcal{S}_{f}[p \hat{u}+(m-k) \hat{v}]= \\
= & \frac{1}{2 \pi} \int_{-k}^{k} d p e^{i p\left(\tau-\tau_{0}\right)} \frac{k}{m} e^{i(m-k)\left(s-s_{0}\right)} \mathcal{S}_{f}[p \hat{u}+(m-k) \hat{v}] .
\end{aligned}
$$

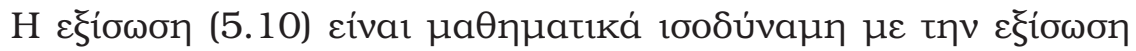

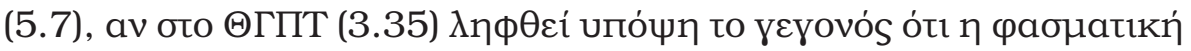

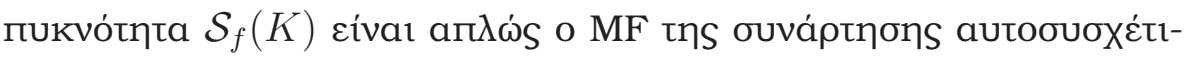
ons $\mathcal{R}_{f}(x)$ [77].

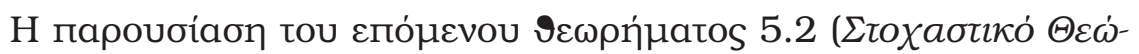

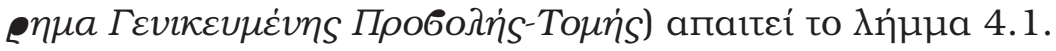

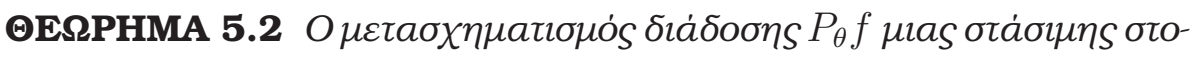

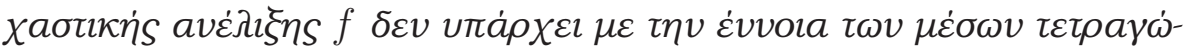

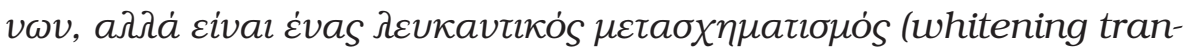

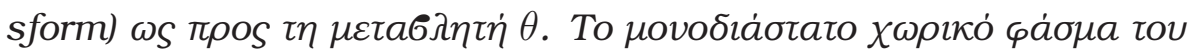

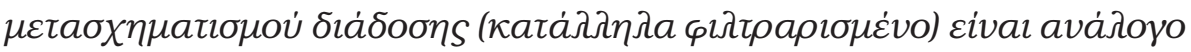

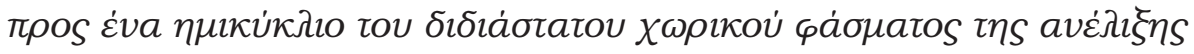




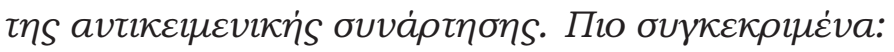

$$
\begin{aligned}
& \mathcal{E}\left\{P_{\theta} f\left(\tau_{0} ; s_{0}\right) \overline{P_{\theta}^{\prime} f}\left(\tau_{0}^{\prime} ; s_{0}^{\prime}\right)\right\}= \\
& =\frac{1}{2 \pi} \int_{-k}^{k} d p e^{i p\left(\tau_{0}-\tau_{0}^{\prime}\right)} \frac{k}{m} e^{i(m-k)\left(s_{0}-s_{0}^{\prime}\right)} \frac{\mathcal{S}_{f}[p \hat{u}+(m-k) \hat{v}]}{\frac{|p|}{4 \pi}} \delta\left(\theta-\theta^{\prime}\right) .
\end{aligned}
$$

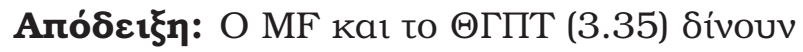

$$
\begin{aligned}
& \mathcal{E}\left\{P_{\theta} f\left(\tau_{0} ; s_{0}\right) \overline{P_{\theta}^{\prime} f}\left(\tau_{0}^{\prime} ; s_{0}^{\prime}\right)\right\}= \\
& =\frac{1}{(2 \pi)^{2}} \int_{-k}^{k} d p \int_{-k}^{k} d p^{\prime} e^{i\left(p \tau_{0}-p^{\prime} \tau_{0}^{\prime}\right)} \mathcal{E}\left\{\widetilde{P_{\theta} f}\left(p ; s_{0}\right) \widetilde{\widetilde{P_{\theta}^{\prime}}}\left(p^{\prime} ; s_{0}^{\prime}\right)\right\}= \\
& =\frac{1}{(2 \pi)^{2}} \int_{-k}^{k} d p \int_{-k}^{k} d p^{\prime} e^{i\left(p \tau_{0}-p^{\prime} \tau_{0}^{\prime}\right)} \frac{k}{m} \frac{k}{m^{\prime}} e^{i\left[(m-k) s_{0}-\left(m^{\prime}-k\right) s_{0}^{\prime}\right]} . \\
& \cdot \mathcal{E}\left\{\tilde{f}[p \hat{u}+(m-k) \hat{v}] \overline{\tilde{f}}\left[p^{\prime} \hat{u}^{\prime}+\left(m^{\prime}-k\right) \hat{v}^{\prime}\right]\right\}= \\
& =\frac{(2 \pi)^{2}}{(2 \pi)^{2}} \int_{-k}^{k} d p \int_{-k}^{k} d p^{\prime} e^{i\left(p \tau_{0}-p^{\prime} \tau_{0}^{\prime}\right)} \frac{k}{m} \frac{k}{m^{\prime}} e^{i\left[(m-k) s_{0}-\left(m^{\prime}-k\right) s_{0}^{\prime}\right]} \cdot \\
& \cdot \mathcal{S}_{f}[p \hat{u}+(m-k) \hat{v}] \delta\left[p \hat{u}+(m-k) \hat{v}-p^{\prime} \hat{u}^{\prime}-\left(m^{\prime}-k\right) \hat{v}^{\prime}\right],
\end{aligned}
$$

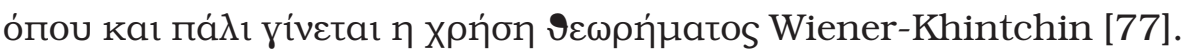

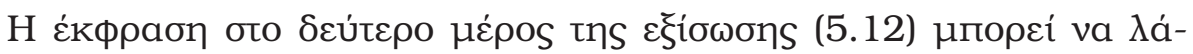

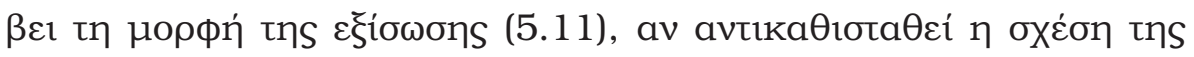

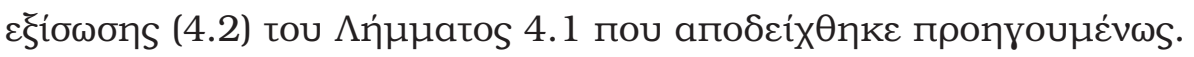

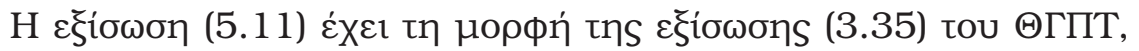

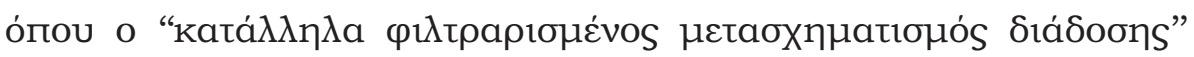

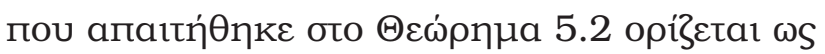

$$
\Pi_{\theta} f\left(\tau_{0} ; s_{0}\right)=\frac{1}{2 \pi} \int_{-k}^{k} d p e^{i p \tau_{0}} \sqrt{\frac{|p|}{4 \pi}} \widetilde{P_{\theta} f}\left(p ; s_{0}\right) .
$$




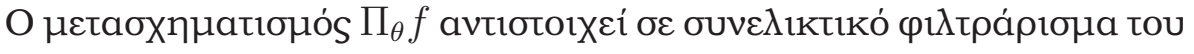

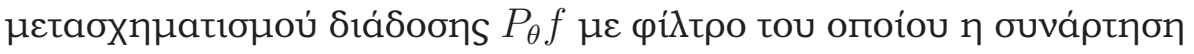

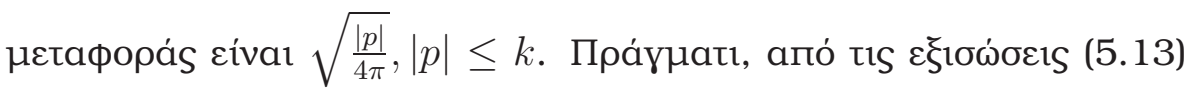
kaı (5.11) paívetaı ótı

$$
\begin{aligned}
& \mathcal{E}\left\{\Pi_{\theta} f\left(\tau_{0} ; s_{0}\right) \overline{\Pi_{\theta}^{\prime}} f\left(\tau_{0}^{\prime} ; s_{0}^{\prime}\right)\right\}= \\
& =\frac{1}{2 \pi} \int_{-k}^{k} d p e^{i p\left(\tau_{0}-\tau_{0}^{\prime}\right)} \frac{k}{m} e^{i(m-k)\left(s_{0}-s_{0}^{\prime}\right)} \mathcal{S}_{f}[p \hat{u}+(m-k) \hat{v}] \delta\left(\theta-\theta^{\prime}\right),
\end{aligned}
$$

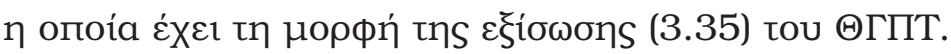

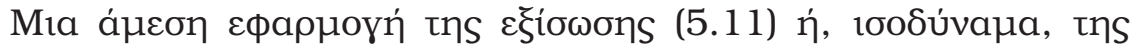

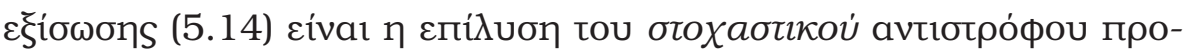
$\beta \lambda$ ń

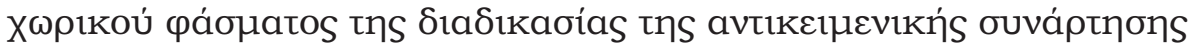

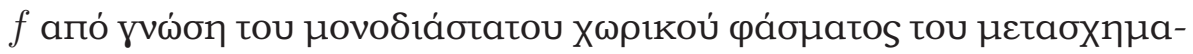

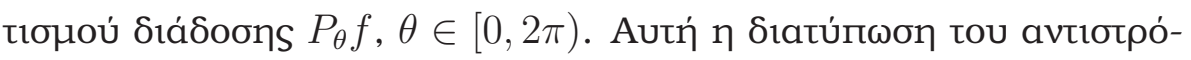

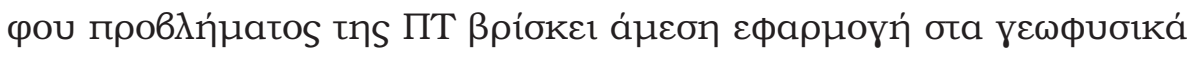

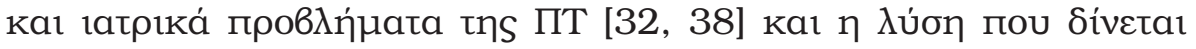

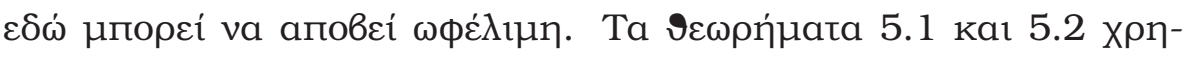

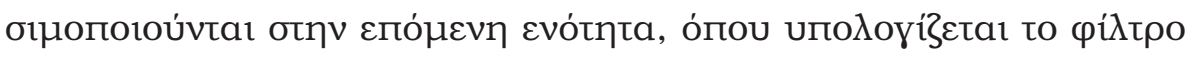

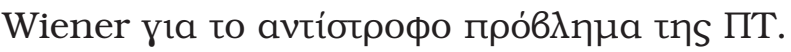

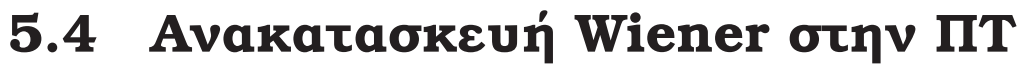

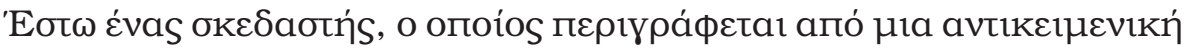

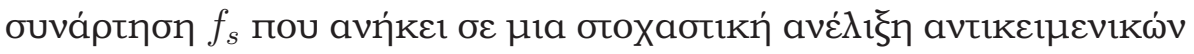




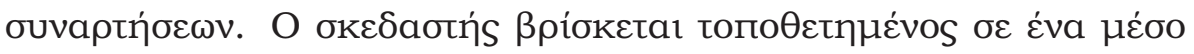

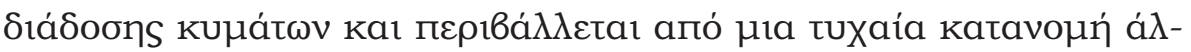

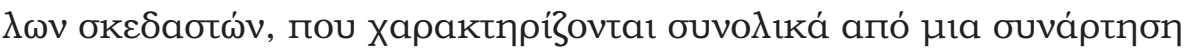

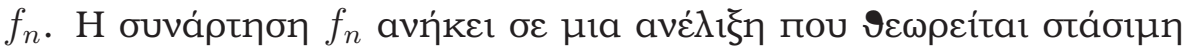

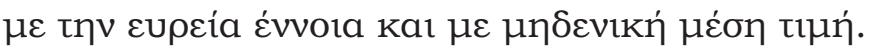

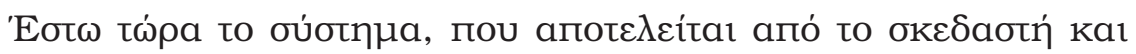

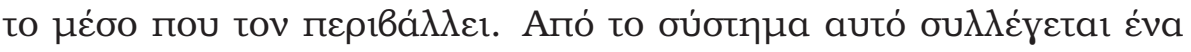

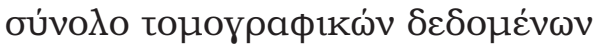

$$
y\left(\tau_{0}, \theta\right)=\alpha\left(\tau_{0}, \theta\right)+\mathcal{N}\left(\tau_{0}, \theta\right) \quad, \quad-\infty<\tau_{0}<\infty \quad, \quad 0 \leq \theta<2 \pi,
$$

ónou

$$
\alpha\left(\tau_{0}, \theta\right)=r_{\theta}\left(\tau_{0}\right) *_{\tau_{0}} P_{\theta} f_{s}\left(\tau_{0} ; s_{0}\right)
$$

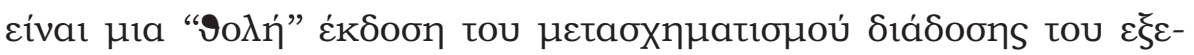

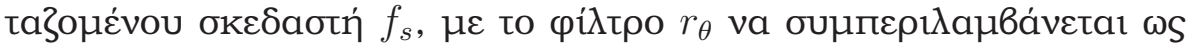

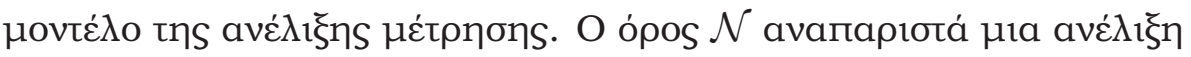

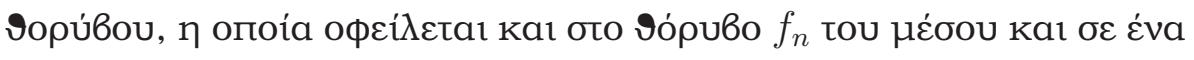

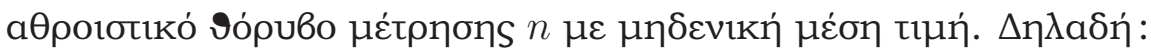

$$
\mathcal{N}\left(\tau_{0}, \theta\right)=r_{\theta}\left(\tau_{0}\right) *_{\tau_{0}} P_{\theta} f_{n}\left(\tau_{0} ; s_{0}\right)+n\left(\tau_{0}, \theta\right) .
$$

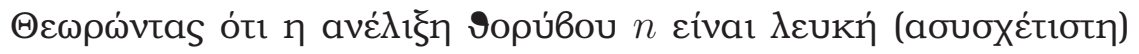

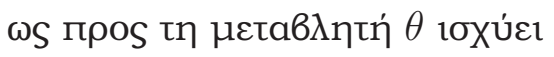

$$
\mathcal{E}\left\{n\left(\tau_{0}, \theta\right) \bar{n}\left(\tau_{0}^{\prime}, \theta^{\prime}\right)\right\}=\mathcal{R}_{n}\left(\tau_{0}-\tau_{0}^{\prime} ; \theta\right) \delta\left(\theta-\theta^{\prime}\right),
$$

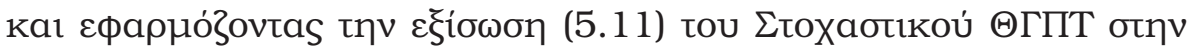




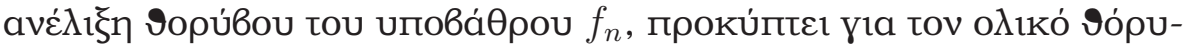

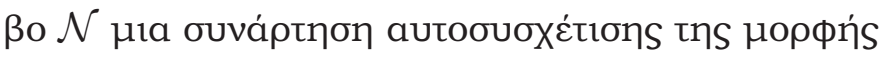

$$
\mathcal{E}\left\{\mathcal{N}\left(\tau_{0}, \theta\right) \overline{\mathcal{N}}\left(\tau_{0}^{\prime}, \theta^{\prime}\right)\right\}=\mathcal{R}_{\mathcal{N}}\left(\tau_{0}-\tau_{0}^{\prime} ; \theta\right) \delta\left(\theta-\theta^{\prime}\right)
$$

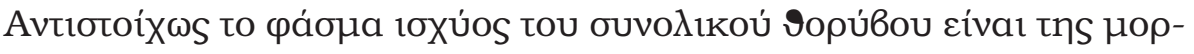

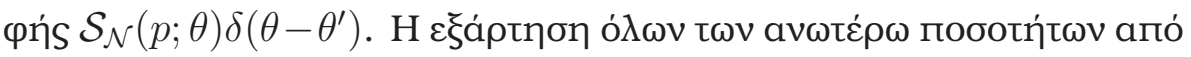

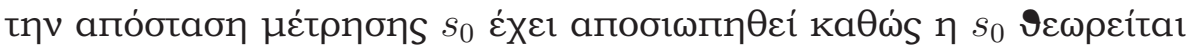

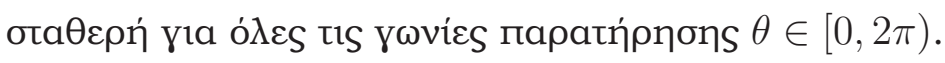

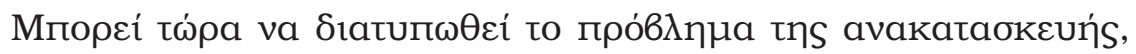

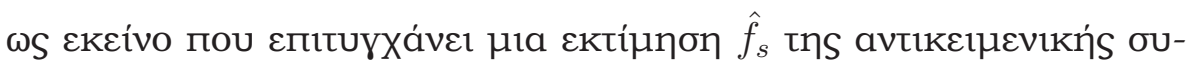

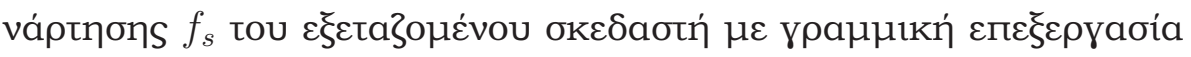

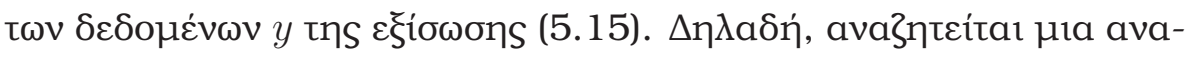

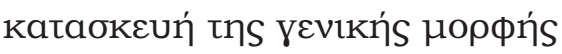

$$
f_{s, \text { rec }}(x)=\int_{0}^{2 \pi} d \theta \int_{-\infty}^{\infty} d \tau_{0} b\left(x ; \tau_{0}, \theta\right) y\left(\tau_{0}, \theta\right),
$$

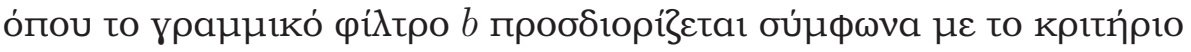

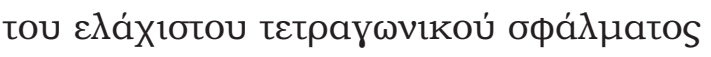

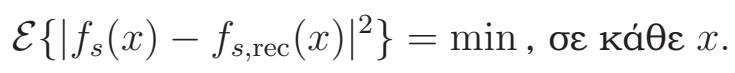

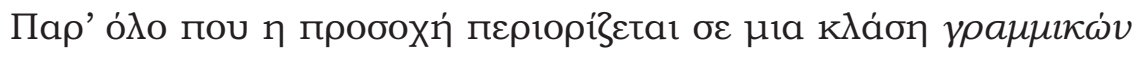

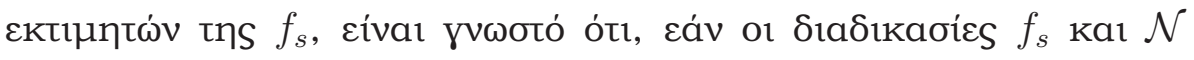

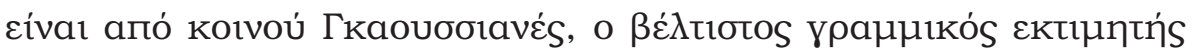

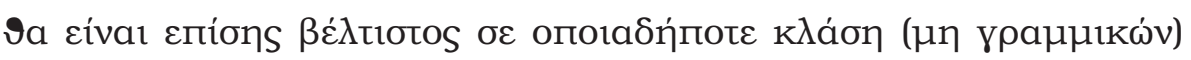

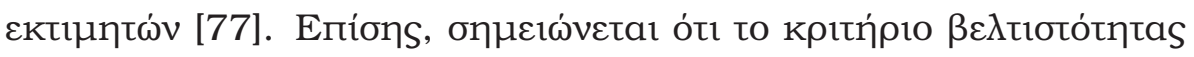

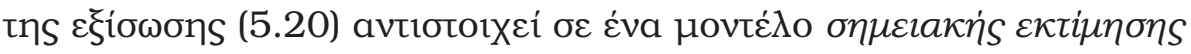




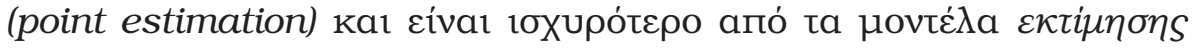

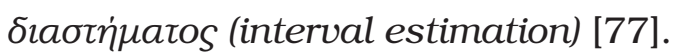

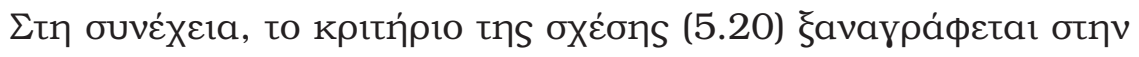

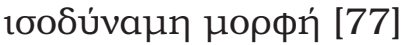

$$
\mathcal{E}\left\{\left[f_{s}(x)-f_{s, \text { rec }}(x)\right] \bar{f}_{s, \text { rec }}(x)\right\}=0, \text { Vla ká } \theta \varepsilon x .
$$

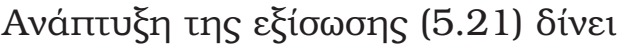

$$
\mathcal{E}\left\{\left|f_{s, \mathrm{rec}}(x)\right|^{2}\right\}=\mathcal{E}\left\{f_{s}(x) \bar{f}_{s, \mathrm{rec}}(x)\right\}, \text { Vla ká } \theta \varepsilon x .
$$

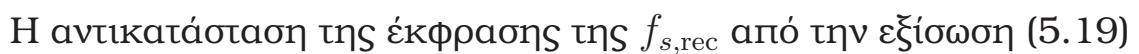

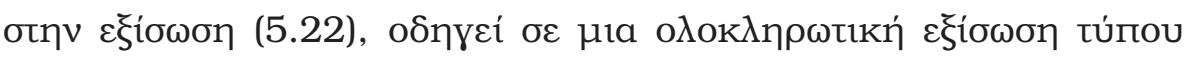
Fredholm [77]

$$
\begin{aligned}
& \int_{0}^{2 \pi} d \theta \int_{-\infty}^{\infty} d \tau_{0} \bar{b}\left(x ; \tau_{0}, \theta\right) . \\
& \quad \cdot \int_{0}^{2 \pi} d \theta^{\prime} \int_{-\infty}^{\infty} d \tau_{0}^{\prime} b\left(x ; \tau_{0}^{\prime}, \theta^{\prime}\right) \mathcal{R}_{y}\left(\tau_{0}-\tau_{0}^{\prime} ; \theta^{\prime}\right) \delta\left(\theta-\theta^{\prime}\right)= \\
& =\int_{0}^{2 \pi} d \theta \int_{-\infty}^{\infty} d \tau_{0} \bar{b}\left(x ; \tau_{0}, \theta\right) \mathcal{R}_{f_{s} y}\left(x ; \tau_{0}, \theta\right), \text { via ká } \theta \varepsilon x
\end{aligned}
$$

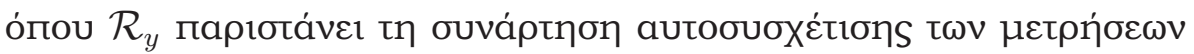

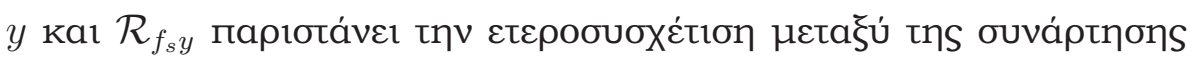

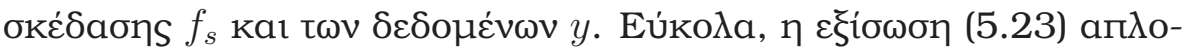
повсіта1 бтр

$$
\int_{-\infty}^{\infty} d \tau_{0}^{\prime} b\left(x ; \tau_{0}^{\prime}, \theta\right) \mathcal{R}_{y}\left(\tau_{0}-\tau_{0}^{\prime} ; \theta\right)=\mathcal{R}_{f_{s} y}\left(x ; \tau_{0}, \theta\right),
$$

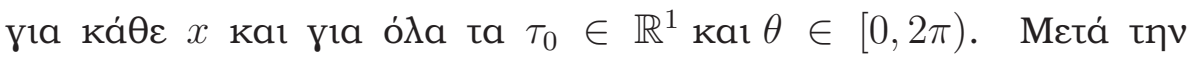

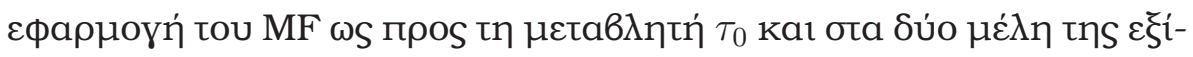

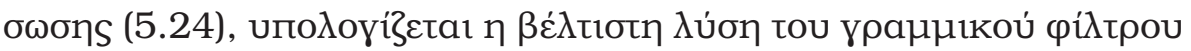




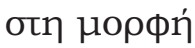

$$
\tilde{b}(x ; p, \theta)=\frac{\mathcal{S}_{f_{s} y}(x ; p, \theta)}{\mathcal{S}_{y}(p ; \theta)} .
$$

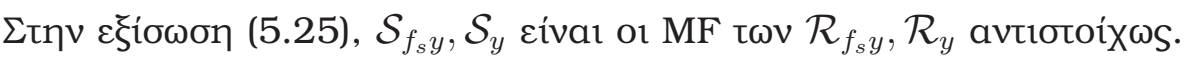

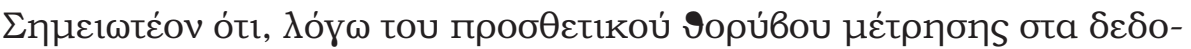

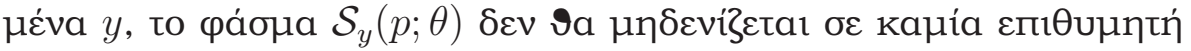
ouxvótnta $p$.

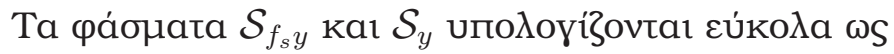

$$
\mathcal{S}_{f_{s} y}(x ; p, \theta)=\overline{\tilde{r}_{\theta}}(p) \mathcal{S}_{f_{s} P_{\theta} f_{s}}(x ; p)
$$

kal

$$
\mathcal{S}_{y}(p ; \theta)=\left|\tilde{r}_{\theta}(p)\right|^{2} \mathcal{S}_{P_{\theta} f_{s}}(p)+\mathcal{S}_{\mathcal{N}}(p ; \theta) .
$$

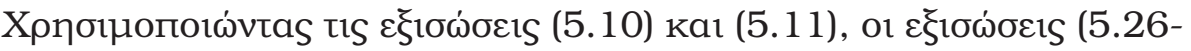

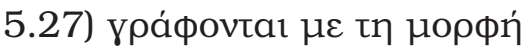

$$
\mathcal{S}_{f_{s} y}(x ; p, \theta)=\overline{\tilde{r}_{\theta}}(p) \frac{k}{m} e^{i(m-k)\left(s-s_{0}\right)} \mathcal{S}_{f_{s}}[p \hat{u}+(m-k) \hat{v}]
$$

Ka1

$$
\mathcal{S}_{y}(p ; \theta)=\left|\tilde{r}_{\theta}(p)\right|^{2} \frac{k}{m} \frac{\mathcal{S}_{f_{s}}[p \hat{u}+(m-k) \hat{v}]}{\frac{|p|}{4 \pi}}+\mathcal{S}_{\mathcal{N}}(p ; \theta)
$$

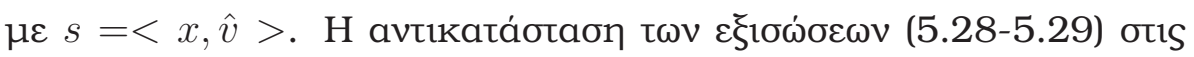

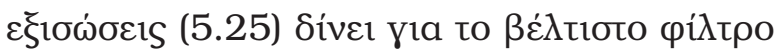

$$
\tilde{b}(x ; p, \theta)=\frac{\overline{\tilde{r}_{\theta}}(p) \frac{k}{m} \mathcal{S}_{f_{s}}[p \hat{u}+(m-k) \hat{v}] e^{i(m-k)\left(s-s_{0}\right)}}{\left|\tilde{r}_{\theta}(p)\right|^{2} \frac{k}{m} \frac{\mathcal{S}_{f_{s}}[p \hat{u}+(m-k) \hat{v}]}{\frac{|p|}{4 \pi}}+\mathcal{S}_{\mathcal{N}}(p ; \theta)}
$$

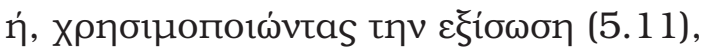

$$
\tilde{b}(x ; p, \theta)=\frac{|p|}{4 \pi} \frac{\overline{\tilde{r}_{\theta}}(p) \mathcal{S}_{P_{\theta} f_{s}}(p)}{\left|\tilde{r}_{\theta}(p)\right|^{2} \mathcal{S}_{P_{\theta} f_{s}}(p)+\mathcal{S}_{\mathcal{N}}(p ; \theta)} e^{i(m-k)\left(s-s_{0}\right)} .
$$




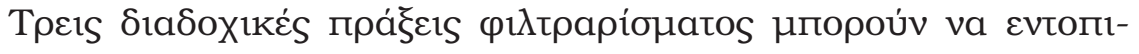

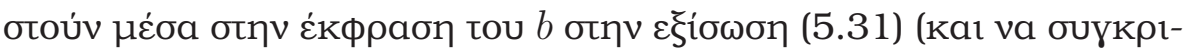

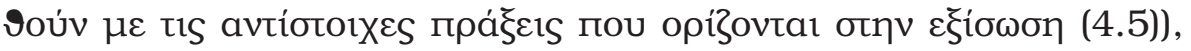

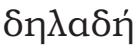

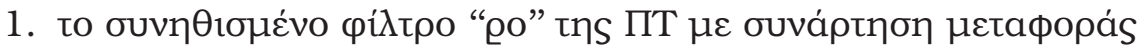
$\frac{|p|}{4 \pi},|p| \leq k$,

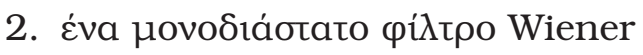

$$
\frac{\overline{\tilde{r}}_{\theta}(p) \mathcal{S}_{P_{\theta} f_{s}}(p)}{\left|\tilde{r}_{\theta}(p)\right|^{2} \mathcal{S}_{P_{\theta} f_{s}}(p)+\mathcal{S}_{\mathcal{N}}(p ; \theta)}
$$

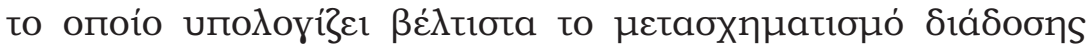

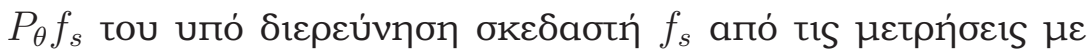
Эópubo $y$, ka1

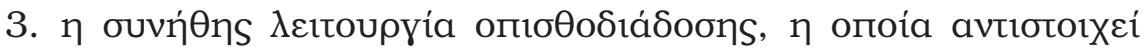

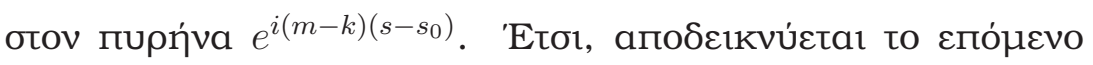

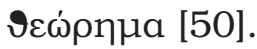

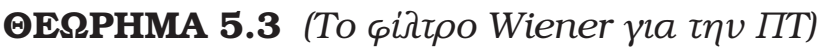

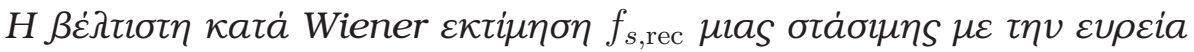

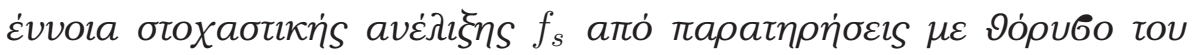

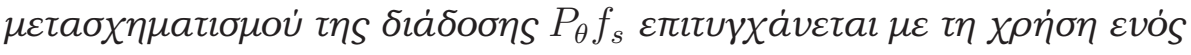
$A \Phi O$

$$
\begin{gathered}
f_{s, \text { rec }}(x)=\frac{1}{2} \frac{1}{(2 \pi)^{2}} \int_{0}^{2 \pi} d \theta \int_{-k}^{k} d p e^{i p \tau}|p| \frac{\bar{r}_{\theta}(p) \mathcal{S}_{P_{\theta} f_{s}}(p)}{\left|\tilde{r}_{\theta}(p)\right|^{2} \mathcal{S}_{P_{\theta} f_{s}}(p)+\mathcal{S}_{\mathcal{N}}(p ; \theta)} \\
e^{i(m-k)\left(s-s_{0}\right)} \tilde{y}(p, \theta)=\frac{1}{2 \pi} \int_{0}^{2 \pi} d \theta B_{\theta}\left(h_{\theta} *_{\tau_{0}} y_{\theta}\right)(x)
\end{gathered}
$$




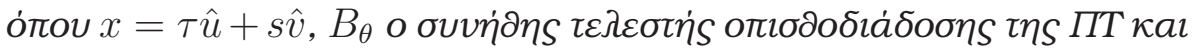

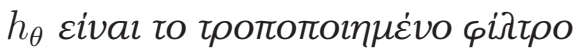

$$
\tilde{h}_{\theta}(p)=\frac{|p|}{4 \pi} \frac{\overline{\tilde{r}_{\theta}}(p) \mathcal{S}_{P_{\theta} f_{s}}(p)}{\left|\tilde{r}_{\theta}(p)\right|^{2} \mathcal{S}_{P_{\theta} f_{s}}(p)+\mathcal{S}_{\mathcal{N}}(p ; \theta)} .
$$

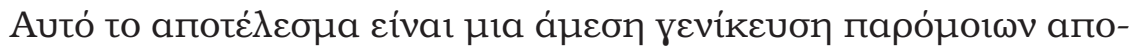

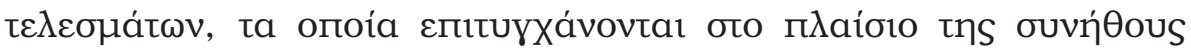

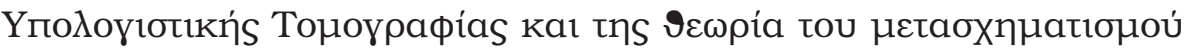

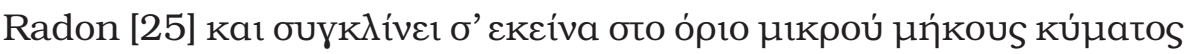
$(\lambda \rightarrow 0$ kaı $k \rightarrow \infty)$.

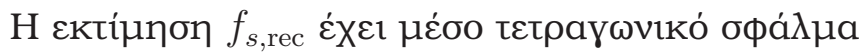

$$
\begin{aligned}
\mathcal{E}\left\{\left|f_{s}-f_{s, \text { rec }}\right|^{2}\right\}= & \frac{1}{(2 \pi)^{2}}\left\{\int d K \mathcal{S}_{f_{s}}(K)-2 \pi \int_{0}^{2 \pi} d \theta\right. \\
& \left.\int_{-k}^{k} d p \tilde{h}_{\theta}(p) \frac{k}{m} \mathcal{S}_{f_{s}}[p \hat{u}+(m-k) \hat{v}]\right\},
\end{aligned}
$$

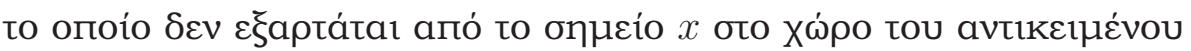

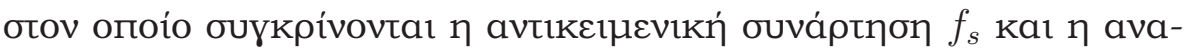

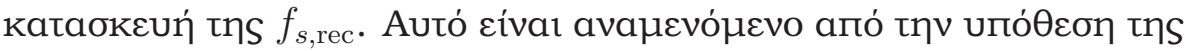

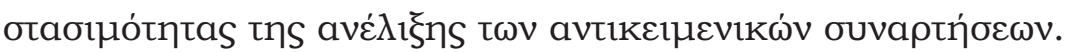

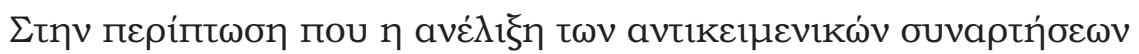

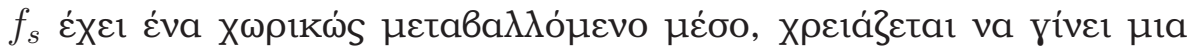

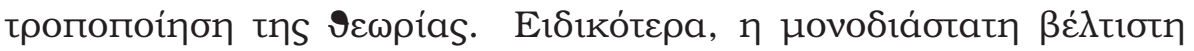

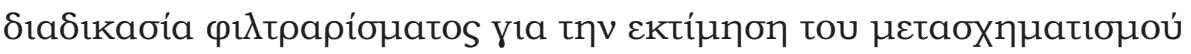

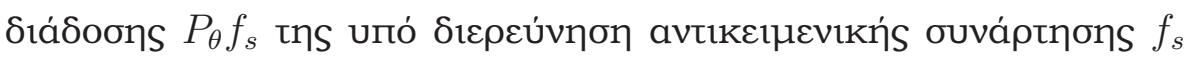

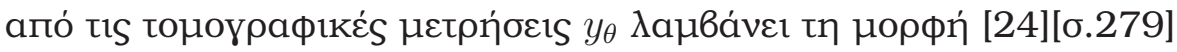

$$
\widehat{P_{\theta} f_{s}}=\mathcal{E}\left\{P_{\theta} f_{s}\right\}+w_{\theta} *_{\tau_{0}}\left[y_{\theta}-\mathcal{E}\left\{y_{\theta}\right\}\right]
$$




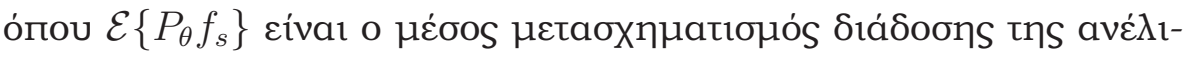

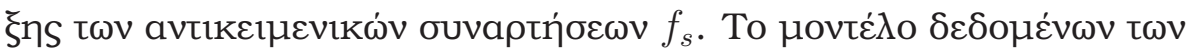

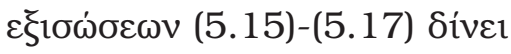

$$
\begin{aligned}
\mathcal{E}\left\{y_{\theta}\right\} & =r_{\theta} *_{\tau_{0}} \mathcal{E}\left\{P_{\theta} f_{s}\right\}+\mathcal{E}\left\{\mathcal{N}_{\theta}\right\} \\
& =r_{\theta} *_{\tau_{0}} \mathcal{E}\left\{P_{\theta} f_{s}\right\}
\end{aligned}
$$

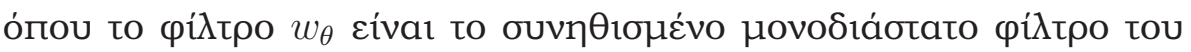
Wiener

$$
\tilde{w}_{\theta}(p)=\frac{\tilde{r}_{\theta}(p) \mathcal{S}_{P_{\theta} f_{s}}(p)}{\left|\tilde{r}_{\theta}(p)\right|^{2} \mathcal{S}_{P_{\theta} f_{s}}(p)+\mathcal{S}_{\mathcal{N}}(p ; \theta)} .
$$

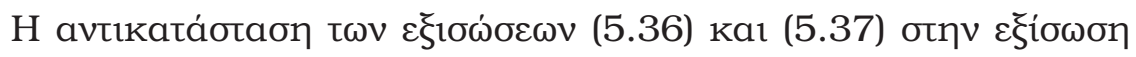
(5.35) Síve1

$$
\widehat{P_{\theta} f_{s}}=\left(\mathcal{I}-w_{\theta} *_{t_{0}} r_{\theta}\right) *_{t_{0}} \mathcal{E}\left\{P_{\theta} f_{s}\right\}+w_{\theta} *_{t_{0}} y_{\theta},
$$

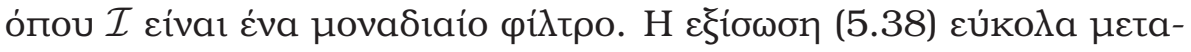

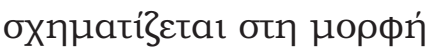

$$
\widehat{P_{\theta} f_{s}}=w_{\theta} *_{t_{0}} y_{\theta}+m_{\theta} *_{t_{0}} \mathcal{E}\left\{P_{\theta} f_{s}\right\}
$$

о́поu

$$
\tilde{m}_{\theta}(p)=\frac{\mathcal{S}_{\mathcal{N}}(p ; \theta)}{\left|\tilde{r}_{\theta}(p)\right|^{2} \mathcal{S}_{P_{\theta} f_{s}}(p)+\mathcal{S}_{\mathcal{N}}(p ; \theta)} .
$$




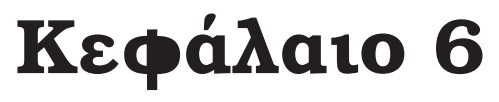

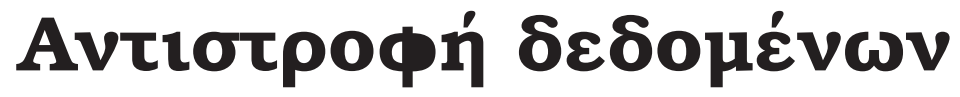

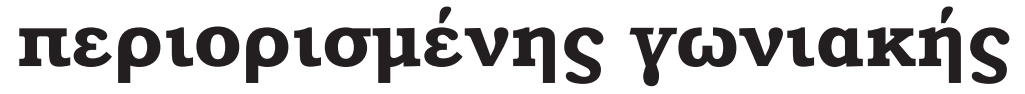

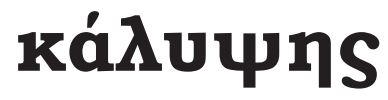

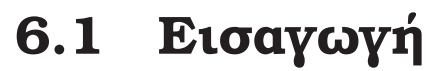

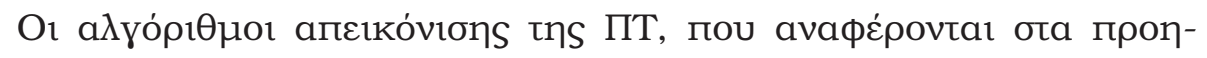

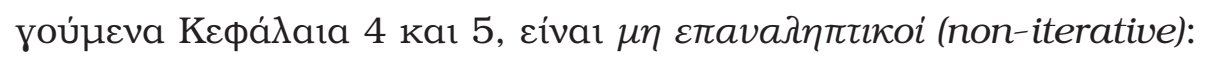

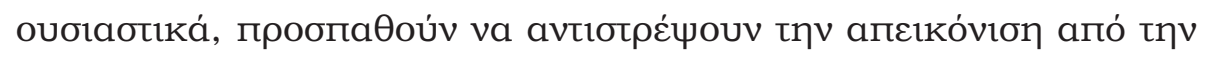

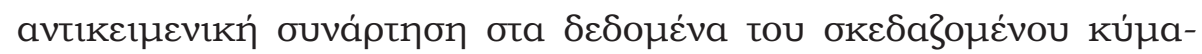

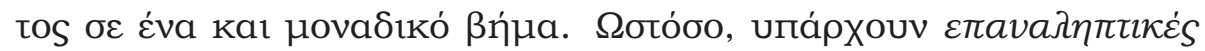

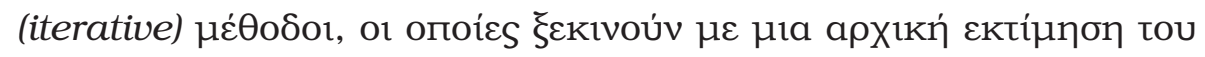

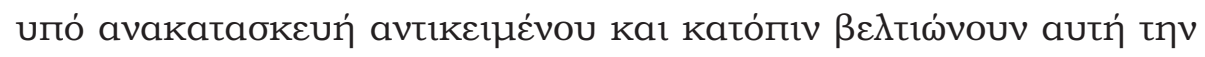

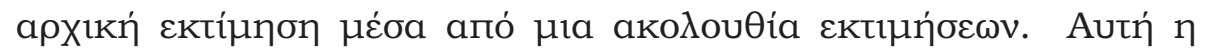




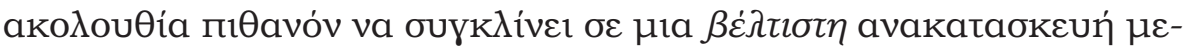

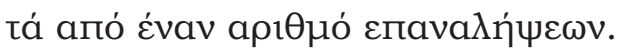

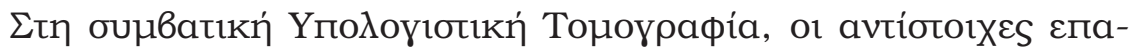

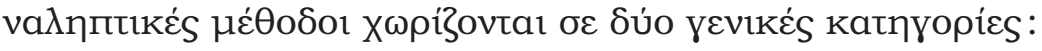

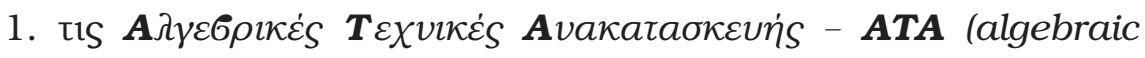
reconstruction techniques) $\mathrm{ka}$

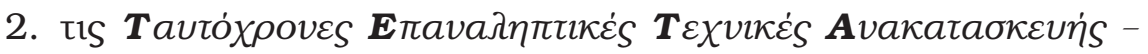
TETA (simultaneous iterative reconstruction techniques).

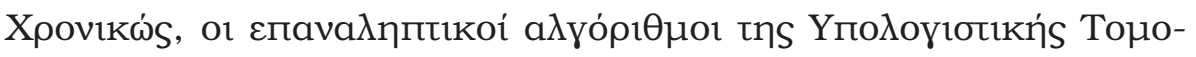

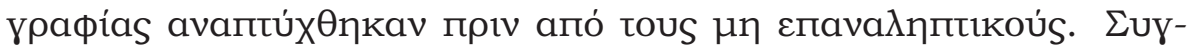

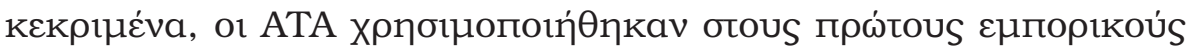

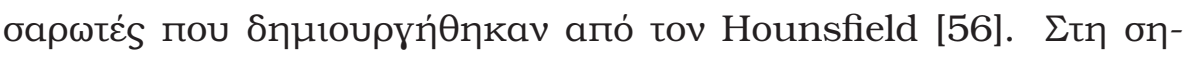

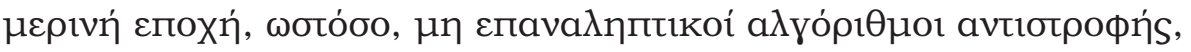

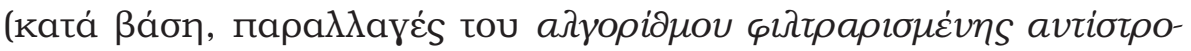

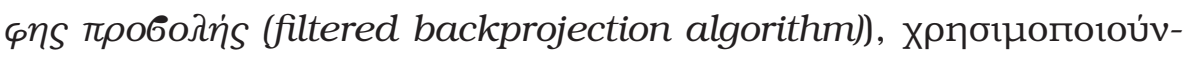

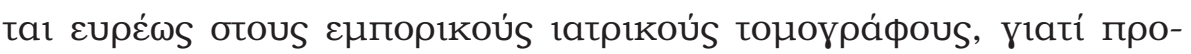

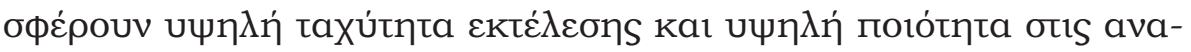

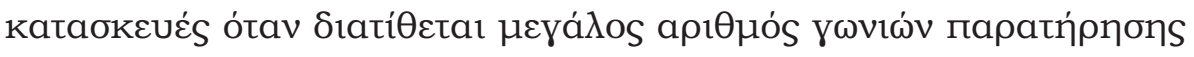

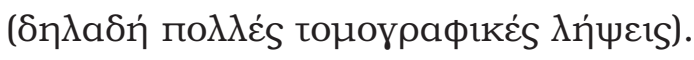

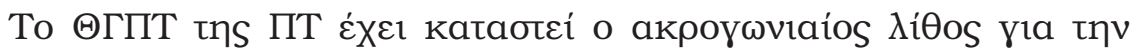

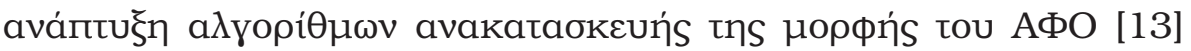

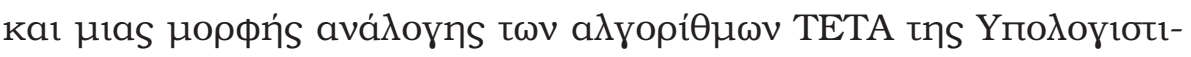

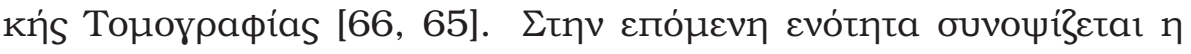

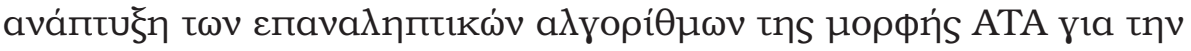




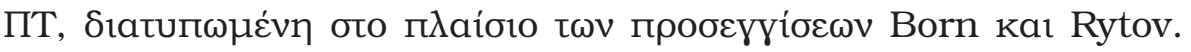

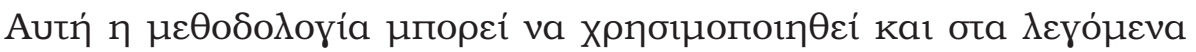

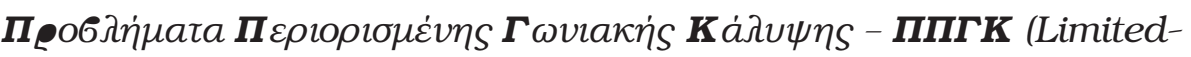

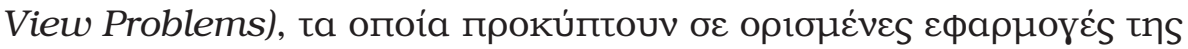

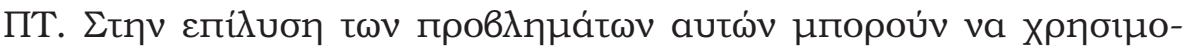

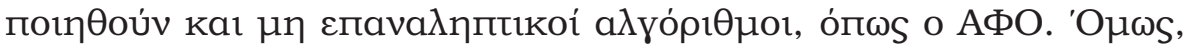

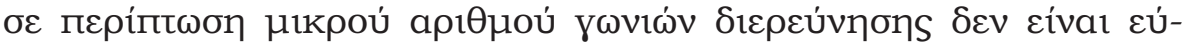

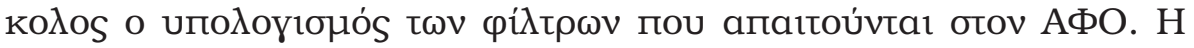

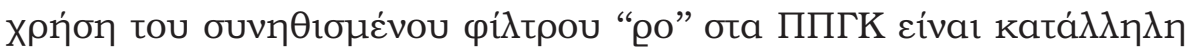

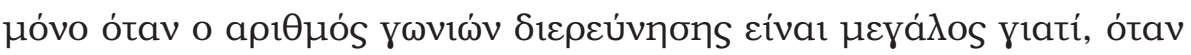

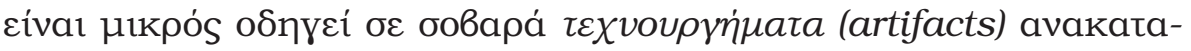

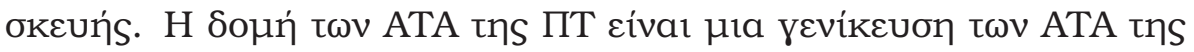

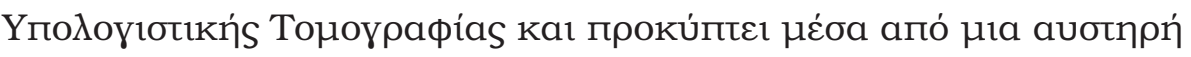

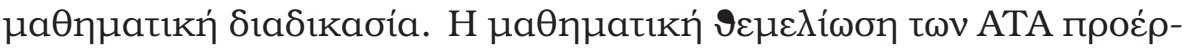

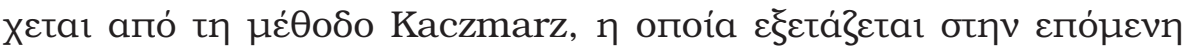

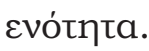

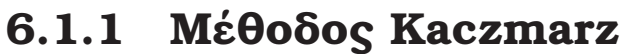

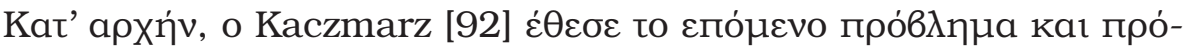

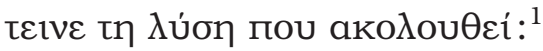

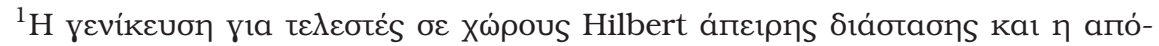

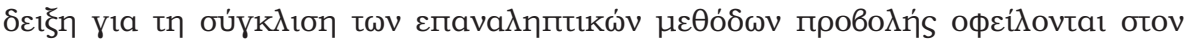

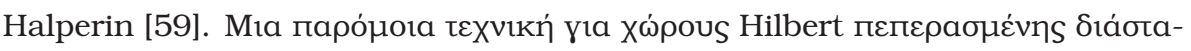

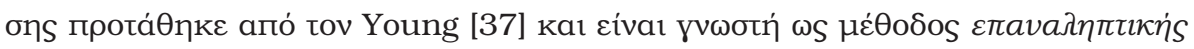

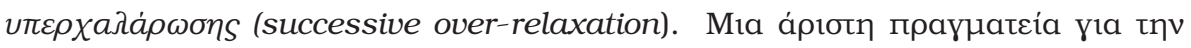




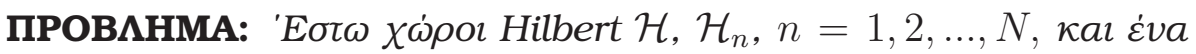

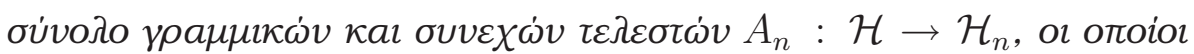

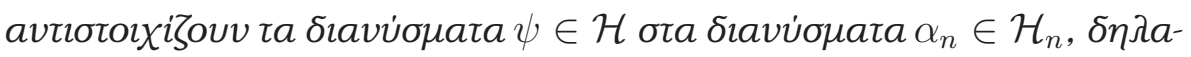

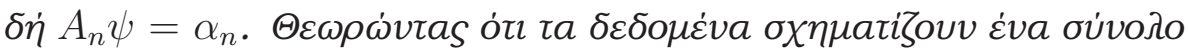

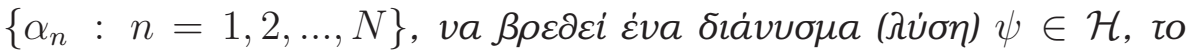

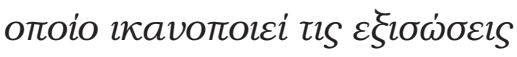

$$
A_{n} \psi=\alpha_{n} \quad n=1,2, \ldots, N
$$

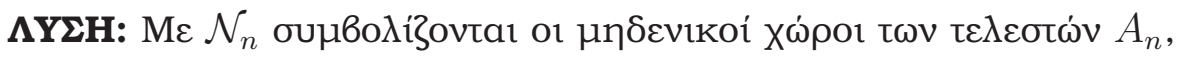

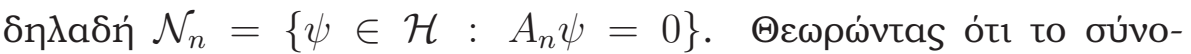

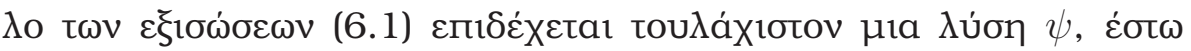

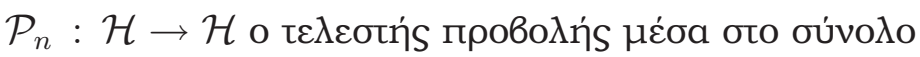

$$
K_{n}=\left\{x: x=\psi+z, z \in \mathcal{N}_{n}\right\}
$$

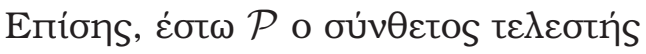

$$
\mathcal{P}=\mathcal{P}_{N} \ldots \mathcal{P}_{1}
$$

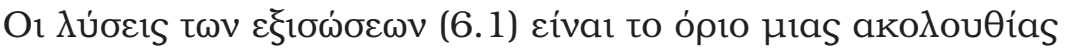

$$
\psi^{(j+1)}=\mathcal{P} \psi^{j}, j=0,1,2, \ldots,
$$

$\mu \varepsilon \psi^{(0)} \in \mathcal{H}$ auӨaipeto.

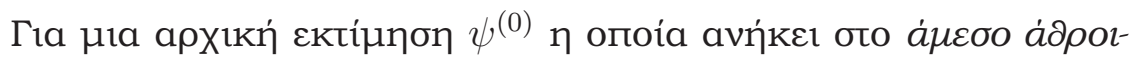

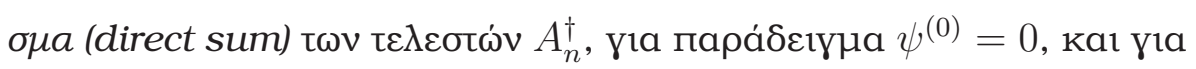

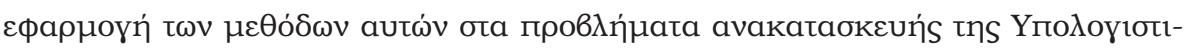

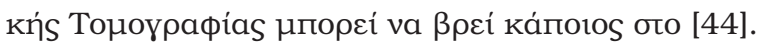




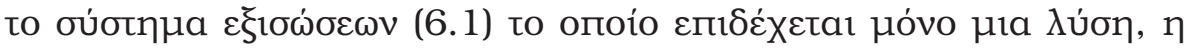

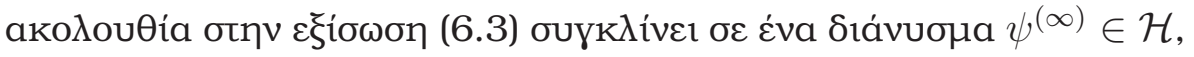

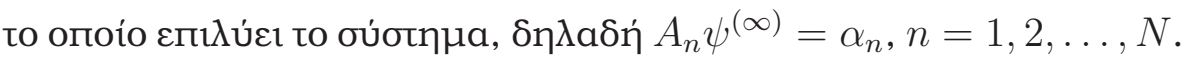

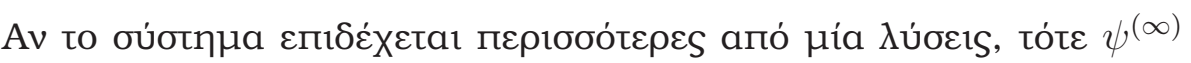

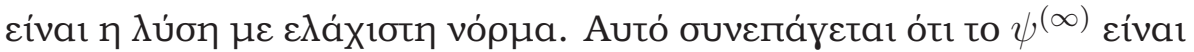

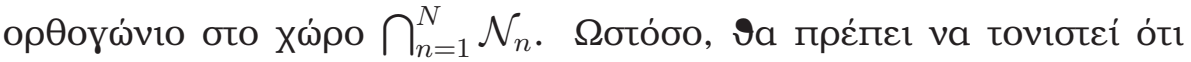

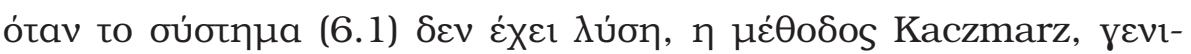

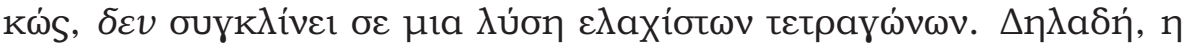

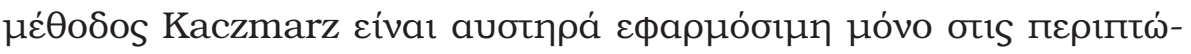

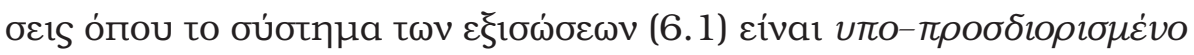

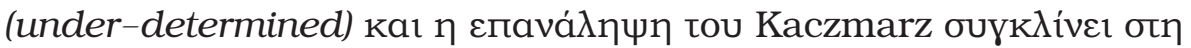

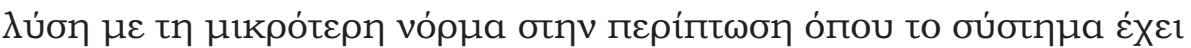

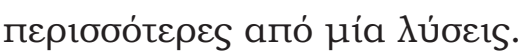

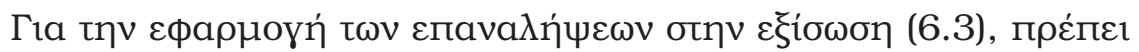

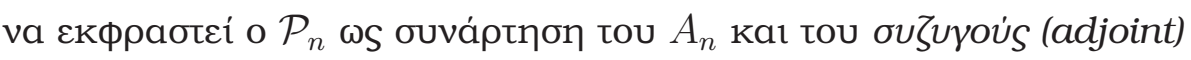

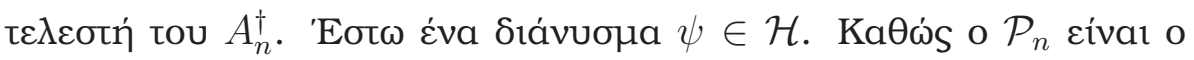

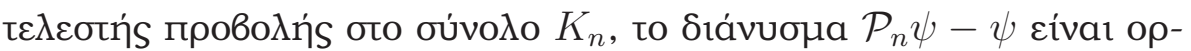

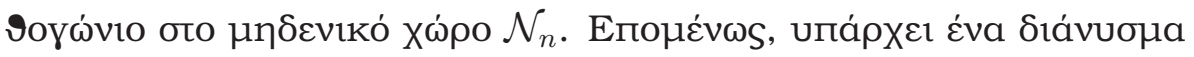

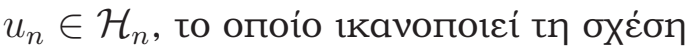

$$
\mathcal{P}_{n} \psi=\psi+A_{n}^{\dagger} u_{n}
$$

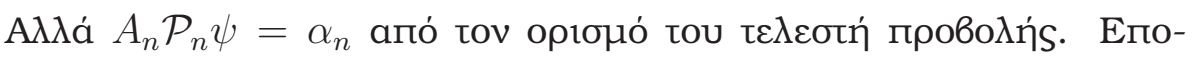

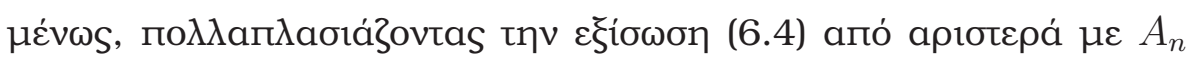

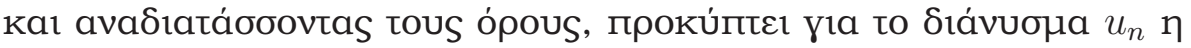




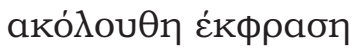

$$
u_{n}=\left(A_{n} A_{n}^{\dagger}\right)^{-1}\left(\alpha_{n}-A_{n} \psi\right) .
$$

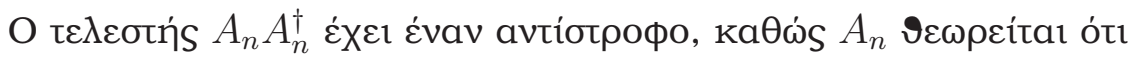

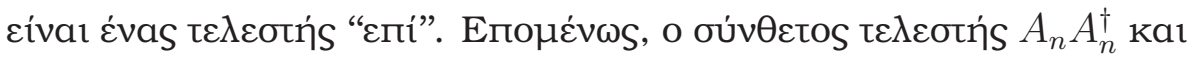

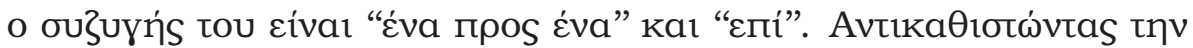

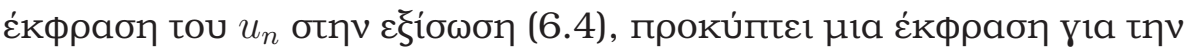

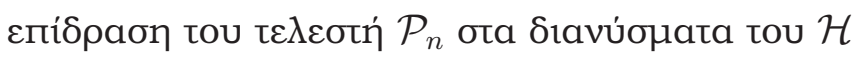

$$
\mathcal{P}_{n} \psi=\psi+A_{n}^{\dagger}\left(A_{n} A_{n}^{\dagger}\right)^{-1}\left(\alpha_{n}-A_{n} \psi\right)
$$

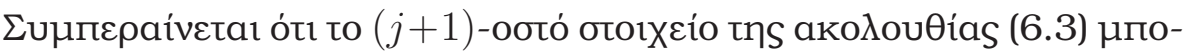

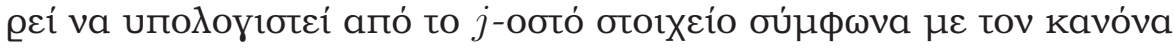

$$
\begin{aligned}
\psi_{0} & =\psi^{(j)} \\
\psi_{n} & =\psi_{n-1}+A_{n}^{\dagger}\left(A_{n} A_{n}^{\dagger}\right)^{-1}\left(\alpha_{n}-A_{n} \psi_{n-1}\right), \quad n=1,2, \ldots, N \\
\psi^{(j+1)} & =\psi_{N} .
\end{aligned}
$$

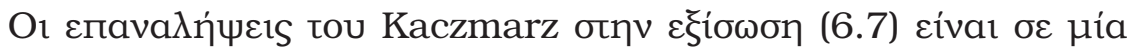

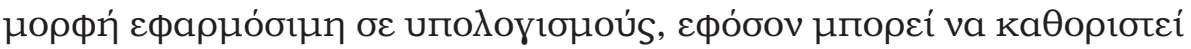

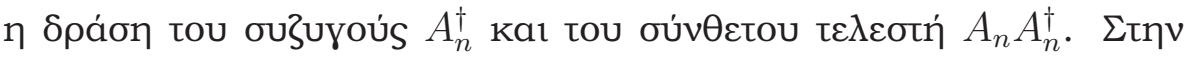

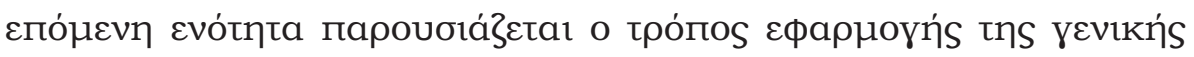

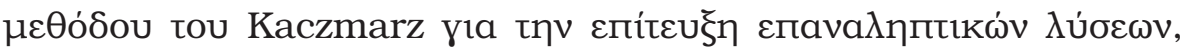

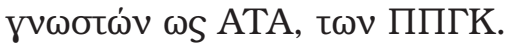




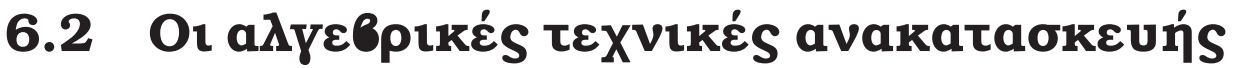 oนทv חT}

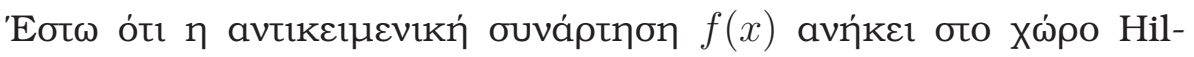

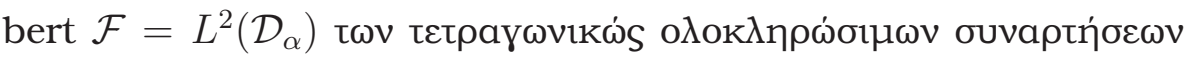

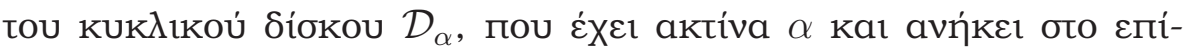

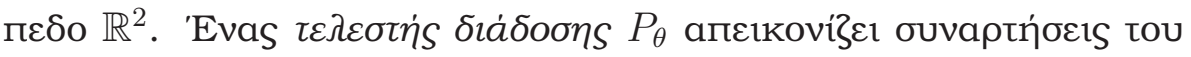

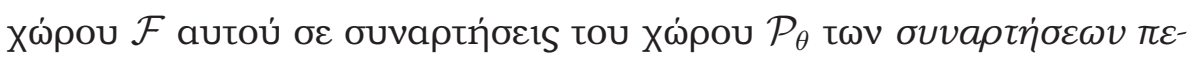

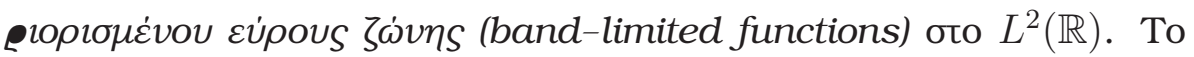

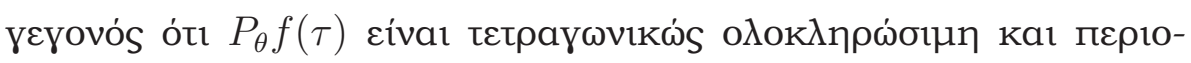

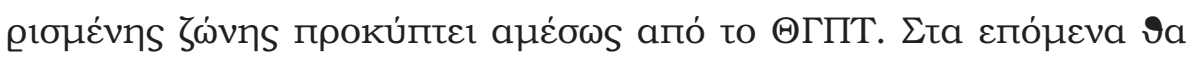

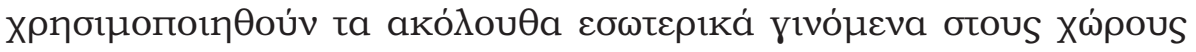

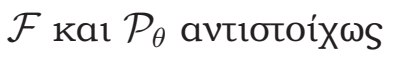

$$
\begin{aligned}
\left(f_{1}, f_{2}\right)_{\mathcal{F}} & =\int f_{1}^{*}(x) f_{2}(x) d x \\
\left(\alpha_{1}, \alpha_{2}\right)_{\mathcal{P}_{\theta}} & =\int_{-\infty}^{\infty} \alpha_{1}^{*}(t) \alpha_{2}(t) d t
\end{aligned}
$$

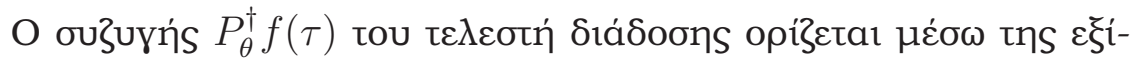
owons

$$
\left(P_{\theta} f, \alpha\right)_{\mathcal{P}_{\theta}}=\left(f, P_{\theta}^{\dagger} \alpha\right)_{\mathcal{F}}
$$

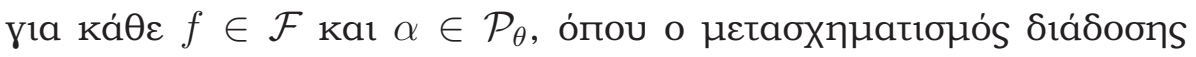

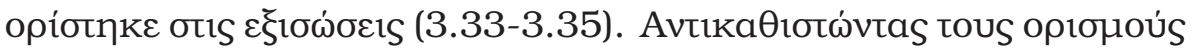




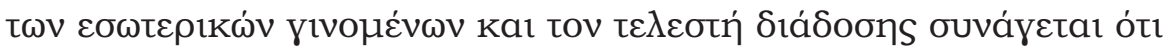

$$
\begin{aligned}
\left(P_{\theta} f, \alpha\right)_{\mathcal{P}_{\theta}} & = \\
& =\int_{-\infty}^{\infty} d \tau\left(P_{\theta} f\right)^{*}(\tau) \alpha(\tau)= \\
& =\frac{1}{2 \pi} \int_{-k}^{k} d p\left(\widetilde{P_{\theta} f}\right)^{*}(p) \tilde{\alpha}(p)= \\
& =\frac{1}{2 \pi} \int_{-\infty}^{\infty} d p \frac{k}{m} e^{-i(m-k) s_{0}} \widetilde{M_{\theta} f}[p \hat{u}+(m-k) \hat{v}] \tilde{\alpha}(p)= \\
& =\frac{1}{2 \pi} \int_{-\infty}^{\infty} d \tau \int_{-L}^{L} d s f^{*}(\tau, s) \int_{-k}^{k} d p \frac{k}{m} \tilde{\alpha}(p) e^{i\left[p \tau+(m-k)\left(s-s_{0}\right)\right]} .
\end{aligned}
$$

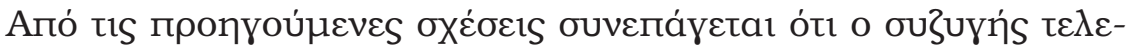

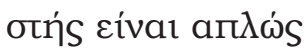

$$
P_{\theta}^{\dagger} \alpha(\tau, s)=\frac{1}{2 \pi} \mathcal{M}_{\theta} \int_{-k}^{k} d p \frac{k}{m} \tilde{\alpha}(p) e^{i\left[p \tau+(m-k)\left(s-s_{0}\right)\right]},
$$

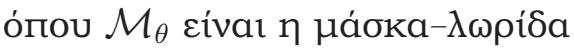

$$
\mathcal{M}_{\theta} f(x)= \begin{cases}f(x), & \text { vıa }|s| \leq a \\ 0, & \text { aגıı่́s, }\end{cases}
$$

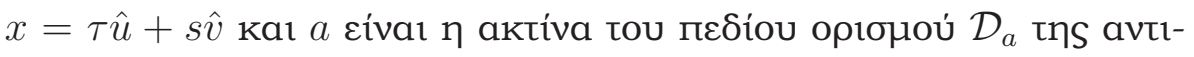

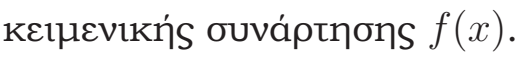

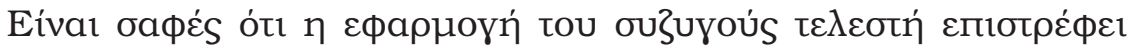

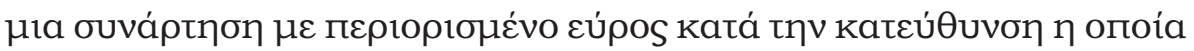

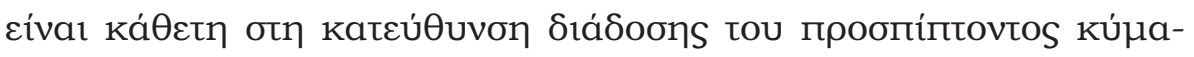

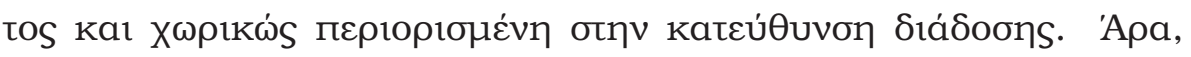

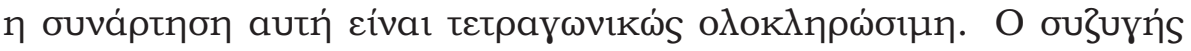




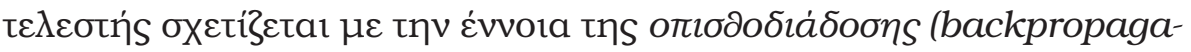

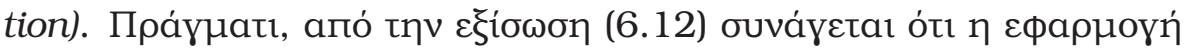

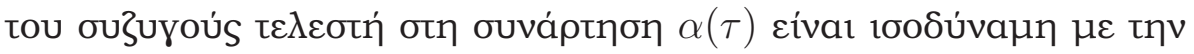

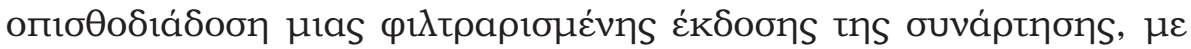

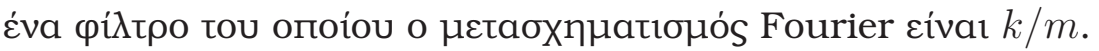

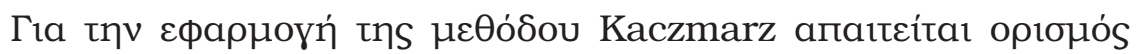

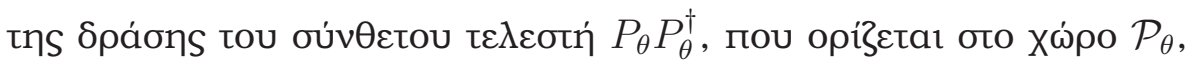

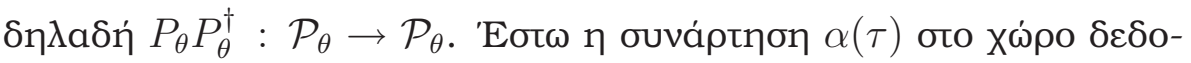

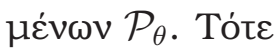

$$
\begin{aligned}
\widetilde{P_{\theta} P_{\theta}^{\dagger} \alpha(p)} & =\frac{k}{m} e^{-i(m-k) s_{0}} \widetilde{P_{\theta}^{\dagger}} \alpha[p \hat{u}+(m-k) \hat{v}] \\
& =2 L \frac{k^{2}}{m^{2}} \tilde{\alpha}(p) .
\end{aligned}
$$

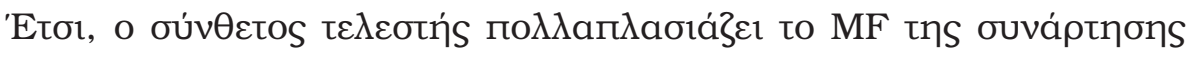

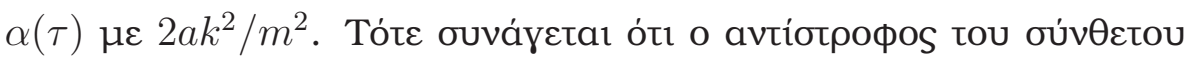

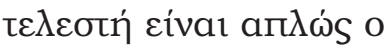

$$
\left(P_{\theta} P_{\theta}^{\dagger}\right)^{-1} \alpha(\tau)=\frac{1}{4 \pi a} \int_{-k}^{k} d p \frac{m^{2}}{k^{2}} \tilde{\alpha}(p) e^{i p \tau} .
$$

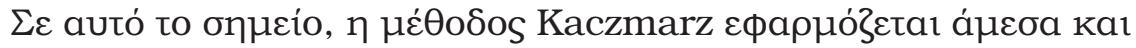

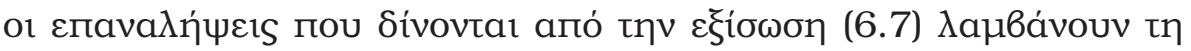
норфи́

$$
\begin{aligned}
& f_{0}=f^{(j)}(x) \\
& f_{n}=f_{n-1}(x)+\frac{1}{4 \pi a} \mathcal{M}_{\theta_{n}} \int_{-k}^{k} d p \frac{m}{k} e^{i\left[p \tau+(m-k)\left(s-s_{0}\right)\right]} . \\
& \cdot\left[\tilde{\alpha}_{\theta_{n}}(p)-P_{\theta_{n}} \widetilde{f_{n-1}}(p)\right] \\
& f^{(j+1)}(x)=f_{N}(x) .
\end{aligned}
$$




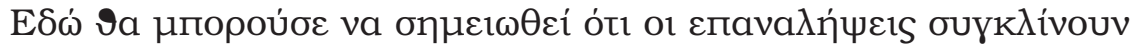

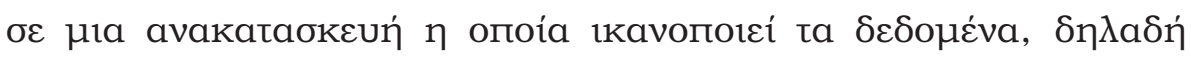

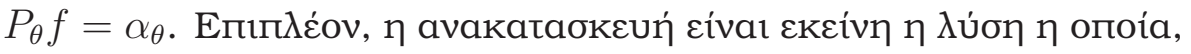

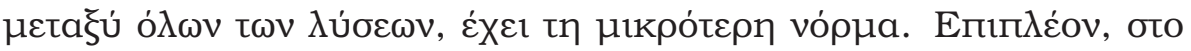

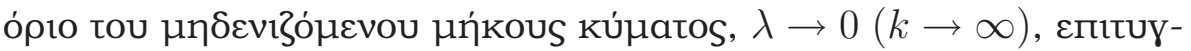
Xávetal

$$
(m-k) \rightarrow 0, \quad m / k \rightarrow 1
$$

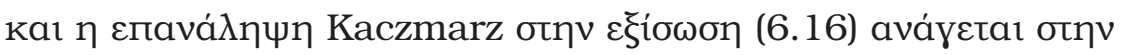

$$
f_{n}(x)=f_{n-1}(x)+\frac{1}{4 \pi a} \mathcal{M}_{\theta_{n}} \int_{-\infty}^{\infty} d p e^{i p \tau}\left[\tilde{\alpha}_{\theta_{n}}(p)-P_{\theta_{n}} \widetilde{f}_{n-1}(p)\right]
$$

ท́

$$
f_{n}(x)=f_{n-1}(x)+\frac{1}{2 a} \mathcal{M}_{\theta_{n}}\left(\alpha_{\theta_{n}}-P_{\theta_{n}} f_{n-1}\right)(x) .
$$

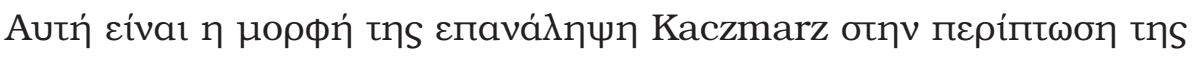

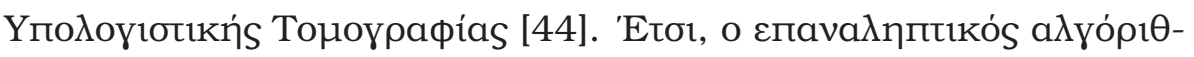

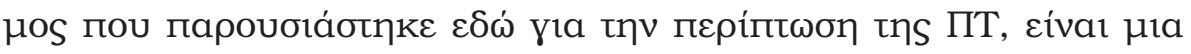

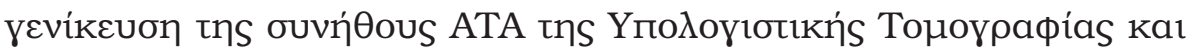

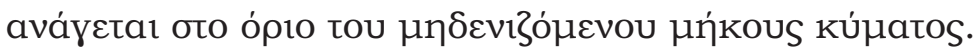

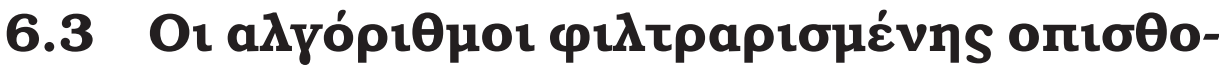

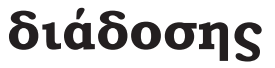

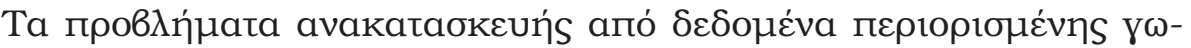

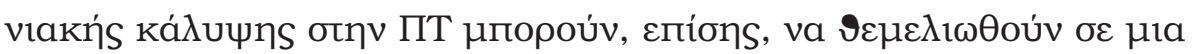

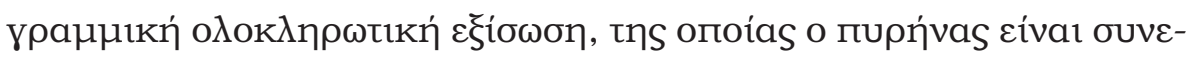

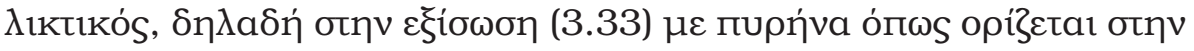




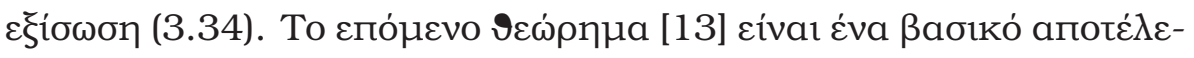

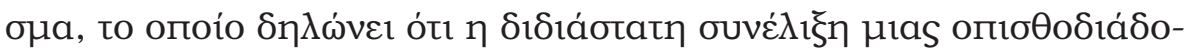

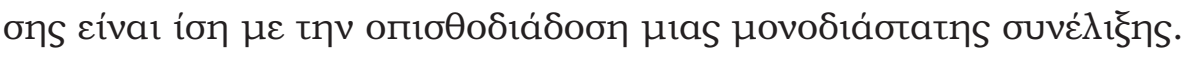

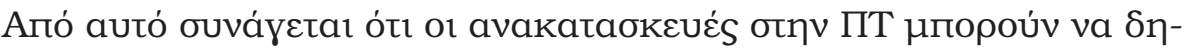

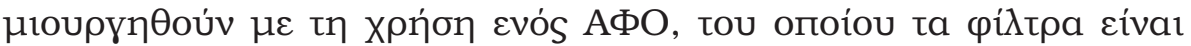

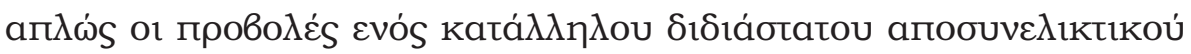

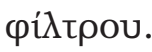

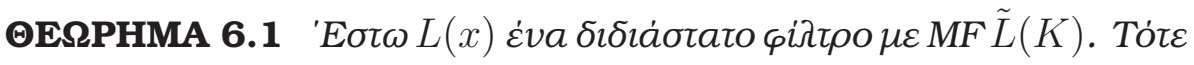
$L * * B_{\theta} \alpha=B_{\theta} l_{\theta} * \alpha$, ó o MF $\operatorname{cival} \tilde{l}_{\theta}(p)=\tilde{L}(p \hat{u}+(k-m) \hat{v})$.

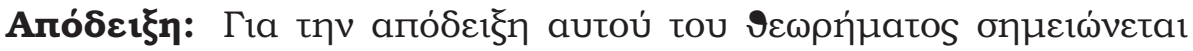

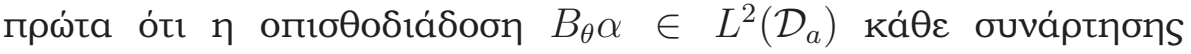

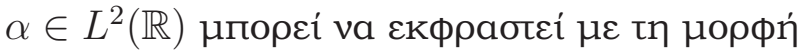

$$
B_{\theta} \alpha(x)=\frac{1}{2 \pi} \int_{-k}^{k} \tilde{\alpha}(p) e^{i\left[p \tau+(m-k)\left(s-s_{0}\right)\right]} d p
$$

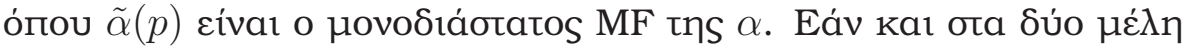

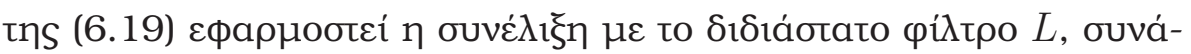
yetal ótı

$$
L * * B_{\theta} \alpha(x)=\frac{1}{2 \pi} \int_{-k}^{k} \tilde{L}[p \hat{u}+(k-m) \hat{v}] \tilde{\alpha}(p) e^{i\left[p \tau+(m-k)\left(s-s_{0}\right)\right]} d p .
$$

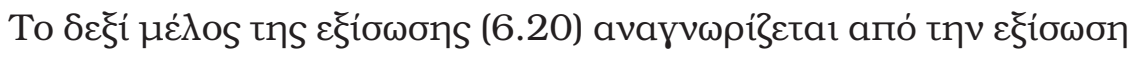

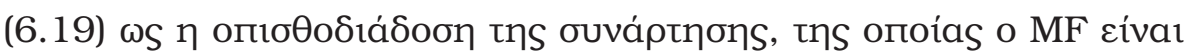

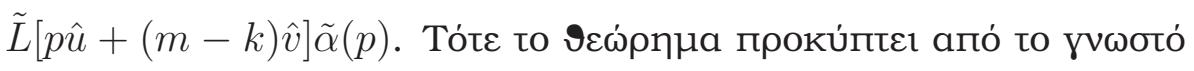




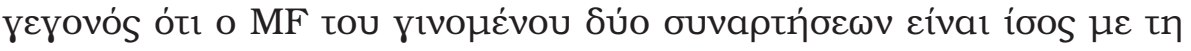

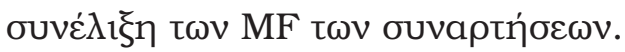

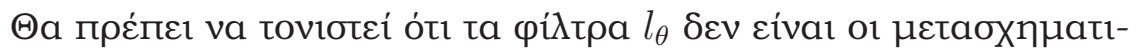

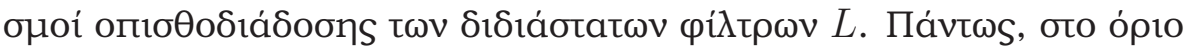

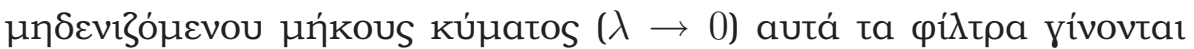

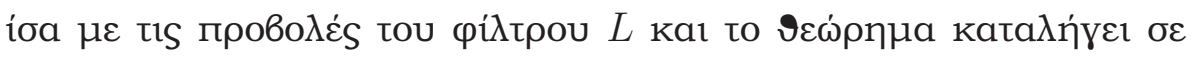

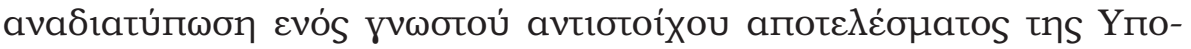

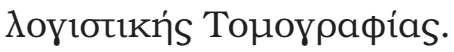

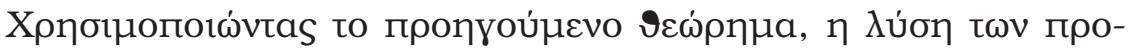

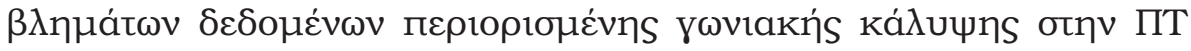

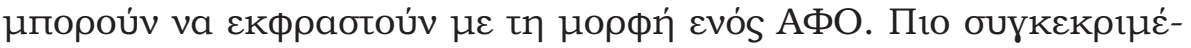
va, ouváyetal ótı

$$
f_{\text {rec }}=L * * \sum_{\theta \in \Theta} B_{\theta} P_{\theta} f=\sum_{\theta \in \Theta} B_{\theta} l_{\theta} * P_{\theta} f .
$$

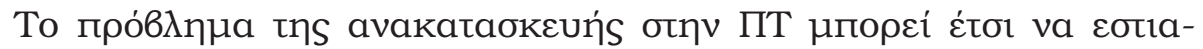

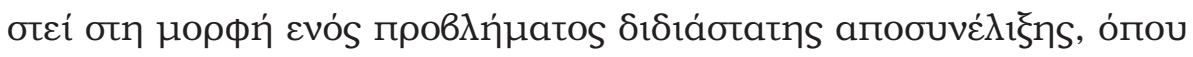

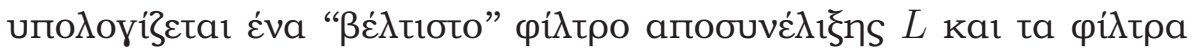

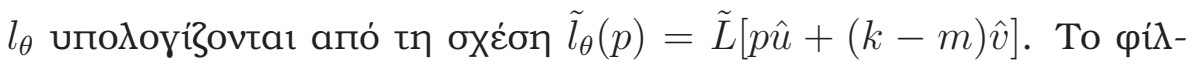

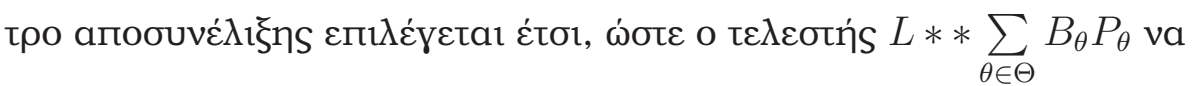

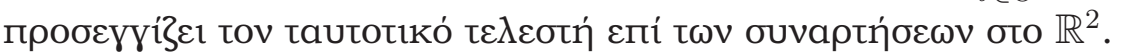




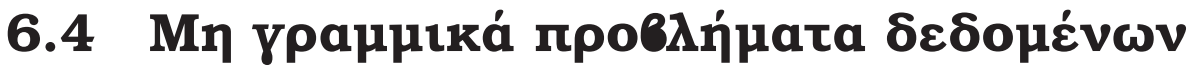

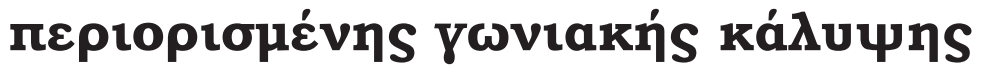

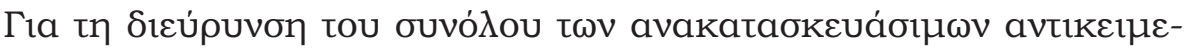

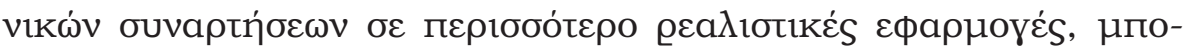

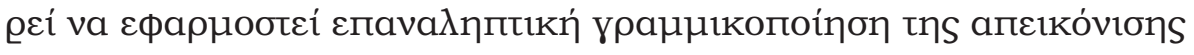

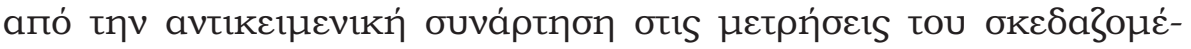

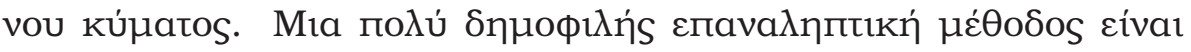

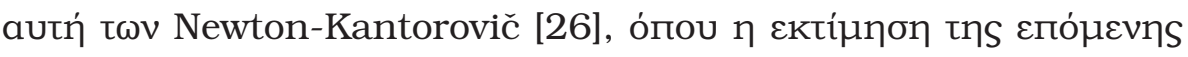

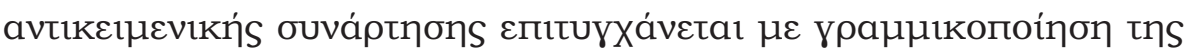

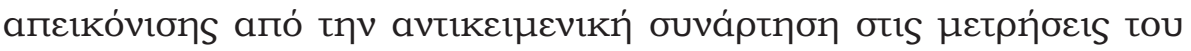

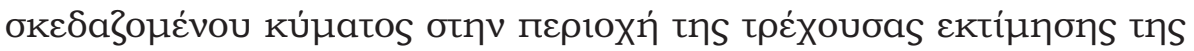

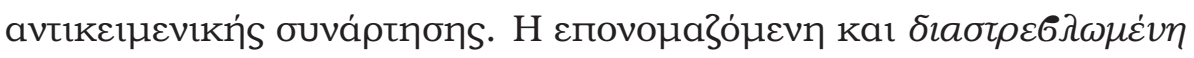

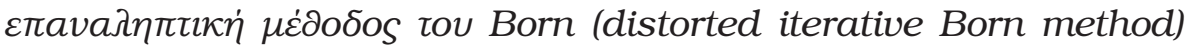

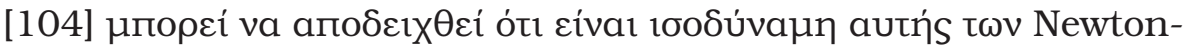

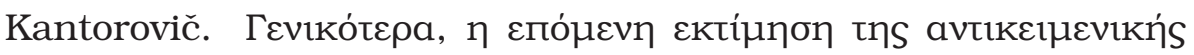

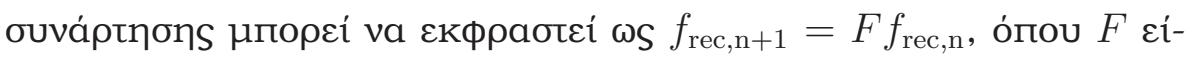

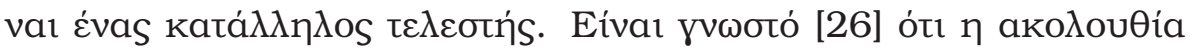

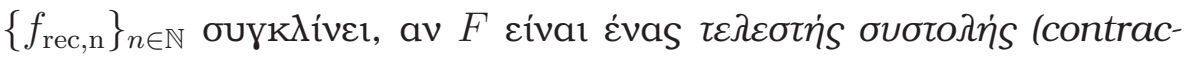

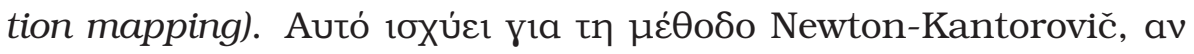

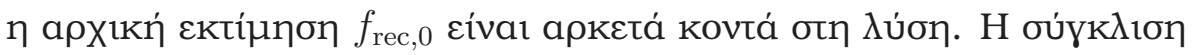

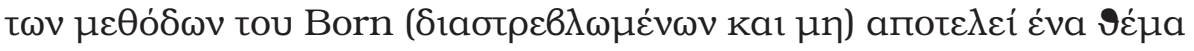

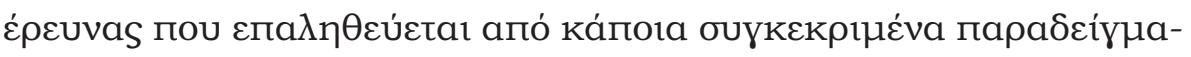

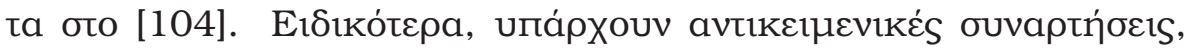

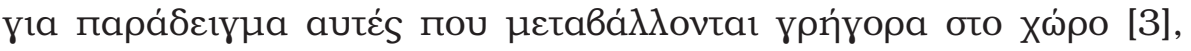




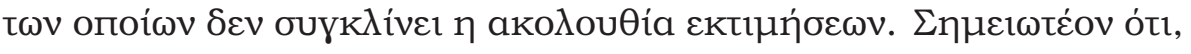

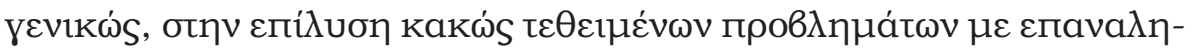

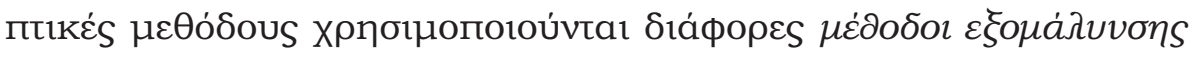
(regularization methods) tns גúons.

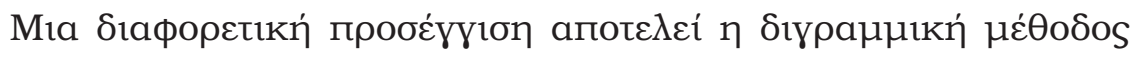

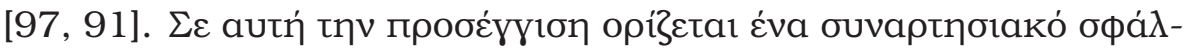

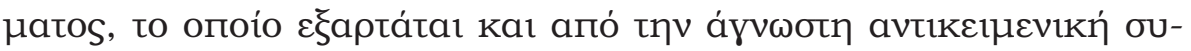

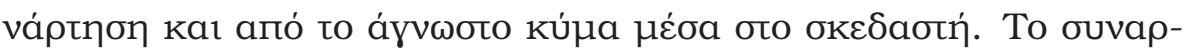

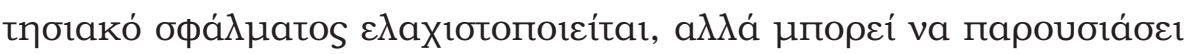

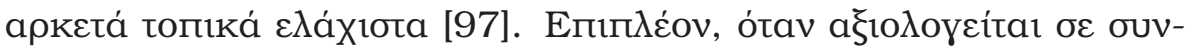

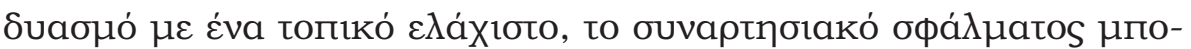

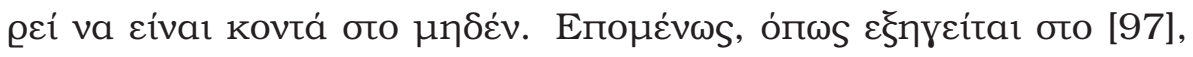

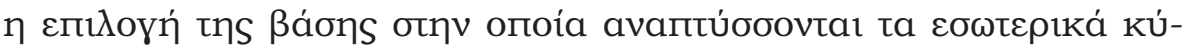

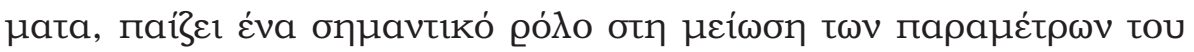

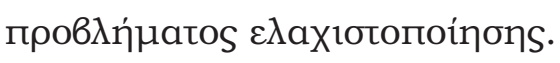

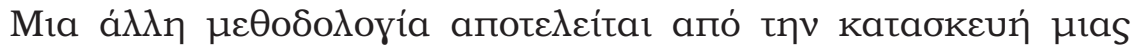

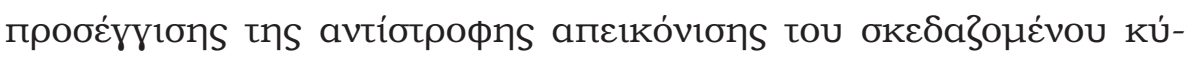

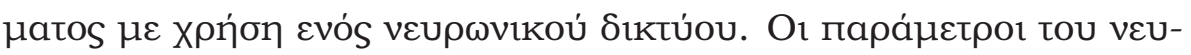

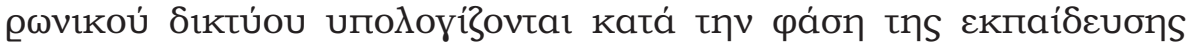

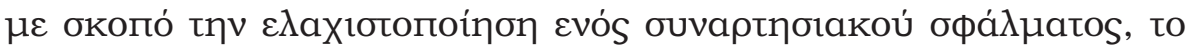

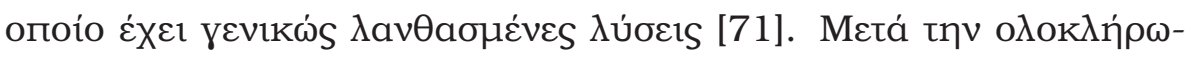

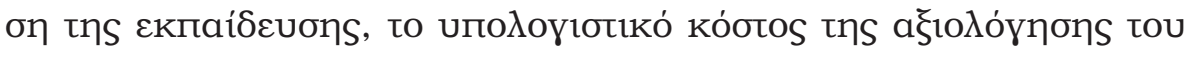

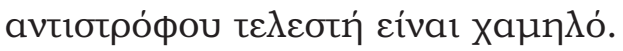

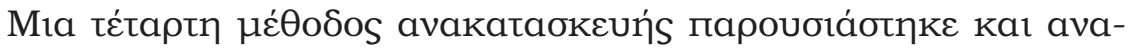

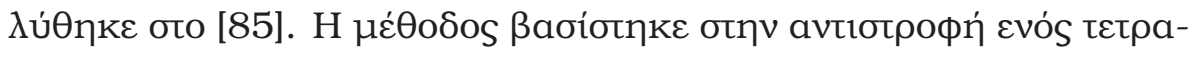




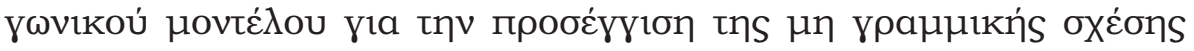

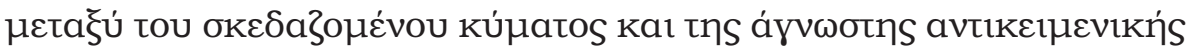

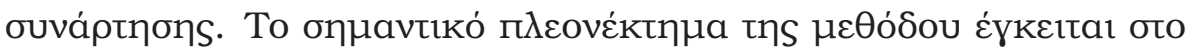

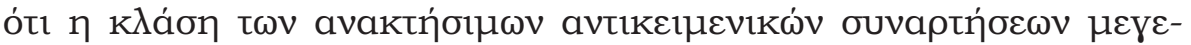

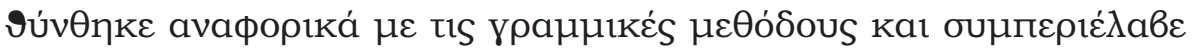

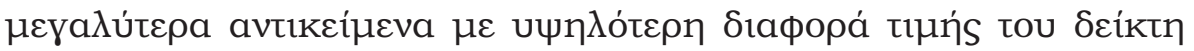

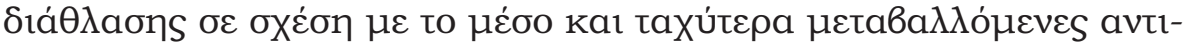

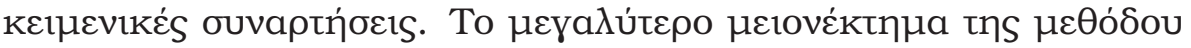

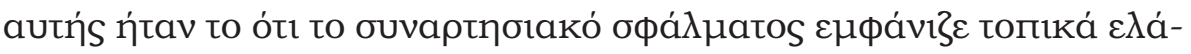

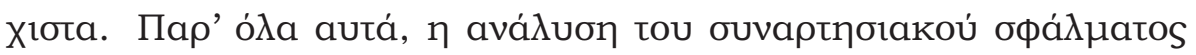

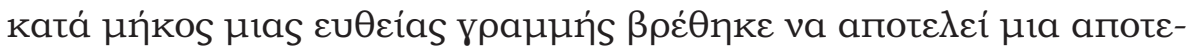

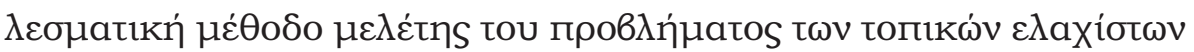
[98].

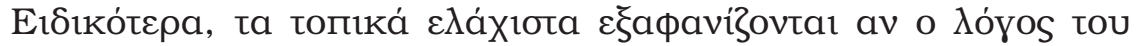

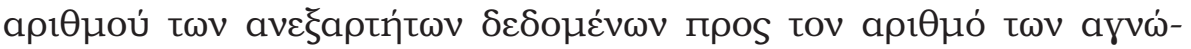

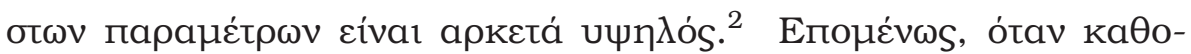

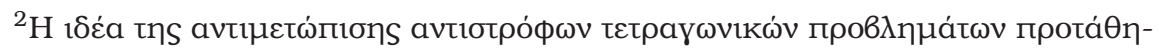

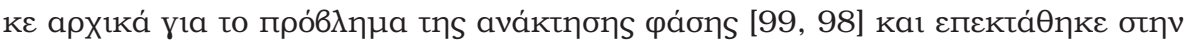

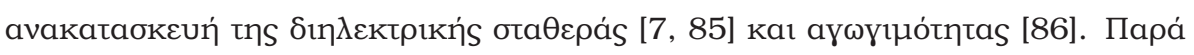

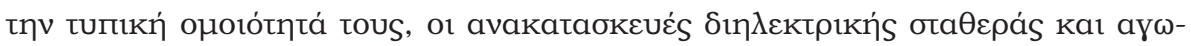

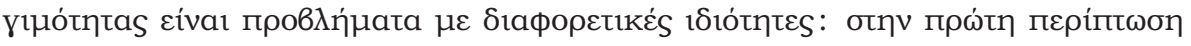

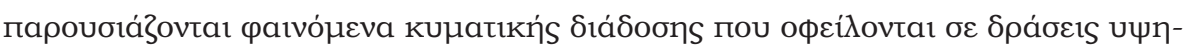

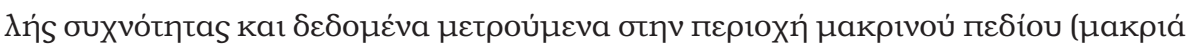

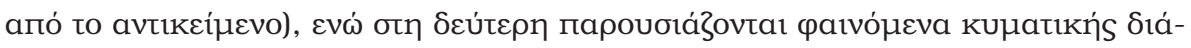

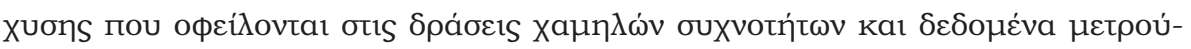

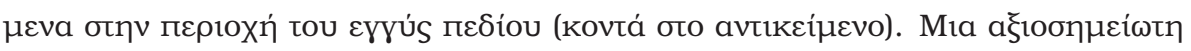

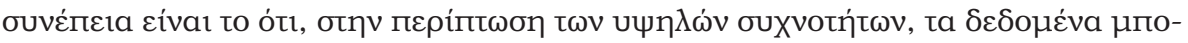




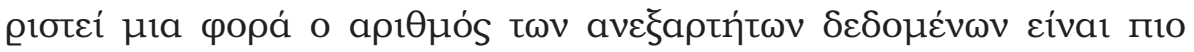

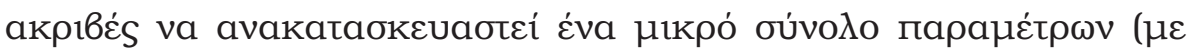

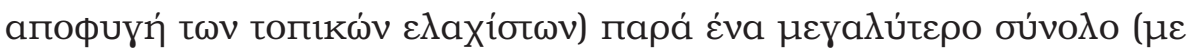

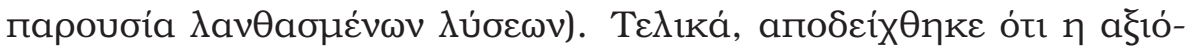

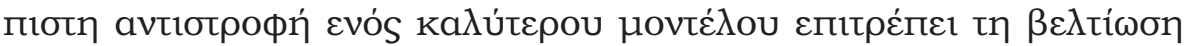

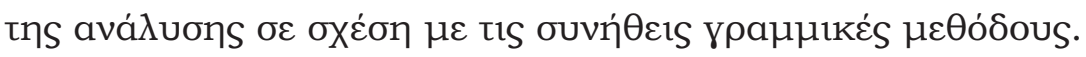

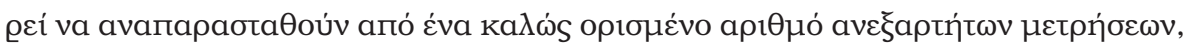

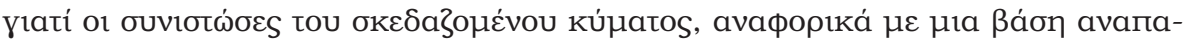

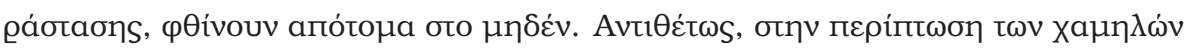

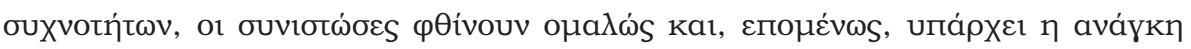

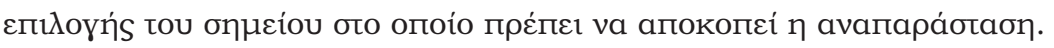




\section{KeфáAato 7}

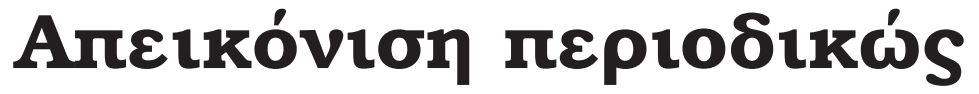

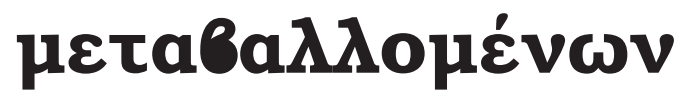

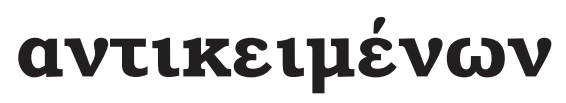

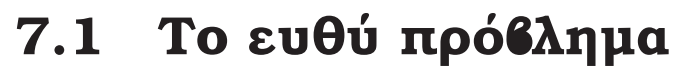

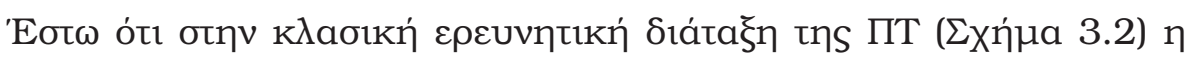

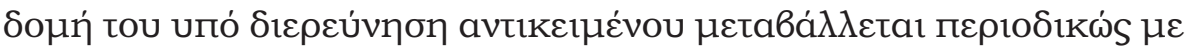

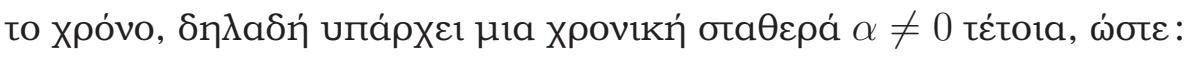

$$
f(x, t)=f(x, t+\alpha), \quad \text { via óla ta } t .
$$

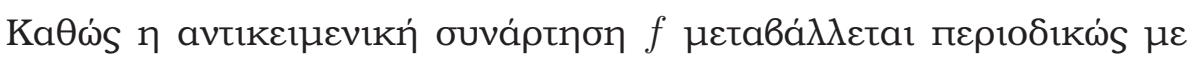

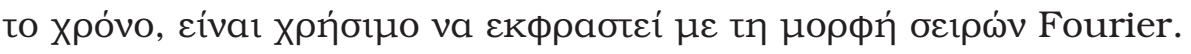




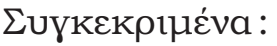

$$
f(x, t)=\sum_{l=-\infty}^{\infty} f_{l}(x) e^{\frac{-i 2 \pi l t}{\alpha}}
$$

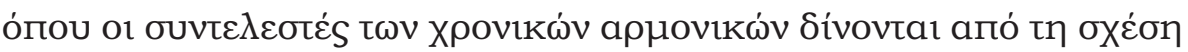

$$
f_{\ell}(x)=\frac{1}{\alpha} \int_{0}^{\alpha} f(x, t) e^{i \frac{2 \pi \ell t}{\alpha}} d t
$$

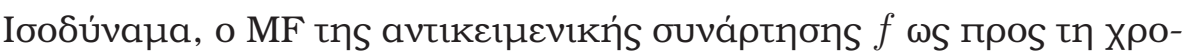

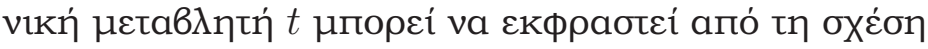

$$
\hat{f}(x, \omega)=\int e^{i \omega t} f(x, t) d t=2 \pi \sum_{\ell=-\infty}^{\infty} f_{\ell}(x) \delta\left(\omega-\frac{2 \pi \ell}{\alpha}\right)
$$

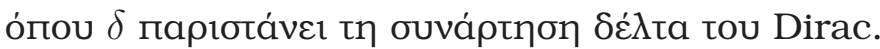

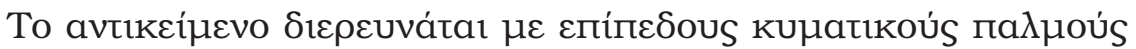

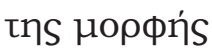

$$
p\left(t-\frac{<x, \hat{v}>}{c}\right)=\frac{1}{2 \pi} \int_{-\infty}^{\infty} d \omega e^{-i \omega\left(t-\frac{<x, \hat{v}>}{c}\right)} \hat{p}(\omega),
$$

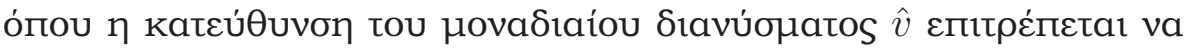

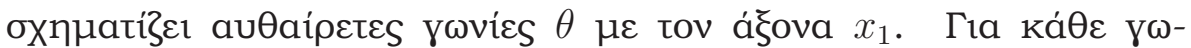

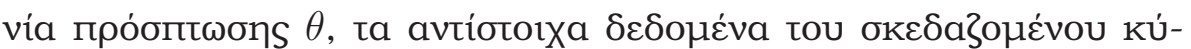

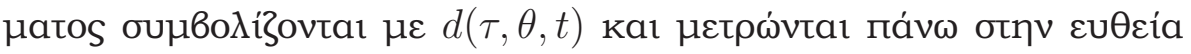

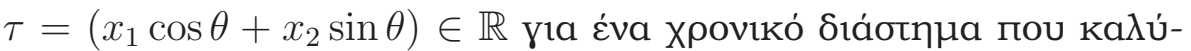

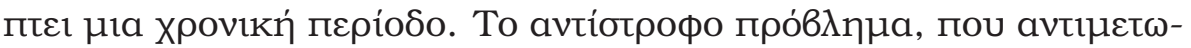

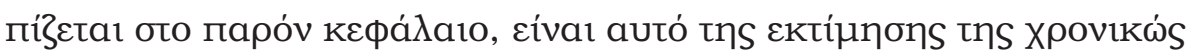

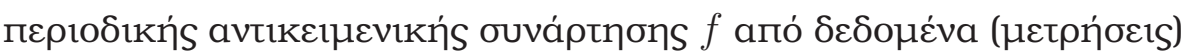

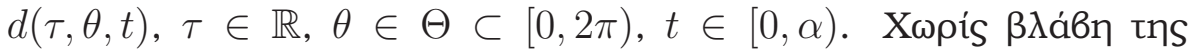




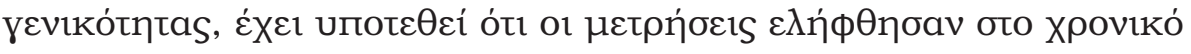
Sıáornua $[0, \alpha){ }^{1}$

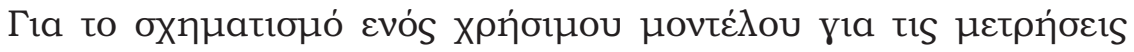

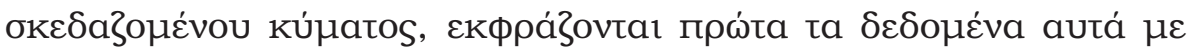

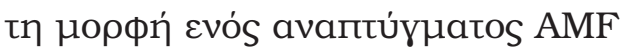

$$
d(\tau, \theta, t)=\frac{1}{(2 \pi)^{2}} \int_{-\infty}^{\infty} d \omega e^{-i \omega t} \int_{-\frac{\omega}{c}}^{\frac{\omega}{c}} d p e^{i p \tau} \tilde{\hat{d}}(p, \theta, \omega)
$$

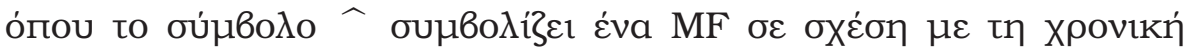

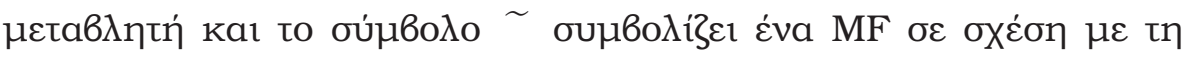
X

$$
\tilde{\hat{f}}\left[p \hat{u}+\left(m-\frac{\omega}{c}\right) \hat{v}, \omega\right]=\int d x e^{-i<\left[p \hat{u}+\left(m-\frac{\omega}{c}\right) \hat{v}\right], x>} \int e^{i \omega t} f(x, t) d t .
$$

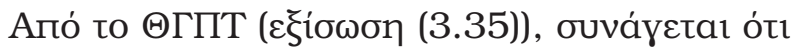

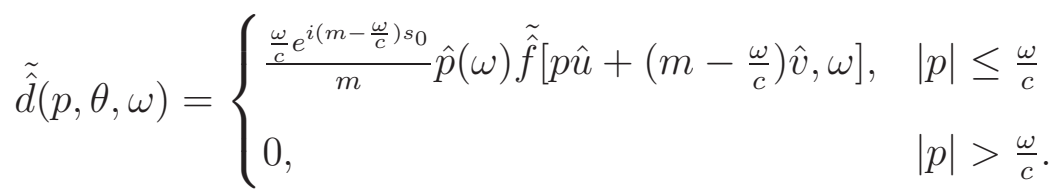

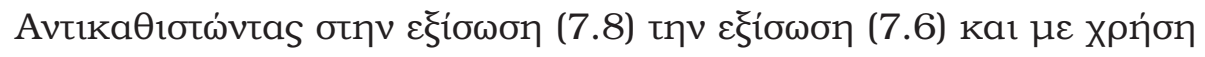

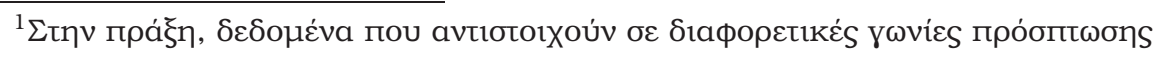

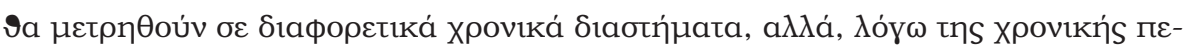

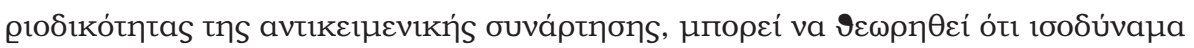

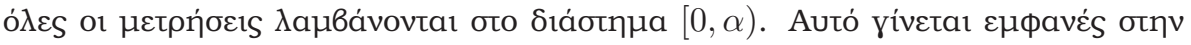

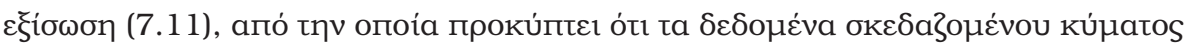

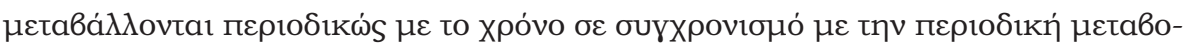
$\lambda \eta \dot{~ t ท s ~ a v t ı k \varepsilon \imath \mu \varepsilon v ı k n ́ s ~ o u v a ́ p t \eta o n s . ~}$
} 
tᄁs ع そ̇ंowons (7.4), ouváyeta1 ót1

$$
\begin{aligned}
d(\tau, \theta, t)=\sum_{\ell=-\infty}^{\infty} e^{-i \frac{2 \pi \ell t}{\alpha}} \hat{p}\left(\frac{2 \pi|\ell|}{\alpha}\right) \frac{|\ell|}{c \alpha} \int_{-\frac{2 \pi|\ell|}{c \alpha}}^{\frac{2 \pi|\ell|}{c \alpha}} d p \\
\cdot e^{i p \tau} \frac{e^{i\left(m-\frac{2 \pi|\ell|}{c \alpha}\right) s_{0}}}{m} \tilde{f}_{\ell}\left[p \hat{u}+\left(m-\frac{2 \pi|\ell|}{c \alpha}\right) \hat{v}\right],
\end{aligned}
$$

о́поu

$$
\tilde{f}_{\ell}(K)=\int e^{-i<K, x>} f_{\ell}(x) d x
$$

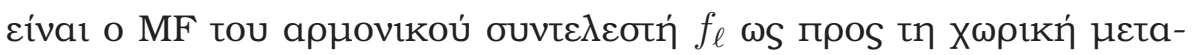
$\beta \lambda \eta т \dot{~} x$.

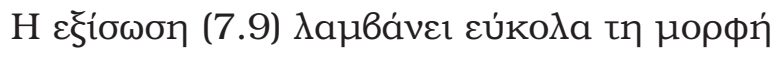

$$
d(\tau, \theta, t)=\sum_{\ell=-\infty}^{\infty} d_{\ell}(\tau, \theta) e^{-i \frac{2 \pi \ell t}{\alpha}}
$$

о́поu

$$
\begin{aligned}
d_{\ell}(\tau, \theta)=\hat{p}\left(\frac{2 \pi \ell}{\alpha}\right) \frac{|\ell|}{c \alpha} \int_{-\frac{2 \pi|\ell|}{c \alpha}}^{\frac{2 \pi|\ell|}{c \alpha}} d p . \\
\quad \cdot e^{i p \tau} \frac{e^{i\left(m-\frac{2 \pi|\ell|}{c \alpha}\right) s_{0}}}{m} \tilde{f}_{\ell}\left[p \hat{u}+\left(m-\frac{2 \pi|\ell|}{c \alpha}\right) \hat{v}\right] .
\end{aligned}
$$

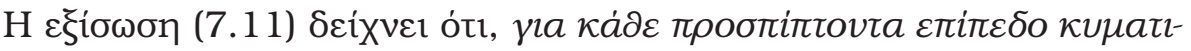

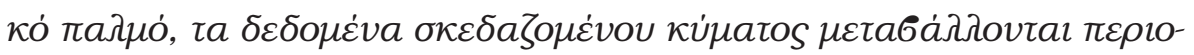

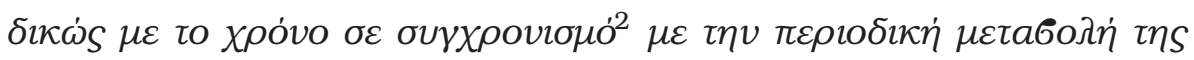

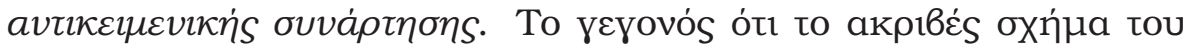

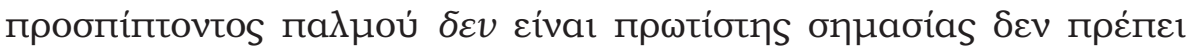

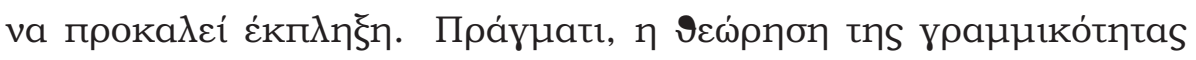

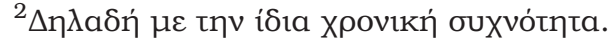




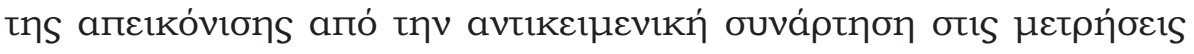

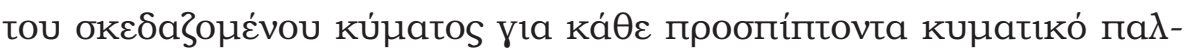

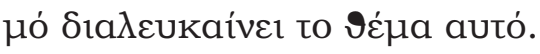

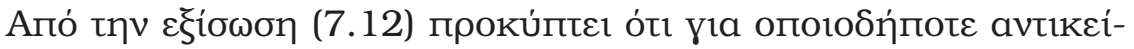

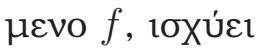

$$
d_{\ell=0}(\tau, \theta) \equiv 0,
$$

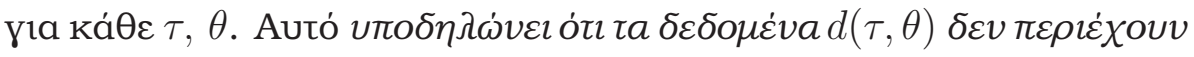

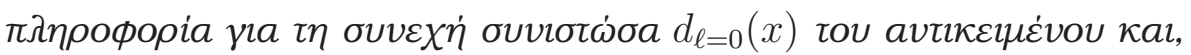

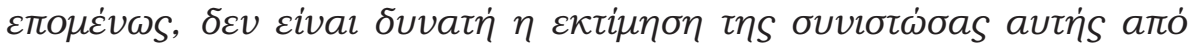

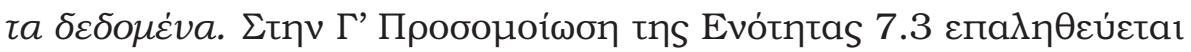

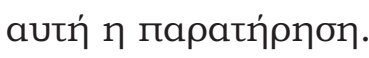

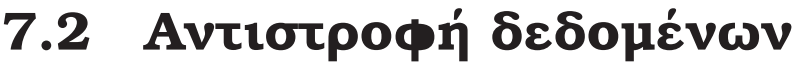

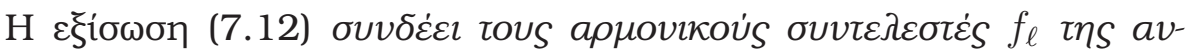

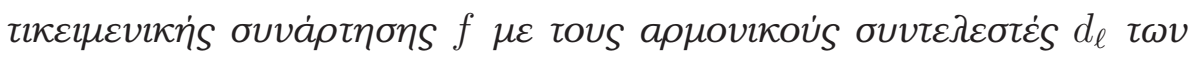

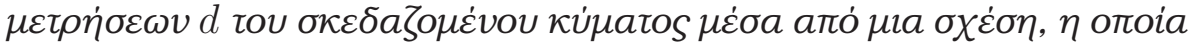

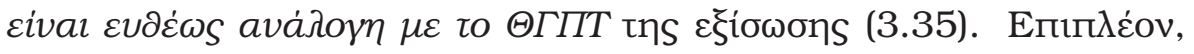

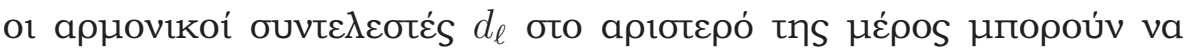

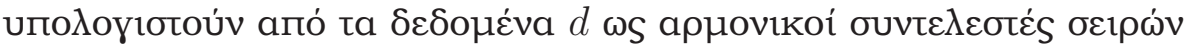
Fourier

$$
d_{\ell}(\tau, \theta)=\frac{1}{\alpha} \int_{0}^{\alpha} e^{i \frac{2 \pi \ell t}{\alpha}} d(\tau, \theta, t) d t
$$

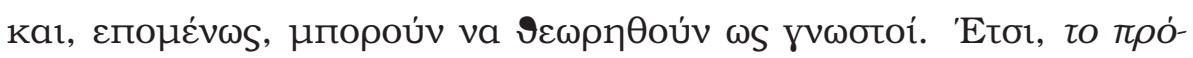

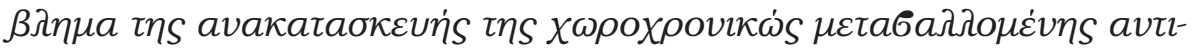

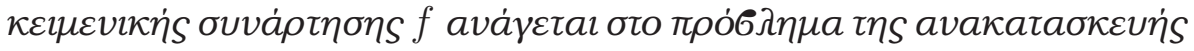




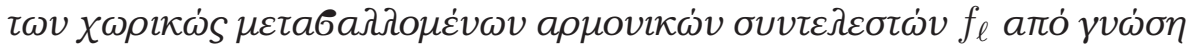

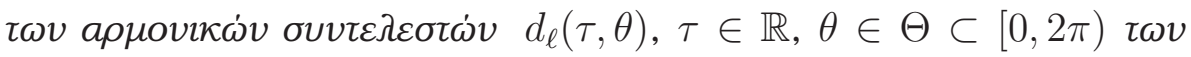
$\mu \varepsilon \tau \rho \dot{\eta} \sigma \varepsilon \omega v$.

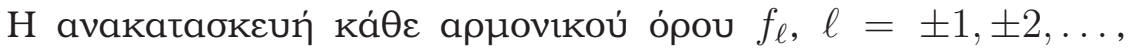

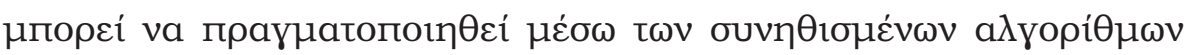

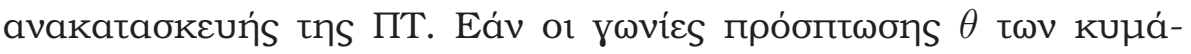

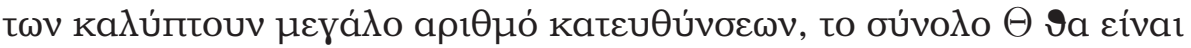

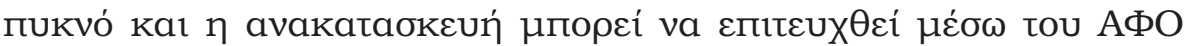

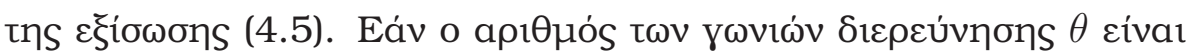

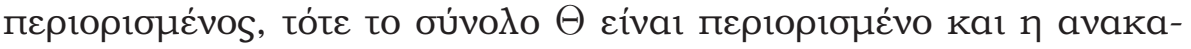

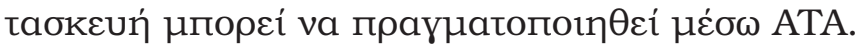

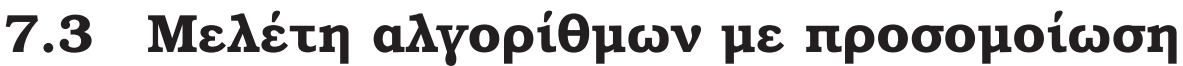

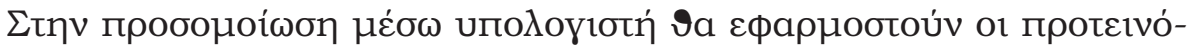

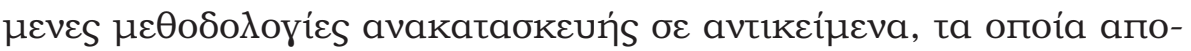

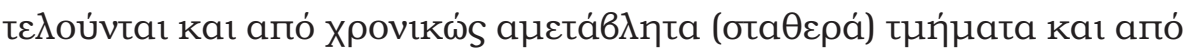

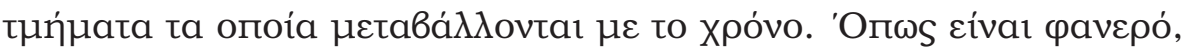

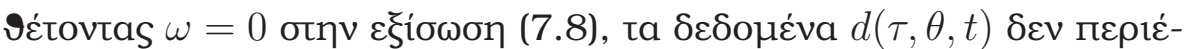

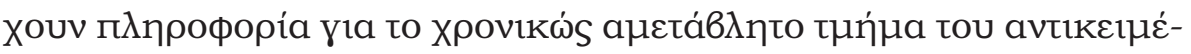

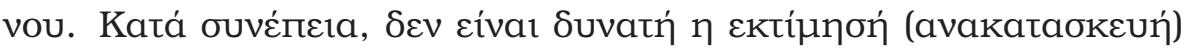

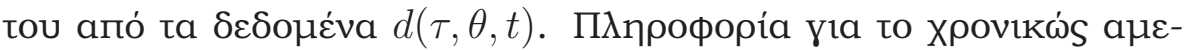

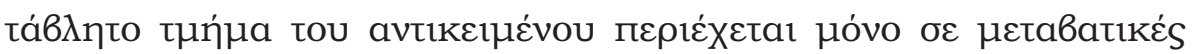

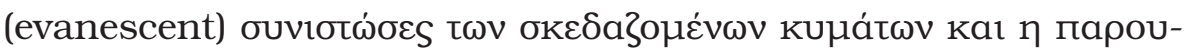

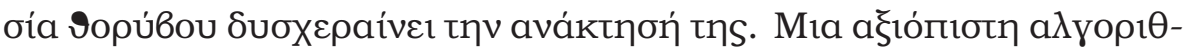




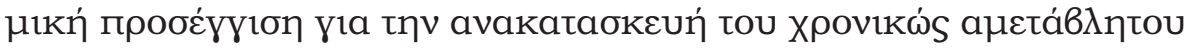

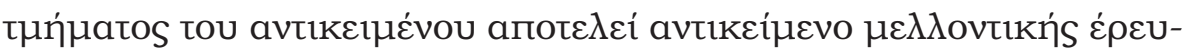
vas.

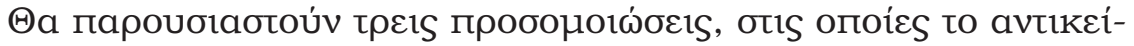

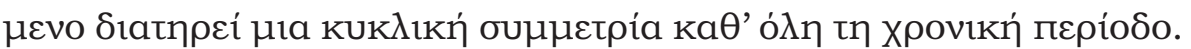

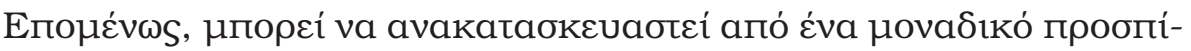

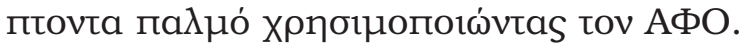

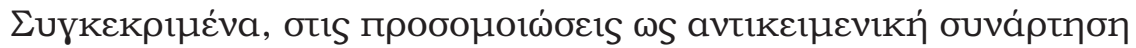

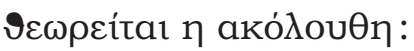

$$
f(x, y, t)=F(x, y) g(t) .
$$

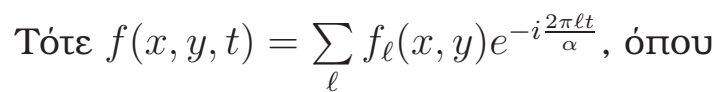

$$
f_{\ell}(x, y)=C_{\ell} F(x, y)
$$

ka1

$$
C_{\ell}=\frac{1}{\alpha} \int_{0}^{\alpha} g(t) e^{i \frac{2 \pi \ell t}{\alpha}} d t
$$

$\Theta \varepsilon \omega \rho \omega \dot{v t a s}$

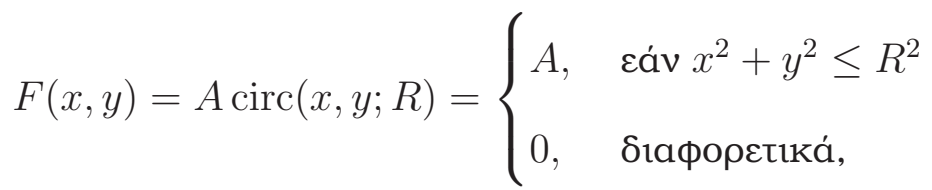

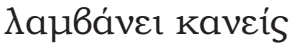

$$
\begin{aligned}
\tilde{F}\left(K_{x}, K_{y}\right) & =2 \pi A \int_{0}^{R} d r r J_{0}\left(r \sqrt{K_{x}^{2}+K_{y}^{2}}\right) \\
& =\frac{2 \pi A R}{K_{x}^{2}+K_{y}^{2}} J_{1}\left(R \sqrt{K_{x}^{2}+K_{y}^{2}}\right) .
\end{aligned}
$$




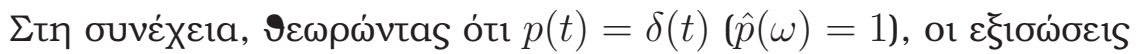
(7.8) kaı (7.12) Sívouv

$$
\begin{aligned}
\tilde{d}_{\ell}(p, \theta) & =\frac{2 \pi|\ell| /(c \alpha)}{2 \pi} \cdot \frac{e^{i\left(m-\frac{2 \pi|\ell|}{c \alpha}\right) s_{0}}}{m} \tilde{f}_{\ell}\left[p \hat{u}+\left(m-\frac{2 \pi|\ell|}{c \alpha}\right) \hat{v}\right] \\
& =\frac{|\ell|}{c \alpha} \cdot \frac{e^{i\left(m-\frac{2 \pi|\ell|}{c \alpha}\right) s_{0}}}{m} \tilde{f}_{\ell}\left[p \hat{u}+\left(m-\frac{2 \pi|\ell|}{c \alpha}\right) \hat{v}\right]
\end{aligned}
$$

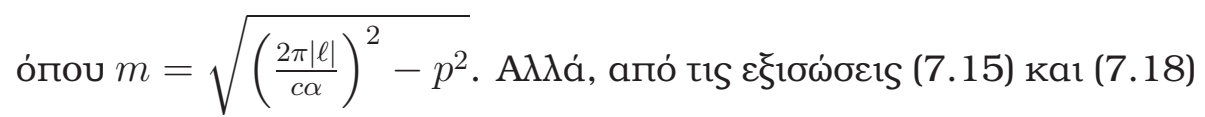

$$
\tilde{f}_{\ell}\left[p \hat{u}+\left(m-\frac{2 \pi|\ell|}{c \alpha}\right) \hat{v}\right]=C_{\ell} \frac{2 \pi A R}{\rho} J_{1}(R \rho),
$$

о́поч $\rho=\sqrt{p^{2}+\left(m-\frac{2 \pi|\ell|}{c \alpha}\right)^{2}}=\sqrt{\frac{4 \pi|\ell|}{c \alpha}\left(\frac{2 \pi|\ell|}{c \alpha}-m\right)}$. 'E七бı

$$
\tilde{d}_{\ell}(p, \theta)=\frac{|\ell|}{c \alpha} \frac{e^{i\left(m-\frac{2 \pi|\ell|}{c \alpha}\right) s_{0}}}{m} C_{\ell} \frac{2 \pi A R}{\rho} J_{1}(R \rho),
$$

$\mu \varepsilon \rho=\sqrt{\frac{4 \pi|\ell|}{c \alpha}\left(\frac{2 \pi|\ell|}{c \alpha}-m\right)}$ kat $\theta \in[0,2 \pi) .^{3}$

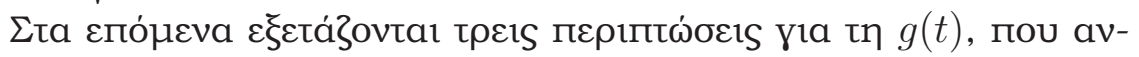

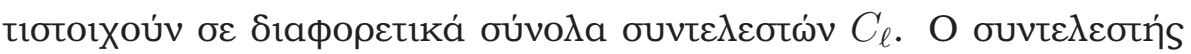

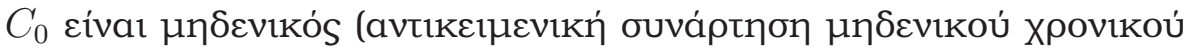

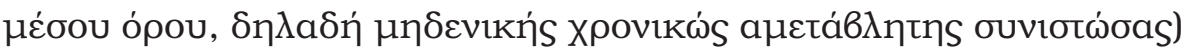

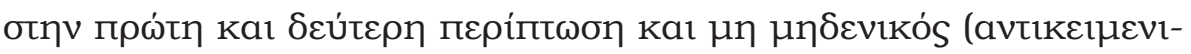

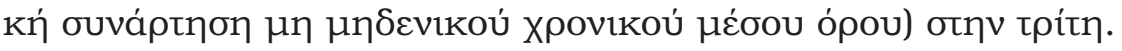

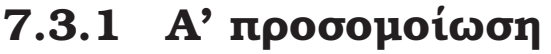

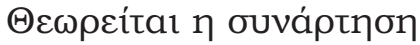

$$
g(t)=\cos \frac{2 \pi q t}{\alpha}, \quad 0 \leq t \leq \alpha,
$$

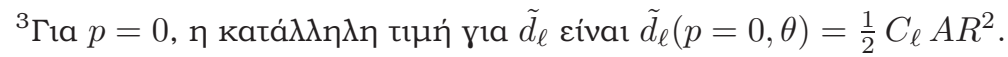




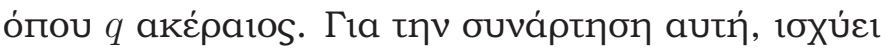

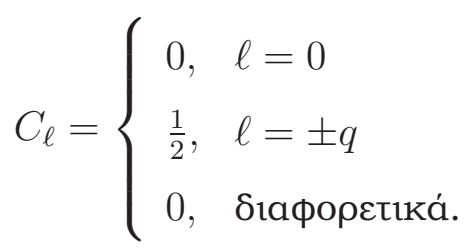

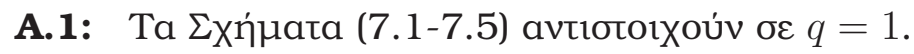

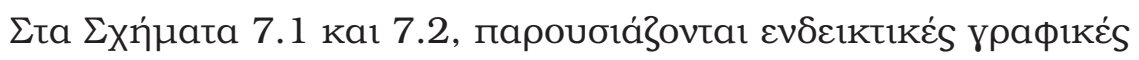

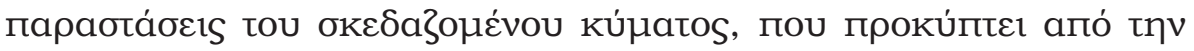

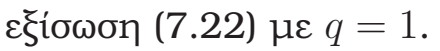

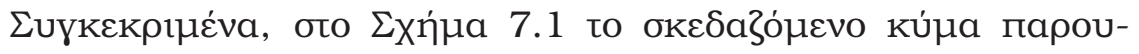

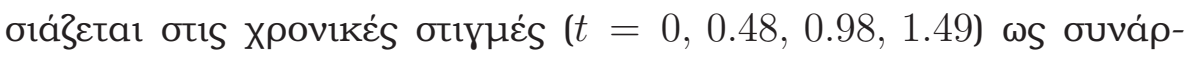

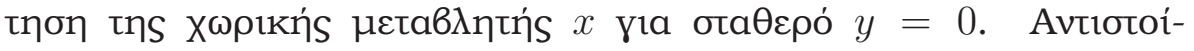

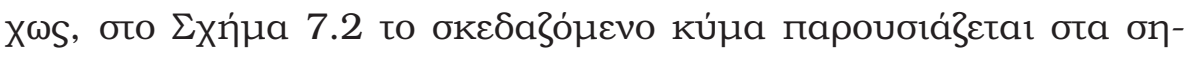
uria $(x=0, y=0),(x=0.50, y=0),(x=0.73, y=0)$,

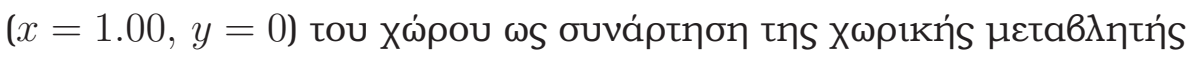
$t$. 

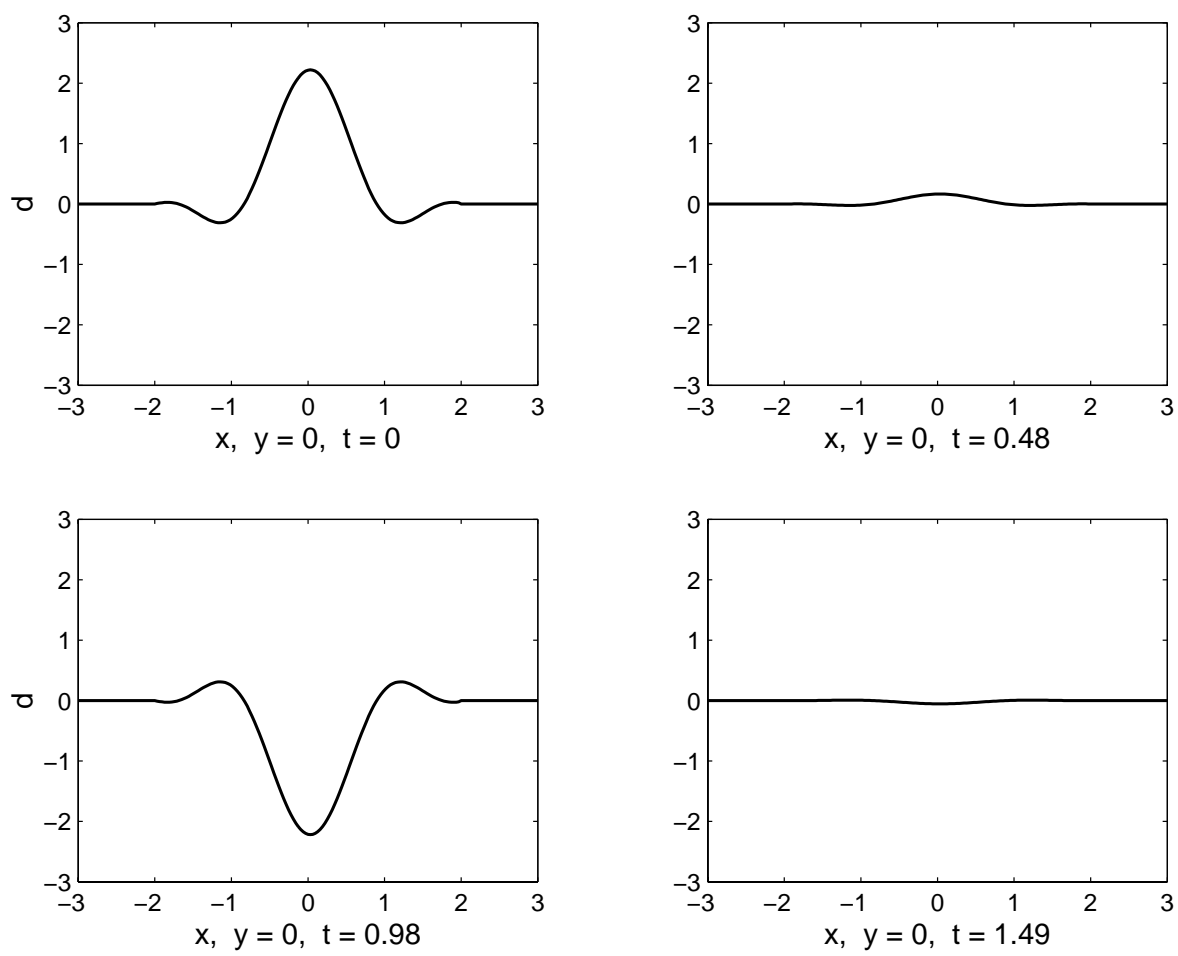

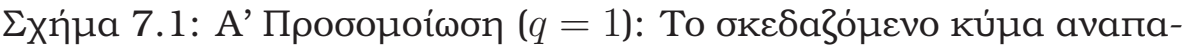

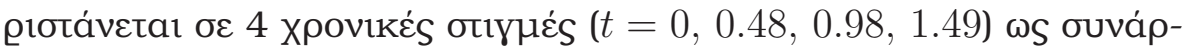

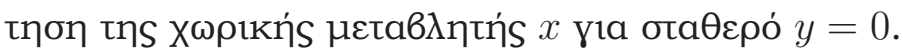



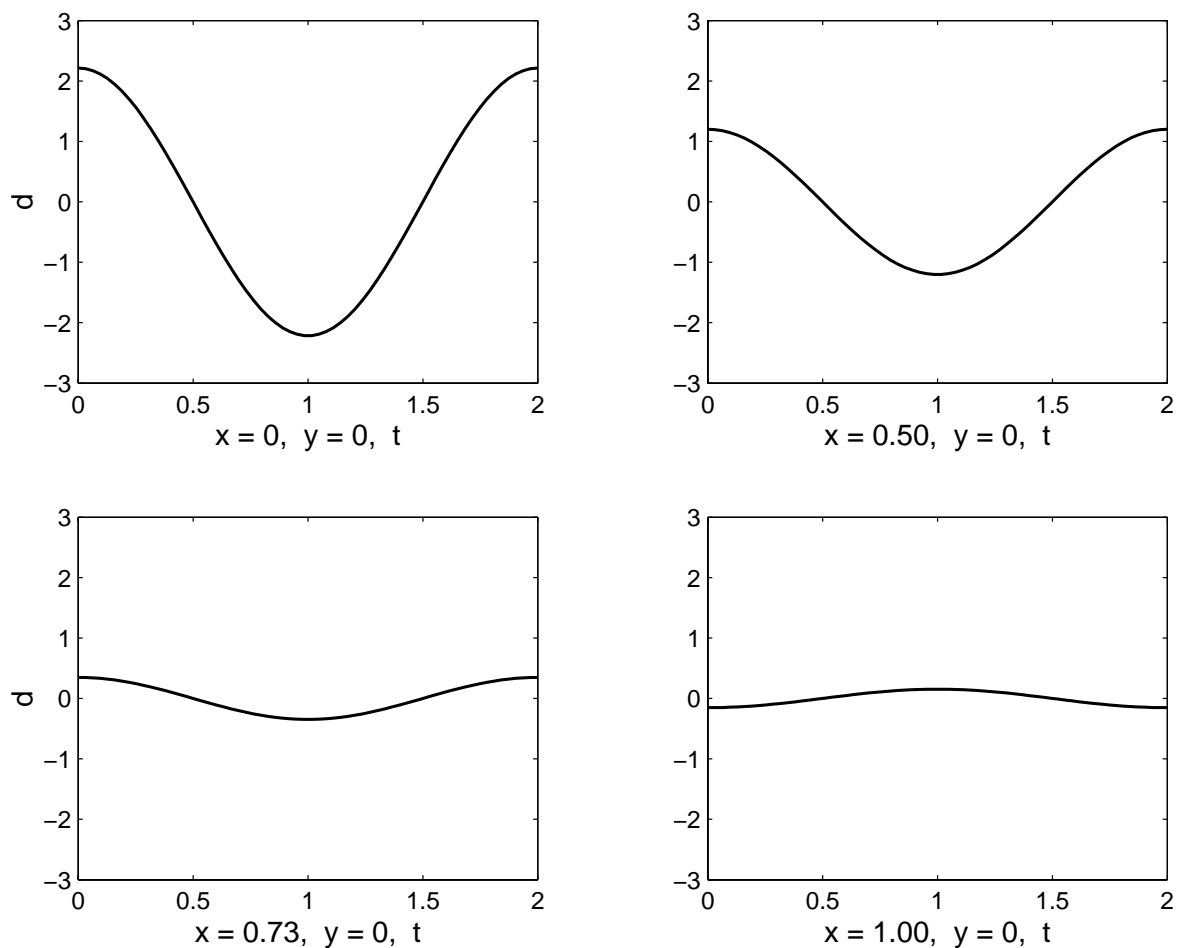

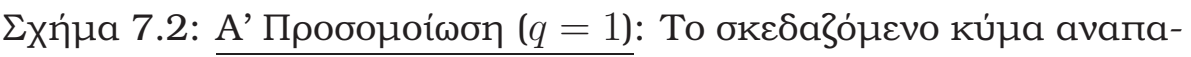

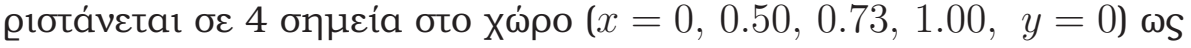

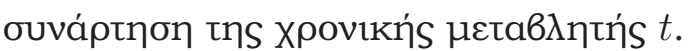

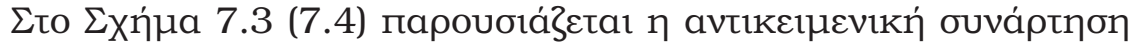

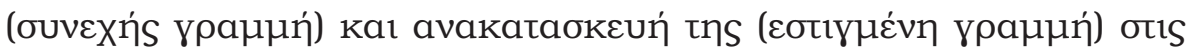

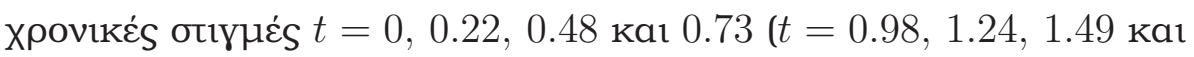

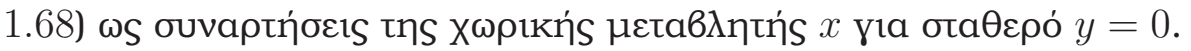



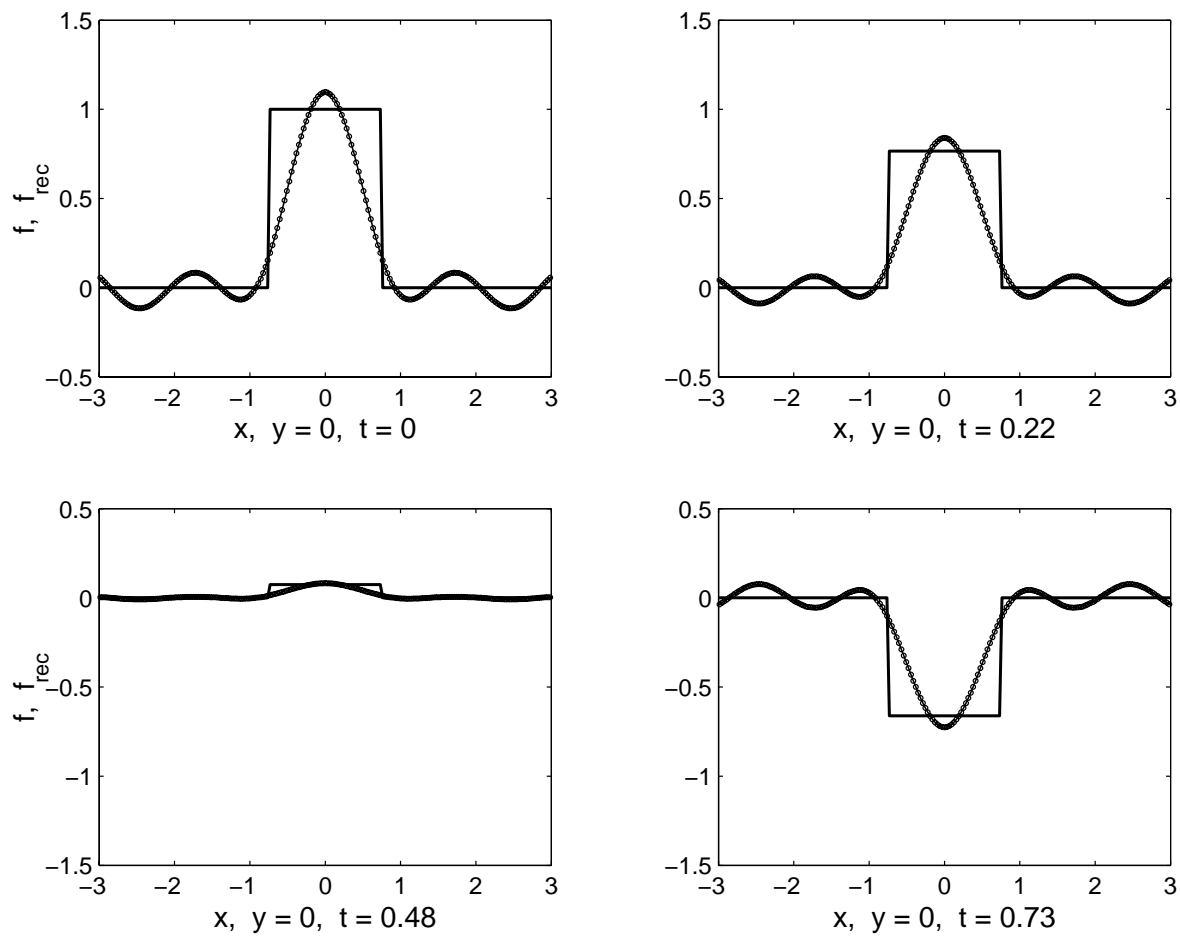

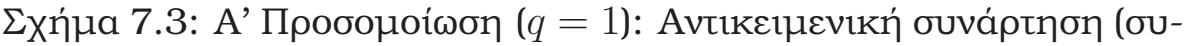

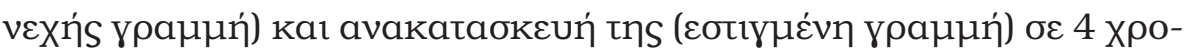

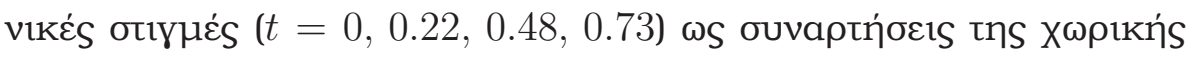

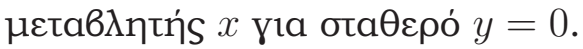



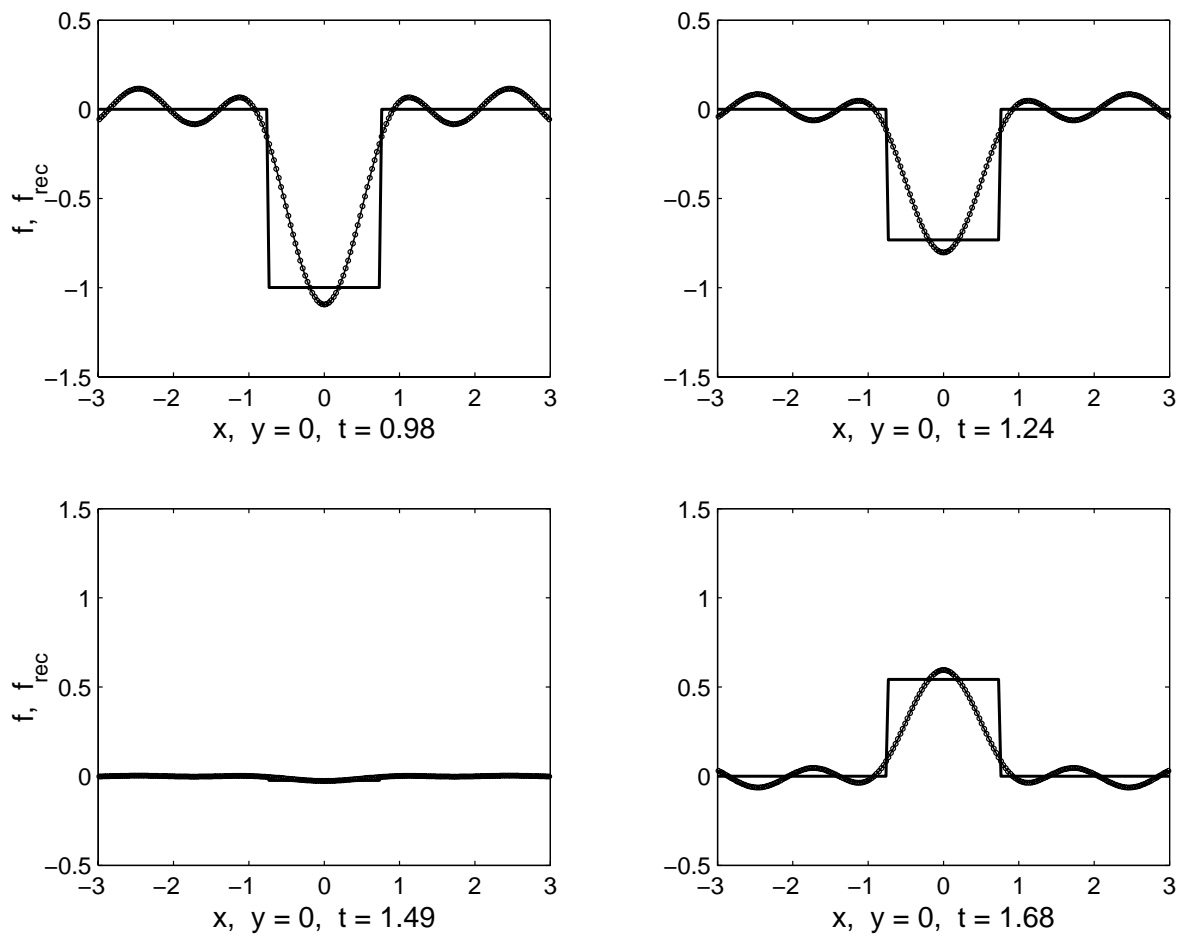

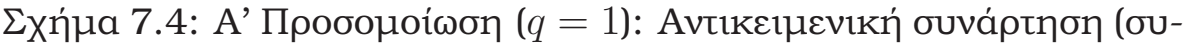

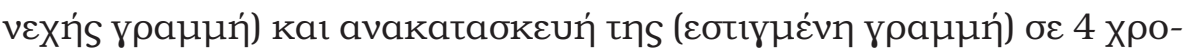

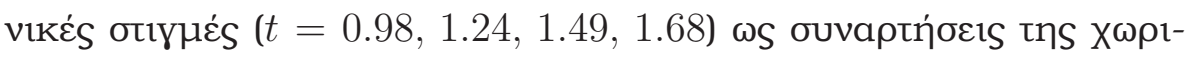

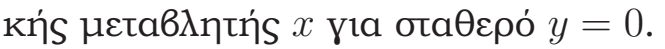




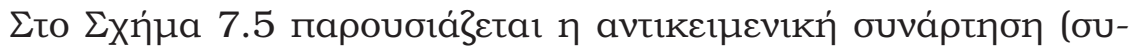

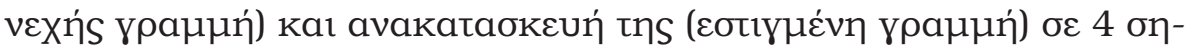

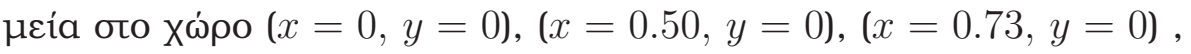

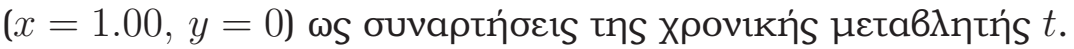
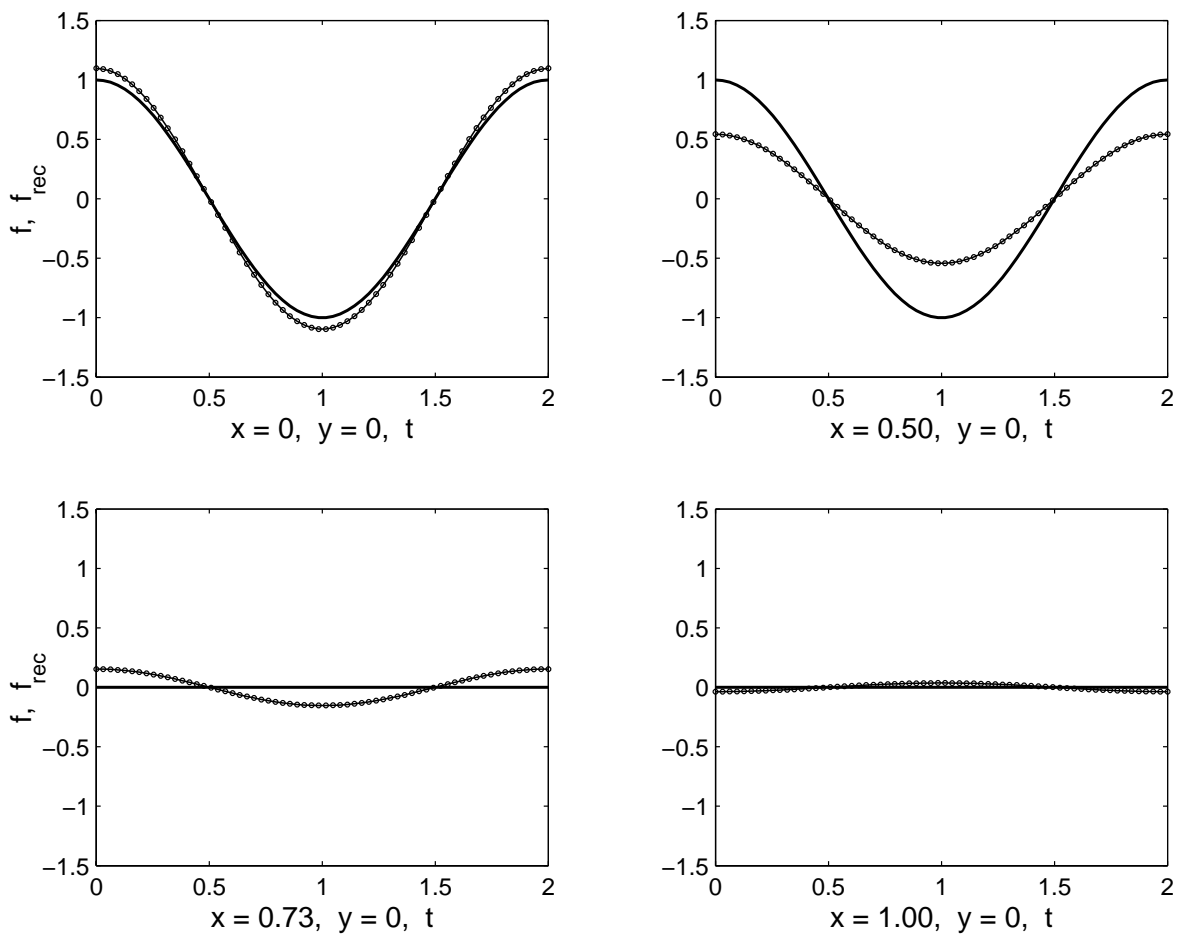

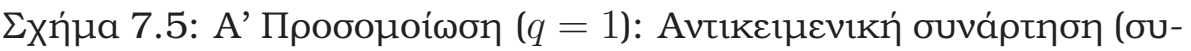

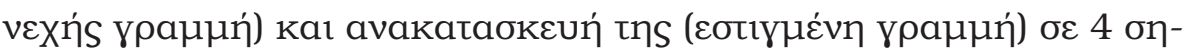

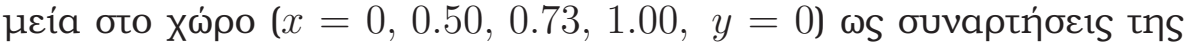

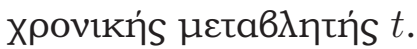




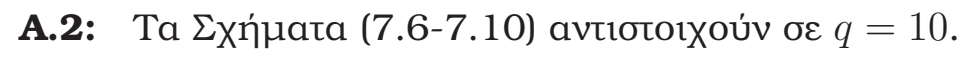

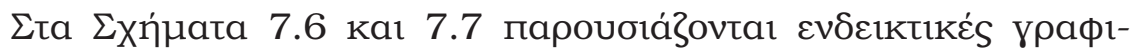

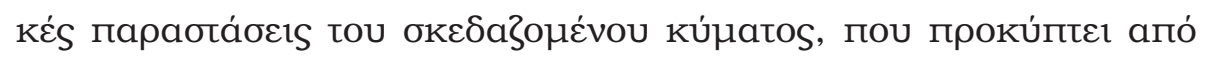

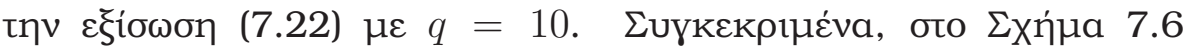

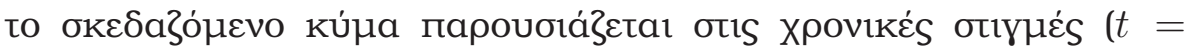

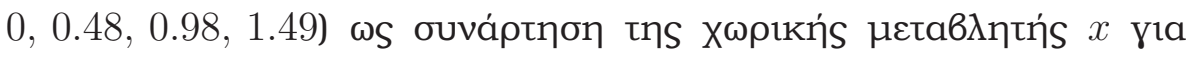

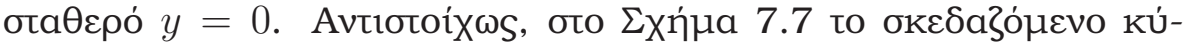

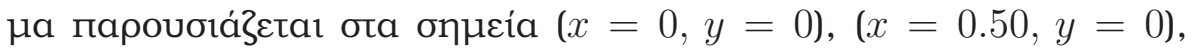

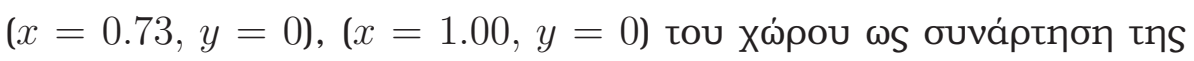
X 

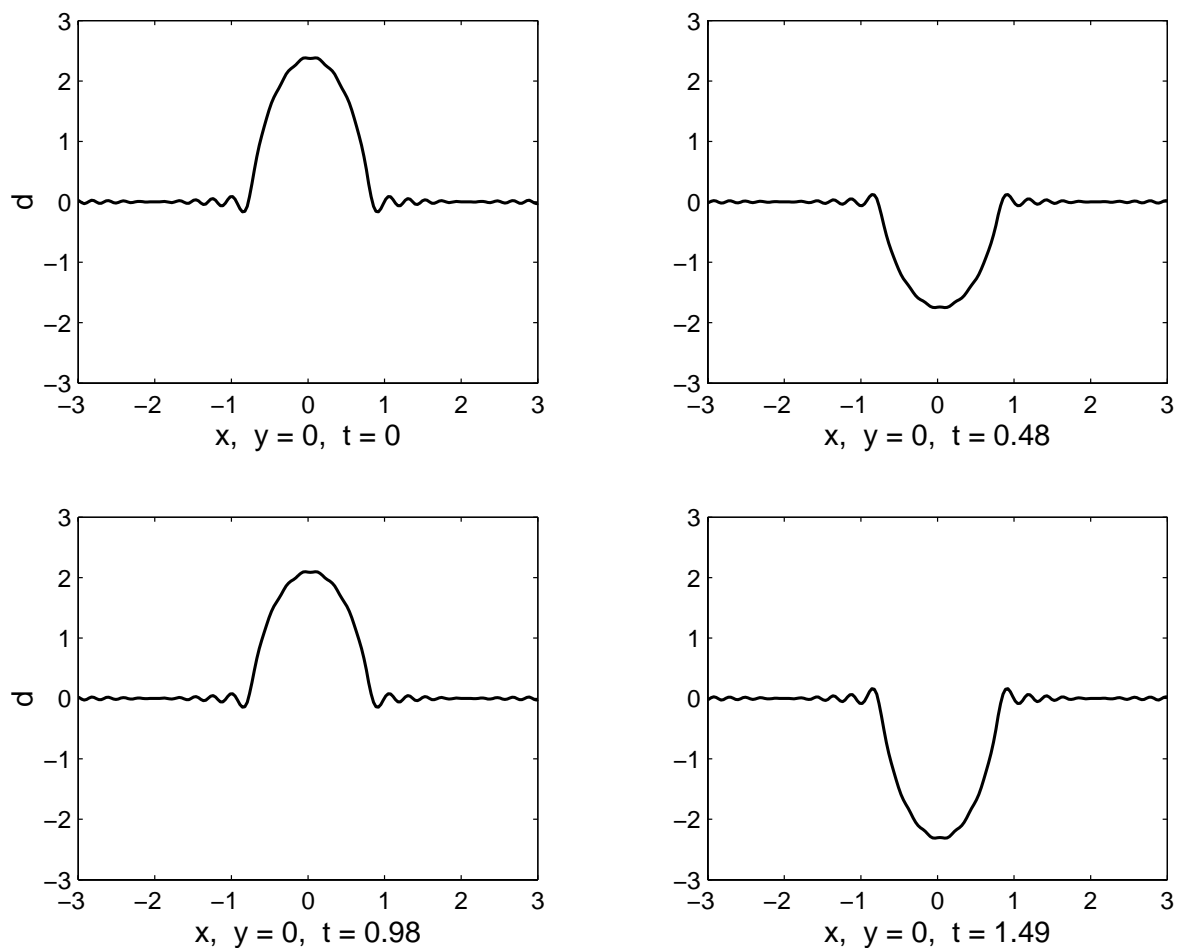

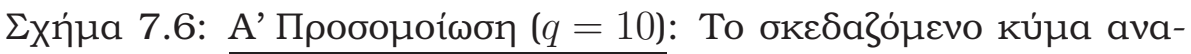

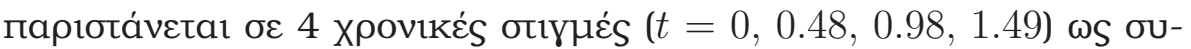

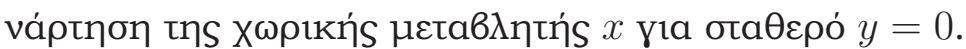

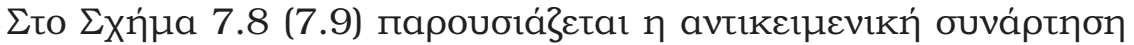

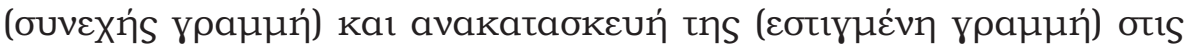

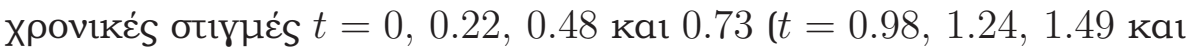

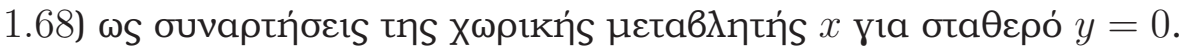



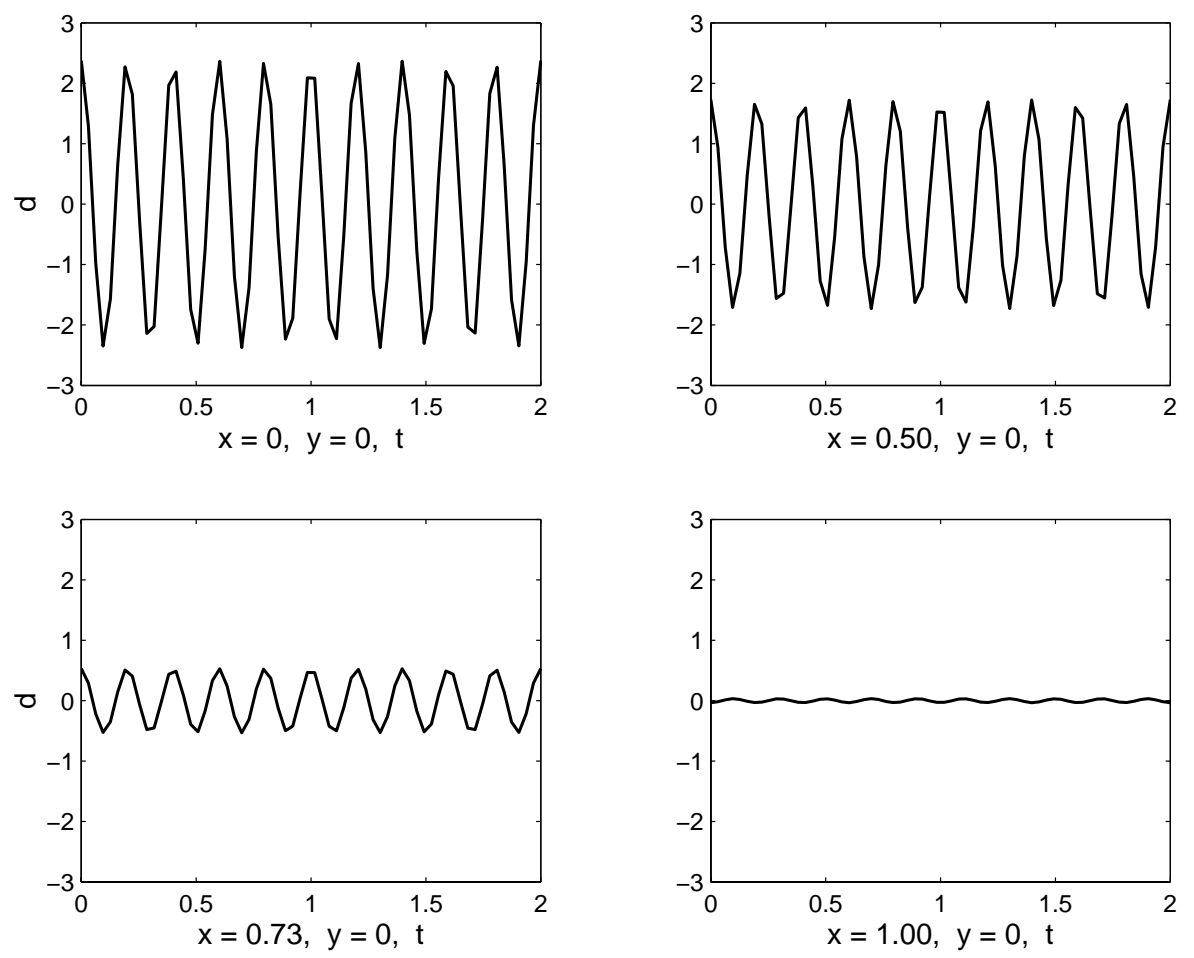

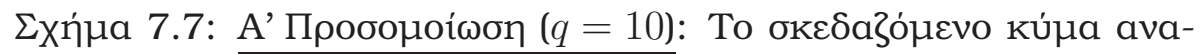

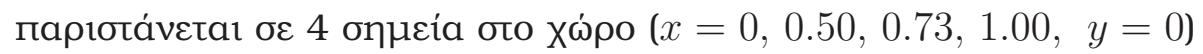

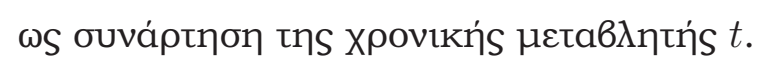



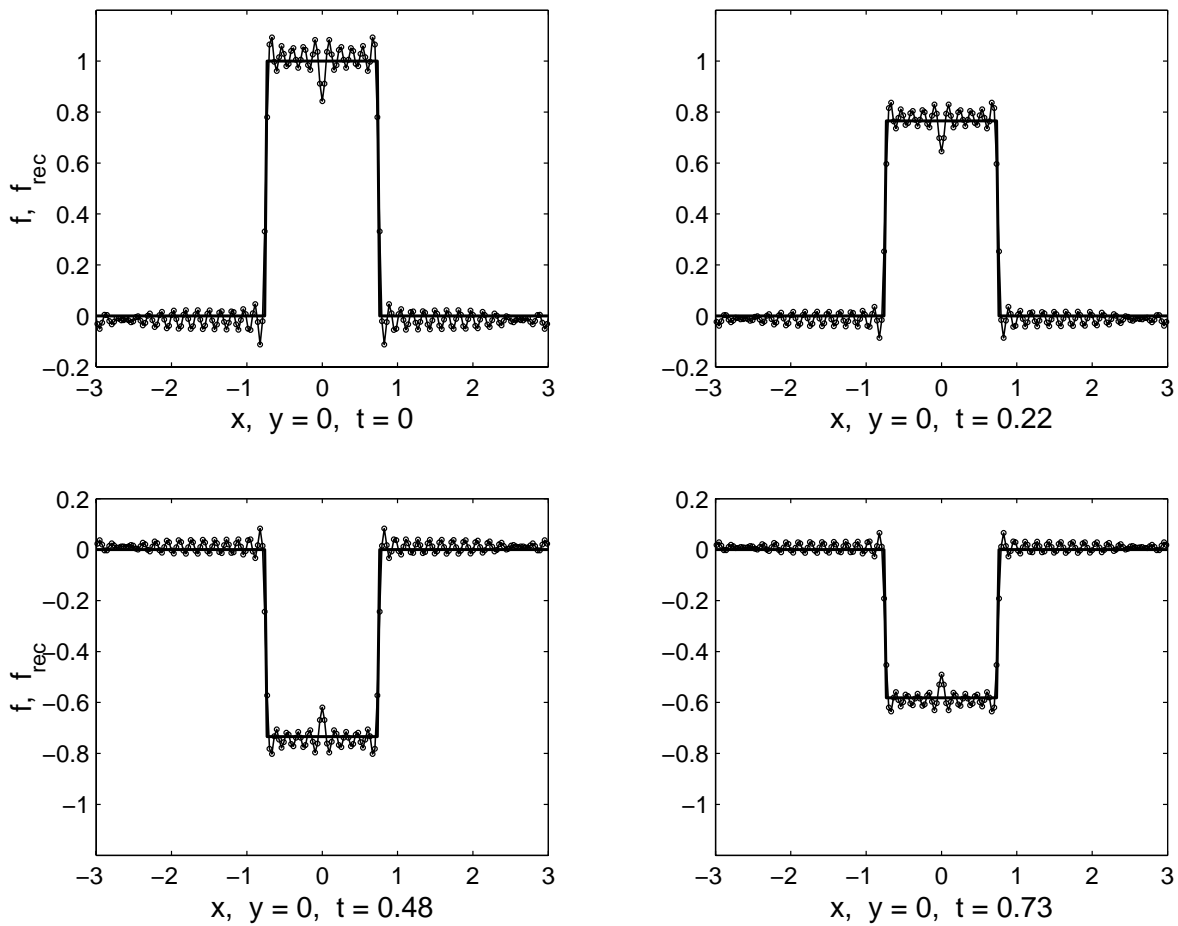

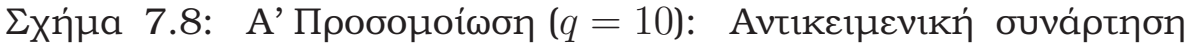

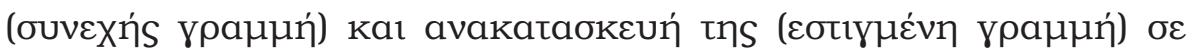

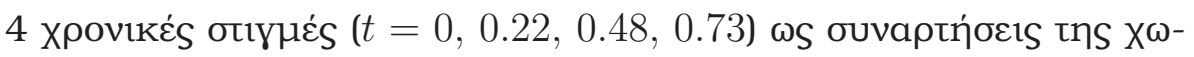

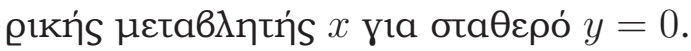



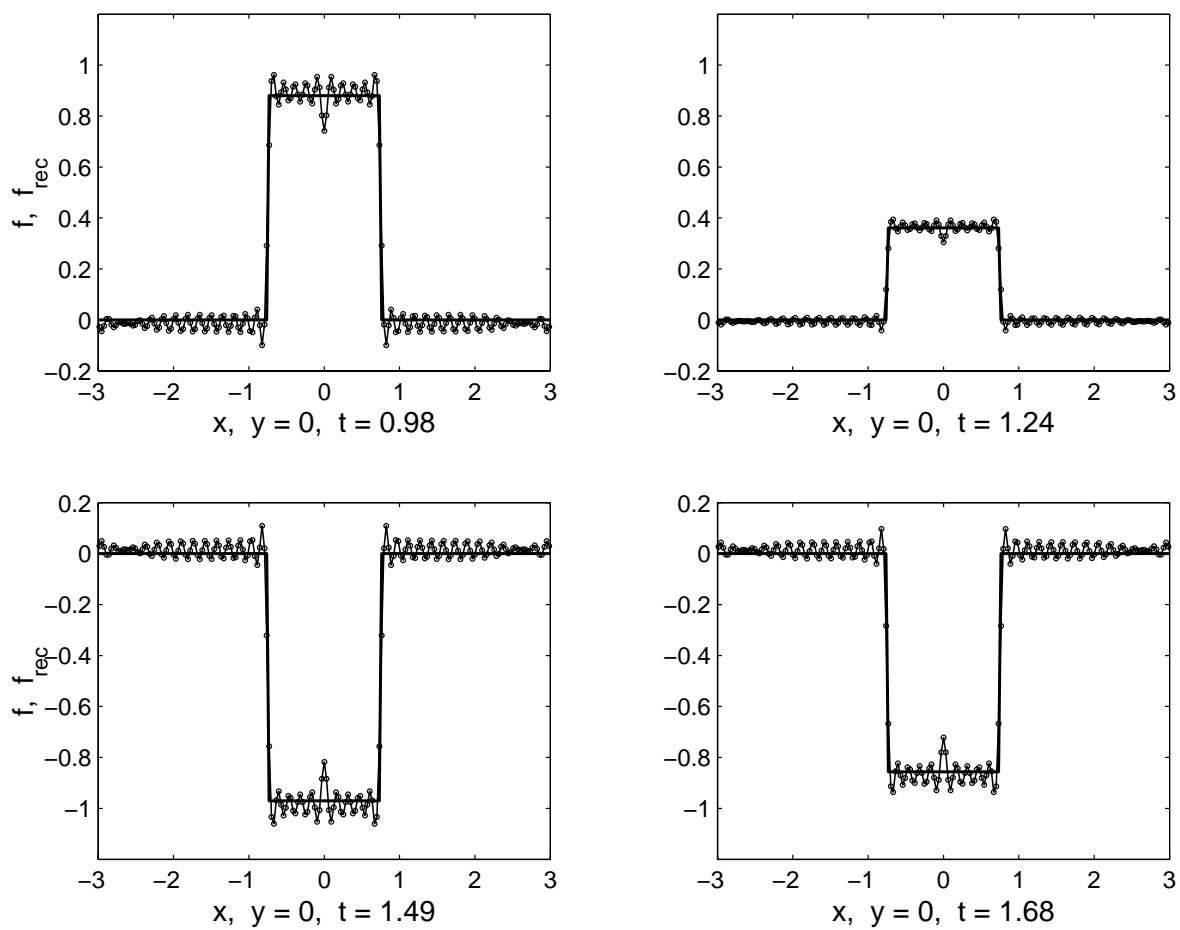

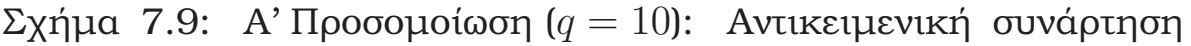

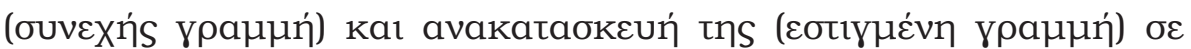

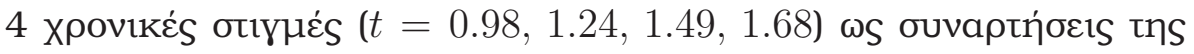

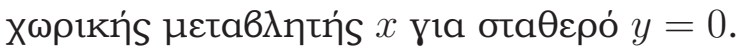




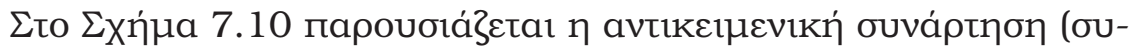

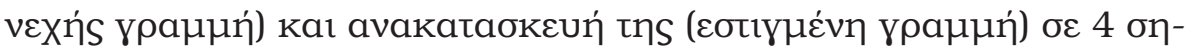

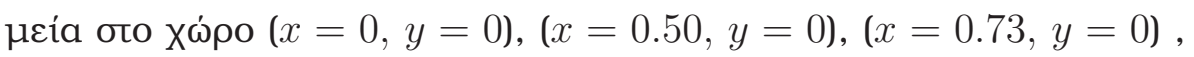

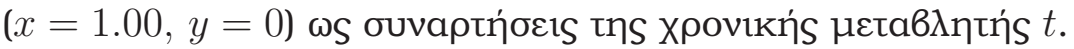
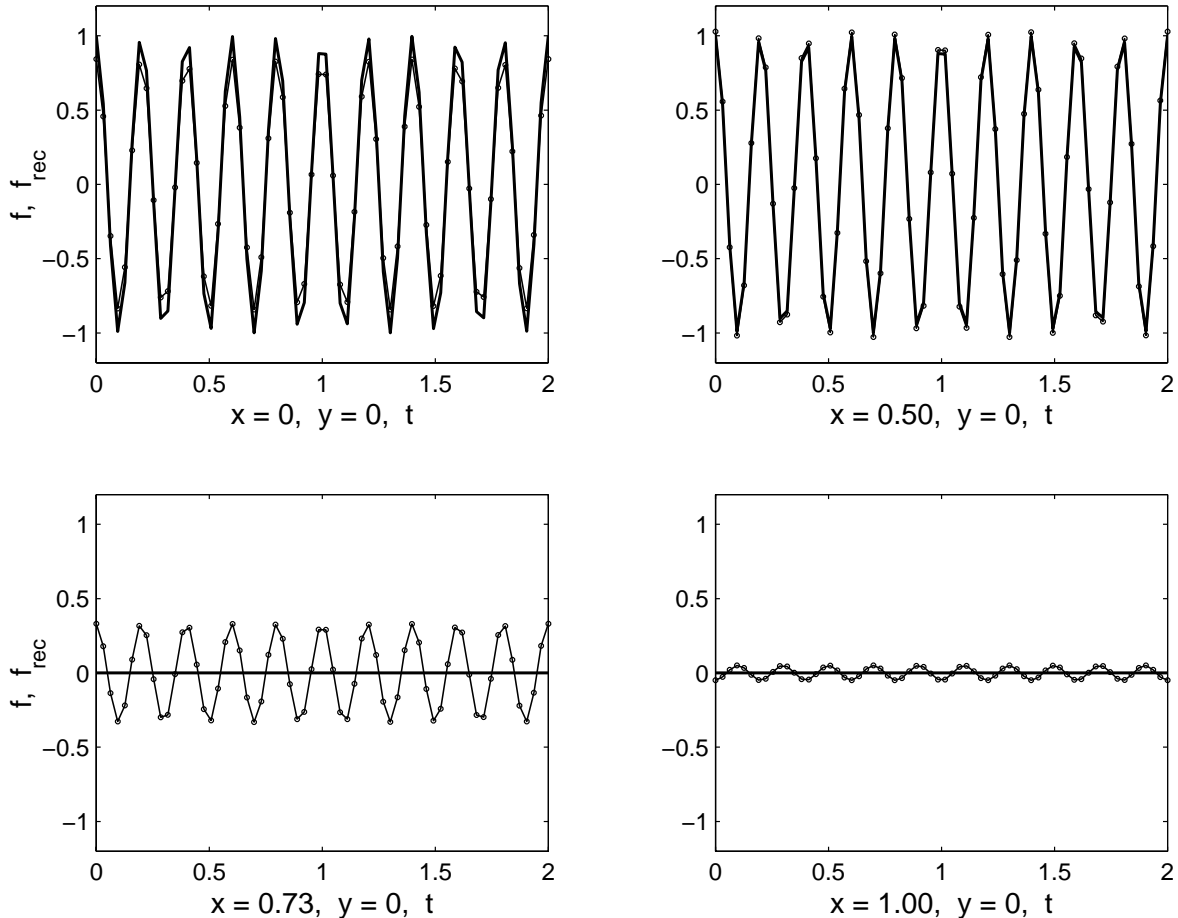

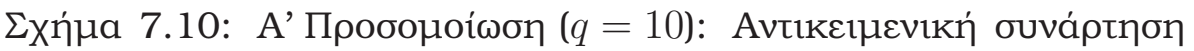

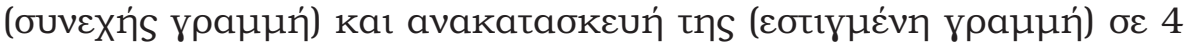

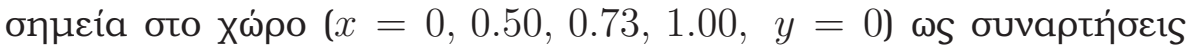

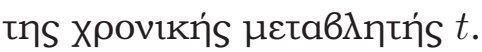




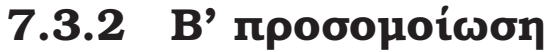

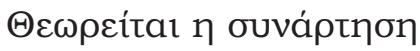

$$
\begin{array}{rl}
g(t)=-0.75 \cos \frac{2 \pi t}{\alpha}+0 & .50 \cos \frac{8 \pi t}{\alpha}+0.35 \cos \frac{12 \pi t}{\alpha}+ \\
& +0.25 \cos \frac{20 \pi t}{\alpha}, \text { vıa } 0 \leq t \leq \alpha . \quad(7.24)
\end{array}
$$

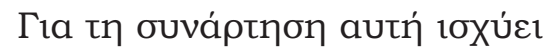

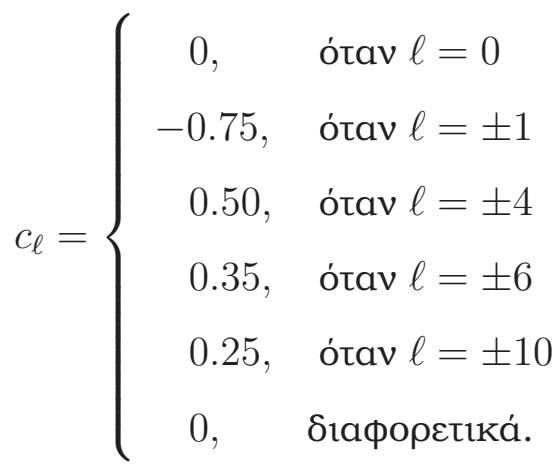



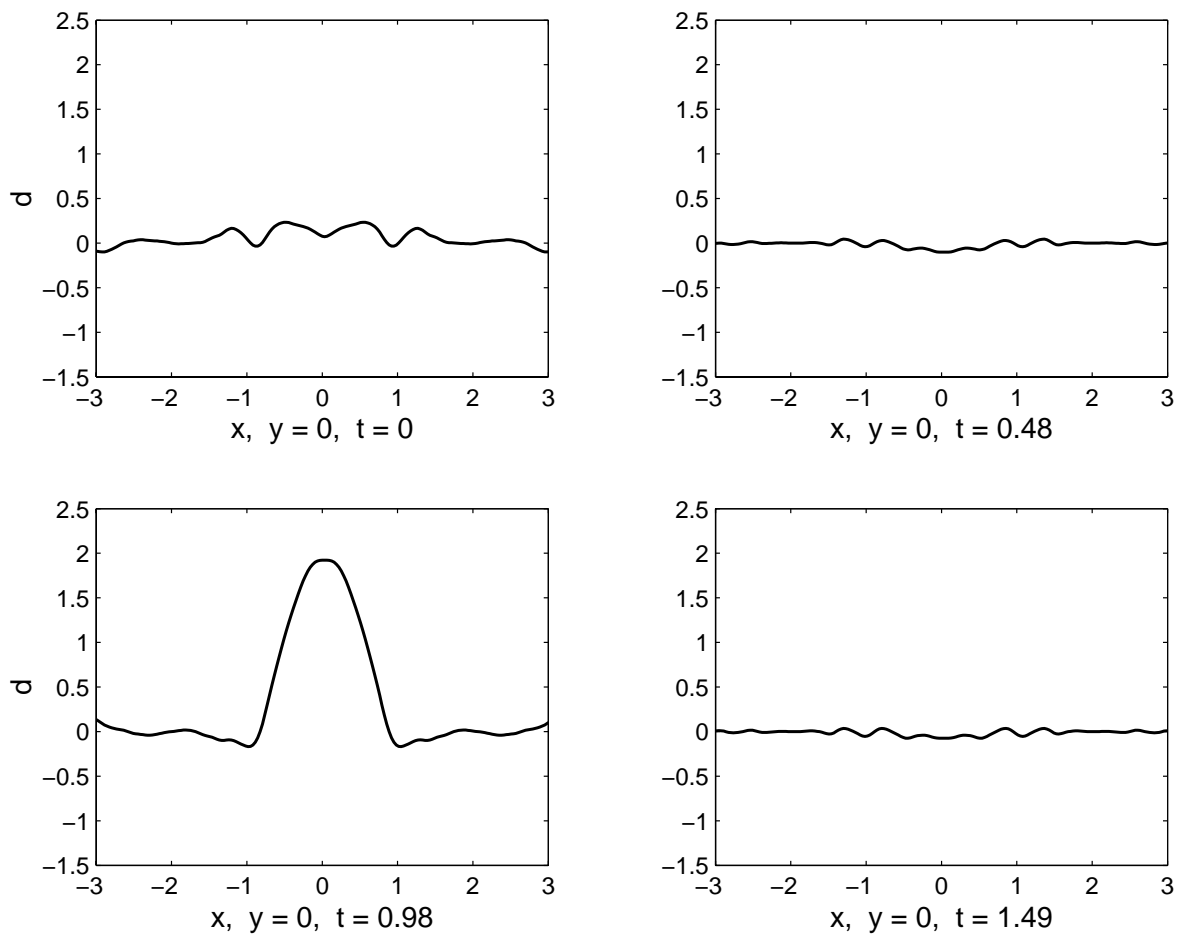

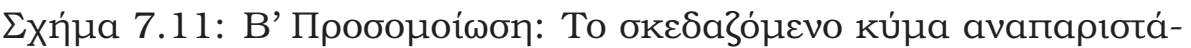

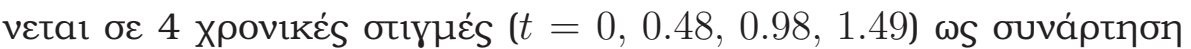

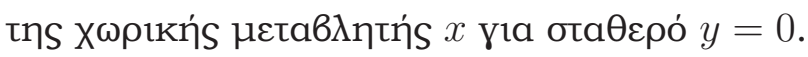

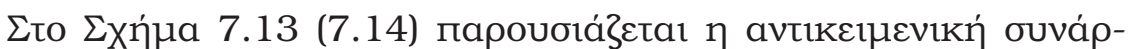

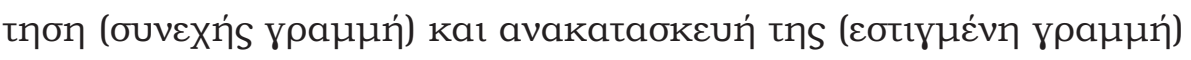

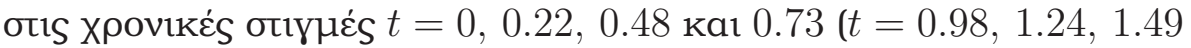

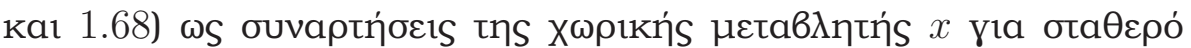
$y=0$. 

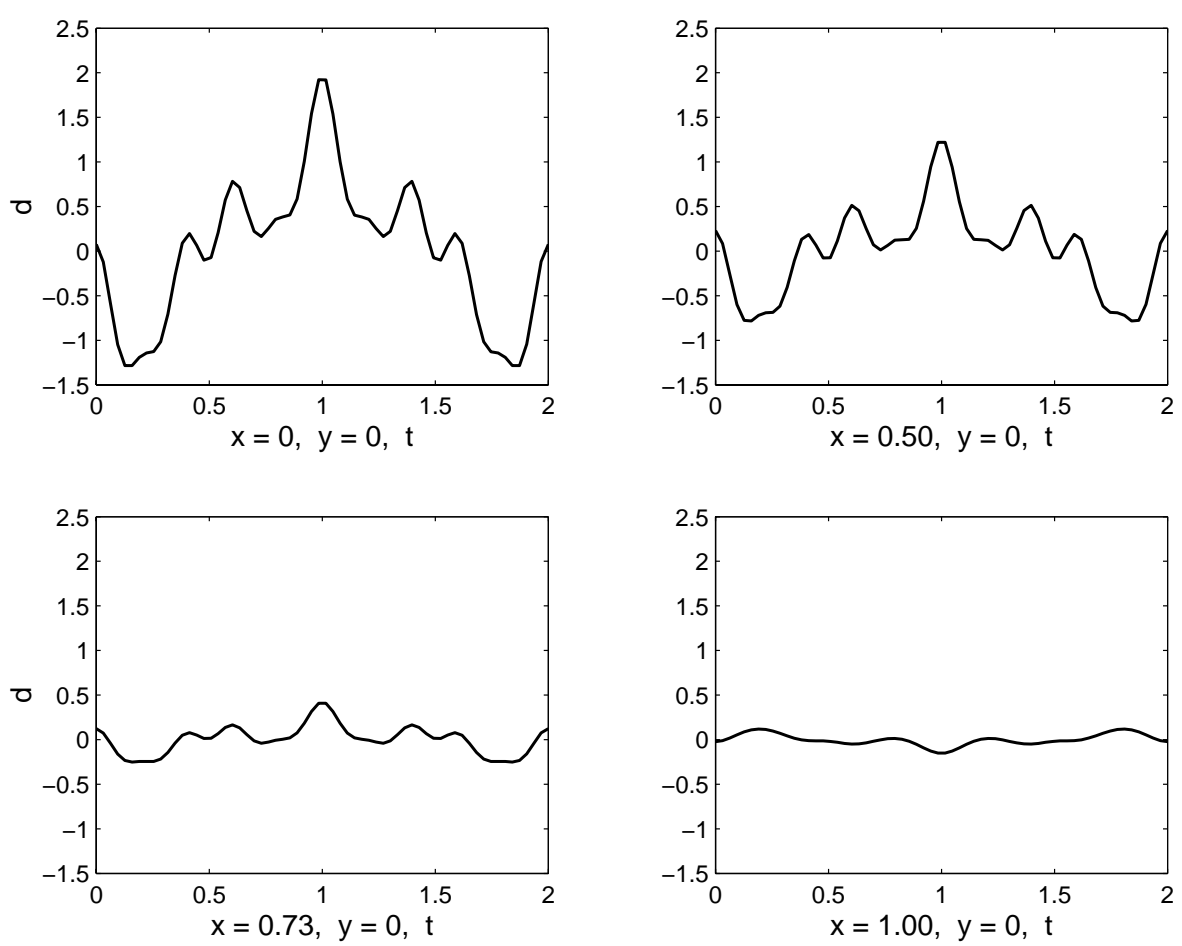

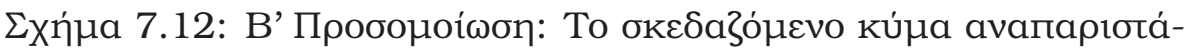

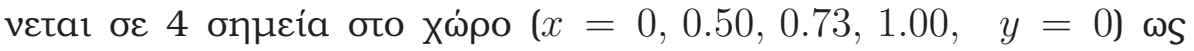

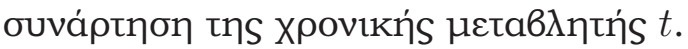



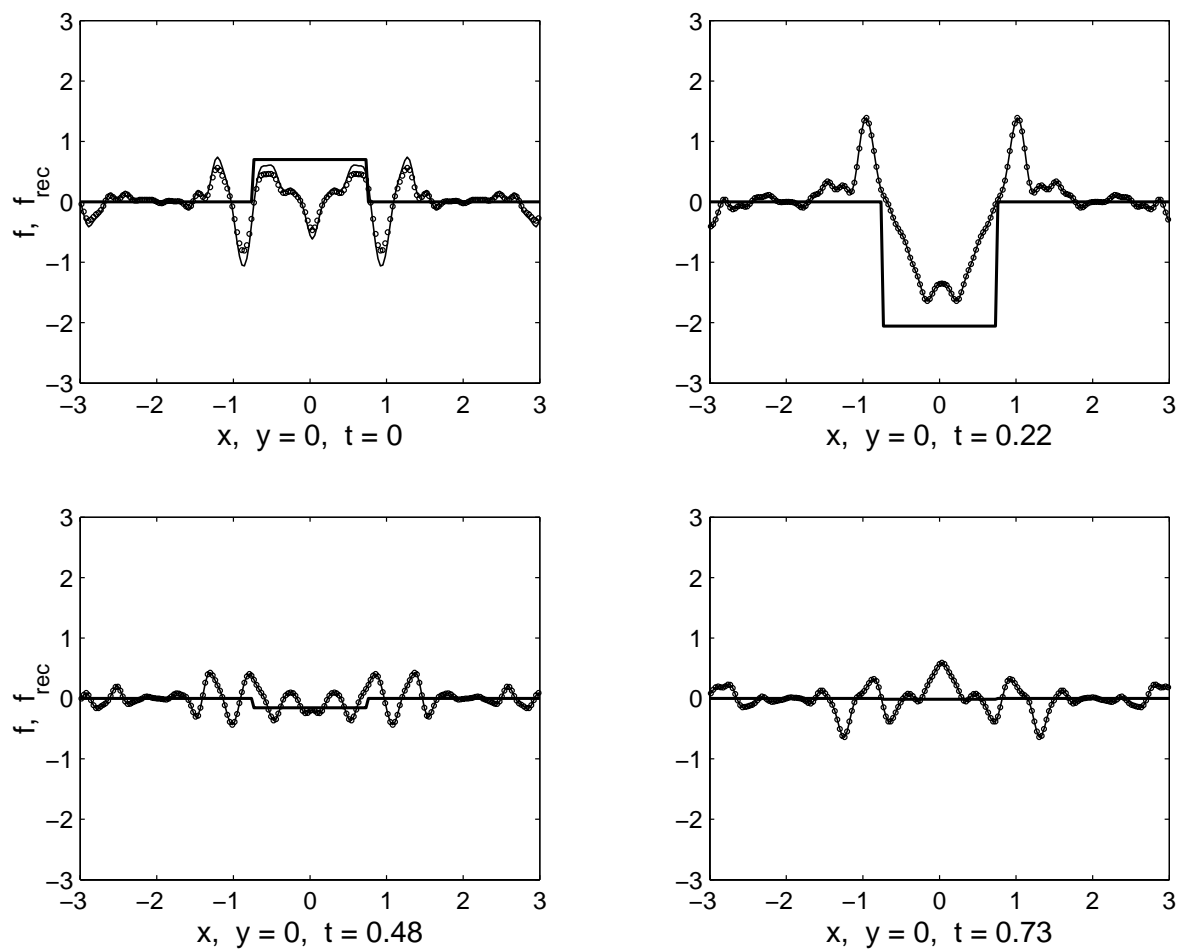

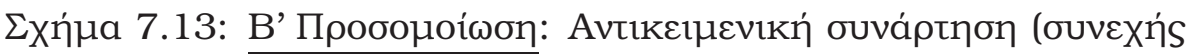

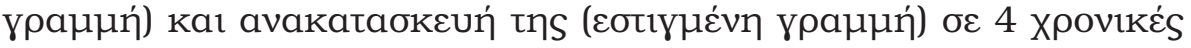

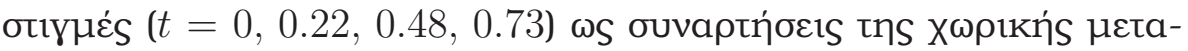

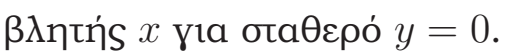



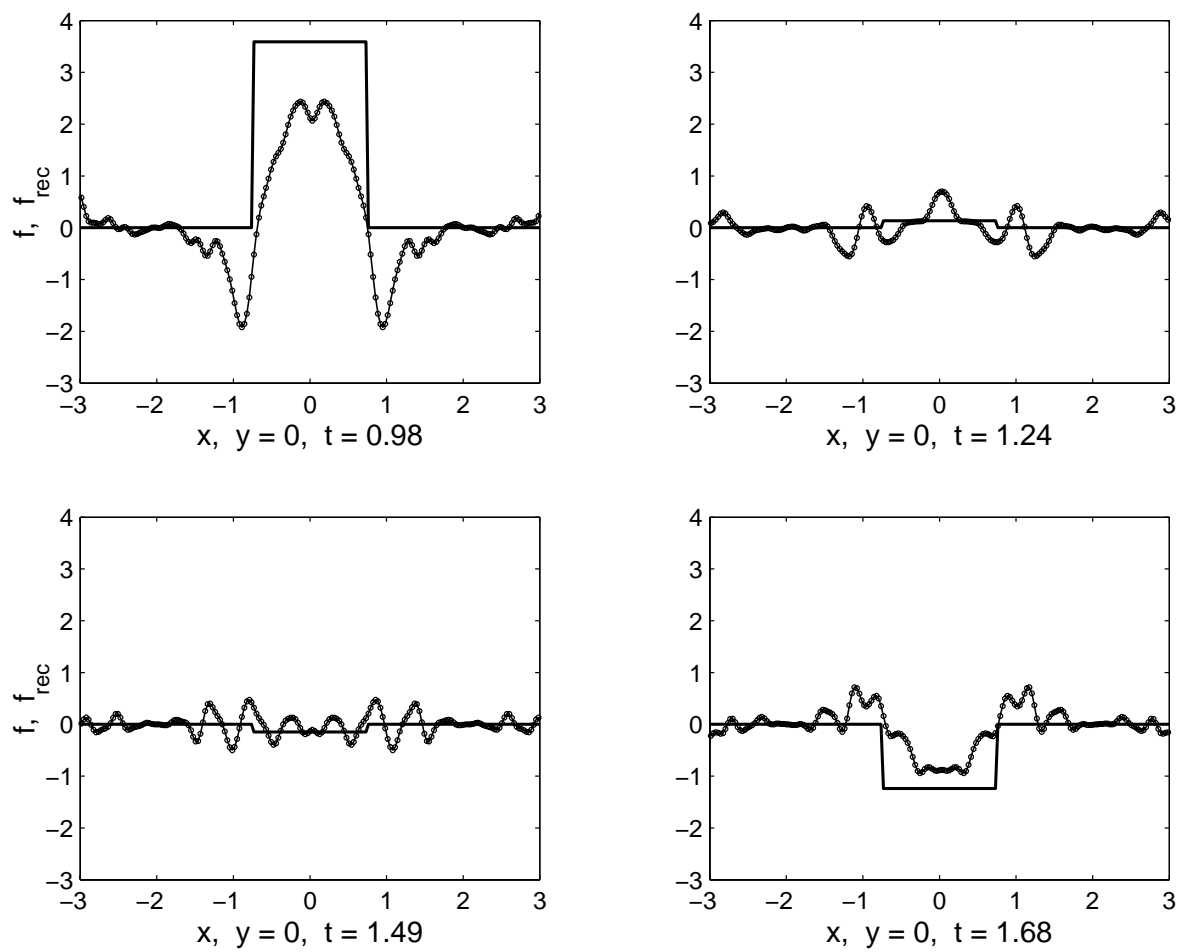

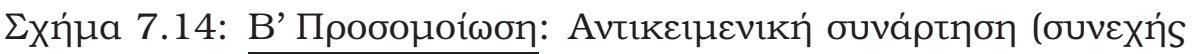

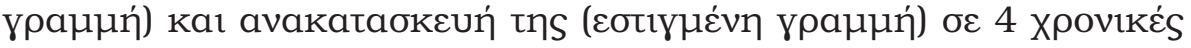

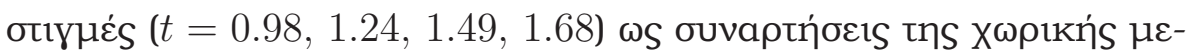

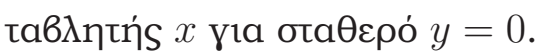




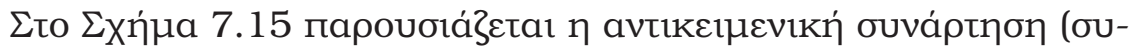

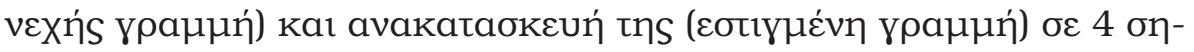

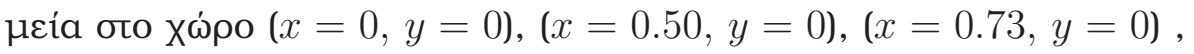

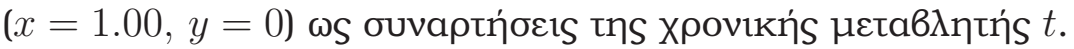
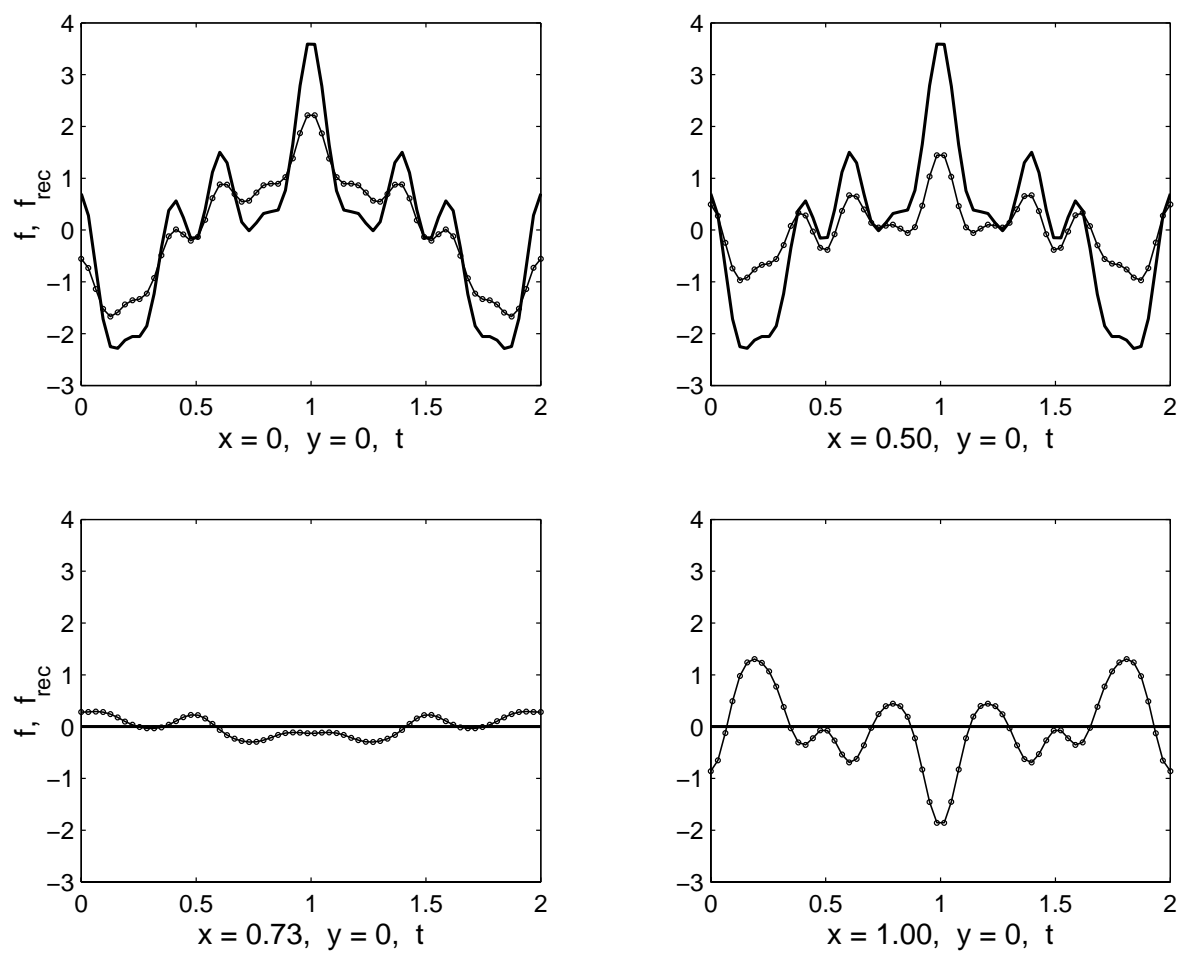

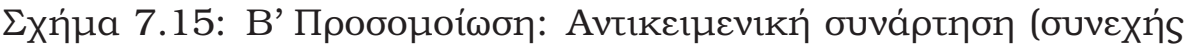

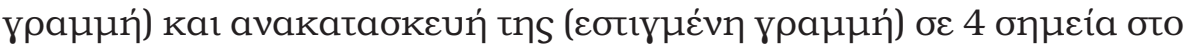

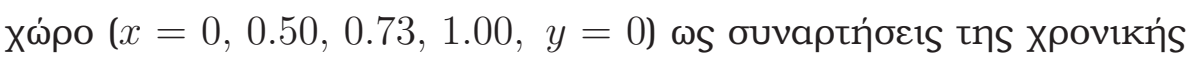

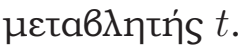




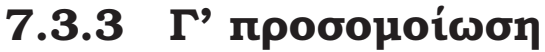

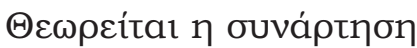

$$
\begin{aligned}
g(t)=1-0.75 \cos \frac{2 \pi t}{\alpha}+ & 0.50 \cos \frac{8 \pi t}{\alpha}+0.35 \cos \frac{12 \pi t}{\alpha}+ \\
& +0.25 \cos \frac{20 \pi t}{\alpha}, \text { via } 0 \leq t \leq \alpha .
\end{aligned}
$$

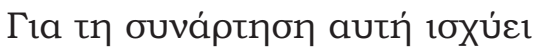

$$
c_{\ell}=\left\{\begin{aligned}
1.00, & \text { ótav } \ell=0 \\
-0.75, & \text { ótav } \ell= \pm 1 \\
0.50, & \text { ótav } \ell= \pm 4 \\
0.35, & \text { ótav } \ell= \pm 6 \\
0.25, & \text { ótav } \ell= \pm 10 \\
0, & \text { Sıафорет1кá. }
\end{aligned}\right.
$$



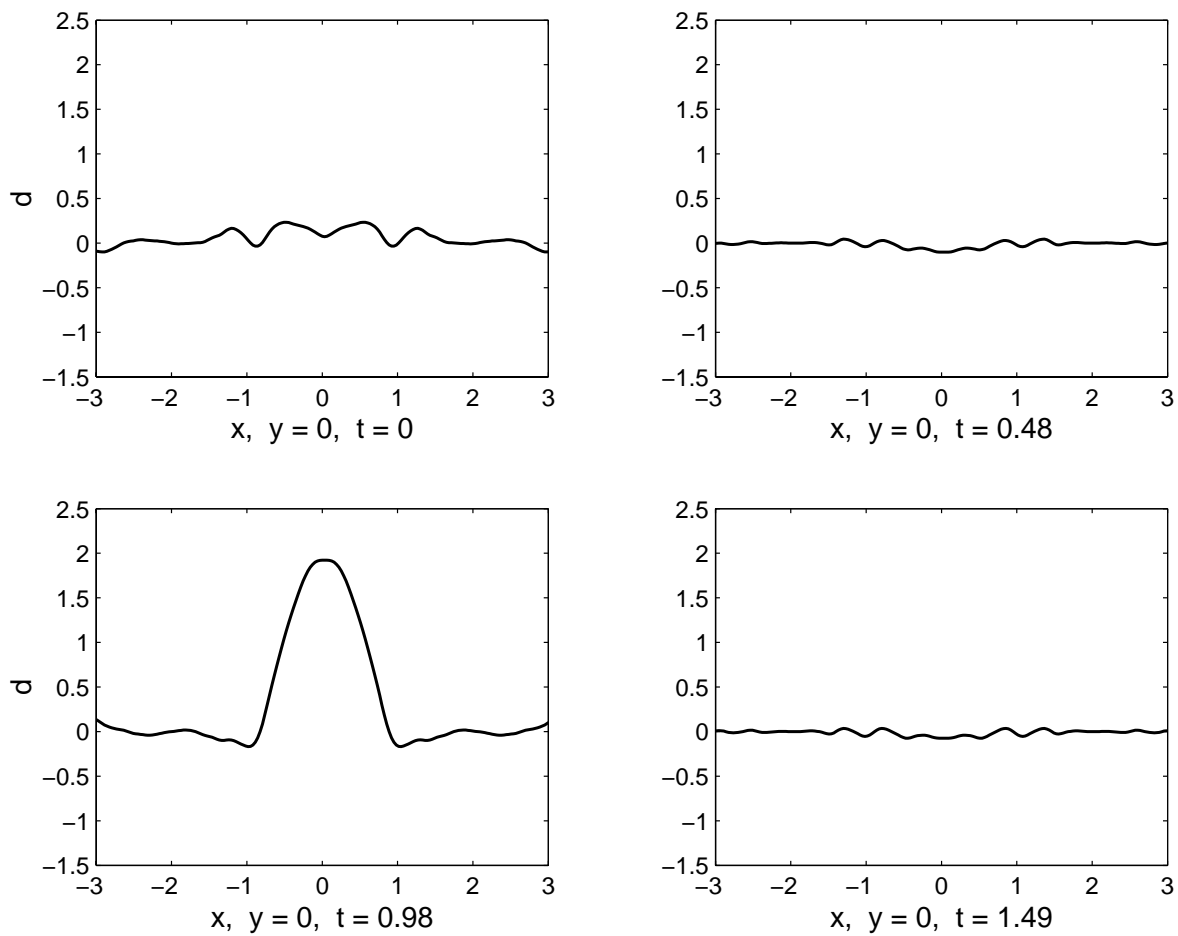

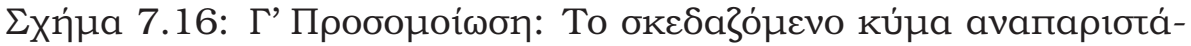

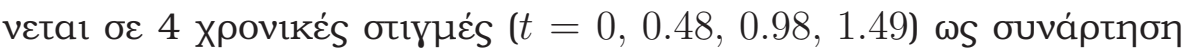

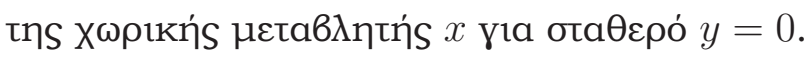

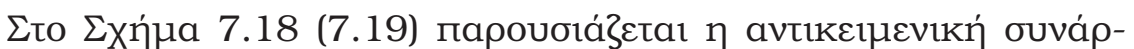

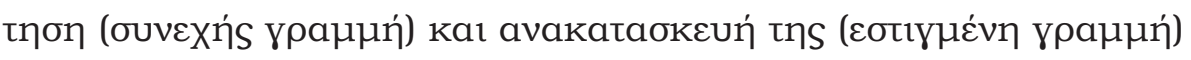

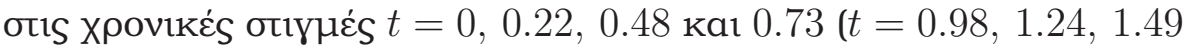

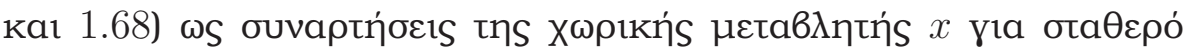
$y=0$. 

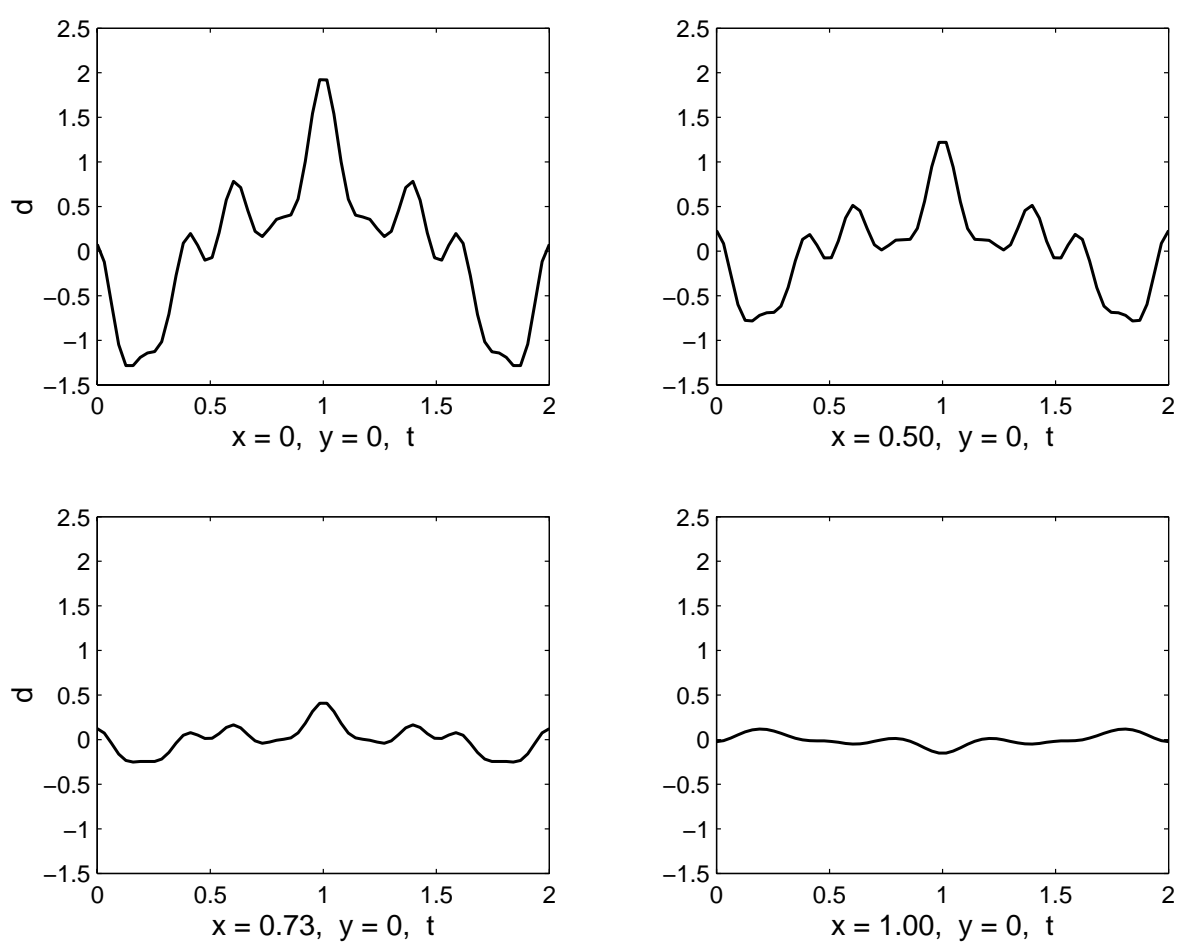

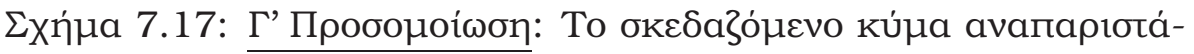

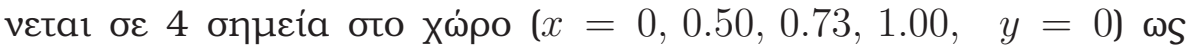

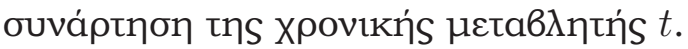



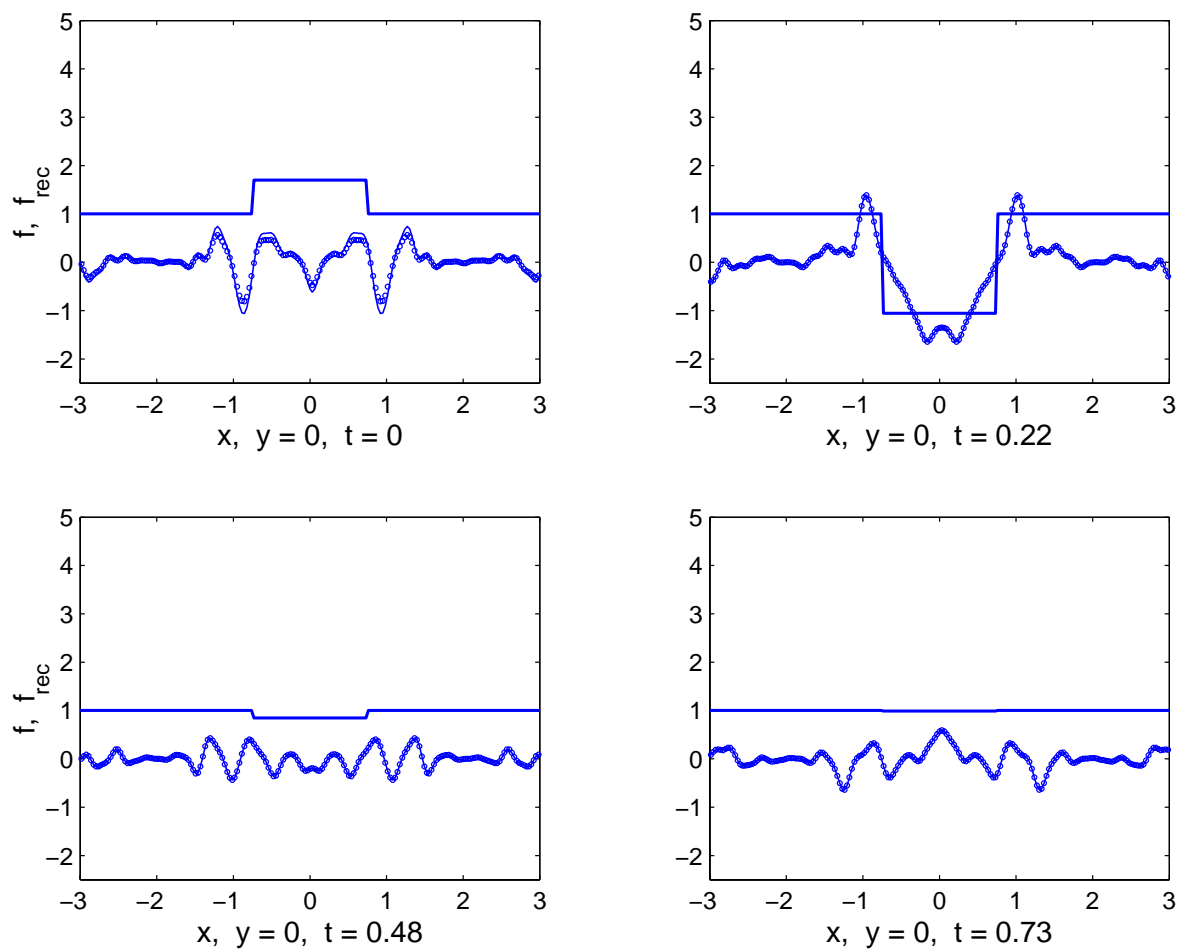

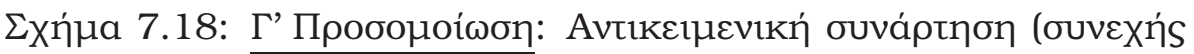

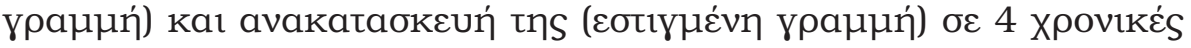

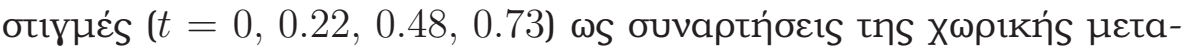

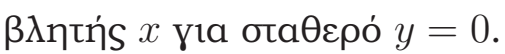



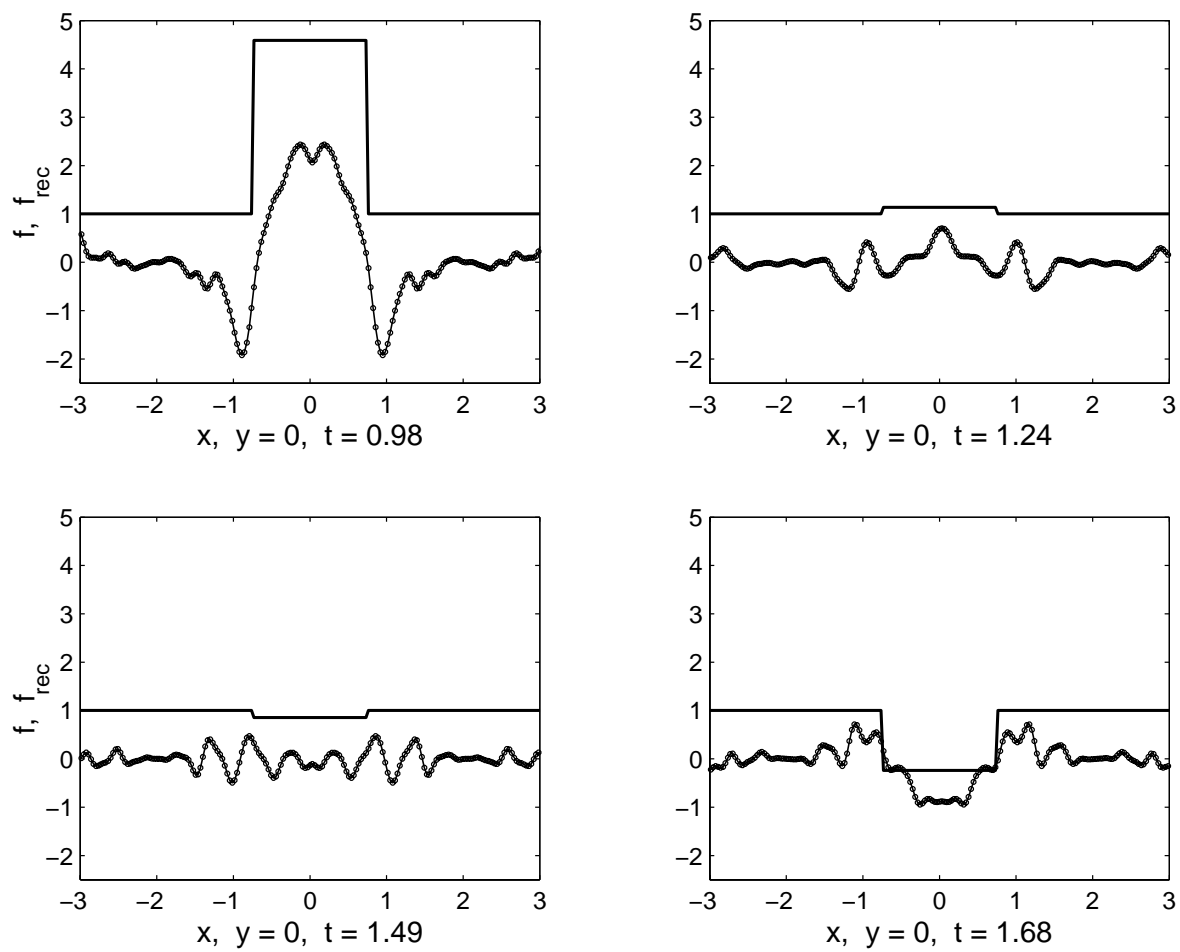

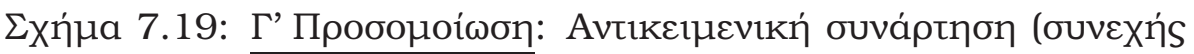

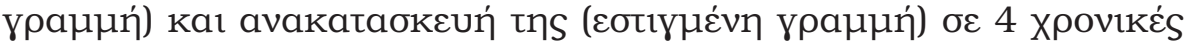

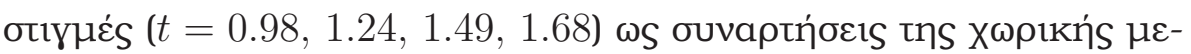

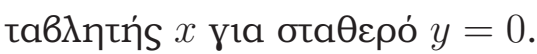




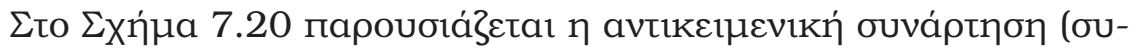

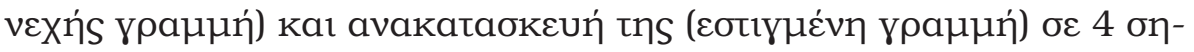

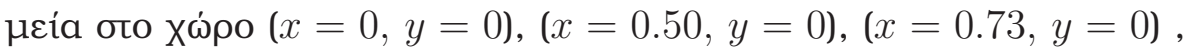

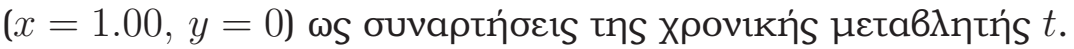
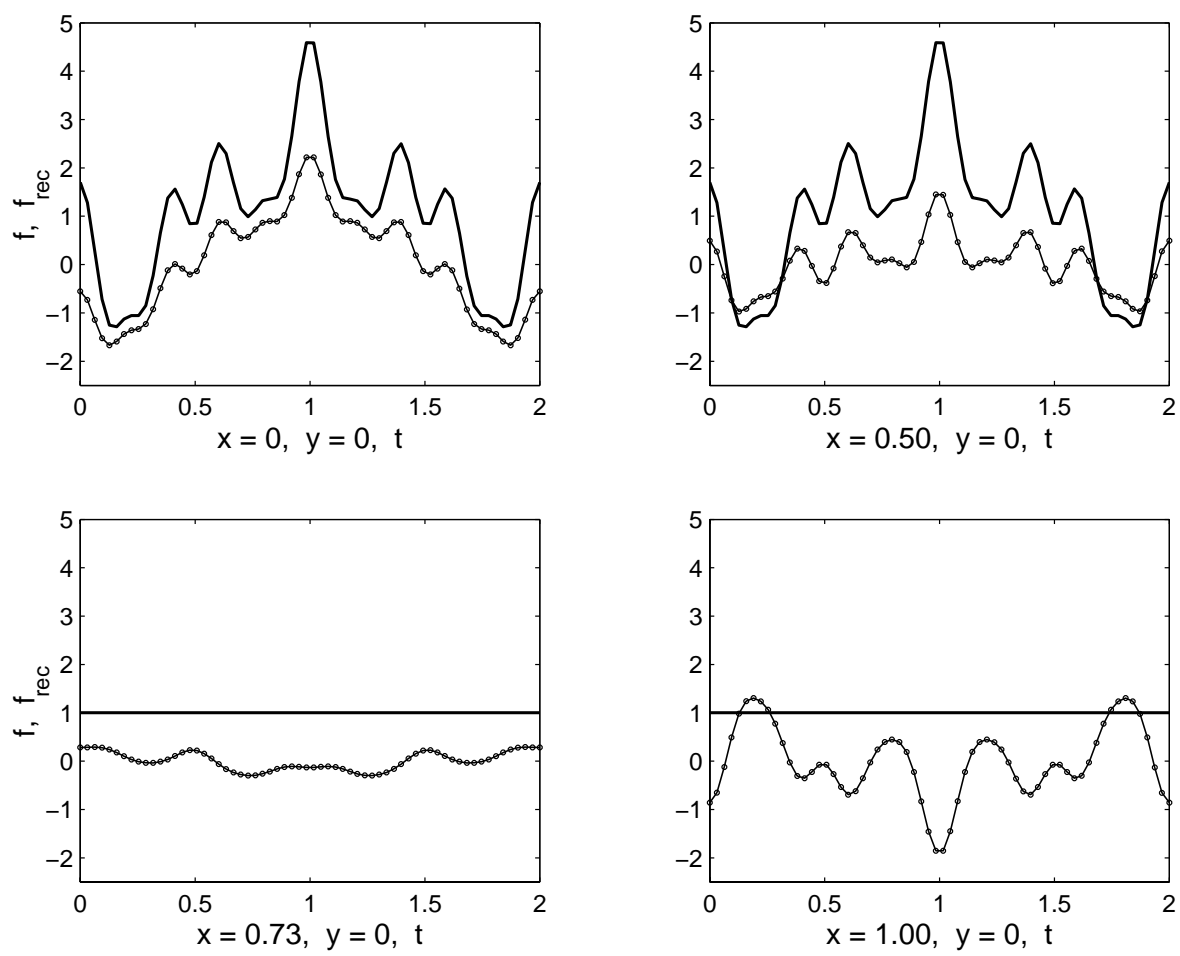

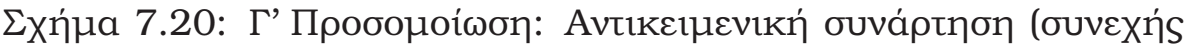

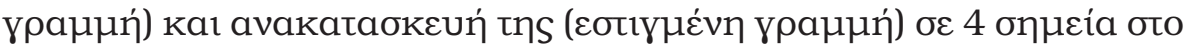

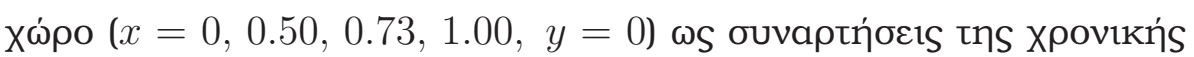

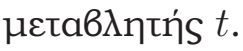




\section{KeфáXaıo 8}

\section{Eríioyos}

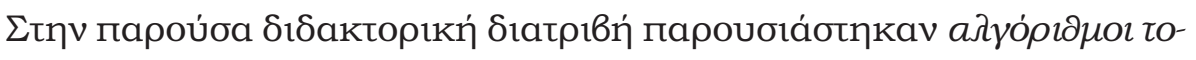

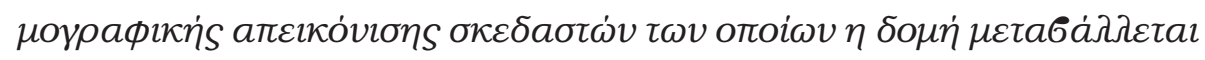

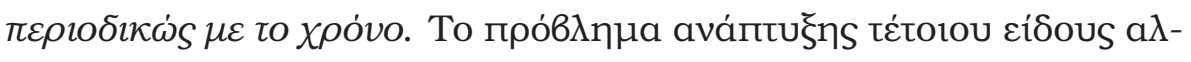

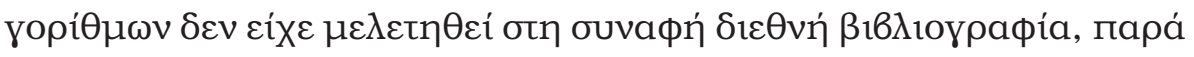

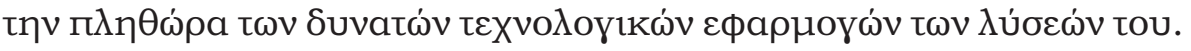

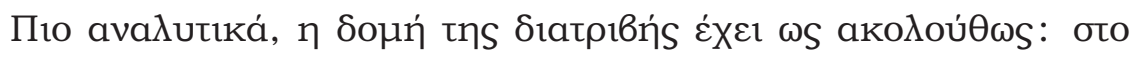

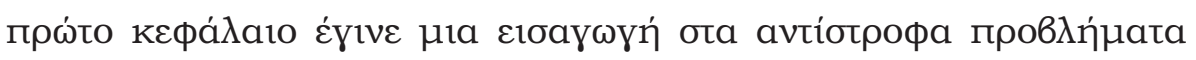

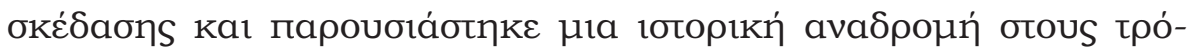

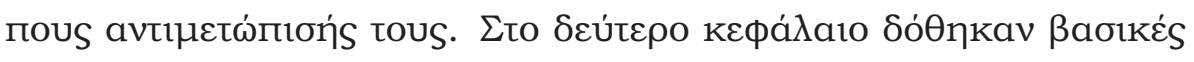

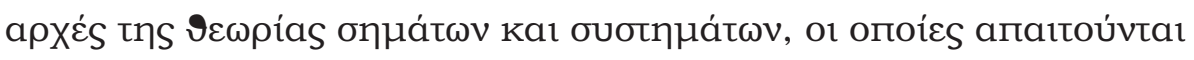

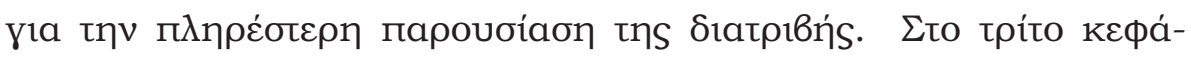

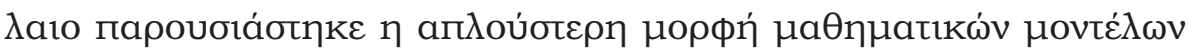

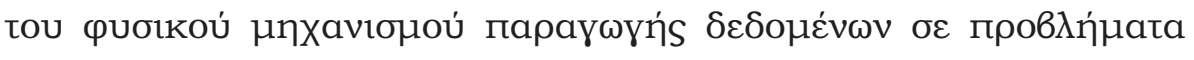

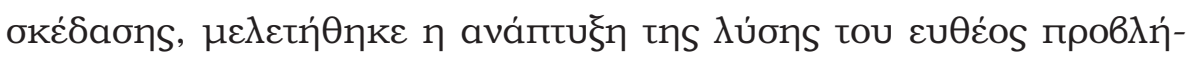

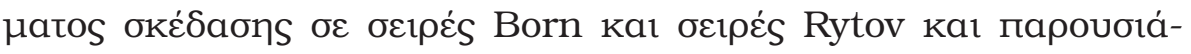




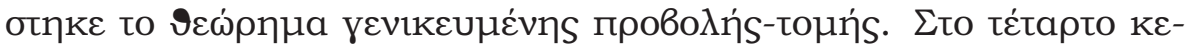

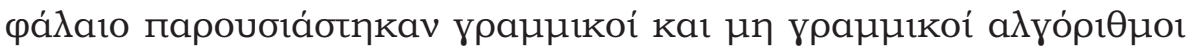

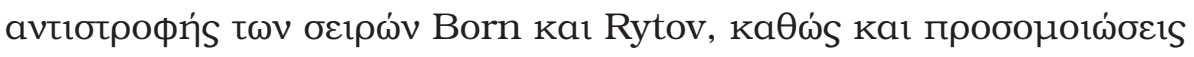

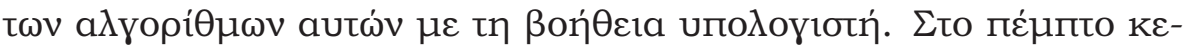

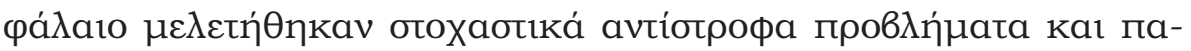

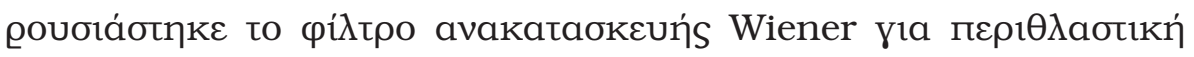

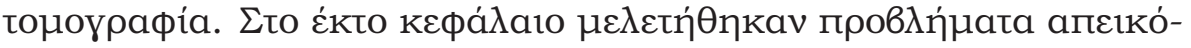

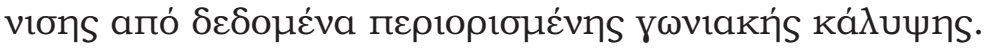

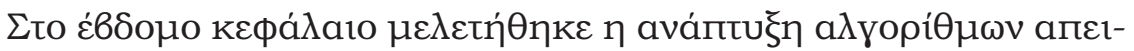

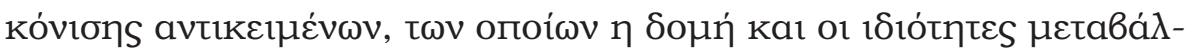

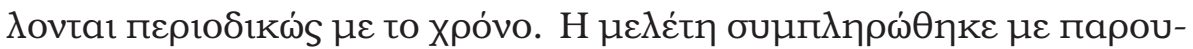

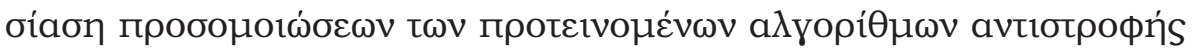

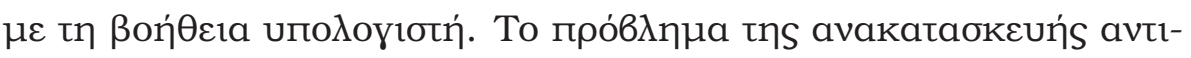

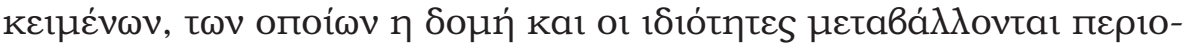

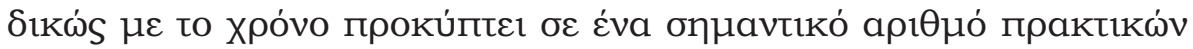

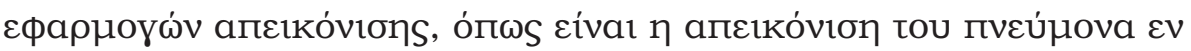

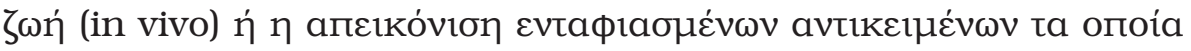

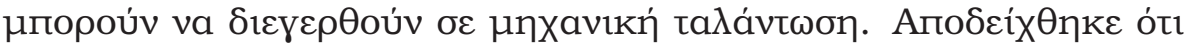

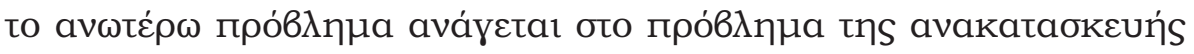

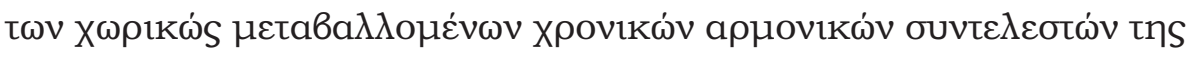

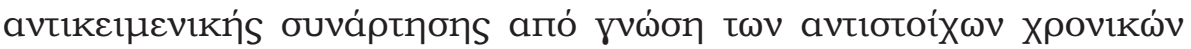

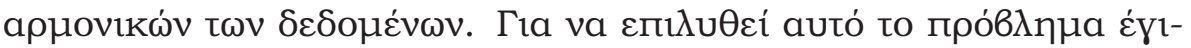

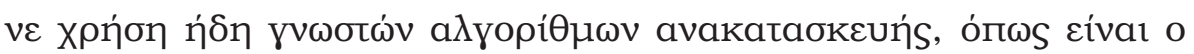

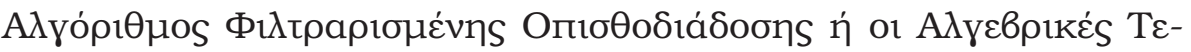

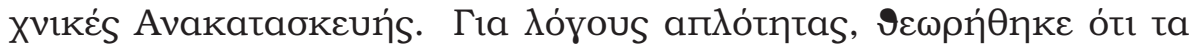




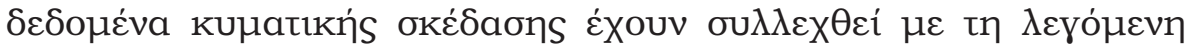

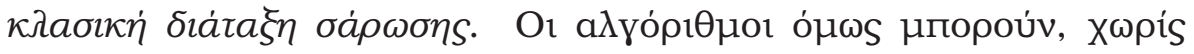

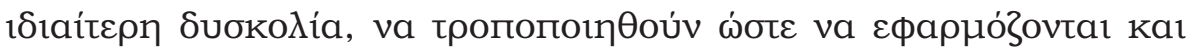

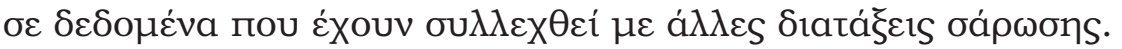

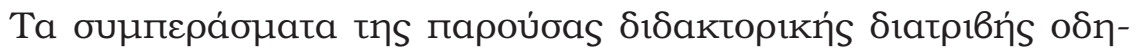

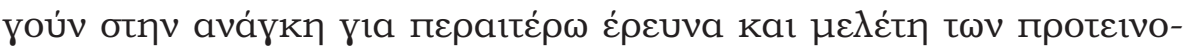

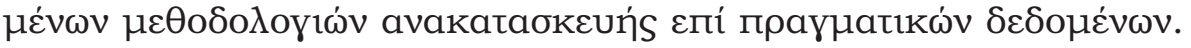

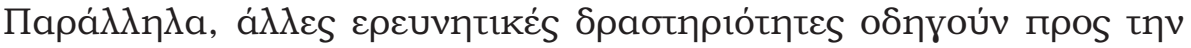

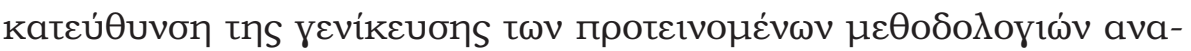

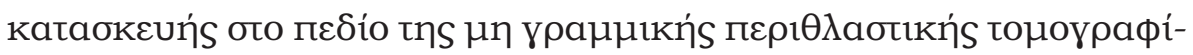

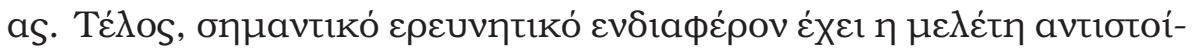

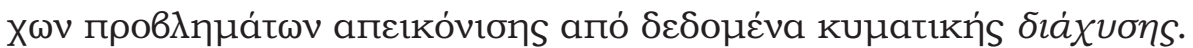




\section{Bibitoypaqia}

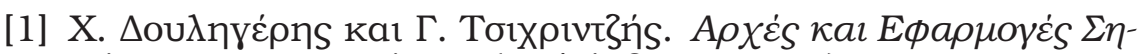

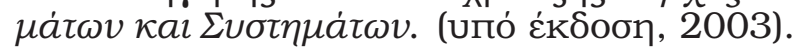

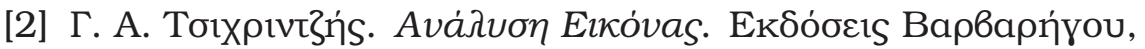
Пвıрaıú́s, 2002.

[3] A. Addivinola, A. Brancasio, G. Leone and R. Pierri. Microwave tomography by a quadratic model: numerical results for the cylindrical case. Eto Proc. Int. Conf. Electromagnetics in Advanced Applications, Turin, 1995.

[4] A B Weglein and F A Gasparotto and P M Carvalho and R H Stolt. An inverse-scattering series method for attenuating multiples in seismic reflection data. Geophysics, 62:19751989, 1997.

[5] A B Weglein and K H Matson. Inverse scattering internal multiple attenuation: an analytic example and subevent

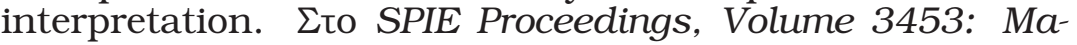

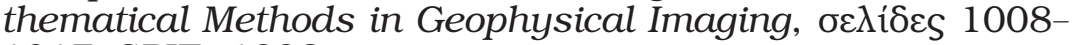
1017. SPIE, 1998.

[6] A Brancaccio and R Pierri. Information content of Born scattered fields: results in the circular cylindrical case. $J$. Opt. Soc. Am. A, 15:1909-1917, 1998.

[7] A. Brancaccio, V. Pascazio and R. Pierri. A quadratic model for inverse profiling: the one-dimensional case. J. Electromag. Waves Appl., 9:673-96, 1995.

[8] A C Kak and M Slaney. Principles of Computerized Tomographic Imaging. IEEE Press, New York, 1988.

[9] A. J. Devaney. Nonuniqueness in the inverse scattering problem. J. Math. Phys., 19:1526, 1978. 
[10] A. J. Devaney. A computer simulation study of diffraction tomography. IEEE Trans. Bio. Eng., BME-30:377, 1982.

[11] A. J. Devaney. A filtered backpropagation algorithm for diffraction tomography. Ultrasonic Imaging, 4:336, 1982.

[12] A. J. Devaney. Geophysical diffraction tomography. IEEE Trans. Geosci. and Remote Sensing, GE-22:3, 1984.

[13] A. J. Devaney. Reconstructive tomography with diffracting wavefields. Inverse Problems, 2:161, 1986.

[14] A. J. Devaney. Structure determination from intensity measurements in scattering experiments. Physical Review Letts., 62:2385, 1989.

[15] A. J. Devaney. The limited-view problem in diffraction tomography. Inverse Problems, 5:501, 1989.

[16] A. J. Devaney. Inverse scattering and diffraction tomography using intensity data. Eto Acoustical Imaging, Vol. 18,

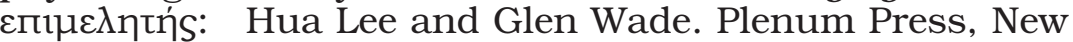
York, 1990.

[17] A. J. Devaney. Diffraction tomographic reconstruction from intensity data. IEEE Trans. Image Processing, IP-1:221228,1992

[18] A. J. Devaney and D. Zhang. Geophysical diffraction tomography in a layered background. Wave Motion, 14:243, 1991.

[19] A J Devaney and E Wolf. A new perturbation expansion for inverse scattering from three-dimensional finite-range potentials. Physics Letters, 89A(6):269-272, 1982.

[20] A J Devaney and G A Tsihrintzis. Maximum likelihood estimation of object location in diffraction tomography. IEEE Trans. Signal Processing, SP-39(3):672, 1991.

[21] A. J. Devaney and G. C. Sherman. Nonuniqueness in inverse source and scattering problems. IEEE Trans. Ant. and Propag., AP-30:1034, 1982.

[22] A. J. Devaney and M. L. Oristaglio. Inversion Procedure for Inverse Scattering within the Distorted Wave Born Approximation. Physical Review Letts., 51:237, 1983.

[23] A J Witten and J E Molyneux and J E Nyquist. Ground penetrating radar tomography: Algorithms and case studies. IEEE Trans. Geo. Rem Sens., GRS-32:461-467, 1994. 
[24] A. K. Jain. Fundamentals of Digital Image Processing. Prentice Hall, Englewood Cliffs, 1989.

[25] A. K. Jain and S. Ansari. Radon transform theory for random fields and optimum image reconstruction from noisy

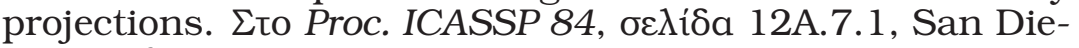
go, California, 1984.

[26] A N Kolmogorov and S V Fomin. Elements de la Theorie des Fonctions et de l' analyse Fonctionelle. Mir, Moscow, 1973.

[27] A Q Howard and W C Chew and M C Moldoveanu. A new correction to the Born approximation. IEEE Trans. Geosci. Remote Sensing, 28(3):394-399, May 1990.

[28] A Witten. Sounding out buried waste. Civil Engineering, 60:62-64, 1990.

[29] A Witten. Application of a maximum likelihood estimator to tunnel detection. Inverse Problems, 7:L49-L55, 1991.

[30] A Witten and D Gillette and W C King and J Sypniewski. Geophysical diffraction tomography at a dinosaur site. Geophysics, 57:187-195, 1992.

[31] A Witten and W C King. Acoustical imaging of subsurface features. J. Envir. Eng., 116:166, 1990.

[32] C L Byrne and M A Fiddy. Images as power spectra: Reconstruction as a Wiener filter approximation. Inverse Problems, 4:399, 1988.

[33] C W Groetsch. The Theory of Tikhonov Regularization fro Fredholm Euations of the First Kind. Pitman, Boston, Massachusetts, 1984.

[34] D. Colton and P. Monk. The inverse scattering problem for acoustic waves in an inhomogeneous medium. Eto Inverse

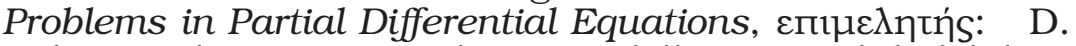
Colton and R. Ewing and W. Rundell. SIAM, Philadelphia, 1990.

[35] D Colton and R Kress. Inverse Acoustic and Electromagnetic Scattering. Springer-Verlag, Berlin, 1998. (2nd edition).

[36] D J Rossi and A S Willsky. Reconstruction from Projections Based on Detection and Estimation of Objects, Parts I and II: Performance Analysis and Robustness Analysis. IEEE Trans. Acous., Speech, and Signal Process., ASSP-32:886, 1984. 
[37] D. M. Young. Iterative solution of large linear systems. Academic Press, New York, 1971.

[38] D Rouseff and R P Porter. Diffraction tomography and the stochastic inverse scattering problem. J. Acoust. Soc. Am., 89:1599, 1991.

[39] D.R. Cox. Renewal Theory. Chapman and Hall, London, 1967.

[40] E J Witterholt and J L Kretzsehmar and K L Jay. The application of crosshole electromagnetic wave measurements to mapping of a steam flood. Eto Proc. Petroleum Society of CIM. Petroleum Society of CIM, 1982.

[41] E. Masry. Alias-free sampling: an alternative conceptualization and its applications. IEEE Trans. Inf. Theory, IT-24 (3):317-246, 1978.

[42] E Wolf. Three dimensional structure determination of semi transparent objects from holographic data. Opt. Commun., 1:153, 1969.

[43] E Wolf. Principles and development of diffraction tomo-

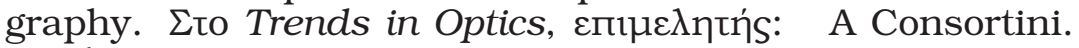
Academic Press, San Diego, 1996.

[44] F Natterer. The Mathematics of Computerized Tomography. Wiley, New York, 1986.

[45] F.J. Beutler and O. A. Leneman. Random sampling of random processes: stationary point processes. Inf. Control, 9 (4):325-46, 1966.

[46] F.J. Beutler and O. A. Leneman. The theory of stationary point processes. Acta Math., 116:159-97, 1966.

[47] G A Tsihrintzis and A J Devaney. Estimation of object location from diffraction tomographic intensity data. IEEE Trans. Signal Processing, SP-39(9):2136, 1991.

[48] G A Tsihrintzis and A J Devaney. Maximum likelihood estimation of object location in diffraction tomography, Part II: Strongly scattering objects. IEEE Trans. Signal Processing, sp-39(6):1466, 1991.

[49] G A Tsihrintzis and A J Devaney. Application of a maximum likelihood estimator in an experimental study of ultrasonic diffraction tomography. IEEE Trans. Med. Imag., MI-12:545-554, 1993. 
[50] G A Tsihrintzis and A J Devaney. Stochastic diffraction tomography: Theory and computer simulation. Signal Processing, 39:49-64, 1993.

[51] G A Tsihrintzis and A J Devaney. Stochastic geophysical diffraction tomography. Int. J. Imag. Syst. Techn., 5:239$242,1994$.

[52] G A Tsihrintzis and A J Devaney. Maximum Likelihood

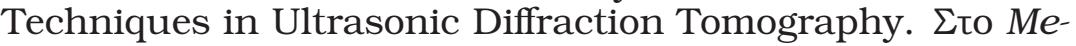

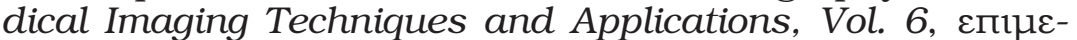

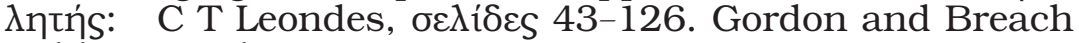
Publ., Newark, NJ, 1998.

[53] G A Tsihrintzis and A J Devaney and E Heyman. Estimation of object location from wideband scattering data. IEEE Trans. Image Proc., IP-8:996-1001, 1999.

[54] G A Tsihrintzis and P M Johansen and A J Devaney. Buried object detection and location estimation from electromagnetic field measurements. IEEE Trans. Ant. Prop., AP47:1742-1744, 1999.

[55] G. Beylkin and M. L. Oristaglio. Distorted-wave Born and distored-wave Rytov approximation. Opt. Commun., 53:213, 1985.

[56] G. N. Hounsfield. Computerized transverse axial scanning tomography: Part I, description of the system. Br. J. Radiol., 46:1016, 1973.

[57] G T Herman. Image Reconstruction from Projections: The Fundamentals of Computerized Tomography. Academic Press, New York, 1980.

[58] H.S. Shapiro and R.A. Silverman. Alias-free sampling of random noise. SIAM J. Appl. Math., 8 (2):245-8, 1960.

[59] I. Halperin. The product of projection operators. Acta Sci. Math., 23:96, 1962.

[60] J A Scales and A Gersztenkorn. Robust methods in inverse theory. Inverse problems, 4:1071-1091, 1988.

[61] J Cullum. The effective choice of the smoothing norm in regularization. Math. Computation, 33(145):149-170, Jan 1979.

[62] J E Molyneux and A J Witten. Diffraction tomographic imaging in a monostatic measurement geometry. IEEE Trans. Geo. Rem Sens., GRS-31:507-51 1, 1993. 
[63] K Chadan and D Colton and L Paivarinta and W Rundell. An Introduction to Inverse Scattering and Inverse Spectral Problems. SIAM, Philadelphia, 1997.

[64] K. Iwata and R. Nagata. Calculation of refractive index distribution from interferograms using the Born and Rytov's approximation. Japan J. Appl. Phys., 14:379, 1974.

[65] K T Ladas. Iterative Methods in Diffraction Tomography. Ph.D. Dissertation, Northeastern University, Boston, Massachusetts, 1991.

[66] K T Ladas and A J Devaney. Generalized ART algorithm for diffraction tomography. Inverse Problems, 7:109, 1991.

[67] K T Ladas and A J Devaney. Iterative methods in geophysical diffraction tomography. Inverse Problems, 8:119-132, 1992.

[68] M Azimi and A C Kak. Distortion in diffraction tomography caused by multiple scattering. IEEE Trans. Med. Imag., MI2:176-195, 1983.

[69] M. Bellanger. Digital Processing of Signals: Theory and practise. John Wiley, New York, 1988.

[70] M Bertero and C De Mol and E R Pike. Linear inverse problems with discrete data II: Stability and regularisation. Inverse problems, 4:573-594, 1988.

[71] M Bianchini and M Gori and M Maggini. On the problem of local minima in recurrent neural networks. IEEE Trans. Neural Network, 5:167-77, 1994.

[72] M H Maleki and A J Devaney and A Schatzberg. Tomographic reconstruction from optical scattered intensities. $J$. Opt. Soc. Am. A, 9:1356-1363, 1992.

[73] M R Luetgen and W C Karl and A S Willsky. Efficient Multiscale Regularization with Applications to Computation of Optical Flow. IEEE Trans. Im. Proc., IP-3:41-64, 1994.

[74] M Slaney and A C Kak and L E Larsen. Limitations of imaging with first-order diffraction tomography. IEEE Trans. Microw. Theory Techn., MTT-32:860-874, 1984.

[75] N Kalouptsidis. Signal Processing Systems: Theory and Design. Wiley, New York, NY, 1978. 
[76] N Sponheim and L-J Gelius and I Johansen and J J Stamnes. Quantitative results in ultrasonic tomography of large objects using line sources and curved detector arrays. IEEE Trans. Ultra., Ferroelec., and Freq. Con., UFFC-38:370, 1991.

[77] N Wiener. The Extrapolation, Interpolation and Smoothing of Stationary Time Series. Wiley, New York, 1949.

[78] P M Morse and H Feshbach. Methods of Theoretical Physics, Parts I,II. McGraw-Hill, New York, 1953.

[79] R B Pratt and M H Worthington. The application of diffraction tomography to cross-hole seismic data. Geophysics, 53:1284, 1988.

[80] R G Newton. Inverse scattering. I. One dimension. J. Math. Phys., 21(3):493, 1980.

[81] R G Newton. Inverse scattering. II. Three dimensions. J. Math. Phys., 21(7):1698, 1980.

[82] R G Newton. Inverse scattering. III. Three dimensions, continued. J. Math. Phys., 22(10):2191, 1981.

[83] R G Newton. Scattering Theory of Waves and Particles. Springer Verlag, Berlin, 1982.

[84] R. K. Mueller and M. Kaveh and G. Wade. Reconstructive tomography and applications to ultrasonics. Proc. IEEE, 67:567, 1979.

[85] R Pierri and A Brancaccio. Imaging of a rotationally symmetric dielectric cylinder by a quadratic approach. J. Opt. Soc. Am. A, 14:2777-2785, 1997.

[86] R. Pierri, G. Rubinacci and A. Tamburrino. A quadratic approach for the reconstruction of conductivity profiles using eddy current. IEEE Trans. Magn., 32:1310-13, 1996.

[87] R T Prosser. Formal solutions of inverse scattering problems. J. Math. Phys., 10:1819-1822, 1969.

[88] R T Prosser. Formal solutions of inverse scattering problems, II. J. Math. Phys., 17:1775-1779, 1976.

[89] R T Prosser. Formal solutions of inverse scattering problems, III. J. Math. Phys., 21:2648-2653, 1980. 
[90] R W Deming and A J Devaney. Diffraction tomography for multi-monostatic ground penetrating radar imaging. Inverse Problems, 13:29-45, 1997.

[91] S Barkeshli and R G Lautzenheizer. An iterative method for inverse scattering problems based on an exact gradient search. Radio Science, 29:1119-1130, 1994.

[92] S Kaczmarz. Angenaehrte Aufloesung von Systemen linea-

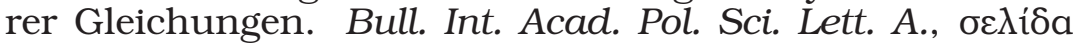
355, 1937.

[93] S. Kawata and Y. Touki and S. Minami. Optical microsco-

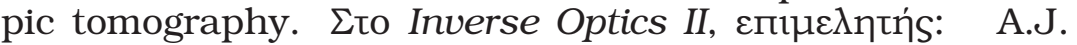
Devaney and R.H.T. Bates. SPIE 558, 1985.

[94] S X Pan and A C Kak. A computational study of reconstruction algorithms for diffraction tomography. IEEE Trans. Acous., Speech, and Signal Process., ASSP-31:1262, 1982.

[95] T B Hansen and P M Johansen. Inversion scheme for ground penetrating radar that takes into account the airsoil interface. IEEE Trans. Geosci. and Remote Sensing, Jan. 2000.

[96] T E Levy and A Witten. Denizens of the Desert. Archaeology

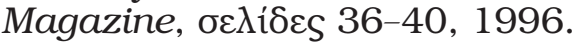

[97] T Isernia and V Pascazio and R Pierri. A non-linear estimation method in tomographic imaging. IEEE Trans. Geosci. Remote Sensing, 35:910-23, 1997.

[98] T. Isernia, G. Leone and R. Pierri. Phase retrieval of radiated fields. Invere Problems, 11:183-203, 1995.

[99] T. Isernia, G. Leone, R. Pierri and F. Soldovieri. On the local minima in phase reconstruction algorithms. Radio Sci., 31:1887-99, 1996.

[100] T Melamed and E Heyman. Spectral analysis of timedomain diffraction tomography. Radio Science, 32:593603, 1997.

[101] T Melamed and E Heyman and L B Felsen. Local spectral analysis of short-pulse excited scattering from weakly inhomogeneous media; Part I: Forward scattering. IEEE Trans. Ant. Prop., AP-47:1208-1217, 1999.

[102] T Melamed and E Heyman and L B Felsen. Local spectral analysis of short-pulse excited scattering from weakly inhomogeneous media; Part II: Inverse scattering. IEEE Trans. Ant. Prop., AP-47:1218-1227, 1999. 
[103] U. Frisch. Wave propagation and scattering in random media. Eтo Probabilistic Methods in Applied Mathematics, вп1-

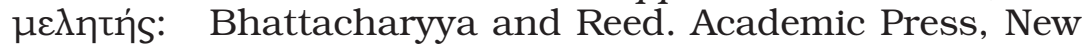
York, 1968.

[104] W C Chew and Y M Wang. Reconstruction of twodimensional permittivity using the distorted Born iterative method. IEEE Trans. Med. Imaging, MI-29:218-225, 1990.

[105] X Pan. Unified reconstruction theory for diffraction tomography, with consideration of noise control. J. Opt. Soc. Am. A, 15:2312-2326, 1998.

[106] Z-Q Lu. JKM perturbation theory, relaxation perturbation theory, and their applications to inverse scattering: Theory and reconstruction algorithms. IEEE Trans. Ultra., Ferroelec., and Freq. Con., UFFC-33:722-730, 1986.

[107] Z-Q Lu and Y-Y Zhang. Acoustical tomography based on the second-order transform perturbation approximation. IEEE Trans. Ultra., Ferroelec., and Freq. Con., UFFC-43:296-302, 1996.

[108] A.H. Zemanian. Distribution Theory and Transform Analysis: An Introduction to Generalized Functions with Application. Dover Publication, New York, 1965. 